UCRL-52863 Rev. 1

Distribution Category UC-38

UCRL- -52863-Rev . 1

DE87 012387

\title{
Conduction Heat Transfer Solutions
}

\author{
James H. VanSant \\ Manuscript date: August 1983
}

\begin{abstract}
DISCLAIMER
This report was prepared as an account of work sponsored by an agency of the United States Government. Neither the United States Government nor any agency thereof, nor any of their emplnyees, makes any wartanly, express or implied, or assomes any legal liability or responsibility for the accuracy, completeness, or usefulness of any information, appiratus, product, or process disclosed, or represents that its use would not infringe privately owned rights. Reference herein to any specific commercial product, process, or service by trade name, trademark, manufacturer, or otherwise does not necessarily constitute or imply its endorsement, recommendation, or favoring by the United States Government or any agency thercof. The views and opinions of authors expressed herein do not necessarily state or reflect those of the United States Government or any agency thereof.
\end{abstract}

\section{LAWRENCE LIVERMORE NATIONAL LABORATORY University of California - Livermore, California - 94550}

Available from: National Technical Information Service - U.S. Department of Commerce 5285 Port Royal Road $\bullet$ Springlield. VA 22161 \$41.50 per copy • (Microliche $\$ 4.50$ ) 
Preface

Nomenclature

Introduction

steady-state solutions

1. Rlane Surface - Steady State

1.1 Solids Bounded by Plane Surfaces . . . . . . . . 1-1

1.2 Solids Bounded by Plane Surfaces

-With Internal Heating

2. Cylindrical surface - steady state

2.1 Solids Bounded by Cylindrical Surfaces

-No Internal Heating

2.2 Solids Bounded by Cylindrical Surfaces

-With Internal Heating

3. Spherical Surface - Steady State

3.1 Solids Bounded by Spherical Surfaces

--No Internal Heating

$1-27$

3.2 Solids Bounded by Spherical Surfaces

-With Internal Heating

4. Traveling Heat Sources

4.1 Traveling Heat Sources . - . . . - . . . . . - 4-1

5. Extended Surface - Steady state

5.1 Extended Surfaces--No Internal lieating . • . . . . 5-1

5.2 Extended Surfaces--With Internal Heating . . . . . 5-31

Transient Solutions

6. Infinite Solids - Transient

6.1 Infinite Solids-No Internal Heating . . . . . . 6-1

6.2 Infinite Solids--With Internal Beating . . . . . . 6-22

7. Semi-Infinite Solids - Transient

7.1 Semi-Infinite Solids--No Internal Heating . . . . . 7-1

7.2 -mi-Infinite Solids--With Irternal Heating . . . . 7-22

8. Plane Surface - Transient

8.1 Solids Bounded by Plane Surfaces -No Internal Heating . . . . . . . . . . . . 8-1

8.2 Solids Bounded by Plane Surfaces -With Internal Heating . . . . . . . . . 8-52 
9. Cylindrical Surface - Transient

9.1 Solids Bounded by Cylindrical Surfaces --No Internal Heating

9.2 Solids Bounded by Cylindrical Surfaces -With Internal Heating

10. Spherical Surface - Transient

10.1 Solids Bounded by Spherical Surfaces --No Internal Heating

10.2 Solids Bounded by Spherical Surfaces -With Internal Heating . . . . . . . . . . . 10-19

11. Change of Phase

11.1 Change of Phase--Plane Interface . . . . . . . . II-I

11.2 Change of Phase--Nonplanar Interface . . . . . . 11-13

12. Traveling Boundaries

12.1 Traveling Boundaries . . . . . . . . . . . . 12-1

Figures and Tables for Solutions . - . . . - . . . . . . - F-l

Miscellaneous Data

13. Mathenatical Functions . . . . . . . . . . . . . 13-1

14. Roots of Scme Characteristic Equations . . . . . . . - 14-1

15. Constants and Conversion Factors . . . . . . . . . . 15-1

16. Convection Coefficients . . . . . . . . . . . . . 16-1

17. Contact Coefficients . . . . . . . . . . . . . . 17-1

18. Thermal properties . . . . . . . . . . . . . . 18-1

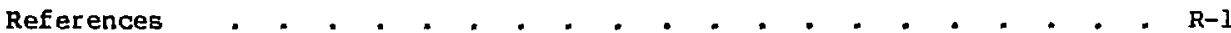


PREFACE

This text is a collection of solutions to a variety of heat conduction problems found in numerous publications, such as textbooks, handbooks, journals, reports, etc. Its purpose is to assemble these solutions into one source that can facilitate the search for a particular problem solution. Generally, it is intended to be a handbook on the subject of heat conduction. Engineers, scientists, technologists, and designers of all disciplines should find this material useful, particularly those who design thermai systems or estimate temperatures and heat transfer rates in structures. More than 500 problem solutions and relevant data are tabulated for easy retrieval. Having this kind of material available can save time and effort in reaching design decisions.

There are twelve sections of solutions which correspond with the class of problems found in each. Geometry, state, boundary conditions, and other categories are used to classify the problems. A case number is assigned to each problem for cross-referencing, and also for future reference. Each problem is concisely described by geometry and condition statements, and many times a descriptive sketch is also included. At least one source reference is given so that the user can review the methods used to derive the solutions. Problem solutions are given in the form of equations, graphs, and tables of data, all of which are also identified by problem case numbers and source references.

The introduction presents a synopsis on the theory, differential equations, and boundary conditions for conduction heat transfer. Some discussion is given on the use and interpretation of solutions. Also, some example problem solutions are included. This material may give the user a review, or even same ingight, on the phenomenology of heat conduction and its applicability to specific problems.

Supplementary data such as mathematical functions, convection correlations, and thermal properties are included for aiding the user in computing numerical values from the solutions. Property data were taken from some of the latest publications relating to the particular properties listed. Only the international system of units (SI) is used. 
Consistency in momenclature and terminology is used throughout, making this text more readable than a collection of different references. Also, dimensionless parameters are frequently used to generalize the applicability of the solutions and to permit easier evaluation of the effects of problem conditions.

Even though some of the equational solutions are lengthy and include several different mathematical functions, this should not pose a formidable task for most users. Modern computers can make complicated calculations easy to perform. Even many electronic calculators can be used to compute complex functions. If, however, these tools are not available, one can resort to hand computing methods. The table of mathematical functions and constants would be useful in that case.

Heat conduction has been studied extensively, and the number of published solutions is large. In fact, there are many solutions that are not included in this text. For example, some solutions are found by a specific computational process that cannot be described briefly. Moreover, new solutions are constantly appearing in technical journals and reports. Nevertheless, this collection contains most of the published solutions.

The differential equations and boundary-condition equations for heat flow are identical in form to those for other phenomena such as electrical fields, fluid flow, and mass diffusion. This similarity gives additional utility to the heat conduction solutions. The user needs only identify equivalence of conditions and terms when selecting a proper solution. This practice is prescribed in many texts on applied mathematics, electrical theory, heat transfer, and mass transfer.

A search for particular solutions has frequently been a tedious and difficult task. Too often, countless hours have been spent in searching for a problem solution. Locating and obtaining a proper reference can require considerable effort. Also, it is frequently necessary to study a theoretical development in order to find the applicable solution. In so doing, there are sometimes misinterpretations which lead to erroneous results. This text should help alleviate some of these problems.

Science gives us information for reaching new frontiers in technology. It is, thus, appropriate to give something back. I hope this text is at least a small contribution. 


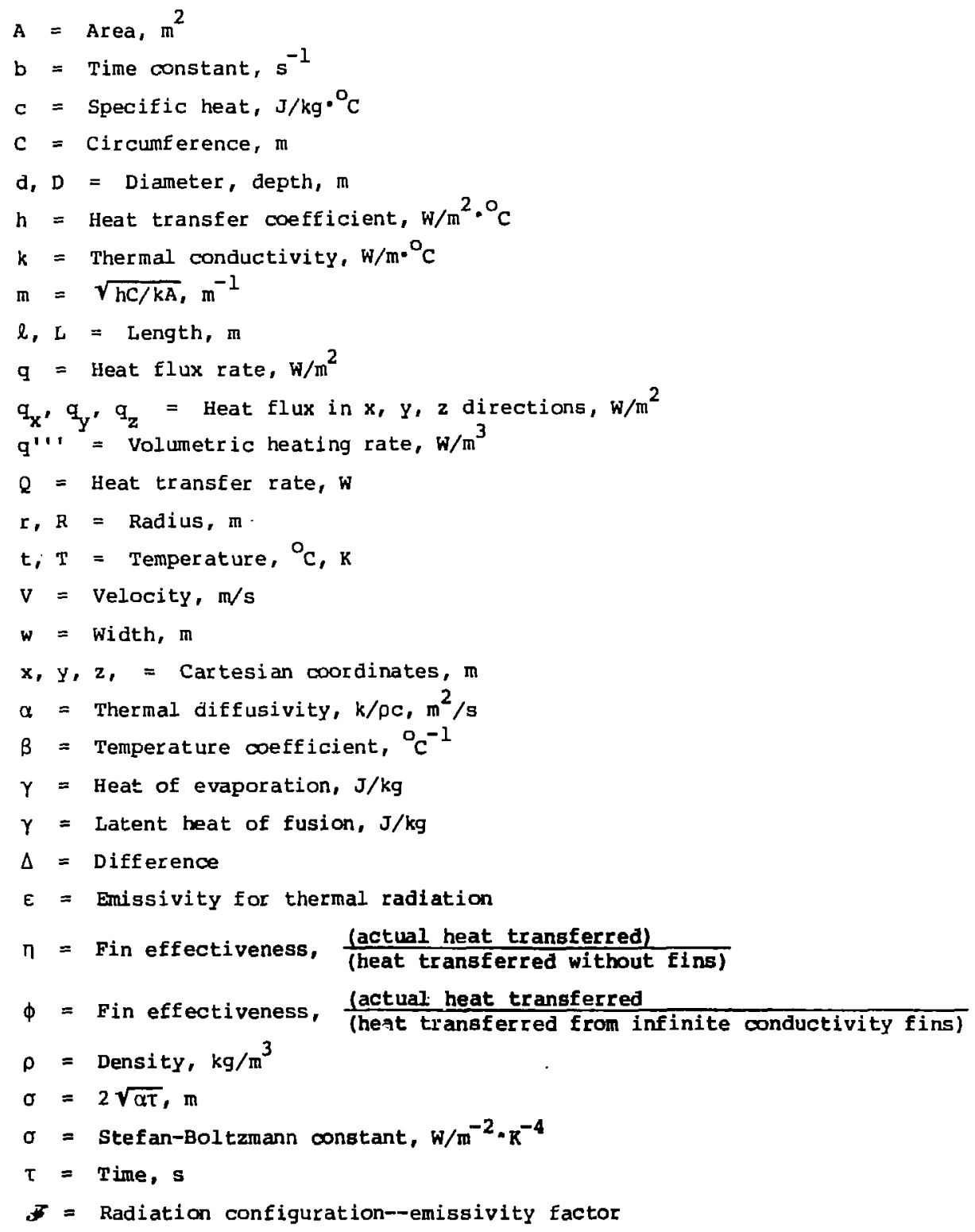


$B i_{\ell}, B i=B i o t$ modulus $=\mathrm{hl} / \mathrm{k}$

$B E_{\ell}, B E=B i F O=\mathrm{ht} / \rho C l$

$\mathrm{FO}_{\ell}$, Fo $=$ Fourier modulus $=\alpha T / \ell^{2}$

Fo $_{\ell}{ }^{*}$ Fo $^{*}=$ Modified Fourier modulus $=1 /(2 \sqrt{\mathrm{FO}})$

$\mathrm{Gr}_{\ell}, \mathrm{Gr}=$ Grashof number $=\mathrm{g} B \Delta \mathrm{t} \ell^{3} / \nu^{2}$

$K i_{\ell}, K i=K i r p i c h e v$ number $=q l / k \Delta t$

$\mathrm{Nu}_{\mathrm{d}}, \mathrm{Nu}=$ Nusselt number $=\mathrm{hd} / \mathrm{k}$

$\mathrm{Pd}_{\ell}, \mathrm{Pd}=$ Predvoditelev modulus $=\mathrm{bl}^{2} / \mathrm{a}$

$\mathrm{PO}_{\ell}$, PO $=$ Pomerantsev modulus $=\mathrm{q}^{\prime} \cdot \mathrm{l}^{2} / \mathrm{k} \Delta \mathrm{t}$

$\operatorname{Pr}=$ Prandtl number $=v / \alpha$

$\mathbf{R}=$ Radius ratio $=r / r_{0}$

$\mathrm{Re}_{\mathrm{d}}$, Re $=$ Reynolds number $=v d / v$

$\mathrm{X}=$ Length ratio $=\mathrm{x} / \mathrm{h}$

$Y=$ Width ratio $=y / W$

$\mathrm{z}=$ Length ratio $=\mathrm{z} / \mathrm{l}$

MATHEMATICAL FUNCTIONS

$\exp =$ Exponential function

$E i=$ Exponential integral

erf $=$ Error function

erfc = Complementary error function

$i^{n}$ erfe $=$ Complementary error function integral

$I_{n}=$ Moäified Bessel function of the first kind

$J_{n}=$ Bessel function of the first kind

$K_{n}=$ Modified Bessel function of the second kind

$\ell_{\mathbf{n}}=$ Natural $\log$

$Y_{n}=$ Bessel function of the second kind

$P_{n}=$ Legendre polynomial of the first kind

$\Gamma=$ Gamma Eunction 
Energy in the form wf heat has been used by man ever since he began walking on this earth. Moreover, the transfer of heat is essential to our very existence. Not only do our own physiological functions require some form of heat transfer, but so do most life-sustaining processes of nature and many mancontrolled activities. The importance of the thermal sciences in the total sphere of science can, thus, hardly be disputed.

Conduction is one of the three principal heat transfer modes, the others being convection and radiation. It is customarily distinguished as being an energy diffusion process in materials which do not contain molecular convection. Kinetic energy is exchanged between molecules resulting in a net transfer between regions of different energy levels, these energy levels are commonly called temperature. Particularly, heat conduction in metals is mainly attributed to the motion of free electrons and in solid electrical insulators to the longitudinal oscillations of atoms. In fluids, the elastic impact of molecules is considered as the heat conduction process.

The process of heat transfer in materials has been studied for many centuries. Even early Greek philosophers, such as Lucretius (c. 98-55 B.C.), meditated on the subject and recorded their conclusions. Much later, the famous mathematical physicist, Joseph B. J. Fourier (1768-1830), developed a mathematical expression that became the basis of practically all heat conduction solutions. He postulated that a local heat flux rate in a material is proportional to the local temperature gradient in the direction of heat flow:

$$
q_{x} \propto \frac{\partial t}{\partial x}
$$

where $q_{x}$ is the heat flow in the $x$-direction per unit area as illustrated in Fig. la. Material properties are accounted for by including a proportionality constant:

$$
q_{y}=-k \frac{\partial t}{\partial x}
$$




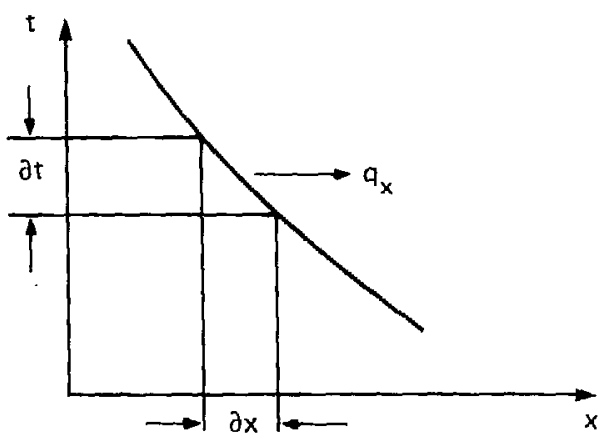

(a)

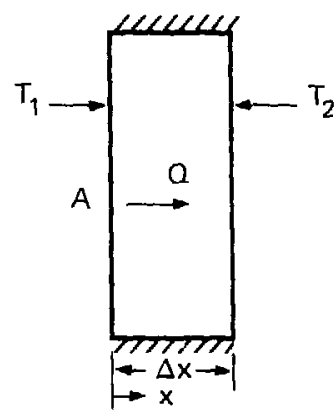

(b)

FIG. 1. Illustration of heat flow and temperature gradient.

where the constant $k$ is called thermal conductivity. (The minus sign must be included to satisfy the second law of thermodynamics.) This equation is called Fourier's law for heterogeneous isotropic continua.

A simpler form of Fourier's law is for homogeneous isotropic continua. For example, consider a plate of this type material having isothermal surfaces and insulated edges as shown in Fig. 1. The plate has a width $\Delta x$, surface area $A$, and thermal conductivity $k$. The heat flow in the plate $i s$ expressed as

$$
Q_{x}=-k A \frac{\left(t_{2}-t_{1}\right)}{\Delta x}
$$

This expression becomes Eg. (2) when $\Delta x$ diminishes to zero.

$$
\frac{Q_{x}}{A}=q_{x}=-k \lim _{\Delta x \rightarrow 0} \frac{\Delta t}{\Delta x}=-k \frac{\partial t}{\partial x} .
$$

The heat flux $q$ is presumed to have both magnitude and direction. Thus, it can be given as a vector, $\bar{q}$ which is normal to an isothermal surface. For example, in Cartesian coordinates

$$
\bar{q}=q_{x} \bar{i}+q_{y} \bar{j}+q_{z} \bar{k}
$$


where $\bar{i}, \bar{j}, \bar{k}$ are unit vectors in tre $x-, y^{-}$, and $z$-directions, respectively. Since Eq. (4) defines $q_{x}=-k \partial t / \partial x$, and similarly $q_{y}=-k \partial t / \partial y, q_{z}=-k \partial t / \partial z$, we can state

$$
\begin{aligned}
& \bar{q}=-k(\bar{i} \partial t / \partial x+\bar{j} \partial t / \partial y+\bar{k} \partial t / \partial z) \\
& \bar{q}=-k \nabla t .
\end{aligned}
$$

In anisotropic continua the direction of the heat flux vector is not necessarily normal to an isothermal surface. Example materials are crystals, laminates, and oriented fiber composites. In suck materials we may assume each component of the heat flux vector to be linearly dependent on all components of the temperature gradient at a point. The vector form of Fourier's law for heterogeneous anisotropic continua becomes

$$
\vec{q}=-k \cdot \nabla t
$$

where $K$ is the conductivity tensor; the components of this tensor are called the conductivity coefficients.

$$
\begin{aligned}
& \text { In Cartesian form, Eq. (8) is } \\
& q_{x}=-\left(k_{11} \frac{\partial t}{\partial x}+k_{12} \frac{\partial t}{\partial y}+k_{13} \frac{\partial t}{\partial z}\right) \\
& q_{y}=-\left(k_{21} \frac{\partial t}{\partial x}+k_{22} \frac{\partial t}{\partial y}+k_{23} \frac{\partial t}{\partial z}\right) \\
& q_{z}=-\left(k_{31} \frac{\partial t}{\partial x}+k_{32} \frac{\partial t}{\partial y}+k_{33} \frac{\partial t}{\partial z}\right) .
\end{aligned}
$$

To compute heat flow by Fourier's law, a thermal conductivity value is needed. It can be estimated from theoretical predictions for some ideal materials, but mostly, it is determined by measurement and Fourier's law, Eq. (2). As illustrated in Fig. 2, thermal conductivity can have a large range, which depends on materials and temperature. For example, copper at $20 \mathrm{~K}$ has a themal conductivity of approximately $1000 \mathrm{w} / \mathrm{m} \cdot \mathrm{K}$ and diatcmaceous earth at $200 \mathrm{~K}$ has a conductivity of $0.05 \mathrm{w} / \mathrm{m}^{\bullet} \mathrm{K}$. Consequently, heat flow in materials can have a very large range, depending on a combined effect of temperature gradient and material property.

Thermal properties of some selected materials are given in Section 18 . 


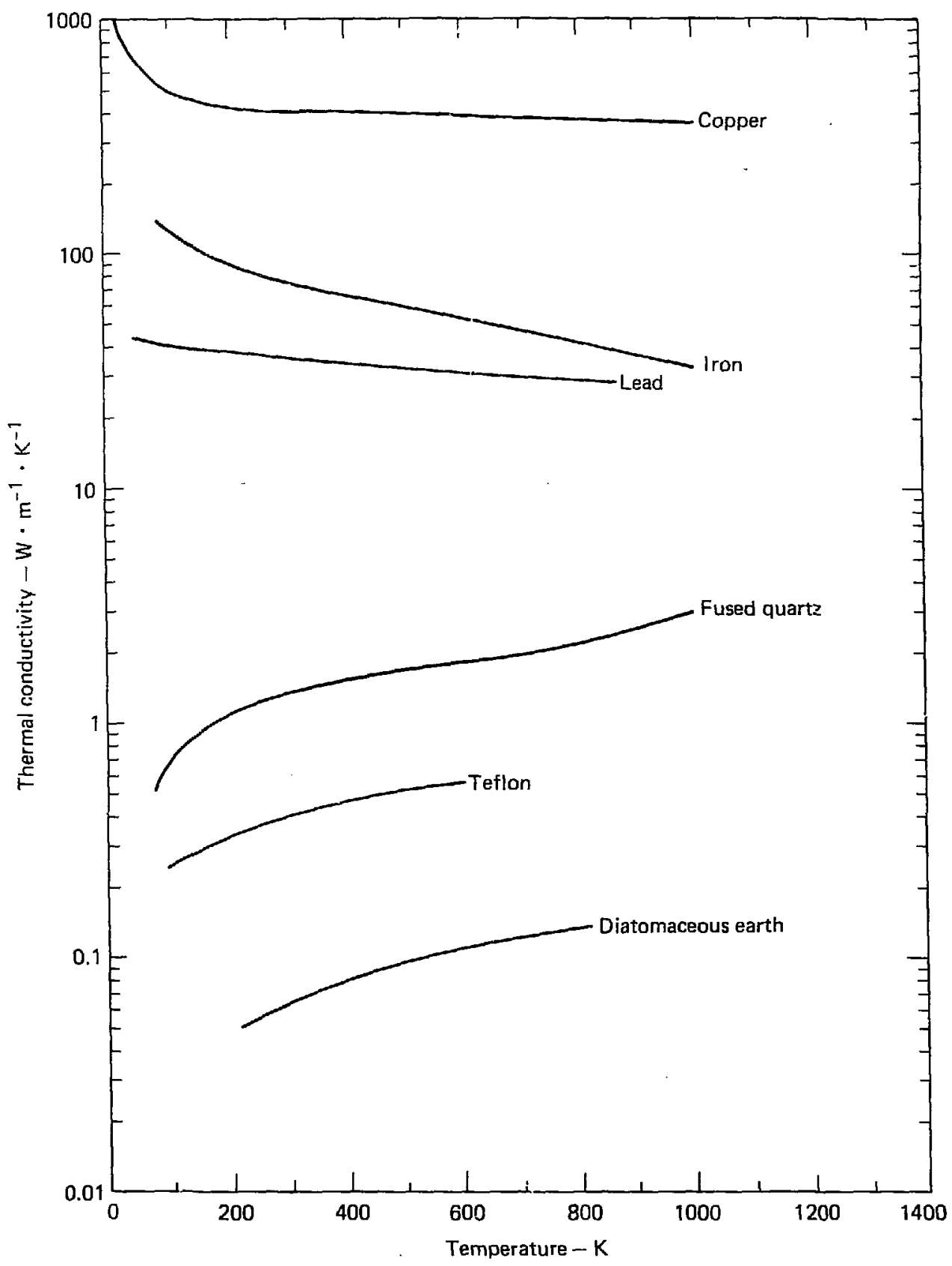

FIG. 2. Thermal conductivity of some selected solids. 
Solutions to heat conduction problems are usually found by some mathematical technique which begins with a differential equation of the temperature field. The appropriate equation should include all energy sources and sinks pertinent to a particular problem. Also, the equation should be expressed in terms of a convenient coordinate system such as rectangular, cylindrical, or spherical. Then analytical or differencing methods can be used to solve for temperature or heat flow.

A common method for deriving the generalized differential equation for heat conduction is to apply the first law of thermodynamics (conservation of energy) to a volume element in a selected coordinate system. By accounting for all the thermal energy transferred through the element faces, the change of internal energy and thermal sources or sinks in the element, and by letting the element dimensions approach zero, the differential equation can be derived. This procedure is typified by the following heat energy accounting of the rectangular solid element shown in Fig. 3 . The net heat flow though its six faces is

$$
Q_{\text {net }}=Q_{x}-Q_{x+\Delta x}+Q_{y}-Q_{y+\Delta y}+Q_{z}-Q_{z+\Delta z}
$$

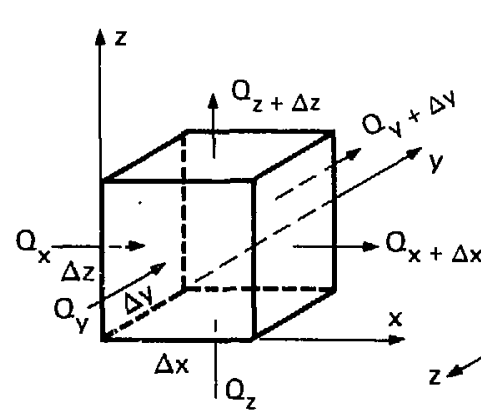

(a)

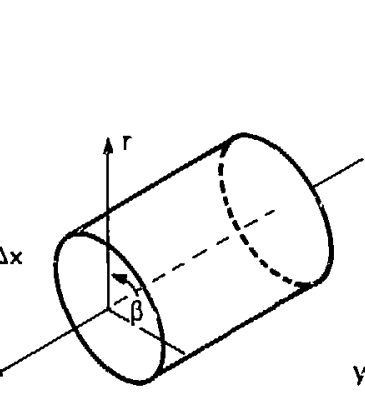

(b)

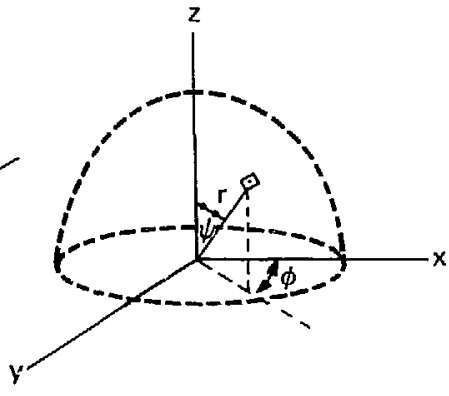

(c)

FIG. 3. Coordinate systems for heat conduction equations: (a) rectangular, (b) cylindrical, (c) spherical. 
where typically

$$
\begin{aligned}
& Q_{x}=-\Delta y \Delta z\left(k_{x} \frac{\partial t}{\partial x}\right)_{x}, \\
& Q_{y}=-\Delta x \Delta z\left(k_{y} \frac{\partial t}{\partial y}\right)_{y}, \\
& Q_{z}=-\Delta x \Delta y\left(k_{z} \frac{\partial t}{\partial z}\right)_{z},
\end{aligned}
$$

and $k_{x}, k_{y}$ and $k_{z}$ are directional conductivities.

An increase in internal energy of the element is cepresented by

$$
\Delta I=\Delta x \Delta y \Delta z \rho c \frac{\partial t}{\partial \tau}
$$

where $t$ is the mean temperature of the element, $\rho$ is the material density, and $c$ is its specific heat.

Internal energy sources can be expressed as

$$
Q^{\prime \prime}=q^{\prime}{ }^{\prime} \Delta x \Delta y \Delta z,
$$

where $q^{\prime \prime \prime}$ is the unit volume source rate. Examples of internal heating in materials are joule, nuclear, or radiation heating. Sumning these energies in accordance with the energy conservation law yields

$\Delta I=Q_{\text {net }}+Q^{\prime \prime \prime}, \quad \rho c \frac{\partial t}{\partial \tau}=\frac{q_{x}-q_{x+\Delta x}}{\partial x}+\frac{q_{y}-q_{q+\Delta y}}{\partial y}+\frac{q_{z}-q_{z+\Delta z}}{\partial z}+q ' \cdots$.

For the limits $\Delta x, \Delta y, \Delta z+0$, we obtain

$$
\rho c \frac{\partial t}{\partial \tau}=q^{\prime \prime}=\frac{\partial q_{x}}{\partial x}-\frac{\partial q_{y}}{\partial y}-\frac{\partial q_{z}}{\partial z}
$$


where

$$
\begin{aligned}
& q_{x}=-k_{x} \frac{\partial t}{\partial x}, \\
& q_{y}=-k_{y} \frac{\partial t}{\partial y}, \\
& q_{z}=-k_{z} \frac{\partial t}{\partial z} .
\end{aligned}
$$

Using Eq. (14) as a general differential equation, we can derive the following specific equations.

\subsection{Rectanqular Coordinate System}

For isotropic heterogeneous media

$\rho c \frac{\partial t}{\partial \tau}=\frac{\partial}{\partial x}\left(k \frac{\partial t}{\partial x}\right)+\frac{\partial}{\partial y}\left(k \frac{\partial t}{\partial y}\right)+\frac{\partial}{\partial z}\left(k \frac{\partial t}{\partial z}\right)+q^{\prime \prime}$.

For isotropic homogeneous media this becomes

$\frac{\partial t}{\partial \tau}=\frac{k}{\rho c}\left[\frac{\partial^{2} t}{\partial x^{2}}+\frac{\partial^{2} t}{\partial y^{2}}+\frac{\partial^{2} t}{\partial z^{2}}\right]+\frac{q^{\prime \prime}}{\rho c}=\alpha \nabla^{2} t+\frac{g^{\prime \prime \prime}}{\rho c}$.

When $q^{\prime \prime}=0$, Eq. (i6) becomes Fourier's equation.

In steady-state conditions, $\partial t / \partial \tau=0$ and $\mathrm{Eq}$. (16) becases the Poisson equation.

When $q^{\prime \prime}=\partial t / \partial \tau=0$, Eq. (16) reduces to the Laplace equation.

Nonisotropic materials, such as laminates, can have directionally sensitive properties. For such materials the conduction differential equation in two dimensions is expressed in the following form:

$$
\begin{aligned}
\rho c \frac{\partial t}{\partial \tau}=\left(k_{\xi} \cos { }^{2} \beta+k_{\eta} \sin ^{2} \beta\right) \frac{\partial^{2} t}{\partial x^{2}} & +\left(k_{\xi} \sin ^{2} B+k_{i, 1} \cos ^{2} \beta\right) \frac{\partial^{2} t}{\partial y^{2}} \\
& +\left(k_{\xi}-k_{\eta}\right)\left(\sin ^{2} B\right) \frac{\partial^{2} t}{\partial x \partial y}+q^{\prime \prime},
\end{aligned}
$$




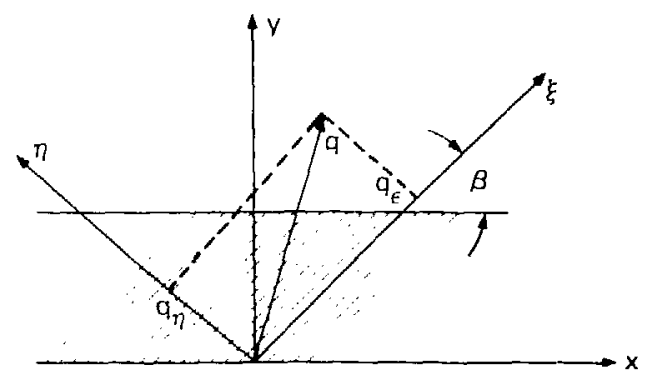

FIG. 4. Coordinate system Eor a nonisotropic medium.

where $k_{\xi}$ and $k_{\eta}$ are directional thermal conductivities, and $\beta$ is the angle of laminations as indicated in Fig. 4. When the geometrical axes of the nonisotropic material are oriented with the principal axes of the thermal conductivities, then Eq. (17) simplifies to the form of Eq. (14)

$$
\rho c \frac{\partial t}{\partial \tau}=k_{x} \frac{\partial^{2} t}{\partial x^{2}}+k_{y} \frac{\partial^{2} t}{\partial y^{2}}+q^{\prime \prime} \cdot
$$

\subsection{Cylizdrical Coordinate System}

Rectangular coordinates can be transformed into cylindrical coordinates by the relations $x=r \cos \theta, y=r \sin \theta$, and $z=z$. The partial differential equations (15) and (16) transformed to cylindrical coordinates are thus

$$
\begin{aligned}
& \nu c \frac{\partial t}{\partial \tau}=\frac{1}{r} \frac{\partial}{\partial r}\left(r k \frac{\partial t}{\partial r}\right)+\frac{1}{r} \frac{\partial}{\partial \theta}\left(k \frac{\partial t}{\partial \theta}\right)+\frac{\partial}{\partial z}\left(k \frac{\partial t}{\partial z}\right)+q^{\prime \prime} \\
& \frac{\partial t}{\partial \tau}=\alpha\left(\frac{\partial^{2} t}{\partial r^{2}}+\frac{1}{r} \frac{\partial t}{\partial r}+\frac{1}{r^{2}} \frac{\partial^{2} t}{\partial \theta^{2}}+\frac{\partial^{2} t}{\partial z^{2}}\right)+\frac{g^{\prime \prime}}{\rho c} .
\end{aligned}
$$

For nonisotropic materials with the conduccivity and geometry axes aligned as in Eq. (18) the differential equation is

$$
\rho c \frac{\partial t}{\partial \tau}=\frac{k_{r}}{\partial r} \frac{\partial}{\partial r}\left(r \frac{\partial t}{\partial r}\right)+\frac{k_{\theta}}{r^{2}} \frac{\partial^{2} t}{\partial \theta^{2}}+k_{z} \frac{\partial^{2} t}{\partial z^{2}}+q^{\prime \prime} \cdot
$$




\subsection{Spherical Coordinate System}

A transformation Erom rectangular to spherical coordinates can be accomplished by substituting the relations $x=r \sin \psi \cos \phi, y=r \sin \psi \sin \phi$ and $z=\cos \psi$ into Eqs. (15) and (16), which yield the partial differential equations for isotropic heterogeneous and homogeneous materials, respectively.

$$
\begin{aligned}
\rho c \frac{\partial t}{\partial \tau}=\frac{1}{r^{2}} \frac{\partial}{\partial r}\left(r^{2} k \frac{\partial t}{\partial r}\right)+\frac{1}{r^{2} \sin ^{2} \psi} \frac{\partial}{\partial \theta}\left(k \frac{\partial t}{\partial \theta}\right) \\
+\frac{1}{r^{2} \sin \psi} \frac{\partial}{\partial \psi}\left(k \sin \psi \frac{\partial t}{\partial \psi}\right)+q^{\prime \prime \prime} \\
\frac{\partial t}{\partial \tau}=\alpha\left(\frac{\partial^{2} t}{\partial r^{2}}+\frac{2}{r} \frac{\partial t}{\partial r}+\frac{1}{r^{2} \sin ^{2} \psi} \frac{\partial^{2} t}{\partial \theta^{2}}+\frac{1}{r^{2} \tan \psi} \frac{\partial t}{\partial \psi}\right)+\frac{q^{\prime \prime \prime}}{\rho c} .
\end{aligned}
$$

The differential equation for nonisotropic materials with aligned conductivity and geometric axes is

$$
\rho c \frac{\partial t}{\partial \tau}=\frac{k_{r}}{r} \frac{\partial^{2} t}{\partial r^{2}}+\frac{k_{\phi}}{r^{2} \sin ^{2} \psi} \frac{\partial^{2} t}{\partial \phi^{2}}+\frac{k_{\psi}}{r^{2} \sin \psi} \frac{\partial}{\partial \psi} \sin \psi \frac{\partial t}{\partial \psi}+q^{\prime} \cdot \cdot
$$

\section{SPECIAL DIFFEREMTIAL EQUATIONS}

Some defining equations can have implied assumptions and boundary conditions. They are usually employed in special cases to simplify the method of solution. However, Fourier's law is the basis for deriving these special equations.

\subsection{Corabined Conduction-Convection}

Thin materials having relatively high thermal conductivity have very small lateral temperature gradients. If the surfaces are convectively heated or cooled, the convection condition becomes part of a heat accounting on a differential element. Referring to Fig. 3 , let $\Delta z$ be the thickness $b$ of $a$ thin solid. On the surface $z$ and $z+\Delta z$, the solid has a convection boundary described by $q=h\left(t-t_{f}\right)$, where $t_{f}$ is the convection fluid temperature. 
Applying the same principles used to develop the general equations for rectangular coordinate systems should result in

$$
\rho c \frac{\partial t}{\partial \tau}+2 \frac{h}{b}\left(t-t_{f}\right)=q^{\prime \prime}+\frac{\partial}{\partial x}\left(k \frac{\partial t}{\partial x}\right)+\frac{\partial}{\partial y}\left(k \frac{\partial t}{\partial y}\right) .
$$

If the geometry is a thin rod of circumference $C$, the appropriate equation is

$$
\rho c \frac{\partial t}{\partial \tau}+\frac{h C}{A}\left(t-t_{E}\right)=q^{\prime \prime}+\frac{\partial}{\partial z}\left(k \frac{\partial t}{\partial z}\right) .
$$

\subsection{Moving heat sources}

The general heat conduction Eq. (15) can also be used for moving heat sources, but a simpler quasi-steady-state equation can be derived by coordinate transformation. If the coordinates are relative to the traveling source, the temperature distributions appear to be stationary. For example, if a point source of strength $Q$ is moving at a velocity $U$ parallel to the $x$-axis, the transformation would be $x=x^{\prime}+U \tau$, where $x^{\prime}$ is the $x$-direction distance from the source. By substitution in Eq. (16) we can obtain

$$
\frac{\partial}{\partial x^{\prime}}\left(k \frac{\partial t}{\partial x^{\prime}}\right)+\frac{\partial}{\partial y}\left(k \frac{\partial t}{\partial y}\right)+\frac{\partial}{\partial z}\left(k \frac{\partial t}{\partial z}\right)+u p c \frac{\partial t}{\partial x^{\prime}}+g^{\prime \prime \prime}=0 .
$$

The applicable equation for a moving source in a thin rod that is convectively cooled is

$$
\frac{\partial}{\partial x^{\prime}}\left(k \frac{\partial t}{\partial x^{\prime}}\right)+U p C \frac{\partial t}{\partial x^{\prime}}+q^{\prime \prime \prime}=\frac{h C}{A}\left(t-t_{f}\right) .
$$

The moving source strength is accounted for in the boundary conditions for a particular problem solution.

\section{BOUNDARY CONDITIONS}

Solutions to heat conduction problems require statements of conditions. For general solutions there must be ;iven at least a definition of the solution region, such as infinite, semi-infinite, quarter-infinite, finite, etc. Additionally, limits can be specified for any of these regions. 
If specific solutions are needed, then complete conditions must be defined. They could include, for example, initial, internal, and surface conditions. Other conditions might include property definitions. Whether deriving a solution or searching for existing solutions, one must decide which conditions are applicable to the problem and how they can be suitably expressed.

\subsection{Initial Condition}

Unsteady-state problems must have an initial condition defined. Mostly, this implies a temperature distribution at $\tau=0$ but, also, internal or surface conditions could have initial values. A problem solution for $\tau>0$ depends, of course, on whatever is specified at $\tau=0$.

\subsection{Surface Conditions}

The most commonly employed surface conditions in heat conduction problems are prescribed surface convection, temperature, heat flux, or radiation. It is even acceptable to prescribe two of these for the same surface, such as combined radiation and convection. other surface conditions could include phase change, ablation, chemical reactions, or mass transfer from a porous solid.

\subsubsection{Convection boundary}

Conduction and convection heat transfer rates on a surface are equated to satisfy continuity of heat flux according to Newton's law:

$$
-k \frac{\partial t\left(x_{b}, \tau\right)}{\partial x}=h\left[t\left(x_{b}, \tau\right)-t_{f}\right] \text {, }
$$

where $t_{f}$ is the temperature of the convection fluid, and $x_{b}$ is the boundary location. The convection coefficient $h$ must be determined fram suitable sources that give predicted values satisfying the conditions of the fluid. (Some correlations of convection coefficients are given in section 16.) The method for defining $h$ can vary depending on the type of convection or the methods prescribed by those researchers who have supplied values for the coefficient. However, $h$ is usually defined as 
TABLE 1. Sample convection coefficient values.

\begin{tabular}{llr}
\hline Fluid & \multicolumn{1}{c}{ Condition } & $\mathrm{h}, \mathrm{W} / \mathrm{m}^{2} \cdot \mathrm{K}$ \\
\hline Air & Free convection on vertical plates & 10 \\
Air & Forced convection on plates & 100 \\
Air & Forced flow in tubes & 200 \\
Steam & Forced flow in tubes & 300 \\
Oil & Forced flow in tubes & 500 \\
Water & Forced flow in tubes & 2,000 \\
Water & Nucleate boiling & 5,000 \\
Liquid helium & Nucleate boiling & 8,000 \\
Steam & Film condensation & 10,000 \\
Liquid metal & Forced flow in tubes & 20,000 \\
Steam & Dropwise condensation & 50,000 \\
Water & Forced convection boiling & 100,000 \\
\hline
\end{tabular}

$$
h=\frac{g}{t\left(x_{b} \cdot \tau\right)-t_{E}},
$$

where $t_{f}$ can be given as

$$
t_{E}=B\left[t\left(x_{b}, \tau\right)-t_{\infty}\right]+t_{\infty},
$$

and where $\beta \leq 1$, and $t_{\infty}$ is the temperature outside the thermal boundary layer of the fluid. In this respect, one must take care to use the proper fluid temperature and convection coefficient.

Some typical order-of-magnitude values for the convection coefficient $h$ are given in Table 1.

\subsubsection{Surface temperature}

Of all boundary conditions, this is probably the simplest in a mathematical sense. It can be variable or constant with respect to position and time. In the real sense, it is very difficult to achieve a prescribed surface temperature, but it can be closely approached by imposing a relatively high convection rate. 


\subsubsection{Heat Elux}

Fourier's law defines the flux on a boundary by

$$
q=-k \frac{\partial t\left(x_{b^{\prime}} \tau\right)}{\partial x}
$$

An adiabatic surface can be defined by either setting $q$ in Eq. (32) or $h$ in Eq. (29) to zero. Inversely, if the solid's temperature distribution has been solved, then Eq. (32) can be used to determine the surface heat flux. Understandably, heat flux can be, in particular cases, time and position dependent.

\subsubsection{Thermal radiation}

Heat transfer from an opaque surface by radiation can be expressed as

$$
\sigma \bar{F}\left[T^{4}\left(x_{b}, \tau\right)-T_{s}^{4}\right]=-k \frac{\partial t\left(x_{b}, \tau\right)}{\partial x},
$$

where $\sigma$ is the stefan-Boltzmann radiation constant, $\$$ is the combined configuration-emissivity factor for muiltiple-surface radiation exchange, and $\mathrm{T}_{\mathbf{S}}$ is the sink or source temperature for radiation. Because $\mathrm{Eq}$. (33) is a nonlinear expression, it is frequently difficult to find exact solutions to problems with this condition.

A common method for dealing with radiation problems is to treat the radiation boundary as a convection boundary. According to Eq. (29) we can write

$$
-k \frac{\partial t\left(x_{b}, \tau\right)}{\partial x}=h_{r}\left[t\left(x_{b}, \tau\right)-t_{s}\right],
$$

where

$$
h_{c}=\sigma\left[T\left(x_{b}, \tau\right)+\tau\right]\left[T^{2}\left(x_{b}, \tau\right)+T_{s}^{2}\right] .
$$

Using this method means that $T\left(x_{b}, \tau\right)$ must First be estimated in order to compute a value of $h_{r}$. After a value for $T\left(x_{b}, \tau\right)$ has been computed from the problem solution, then the estimated value for $h_{r}$ can be improved. This, of course, becomes an iterative process. 


\subsection{Interface Conditions}

\subsubsection{Contact}

Two contacting solids, either similar of dissimilar, will almost always have some interface thermal resistance to heat flow between them. The magnitude of this resistance can depend greatly on the condition of the two contacting surfaces. Properties that can effect the surface condition include cleanliness, roughness, waviness, yield strength, contact pressure, and the thermal conductivities of the solids and interstitual fluid. Since there are so many influences on the contact thermal, resistance, it is difficult to theoretically predict its value. Consequently, experimental results are frequently used. Same representative values of the inverse thermal contact resistance, commonly referred to as the thermal contact coefficient, are given in Section 17.

The generally accepted definition of the contact coefficient is

$$
h_{c}=\frac{q}{\Delta t}
$$

where $q$ is the steady heat flux corresponding to a fictitious interface temperature drop of $\Delta t_{i}$ defined by extrapolating the virtual linear temperature gradient in each solid to the contact centerline. This temperature drop, which is illustrated in Fig. 5, would diminish to zero if the interfaces were in perfect contact.

\subsubsection{Phase change}

Other interface conditions include those caused by endothermic reactions such as melting, solidification, sublimation, vaporization, and chemical dissuciation.

A statement of an interface reaction condition defines the difference of heat flux across the interface. If the interface which separates two phases of a material is located at $x=x_{i}$, the heat balance for a phase change reaction is given in the form

$$
k_{2} \frac{\partial t_{2}\left(x_{i}\right)}{\partial x}-k_{1} \frac{\partial t_{1}\left(x_{i}\right)}{\partial x}=\gamma p_{2} \frac{d x_{i}}{d \tau} .
$$




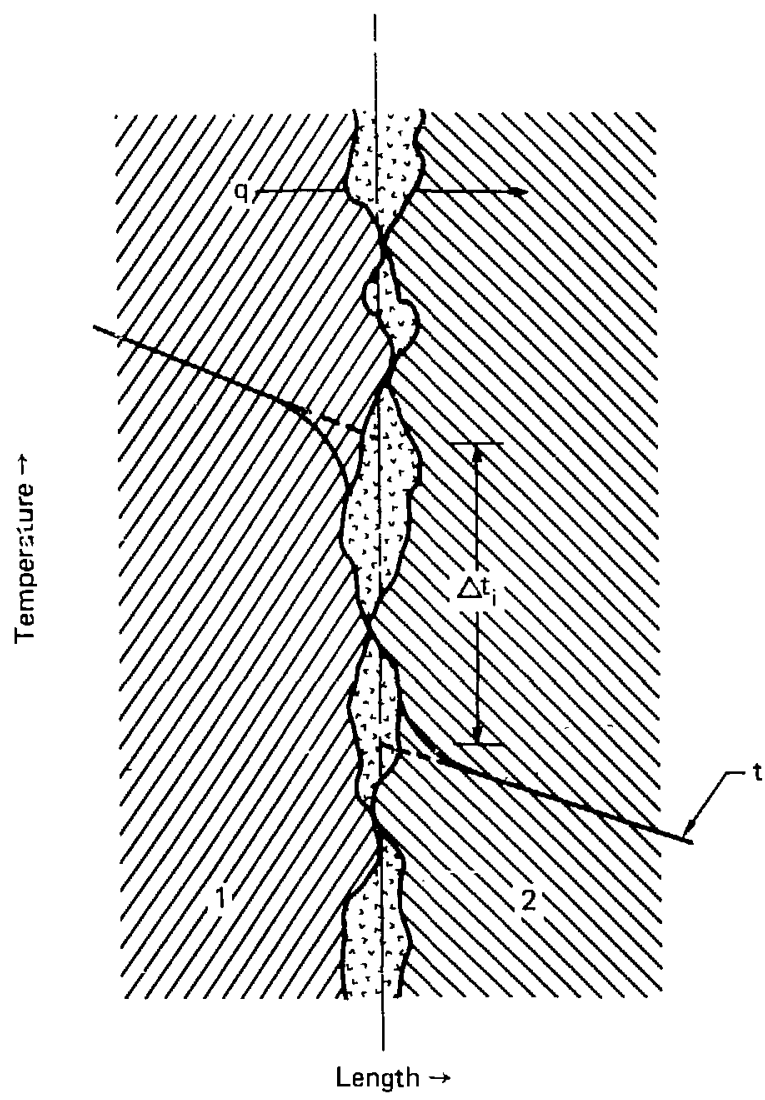

FIG. 5. Illustration of interface contact between solids.

where $Y$ is the latent heat or chemical heat capacity, and subscripts 1 and 2 refer to the two phases.

\subsection{Solutions}

\subsection{Extending solutions}

A solution can be retrieved after identifying a problem by boundary conditions, geometry, and other pertinent data. Usually, a temperature solution is given, but heat flow can be derived from the temperature 
distribution by using Fourier's law, i.e. Eq. (2). If cumulative heat flow is required, a time and surface integration of local heat flux is necessary.

$$
Q=\int_{s} \int_{0}^{\tau} \frac{\partial t\left(s_{l} \tau\right)}{\partial n} d \tau d s,
$$

where $\mathrm{n}$ is the direction normal to the surface $\mathrm{s}$.

Steady-state solutions can be considered as the infinite-time condition for unsteady-state solutions. That is, problems which have a time-asymptotic solution exhibit steady-state solutions for $\tau+\infty$. Thus, steady-state solutions can be derived from transient solutions.

A steady surface temperature condition can be implied from a convection boundary condition. For $h \rightarrow \infty$, the surface temperature approaches the fluid temperature. Therefore, a solution which includes a convection boundary can be transformed into a constant temperature boundary solution by solving for the implied limiting case.

\subsection{Dimensionless parameters}

Grouping particular variables yields dimensionless numbers that can be useful. Symbolically, they can shorten an equational expression. But, they can also give insight to the behavior of heat transfier in a particular problem.

One very useful parameter is the Biot number, $B i=h l / k$, which results from convection boundary conditions. This parameter is proportional to the ratio of the conduction resistance to the convection resistance. Thus, we could say that for

$B i>1$, conduction is highest resistance to heat transfer,

$\mathrm{Bi}<1$, convection is aighest resistance to heat transfer,

$B i \ll 1$, the solid behaves like $k \simeq \infty$.

Another dimensionless parameter is the Fourier number, Fo $=\alpha \tau, l^{2}$, which is found in cransient solutions. This number is a dimensionless time value, but it is also considered an indicator of the degree of thermal penetration into a solid. Since $\alpha \tau / l^{2}=(k \tau \Delta t / l) /(\rho c \ell \Delta t)$, it is proportional to the ratio of condurtion heat transferred to thernial capacity. Thus, an increasing Fo value impiies approaching thermal equilibrium. 
The product of B1 and Fo numbers ylelds the parameter Bf $=\mathrm{hT} / \mathrm{pcl}$ which occurs in transient problens having a convection boundary. This is also a dimensionless time parameter, but it is based on convection heat transfer instead of conduction as in the Fourier number.

Solutions to problens having an internal heat source g'' usually have a dimensionless heating parameter called the Pomerantsev modulus Po $=q^{\prime \prime} \cdot l^{2} / k \Delta t$. This number is a ratlo of internal heating to heat conduction rates. Large values of Po imply large temperature dirferences will occur in the solid.

The parameter $\mathrm{FO}^{\star}=\ell / 2 \sqrt{\alpha \tau}$ is a form of the reciprocal of the Fourier number and occurs in many solutions for transient temperatures in semi-infinite solids.

Wher time dependent boundary conditions have a time constant, the solution will frequently include a dimensionless group called the Predvoditelev modulus, $\mathrm{Pd}=\mathrm{b} \mathrm{l}^{2} / \mathrm{d}$, where $\mathrm{b}$ is the inverse time constant. Small values of Pd imply a slow changing condition. It signifies the ratio of the change rate of the boundary condition to the change rate of the solid temperature.

\subsection{Example Problems}

\subsubsection{Steady heat-transfer in a pipe wall}

Hot water flows at $0.5 \mathrm{~m} / \mathrm{s}$ in a $2.5 \mathrm{~cm} \mathrm{i.d.,} 2.66 \mathrm{~cm} 0 . d$. smooth copper pipe. The pipe is horizontal in still air and covered with a 1-cm layer of polystyrene foam insulation. For a $65^{\circ} \mathrm{C}$ water temperature and $20^{\circ} \mathrm{C}$ air temperature, estimate the heat loss rate per unit length. The solution given in case 2.1.2 is

$$
q=\frac{2 \pi\left(t_{1}-t_{2}\right)}{\frac{1}{k_{1}} \ln \frac{r_{2}}{r_{1}}+\frac{1}{k_{2}} \ln \frac{r_{3}}{r_{2}}+\frac{1}{r_{1} h_{1}}+\frac{1}{r_{3} h_{3}}} .
$$


From the problem description

$t_{1}=E 5^{\circ} \mathrm{C}$

$\mathrm{t}_{4}=20^{\circ} \mathrm{C}$

$r_{1}=1.25 \mathrm{~cm}$

$\mathrm{r}_{2}=1.33 \mathrm{~cm}$

$r_{3}=2.33 \mathrm{~cm}$

$k_{1}=400 \mathrm{w} / \mathrm{m}^{\circ}{ }^{\circ} \mathrm{C}$ (from Table 18.1 )

$k_{2}=0.038 \mathrm{~W} / \mathrm{m}^{\circ}{ }^{\circ} \mathrm{C}$ (from Table 18.2)

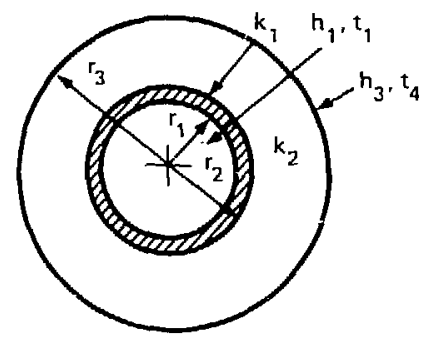

$\mathrm{ha} / \mathrm{k}=0.0155 \mathrm{Pr}^{0.5} \mathrm{Re}^{0.9}$ (f zom sect. 16.1)

$h_{1}=\left(k_{\text {water }} / 2 r_{1}\right)\left(0.0155 \mathrm{Pr}^{0.5} \mathrm{Re}^{0.9}\right)$

$k_{\text {water }}=0.659 \mathrm{~W} / \mathrm{m}^{\circ}{ }^{\circ} \mathrm{C}$ (at $65^{\circ} \mathrm{C}$ )

$\mathrm{Pr}=2.73$

$\operatorname{Re}=2 \rho \vee r l / \mu$

$\rho=980 \mathrm{~kg} / \mathrm{m}^{3}$

$\mathrm{v}=0.5 \mathrm{~m} / \mathrm{s}$

$\mu=4.3 \times 10^{-4} \mathrm{~kg} / \mathrm{m} \cdot \mathrm{s}$

$\operatorname{Re}=\frac{2(980)(0.5)(0.0125)}{4.3 \times 10^{-4}}=28488$

$h_{1}=(0.659 / 0.025)(0.0155)(2.73)^{0.5}(28488)^{0.9}=6895 \mathrm{~W} /\left(\mathrm{m}^{2}-{ }^{\circ} \mathrm{C}\right)$

$\mathrm{h}_{3}=\left(\mathrm{k}_{\mathrm{air}} / 2 \mathrm{r}_{3}\right) \mathrm{c}\left(\mathrm{Gr}_{\mathrm{d}^{\mathrm{Pr}}}\right)^{\mathrm{m}}$ (from Sect. 16.8)

$k_{\text {air }}=0.025 \mathrm{w} / \mathrm{m}^{\circ}{ }^{\circ} \mathrm{C}$

Pr $=0.71$

$\mathrm{Gr}=g \beta\left(t_{4}-t_{3}\right)\left(2 r_{3}\right)^{3} / \nu^{2}$

$g=9.8 \mathrm{in} / \mathrm{s}$

$B=1 / T_{4}=1 / 293 \mathrm{~K}^{-1}$

$v=16.55 \times 10^{-6} \mathrm{~m}^{2} / \mathrm{s}$

$\mathrm{Gr}_{d^{\mathrm{Pr}}}=\frac{(9.8)(0.0466)^{3}(0.71)}{(293)\left(16.55 \times 10^{-6}\right)^{2}}=8774,\left(t_{4}-t_{3}=1{ }^{\circ} \mathrm{C}\right)$

$\mathrm{C}=1.14, \mathrm{~m}=1 / 7$ (from Table 16.3)

$h_{3}=(0.025 / 0.0466)(1.14)(8774)^{1 / 7}=2.24 \mathrm{~W} / \mathrm{m}^{2} \cdot{ }^{\circ} \mathrm{C}$

$$
\begin{aligned}
q= & \frac{2 \pi(65-20)}{\frac{1}{400} \ln \left(\frac{1.33}{1.25}\right)+\frac{1}{0.038} \ln \left(\frac{2.33}{1.33}\right)+\frac{1}{(0.0125)(6895)}+\frac{1}{(0.233)} \frac{1}{(2.24)}} \\
& =\frac{2 \pi(45)}{1.55 \times 10^{-4}+14.76+0.022+19.16}=8.33 \mathrm{w} / \mathrm{m}
\end{aligned}
$$




$$
t_{3}-t_{4}=\frac{q}{2 \pi r_{3} h_{3}}=\frac{8.33}{2 \pi(0.0233)(2.24)}=25.4^{\circ} \mathrm{C} \text {. }
$$

Using this new estimate of $\left(t_{4}-t_{3}\right)$, we can recalculate $h_{3}$.

$$
\begin{aligned}
& \mathrm{h}_{3}=2.24(25.4)^{1 / 7}=3.56 \mathrm{~W} / \mathrm{m}^{2} \cdot{ }^{\circ} \mathrm{C}, \\
& \mathrm{q}=10.54 \mathrm{~W} / \mathrm{m} .
\end{aligned}
$$

Additional iterations on $h_{3}$ would little improve this result.

Note that the copper tube and water film have a small effect on the results because they present little resistance to heat transfer by comparison to the insulation and air film.

\subsubsection{Transient heat conduction in a slab}

A billet of 304 stainless steel measuring $2 \times 2 \times 0.1 \mathrm{~m}$ thick and having a uniform temperature of $30^{\circ} \mathrm{C}$ is heated by sudden immersion into a $450^{\circ} \mathrm{C}$ molten salt bath. The mean convection coefficient is $350 \mathrm{w} / \mathrm{m}^{2} \cdot{ }^{\circ} \mathrm{C}$. Determine the time required for the center temperature of the billet to reach $400^{\circ} \mathrm{C}$. The solution is found in Section 8.1 (case 8.1 .7 and Fig. 8.4a):

$\frac{t-t_{F}}{t_{i}-t_{F}}=\frac{400-450}{30-450}=0.119$.

From Table 18.1

$k=21 \mathrm{w} / \pi 1^{\circ} \mathrm{C}$,

$\alpha=7 \times 10^{-6} \mathrm{~m}^{2} / \mathrm{s}$,

$\frac{k}{h l}=\frac{21}{(350)(0.05)}=1.2$.

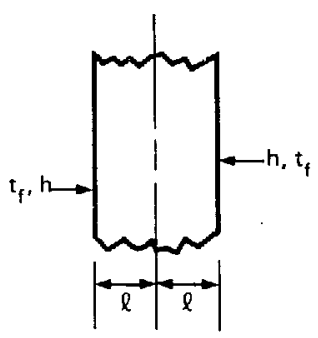

From Fig. $8.4 \mathrm{a}$

$\alpha \tau / \ell^{2}=3.4$,

$\tau=\frac{3.4 \ell^{2}}{\alpha}=\frac{(3.4)(0.05)^{2}}{7 \times 10^{-6}}=1214 \mathrm{~s}$. 


\subsubsection{Transient heat conduction in a semi-infinite plate}

For the conditions given in 5.3.2, find the billet center temperature at $0.05 \mathrm{~m}$ from the edges and sides of the billet.

The solution is found in case 7.1 .21 and Fig. 9.4a for a semi-infinite plate and expressed as $T=(P)(F o) \cdot S(X)$. Values of $P(F o)$ are given in $F i g .8 .4$ and values of $S(X)$ are given in Fig. 7.2.

$$
\begin{aligned}
& \mathrm{Bi} \sqrt{\mathrm{Fo}}=\frac{\mathrm{h} \sqrt{\alpha \tau}}{\mathrm{k}}=\frac{350 \sqrt{\left(7 \times 10^{-6}\right)(1214)}}{21}=1.54 \\
& \mathrm{Fo}_{\mathrm{x}}^{*}=\frac{\ell}{2 \sqrt{\alpha \tau}}=\frac{0.05}{2 \sqrt{\left(7 \times 10^{-6}\right)(1214)}}=0.27
\end{aligned}
$$

If $\left(T=\left(t-t_{f}\right) /\left(t_{i}-t_{f}\right)\right.$,

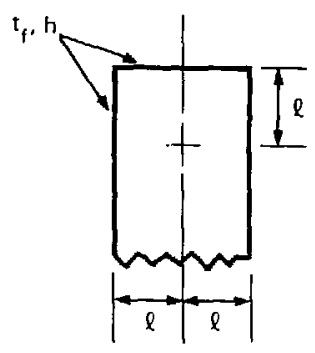

$$
S(X)=1-0.45=0.55 \text { (from Fig. 7.2). }
$$

The $P(F o)$ value is given in 5.3.2. Thus, the solution is

$$
\begin{aligned}
& \frac{t-t_{E}}{t_{i}-t_{f}}=P(F O) S(X)=(0.119)(0.55)=0.066 \\
& t=(0.066)(30-450)+450=423^{\circ} \mathrm{C}
\end{aligned}
$$

\subsubsection{Exterided surface steady-state heat transfer}

A $160{ }^{\circ} \mathrm{C}$ uniform-temperature copper plate has a long rectangular rib brazed to it. All surfaces are convectively cooled by $30^{\circ} \mathrm{C}$ air having a convection coefficient of $53 \mathrm{w} /\left(\mathrm{m}^{2} \cdot{ }^{\circ} \mathrm{C}\right)$. The $\mathrm{r}$ ib is yellow brass extending $4 \mathrm{~cm}$ from the flat surface and $2 \mathrm{~cm}$ wide. Estimate the additional heat loss from the flat surface caused by the rib.

The solution for temperature distribution in the $r i b$ is given in Case 1.1 .17 for $f(x)=t_{i}, w=b$ and $\ell=a$.

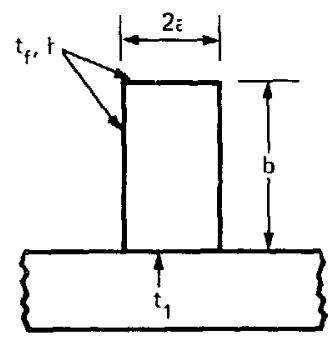




$$
\begin{aligned}
& \frac{t-t_{f}}{t_{1}-t_{f}}=2 \sum_{n=1}^{\infty} \frac{B i \cos \left(\lambda_{n} X\right)\left\{\lambda_{n} \cosh \left[\lambda_{n}(B-y)\right]+B i \sinh \left[\lambda_{n}(B-y)\right]\right\}}{\cos \left(\lambda_{n}\right)\left(B i^{2}+\lambda_{n}^{2}+B i\right)\left[\lambda_{n} \cosh \left(\lambda_{n} B\right)+B i \sinh \left(\lambda_{n} B\right)\right]} \\
& \lambda_{n} \tan \left(\lambda_{n}\right)=B i \quad \text { (characteristic equation) } \\
& B=b / a=4 / 1=4 \\
& x=x / a, Y=y / a \\
& B i=\frac{h a}{k}=\frac{(53)(0.01)}{130}=0.004 \quad \text { (k value from Table 18.1) }
\end{aligned}
$$

From Table 24.1

$$
\lambda_{1}=0.0632, \lambda_{2}=3.1429, \lambda_{3}=6.2838, \lambda_{4}=9.4252, \lambda_{5}=12.5667 .
$$

Heat loss form the convectively cooled surfaces is determined by Eq. 32 applied to the boundary $\mathrm{y}=0$.

$$
\begin{aligned}
q & =2 k \int_{0}^{a} \frac{\partial t(x, 0)}{\partial y} d x \\
& =4 k\left(t_{i}-t_{f}\right) \sum_{n=1}^{\infty} \frac{B i \tan \left(\lambda_{n}\right)\left(\lambda_{n} \sinh \left(\lambda_{n} B\right)+B i \cosh \left(\lambda_{n} B\right)\right]}{\left(\lambda_{n}^{2}+B i\right)\left[\lambda_{n} \cosh \left(\lambda_{n} B\right)+B i \sinh \left(\lambda_{n} B\right)\right]} \\
& =666 \mathrm{~W} / \mathrm{m}
\end{aligned}
$$

Heat loss without the rib attached would be

$$
q=h A\left(t_{1}-t_{f}\right)=53(0.02)(160-30)=138 \mathrm{w} / \mathrm{m} \text {. }
$$

The additional heat loss is thus

$$
\Delta q=666-138=\underline{528 \mathrm{~W} / \mathrm{m}} \text {. }
$$

\subsubsection{Rectangular fin heat transfer}

Use the straight rectangular fin solution to estimate heat loss from the rib described in 5.3.4.

The solution is found in Case 5.1 .4 and Fig. 5.2.

$$
\begin{aligned}
& \mathrm{m}=\sqrt{\frac{h}{k a}}=\sqrt{\frac{53}{(130)(0.01)}}=6.385 \mathrm{~m}^{-1} \\
& l_{c}=0.04+0.01=0.05 \mathrm{~m} \\
& m \ell_{c}=0.3193
\end{aligned}
$$




\subsubsection{Semi-infinite plate heat transfer}

Find an equation for the heat transfer rate through the edge of the semiinfinite plate described in case 1.1 .5 with $f(x)=t_{2}$.

Using the given temperature solution and Egs. (2) and (37) we can find the heat transfer in the following manner:

$$
\begin{aligned}
& q_{Y}=-k \frac{\partial t}{\partial Y}=-\frac{k}{l}\left(t_{2}-t_{1}\right) \frac{\partial T}{\partial Y} \text {, where } T=\frac{t-t_{1}}{t_{2}-t} \\
& \left.\frac{\partial T}{\partial Y}\right|_{Y}=0=-2 \sum_{n=1}^{\infty} \sin (n \pi x)[1-\cos (n \pi)] \\
& Q_{Y} \mid Y=0 \\
& =l \int_{0}^{1} q_{Y} \mid Y=0 d x=2 k\left(t_{2}-t_{1}\right) \int_{0}^{1} \sum_{n=1}^{\infty} \sin (n \pi X)[1-\cos (n \pi)] d x \\
& =2 k\left(t_{2}-t_{1}\right) \sum_{n=1}^{\infty} \frac{[1-\cos (n \pi)]^{2}}{n \pi}=\frac{g}{\pi} k\left(t_{2}-t_{1}\right) \sum_{n=1}^{\infty} \frac{1}{n}, n=1,3,5, \ldots
\end{aligned}
$$


Section 1.1. Solids Bounded by Plane Surfaces-No Internal Heating.

$\begin{array}{lll}\text { Case No. References Solution } & \text { Destiption }\end{array}$

\begin{tabular}{lll}
\hline 1.1 .1 & $\begin{array}{l}\text { Convectively heated and } \\
\text { p. 3-103 cooled plate. }\end{array}$ & $q=\frac{h_{1}\left(t_{l}-t_{2}\right)}{B i_{1}+1+\left(h_{1} / h_{2}\right)}$ \\
$\frac{t-t_{1}}{t_{2}-t_{1}}=\frac{B i_{1}+1}{B i_{1}+1+\left(h_{1} / h_{2}\right)}$
\end{tabular}

$\begin{array}{lll}\text { I } & 1.1 .2 & 19, \\ & & \text { p. } 3-103\end{array}$

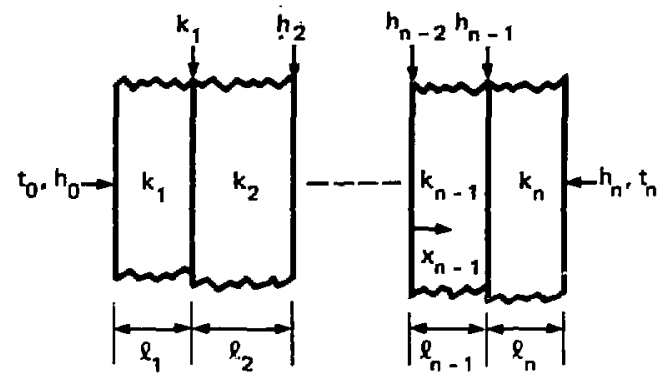

$q=\frac{\left(t_{0}-t_{n}\right)}{\sum_{i=1}^{n}\left(w_{i} / k_{i}+1 / h_{i}\right)+1 / h_{0}}$

Temp in the jth layer:

$$
\frac{t_{j}-t_{0}}{t_{n}-t_{0}}=\frac{\sum_{i=1}^{j-1}\left(l_{i} / k_{i}+1 / h_{i}\right)+\left(x_{j} / k_{j}\right)+\left(1 / h_{0}\right)}{\sum_{i=1}^{n}\left(l_{i} / k_{i}+1 / h_{i}\right)+\left(1 / h_{0}\right)}, j>1
$$


Section 1.1. Solids Bounded by Plane Surfaces--No Internal Heating.

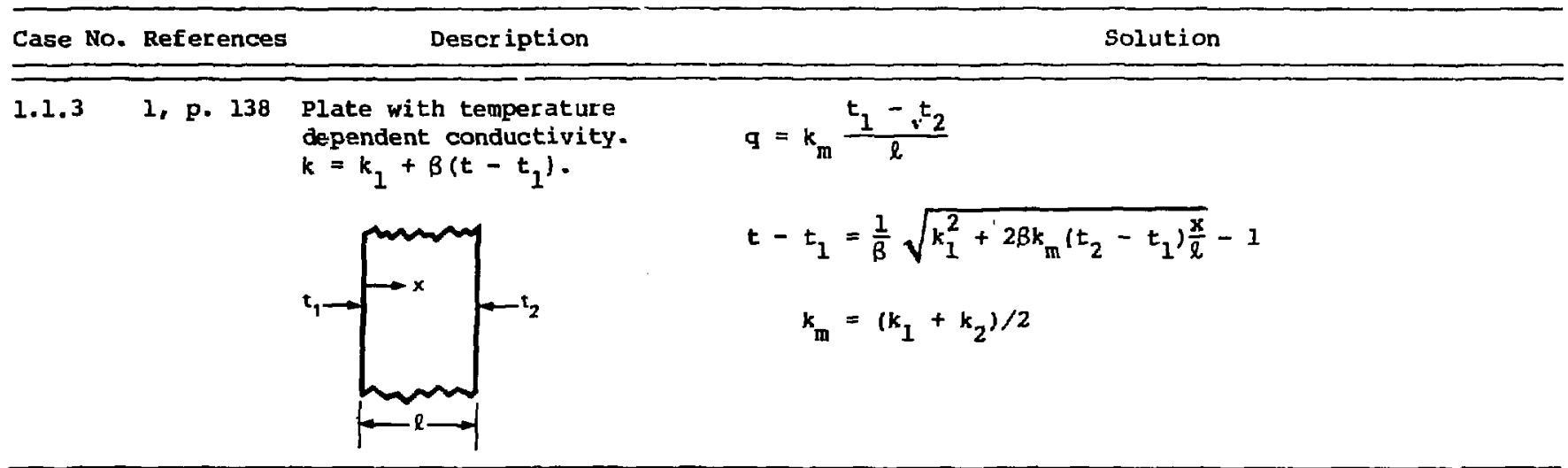

$\stackrel{\leftrightarrow}{1.1 .4}$ 2, p. 221 Porous plate with internal fluid flow.

$t=t_{1}, x=0$.

$\frac{t-t_{0}}{t_{2}-t_{0}}=\exp \left[-\xi_{p} \delta(1-x / \delta)\right], 0 \leq x \leq \delta$

$t=t_{2}, x=\delta$.

$P=$ Porosity.

$\frac{t-t_{0}}{t_{2}-t_{1}}=\frac{\exp \left(\xi_{E} x\right)}{\exp \left[\xi_{p} \delta-1\right]},-\infty \leq x \leq 0$

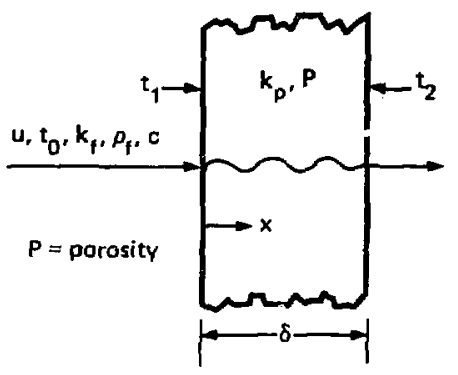

Mean temp:

$\begin{aligned} \frac{t_{m}-t_{0}}{t_{2}-t_{0}} & =\frac{1}{\xi_{p} \delta}\left[1-\exp \left(-\xi_{p} \delta\right)\right], 0 \leq x \leq \delta \\ \xi_{P} & =\frac{\rho_{f} U C}{k_{E}(1-P)}, \xi_{f}=\frac{\rho_{f} U C}{k_{E}(1-P)}\end{aligned}$

(See Fig. 1.1) 
Section 1.1. Solids Bounded by Plane Surfaces--No Internal Heating.

\begin{tabular}{lll}
\hline Case No. References & Description Solution
\end{tabular}

1.1.5 2, p. 122 Semi-infinite plate.

9, p. $164 t=t_{1}, x=0, l, y \geq 0$.

$t=f(x), 0>x>l, y=0$.

$t-t_{1}=2 \sum_{n=1}^{\infty} \exp (-n \pi x) \sin (n \pi x)$

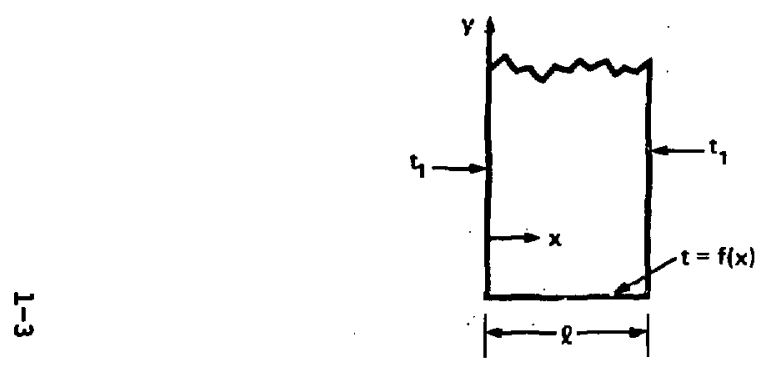

$$
x \int_{0}^{1}\left[f(x)-t_{i}\right] \sin (n \pi x) d x
$$

For $f(x)=t_{2}$

$\frac{t-t_{1}}{t_{2}-t_{1}}=\frac{2}{\pi} \sum_{n=1}^{\infty} \frac{1}{n} \exp (-n \pi Y) \sin (n \pi X)[1-\cos (n \pi)]$

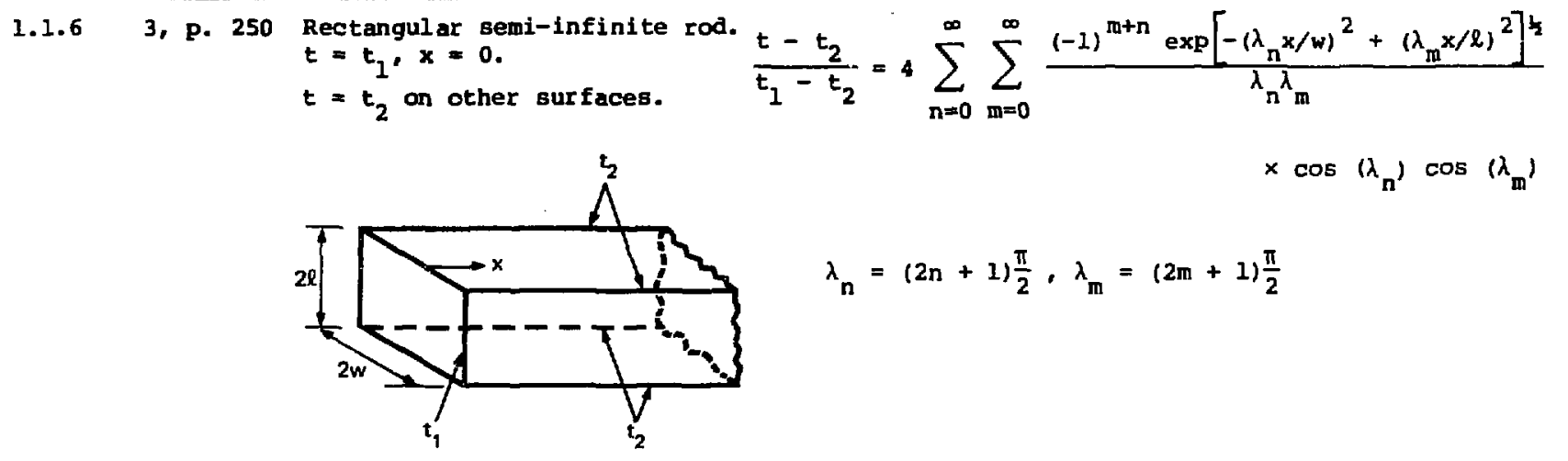


Section 1.1. Solids Bounded by Plane Surfaces--No Internal Heating.

\section{Case No. References}

Description

Solution

\begin{tabular}{|c|c|c|c|}
\hline 1.1 .7 & 2, p. 130 & $\begin{array}{l}\text { Rectangular infinite rod. } \\
t=F_{1}(x), 0<x<\ell, y=0 . \\
t=F_{2}(x), 0<x<\ell, y=w . \\
t=G_{1}(x), x=0,0<y<w . \\
t=G_{2}(x), x=\ell, 0<y<w .\end{array}$ & $\begin{array}{l}t=t_{I}+t_{I I}+t_{I I I}+t_{I V} \\
t_{I}=2 \sum_{n=1}^{\infty} \frac{\sinh (n \pi Y / L)}{\sinh (n \pi / L)} \sin (n \pi X L) \int_{0}^{1} F_{I}(X) \sin (n \pi X) d X \\
t_{I I}=2 \sum_{n=1}^{\infty} \frac{\sinh \left[\frac{n \pi}{L}(I-Y)\right]}{\sinh (n \pi / L)} \sin (n \pi X L) \int_{0}^{I} F_{2}(X) \sin (n \pi X) d X \\
t_{I I I}=2 \sum_{n=1}^{\infty} \frac{\sinh (n \pi Y / L)}{\sinh (n \pi / L)} \sin (n \pi Y) \int_{0}^{I} G_{1}(Y) \sin (n \pi Y) d Y \\
t_{I V}=2 \sum_{n=1}^{\infty} \frac{\sinh [n \pi L(I-X)]}{\sinh (n \pi L)} \sin (n \pi Y) \int_{0}^{I} G_{2}(Y) \sin (n \pi Y) d Y \\
\frac{t-t_{1}}{t_{2}-t_{1}}=\frac{4 / w}{\pi} \sum_{n=1}^{\infty} \frac{\cosh \left[\frac{n \pi}{L}\left(\frac{1}{2}-Y\right)\right] \sin (n \pi X),}{n \cosh \left(\frac{\pi}{2 L}\right)}\end{array}$ \\
\hline
\end{tabular}

$t=1(x), 0<x<\theta_{1}, y=w$

$t=F_{2}(x), 0<x<\ell, y=w$.

$t=G_{2}(x), x=l, 0<y<w$.

$t_{I I}=2 \sum_{n=1}^{\infty} \frac{\sinh \left[\frac{n \pi}{L}(1-Y)\right]}{\sinh (n \pi / L)} \sin (n \pi x L) \int_{0}^{l} F_{2}(x) \sin (n \pi x) d x$

5

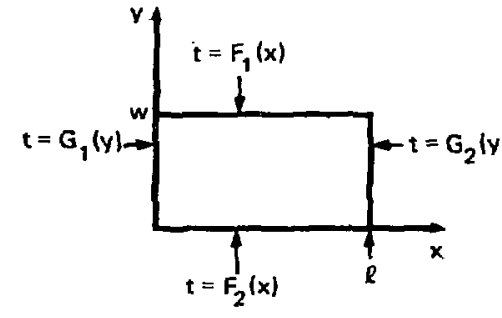

$$
\begin{aligned}
& t_{I V}=2 \sum_{n=1}^{\infty} \frac{\sinh [n \pi L(I-X)]}{\sinh (n \pi L)} \sin (n \pi Y) \int_{0}^{l} G_{2}(Y) \sin (n \pi Y) d Y \\
& L=\ell / W \\
& \frac{t-t_{1}}{t_{2}-t_{1}}=\frac{4}{\pi} \sum_{n=1}^{\infty} \frac{\cosh \left[\frac{n \pi}{L}\left(\frac{1}{2}-Y\right)\right]}{n \cosh \left(\frac{\pi}{2 L}\right)} \sin (n \pi X), \\
& F_{1}(x)=F_{2}(x)=t_{2}, G_{2}(Y)=G_{1}(Y)=t
\end{aligned}
$$


Section 1.1. Solids Bounded by Plane Surfaces--No Internal Heating.

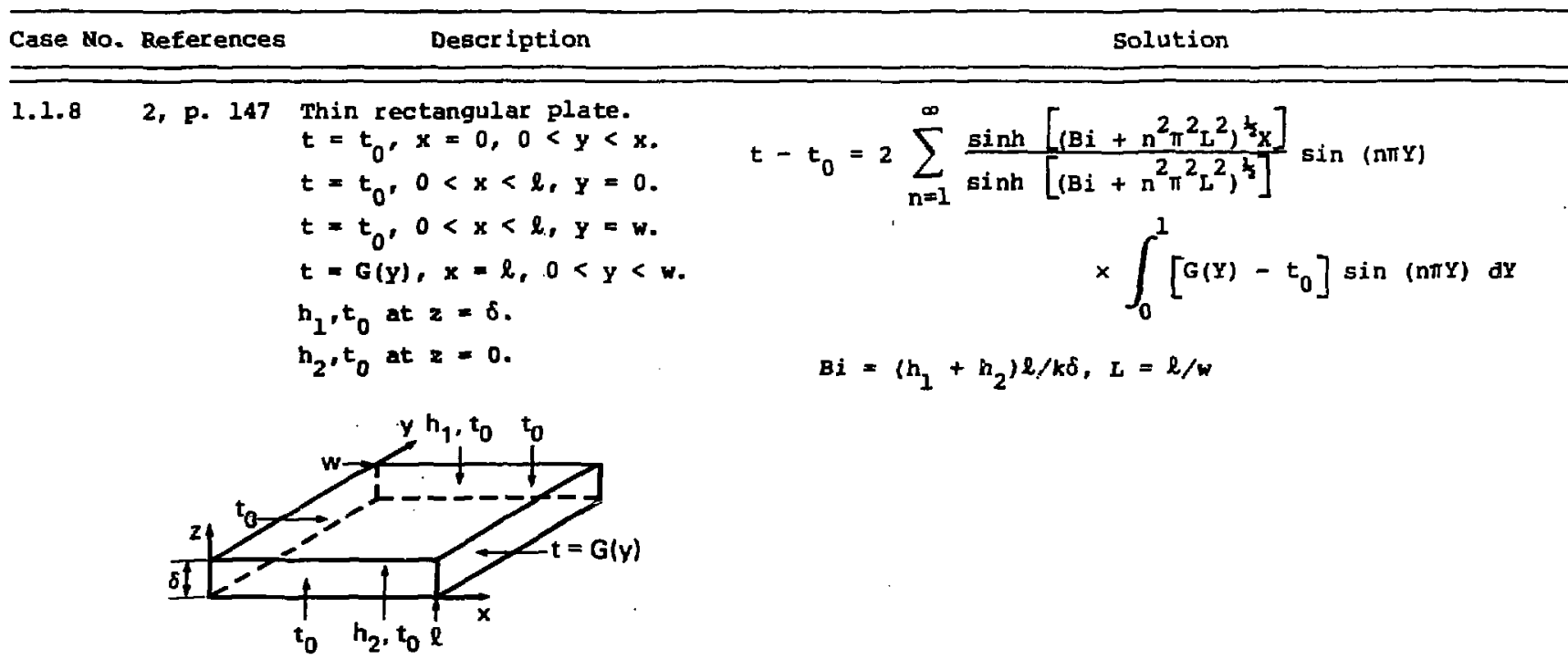

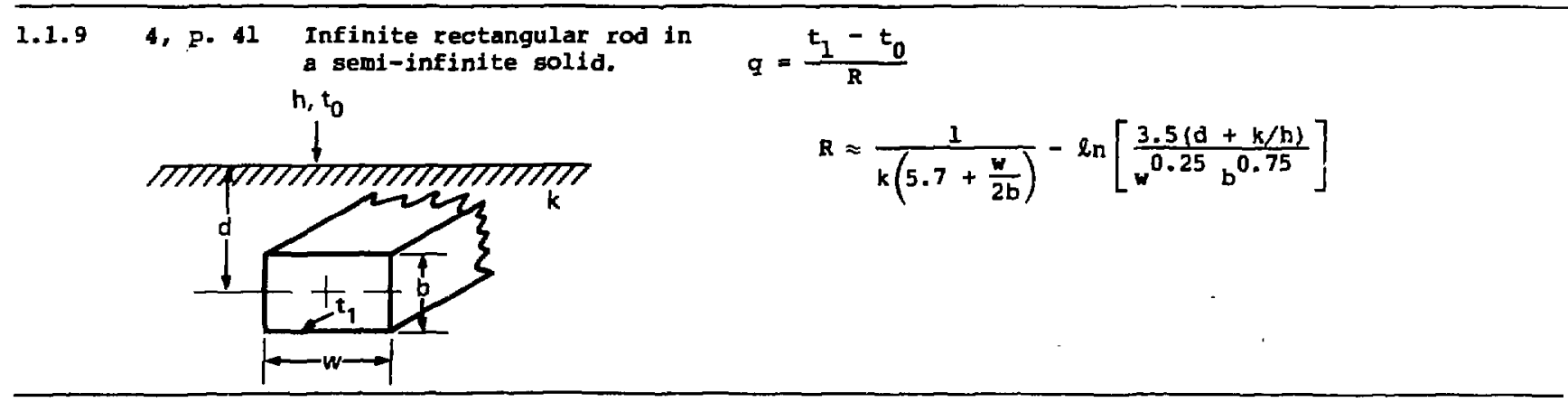


Section 1.1. Solids Bounded by Plane Surfaces--No Internal Heating.

Case No. References $\quad$ Description Solution

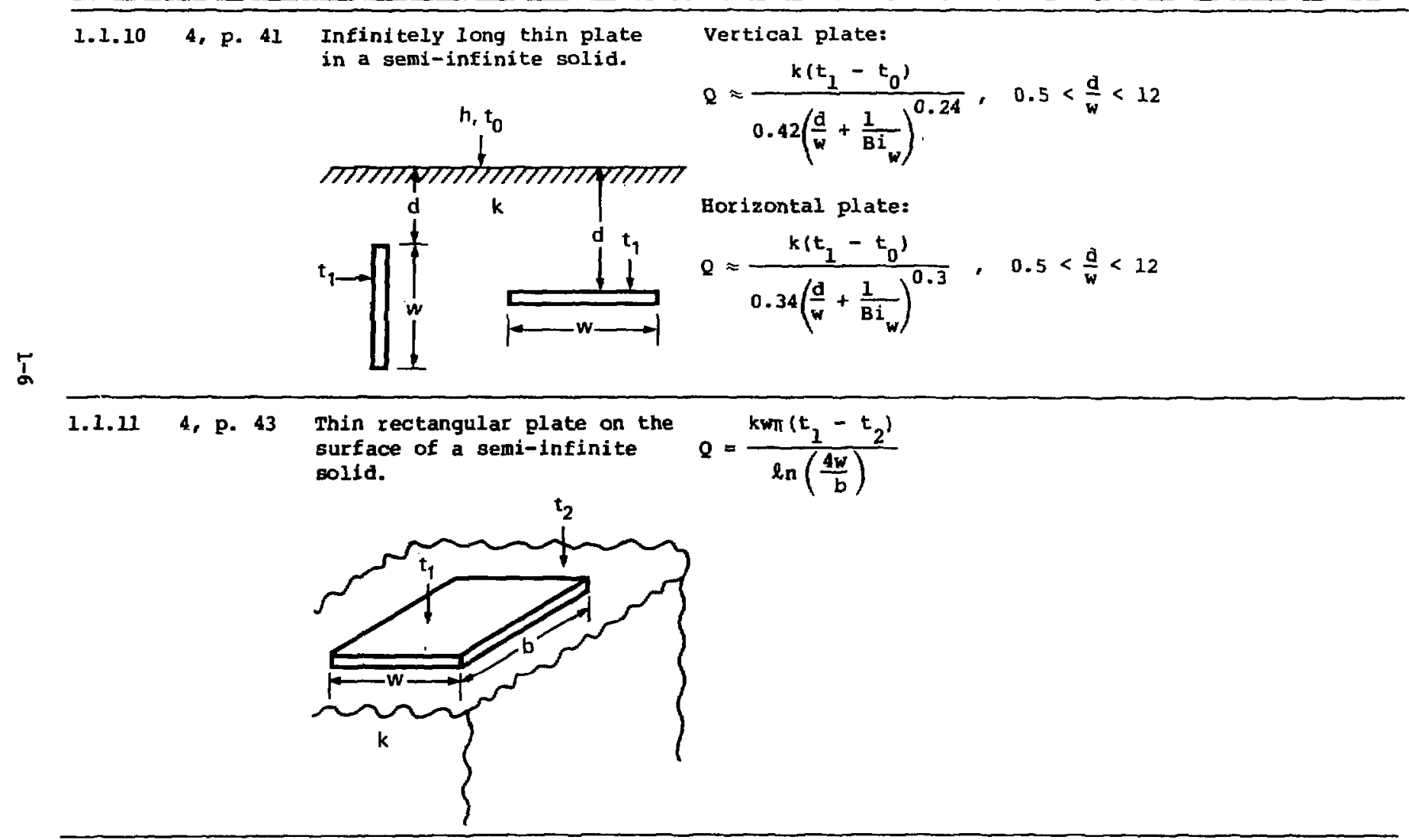


Section 1.1.: Solids Bounded by Plane Surfaces--No Internal Heating.

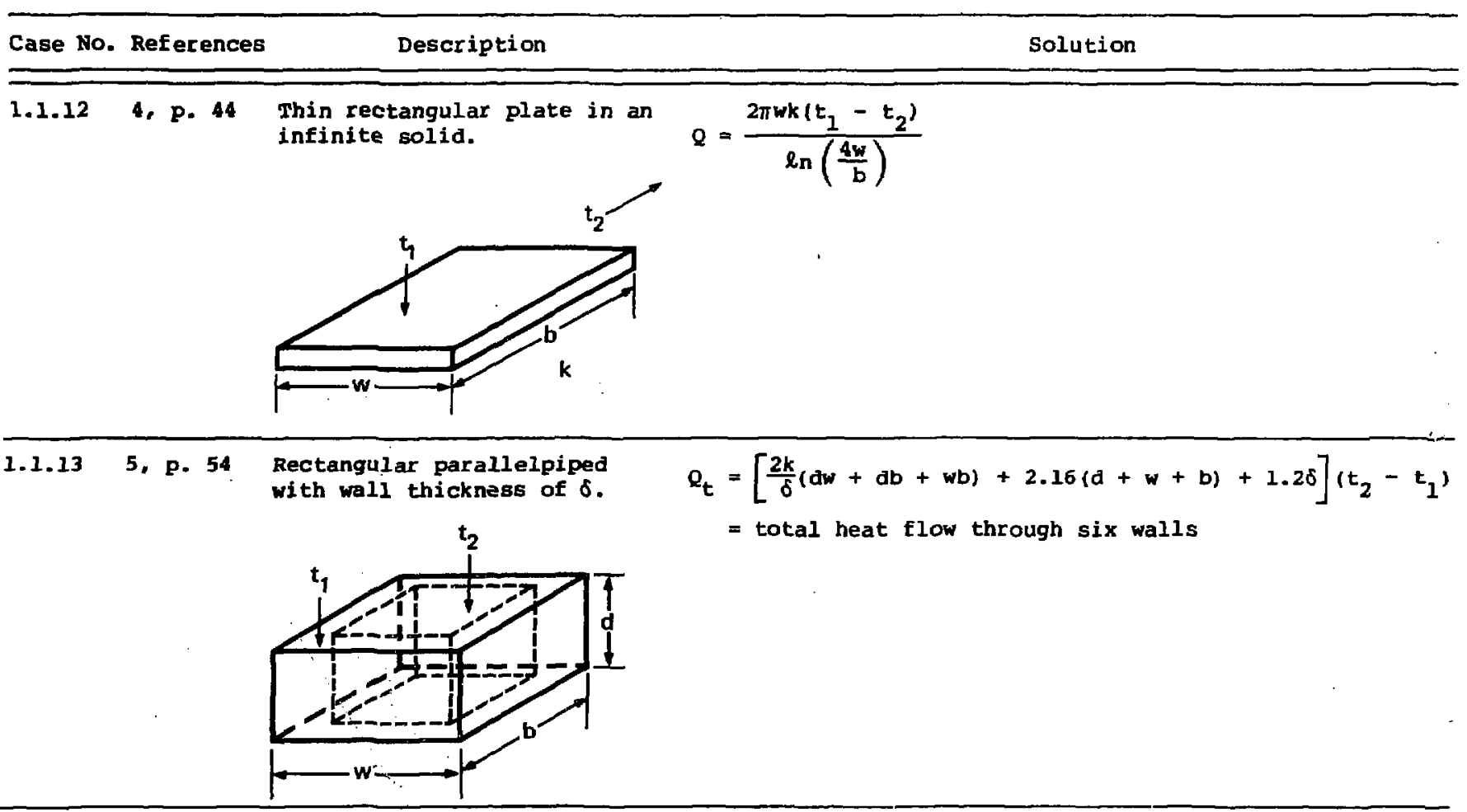


Section 1.1. Solids Bounded by Plane Surfaces--No Internal Heating.

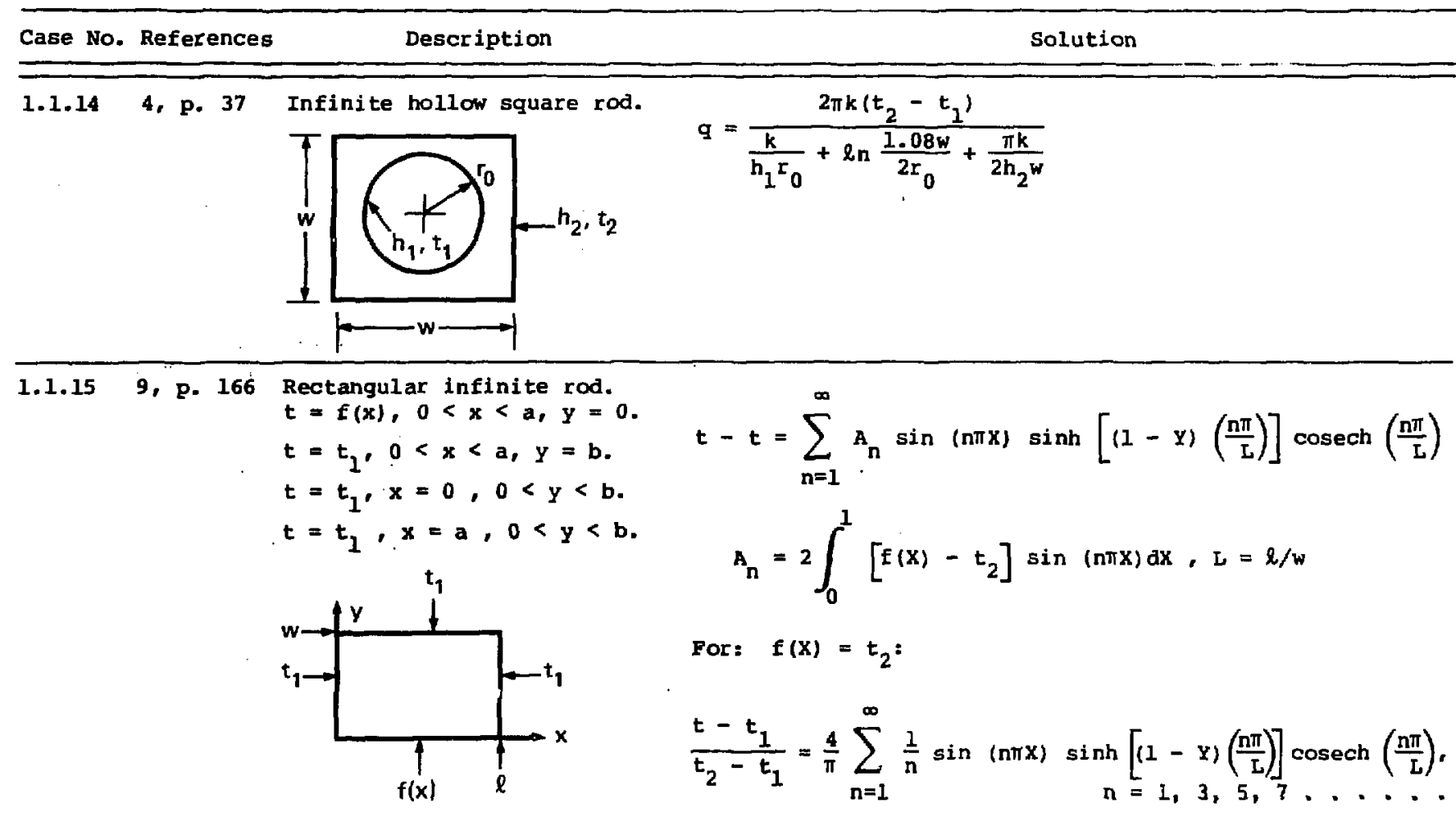


Section 1.1. Solids Bounded by Plane Surfaces--No Internal Heating.

\section{Case No. References}

Description

Solution

1.1.169, 9. 167 Rectangular infinite rod.

$t=f(x), 0<x<\ell, y=0$.

$q_{y}=0,0<x<\ell, y=w$.

$\mathrm{q}_{\mathrm{x}}=0, \mathrm{x}=0,0<\mathrm{y}<\mathrm{w}$.

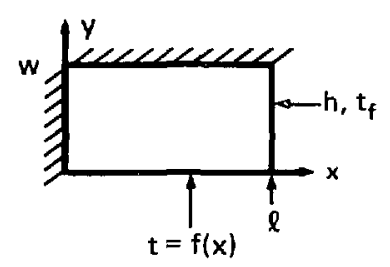

$$
\begin{gathered}
t-t_{f}=2 \sum_{n=1}^{\infty} \frac{\left(B i^{2}+\lambda_{n}^{2}\right) \cos \left(\lambda_{n} X\right) \cosh \left[(1-Y) W \lambda_{n}\right]}{\left[\left(B i^{2}+\lambda_{n}^{2}\right)+B i\right] \cosh \left(\lambda_{n} W\right)} \\
\quad \times \int_{0}^{l}\left[f(X)-t_{f}\right] \cos \left(\lambda_{n} X\right) d x \\
\text { For: } f(x)=t_{1}: \\
\frac{t-t_{f}}{t_{1}-t_{f}}=2 B i \sum_{n=1}^{\infty} \frac{\cos \left(\lambda_{n} X\right) \cosh \left[(1-Y) W \lambda_{n}\right]}{\left[\left(B i^{2}+B_{n}^{2}\right)+B i\right] \cos \left(\lambda_{n}\right) \cosh \left(\lambda_{n} W\right)}
\end{gathered}
$$


Section 1.1. Solids Bounded by Plane Surfaces--No Internal Heating.

\begin{tabular}{lll}
\hline Case No. References & Description & Solution \\
\hline
\end{tabular}

1.1.17 9, p. 168 Case 1.1.16 with $q_{y}=0$,

$y=w, 0<x<\ell$ is replaced

by convection boundary $h, t_{f}$.

$$
\begin{gathered}
t-t_{E}=2 \sum_{n=1}^{\infty} \frac{\left(B i^{2}+\lambda_{n}^{2}\right) \cos \left(\lambda_{n} X\right)\left\{\lambda_{n} \cosh \left[\lambda_{n}(W-y)\right]+B i \sinh \left[\lambda_{n}(W-Y)\right]\right\}}{\left[\left(B i^{2}+\lambda_{n}^{2}\right)+B i\right]\left\{\lambda_{n} \cosh \left(\lambda_{n} W\right)+B i \sinh \left(\lambda_{n} W\right)\right\}} \\
\quad x \int_{0}^{1}\left[E(X)-t_{f}\right] \cos \left(\lambda_{n} X\right) d x \\
\text { For: } f(x)=t_{1}: \\
\frac{t-t_{f}}{t_{1}-t_{E}}=2 \sum_{n=1}^{\infty} \frac{\operatorname{Bos}\left(\lambda_{n}\right)\left[\left(B i^{2}+\lambda_{n}^{2}\right)+B i\right]\left[\lambda_{n} \cosh \left(\lambda_{n} W\right)+B i \sinh \left(\lambda_{n} W\right)\right]}{\cos \left(\lambda_{n} X\right)\left\{\lambda_{n} \cosh \left[\lambda_{n}(W-Y)\right]+B i \sinh \left[\lambda_{n}(W-Y)\right]\right\}}
\end{gathered}
$$


Section 1.1. Solids Bounded by Plane Surfaces--No Internal Heating.

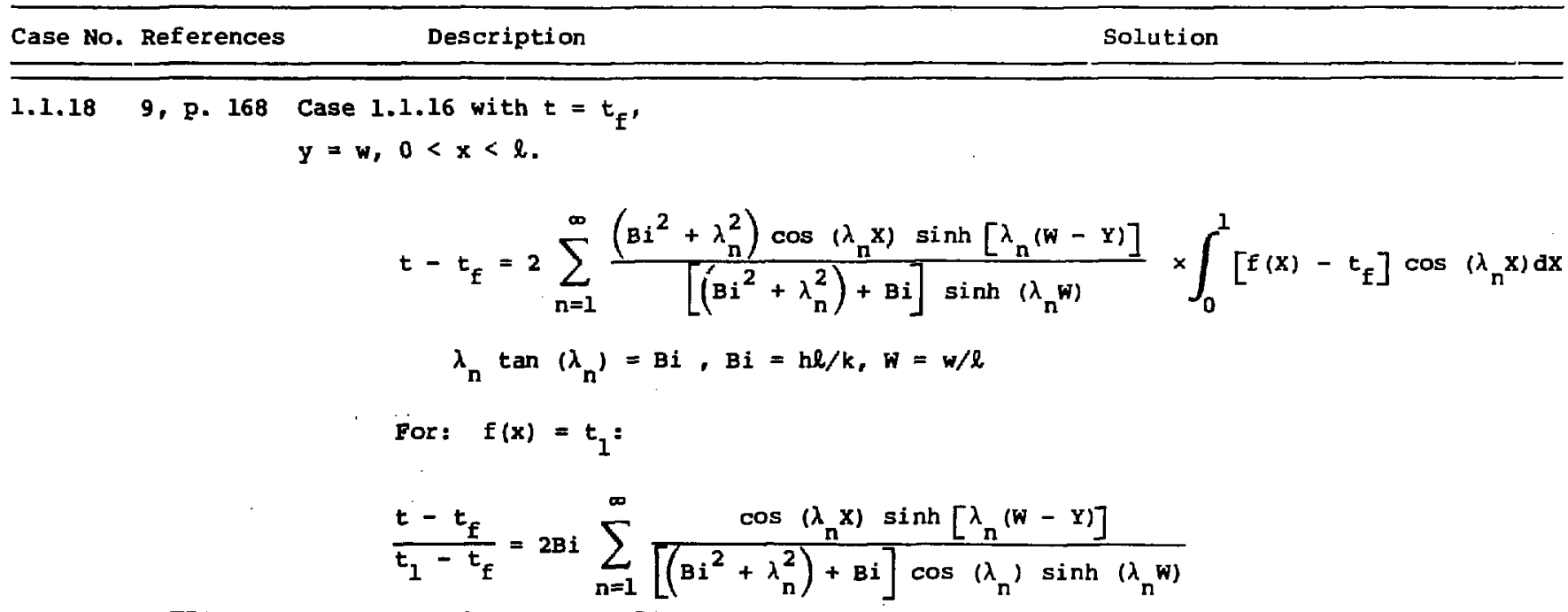

\section{1 .199$, p. 169}

Case 1.1.16 with

$t=t_{1}, y=0,0<x<l$.

$t=t_{2}, y=w, 0<x<l$.

$\frac{t-t_{f}}{t_{1}-t_{f}}=2 B i \sum_{n=1}^{\infty} \frac{\cos \left(\lambda_{n} X\right)\left\{\sinh \left[\lambda_{n}(W-Y)\right]-\sinh \left(\lambda_{n} Y\right)\left(t_{2}-t_{f}\right) /\left(t_{1}-t_{f}\right)\right\}}{\left[\left(B i^{2}+\lambda_{n}^{2}\right)+B i\right] \cos \left(\lambda_{n}\right) \sinh \left(\lambda_{n} W\right)}$

$\lambda_{n} \tan \left(\lambda_{n}\right)=B i, w=w / \ell$ 
Section 1.1. Solids Bounded by Plane Surfaces--No Internal Heating.

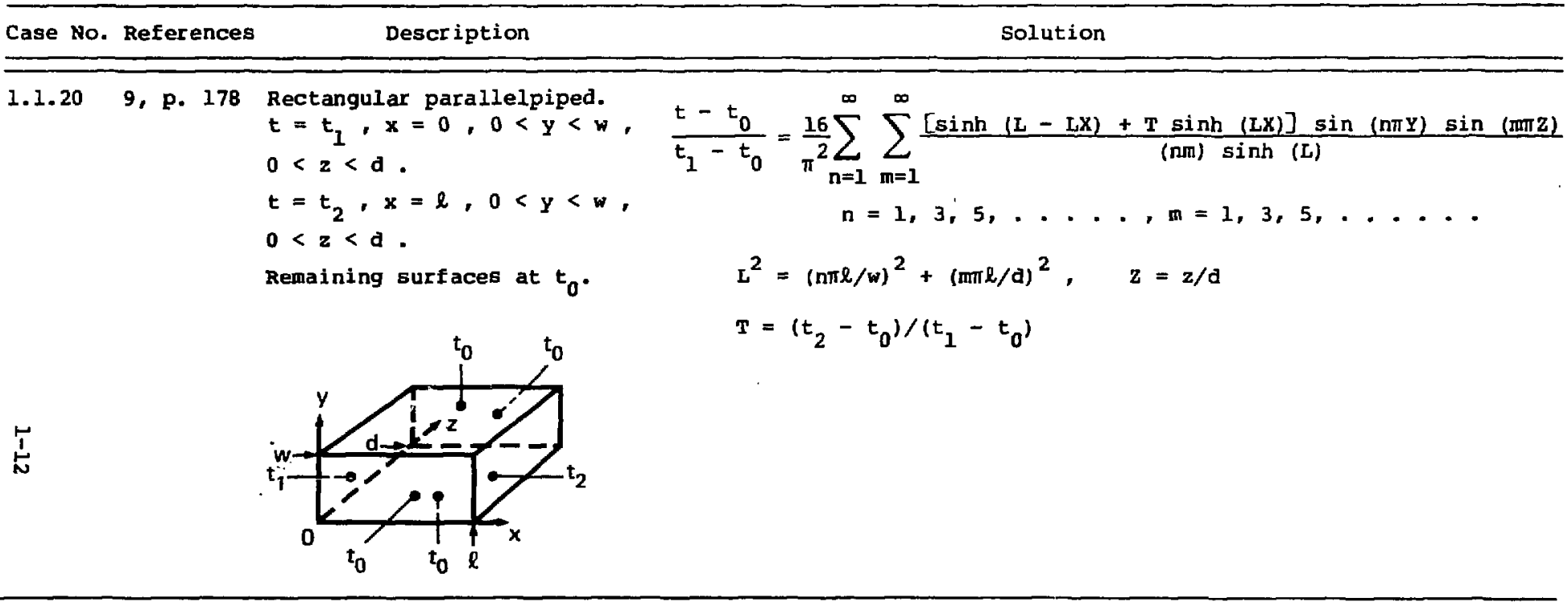


Section 1.1. Solids Bounded by Plane Surfaces--No Internal Heating.

\begin{tabular}{|c|c|c|}
\hline Case No. & References & Descr iption \\
\hline 1.1 .21 & 9, p. 179 & 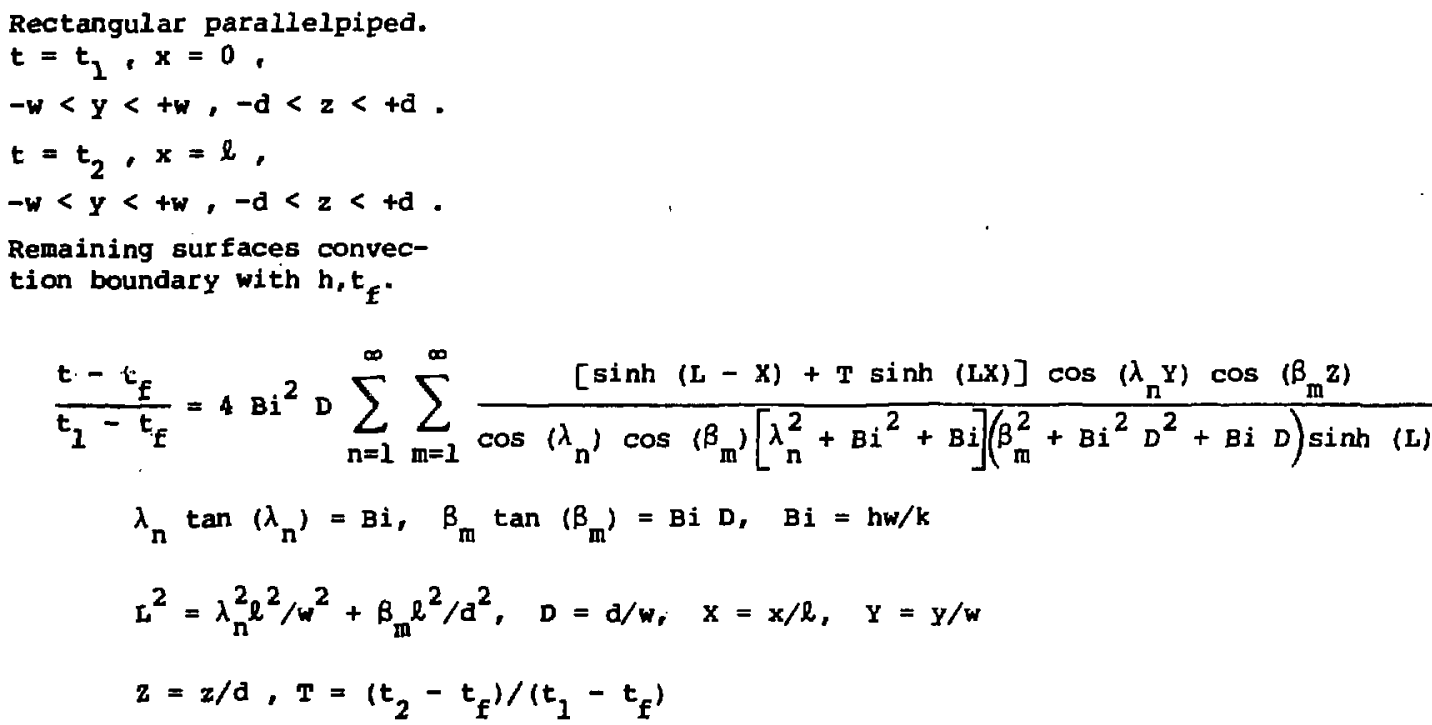 \\
\hline
\end{tabular}


Section 1.1. Solids Bounded by Plane Surfaces--No Internal fleating.

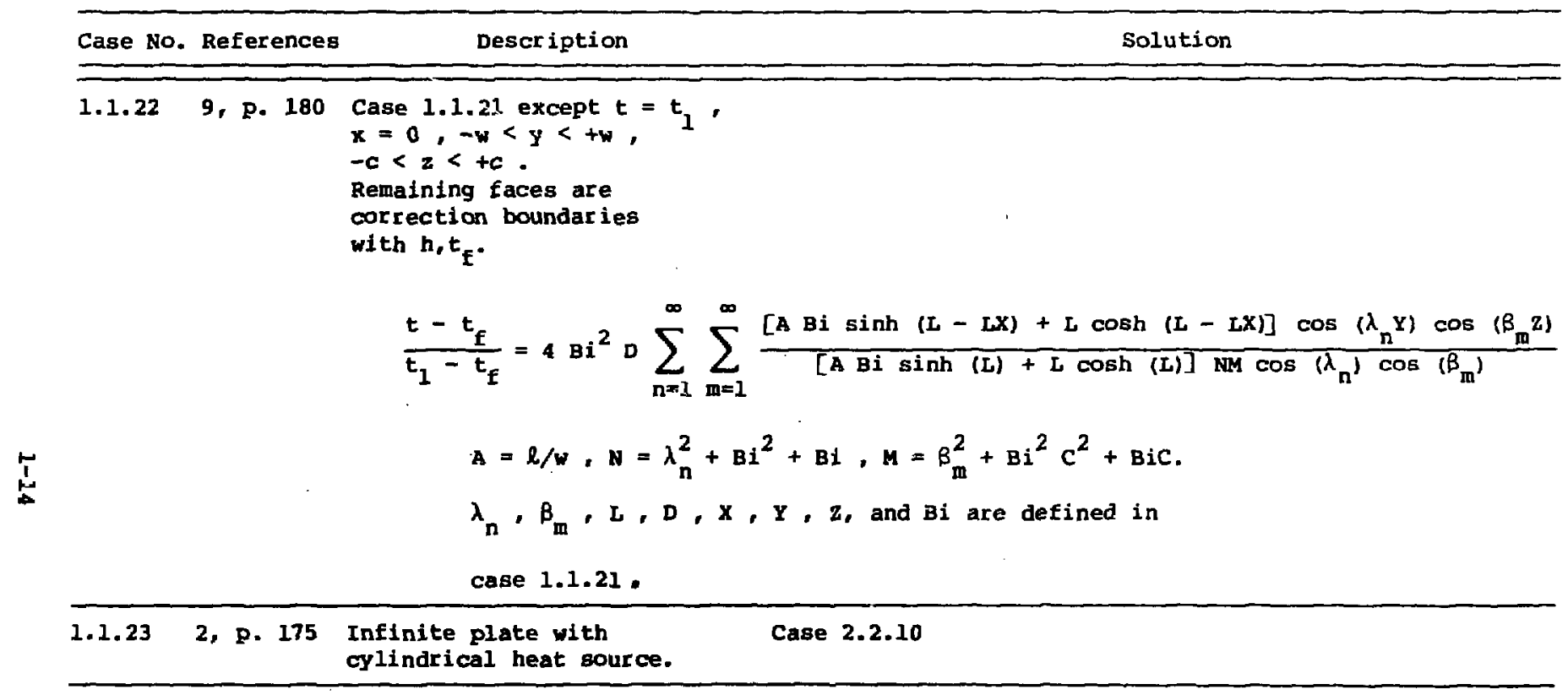


Section 1.1. Solids Bounded by Plane Surfaces--No Internal Heating.

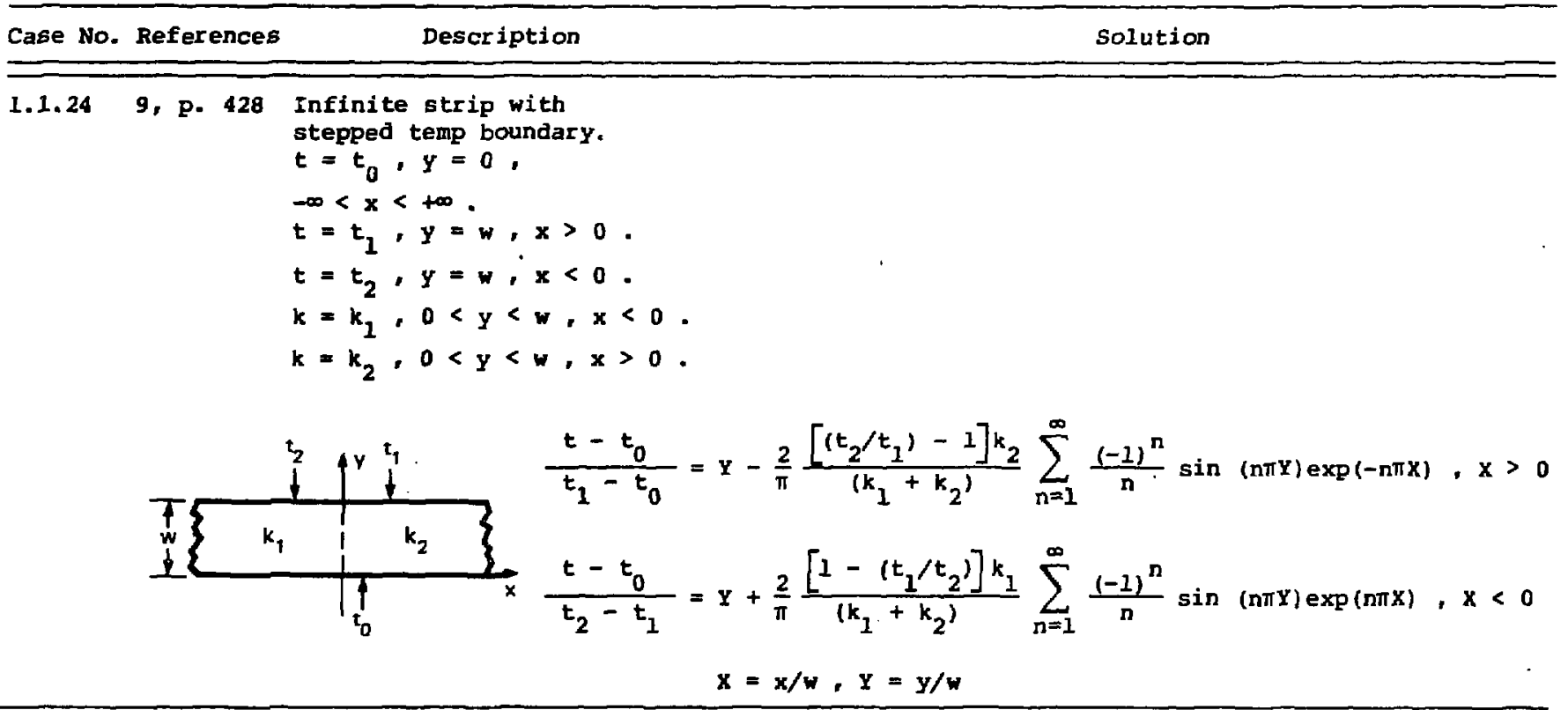


Section 1.1. Solids Bounded by Plane Surfaces--No Internal Heating.

\section{Case No. References \\ -}

1.1 .25

9. P. 452

Heated planes on a
semi-infinite medium.
$t=t$

$t=t_{1}, x \leq-l, y=0$.

$t=t_{2}, x \geq+l, y=0$.

$q_{y}=0,-l<x<+l$,

$y=0$.

告

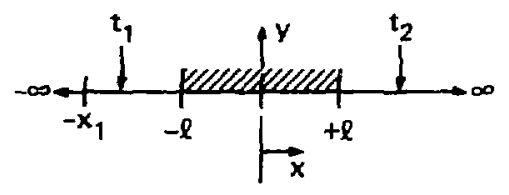

Solution

$$
Q=\frac{k}{\pi} \cosh ^{-1}\left(\frac{x_{1}}{l}\right)\left(t_{1}-t_{2}\right),-x_{1}<x<-l
$$

Heat flow from bottom side of semi-infinite plane: $Q=k\left[\left(x_{1} / s\right)+(1 / \pi)\right]\left(t_{1}-t_{2}\right), 0<x<x_{1}$. an Infinite medium.

$t=t_{1}, x \geq 0, y=s$.

$t=t_{2},-\infty<x+\infty$.

Heat flow from top side of semi-infinite plane:

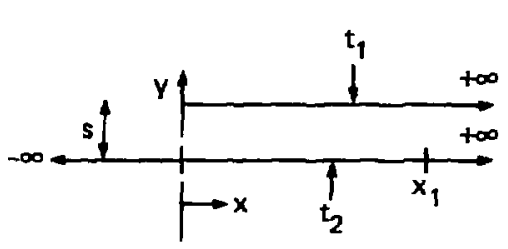

$Q=\frac{k}{\pi} \ln \left[\left(\pi x_{1} / s\right)+1\right]\left(t_{1}-t_{2}\right), 0<x<x_{1}$. 
Section 1.1. Solids Bounded by Plane Surfaces--No Internal Heating.

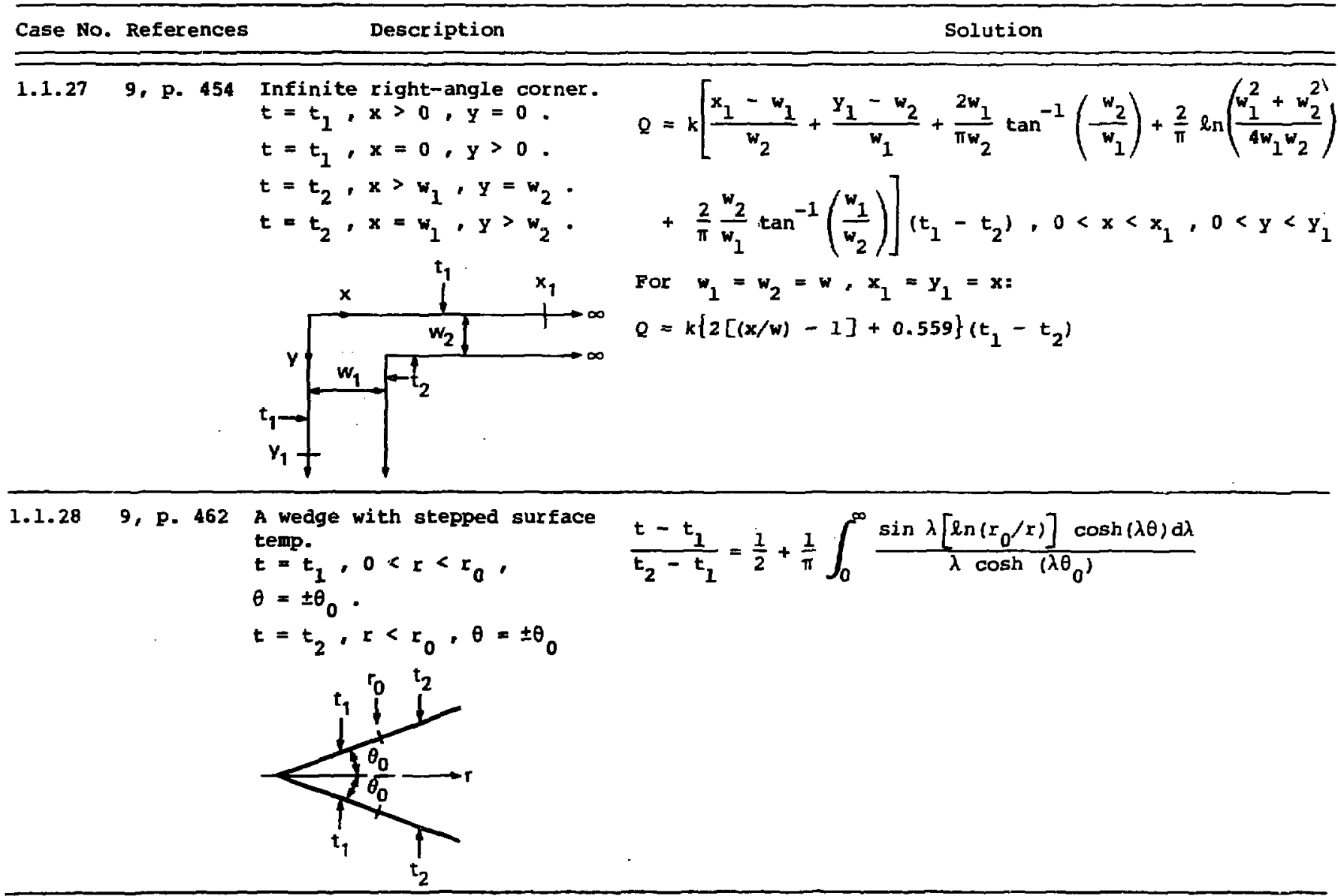


Section 1.1. Solids Bounded by Plane Surfaces--No Internal Heating.

\begin{tabular}{|c|c|c|c|}
\hline Case No. & References & Description & Solution \\
\hline 1.1 .29 & 15 & $\begin{array}{l}\text { Semi-infinite strip with } \\
\text { convection boundary. } \\
t=t_{1}, x=0, w, y=0 \text {. } \\
\text { convection boundary at } \\
y=0 \text {. }\end{array}$ & $\begin{array}{l}\frac{t-t_{1}}{t_{f}-t_{1}}=4 \sum_{n=1}^{\infty} \frac{\exp (-n \pi Y) \sin (n \pi x)}{n \pi+\left(n^{2} \pi^{2} / B i\right)}, n=1,3,5, \ldots . . \\
\text { Heat transfer into strip at } y=0: \\
\frac{Q}{k\left(t_{1}-t_{f}\right)}=\frac{8}{\pi} \sum_{n=1}^{\infty} \frac{1}{n+\left(n^{2} \pi / B i\right)}, n=1,3,5, \ldots . . \\
\text { See Tables } 1.2 a \text { and } 1.2 b .\end{array}$ \\
\hline
\end{tabular}

Periodic strip heated plate $q_{x}(w, y)=0$,

on $2 b$ wide strips. $t(w, y)=t_{s}$, on $(2 a-b)$ wide strips spaced $2 a$ on centers.

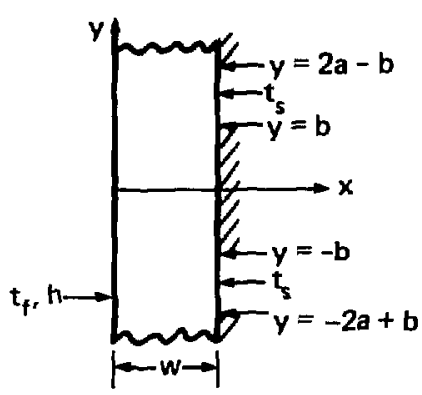

See Fig. 1.6a and $b$ for values of maximum differences on $y=0$ surface, i.e. $[t(0, a)-t(0,0)]=\Delta t_{m^{\prime}}$ and heat transfer. 
Section 1.1. Solids Bounded by Plane Surfaces--No Internal Heating.

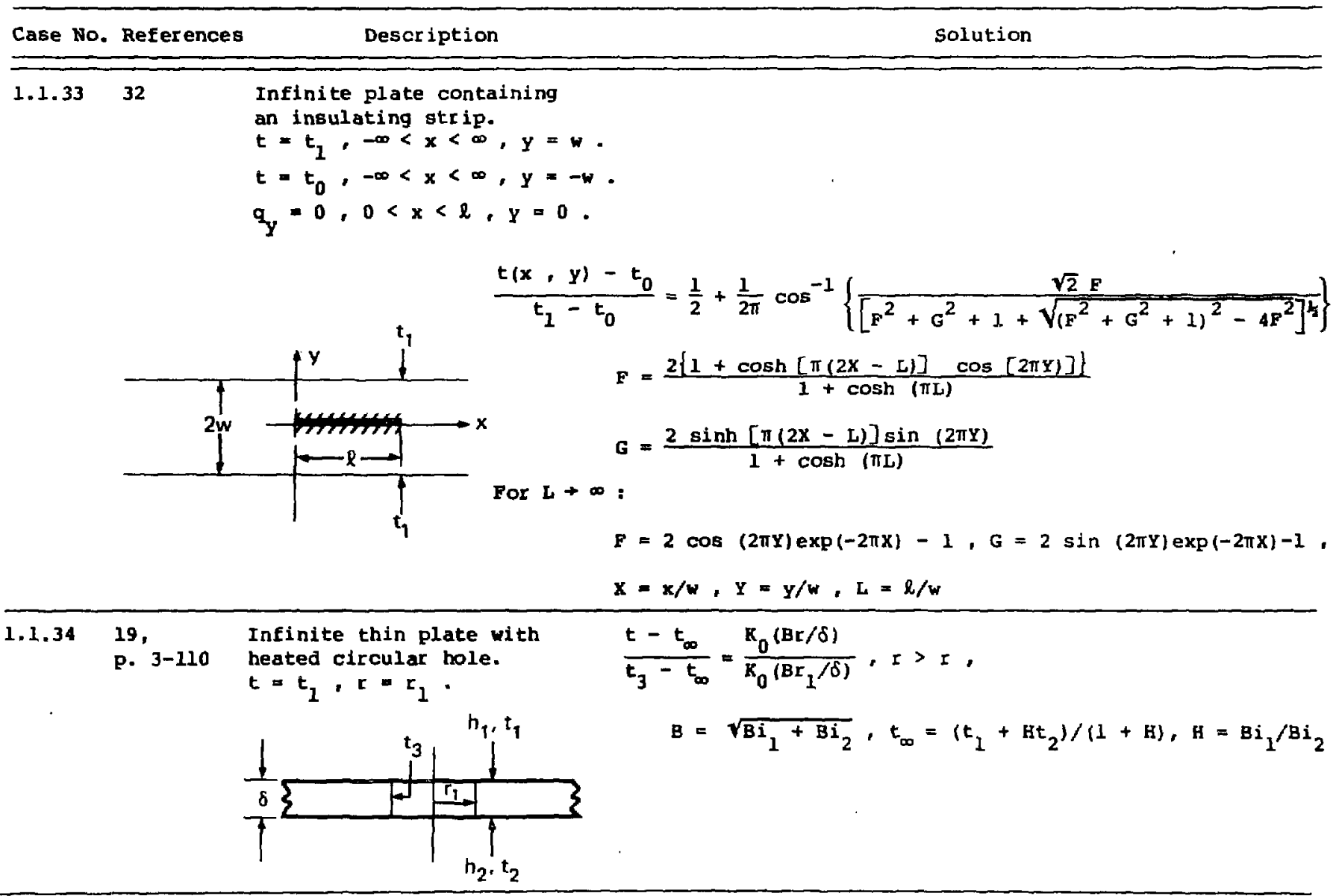


Section 1.1. Solids Bounded by Plane Surfaces--No Internal Heating.

\begin{tabular}{lll}
\hline Case No. References & Description & Solution \\
\hline
\end{tabular}

\begin{tabular}{llll}
\hline 1.1 .35 & $\begin{array}{l}19, \\
\text { p. } 3-110\end{array}$ & $\begin{array}{l}\text { Case } 1.1 .34 \text { with } t_{3} \\
\text { replaced by a heat } \\
\text { source of strength } q .\end{array}$ & $\frac{k \delta\left(t-t_{\infty}\right)}{q}=\frac{k_{0}(B r / \delta)}{2 \pi\left(B r_{1} / \delta\right) k_{1}\left(B r_{1} / \delta\right)}, r>r_{1}$ \\
\end{tabular}

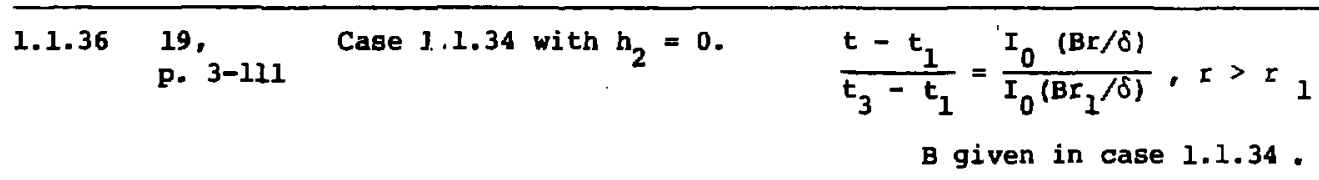

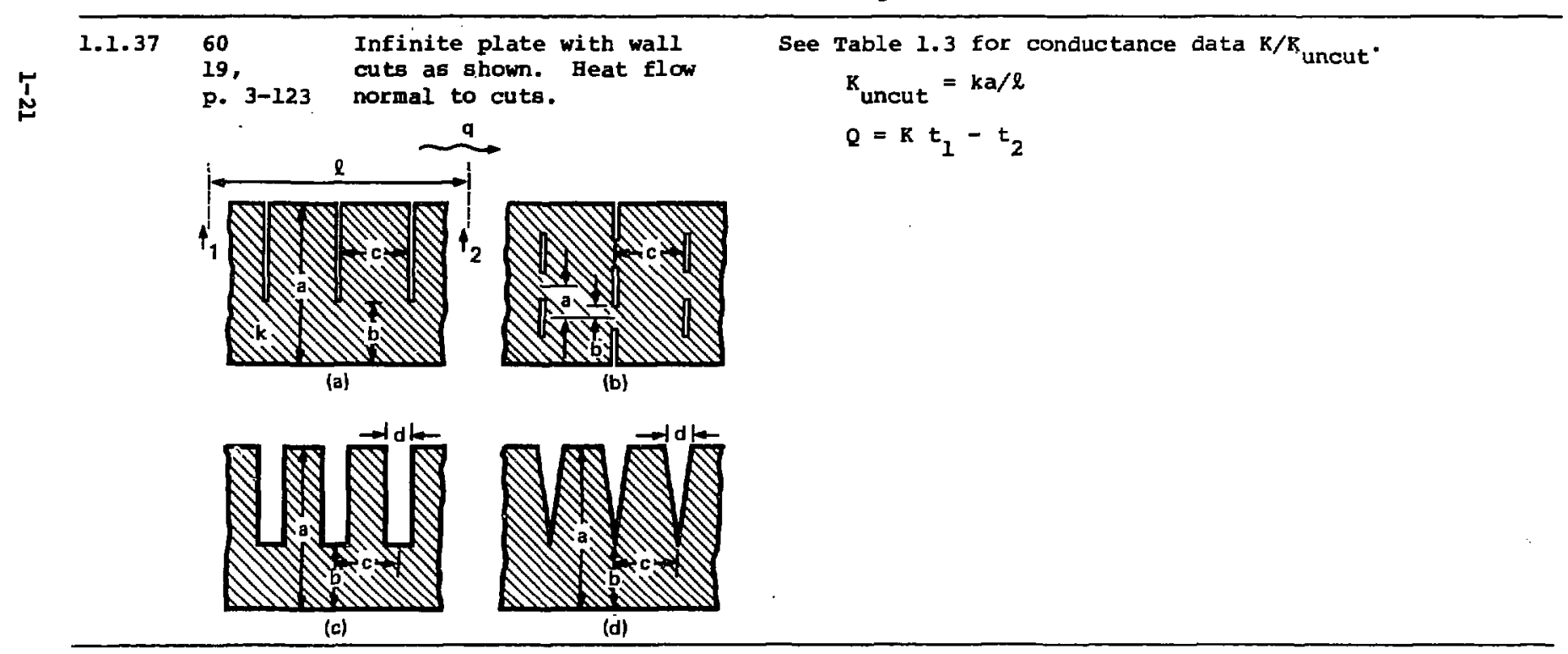


Section 1.1. Solids Bounded by Plane Surfaces--No Internal Heating.

\begin{tabular}{|c|c|c|c|}
\hline Case No. & References & Description & Solution \\
\hline 1.1 .37 .1 & 84 & $\begin{array}{l}\text { Infinite medium with } \\
\text { single and multiple } \\
\text { insulating cuts. }\end{array}$ & See Ref. 84 for temperature and heat flow solutions. \\
\hline 1.1 .38 & $\begin{array}{l}61 \\
19, \\
\text { p. } 3-124\end{array}$ & $\begin{array}{l}\text { Case } 1.1 .37 \text {, cut (c), } \\
\text { with only one cut. }\end{array}$ & $\begin{array}{l}\text { Conductance: } \\
\left.K=\frac{k}{[(l-d) / a]+(d / b)+(4 / \pi) \ln [\sec (\pi / 2)[1-(b / a)]}\right\} \\
Q=K t_{1}-t_{2}\end{array}$ \\
\hline 1.1 .39 & $\begin{array}{l}62 \\
19 . \\
\text { p. 3-126 }\end{array}$ & $\begin{array}{l}\text { Infinite rib on an } \\
\text { infinite plate with } \\
\text { convection. } \\
t=t_{w}, x= \pm \delta / 2, y>\ell . \\
t=t_{w}, x>|\delta / 2|, y=\ell . \\
\text { Convection boundary at } y=0 .\end{array}$ & See Fig. 1.10 for ternp at $x=0, y \geq 0$ \\
\hline
\end{tabular}


Section 1.1. Solids Bounded by Plane Surfaces--No Internal Heating.

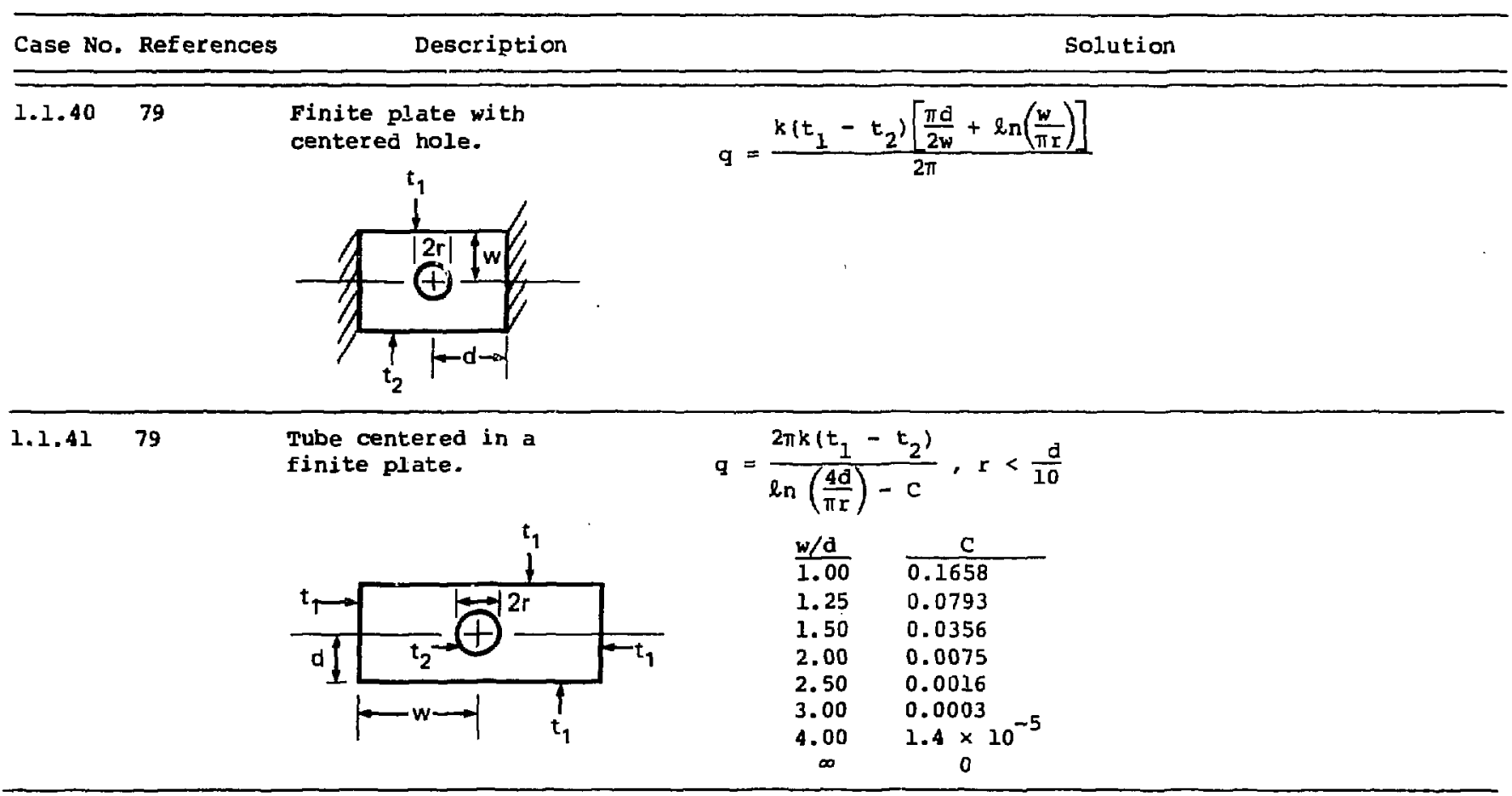


Section 1.1. Solids Bounded by Plane Surfaces--No Internal Heating.

\begin{tabular}{|c|c|c|c|c|c|}
\hline Case No. & References & Description & \multicolumn{3}{|c|}{ Solution } \\
\hline 1.1 .42 & 79 & $\begin{array}{l}\text { Tube in a multi-sided } \\
\text { infinite solid. }\end{array}$ & $\begin{aligned} q= & \frac{2 \pi k\left(t_{1}-t_{2}\right)}{\ln \left(\frac{r_{2}}{r_{1}}\right)-c(n)} \\
& n=\text { No. sides } \\
& r_{1}<r_{2} / 10 \text { for } n=3\end{aligned}$ & $\begin{array}{r}n \\
3 \\
4 \\
5 \\
6 \\
7 \\
8 \\
9 \\
10 \\
\infty\end{array}$ & $\begin{array}{c}\frac{C(n)}{0.5696} \\
0.2708 \\
0.1606 \\
0.1067 \\
0.0761 \\
0.0570 \\
0.0442 \\
0.0354 \\
0\end{array}$ \\
\hline
\end{tabular}

$\frac{1.1 .43 \quad 79}{1.1 .44 \quad 87}$

$q=\frac{2 \pi k\left(t_{1}-t_{2}\right)}{0.93 \ln (w / d)-0.0502}, w / d>1.4$

$g=\frac{2 \pi k\left(t_{1}-t_{2}\right)}{0.785 \ln (w / d)}, w / d<1.4$

$q=S k\left(t_{1}-t_{0}\right)$

See Fig. 1.11 for values of $S$.

$2 \mathrm{~b}$ 
Section 1.1. Solids Bounded by Plane Surfaces--No Internal Heating.

Case No. References

Description

Solution

占 
Section 1.1. Soliós Bounded by Plane Surfaces-No Internal Heating.

Case No. References Description

Solution 
Section 1.2. Solids Bounded by Plane Surfaces--With Internal Heating.

\section{Case No. References}

Description

Solution

\begin{tabular}{llll}
\hline 1.2 .1 & 1, & Infinite plate. & $t-t_{1}$ \\
& p. 169 & $\begin{array}{l}t=t_{1}, x=0 . \\
t\end{array}$ & \\
& & & $t_{2}, x=\ell . t_{1}$
\end{tabular}

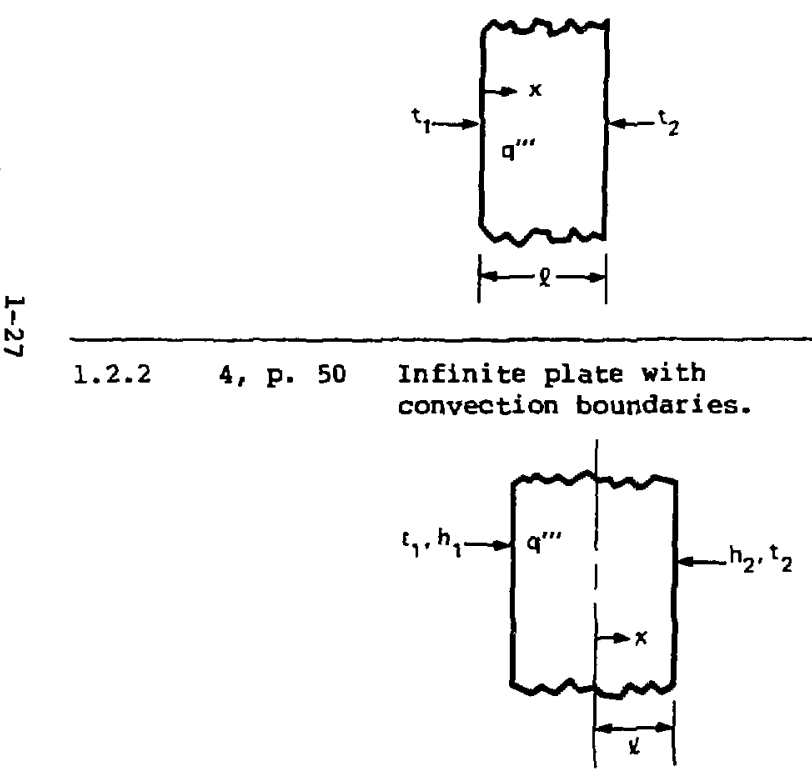

$$
\begin{array}{r}
\frac{t-t_{2}}{t_{1}-t_{2}}=\frac{1-P O\left(1 / B i_{2}+1\right)}{1+B i_{2}+H}+\frac{P O}{B i_{2}}+\frac{P O}{2}\left(1-x^{2}\right) \\
+\frac{B i_{1}\left[1+P O\left(1 / B i_{2}+1 / 2\right)\right](1-X)}{1+B i_{1}+H}
\end{array}
$$$$
H=h_{2} / h_{1}
$$ 
Section 1.2. Solids Bounded by Plane Surfaces--With Internal Heating.

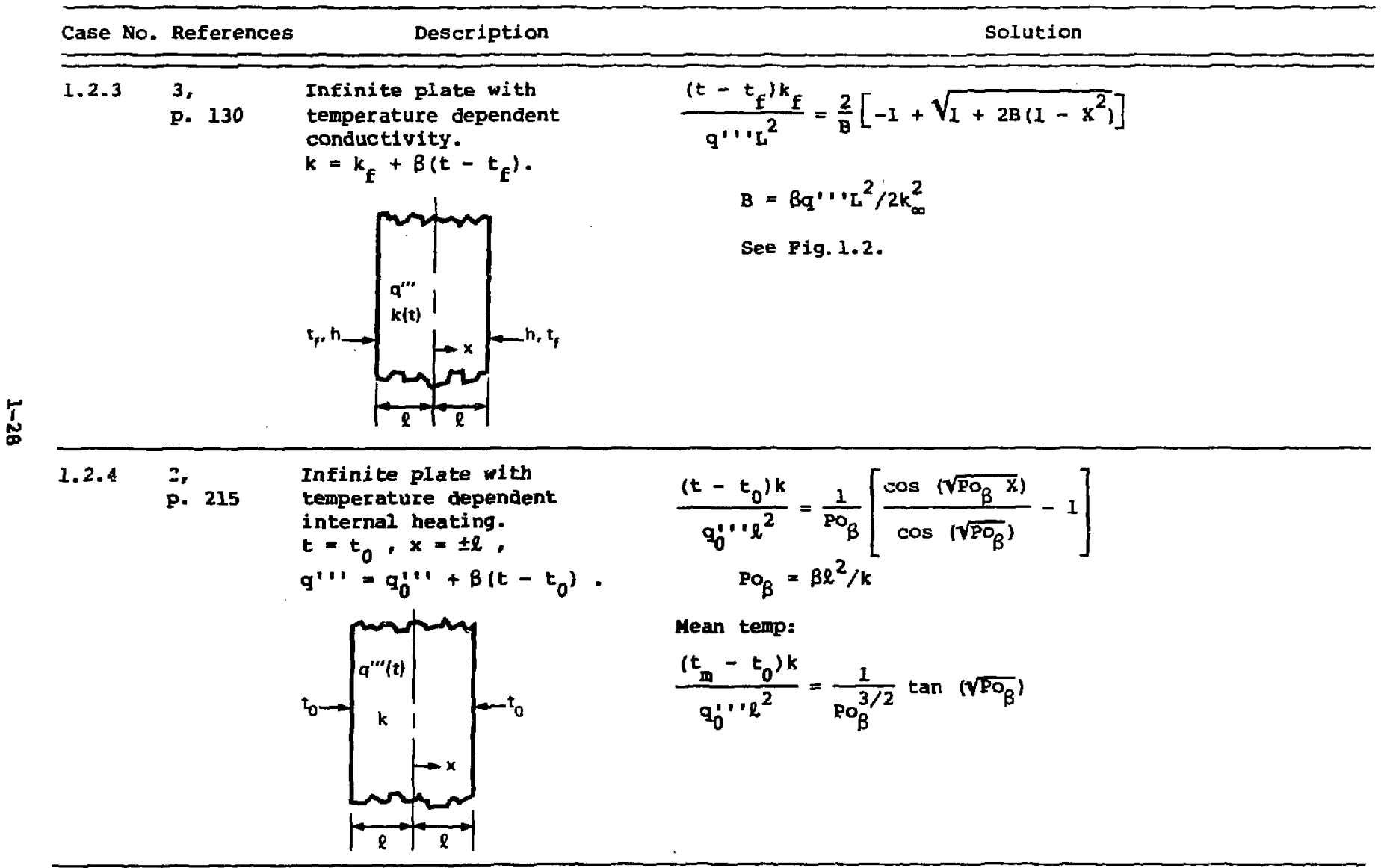


Section 1.2. Solids Bounded by Plane Surfaces--With Internal Heating.

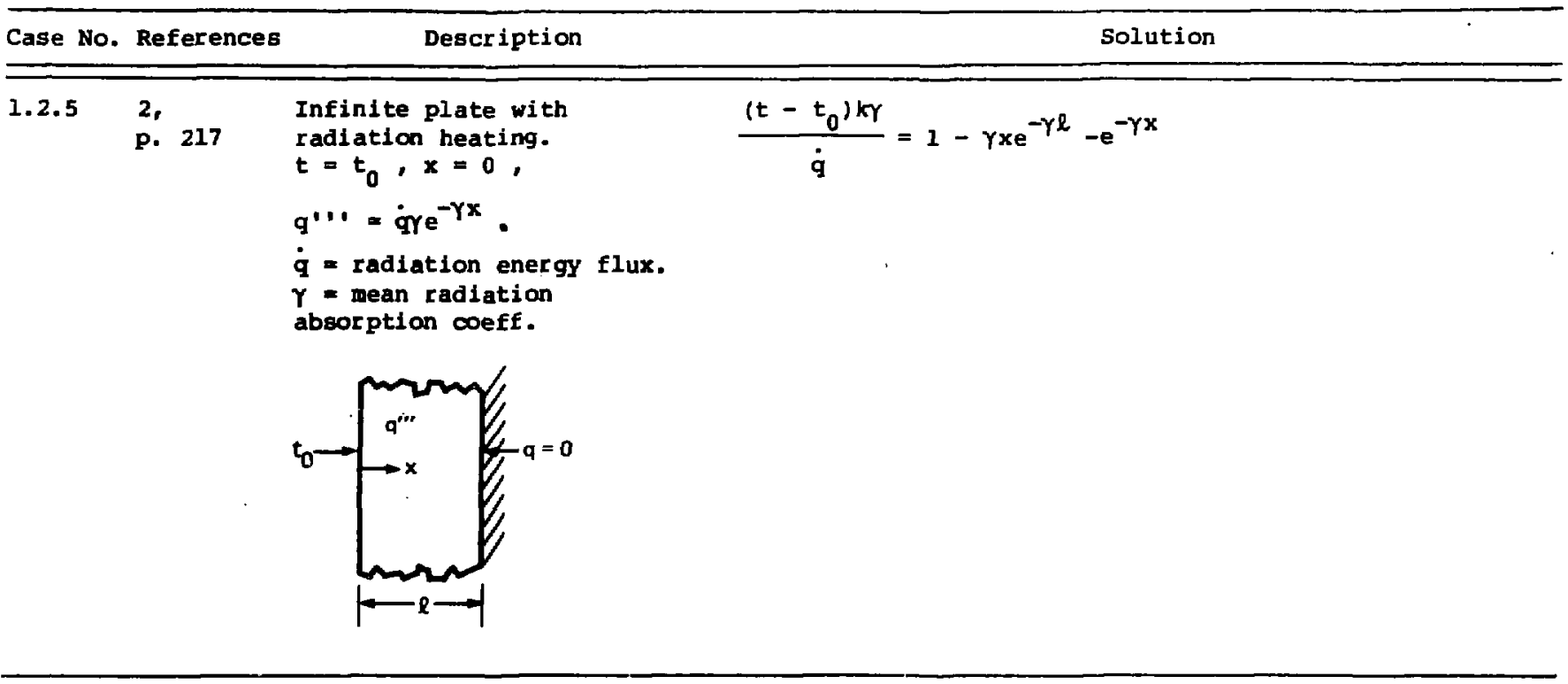


Section 1.2. Solids Bounded by Plane Surfaces--With Internal Heating.

\begin{tabular}{|c|c|c|c|}
\hline Case No. & References & Description & Solution \\
\hline 1.2 .6 & $\begin{array}{l}\text { 2, } \\
\text { p. } 223\end{array}$ & $\begin{array}{l}\text { Porous plate with } \\
\text { internal fluid flow. } \\
P=\text { Porosity. }\end{array}$ & 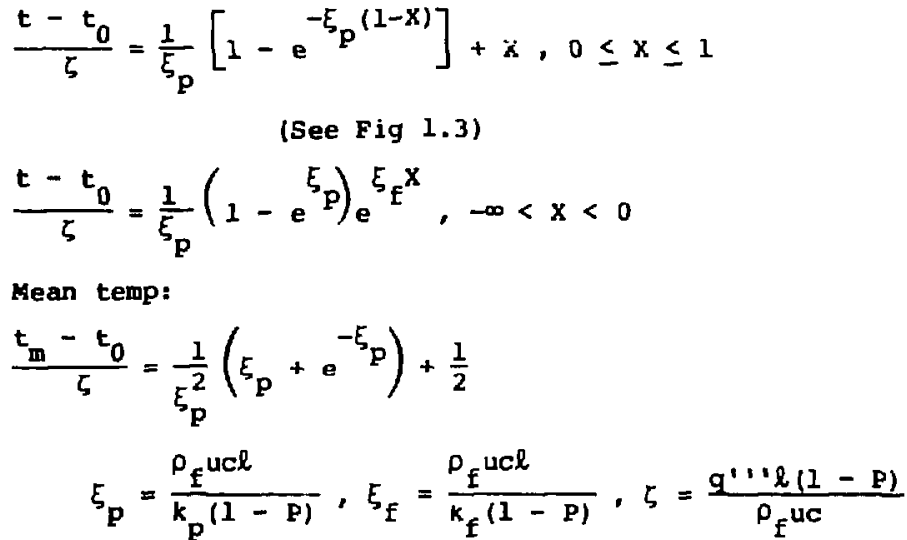 \\
\hline
\end{tabular}


Section 1.2. Solids Bounded by Plane Surfaces--with Internal Heating.

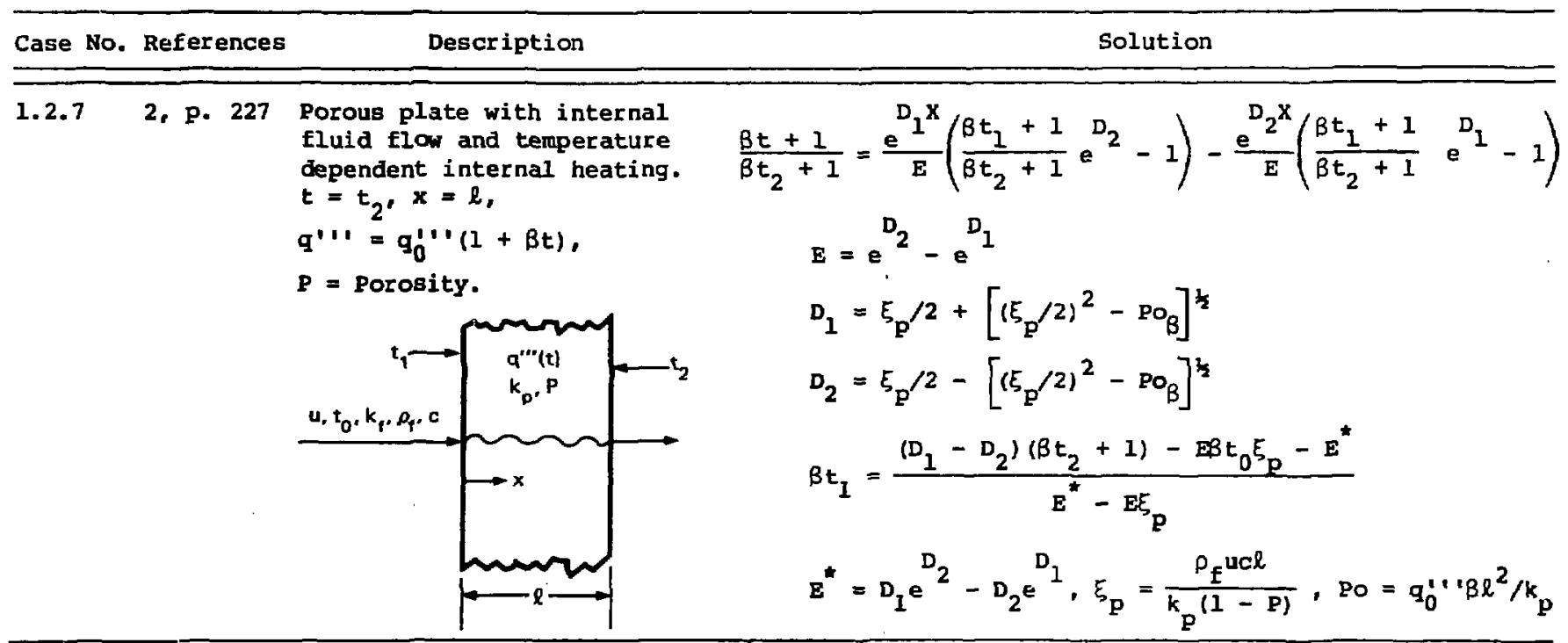

1.2.8 3, p. 220 Rectangular rod.

$t=t_{0}, x= \pm b,-a<y<+a \cdot \frac{\left(t-t_{0}\right) k}{q+a^{2}}=\frac{1}{2}\left(1-y^{2}\right)-2$

$t=t_{0},-b<x<+b, y= \pm a$.

$\times \sum_{n=0}^{\infty} \frac{(-1)^{n} \cosh \left[(2 n+1) \frac{\pi}{2} x\right] \cos \left[(2 n+1) \frac{\pi}{2} x\right]}{\left[(2 n+1) \frac{\pi}{2}\right]^{3} \cosh \left[(2 n+1) \frac{\pi}{2^{B}}\right]}$

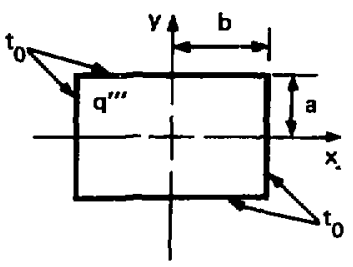

See Fig. 1.4, Table 1.1.

$Y=y / a, X=x / a, B=b / a$ 
Section 1.2. Solids Bounded by Plane Surfaces--With Internal Heating.

Case No. References Description Solution

2.930 po 469 Infinite triangular bar.
$t=t_{0}, x= \pm y$.

$t=t_{0}, x=l$.

$$
\begin{aligned}
& \frac{\left(t-t_{0}\right) k}{q^{\prime}+\ell^{2}}=\frac{5}{16}\left(x^{2}-y^{2}\right) \text { ln }\left(\frac{1}{x}\right) \\
& Y=y / \ell
\end{aligned}
$$

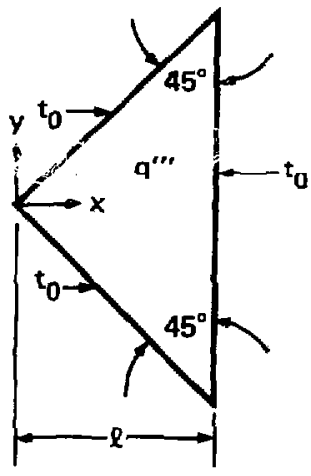


Section 1.2. Solids Bounded by Plane Surfaces--With Internal Heating.

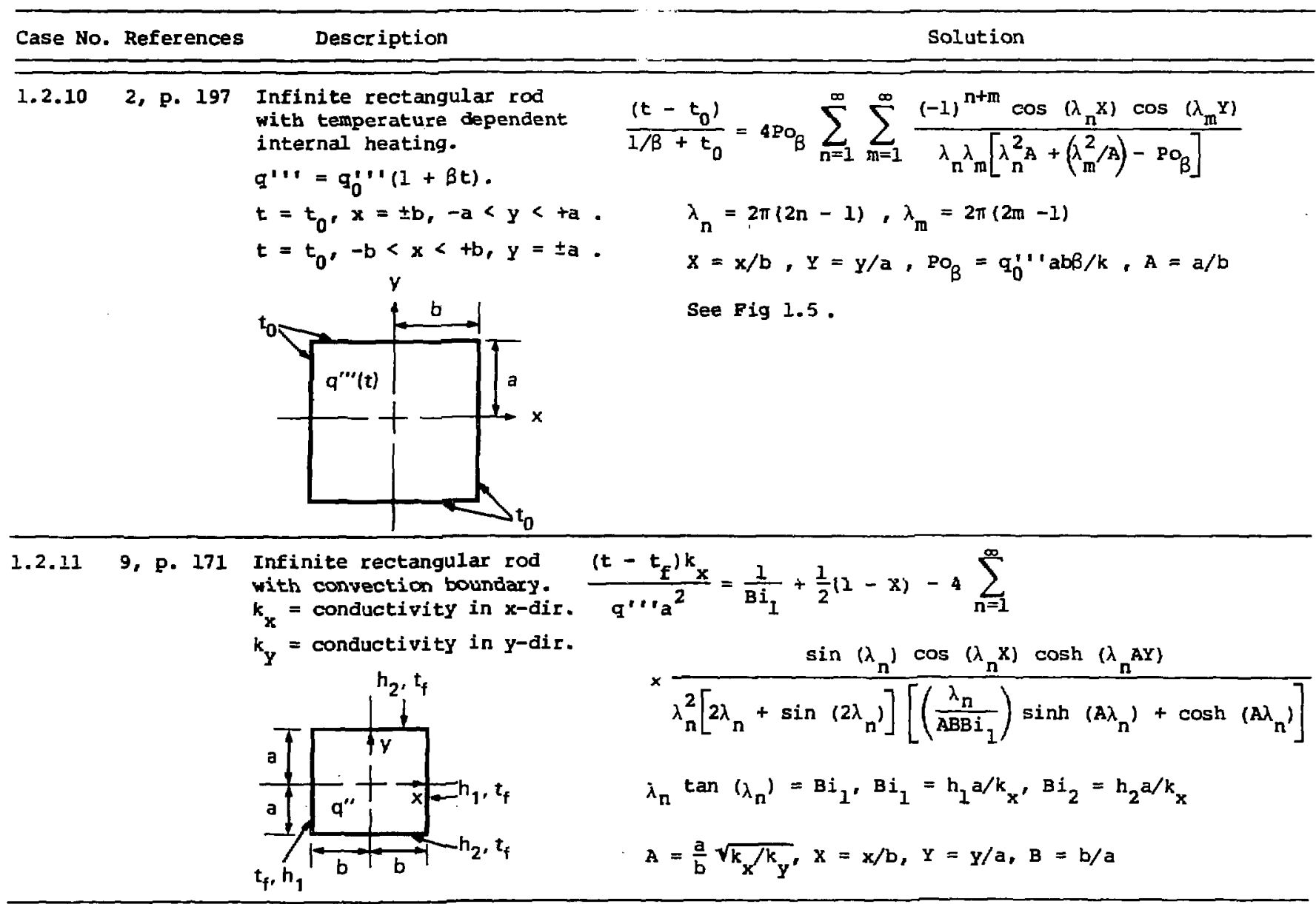


Section 1.2. Solids Bounded by Plane Surfaces-With Internal Beating.

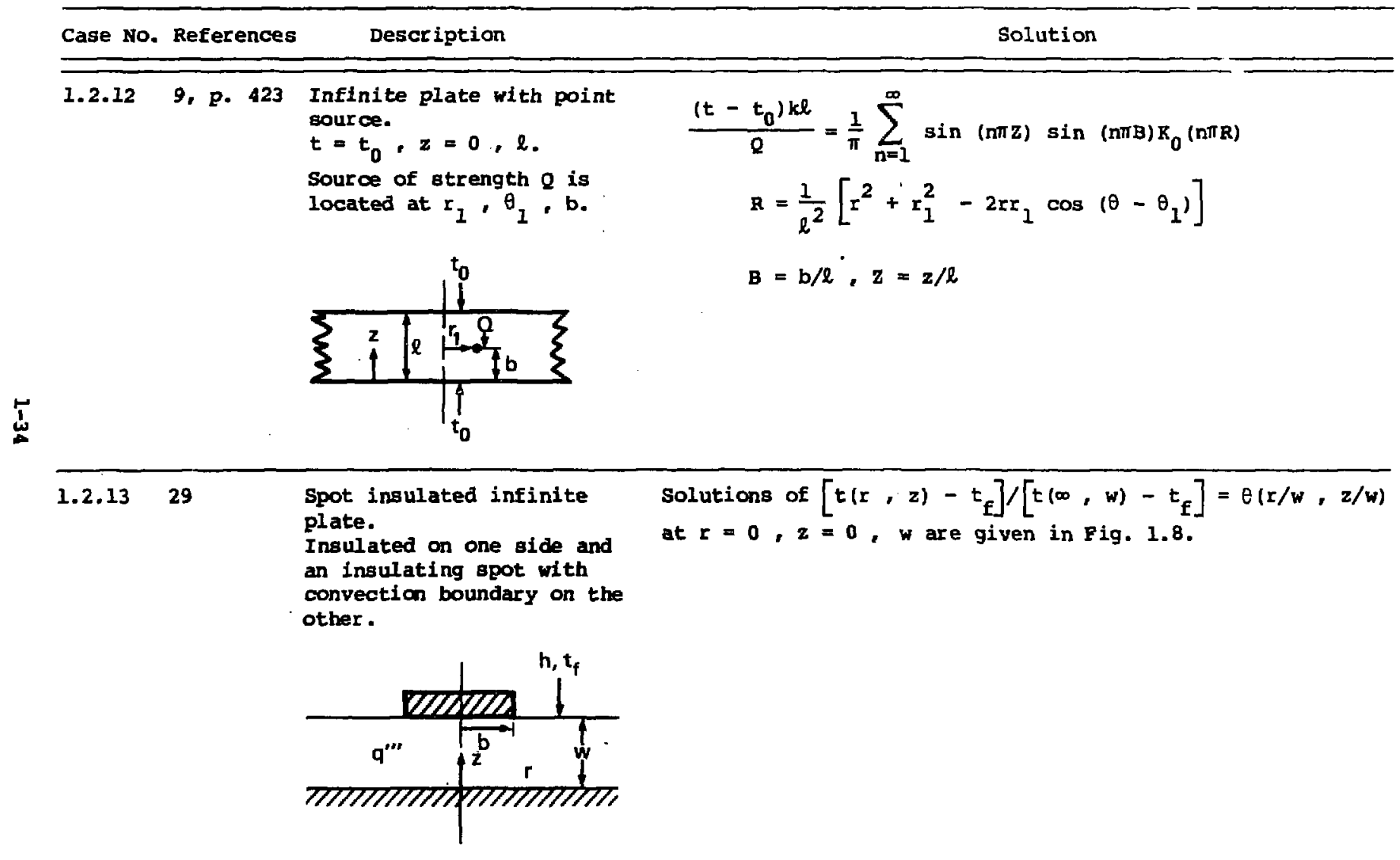


Section 1.2. Solids Bounded by Plane Surfaces-With Internal Beating. 
Section 1.2. Solids Bounded by Plane Surfaces--With Internal Heating.

\begin{tabular}{lll}
\hline Case No. References & Description & Solutiun \\
\hline
\end{tabular}




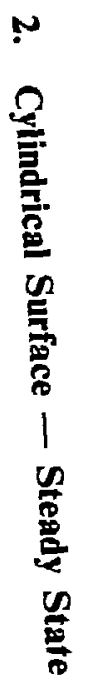


Section 2.1. Solids Bounaed by Cylindrical Surfaces--No Internal Heating.

$\frac{\text { Case No. References }}{\text { 2.1.1 4, p. } 37 \text { Infinite hollcw cylinder. } q=\frac{2 \pi k\left(t_{0}-t_{i}\right)}{\ln \left(r_{0} / r_{i}\right)+\left(1 / B i_{i}\right)+\left(1 / B i_{0}\right)}}$

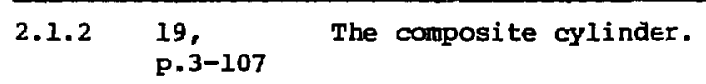

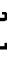

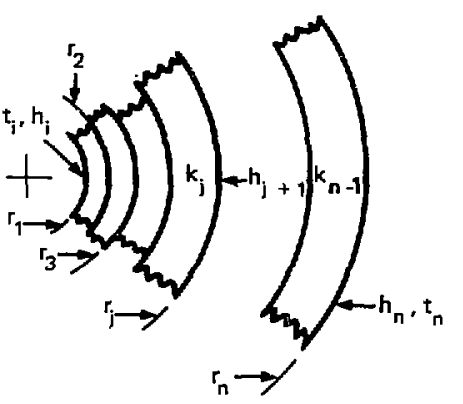

$$
q=\frac{2 \pi\left(t_{n}-t_{1}\right)}{\sum_{i=1}^{n-1} \frac{1}{k_{i}} \ln \left(\frac{r_{i}+1}{r_{i}}\right)+\sum_{i=1}^{n} \frac{1}{r_{i} h_{i}}}
$$

Temp in the jth layer:

$$
\begin{aligned}
& \frac{t_{j}-t_{1}}{t_{n}-t_{1}}= \\
& \frac{\sum_{i=1}^{j-1}\left[\frac{1}{k_{i}} \ln \left(\frac{r_{i}+1}{r_{i}}\right)+\frac{1}{r_{i} h_{i}}\right]+\frac{1}{k_{j}} \ln \left(\frac{r}{r_{j}}\right)+\frac{1}{r_{j} h_{j}}}{\sum_{i=1}^{n-1} \frac{1}{k_{i}} \ln \left(\frac{r_{i}+1}{r_{i}}\right)+\sum_{i=1}^{n} \frac{1}{r_{i} h_{i}}}, j>1
\end{aligned}
$$


Section 2.1. Solids Bounded by Cylindrical Surfaces--No Internal Heating

2.1.3 3, p. 121 Insulated tubes.

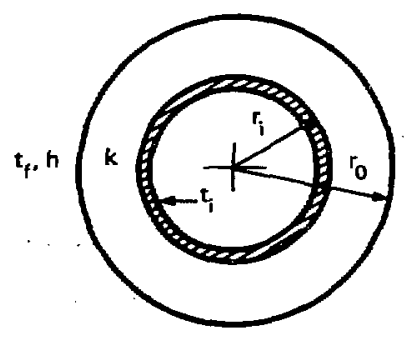

$$
q=\frac{2 \pi k\left(t_{i}-t_{E}\right)}{\ln \left(\frac{r_{0}}{r_{i}}\right)+\frac{1}{B i_{i}}}
$$

See Fig. 2.1

Max heat Ioss occurs when $r_{0}=k / h$.

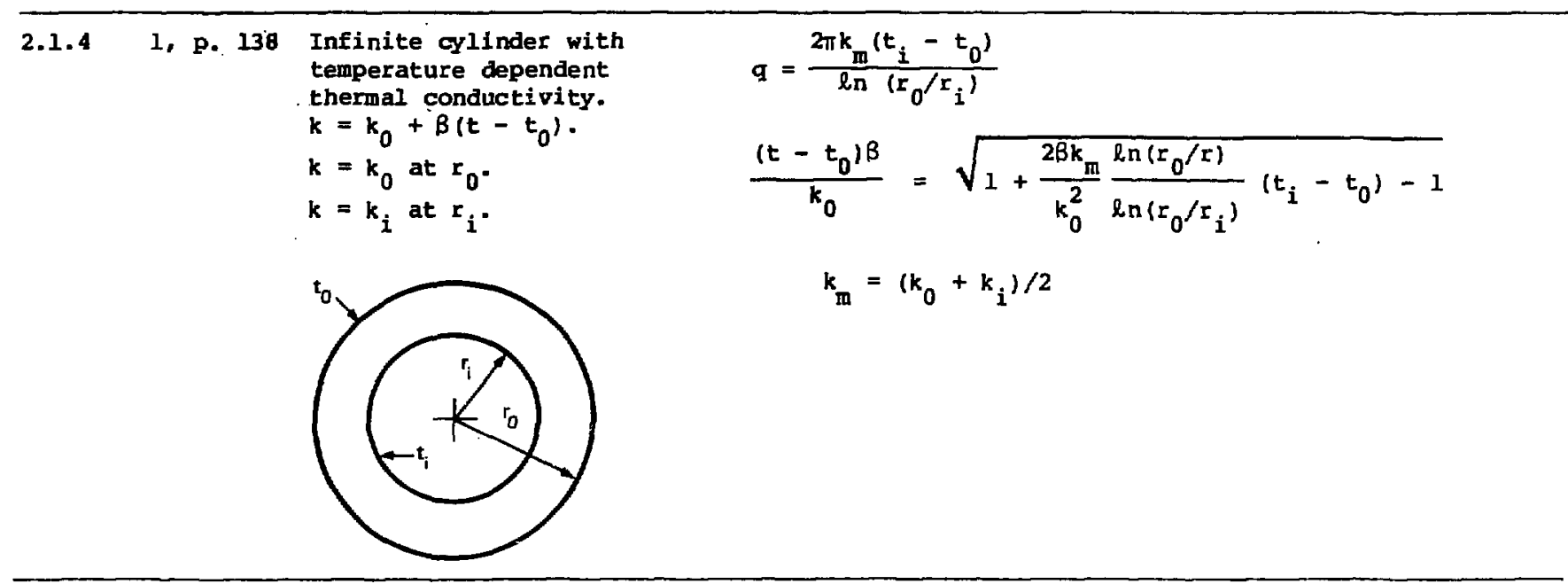


Section 2.1. Solids Bounded by Cylindrical Surfaces--No Internal Heating.

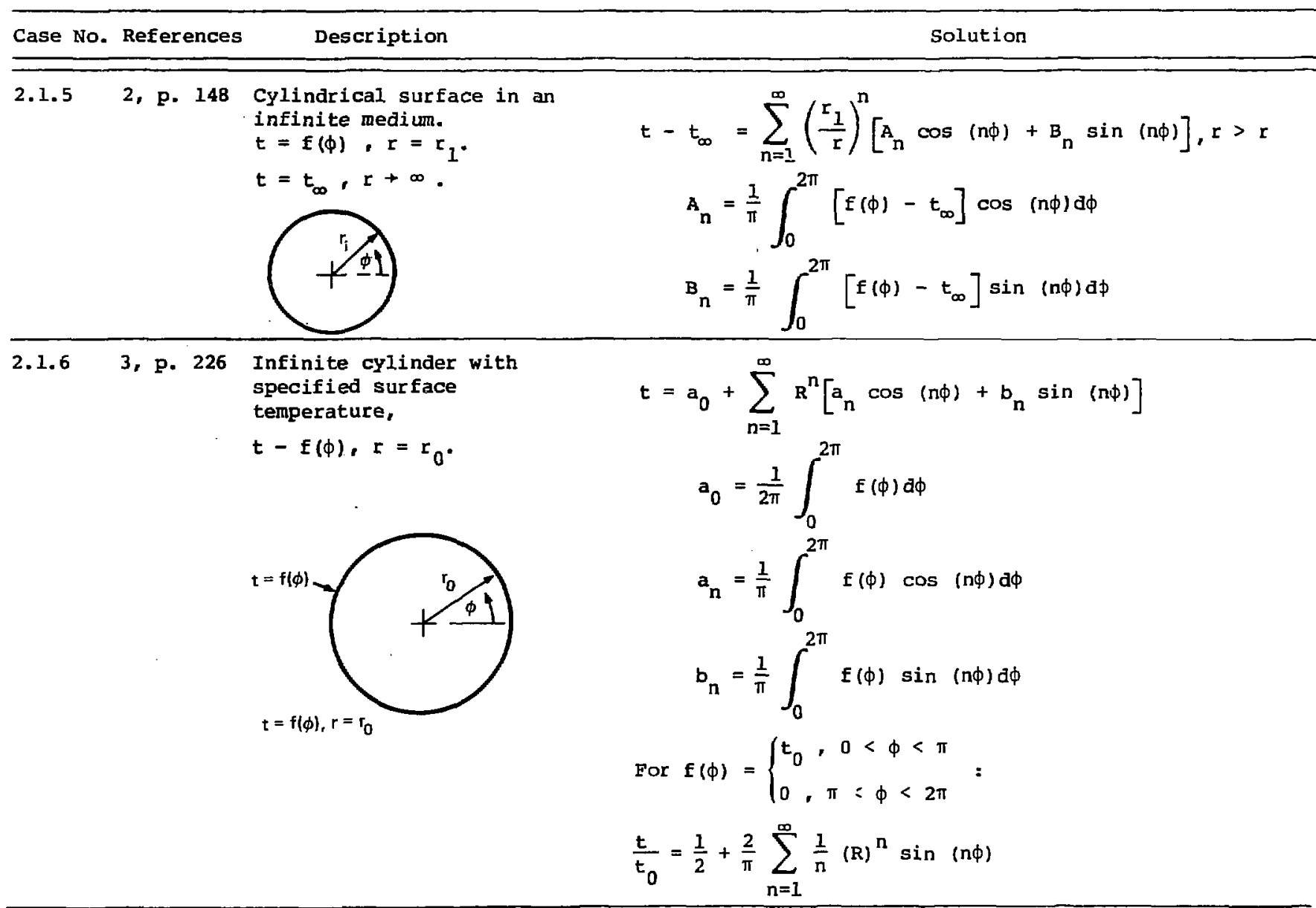


Section 2.1. Solids Bounded by Cylindrical Surfaces--No Internal Heating.

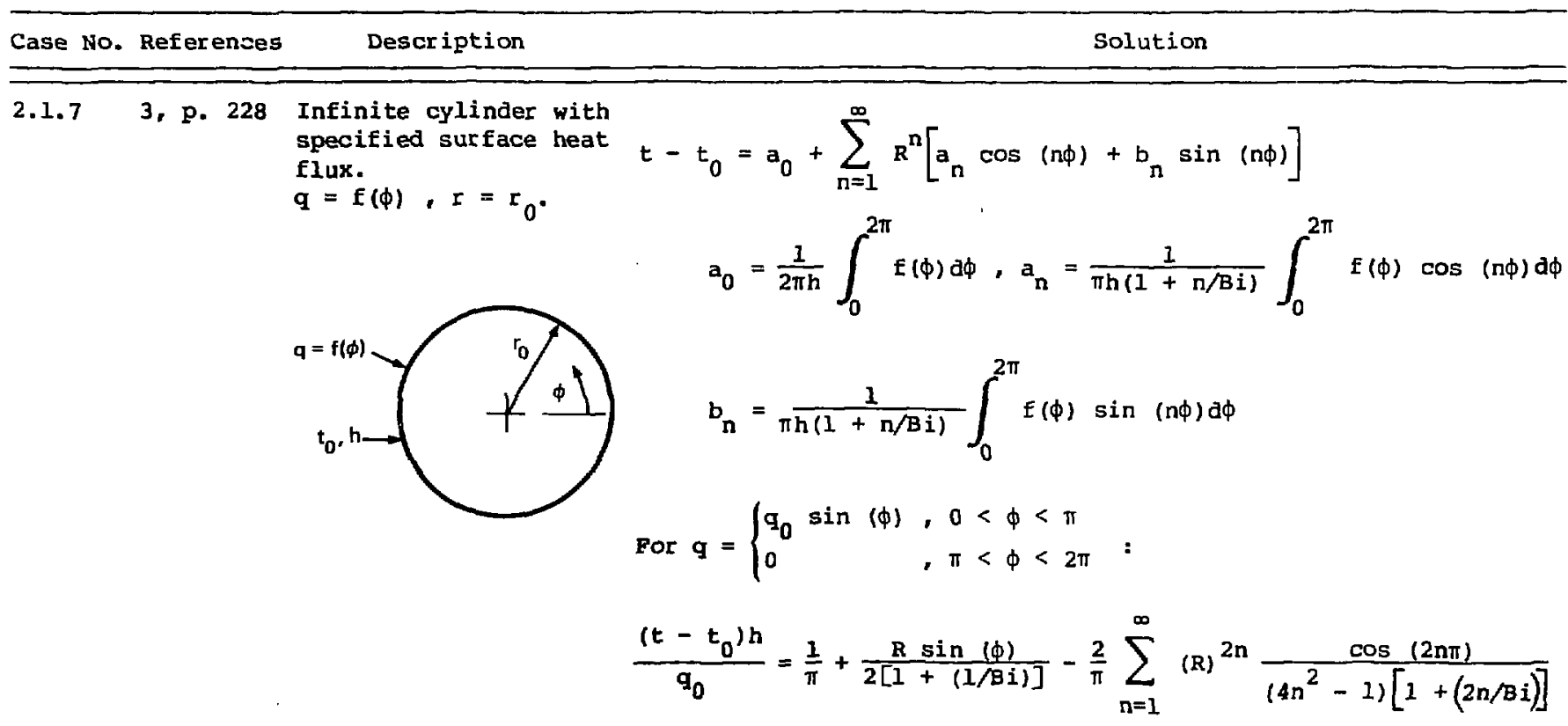


Section 2.1. Solids Bounded by Cylindrical Surfaces-No Internal Heating.

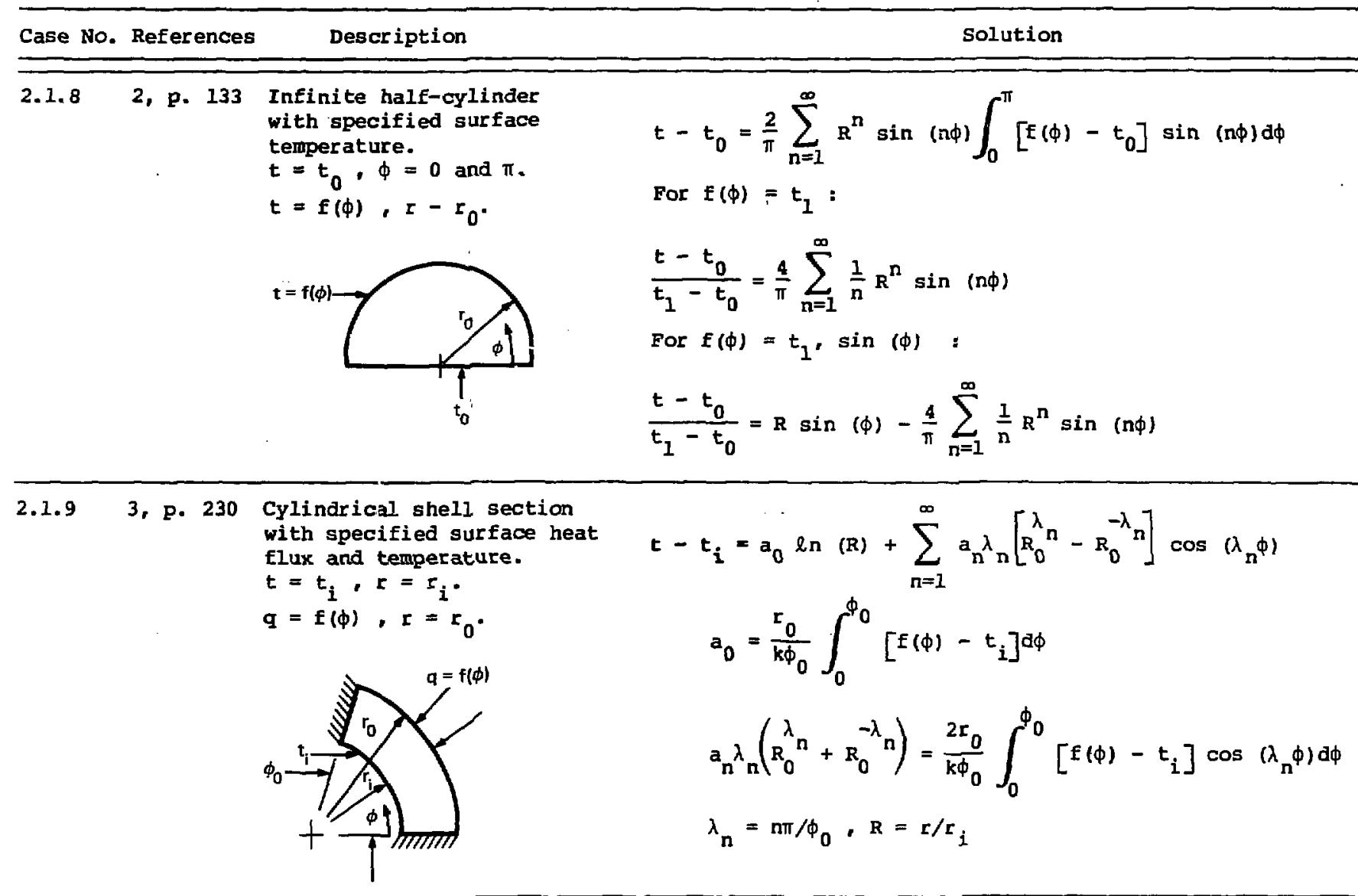


Section 2.1. Solids Bounded by Cylindrical Surfaces--No Internal Heating.

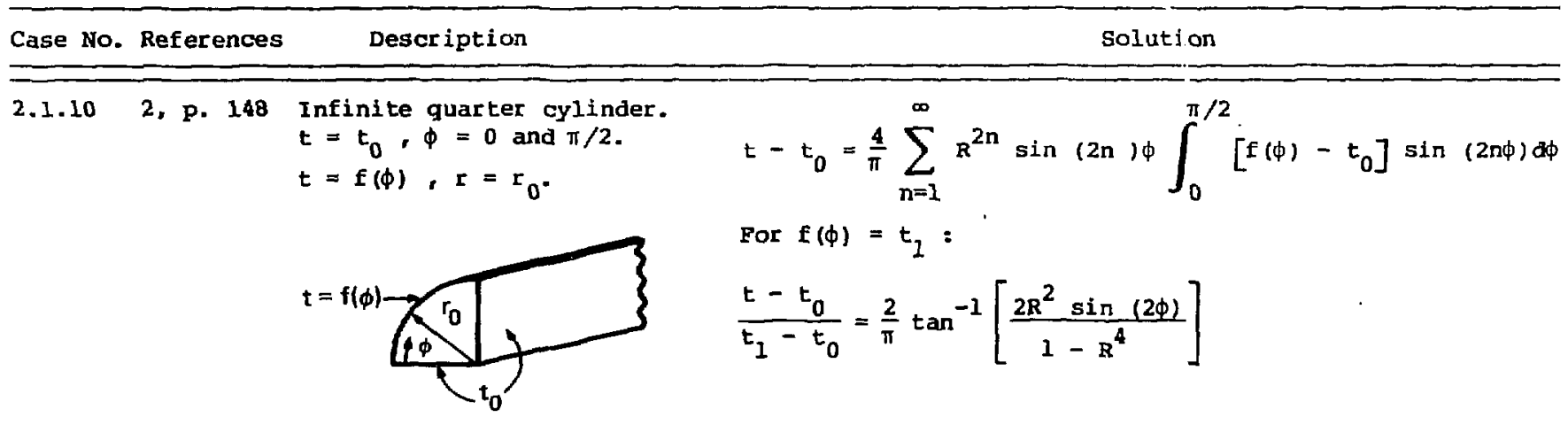
surface temperatures. $t=t_{1}, z=0$.

$t=t_{1}, r=r_{0}$.

$t=t_{2}, z=\ell$.

$$
\begin{aligned}
& \frac{t-t_{1}}{t_{2}-t_{1}}=2 \sum_{n=1}^{\infty} \frac{\sinh \left(\lambda_{n} 2\right) J_{0}\left(\lambda_{n} R\right)}{\lambda_{n} \sinh \left(\lambda_{n} L\right) J_{1}\left(\lambda_{n}\right)} \\
& J_{0}\left(\lambda_{n}\right)=0, z=z / r_{0}, R=r / r_{0}, L=\ell / r_{0}
\end{aligned}
$$

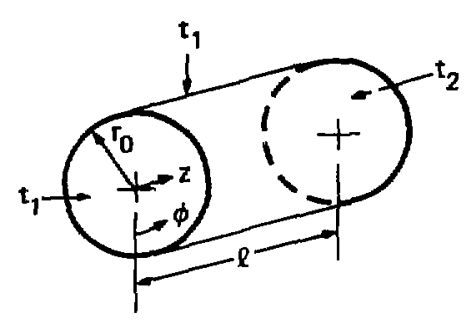

For $t_{2}=E(r)$ :

$t-t_{1}=\frac{2}{r_{0}^{2}} \sum_{n=1}^{\infty} \frac{\sinh \left(\lambda_{n} z\right) J_{0}\left(\lambda_{n} R\right)}{\sinh \left(\lambda_{n} L\right) J_{1}^{2}\left(\lambda_{n}\right)} \int_{0}^{l} R\left[f(R)-t_{1}\right]_{0}\left(\lambda_{n} R\right) d R$ 
Section 2.1. Solids Bounded by Cylindrical Surfaces--No Internal Heating.

Case No. References Description Solution

\begin{tabular}{|c|c|c|c|c|}
\hline 2.1 .12 & 3 , p. 253 & $\begin{array}{l}\text { Case 2.1.11 with } \\
t=t_{1}, r=0, r=\ell \\
t=t_{1}, r=r_{0}, 0<\phi<\pi \\
t=t_{0}, r=r_{0}, \pi<\phi<2 \pi\end{array}$ & $\begin{aligned} \frac{t}{t_{1}}-\frac{t_{0}}{-t_{0}}=\frac{2}{\pi} \sum_{n=1}^{\infty} \frac{I_{0}\left(\lambda_{n} R\right) \sin \left(\lambda_{n} z\right)}{n I_{0}\left(\lambda_{n} R_{0}\right)} \\
+\frac{8}{\pi^{2}} \sum_{n=1}^{\infty} \sum_{m=1}^{\infty} \frac{I_{m}\left(\lambda_{n} R\right)}{}\end{aligned}$ & $\frac{\sin \left(\lambda_{n} z\right) \sin (m \phi)}{\operatorname{nmI}\left(\lambda_{n} R_{0}\right)}$ \\
\hline
\end{tabular}

2.1.13 3, p. 234 Semi-infinite rod with variable end temperature.

$t=E(r), z=0$.

$t=t_{0}, r=r_{0}$.

$$
\begin{aligned}
t-t_{0} & =\sum_{n=1}^{\infty} A_{n} J_{0}\left(\lambda_{n} R\right) \exp \left(-\lambda_{n} z\right) \\
A_{n} & =\frac{2 \int_{0}^{1} R\left[f(R)-t_{0} J_{0}\left(\lambda_{n} R\right) d R\right.}{J_{1}^{2}\left(\lambda_{n}\right)} \\
z & =z / x_{0}, J_{0}\left(\lambda_{n}\right)=0, \lambda_{n}>0
\end{aligned}
$$

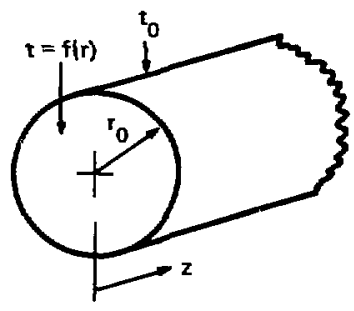

If $f(r)=t_{1}$ :

$\frac{t-t_{0}}{t_{1}-t_{0}}=2 \sum_{n=1}^{\infty} \frac{\exp \left(-\lambda_{n} 2\right) J_{0}\left(\lambda_{n}^{R}\right)}{\lambda_{n} J_{1}\left(\lambda_{n}\right)}$ 
Section 2.1. Solids Bounded by Cylindrical Surfaces--No Internal Heating.

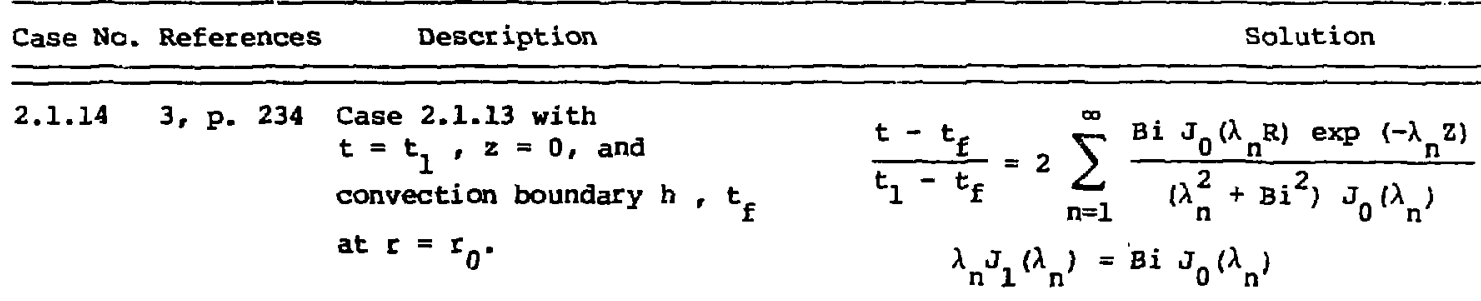

2.1.15 3, p. 238 Infinite rod with a traveling boundary between two temperature zones.

$t=t_{1}, r=r_{0}, z<0$.

$t=t_{2}, r=r_{0}, z>0$.

velocity of boundary $(z=0)=v$.

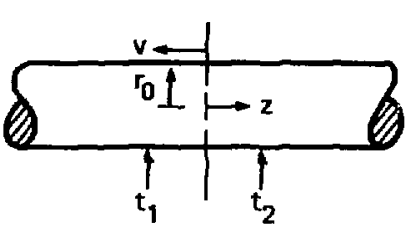

$\frac{t-t_{2}}{t_{1}-t_{2}}=1-\sum_{n=1}^{\infty}\left[1-\frac{1}{\left[1+\left(\lambda_{n} / P\right)^{2}\right]^{1 / 2}}\right] \frac{J_{0}\left(\mu_{n} R\right)}{\lambda_{n} J_{1}\left(\lambda_{n}\right)}$

$x \exp \left[\left\{1+\left[1+\left(\lambda_{n} / P\right)^{2}\right]^{3 / 2}\right\} P z\right], z<0$

$\frac{t-t_{2}}{t_{1}-t_{2}}=\sum_{n=1}^{\infty}\left[1+\frac{1}{\left[1+\left(\lambda_{r} / D\right)^{2}\right]^{1 / 2}}\right] \frac{J_{0}\left(\lambda_{n} R\right)}{\lambda_{n} J_{1}\left(\lambda_{n}\right)}$

$x \exp \left[\left\{1-\left[1+\left(\lambda_{n} / P\right)^{2}\right] 1 / 2 \mid P z\right], z>0\right.$

$\mathrm{z}=\mathrm{z} / \mathrm{r}_{0}, \mathrm{P}=\mathrm{vr}_{0} / \alpha_{\mathrm{r}} \mathrm{J}_{0}\left(\lambda_{\mathrm{n}}\right)=0$ 
Section 2.1. Solids Bounded by Cylindrical Surfaces--No Internal Heating.

\begin{tabular}{|c|c|c|c|}
\hline Case No. & References & Description & Solution \\
\hline 2.1 .16 & 3, p. 238 & $\begin{array}{l}\text { Finite rod with band heating. } \\
q_{z}=0, z= \pm b \text {. } \\
\text { Convection boundary at } r=r_{0^{\prime}} \\
\text {-b<z<+b. } \\
\text { Surface heating }\left(q_{s}\right) \text { at } r=r_{0^{\prime}} \\
-\ell<z<+l \text {. }\end{array}$ & $\begin{aligned} \frac{t-t_{f}}{g_{s} I_{0} / k} & =\frac{L}{B i}+2 \sum_{n=1}^{\infty} \frac{\sin \left(\lambda_{n} L\right) I_{0}\left(\lambda_{n} \rho R\right) \cos \left(\lambda_{n} z\right)}{\lambda_{n}\left[B i I_{0}\left(\lambda_{n} \rho\right)+\left(\lambda_{n} \rho\right) I_{1}\left(\lambda_{n} \rho\right)\right]} \\
\lambda_{n} & =n \pi, B i=h r_{0} / k, L=l / b, z=z / b \\
R & =r / r_{0}, \rho=r_{0} / b\end{aligned}$ \\
\hline
\end{tabular}

2.1.17 4, p. 37 Eccentric hollow cylinder.

$t=t_{2}, r=r_{2}$.

$t=t_{1}, r=r_{1}$

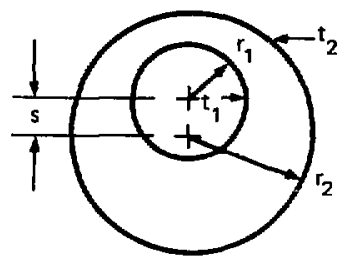

$$
\begin{gathered}
q=\frac{2 \pi k\left(t_{2}-t_{1}\right)}{\ln \left[\frac{\sqrt{(R+1)^{2}-s^{2}}+\sqrt{(R-1)^{2}-s^{2}}}{\sqrt{(R+1)^{2}-s^{2}}-\sqrt{(R-1)^{2}-s^{2}}}\right]} \\
R=r_{2} / r_{1}, s=s / r_{1}
\end{gathered}
$$

See Ref. 80 for other solutions to eccentric cylinder. 
Section 2.1. Solids Bounded by Cylindrical Surfaces--No Internal Eeating.

Case No. References Description Solution

2.1.18 4, p. 39 Pipe in semi-infinite solid.

$d>4 r_{1}$.

$q=\frac{2 \pi k_{1}\left(t_{2}-t_{1}\right)}{\frac{1}{B i_{1}}+\ln \left(\frac{r_{2}}{r_{1}}\right)+\frac{1}{R} \ln \left[2 D+\frac{2 K D}{B i_{2}}\right]}$

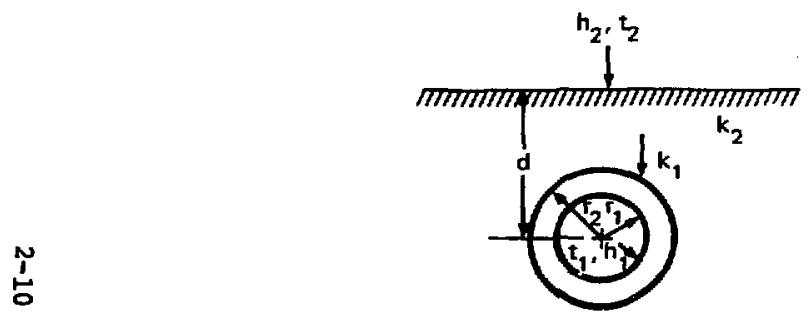

$$
B i_{1}=\frac{h_{1} r_{1}}{k_{1}}, B i_{2}=\frac{h_{2} d}{k_{2}}, K=\frac{k_{2}}{k_{1}}, D=\frac{d}{r_{2}}
$$

2.1.19 4, p. 40 Row of pipes in semiinfinite solid.

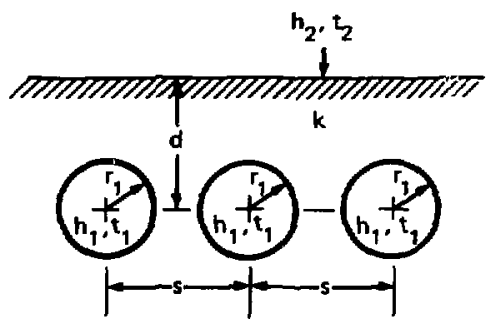

$q=\frac{2 \pi k\left(t_{2}-t_{1}\right)}{\frac{1}{R 1_{1}}+\ln \left[\pi \frac{d}{D r_{1}} \sinh \left(\pi 2\left[D+\frac{D}{B i_{2}}\right]\right)\right]}$, for one pipe. $B i_{1}=\frac{h_{1} r_{1}}{k}, B i_{2}=\frac{h_{2} d}{k}, D=\frac{d}{s}$ 
Section 2.1. Solids Bounded by Cylindrical Surfaces--No Internal Heating.

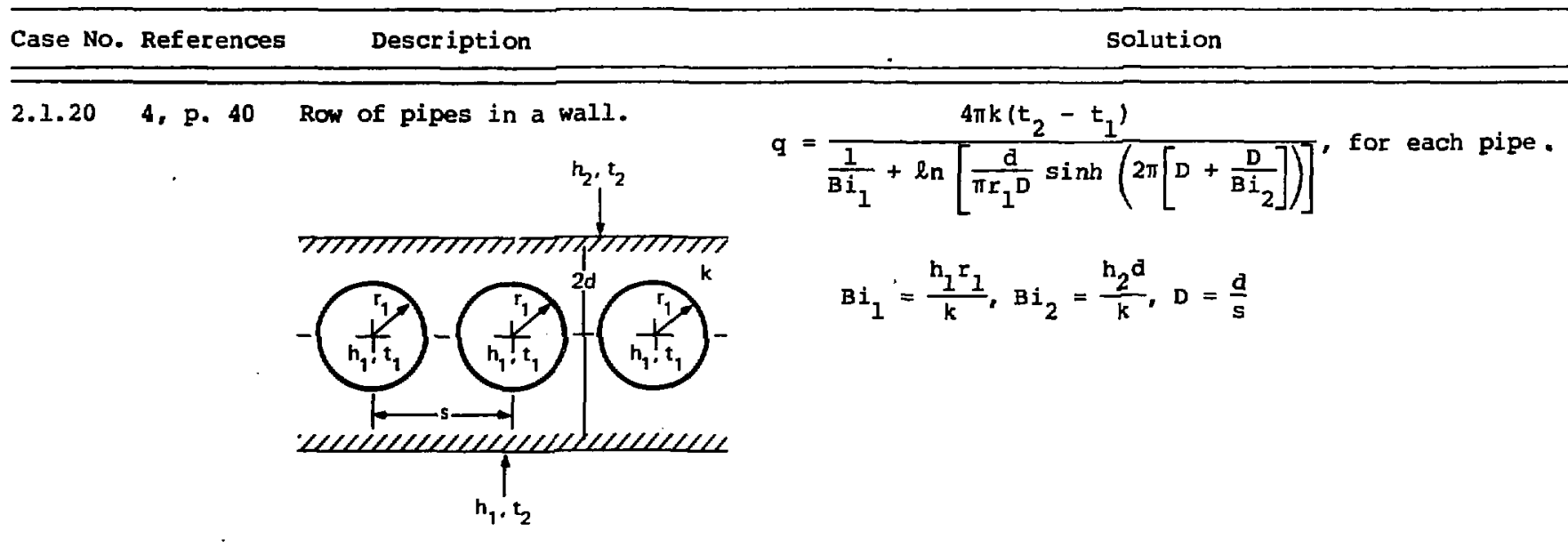

2.1.21 4, p. 38 Two pipes in a semiinfinite solid.

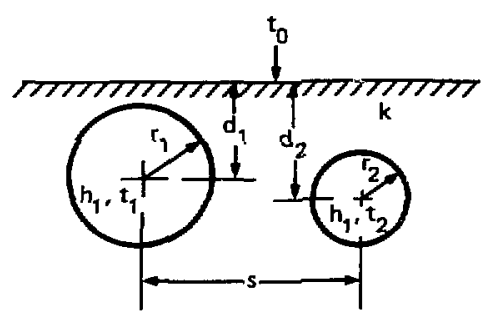

$$
q_{1}=\frac{2 \pi k\left(t_{1}-t_{0}\right) z_{1}}{z_{2}}
$$

$$
\begin{aligned}
& z_{1}=\ln \left(\frac{2 d_{2}}{r_{2}}\right)-\frac{t_{2}-t_{0}}{t_{1}-t_{0}} \ln \sqrt{\frac{s^{2}+\left(d_{1}+d_{2}\right)^{2}}{s^{2}+\left(d_{1}-d_{2}\right)^{2}}} \\
& z_{2}=\ln \left(\frac{2 d_{1}}{r_{1}}\right) \ln \frac{2 d_{2}}{r_{2}}+\left(\ln \sqrt{\frac{s^{2}+\left(d_{1}+d_{2}\right)}{s^{2}+\left(d_{1}-d_{2}\right)^{2}}}\right)^{2}
\end{aligned}
$$

For $q_{2}$, interchange indices 1 and 2 . 
Section 2.1. Solids Bounded by Cylindrical Surfaces--No Internal Heating.

\begin{tabular}{|c|c|c|c|}
\hline Case No. & References & Descr iption & Solution \\
\hline 2.1 .22 & $\begin{array}{ll}4, & \text { p. } 43 \\
9, & \text { p. } 215\end{array}$ & $\begin{array}{l}\text { Circular disk on the } \\
\text { surface of a semi-infinite } \\
\text { solid. } \\
t=t_{1}, z+\infty \text {. }\end{array}$ & $\begin{array}{l}q=4 r_{0} k\left(t_{2}-t_{1}\right) \\
\frac{t-t_{1}}{t_{2}-t_{1}}=\frac{2}{\pi} \sin ^{-1}\left[\frac{2}{\left[(R-1)^{2}+z^{2}\right]^{1 / 2}+\left[(R+1)^{2}+z^{2}\right]^{1 / 2}}\right. \\
z=2 / r_{0}\end{array}$ \\
\hline 2.1 .23 & 4, p. 44 & $\begin{array}{l}\text { Circular disk in an } \\
\text { infinite solid. } \\
t=t_{1}, 2+ \pm \infty_{*} \\
\end{array}$ & $q=8 r_{0} k\left(t_{2}-t_{1}\right)$ \\
\hline 2.1 .24 & 4, p. 42 & $\begin{array}{l}\text { Circular ring in a semi- } \\
\text { infinite solid. }\end{array}$ & $q=\frac{4 \pi^{2} r_{2}\left(t_{1}-t_{0}\right)}{\ln \left(\frac{8 r_{2}}{r_{1}}\right)+\ln \left[\frac{4 r_{2}}{d\left(1+\frac{1}{B i_{d}}\right)}\right]}, r_{1} \ll d \ll r_{2}$ \\
\hline
\end{tabular}


Section 2.1. Solids Bounded by Cylindx' רl Surfaces--No Internal Heating.

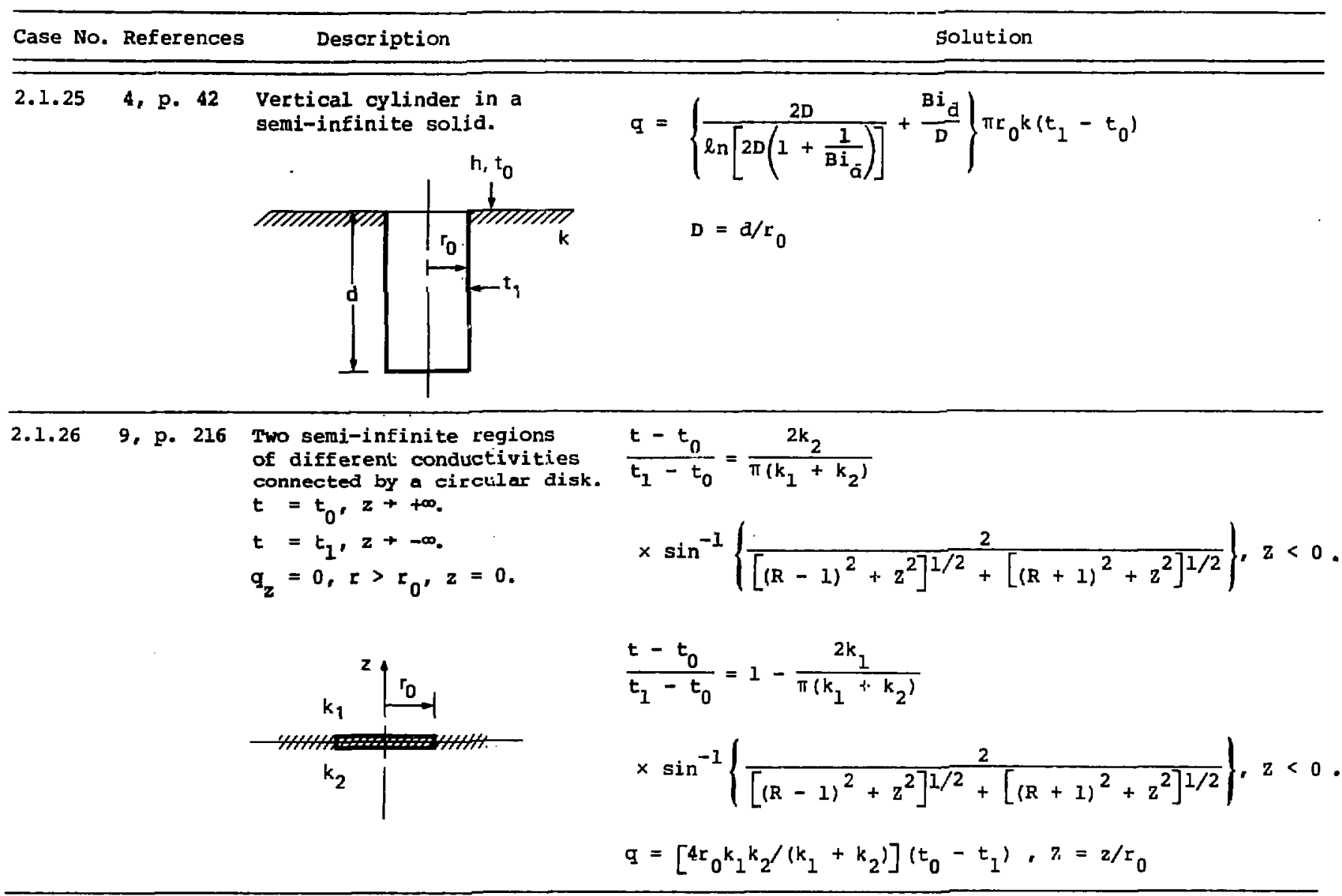


Section 2.1. Solids Bounded by Cylindrical Surfaces--No Internal Heating.

\begin{tabular}{|c|c|c|c|}
\hline Case No. & References & Description & Solution \\
\hline 2.1 .27 & $\begin{array}{l}9, \text { p. } 218 \text {, } \\
69\end{array}$ & $\begin{array}{l}\text { Finite cylinder with: } \\
t=f(r), z=0,0<r<r_{0^{\circ}} \\
t=t_{0}, z=l, 0<r<r_{0^{\circ}} \\
\text { Convection boundary at } \\
r=r_{0}, 0<z<l, \\
\text { with } h, t_{0} .\end{array}$ & $\begin{aligned} t-t_{0} & =\sum_{n=1}^{\infty} c_{n} \frac{J_{0}\left(R \lambda_{n}\right) \sinh \left([1-2] L \lambda_{n}\right)}{\sinh \left(L \lambda_{n}\right)} \\
c_{n} & =\frac{2 \lambda_{n}^{2}}{\left(B i^{2}+\lambda_{n}^{2}\right) J_{0}^{2}\left(\lambda_{n}\right)} \int_{0}^{1} R\left[E(R)-t_{0}\right]_{0}^{J_{0}\left(R \lambda_{n}\right) d R} \\
L & =\ell / r_{0}, B i=h r_{0} / k, \lambda_{n} J j\left(\lambda_{n}\right)+B i J_{0}\left(\lambda_{n}\right)=0, \lambda_{n}>0 \\
\text { For } f(r) & =t_{1}: \\
\frac{t-t_{0}}{t_{1}-t_{0}} & =2 B i \sum_{n=1}^{\infty} \frac{J_{0}\left(R \lambda_{n}\right) \sinh \left([--z] L \lambda_{n}\right)}{\left(B i^{2}+\lambda_{n}^{2}\right) J_{0}\left(\lambda_{n}\right) \sinh \left(L \lambda_{n}\right)}\end{aligned}$ \\
\hline
\end{tabular}

2.1.28 9, p. 219 Case 2.1.27 with $t=f(r)$, $z=0,0<r<r_{0}$.

Remaining surfaces convection boundaries $h, t_{0}$.

$$
\begin{aligned}
t-t_{0}=\sum_{n=1}^{\infty} c_{n} J_{0}\left(R \lambda_{n}\right) \\
\quad \times \frac{\lambda_{n} \cosh \left([1-z] L \lambda_{n}\right)+B i \sinh \left([1-z] L \lambda_{n}\right)}{\lambda_{n} \cosh \left(L \lambda_{n}\right)+B i \sinh \left(L \lambda_{n}\right)}
\end{aligned}
$$


Section 2.1. Solids Bounded by Cylindrical Surfaces--No Internal Heating.

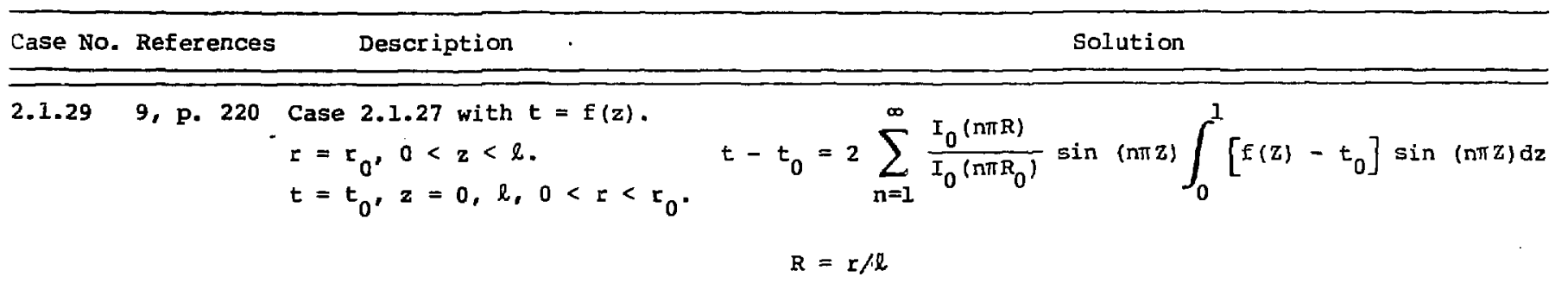

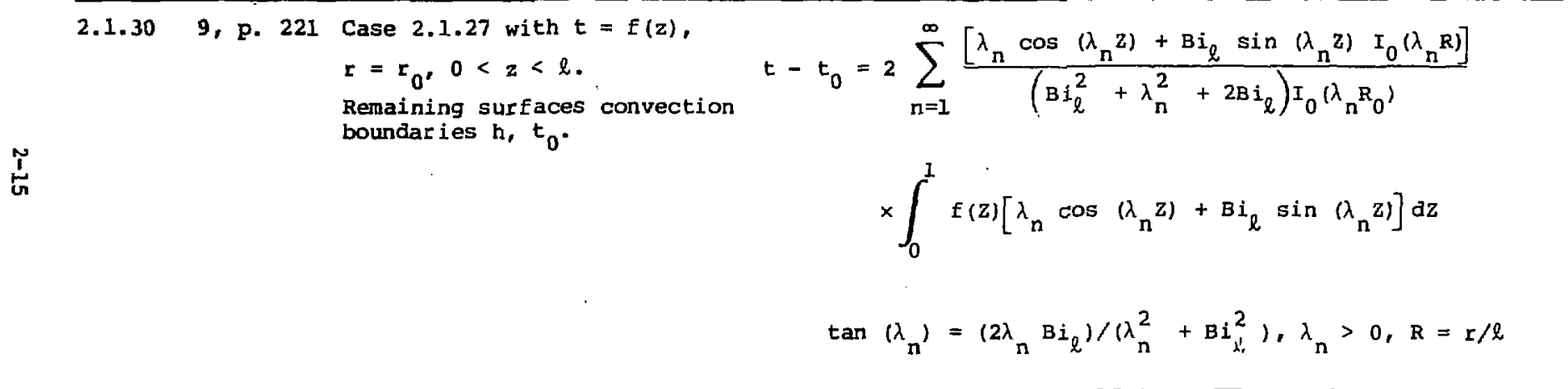


Section 2.1. Solids Bounded by Cylindrical Surfaces--No Internal Heating.

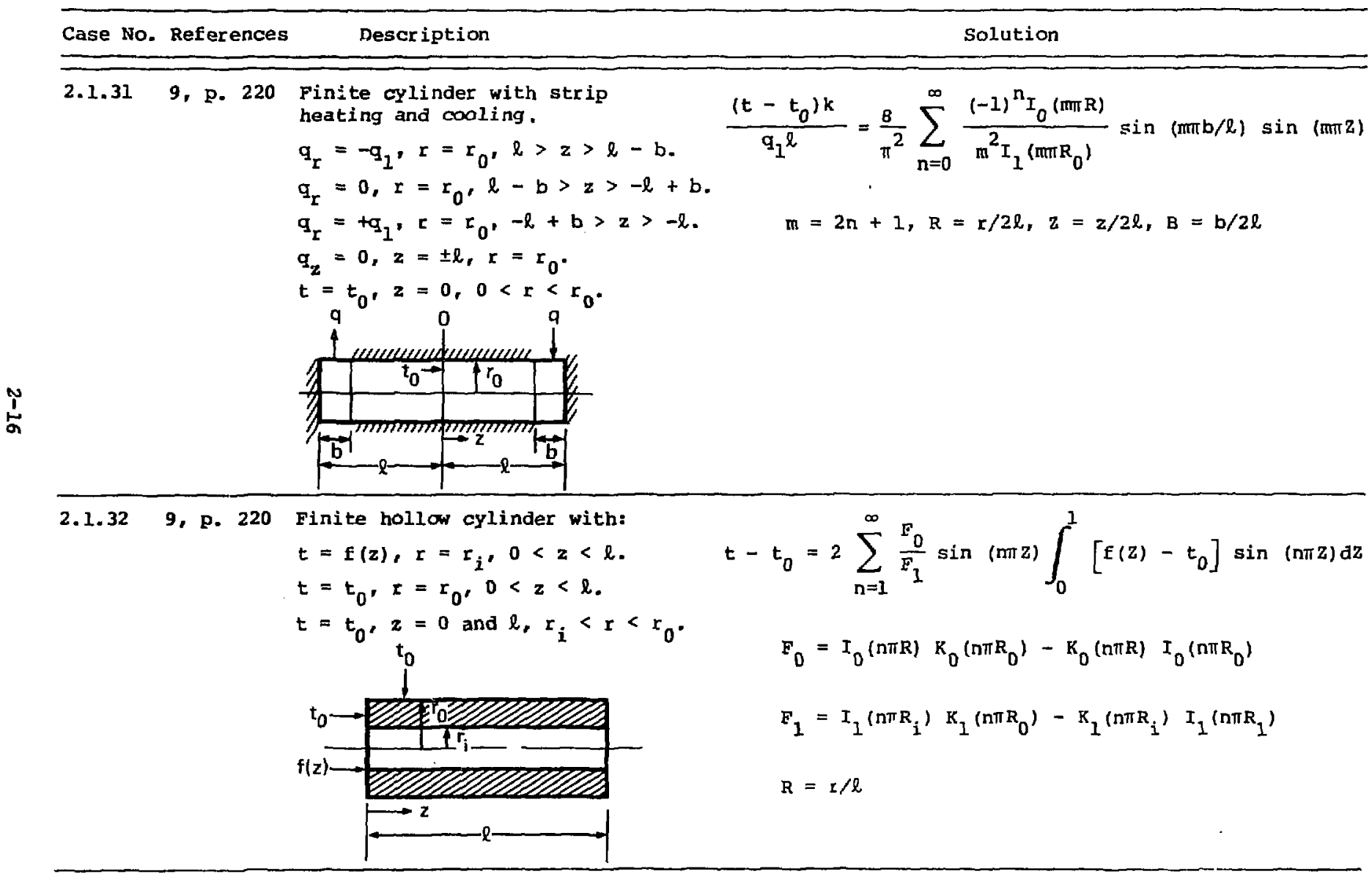


Section 2.1. Solids Bounded by Cylindrical Surfaces--No Internal Heating.

\begin{tabular}{|c|c|c|c|}
\hline Case No. & References & Description & Solution \\
\hline 2.1 .33 & 9, p. 221 & $\begin{array}{l}\text { Case } 2.1 .32 \text { with } q_{r}=f(z), \\
r=r_{i}, 0<z<\ell . \\
\text { Remaining surfaces at } t_{0^{*}}\end{array}$ & $\begin{array}{l}t-t_{0}=-\frac{2 l}{k \pi} \sum_{n=1}^{\infty} \frac{P_{0}}{n F_{1}} \sin (n \pi z) \int_{0}^{1} f(z) \sin (n \pi z) d z \\
F_{0} \text { defined in case } 2.1 .32 \\
F_{1}=I_{1}\left(n \pi R_{i}\right) k_{0}\left(n \pi R_{0}\right)+R_{I}\left(n \pi R_{i}\right) I_{0}\left(n \pi R_{0}\right) \\
\text { For } f(z)=q_{1}, w<z<(l-w) \\
\quad=0,0<z<w \text { and }(l-w)<z<l: \\
\frac{\left(t-t_{0}\right) k}{q_{1} l}=\sum_{n=1}^{\infty} \frac{F_{0}}{n^{2} F_{1}} \cos (n \pi w) \sin (n \pi z), n=1,3,5, . . \\
w=w / l\end{array}$ \\
\hline
\end{tabular}


Section 2.1. Solids Bounded by Cylindrical Surfaces--No Internal Heating.

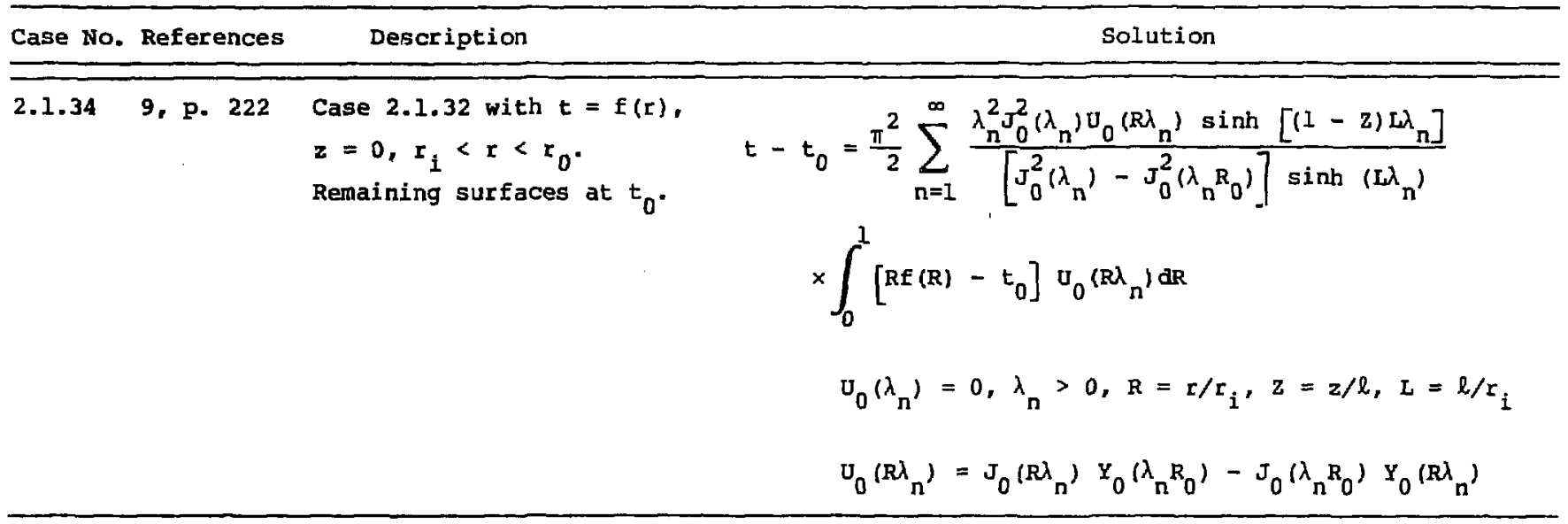

2.1.35 9, p. 222 Case 2.1.32 with $t=f(z)$, $r=r_{i}, 0<z<\ell$. Remaining surfaces convection boundary with $h, t_{f}$.

$$
\begin{gathered}
t-t_{f}=2 \sum_{n=1}^{\infty} \frac{\left[\lambda_{n} \cos \left(\lambda_{n} z\right)+B i \sin \left(\lambda_{n} z\right)\right] G(R, n)}{\left[\lambda_{n}^{2}+B i_{\ell}^{2}+2 B i_{\ell}\right] G\left(r_{0}, n\right)} \\
\quad \int_{0}^{I}\left[f(z)-t_{f}\right]\left[\lambda_{n} \cos \left(\lambda_{n} Z\right)+B i_{\ell} \sin \left(\lambda_{n} z\right)\right] d z \\
\tan \left(\lambda_{n}\right)=2 \lambda_{n} B i_{\ell} /\left(\lambda_{n}^{2}-B i_{\ell}^{2}\right), \lambda_{n}>0 \\
G(R, n)=I_{0}\left(R \lambda_{n}\right)\left[\lambda_{n} K_{1}\left(\lambda_{n} R_{0}\right)-B i_{\ell} K_{0}\left(\lambda_{n} R_{0}\right)\right] \\
\quad+K_{0}\left(R \lambda_{n}\right)\left[\lambda_{n} I_{1}\left(\lambda_{n} R_{0}\right)+B i I_{0}\left(\lambda_{n} R_{0}\right)\right]
\end{gathered}
$$


Section 2.1. Solids Bounded by Cylindrical Surfaces--No Internal Heating.

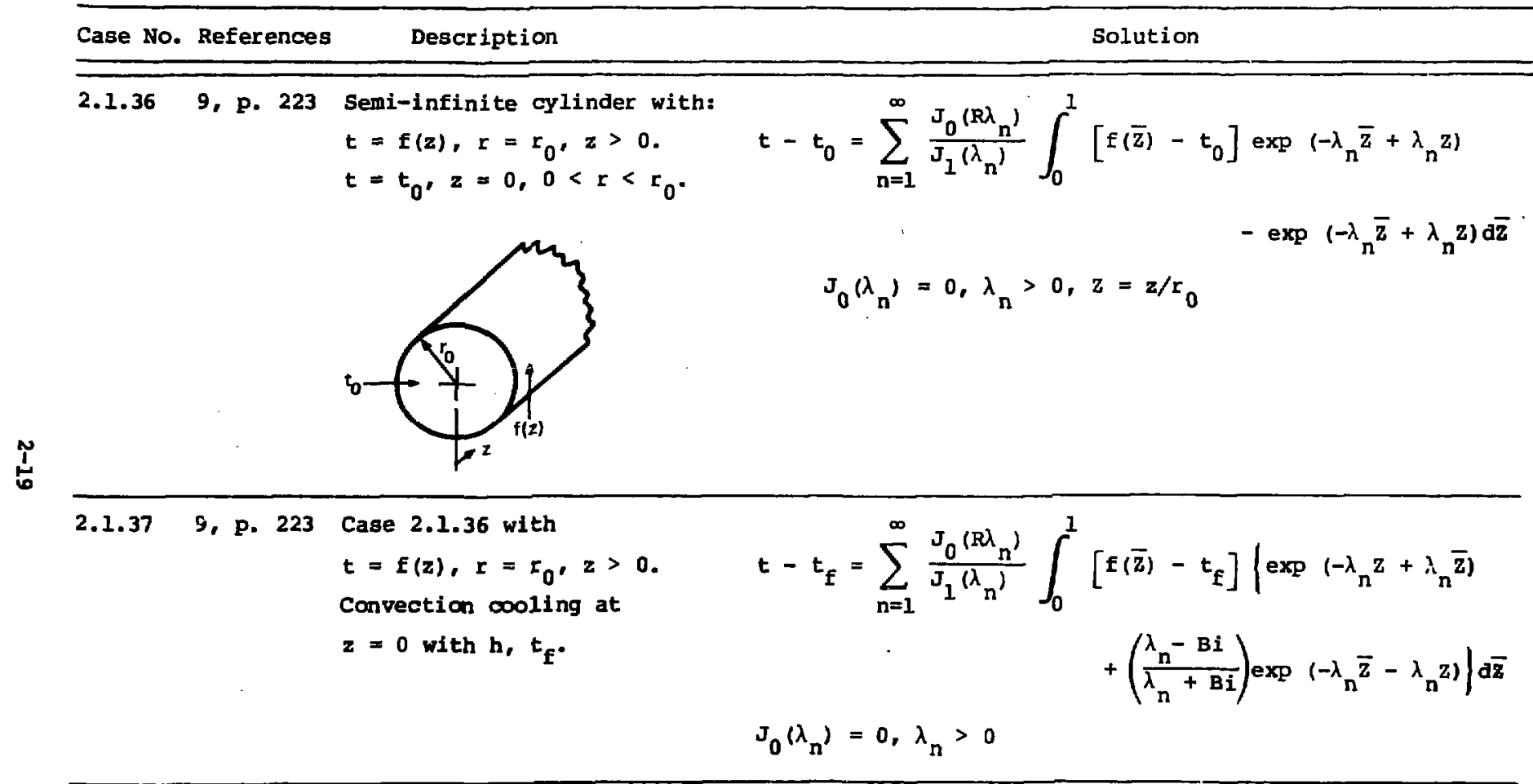


Section 2.1. Solids Bounded by Cylindrical Surfaces--No Internal Heating.

\begin{tabular}{|c|c|c|c|}
\hline Case No. & References & Description & Solution \\
\hline 2.1 .38 & 9. p. 223 & $\begin{array}{l}\text { Rinite cylinder with heated } \\
\text { disks on ends. } \\
\mathrm{q}_{2}=+\mathrm{q}_{1}, \mathrm{z}=0, \mathrm{\ell}, 0<\mathrm{r}<\mathrm{r}_{1} \text {. } \\
\text { Remaining surfaces insulated. }\end{array}$ & $\begin{array}{l}\frac{\left(t_{m_{1}, 0}-t_{m_{l} \ell}\right) k}{q_{1} l}=1-\frac{16}{\pi^{2}} \sum_{n=1}^{\infty} \frac{I_{1}\left(n \pi R_{1}\right)}{n^{2} I_{1}\left(n \pi R_{0}\right)}\left[I_{1}\left\langle n \pi R_{0}\right) K_{1}\left(n \pi R_{1}\right)\right. \\
\left.\quad-k_{1}\left(n \pi R_{0}\right) I_{1}\left(n \pi R_{1}\right)\right], n=1,3,5, \ldots \ldots \\
R=I / l \\
t_{m, 0}=\text { mean temp. over region } 0<I<r_{1}, z=0 \\
t_{m, l}=\text { mean temp. over region } 0<r<r_{1}, z=l\end{array}$ \\
\hline
\end{tabular}

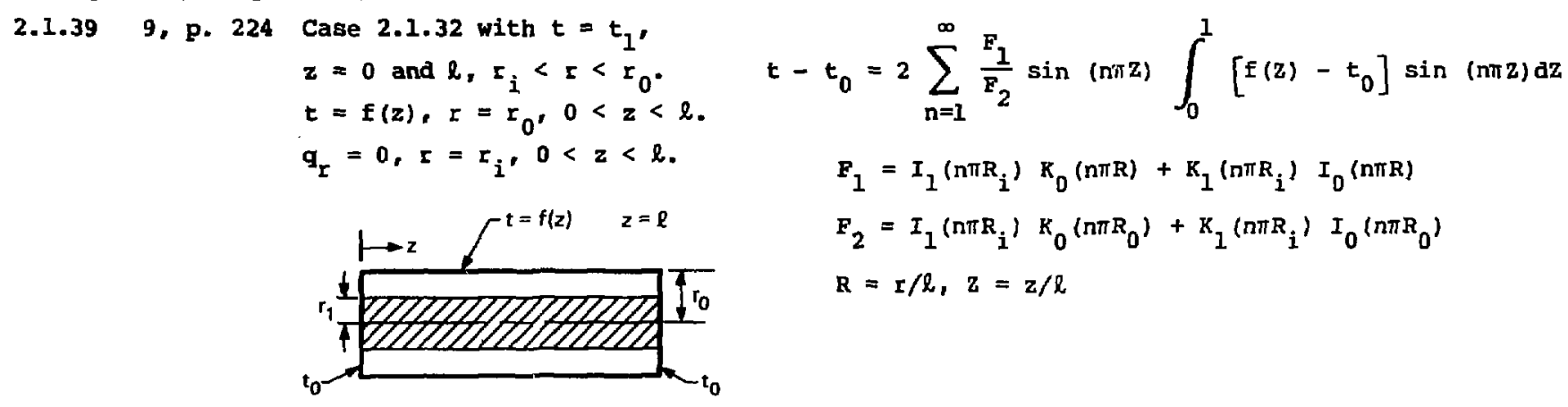


Section 2.1. Solids Bounded by Cylindrical Surfaces--No Internal Heating.

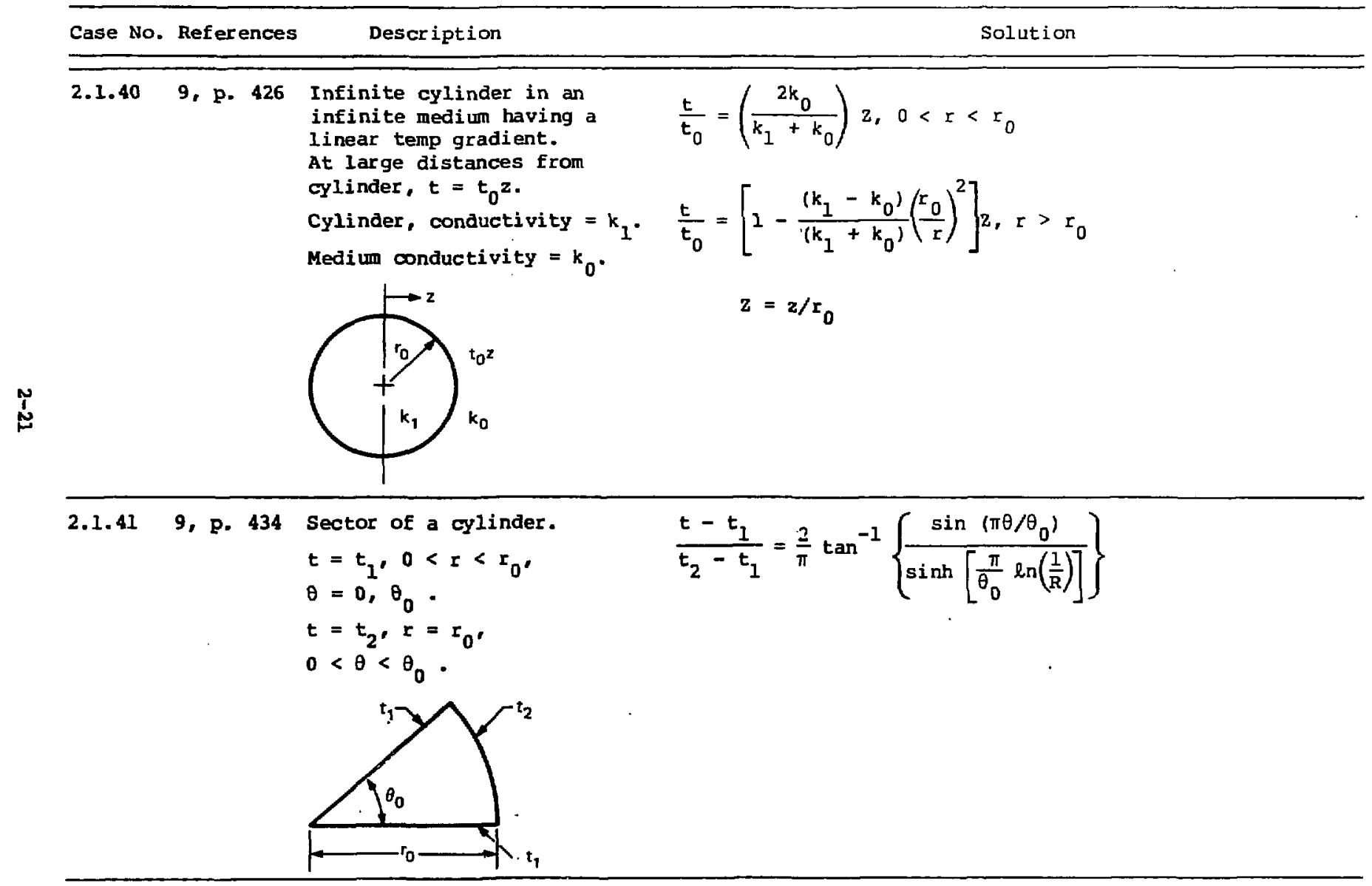


Section 2.1. Solids Bounded by Cylindrical Surfaces--No Internal Heating.

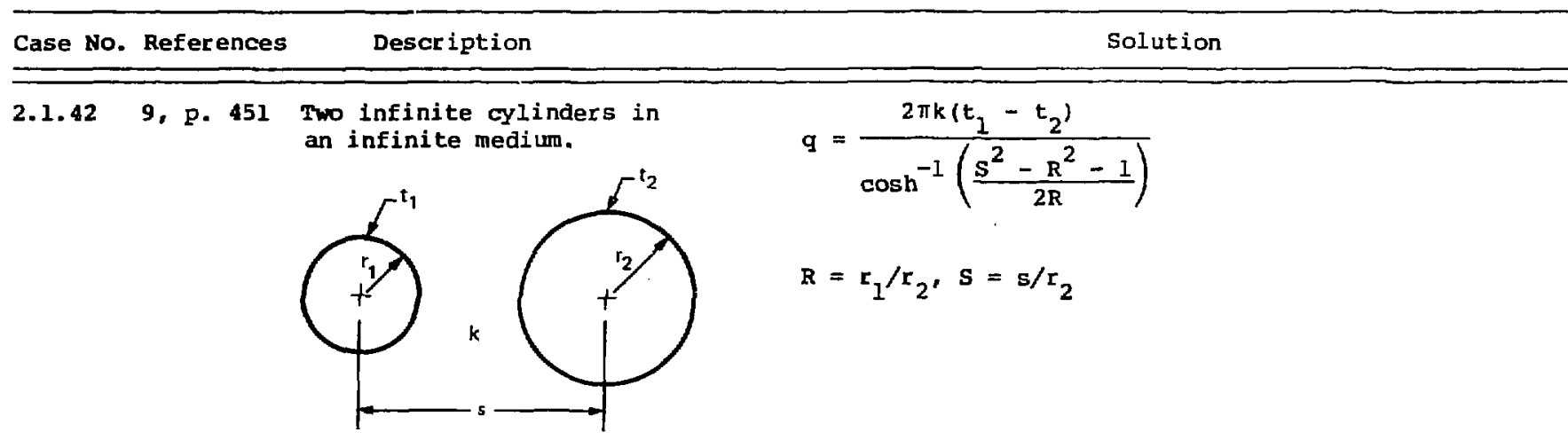

2.1.43 9, p. 462 Spot heated semi-infinite solid.

$q_{z}=q_{0}, 0<r<r_{0}, z=0$.

$q_{z}=0, I>I_{0}, z=0$.

$\frac{\left(t-t_{\infty}\right) k}{r_{0} q_{0}}=\int_{0}^{\infty} \exp (-\lambda z) J_{0}(\lambda R) J_{l}(\lambda) \frac{d \lambda}{\lambda}$

$t=t_{\infty}, r>0, z+\infty$.

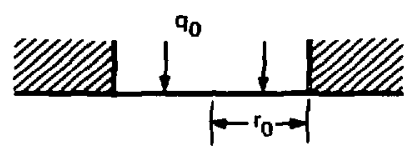


Section 2.1. Solids Bounded by Cylindrical Surfaces--No Internal Heating.

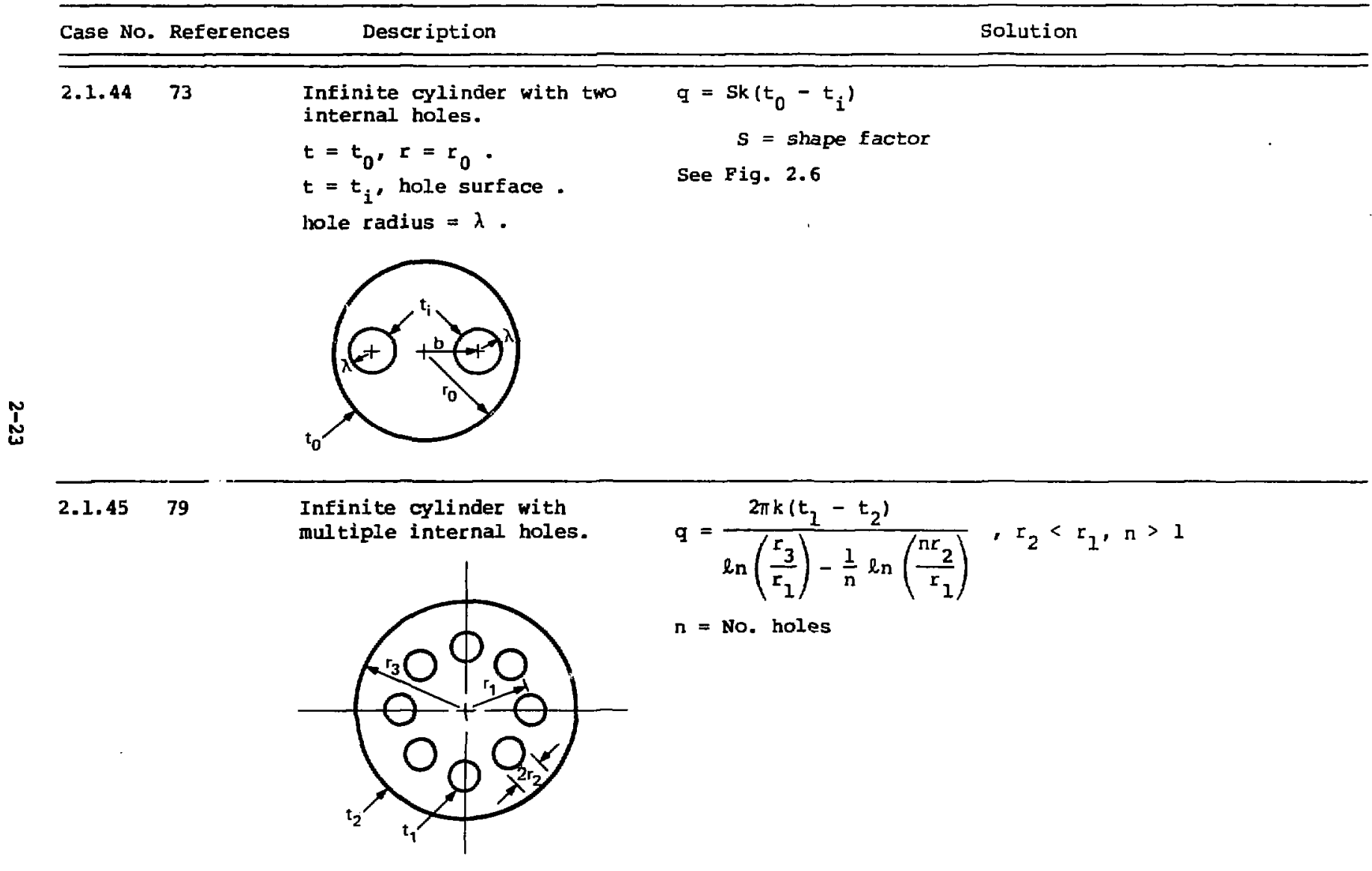


Section 2.1. Solids Bounded by Cylindrical Surfaces--No Internal Heating.

Case No. References

Description

Solution

2.1.46 $79 \quad$ Ellipsoidal pipe.

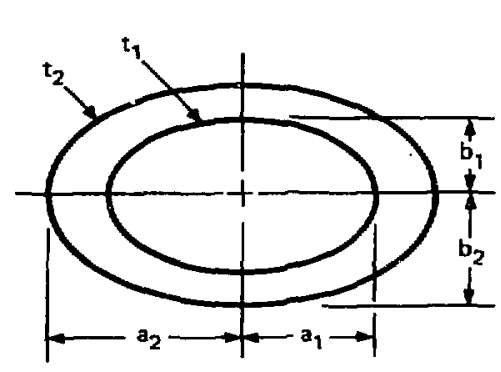

$$
q=\frac{2 \pi k\left(t_{1}-t_{2}\right)}{\ln \left(\frac{a_{2}+b_{2}}{a_{1}+b_{1}}\right)}
$$

$N$

2.1.47 $79 \quad \begin{aligned} & \text { Ellipsoid with a constant } \\ & \text { temperature slot. }\end{aligned} \quad \mathrm{a}=\frac{2 \pi k\left(t_{1}-t_{2}\right)}{\ln \left(\frac{a+b}{c}\right)}$

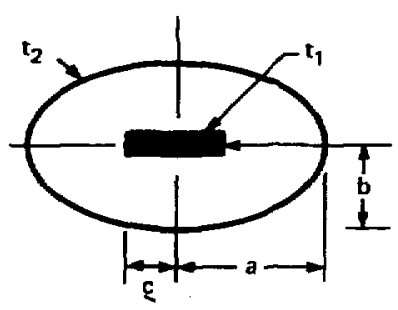


Section 2.1. Solids Bounded by Cylindrical Surfaces--No Internal Beating.

\begin{tabular}{|c|c|c|c|}
\hline Case No. & References & Description & Solution \\
\hline 2.1 .48 & $\oplus$ & $\begin{array}{l}\text { center tube row in an } \\
\text { inite plate. }\end{array}$ & $\begin{array}{l}q=\frac{2 \pi k\left(t_{1}-t_{2}\right)}{\ln \left(\frac{\theta_{2}(a / 2 d)}{\theta_{i}(0)}\right)+\ln \left(\frac{4 d}{\theta}\right)} \\
\theta_{2}(a / d)=\sum_{n=1}^{\infty} \exp \left[i \pi s\left(n+\frac{1}{2}\right)^{2} / 2 d\right] \cos [(2 n+1) \pi a / 2 d] \\
\theta_{1}^{\prime}(0)=\pi \theta_{2} \theta_{3} \theta_{0} \\
\theta_{2}(0)=\sum_{n=1}^{\infty} \exp \left[i \pi s\left(n+\frac{1}{2}\right)^{2} / 2 d\right] \\
\theta_{3}=1+\sum_{n=1}^{\infty} \exp \left(i \pi s n^{2} / 2 d\right) \\
\theta_{0}=1+2 \sum_{n=1}^{\infty}(-1)^{n} \exp ^{\left(i \pi s n^{2} / 2 d\right)}\end{array}$ \\
\hline
\end{tabular}


Section 2.1. Solids Bounded by Cylindrical Surfaces--No Internal Heating.

\section{Case No. References}

$2.1 .4979 \quad$ Single off-center tube in an infinite plate.

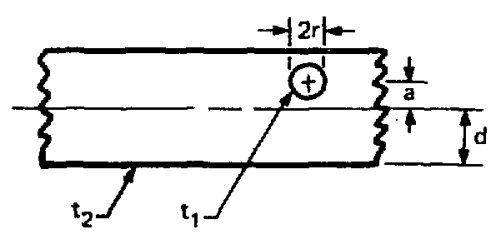

$$
q=\frac{2 \pi k\left(t_{1}-t_{2}\right)}{\ln \left[\cot \left[\frac{\pi(d+a)}{4 d}\right]\right\}+\ln \left(\frac{4 d}{\pi r}\right)}
$$

N

$2.1 .50 \quad 73$

Tube centered in a finite plate.

$$
q=\frac{2 \pi k\left(t_{1}-t_{2}\right)}{\ln \left(\frac{w}{\pi r}\right)+\frac{\pi d}{2 w}}
$$

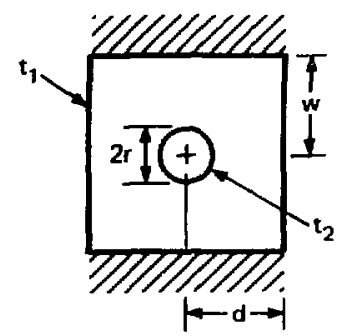


Section 2.1. Solids Bounded by Cylindrical Surfaces--No Internal Heating.

\begin{tabular}{lll} 
Case No. References & Description \\
\hline 2.1 .51 & 79 & $\begin{array}{l}\text { Heat flow between two pipes } \\
\text { in an insulated infinite plate. }\end{array}$
\end{tabular}$q=\frac{2 \pi k\left(t_{1}-t_{2}\right)}{\frac{\pi a}{w}+\ln \left(\frac{w}{\pi r}\right)}$

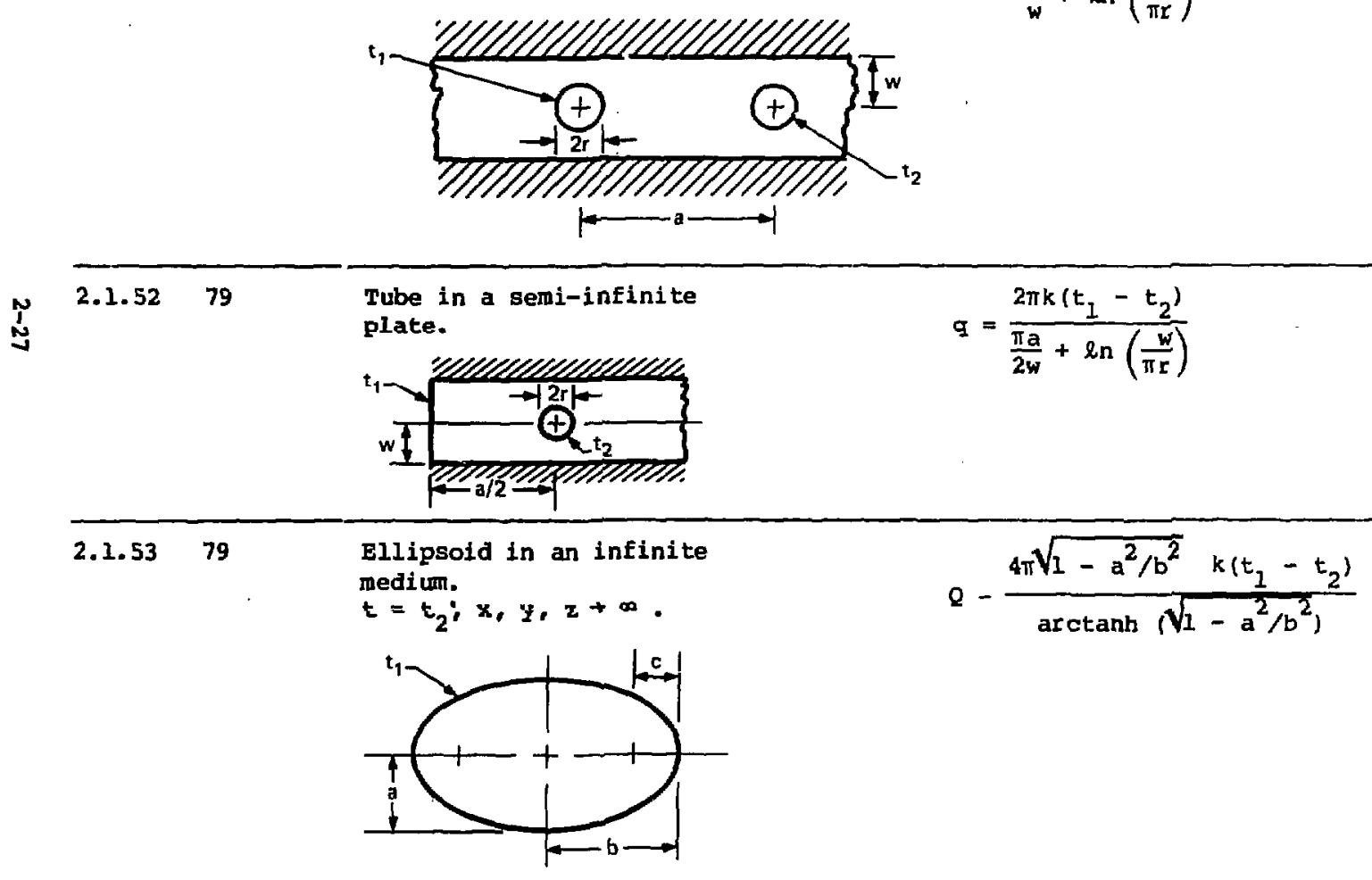


Section 2.1. Solids Bounded by Cylindrical Surfaces--No Internal Heating.

\section{Case No. References}

$$
\text { scription }
$$

Solution

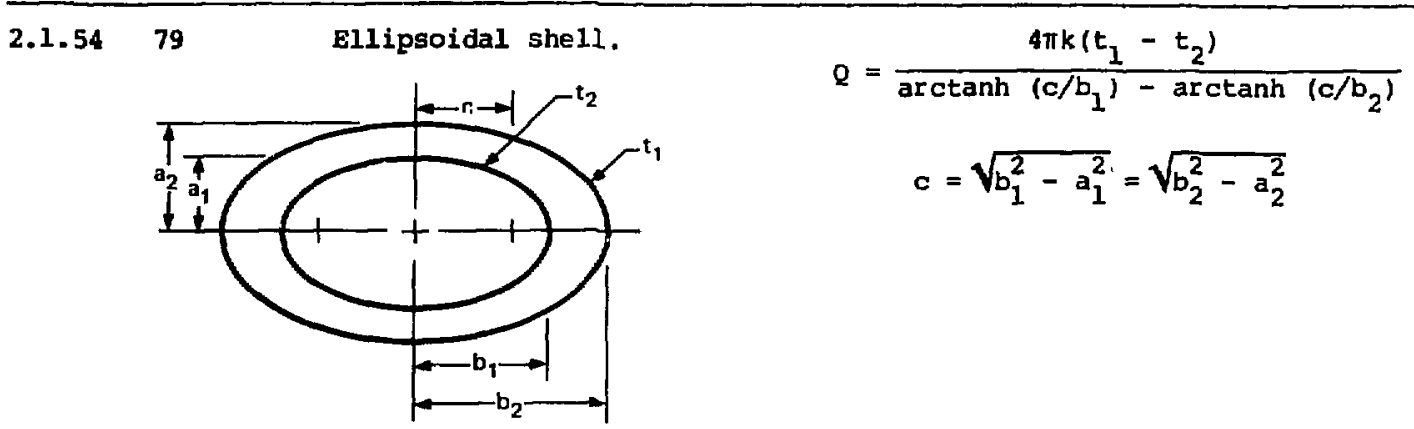

Rod in an infinite medium.

$t=t_{2} ; x, y, z+\infty$.

$Q=\frac{4 \pi k\left(t_{1}-t_{2}\right)}{\ln (2 \ell / r)}, \frac{r}{\ell}<0.1$

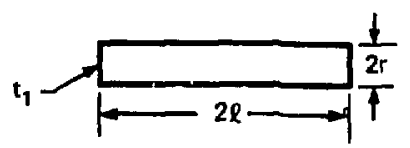

\begin{tabular}{|c|c|c|c|c|}
\hline 2.1 .56 & 79 & $\begin{array}{l}\text { Short cylinder in an infinite } Q=c k\left(t_{1}-t_{2}\right) \\
\text { med. m. } \\
t=t_{2} ; x, y, z+\infty .\end{array}$ & $\begin{array}{l}\ell / r \\
0 \\
0.25 \\
0.5 \\
1.0 \\
2.0 \\
4.0\end{array}$ & $\begin{array}{c}c \\
B \\
10.42 \\
12.11 \\
14.97 \\
19.87 \\
27.84\end{array}$ \\
\hline
\end{tabular}


Section 2.1. Solids Bounded by Cylindrical Surfaces-No Internal Heating.

\begin{tabular}{|c|c|c|c|}
\hline Case No. & References & Description & Solution \\
\hline 2.1 .57 & 79 & $\begin{array}{l}\text { Toroidal ring in an infinite } \\
\text { medium. } \\
t=t_{2} ; x, y, z \rightarrow \infty\end{array}$ & $Q=\frac{4 \pi^{2} k\left(t_{1}-t_{2}\right)}{\ln \left(8 r_{1} / r_{2}\right)}, r_{1} / r_{2}>20$ \\
\hline 2.1 .58 & 79 & $\begin{array}{l}\text { Thin flat ring in an infinite } \\
\text { medium. } \\
t=t_{2} ; x, y, z \rightarrow \infty\end{array}$ & $Q=\frac{4 \pi^{2} k\left(t_{1}-t_{2}\right)}{\ln \left(16 r_{1} / r_{2}\right)}, r_{1} / r_{2}>10$ \\
\hline 2.1 .59 & 79 & $\begin{array}{l}\text { Two parallel rods in an } \\
\text { infinite medium. }\end{array}$ & $\begin{array}{l}Q=\frac{4 \pi k\left(t_{1}-t_{2}\right)}{2\left[\ln \left(\frac{2 l}{r}\right)\left(\frac{\sqrt{s^{2}+\ell^{2}-1}}{s}\right)\right]}, \quad \begin{array}{l}s>5 r \\
l>r\end{array} \\
\text { For parallel strips of width } 2 w, \text { use } r=w / 2 .\end{array}$ \\
\hline
\end{tabular}


Section 2.1. Solids Bounded by Cylindrical Surfaces--No Internal Heating.

Case No. References Description Solution

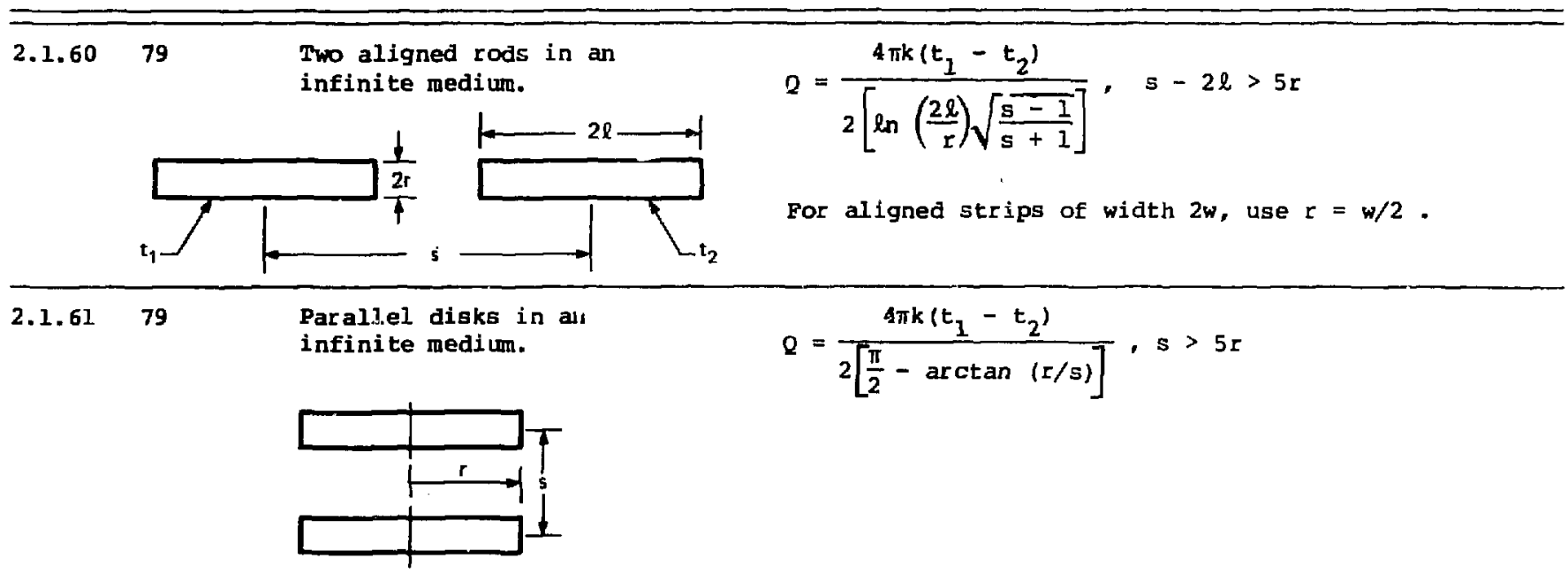

Infinite cylinder with
symetric isothermal caps. $\quad q=\frac{k \pi\left(t_{1}-t_{2}\right)}{2 \ln [2(1+\cos \theta) / \sin \theta]}$


Section 2.1. Solids Bounded by Cylindrical Surfaces--No Internal Heating.

Case No. References Description Solution


Seution 2.1. Solids Bounded by Cylindrical Surfaces--No Internal Heating.

Case No. References Description Solution

$\stackrel{N}{\dot{u}}$ 
Section 2.2. Solids Bounded by Cylindrical Surfaces--With Internal Heating.

\begin{tabular}{|c|c|c|c|}
\hline Case No. & References & Description & Solution \\
\hline 2.2 .1 & 4,51 & $\begin{array}{l}\text { Infinite cylinder with } \\
\text { convection boundary. } \\
t_{0}, h\end{array}$ & $\frac{\left(t-t_{0}\right) k}{q^{\prime} \cdot r_{0}^{2}}=\frac{1}{4}\left[\frac{2}{B i}+1-R^{2}\right]$ \\
\hline 2.2 .2 & 4,51 & $\begin{array}{l}\text { Hollow infinite cylinder with } \\
\text { with convection boundary on } \\
\text { outside gurface. } \\
q_{r}=0, r=r_{i} \text {. }\end{array}$ & $\begin{array}{l}\frac{\left(t-t_{E}\right) k}{q \prime \prime r_{0}^{2}}=\frac{1}{4}\left\{\frac{2}{B i}\left[1-R_{1}^{2}\right]+1-R^{2}+2 R_{i}^{2} \ln (R)\right\} \\
R=r / r_{i}, B i=h r_{0} / k\end{array}$ \\
\hline
\end{tabular}


Section 2.2. Solids Bounded by Cylindrical Surfaces--With Internal Heating.

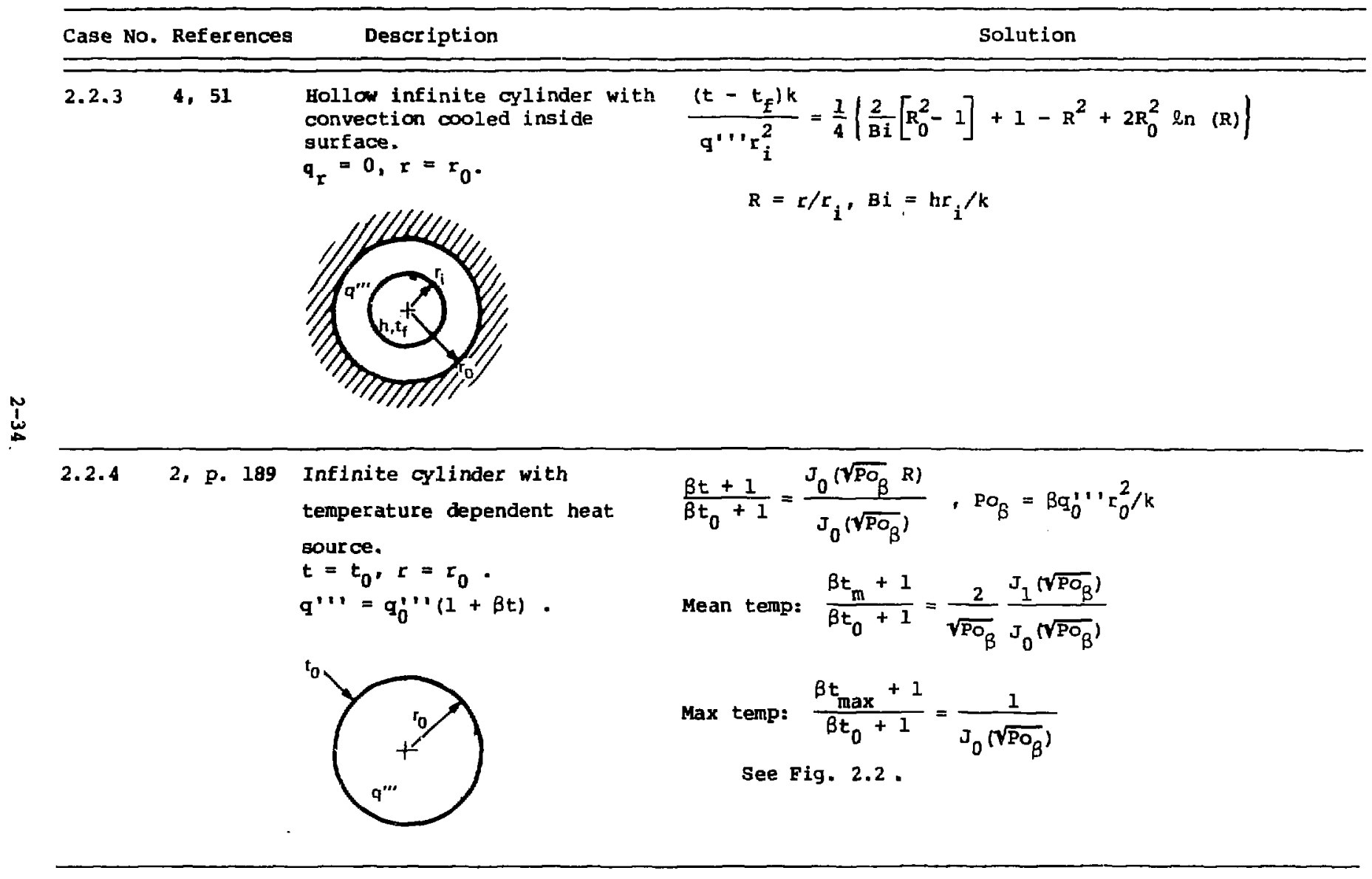


Section 2.2. Solids Bounded by Cylindrical Surfaces-With Internal Heating.

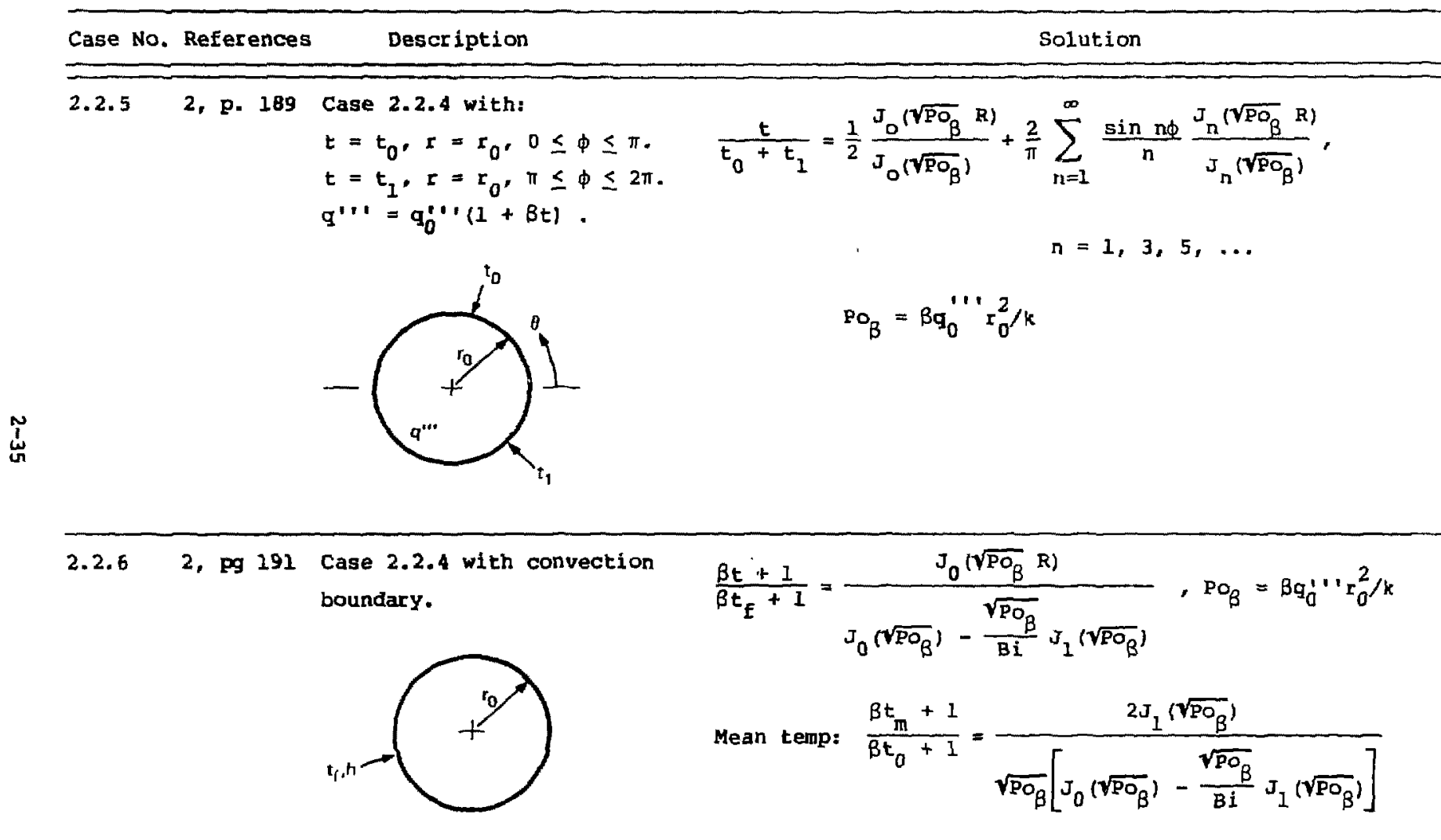

See Ref. 85 for nonuniform convection boundary solution. 
Section 2.2. Solids Bounded by Cylindrical Surfaces--With Internal Heating.

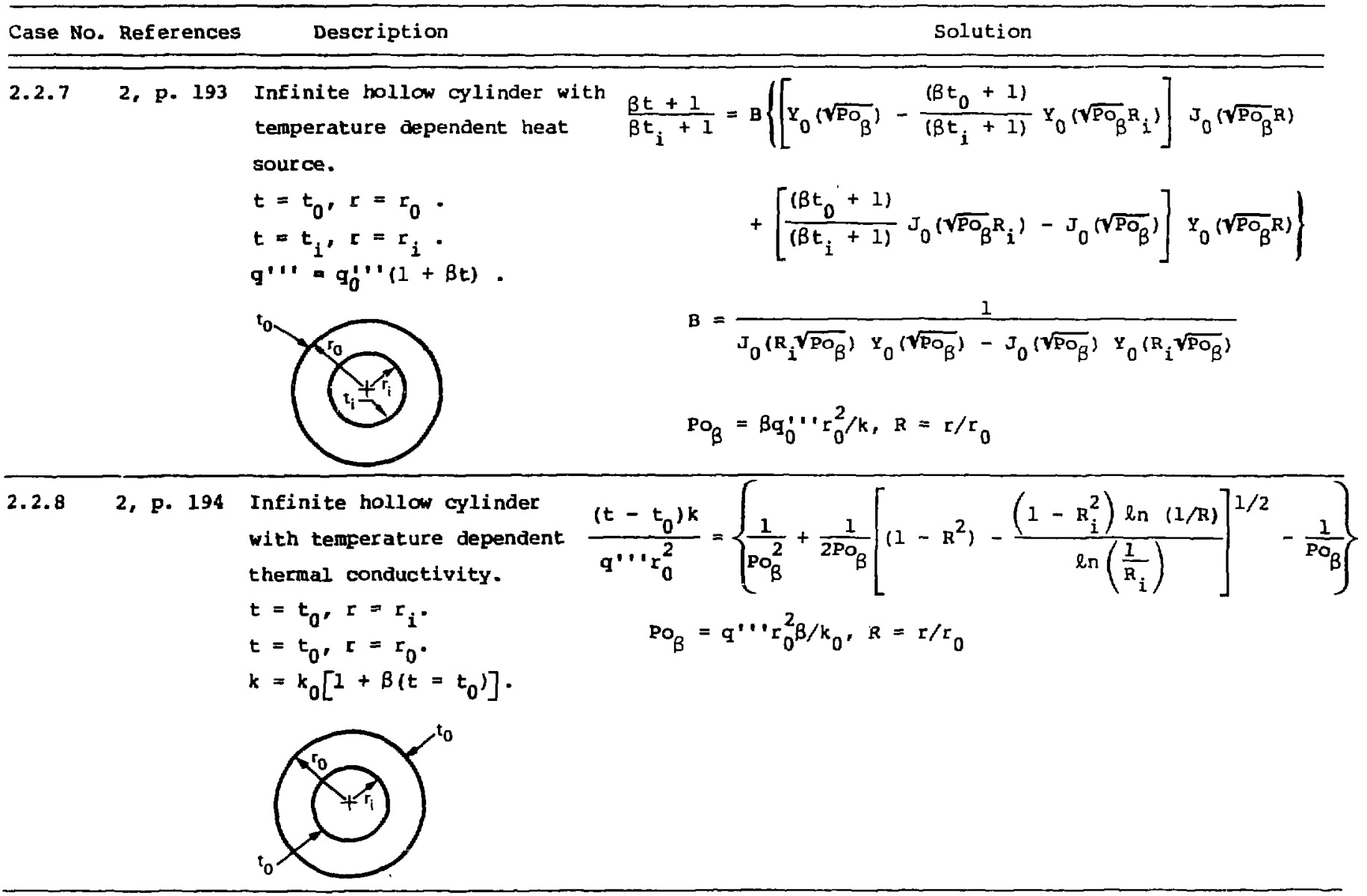


Section 2.2. Solids Bounded by Cylindrical Surfaces--With Internal Heating.

Case No. References

Description

Solution

2.2.9 6, p. 272 Electrically heated wire with temperature dependent thermal and electrical conductivities.

$$
\begin{aligned}
\frac{t-t_{0}}{B} & =R\left\{I+B\left[\frac{R}{B}-\frac{\beta_{e}}{\beta_{t}} \frac{(2+R)}{I 6}\right]\right\} \\
B & =\frac{k_{e 0} r_{0}^{2} E^{2} B_{t}}{k_{t 0^{L}} L^{2}}, R=I-\left(r^{2} / r_{0}^{2}\right) \\
k_{t} & =\text { thermal conductivity } \\
k_{e} & =\text { electrical conductivity } \\
E & =\text { voltage drop over length L }
\end{aligned}
$$$$
t=t_{0} \cdot r=r_{0} \text {. }
$$$$
k_{t} / k_{t 0}=1+\beta_{t}\left(t-t_{0}\right) \text {. }
$$$$
k_{\mathrm{e}} / k_{\mathrm{e} 0}=1+\beta_{\mathrm{e}}\left(t-t_{0}\right) \text {. }
$$

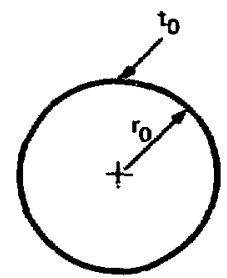

$\hat{d}$

2.2.10 2, p. 175 Cylindrical heat source in an infinite plate.

$t=t_{\infty}, c+\infty$.

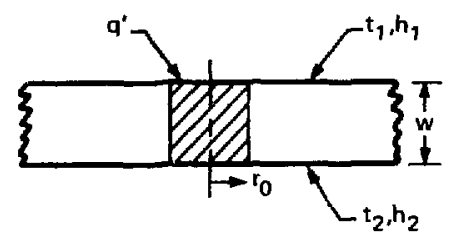

$\frac{t-t_{\infty}}{q^{2} / k W}=\frac{k_{0}[R B]}{2 \pi B k_{1}[B]}$

See Fig. 2.3

$$
\begin{aligned}
& B=r_{0} \sqrt{\left(h_{1}+h_{2}\right) / k w} \\
& t_{\infty}=\frac{h_{1} t_{1}+h_{2} t_{2}}{h_{1}+h_{2}} \\
& q^{\prime}=\text { heating rate in cylindrical source }
\end{aligned}
$$


Section 2.2. Solids Bounded by Cylindrical Surfaces--With Internal Heating.

Case No. References Description Solution

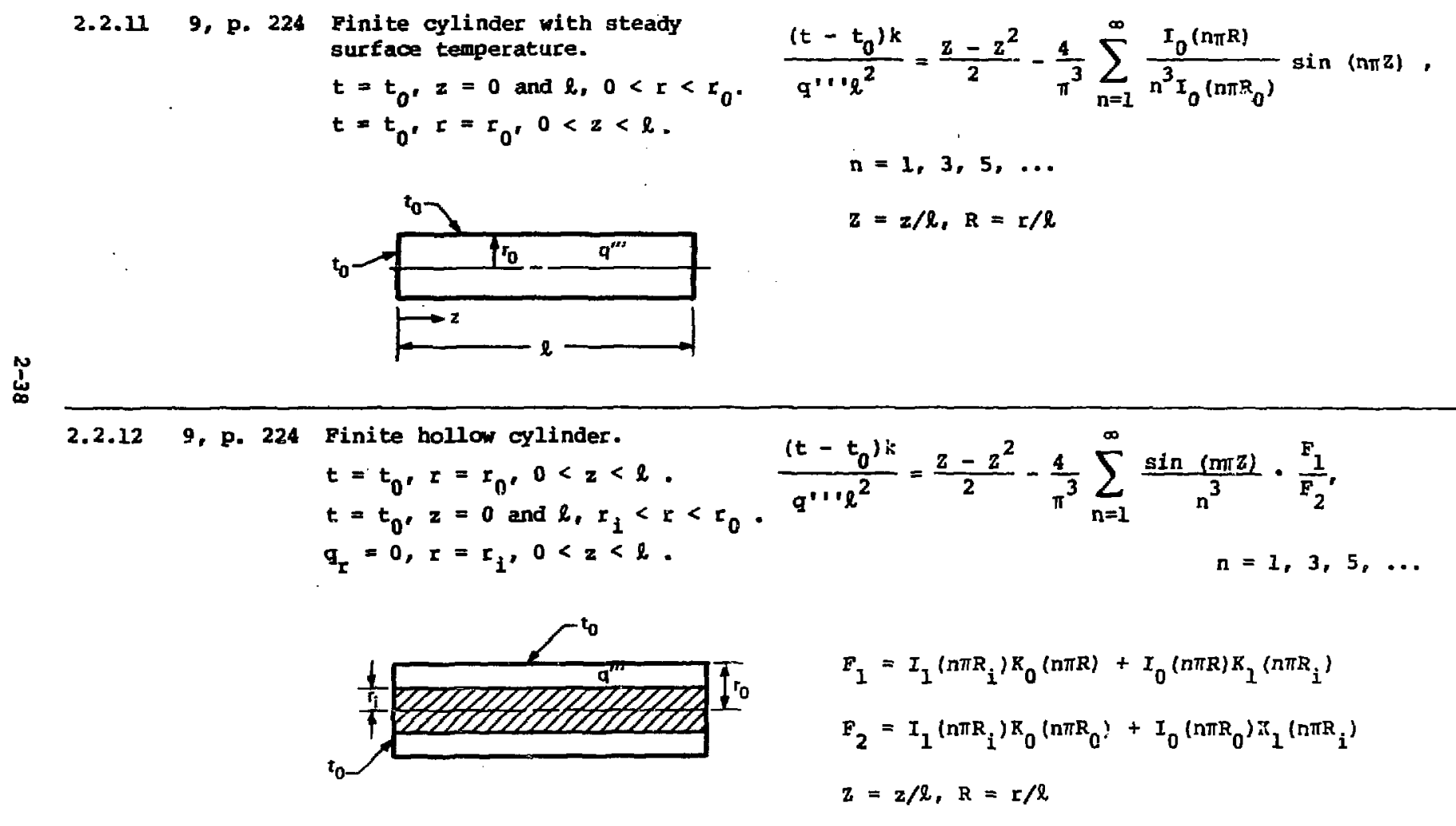


Section 2.2. Solids Bounded by Cylindrical Surfaces--With Internal Heating.

\begin{tabular}{|c|c|c|c|c|}
\hline Case No & Refer & ences & Description & Solution \\
\hline 2.2 .13 & 9, p. & 224 & $\begin{array}{l}\text { Case } 2.2 .11 \text { with } \\
q^{\prime \prime}=q_{0}^{\prime \prime}\left[1+\beta\left(t-t_{0}\right)\right]\end{array}$ & 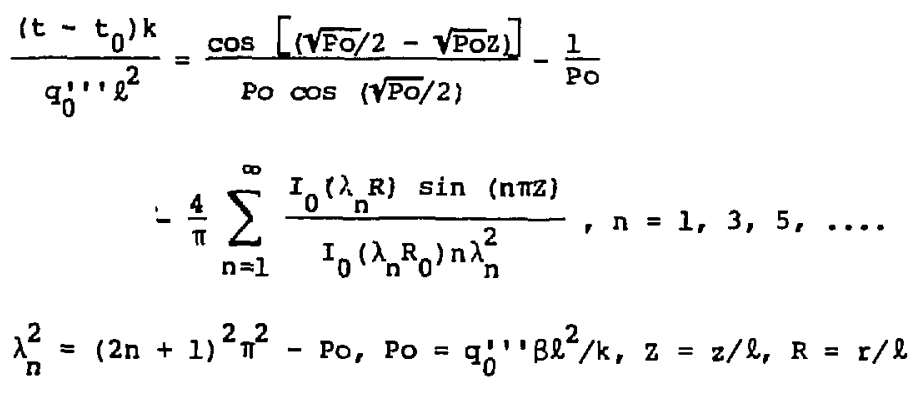 \\
\hline 2.2 .14 & 9, p. & $\longrightarrow$ z & $\begin{array}{l}\text { Finite cylinder with line heat } \\
\text { source. } \\
t=t_{0}, r=r_{0}, 0<z<\ell . \\
t=t_{0}, 0<r<r_{0}, z=0, \ell \text {. } \\
\text { Line source of strength } g^{\prime} \text { per } \\
\text { unit length is located at } r_{1}, \theta_{1}, \\
b<z<\ell-b . \\
\qquad t_{0}\end{array}$ & 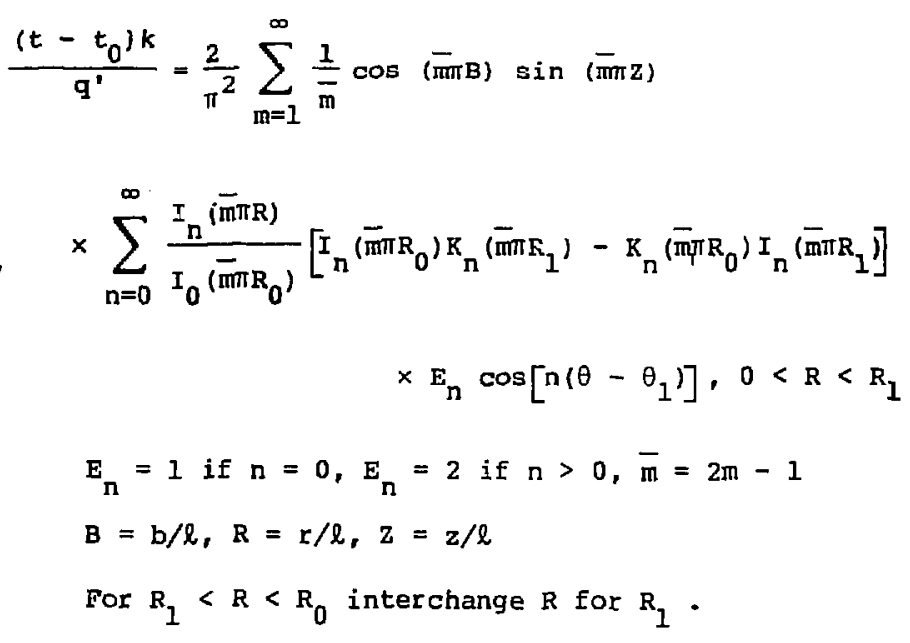 \\
\hline
\end{tabular}


Section 2.2. Solids Bounded by Cylindrical Surfaces--With Internal Heating.

\begin{tabular}{|c|c|c|c|}
\hline Case No. & References & Description & Solution \\
\hline 2.2 .15 & 9, p. 423 & $\begin{array}{l}\text { Case } 2.2 .14 \text { with a point } \\
\text { source of strength } Q \text { located } \\
\text { at } r_{1}, \theta_{1}, z_{1}\end{array}$ & $\begin{array}{l}\frac{\left(t-t_{0}\right) k l}{Q}=\frac{1}{\pi} \sum_{m=1}^{\infty} \sin (m \pi z) \sin \left(m \pi z_{1}\right) \\
\quad \times \sum_{n=0}^{\infty} E_{n} \frac{I_{n}(m \pi R)}{I_{n}\left(m \pi R_{0}\right)}\left[I_{n}(m \pi R) k_{n}\left(m \pi R_{1}\right)-K_{n}(m \pi R) I_{n}\left(m \pi R_{1}\right)\right] \\
\text { For } R_{1}<R<R_{0}, \text { interchange R for } R_{1} \cdot \\
R=r / l, 2=z / l_{r} E_{n}=1 \text { if } n=0, E_{n}=2 \text { if } n>0\end{array}$ \\
\hline
\end{tabular}

2.2.16 9, P. 423 Infinite cylinder with point source of strength $Q$. $t=t_{0}, r=r_{0}$. Source located at $r_{1}, \theta_{1}$, $\mathbf{z}=0$.

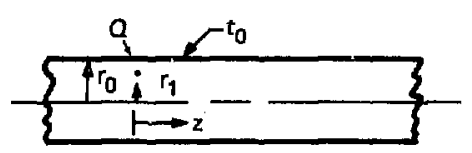

$$
\begin{aligned}
& \frac{\left(t-t_{0}\right) k r_{0}}{Q}=\frac{1}{2 \pi} \sum_{n=-\infty}^{\infty} \cos \left[n\left(\theta-\theta_{1}\right)\right] \\
& \quad \times \sum_{\lambda>0}^{\infty} \frac{\exp \left(-\lambda|z| i J_{n}\left(\lambda_{n} R\right) J_{n}\left(\lambda_{n} R_{1}\right)\right.}{\lambda\left[\frac{n}{\lambda} J_{n}(\lambda)-J_{n+1}(\lambda)\right]^{2}} \\
& J_{n}\left(\lambda_{n}\right)=0, z=z / r_{0}, R=r / r_{0}
\end{aligned}
$$


Section 2.2. Solids Bounded by Cylindrical Surfaces--With Internal Heating.

\begin{tabular}{|c|c|c|c|}
\hline Case No. & References & Description & Solution \\
\hline 2.2 .17 & 16 & $\begin{array}{l}\text { Bollow cylinder with } \\
\text { temperature dependent } \\
\text { heating and thermal } \\
\text { conductivity. } \\
t=t_{0}, r=r_{0} \\
q_{r}=0, r=r_{0} \\
k=k_{0}, r=r_{0} \\
q^{\prime \prime \prime}=q_{0}^{\prime \prime}, r=0 \\
k=k_{0}+a\left(t_{0}-t\right) \\
g^{\prime \prime \prime}=q_{0}^{\prime \prime \prime}+b\left(t_{0}-t\right)\end{array}$ & $\begin{array}{l}\text { Solution by numerical method. } \\
\text { See Fig. } 2.4 \text { for values of } t \text { at } r=r_{i} \text {. } \\
\qquad \rho_{i}=I-\left(r_{i} / r_{0}\right), K=k_{0} / a t_{0}, R=a t_{0} / b r_{0}^{2}, G=q_{0}^{\prime \prime \prime} / b t_{0} \\
\text { For } a=b=0 \text { : } \\
\frac{\left(t_{0}-t_{i}\right) k_{0}}{q_{0}^{\prime \prime \prime} r_{0}^{2}}=\frac{1}{4}\left(1-\rho_{i}\right)^{2}+\frac{1}{2} \ln \left(\frac{1}{l-\rho_{i}}\right)-\frac{1}{4} \\
\text { (Eq. (5) in Fig. 2.4) }\end{array}$ \\
\hline
\end{tabular}


Section 2.2. Solids Bounded by Cylindrical Surfaces--With Internal Heating.

Case No. References Description Solution

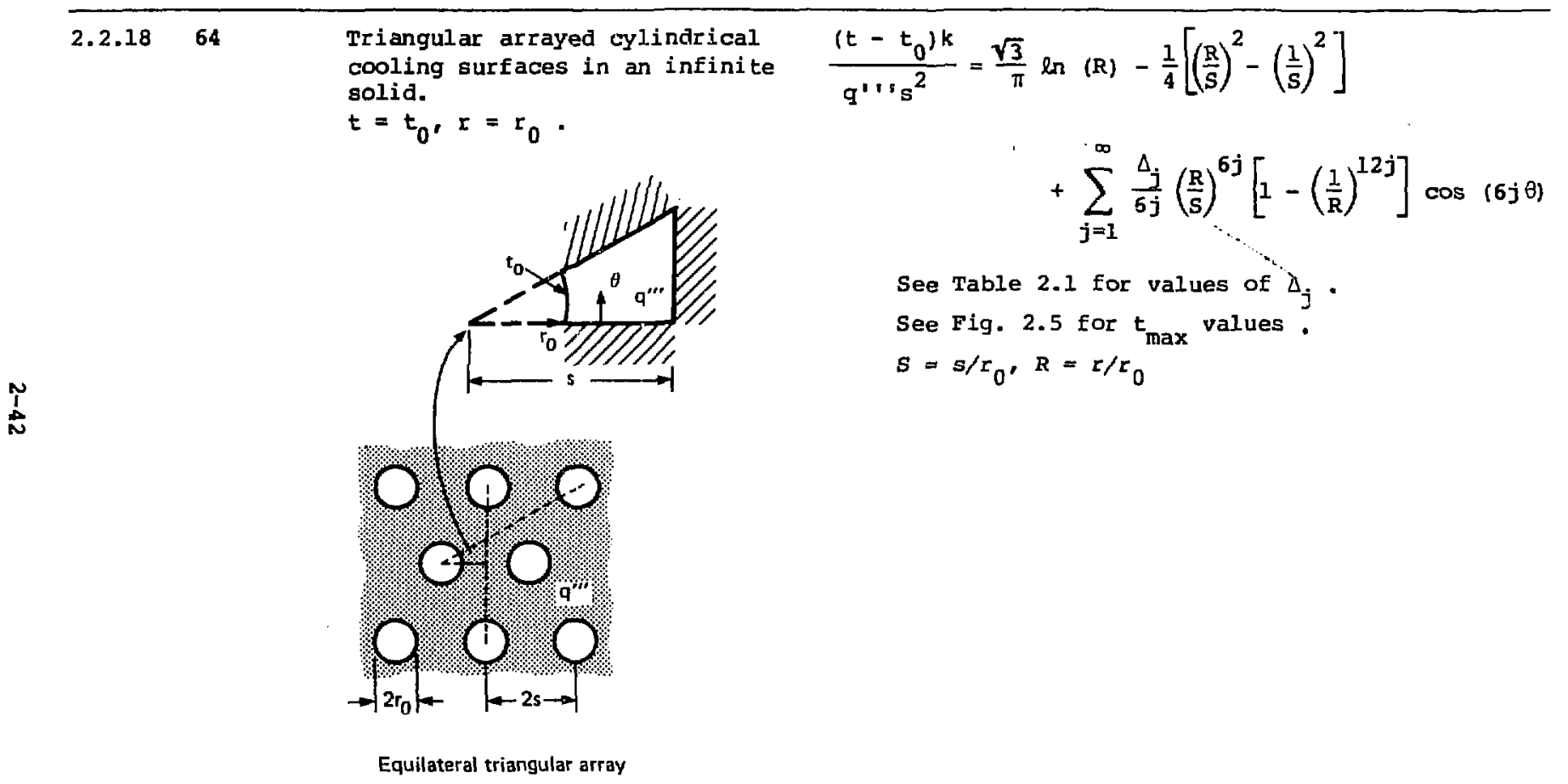


Section 2.2. Solids Bounded by Cylindrical Surfaces--With Internal Heating.

Case No. References Description Solution

2.2.19 $64 \quad$ Case 2.2.18 with a square array of holes.

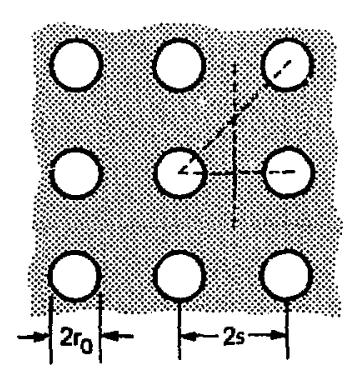

Square array

$$
\frac{\left(t-t_{0}\right) k}{q^{\prime} \cdot s^{2}}=\frac{2}{\pi} \ln (R)-\frac{1}{4}\left[\left(\frac{R}{s}\right)^{2}-\left(\frac{1}{s}\right)^{2}\right]
$$

$+\sum_{j=1}^{\infty} \frac{\delta_{j}}{4 j}\left(\frac{R}{S}\right)^{4 j}\left[1-\left(\frac{1}{R}\right)^{8 j}\right] \cos (4 j \theta)$

See Table 2.2 for values of $\delta_{j}$.

See Fig. 2.5 for $t_{\max }$ values. 
Section 2.2. Solids Bounded by Cylindrical Surfaces -With Internal Heating.

Case No. References Description

Solution

$2.2 .20 \quad 67$

Eccentric, hollow, infinite See Ref. 67 for equational solution. cylinder.
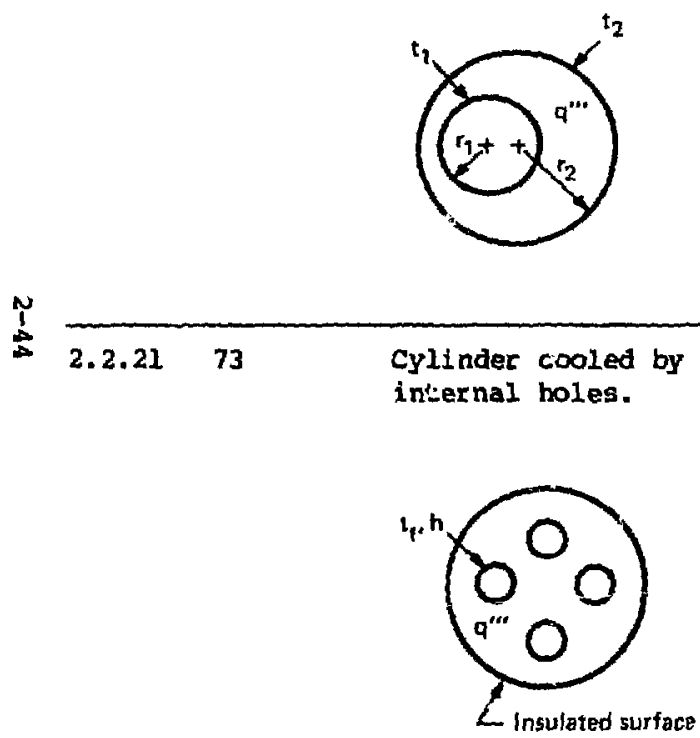
Section 2.2. Sollds Bounded by Cylindrical Surfaces--With Internal Heating.

Case No. References Description Solution

is 
Section 2.2. Solids Bounded by Cylindrical Surfaces-with Internal Heating.

Case No. References Description Solution

$\stackrel{\tilde{I}}{\alpha}$ 
REPRODUCED FAOM BEST AVAILABLE COPY 
Section 3.1. Solids Bounded by Spherical Surfaces--No Internal Heating.

Case No. References Description Solution

3.1.1. 4, p. 37 Spherical shell.

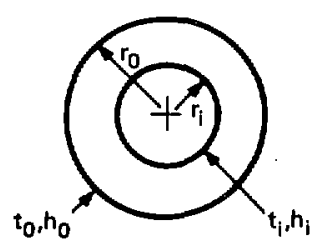

$$
\begin{aligned}
& Q=\frac{4 \pi r_{0} k\left(t_{i}-t_{0}\right)}{\left(r_{0} / r_{i}\right)-1+\left(r_{0} / r_{i}\right)\left(k / h_{i} r_{i}\right)+k / h_{0} r_{0}} \\
& \frac{t-t_{0}}{t_{i}-t_{0}}=\frac{\left(r_{0} / r\right)-1}{\left(r_{0} / r_{i}\right)-1+\left(r_{0} / r_{i}\right)\left(k / r_{1} h_{i}\right)+k / r_{0} h_{0}}
\end{aligned}
$$

3.1.2 $92 \quad$ Composite sphere.

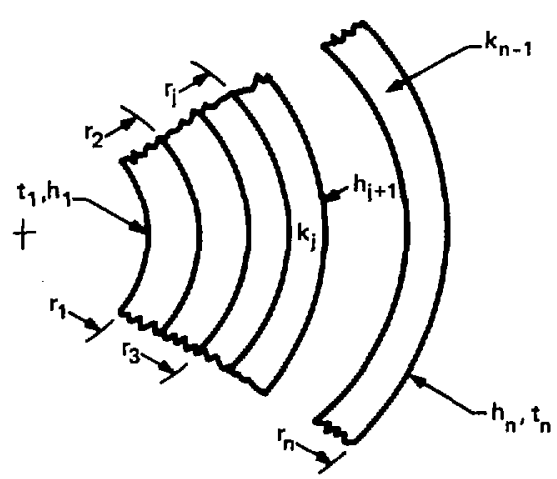

$$
\begin{aligned}
& 0=\frac{4 \pi\left(t_{1}-t_{n}\right)}{\sum_{i=1}^{n-1} \frac{1}{k_{1}}\left(\frac{1}{r_{i}}-\frac{1}{r_{i+1}}\right)+\sum_{i=1}^{n} \frac{1}{r_{i}^{2} h_{i}}} \\
& \frac{t_{j}-t_{1}}{t_{n}-t_{1}}=\frac{\sum_{i=1}^{j-1}\left[\frac{1}{k_{i}}\left(\frac{1}{r_{i}}-\frac{1}{r_{i+1}}\right)+\frac{1}{r_{i}^{2} h_{i}}\right]+\frac{1}{k_{j}}\left(\frac{1}{r_{j}}-\frac{1}{r}\right)}{\sum_{i=1}^{n-1} \frac{1}{k_{i}}\left(\frac{1}{r_{i}}-\frac{1}{r_{i+1}}\right)+\sum_{i=1}^{n} \frac{1}{h_{i} r_{i}^{2}}}, j>1
\end{aligned}
$$

$t_{j}=$ local temperature in $j^{t h}$ layer 
Section 3.1. Solids Bounded by Spherical Surfaces--No Internal Heating.

\section{Case No. References}

Description

Solution

3.1.3 1. p. 139 Sphere with temperature-

dependent thermal conductlvity.

$Q=\frac{4 \pi r_{0} k_{m}\left(t_{i}-t_{0}\right)}{\left(r_{0} / r_{i}\right)-1}$

$t=t_{1}, I=r_{1}$.

$t=t_{0}, r=r_{0}$.

$k_{m}=\left(k_{0}+k_{i}\right) / 2$

$k=k_{0}+B\left(t-t_{0}\right)$.

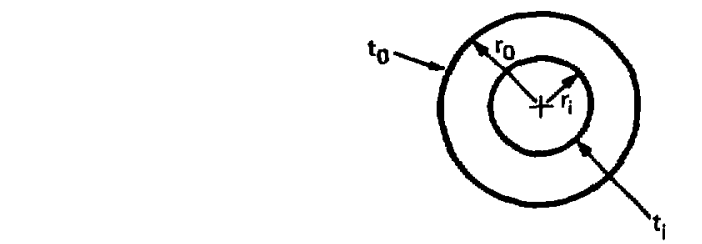

w

$$
\begin{aligned}
k & =k_{0}, t=t_{0} \\
k & =k_{i}, t=t_{i} \\
\frac{t-t_{0}}{k_{0} / B} & =\sqrt{I+2 B\left(t_{i}-t_{0}\right) \frac{k_{m}}{k_{0}^{2}} \frac{\left(r_{0} / r-1\right)}{\left(r_{0} / r_{1}-1\right)}-1}
\end{aligned}
$$

3.1 .42 , p. 137 Sphere with variable Burface temperature. $t=f(\theta), r=r_{0}$.

$t=\sum_{n=0}^{\infty} a_{n}{ }^{n} p_{n}(\cos \theta)$

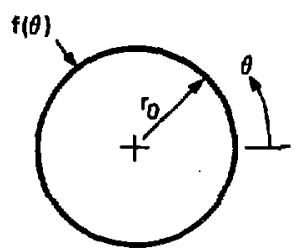
$a_{n}=\frac{2 n+1}{2 r_{0}} \int_{0}^{\pi} f(\theta) p_{n}(\cos \theta) \sin \theta d \theta$ 
Section 3.1. Solids Bounded by Spherical Surfaces-No Internal Heating.

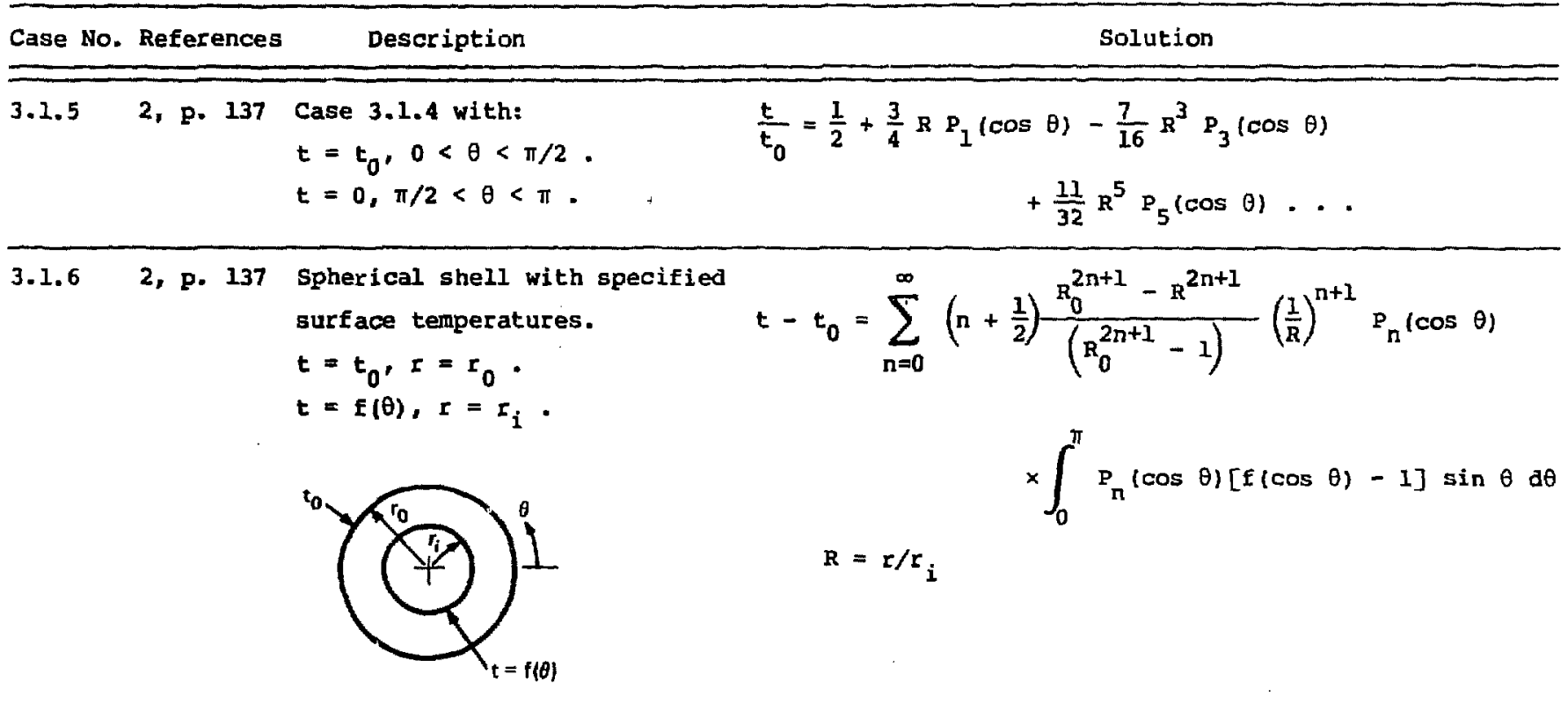


Section 3.1. Solids Bounded by Spherical Surfases--No Internal Heating.

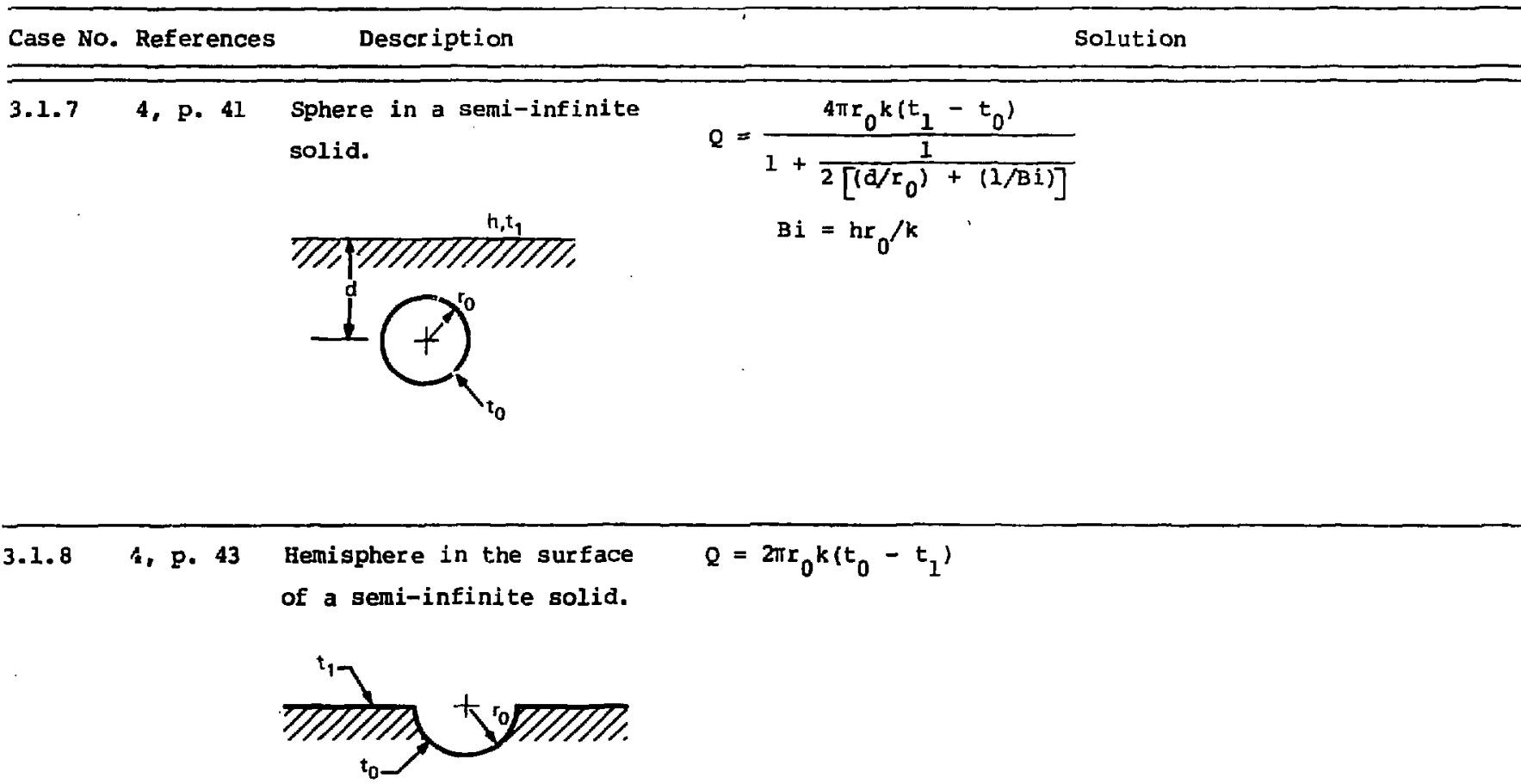


Section 3.1. Solids Bounded by Spherical Surfaces--No Internal Heating.

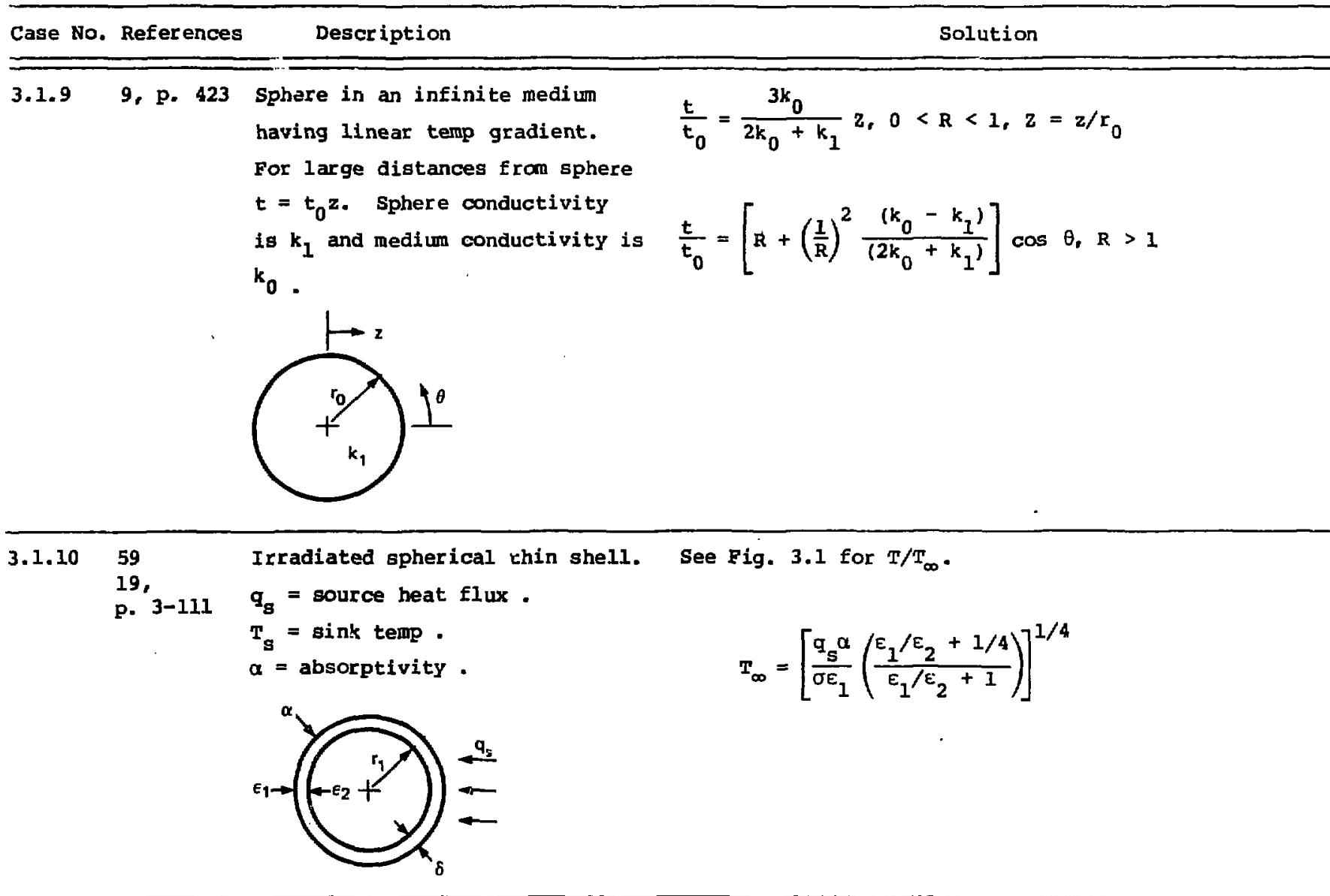


Section 3.1. Solids Bounded by Spherical Surfaces-No Internal Heating.

Case No. References Description Solution

3.1.11 $79 \quad$ Sphere in an infinite medium. $Q=4 \pi r_{0} k\left(t_{1}-t_{2}\right)$

$t=t_{2}, r+\infty$.

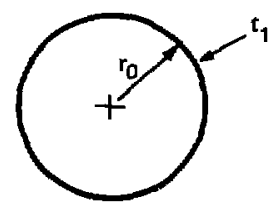

$\breve{\omega}$

Two spheres separated a large

distance in an infinite medium.

$Q=\frac{4 \pi r k\left(t_{1}-t_{2}\right)}{2(1-r / s)}, s>5 r$

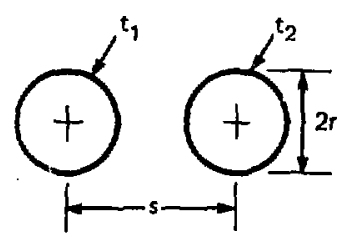

for $s>2 I$, error $\simeq 18$ 
Section 3.1. Solids Bounded by Spherical Surfaces--No Internal Heating.

Case No. References Description Solution

\begin{tabular}{ll}
3.1 .1379 & $\begin{array}{l}\text { Two spheres separated a small } \\
\text { distance (see case } 3.1 .12) \text { in } \\
\text { an infinite medium. }\end{array}$ \\
\hline 3.1 .14 & $\begin{array}{l}\text { Two spheres of different radii } \\
\text { in an infinite medium. }\end{array}$
\end{tabular}


Section 3.1. Solids Bounded by Spherical surfaces--No Internal Beating.

Case No. References Description Solution


Section 3.1. Solids Bounded by Spherical Surfaces--No Internal Heating. Solution Case No. References Description 
Section. 3.2. Solids Bounded by Spherical Surfaces--With Internal Heating.

Case No. References Description Solution

\begin{tabular}{|c|c|c|c|c|}
\hline \multirow[t]{2}{*}{3.2 .1} & & $\begin{array}{l}\text { Spherical shell with specified } \\
\text { inside surface heat flux. } \\
t=t_{0}, r=r_{0} \\
q_{r}=q_{i}, r=r_{i}\end{array}$ & $\begin{array}{l}\frac{\left(t-t_{0}\right) k}{q_{i} r_{i}}=\frac{q^{\prime}{ }^{\prime} r_{i}}{6 q_{i}}\left[\frac{2\left(R-R_{0}\right)}{R_{0}}+R_{0}^{2}-R^{2}\right]+ \\
R=r / r_{i}\end{array}$ & $\frac{R_{0}-R}{R_{0}}$ \\
\hline & 1, p. 190 & $\begin{array}{l}\text { Sphere with temperature } \\
\text { dependent heating. } \\
t=t_{0}, r=r_{0} . \\
g^{\prime \prime \prime}=q_{0}^{\prime} '+\beta\left(t-t_{0}\right) .\end{array}$ & $\begin{array}{l}\frac{\left(t-t_{0}\right) \beta}{q_{0}^{\prime \prime}}=\frac{1}{R} \frac{\sin (R \sqrt{P O})}{\sin (\sqrt{P O})}-1, P O=\beta r_{0}^{2} / k \\
\frac{t-t_{0}}{t_{\max }-t_{0}}=1-R^{2}, P O=0 \\
\frac{t-t_{0}}{t_{\max }-t_{0}}=\frac{\sin (\pi R)}{\pi R}, \sqrt{P O}=\pi \\
\frac{\left(t_{\max }-t_{0}\right) B}{q_{0}^{\prime \prime \prime}}=\frac{\sqrt{P O}}{\sin (\sqrt{P O})}-1\end{array}$ & \\
\hline
\end{tabular}


Section 3.2. Solids Bounded by Spherical Surfaces--With Internal Heating.

Case No. References Description Solution

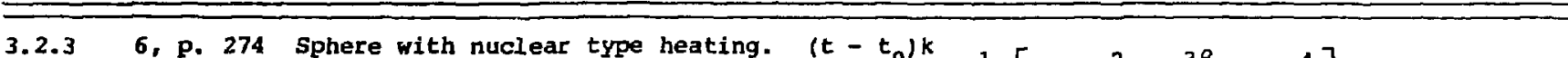

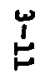
$t-t_{0}, r=r_{0}$.
$q^{\prime \prime}=q_{0}^{\prime \prime}\left[1-\beta\left(r / r_{0}\right)^{2}\right]$.

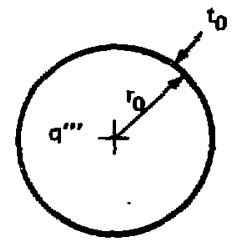

3.2.4 9, p. 232 Solid sphere with convection boundary.

$\frac{\left(t-t_{f}\right) k}{q^{\prime \prime} r_{0}^{2}}=\frac{1}{6}\left[1-R^{2}+(2 / B i)\right]$

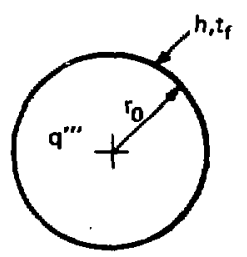

See Ref. 85 for nonuniform convection boundary solution. 
Section 3.2. Solids Bounded by Spherical Surfaces--With. Internal Heating.

\begin{tabular}{|c|c|c|c|}
\hline Case No. & References & Description & Solution \\
\hline 3.2 .5 & 9, p. 232 & $\begin{array}{l}\text { Solid sphere in an infinite } \\
\text { medium. } \\
k=k_{0}, 0<r<r_{0} . \\
k=k_{1}, r>r_{0} . \\
q^{\prime \prime}=q_{0}^{\prime \prime}, 0<r<r_{0} . \\
q^{\prime \prime}=0, r>r_{0} . \\
h=\text { contact coefficient at } r=r_{0} . \\
t=t_{\infty} r>\infty \text {. }\end{array}$ & $\begin{array}{l}\frac{\left(t-t_{\infty}\right) k_{0}}{q_{0}^{\prime \prime} r_{0}^{2}}=\frac{1}{6}\left(1-R^{2}+2 / B i+2 k_{0} / k_{1}\right), 0<R<1 \\
\frac{\left(t-t_{\infty}\right) k}{q_{0}^{\prime \prime} r_{0}^{2}}=1 / 3 R, r>r_{0} \\
B i=h r_{0} / k_{0}\end{array}$ \\
\hline
\end{tabular}


Section 3.2. Solids Bounded by Spherical Surfaces--with Internal Heating.

Case No. References Description

Solution

$\underset{\omega}{\omega}$ 
Section 3.2. Solids Bounded by Spherical Surfaces-With Internal Heating.

Case No. References Description Solution

$\underset{⿱ 亠 䒑}{\mathbf{L}}$ 


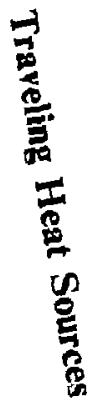

REPRODUCED FROM

BEST AVAILABLE COPY

$\therefore$ 
Section 4.1. Traveling Heat Sources.

\section{Case No. References}

Description

Solution

2, p. 189

9. p. 267

Traveling line heat source in

an infinite solid.

$$
\frac{\left(t-t_{0}\right) k}{q^{\prime}}=\frac{1}{2 \pi} \exp \left(-\frac{v x}{2 \alpha}\right) k_{0}\left(\frac{v}{2 \alpha} \sqrt{x^{2}+y^{2}}\right)
$$

$t=t_{0}, x \rightarrow \infty, y+ \pm \infty$

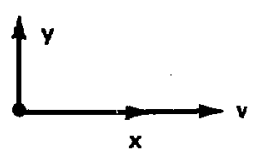

$$
\begin{aligned}
& v_{1}=\text { velocity of line source } \\
& q^{\prime}=\text { heating rate in line per } \\
& \text { unit length }
\end{aligned}
$$

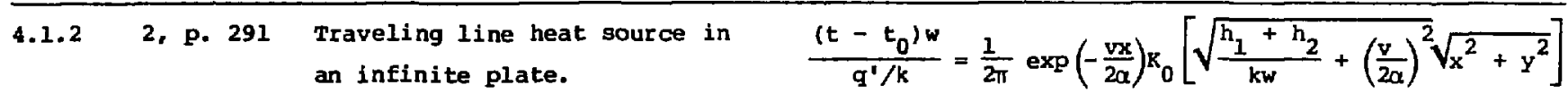

$t=t_{0}, x+\infty, y+ \pm \infty$

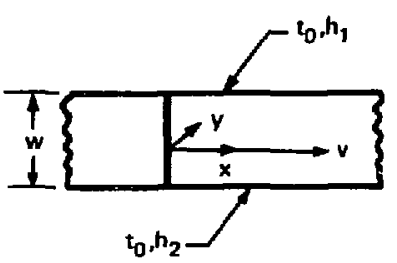

$v=$ velocity of line source

$q^{\prime}=$ heating rate of line 
Section 4.1. Traveling Heat Sources.

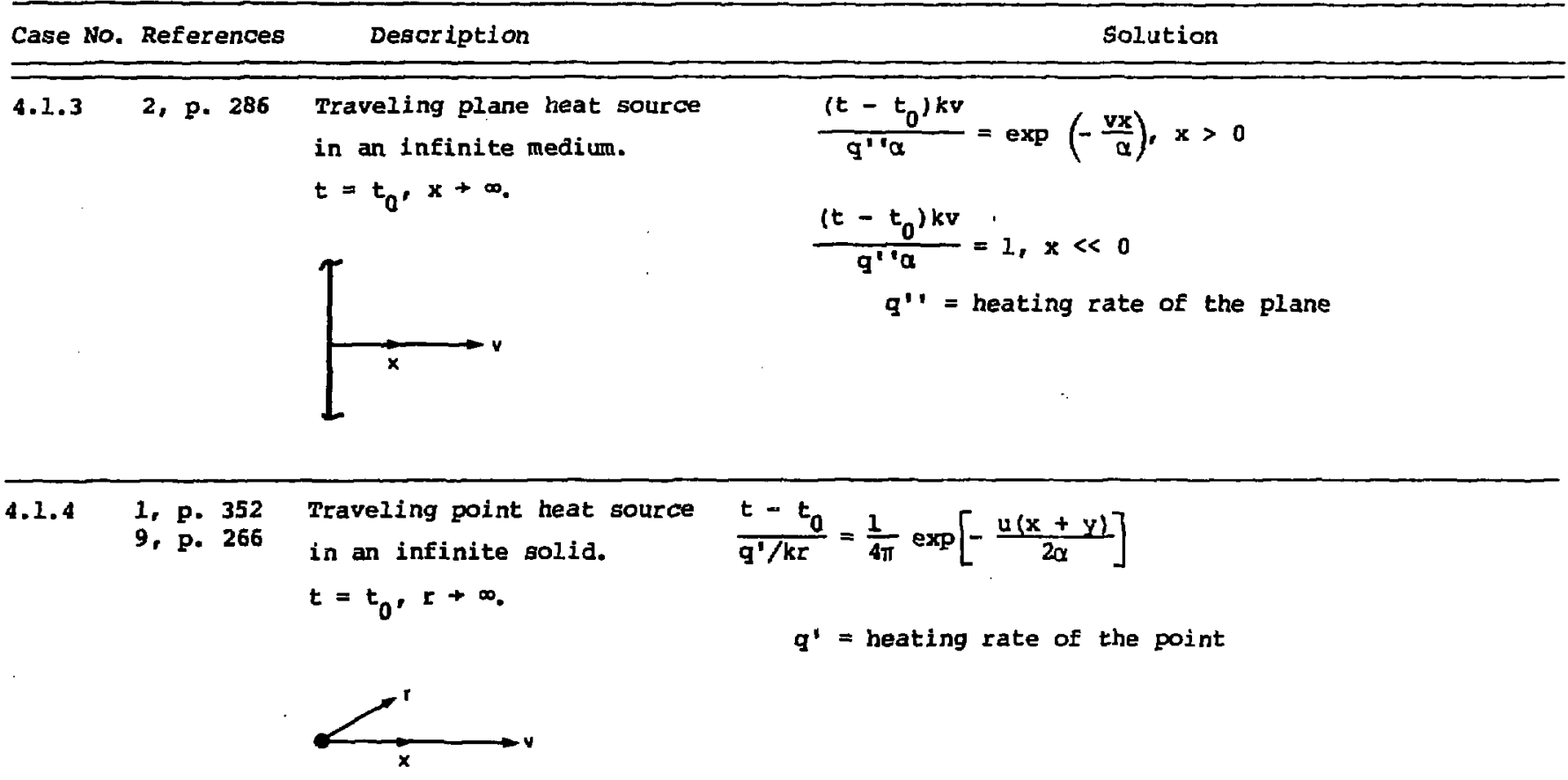


Section 4.1. Traveling Heat Sources.

\begin{tabular}{|c|c|c|c|}
\hline Case No. & References & Description & Solution \\
\hline 4.1 .5 & 9, p. 268 & $\begin{array}{l}\text { Traveling plane source in } \\
\text { an infinite rod. } \\
t=t_{0}, x+ \pm \infty\end{array}$ & $\begin{array}{l}\frac{\left(t-t_{0}\right) k C}{q^{\prime}}=\frac{\alpha C}{A U} \exp \left(\frac{v x-|x| U}{2 \alpha}\right) \\
U=\left(v^{2}+4 \alpha^{2} h C / k A\right)^{1 / 2} \\
v=\text { velocity of source } \\
q^{\prime}=\text { heating rate of source } \\
C=\text { circumference of rod } \\
A=\text { cross-sectional area }\end{array}$ \\
\hline 4.1 .6 & 9, p. 268 & 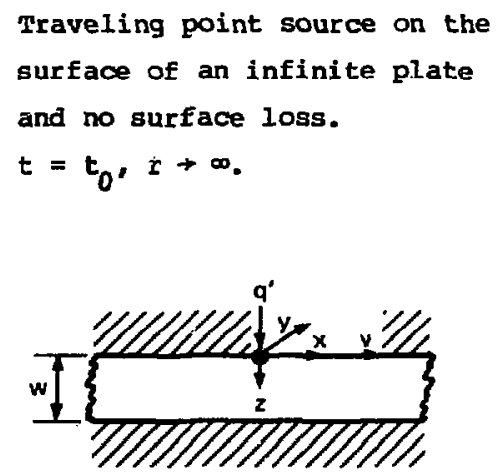 & $\begin{array}{l}\frac{\left(t-t_{0}\right) k w}{q^{\prime}}=\frac{1}{2 \pi} k_{0}\left(\frac{v r}{2 \alpha}\right)+\frac{1}{\pi} \sum_{n=1}^{\infty} k_{0}\left\{\frac{v r}{2 \alpha}\left[1+\left(\frac{2 \alpha n \pi}{v w}\right)^{2}\right]^{1 / 2}\right\} \\
\quad \times \cos \left(\frac{n \pi z}{w}\right) \exp (v x / 2 \alpha) . \\
r^{2}=x^{2}+y^{2} \\
v=\text { source velocity in } x \text {-direction } \\
q^{\prime}=\text { heating rate of point source }\end{array}$ \\
\hline
\end{tabular}


Section 4.1. Traveling Heat sources.

Case No. References Description Solution

\begin{tabular}{|c|c|c|c|}
\hline 4.1 .7 & 9, p. 268 & $\begin{array}{l}\text { Traveling line source on the } \\
\text { surface of an infinite plate } \\
\text { with no surface losses. } \\
t=t_{0}, x+ \pm \infty .\end{array}$ & $\begin{array}{l}\frac{\left(t-t_{0}\right) v w k}{g^{\prime} \alpha}=1+\sum_{n=1}^{\infty} \frac{2}{N} \cos (m z / w)(1-N) \exp (v x / 2 \alpha) \\
N=\left[1+(2 a n \pi / v w)^{2}\right]^{1 / 2} \\
v=\text { source velocity in the } \\
x \text {-direction } \\
q^{\prime}=\text { heating rate of source }\end{array}$ \\
\hline
\end{tabular}
source on the surface of a Bemi-infinite solid with no $\frac{\left(t-t_{0}\right) k v}{q^{\prime} \alpha}=\frac{2}{\pi} \int_{x \rightarrow B}^{x+B} \exp (\lambda) k_{0}\left[\left(z^{2}+\lambda^{2}\right)^{1 / 2}\right] d \lambda$ surface losses.

$t=t_{0}, x+ \pm \infty$.

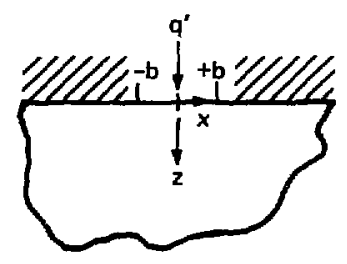

$\mathrm{x}=\mathrm{vx} / 2 \alpha, \mathrm{z}=\mathrm{vz} / 2 \alpha, \quad \mathrm{B}=\mathrm{bv} / 2 \alpha$

See Fig. 4.1

$v=$ strip velocity in the

$x$-airection

$q^{\prime}=$ heating rate of strip 
Section 4.1. Traveling Heat sources.

Case No. References Description Solution

\section{Zase No. ReEerences}

4.1.9 $30 \quad$ Traveling infinite strip source

on a semi-infinite solid with convection boundary.

$\frac{\left(t(x, z)-t_{f}\right) k \pi v}{20 q_{S}}=\int_{x-L}^{x+L} k_{0} e^{-m \sqrt{z^{2}+m^{2}}} d m$

$t=t_{f}, x+ \pm \infty$, and $z$.

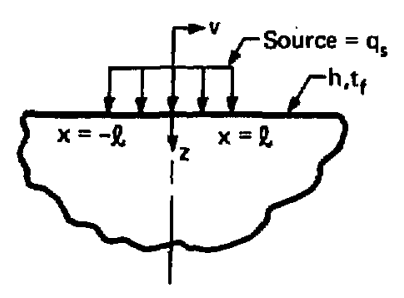

$-\pi H \exp (H z) \int_{0}^{\infty} \tau \exp \left(B^{2} \tau^{2}\right) \operatorname{erfc}(z / 2 \tau+H \tau)$

$x\{\operatorname{erf}[(X+L) / 2 \tau+\tau]-\operatorname{erf}[(X-L) / 2 \tau+\tau]\} d \tau$

$v=$ veloaity of strip

$\mathrm{H}=2 \alpha \mathrm{h} / \mathrm{kv}, \mathrm{L}=\mathrm{v} \ell / 2 \alpha, \mathrm{X}=\mathrm{vx} / 2 \alpha, z=\mathrm{vz} / 2 \alpha$

2.

See Fig. 4.2. 


\section{Section 4.1. Traveling Heat Sources.}

\section{Case No. References}

Description

Solution

4.1.10 30 Traveling band source on an
infinite cylinder with convection cooling.

$$
\frac{\left(t-t_{f}\right) \pi k}{r_{0} q_{s}} \simeq \frac{\phi}{B i}+\frac{2}{\sqrt{R}} \sum_{n=1}^{\infty} \sin (n \phi) \exp [-W(1-R) / \sqrt{2}]
$$

$$
\times\left(\frac{W \cos \left|W^{2} F O-n \phi-[W(1-R) / \sqrt{2}]-\pi / 4\right|+B i \cos \left[W^{2} F O-n \phi-W(1-R) / \sqrt{2}\right]}{n\left(W^{2}+B i^{2}+\sqrt{2} W B i\right)}\right)
$$

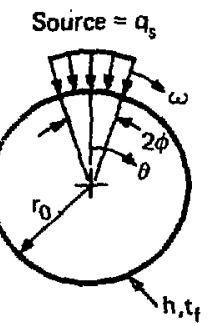

$W=I_{0} \sqrt{n \omega / \alpha}$

$\omega=$ rotation speed of source

$2 \phi=$ angular width of source

See Fig. 4.3 a and b. 
Section 4.1. Traveling Heat Sources.

Case No. References Description Solution

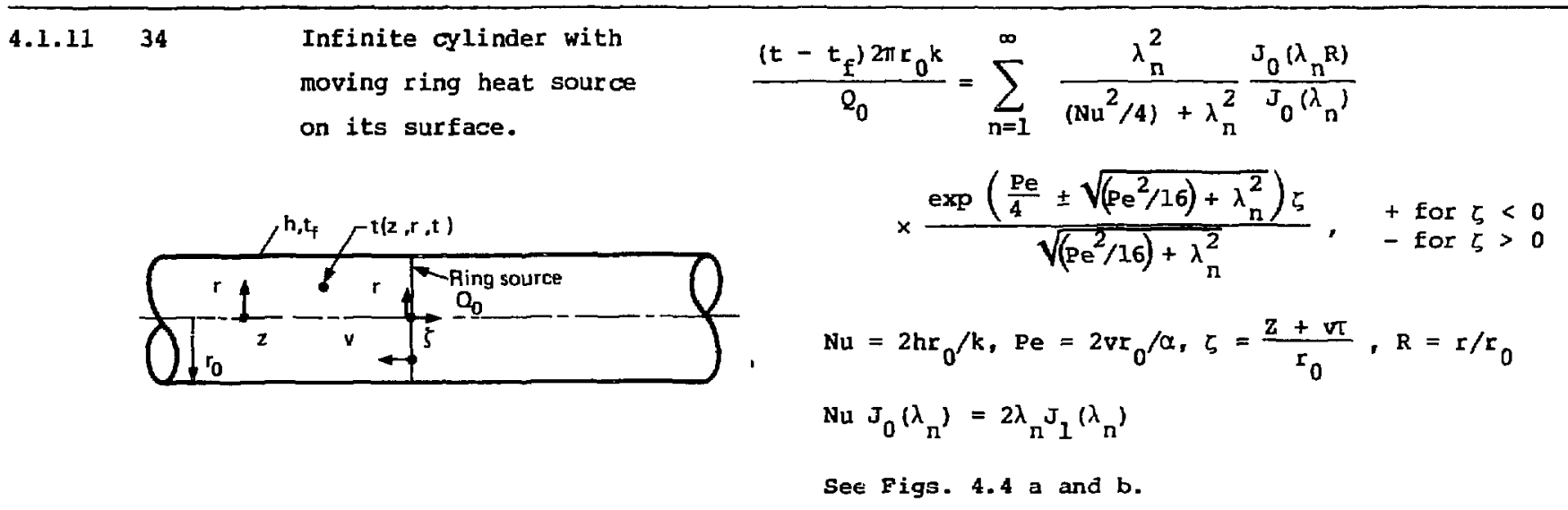


Section 4.1. Traveling Heat Sources.

Case No. References Description Solution

$\begin{aligned} & \begin{array}{l}\text { Infinite cylinder with } \\ \text { a moving ring heat source on } \\ \text { its inner surface. }\end{array}\end{aligned}$

$$
\frac{\left(t-t_{f}\right) 2 \pi I_{i} k}{Q_{0}}=\sum_{n=1}^{\infty} A_{n}\left[J_{0}\left(\lambda_{n} R\right)-\frac{J_{1}\left(\lambda_{n}\right)}{Y_{1}\left(\lambda_{n}\right)} Y_{0}\left(\lambda_{n} R\right)\right] .
$$$$
\lambda_{n}\left[J_{1}\left(\lambda_{n} R_{0}\right) Y_{1}\left(\lambda_{n}\right)-J_{1}\left(\lambda_{n}\right) Y_{1}\left(\lambda_{n} R_{0}\right)\right]
$$$$
=\frac{1}{2} N u\left[J_{0}\left(\lambda_{n} R_{0}\right) Y_{1}\left(\lambda_{n}\right)-J_{1}\left(\lambda_{n}\right) Y_{0}\left(\lambda_{n} R_{0}\right)\right] \text {. }
$$$$
A_{n}=\frac{c_{n}{ }^{2}}{\sqrt{\left(P e^{2} / 16\right)+\lambda_{n}^{2}}} \exp \left[(P e / 4) \pm \sqrt{\left(P e^{2} / 16\right)+\lambda_{n}^{2}}\right] \text {. }
$$

$$
\begin{aligned}
& C_{n}=\frac{\left[J_{0}\left(\lambda_{n}\right) Y_{1}\left(\lambda_{n}\right)-J_{1}\left(\lambda_{n}\right) Y_{0}\left(\lambda_{n}\right)\right] Y_{2}\left(\lambda_{n}\right)}{B_{n}} \\
& B_{n}=\frac{R_{0}^{2}}{2}\left\{\left[J_{0}\left(\lambda_{n} R_{0}: Y_{1}\left(\lambda_{n}\right)-J_{1}\left(\lambda_{n}\right) Y_{0}\left(\lambda_{n} R_{0}\right)\right]^{2}\right.\right.
\end{aligned}
$$$$
\left.+\left[J_{1}\left(\lambda_{n}\right) Y_{1}\left(\lambda_{n} R_{0}\right)-\Psi_{1}\left(\lambda_{n}\right) J_{1}\left(\lambda_{n} R_{0}\right)\right]^{2} \lambda_{n}^{2}\right\}
$$$$
-\frac{1}{2}\left[J_{0}\left(\lambda_{n}\right) Y_{1}\left(\lambda_{n}\right)-J_{1}\left(\lambda_{n}\right) Y_{0}\left(\lambda_{n}\right)\right]^{2}
$$

$\mathrm{R}=\mathrm{r} / \mathrm{r}_{\mathrm{i}}, \mathrm{Nu}=2 \mathrm{hr}_{\mathrm{i}} / \mathrm{k}, \mathrm{Pe}=2 \mathrm{Vr}_{\mathrm{i}} / \alpha, \zeta=\frac{z+\mathrm{v \tau}}{\mathrm{r}_{\mathrm{i}}}$ 
Section 4.1. Traveling ieat Sources.

\begin{tabular}{|c|c|c|c|c|c|c|c|}
\hline Case No. & References & Description & & Solutior: & & & \\
\hline 4.1 .13 & 70 & $\begin{array}{l}\text { Infinite cylinder traveling } \\
\text { through temperature zones. }\end{array}$ & Temperature solution & given in & source & Ref. & 70. \\
\hline
\end{tabular}

4.1.14 71 Traveling plane source in a
thin rod with change of phase.


Section 4.1. Traveling Heat Sources.

Case No. References Description

Solution

$\stackrel{\hat{\leftrightarrow}}{\circ}$ 
Section 4.1. Traveling Heat Sources.

Case No. References Description

Solution

$\stackrel{i}{\mathfrak{H}}$ 
Section 5.1. Extended Surfaces--No Internal Heating.

\section{Case No, References} Description

5.1 .1 l. p. 209 Infinite rod.

$t=t_{0}, x=0$.

$t=t_{E}, x+\infty$.

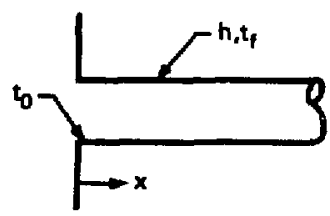

$\frac{t-t_{E}}{t_{D}-t_{E}}=\exp (-\operatorname{mx})$

$\mathrm{m}=\sqrt{\frac{\mathrm{hC}}{\mathrm{kA}}}$

u

5.1 .27$, p. 43 Finite rod, insulated end.

$t=t_{0}, x=0$.

$q_{x}=0, x=l$.

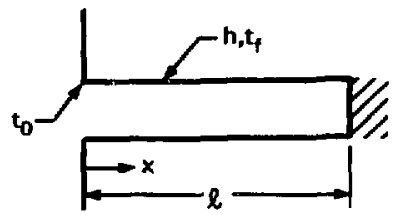

Total heat loss:

$Q=\sqrt{h C k A} \tan (m \ell)\left(t_{D}-t_{E}\right)$

$\frac{t-t_{f}}{t_{0}-t_{f}}=\frac{\cosh [m(l-x)]}{\cosh (m l)}$

See Fig. 5.1. 
Section 5.1. Extended Surfaces-No Internal Heating.

Case No. References Description Solution

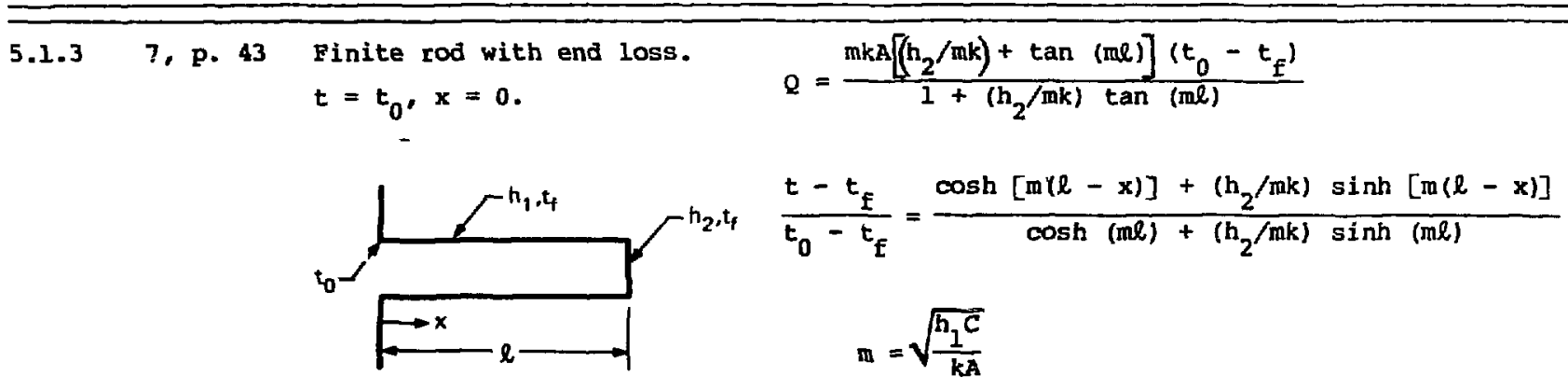

ur

具 5.1 .17$, p. 44 straight rectangular fin.
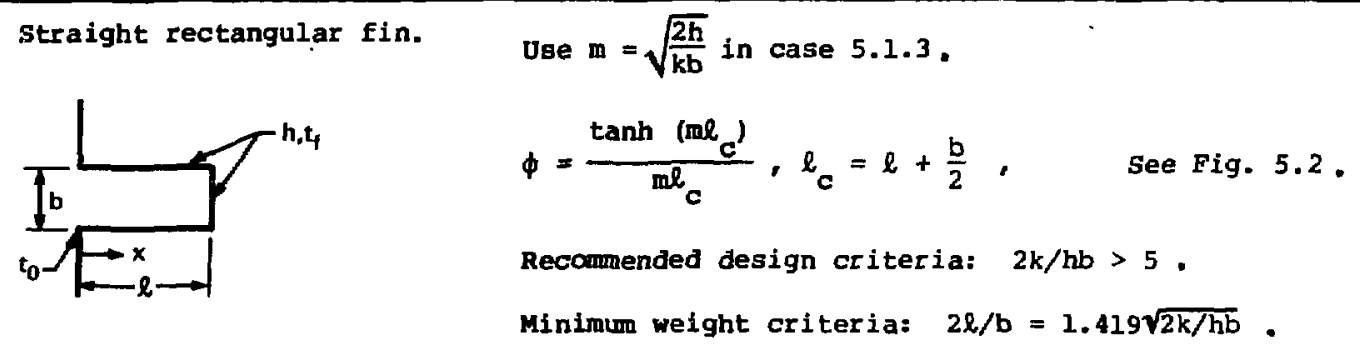
Section 5.1. Extended Surfaces--No Internal Heating.

Case NO. References Description Solution

5.1.5 7, p. 52 straight triangular fin.

$q=\sqrt{2 h k b} \frac{I_{1}(2 \sqrt{\beta l})}{I_{0}(2 \sqrt{\beta l})}, \beta=\frac{2 h l}{k b}$

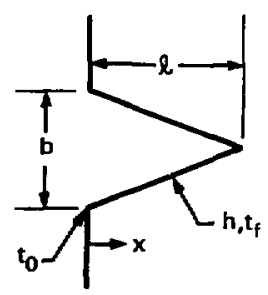

$\frac{t-t_{f}}{t_{0}-t_{f}}=\frac{I_{0}(2 \sqrt{B x})}{I_{0}(2 \sqrt{B l})}, \phi=\frac{1}{\sqrt{B l}} \frac{I_{1}(2 \sqrt{B l})}{I_{0}(2 \sqrt{B l})}$, See Fig. 5.2.

Optimum $\ell / b$ ratio: $\ell / b=0.655 \sqrt{\frac{2 k}{h b}}$.

w

5.1 .61 , p. 228 straight fin of minimum mass.

$\frac{t-t_{f}}{t_{0}-t_{f}}=1-\frac{h\left(t_{0}-t_{f}\right) x}{q^{\prime}}$,

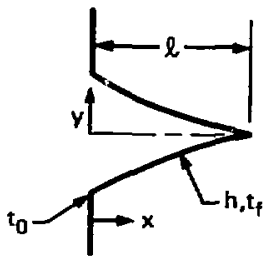

$q^{\prime}=$ total heat loss.

Profile:

$y=\frac{h}{2 k}\left(x-\frac{g^{\prime}}{h\left(t_{0}-t_{f}\right)}\right)^{2}$

$\ell=\frac{g^{\prime}}{h\left(t_{0}-t_{f}\right)}$ 
Section 5.1. Extended Surfaces--No Internal Heating.

Case No. References Description Solution

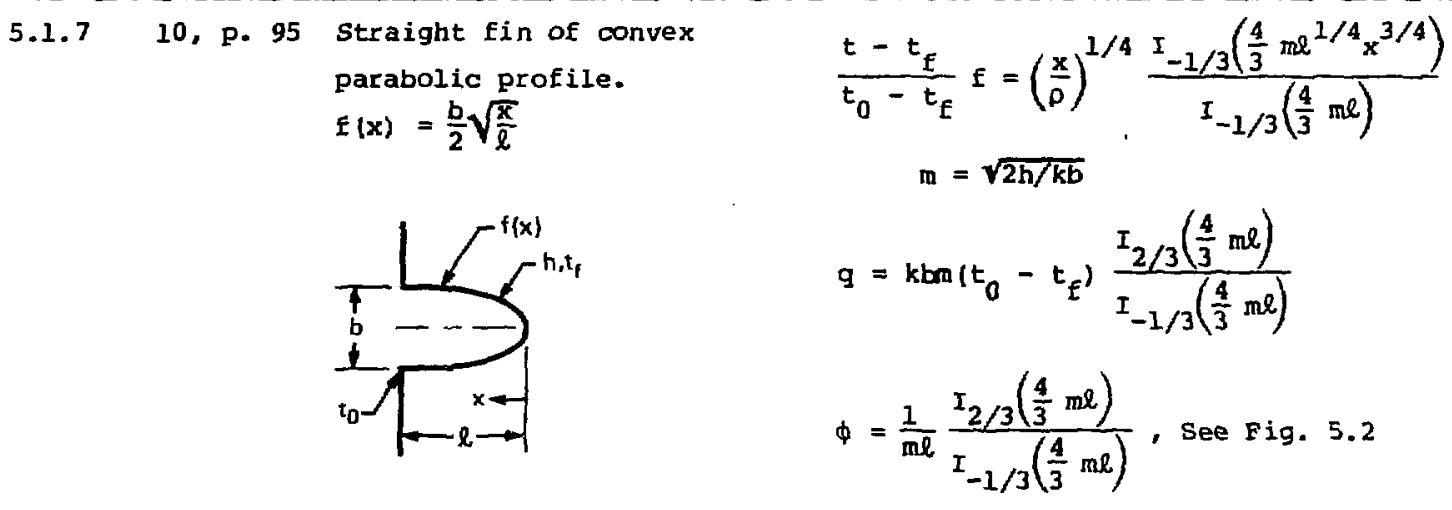

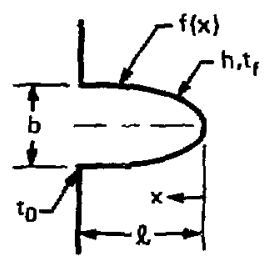

I

$$
\begin{aligned}
& \begin{array}{lll}
5.1 .8 & \text { lo, p. } 175 \text { straight fin of trapezoidal } & t-t_{f} \\
\text { parabolic profile. } & \frac{x}{t_{0}-t_{f}}=\left(\frac{x}{l}\right)^{\beta}
\end{array} \\
& f(x)=\frac{b}{2}\left(\frac{x}{l}\right)^{2} \\
& B=-\frac{1}{2}+\frac{1}{2} \sqrt{1+4 m^{2} h^{2}} \\
& \text { (leif) } \\
& q=\frac{k b \beta}{l}\left(t_{0}-t_{f}\right) \\
& \phi=\frac{1}{\beta+1}, \quad \text { See Ecg. } 5.2
\end{aligned}
$$


Section 5.1. Extended Surfaces-No Internal Heating.

Case No. References Description Solution

5.1.9 10, p. 175 Straight fin of trapezoidal profile.

$\frac{t-t_{f}}{t_{0}-t_{f}}=\frac{K_{1}\left(\beta_{e}\right) I_{0}(\beta)+I_{1}\left(\beta_{e}\right) K_{0}(\beta)}{I_{0}\left(\beta_{c}\right) K_{1}\left(\beta_{e}\right)+I_{1}\left(\beta_{e}\right) K_{0}\left(\beta_{c}\right)}$

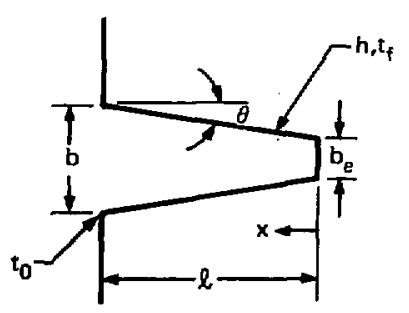

$$
\begin{aligned}
& q=\frac{h \beta_{c}\left(t_{0}-t_{f}\right)}{A^{2} \cos \theta}\left[\frac{K_{1}\left(\beta_{e}\right) I_{1}\left(\beta_{c}\right)-I_{1}\left(\beta_{e}\right) K_{1}\left(\beta_{c}\right)}{K_{1}\left(\beta_{e}\right) I_{0}\left(\beta_{c}\right)+I_{1}\left(\beta_{e}\right) K_{0}\left(\beta_{c}\right)}\right] \\
& \phi=\frac{\beta_{c}}{2 \mathrm{H}^{2} \ell_{c}} \frac{K_{l}\left(\beta_{e}\right) I_{I}\left(\beta_{c}\right)-I_{I}\left(\beta_{e}\right) K_{I}\left(\beta_{c}\right)}{K_{l}\left(\beta_{e}\right) I_{0}\left(\beta_{c}\right)+I_{1}\left(\beta_{e}\right) K_{0}\left(\beta_{c}\right)}
\end{aligned}
$$$$
B=2 B \sqrt{x+\frac{b_{e}(1-\tan \theta)}{2 \tan \theta}}, B_{e}=2 B \sqrt{\frac{b_{e}(1-\tan \theta)}{2 \tan \theta}}
$$$$
\beta_{c}=2 \mathrm{H} \sqrt{l_{c}+\frac{b_{e}^{(1-\tan \theta)}}{2 \tan \theta}}, H=\sqrt{h / k \sin \theta},
$$$$
\ell_{c}=\ell+\frac{b_{e}}{2}
$$ 
Section 5.1. Extended Surfaces--No Internal Heating.

Case No. References Description Solution

5.1.10 7, p. 54 Cylindrical fin--rectangular profile.

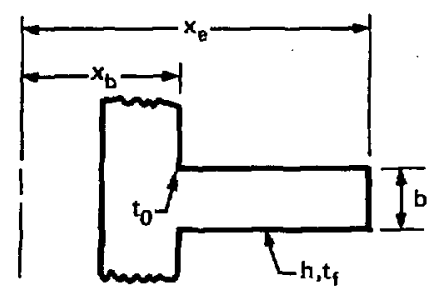

ún

$$
Q=2 \pi x_{b} b k m\left(t_{0}-t_{f}\right) \frac{I_{1}\left(x_{e} m\right) R_{1}\left(x_{b} m\right)-k_{1}\left(x_{e} m\right) I_{1}\left(x_{b} m\right)}{I_{1}\left(x_{e} m\right) R_{0}\left(x_{b} m\right)+K_{1}\left(x_{e} m\right) I_{0}\left(x_{b} m\right)}
$$$$
m=\sqrt{2 h / k b}
$$$$
\frac{t-t_{f}}{t_{0}-t_{f}}=\frac{k_{1}\left(x_{e} m\right) I_{0}(r m)+I_{1}\left(x_{e} m\right) k_{0}(r m)}{k_{1}\left(x_{e} m\right) I_{0}\left(x_{b} m\right)+I_{1}\left(x_{e} m\right) K_{0}\left(x_{b} m\right)}
$$$$
\phi=\frac{2}{x_{b} m\left[1-\left(x_{e} / x_{b}\right)^{2}\right]}\left[\frac{I_{1}\left(x_{b} m\right)-\beta R_{1}\left(x_{b} m\right)}{I_{0}\left(x_{b} m\right)+\beta K_{0}\left(x_{b} m\right)}\right] \text {, See Fig. } 5.3
$$

$$
B=I_{1}\left(x_{e} / x_{b}\right) / K_{l}\left(x_{e} / x_{b}\right)
$$

Minimum material: use Fig. 5.4.

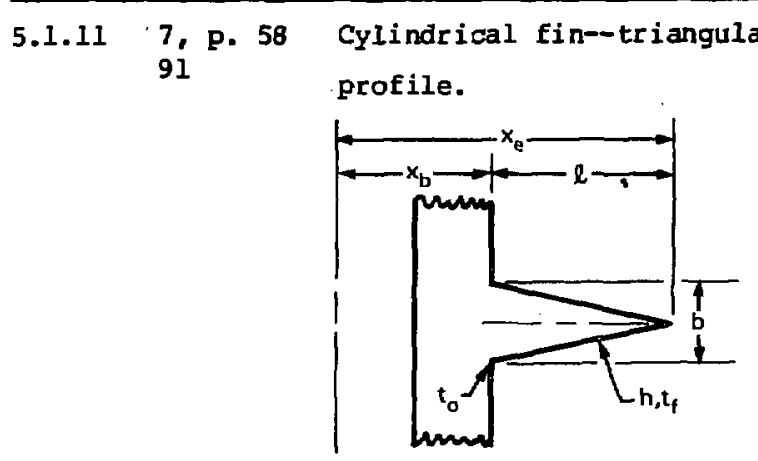


Section 5.1. Extended Surfaces--No Internal Heating.

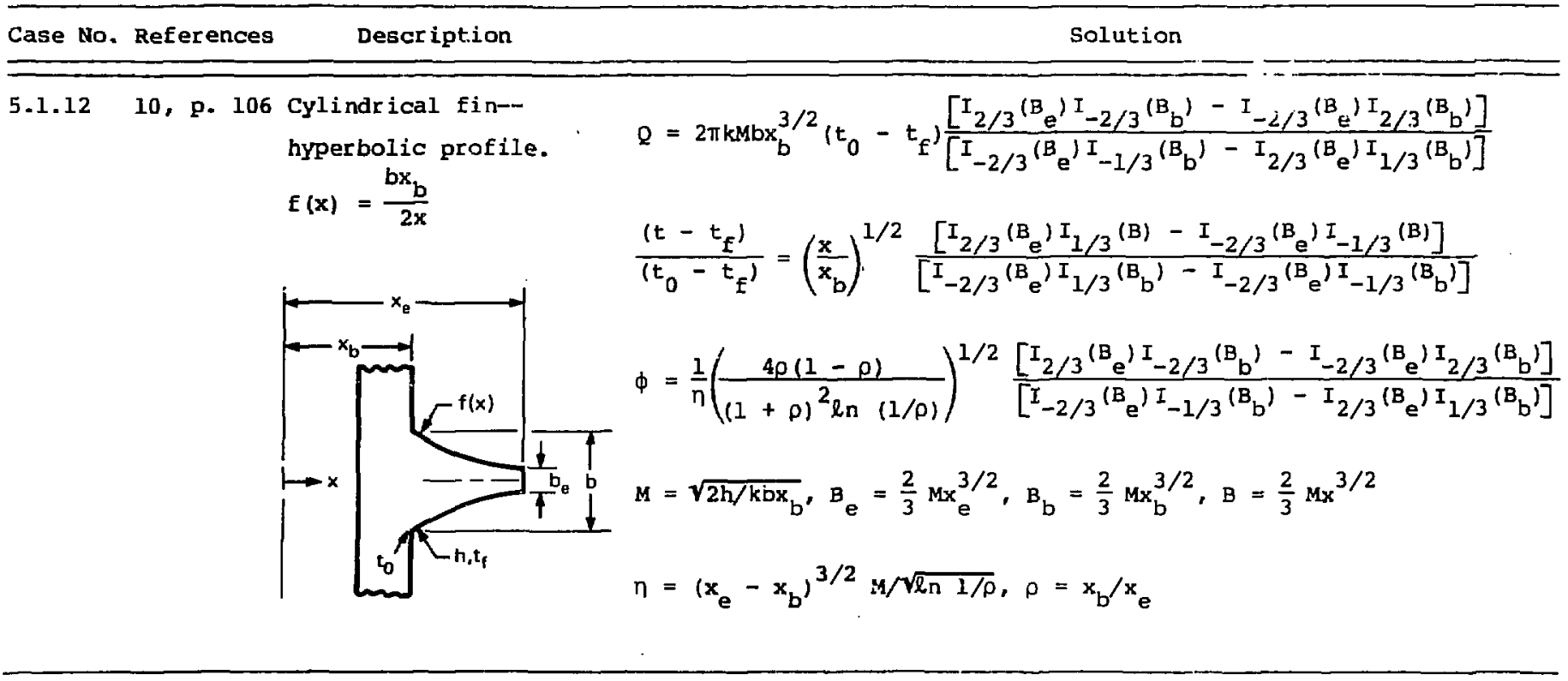


Section 5.1. Extended Surfaces--No Internal Heating.

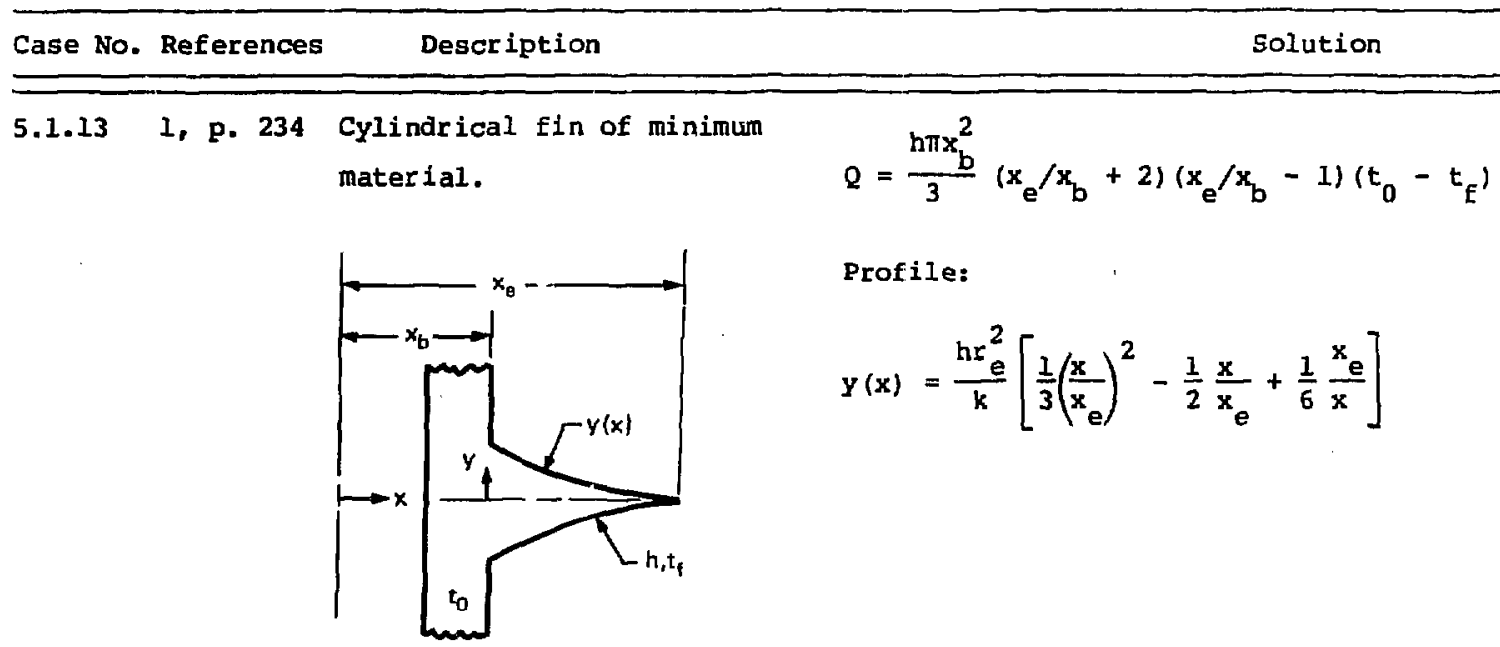

5.1.14 10, p. 114 Pin fins--cylindrical type.

$$
\begin{aligned}
& Q=\frac{\pi}{4} k d^{2} m \tanh (m l)\left(t_{0}-t_{f}\right) \\
& \phi=\frac{\tanh (m l)}{m \ell}, m=\sqrt{4 h / k d}, \text { see Fig. } 5.1
\end{aligned}
$$


Section 5.1. Extended Surfaces--No Internal Heating.

\begin{tabular}{lll}
\hline Case No. References & Description & Solution \\
\hline
\end{tabular}
5.1 .1510$, p. 115 Pin fins-rectangular type. Same as for case 5.1 .14 except $\left(\frac{\pi}{4} d^{2}\right)$ is replaced by (bd)
Depth of fin $=b$.

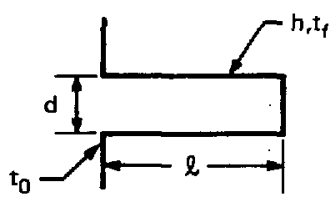

$$
\begin{aligned}
& Q=\frac{\pi k b^{2} M}{B l} \cdot \frac{I_{2}(M)}{I_{1}(M)} \cdot\left(t_{0}-t_{f}\right) \\
& \frac{t-t_{f}}{t_{0}-t_{f}}=\sqrt{\frac{l}{x}} \frac{I_{1}(M \sqrt{x / l})}{I_{1}(M)}, \text { see Fig. 5.1 } \\
& \phi=\frac{4 I_{2}(M)}{M I_{1}(M)}, M=2 \sqrt{2} m, m=\sqrt{2 h / k b}
\end{aligned}
$$


Section 5.1. Extended Surfaces-No Internal Heating.

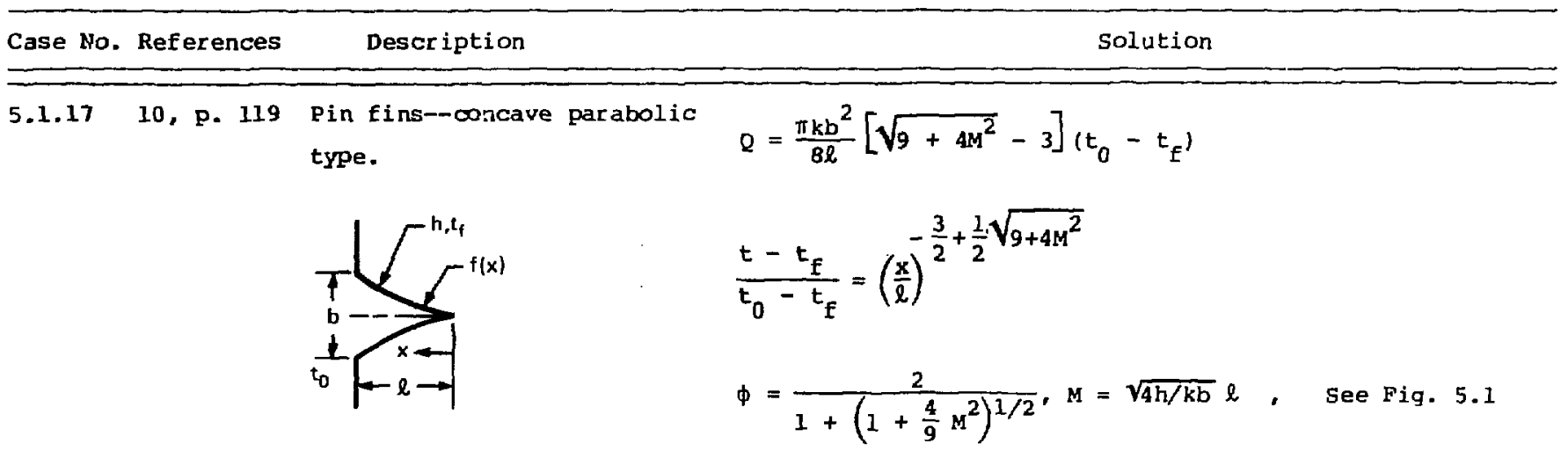

站

5.1.18 10, p. 121 Pin fins--convex parabolic type.

$Q=\frac{3 k \pi b^{2} M}{16 \ell} \frac{I_{I}(M)}{I_{0}(M)}\left(t_{0}-t_{f}\right)$

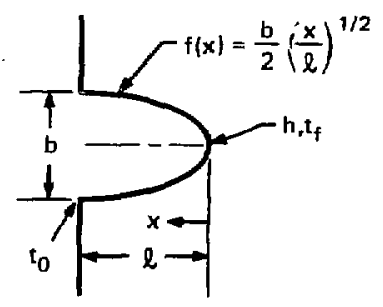

$\frac{t-t_{E}}{t_{0}-t_{E}}=\frac{I_{0}[M(x / \ell)]^{3 / 4}}{I_{0}(M)}, M=\frac{8 \ell}{3} \sqrt{\frac{k}{k b}}$

$\phi=\frac{2}{M} \frac{I_{1}(M)}{I_{0}(M)}$, see Fig. 5.1 
Section 5.1. Bxtended Surfaces--No Internal Heating.

5.1 .1910$, p. 134 Infinite fin heated by square

See Fig. 5.6 arrayed round rods.
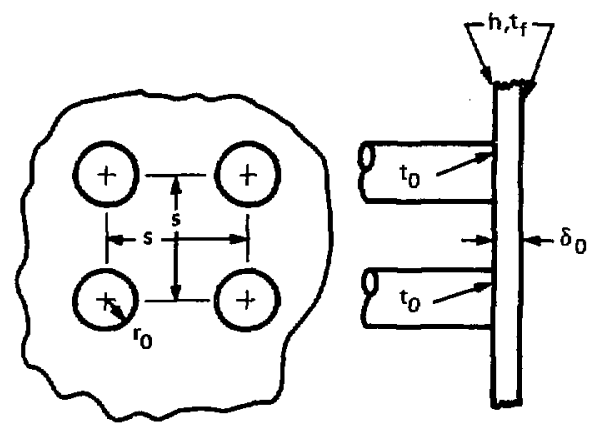

Infinite fin heated by equilateral-tri angul ar arrayed round rods.
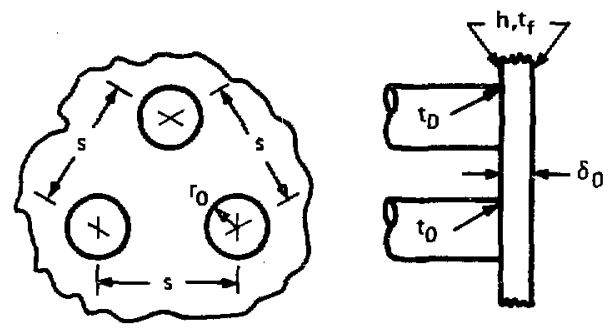
Section 5.1. Extended Surfaces-No Internal Heating.

\section{Case No. References}

Description

Solution

5.1 .2110$, p. 185 Straight rectangular $f$ in with nonuniform cooling. $h(x)=(\gamma+1) h_{a}\left(\frac{x}{l}\right)^{\gamma}$.

$h_{a}=a y$ coeff.

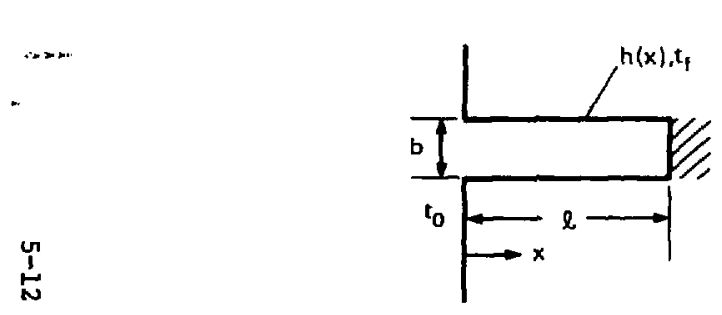

$$
\frac{t-t_{f}}{t_{0}-t_{f}}=\frac{\Gamma\left(\frac{\gamma+1}{\gamma+2}\right)}{2^{1 /(\gamma+2)}} u^{1 /(\gamma+2)}\left[I I_{-\frac{1}{\gamma+2}}^{(U)+\frac{-\frac{\gamma+1}{\gamma+2}}{I^{\left(U_{\ell}\right)}}} \frac{\gamma+1}{\gamma+2}^{\left(U_{\ell}\right)} I_{\frac{1}{\gamma+2}}(U)\right]
$$$$
\phi=\left[\frac{(\gamma+2)^{Y}(\gamma+1)}{(m \ell)^{2(\gamma+1)}}\right]^{1 /(\gamma+2)}\left[\frac{I(\gamma+1) /(\gamma+2)\left(U_{\ell}\right)}{I-(\gamma+1) /(\gamma+2)}{ }^{\left(U_{\ell}\right)}\right]\left[\frac{\Gamma \frac{\gamma+1}{\gamma+2}}{\Gamma \frac{1}{\gamma+2}}\right]
$$$$
u=\frac{2 \sqrt{\gamma+1}}{\gamma+2} m l^{-\gamma / 2} x^{(\gamma+2) / 2}
$$$$
v_{\ell}=\frac{2 \sqrt{\gamma+I}}{\gamma+2} m, m=\sqrt{2 h_{a} / k b}
$$

\section{1 .2210$, p. 191 Same conditions as case}

5.1 .21 with:

$h(x)=h_{a}\left[\frac{(1+a)(x / l+c)^{a}}{(1+c)^{a+1}-c^{a+1}}\right]$

$\frac{t-t_{E}}{t_{0}-t_{E}}=\left(\frac{U}{U_{0}}\right)^{n}\left[\frac{I_{n}(U) I_{2-n}\left(U_{\ell}\right)-I_{-n}(U) I_{n-I}\left(U_{\ell}\right)}{I_{n}\left(U_{0}\right) I_{1-n}\left(U_{\ell}\right)-I_{-n}\left(U_{0}\right) I_{n-1}\left(U_{\ell}\right)}\right]$

$\phi=\frac{2(1-n)}{u_{0}\left[1-\left(u_{\ell} / u_{0}\right)^{2(1-n)}\right]}\left[\frac{I_{n-1}\left(u_{0}\right) I_{l-n}\left(u_{\ell}\right)-I_{l-n}\left(u_{0}\right) I_{n-1}\left(u_{l}\right)}{I_{n}\left(u_{0}\right) I_{1-n}\left(u_{l}\right)-I_{-n}\left(u_{0}\right) I_{n-1}\left(u_{l}\right)}\right]$

$u=2 n\left(\frac{1-n}{n}\right)^{1 / 2}\left[\frac{(x / l+c)^{1 / n}}{(1+c)^{(1-n) / n}-c^{(1-n) / n}}\right] m l$

$u_{0}=u, x=0 ; u_{\ell}=u, x=\ell, n=\frac{1}{2 a}, m=\sqrt{2 h_{a} / k b}$ 
Section 5.1. Extended Surfaces-No Internal Heating.

\section{Case No. References}

Description

Solution

$5.1 .2310, p .187$ Same conditions as case 5.1 .22
with:
\[ h(x)=h_{a}\left[\frac{1-a \exp (-c x / \ell)}{1-(a / c)[1-\exp (-c)]}\right] . \]

$$
\begin{aligned}
& n=\frac{2 m l}{c}, u=n \sqrt{a} \exp \left(-\frac{x c}{2 l}\right) \\
& \psi=n \sqrt{a} \exp (c), B=n \sqrt{a}
\end{aligned}
$$

If $\mathrm{n}$ is an integer:

$$
\begin{aligned}
& Q=\frac{\sqrt{a}}{2 m l\{1-(a / c)[1-\exp (-c)]\}} \cdot \frac{A_{1}-A_{2}}{A_{3}-A_{4}} \\
& A_{1}=\left[Y_{n-1}(\psi)-Y_{n+1}(\psi)\right]\left[J_{n-1}(B)-J_{n+1}(B)\right] \\
& A_{2}=\left[J_{n-1}(\psi)-J_{n+1}(\psi)\right]\left[Y_{n-1}(\beta)-Y_{n+1}(\beta)\right] \\
& A_{3}=J_{n}(\beta)\left[Y_{n-1}(\psi)-Y_{n+1}(\psi)\right] \\
& A_{4}=Y_{m}(\beta)\left[J_{n-1}(\psi)-J_{n+1}(\psi)\right]
\end{aligned}
$$

If $\mathrm{n}$ is not an integer:

$$
\begin{aligned}
\phi= & \frac{c}{\left.2(m l)^{2} \mid 1-(a / c)[1-\exp (-c)]\right\}} \cdot \frac{A_{1}-A_{2}}{A_{3}-A_{4}} \\
A_{1} & =\left[-n J_{-n}(\psi)-\psi J_{1-n}(\psi)\right]\left[-n J_{n}(B)+B J_{n-1}(B)\right] \\
A_{2} & =\left[-J_{n}(\psi)+\psi J_{n-1}(\psi)\right]\left[-n J_{-n}(B)-\beta J_{1-n}(\beta)\right] \\
A_{3} & =J_{n}(\beta)\left[-n J_{-n}(\psi)-\psi J_{1-n}(\psi)\right] \\
A_{4} & =J_{-n}(B)\left[-n J_{n}(\psi)+\psi J_{n-1}(\psi)\right]
\end{aligned}
$$


Section 5.1. Extended Surfaces--No Internal Heating.

Case No. References Description Solution

5.1 .2410$, p. 205 straight fin of rectangular profile radiating to free space.

$t=t_{0}, x=0$.

$T=T_{e^{\prime}} x=\ell$.

Space temp $=0 \mathrm{~K}$.

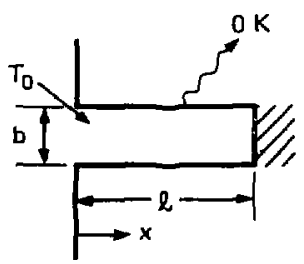

$\ell \sqrt{\frac{20 \delta E T^{3}}{k b}}=B(0.3,0.5)-B_{v}(0.3,0.5)$

$\mathrm{B}=$ complete beta function

$B_{u}=$ incomplete beta function

$Q=2 k b \sqrt{\frac{\sigma \varepsilon}{5 k b}\left(T_{0}^{5}-T_{e}^{5}\right)}, \quad$ see Figs. 5.8 and 5.9

$\phi=\frac{2}{\ell} \sqrt{\frac{1 / \mathrm{z}^{3}-1 / \mathrm{z}^{8}}{20 \sigma \epsilon \mathrm{T}_{\mathrm{e}}^{3} / k b}}, \mathrm{z}=\mathrm{T}_{0} / \mathrm{T}_{\mathrm{e}}$

Optimum length: $b / \ell^{2}=2.486 \sigma \in \mathrm{T}_{0}^{3} / \mathrm{k}$ 
Section 5.1. Extended Surfaces--No Internal Heating.

Case No. References Description Solution

5.1 .2510$, p. 211 straight fin of rectangular profile radiating to nonfree space.

$\frac{Q l}{k b T_{0}}=\sqrt[l]{\frac{2 K_{1} T_{0}^{3}}{5 k b z^{5}}} \sqrt{z-1}\left[z^{4}+z^{3}+z^{2}+z+I-\frac{5 z^{4} K_{2}}{K_{1} T_{0}^{4}}\right]^{1 / 2}$

$T=T_{0}, \mathbf{x}=0$.

$T=T_{e}, x=\ell$.

$K_{2}=$ incident radiation

$\phi=\frac{Q \ell / k b T_{0}}{2 \sigma \varepsilon \ell^{2} T_{0}^{3} / k b}, K_{1}=2 \sigma \varepsilon, z=T_{0} / T_{e}$

$\stackrel{u}{1}$

absorbed from surroundings.

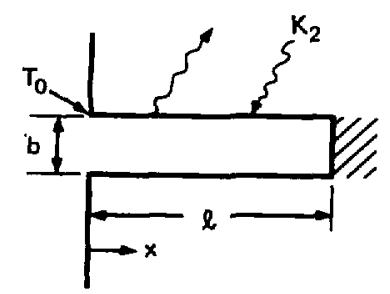

See Figs. 5.10 and 5.11

Optimum length: $\quad b / l^{2}=2.365 \sigma E T_{0}^{3} / k$ 
Section 5.1. Extended Surfaces--No Internal Heating.

Case No. References Description solution

5.1 .2610$, p. 218 Straight trapezoidal fin radiating to nonfree space. $T=T, x=0$.

$R_{2}=$ incident radiation absorbed from surroundings.

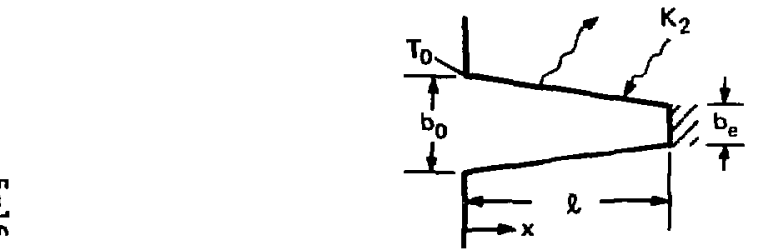

See Figs. 5.12a, 5.12b, 5.12c, and 5.12d.

$\lambda=b_{e} / b_{0}=0.75,0.50,0.25$, and 0 (triangular fin). $R_{1}=2 \sigma \varepsilon$.

5.1.27 10, P. 228 straight fin of least material Profile: radiating to free space. $\mathbf{T}=0, \mathbf{x}=0$.

$E(x)=\frac{3 Q^{2}}{2 k \sigma \in T_{0}^{5}}\left(\frac{x}{\ell}\right)^{7 / 2}, l=\frac{3 Q}{2 \sigma \varepsilon T_{0}^{4}}$

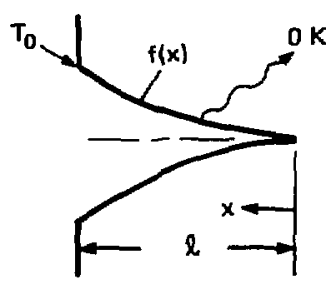

$\frac{T}{T_{0}}=\left(\frac{x}{l}\right)^{1 / 2}$ 
Section 5.1. Extended Surfaces--No Internal Heating.

Case No. References Description Solution

5.1.28 10, p. 230 Straight fin with constant Profile:

temperature gradient

radiating to nonfree space. $f(x)=\frac{\mathrm{K}_{1} \ell^{2} \mathrm{~T}_{0}^{3}}{10 \mathrm{kz}^{3}(z-1)^{2}}\left[\left[1+\frac{x}{\ell}(z-1)\right]^{5}-1-\frac{5 \mathrm{~K}_{2} \mathrm{z}^{4}}{\mathrm{~K}_{1} \mathrm{~T}_{0}^{8}}\left[\frac{\mathrm{x}}{\ell}(\mathrm{z}-1)\right]\right\}$
$\frac{\mathrm{dT}}{\mathrm{dx}}=\frac{\mathrm{T}_{0}-\mathrm{T}_{\mathrm{e}}}{\ell}$.

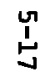

$$
Q=\frac{K_{1} l T_{e}^{4}\left(z^{5}-1\right)}{5(2-1)}-K_{2} \ell
$$

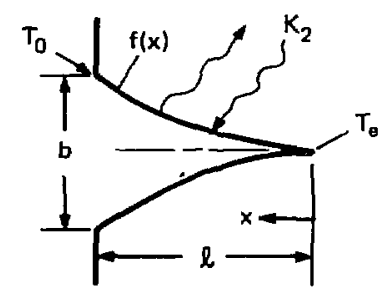

$$
\begin{aligned}
& \mathrm{z}=\mathrm{T}_{0} / \mathrm{T}_{\mathrm{e}} \\
& \mathrm{K}_{1}=2 \sigma \varepsilon \\
& \phi=\frac{\mathrm{z}^{5}-1}{5 z^{5}(z-1)}-\frac{\mathrm{K}_{2}}{\mathrm{~K}_{1} \mathrm{~T}_{0}^{4}}
\end{aligned}
$$

$\mathrm{R}_{2}=$ incident radiation absorbed

$T_{e, \text { min }}=\left(K_{2} / K_{1}\right)^{1 / 4}$, See Figs. 5.13 and 5.14 
Section 5.1. Extended Surfaces--No Internal Heat:-

Case No. References Description Solution

5.1.29 10, Radial fln of trapezoidal

p. 247 profile radiating to nonfree space.

$\mathbf{T}=\mathbf{T}_{0}, \mathbf{r}=\mathbf{r}_{0^{\circ}}$

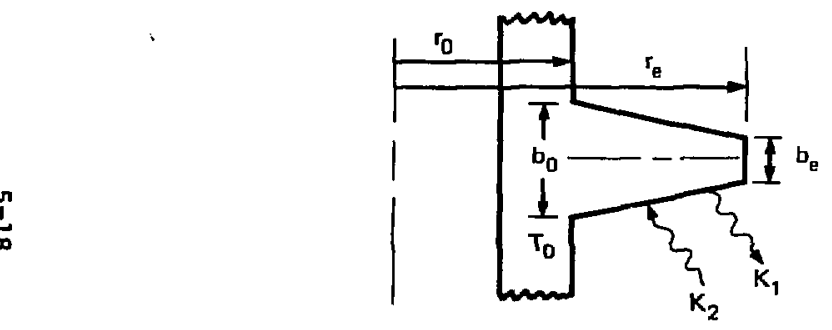

Fin efficiency: (See Figs. 5.15a-5.15l)

$\lambda=b_{\mathrm{e}} / \mathrm{b}_{0}=0,0.5,0.75,1.0$

$\mathrm{K}_{2} / \mathrm{K}_{1} \mathrm{~T}_{0}^{4}=0,0.2,0.4$

Profile No. $=\mathrm{K}_{1} \mathrm{~T}_{0}^{3} \mathrm{r}_{0}^{2} / \mathrm{kb}_{0}$

$\mathrm{K}_{1}=\sigma \varepsilon_{,} \mathrm{K}_{2}=$ incident radiation absorbed 
Section 5.1. Extended Surfaces- No Internal Heating.

\section{Case No. References}

Description

Solution

5.1.30 10, The capped cylinder,

p. 275 convection boundary.

$t=t_{0}, x=\ell$.

Insulated inside surfaces.

1
$\vdots$
\[ \]
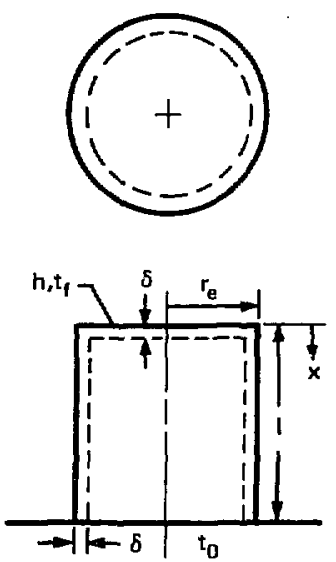

$\phi=\frac{\sinh (m \ell)+[\cosh (m l)-1]\left[I_{1}\left(m r_{e}\right) / I_{0}\left(m r_{e}\right)\right]}{m l\left\{\cosh (m l)+\sinh (m l)\left[I_{1}\left(m r_{e}\right) / I_{0}\left(m r_{e}\right)\right]\right\}}$

Top:

$\frac{t-t_{f}}{t_{0}-t_{f}}=\frac{I_{0}(m r) / I_{0}\left(m r_{e}\right)}{\cosh (m l)+\sinh (m l)\left[I_{l}\left(m r_{e}\right) / I_{0}\left(m r_{e}\right)\right]}$

$\phi=\frac{2 I_{1}\left(m r_{e}\right) / I_{0}\left(m r_{e}\right)}{m r_{e}\left(\cosh (m \ell)+\sinh (m l)\left[I_{1}\left(m r_{e}\right) / I_{0}\left(m r_{e}\right]\right)\right.}$

$m=\sqrt{h / k \delta}$, see Figs. 5.16a, and 5.16b

\section{Side:}

$\frac{t-t_{f}}{t_{0}-t_{f}}=\frac{\cosh (m x)+\sinh (m x)\left[I_{1}\left(m r_{e}\right) / I_{0}\left(m r_{e}\right)\right]}{\cosh (m l)+\sinh (m l)\left[I_{1}\left(m r_{e}\right) / I_{0}\left(m r_{e}\right)\right]}$ 
Section 5.1. Extended Surfaces--No Internal Heating.

Case No. References Description Solution

5.1.31 10, Doubly heated straight,

p. 407 rectangular fin. $t=t_{0}, x=0$.

$t=t_{l}, x=\ell$.

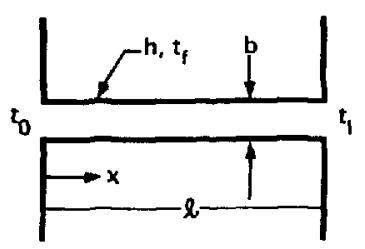

$$
\frac{t-t_{f}}{t_{D}-t_{f}}=\exp (m x)-2 B \sinh (m x)
$$$$
g_{0}=\operatorname{kbm}(28-I)\left(t_{0}-t_{f}\right)
$$$$
q_{l}=k b m\left[\exp (m l)-2 B \cosh (m l)\left(t_{0}-t_{f}\right)\right]
$$

$$
B=\frac{\exp (m l)-\left(t_{l}-t_{f}\right) /\left(t_{0}-t_{f}\right)}{2 \sinh (m l)}, m=\sqrt{2 h / k b}
$$

See Fig. 5.17 
Section 5.1. Extended Surfaces--No Internal Heating.

\begin{tabular}{|c|c|c|c|}
\hline Case No. & References & Description & Solution \\
\hline 5.1 .32 & $\begin{array}{l}\text { 10, } \\
\text { p. } 394\end{array}$ & $\begin{array}{l}\text { single } T \text {, straight, } \\
\text { rectangular fin. } \\
t=t_{0}, x=b . \\
\text { All surfaces convectively } \\
\text { cooled by } h, t_{f^{*}}\end{array}$ & 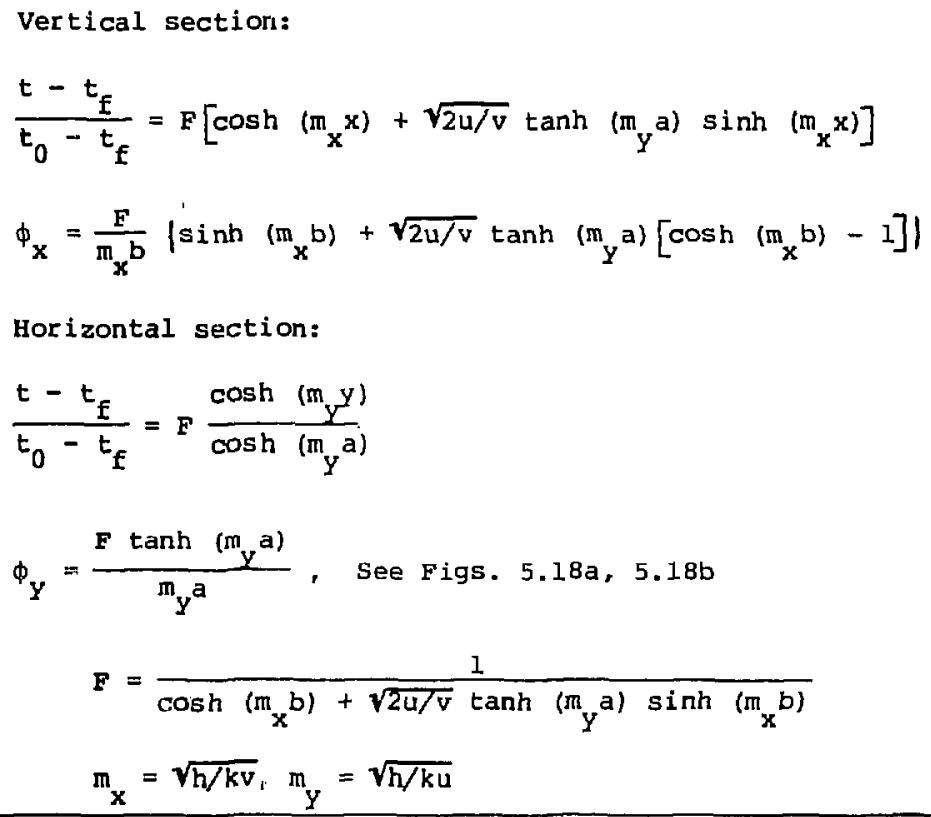 \\
\hline
\end{tabular}


Section 5.1. Extended Surfaces--No Internal Heating.

5.1.33 10, Double T, straight,

p. 413 rectangular fin.

$t=t_{0}, x_{1}=b_{1}$.

Al]. surfaces convectively cooled by $h, t_{f}$.

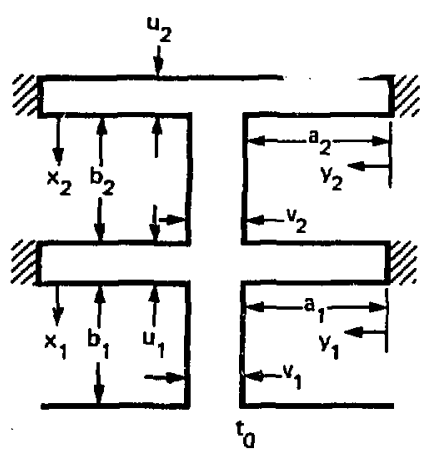

\section{Vertical sections:}

$\frac{t-t_{f}}{t_{0}-t_{f}}=F_{1}\left[\cosh \left(m_{x I} x_{1}\right)+k_{1} \sinh \left(m_{x I} x_{1}\right)\right], 0<x_{1}<b_{1}$.

$\frac{t-t_{f}}{t_{0}-t_{f}}=F_{1} F_{2}\left[\cosh \left(m_{x 2} x_{2}\right)+\sqrt{2 u_{2} / v_{2}} \tanh \left(m_{Y 2} a_{2}\right)\right.$ $x \sinh \left(m_{x 2} x_{2}\right), 0<x_{2}<b_{2}$.

$\phi_{x 1}=\frac{F_{1}}{m_{x 1} b_{1}}\left[\sinh \left(m_{x 1} b_{1}\right)+k_{1}\left(\cosh \left(m_{x 1} b_{1}\right)-1\right)\right], 0<x_{1}<b_{1}$.

$\phi_{x 2}=\frac{F_{1} F_{2}}{m_{x 2} b_{2}}\left[\sinh \left(m_{x 2} b_{2}\right)+\sqrt{2 u_{2} / v_{2}} \tanh \left(m_{y 2} a_{2}\right)\right.$ $\left.x\left(\cosh \left(m_{x 2} b_{2}\right)-1\right)\right], 0<r_{2}<b_{2}$.

Horizontal section:

$\frac{t-t_{f}}{t_{0}-t_{f}}=F_{1} \frac{\cosh \left(m_{y 1} y_{1}\right)}{\cosh \left(m_{y 1} a_{1}\right)}, 0<y_{1}<a_{1}$

$\frac{t-t_{f}}{t_{0}-t_{E}}=F_{1} F_{2} \frac{\cosh \left(m_{y 2} y_{2}\right)}{\cosh \left(m_{y 2} a_{2}\right)}, 0<y_{2}<a_{2}$

$\phi_{y l}=\frac{F_{1}}{m_{Y 1} a_{1}} \tanh \left(m_{Y 1} a_{1}\right), 0<y_{1}<a_{1}$

$\phi_{Y 2}=\frac{F_{1} F_{2}}{m_{y 2} a_{2}} \tanh \left(m_{y 2} a_{2}\right), 0<y_{2}<a_{2}$ 
Section 5.1. Extended Surfaces--No Internal Heating.

\begin{tabular}{lcl}
\hline Case No. References & Description & Solution \\
\hline 5.1 .33 continued & $F_{1}=\frac{1}{\cosh \left(m_{x 1} b_{1}\right)+B \sinh \left(m_{x 1} b_{1}\right)}$ \\
$F_{2}=\frac{1}{\cosh \left(m_{x 2} b_{2}\right)+\sqrt{2 u_{2} / v_{2}} \tanh \left(m_{y 2} a_{2}\right) \sinh \left(m_{x 2} b_{2}\right)}$ \\
$B=F_{2} \sqrt{v_{2} / v_{1}}\left[\sinh \left(m_{x 2} b_{2}\right)+\sqrt{2 u_{2} / v_{2}} \tanh \left(m_{Y 2} a_{2}\right)\right.$ \\
$x \cosh \left(m_{x 2} b_{2}\right)+2 \sqrt{u_{1} / v_{1}} \tanh \left(m_{Y 1} b_{1}\right)$ \\
$m_{x 1}=\sqrt{2 h / k v_{1}}, m_{x 2}=\sqrt{2 h / k v_{2}}$ \\
$m_{y 1}=\sqrt{2 h / k u_{1}}, m_{y 2}=\sqrt{2 h / k u_{2}}$
\end{tabular}


Section 5.1. Extended Surfaces--No Internal Heating.

\section{Case No. References}

5.1.34 2, Insulated wires adjoined on

p. 179

a convectively cooled plate

(e.g., thermocouple wires).

$$
\frac{t_{0}-t_{\infty}}{t_{f I}-t_{\infty}}=\frac{I}{I+\left[B R_{I}\left(I_{w} H\right) / R_{0}\left(I_{w} H\right)\right]}
$$

$t=t_{\infty} r+\infty$.

wire radius $=r_{w}$.

Wire insulation thickness $=\delta$.

$$
B=\frac{2}{\sqrt{k_{w l}}+\sqrt{k_{w 2}}}\left[\frac{k_{p} w\left(h_{1}+h_{2}\right)\left(1 / h_{i}+\delta / k_{i}\right)}{r_{w}}\right]^{1 / 2}
$$

wire therm. cond. $=k_{w}$.

Insulation therm. cond, $=k_{i}$.

$H=\sqrt{2\left(h_{1}+h_{2}\right) / k w}$

plate therm. cond. $=k_{p}$.

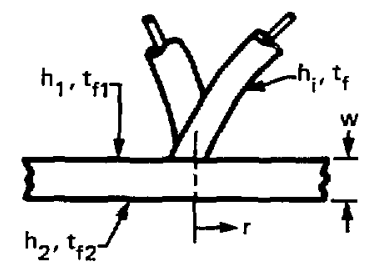


Section 5.1. Extended Surfaces--No Internal Heating.

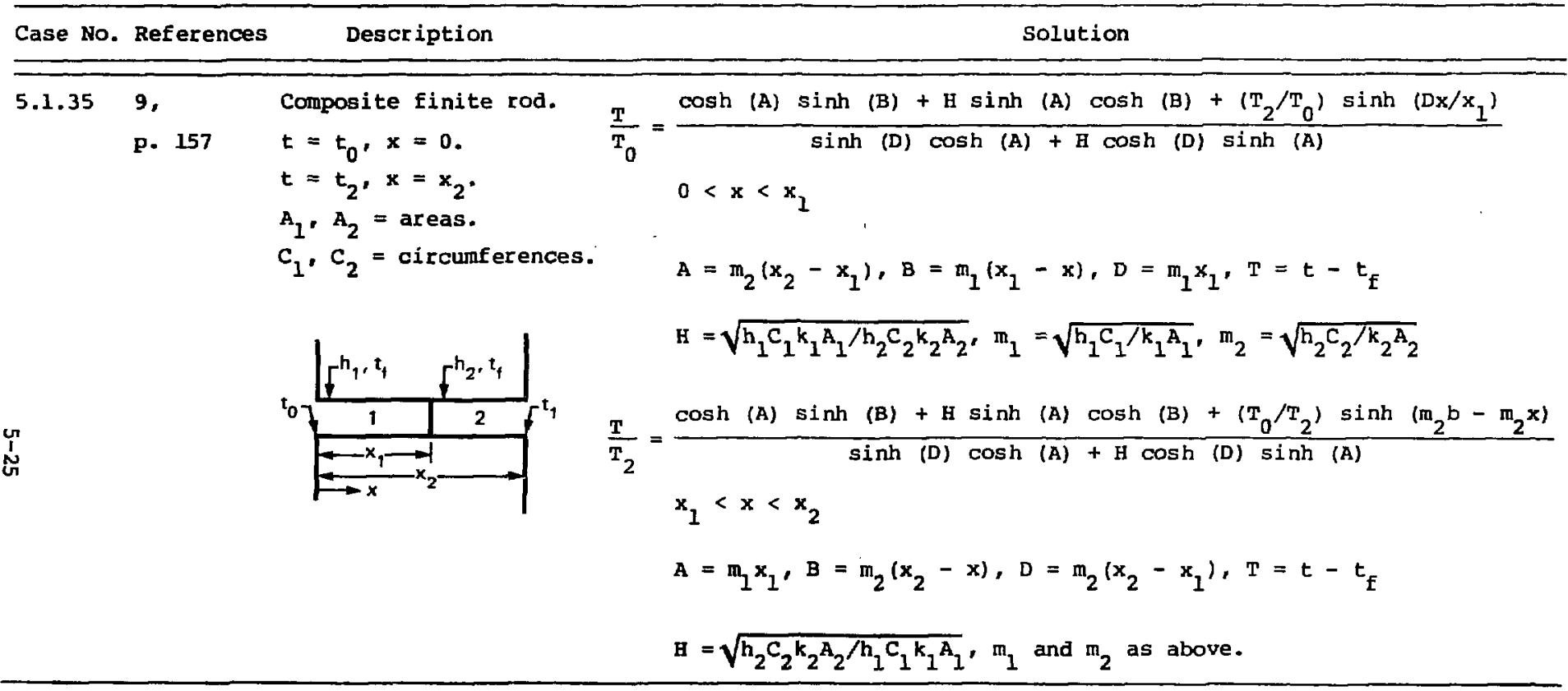


Section 5.1. Extended Surfaces--No Internal Heating.

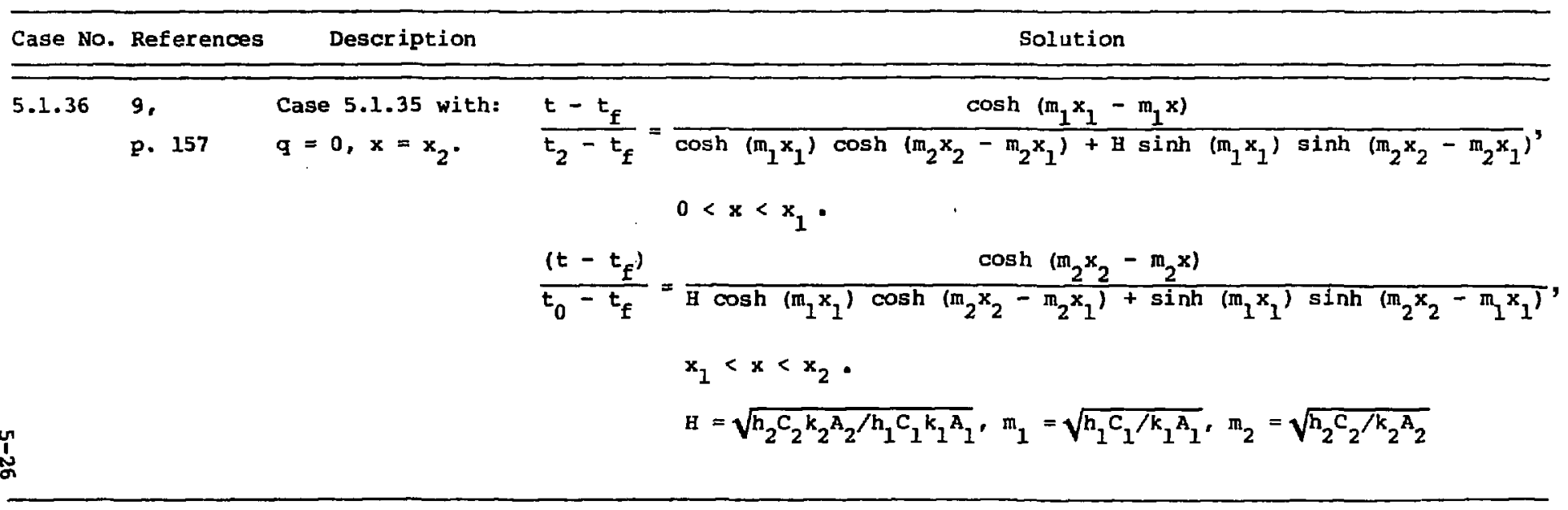


Section 5.1. Extended Surfaces--No Internal Heating.

\begin{tabular}{lll}
\hline Case No. References & Description & Solution \\
\hline
\end{tabular}

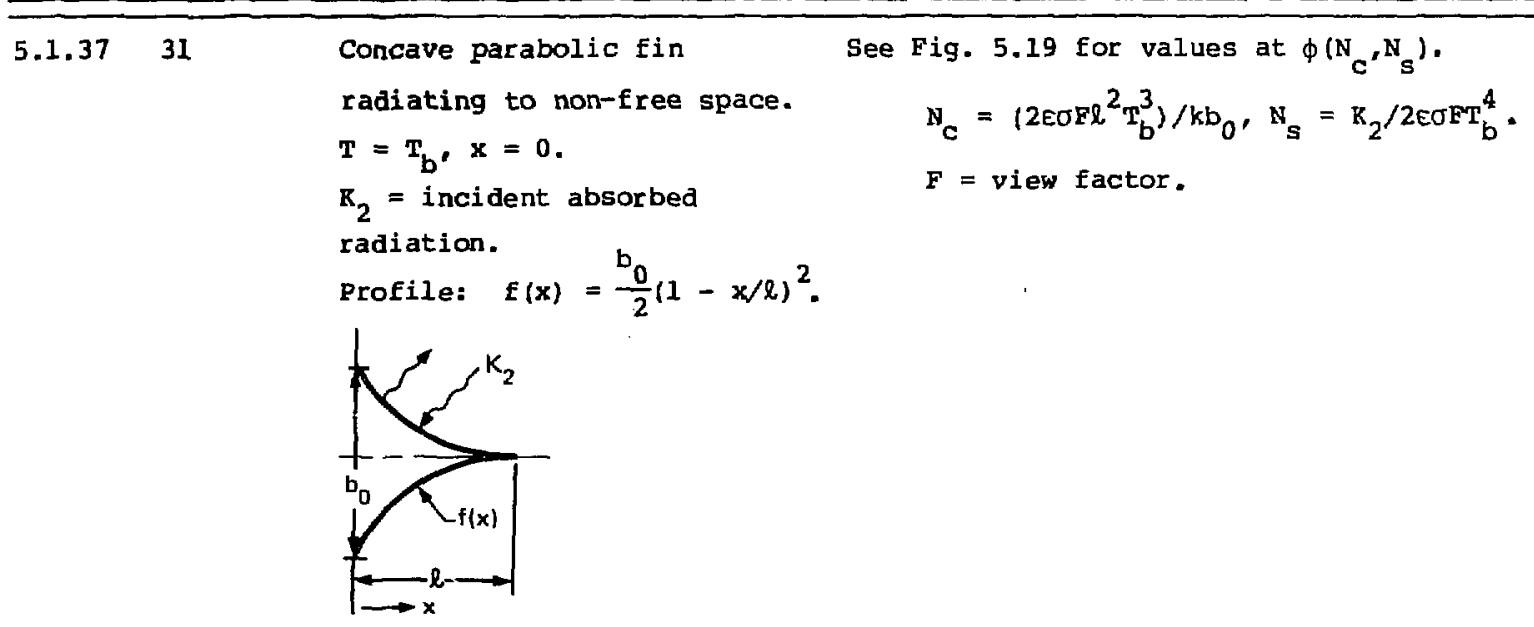

\begin{tabular}{|c|c|c|c|}
\hline 5.1 .38 & 31 & $\begin{array}{l}\text { Convex parabolic } f \text { in } \\
\text { radiating to non-free space. } \\
\text { Conditions same as for case } \\
5.1 .37 \text { except profile is: } \\
f(x)=\frac{b_{0}}{2} \sqrt{1-x / l}\end{array}$ & $\begin{array}{l}\text { See Fig. } 5.20 \text { for values of } \phi\left(N_{c}, N_{s}\right) \text {. } \\
N_{c} \text { and } N_{s} \text { given in case } 5.1 .37\end{array}$ \\
\hline 5.1 .39 & 81 & $\begin{array}{l}\text { straight rectangular fin of } \\
\text { variable conductivity. } \\
k=k_{0}\left[1+B\left(t-t_{0}\right)\right]\end{array}$ & $\begin{array}{l}\text { See Ref. } 81 \text { for approximate temperature and efficiency } \\
\text { solutions. }\end{array}$ \\
\hline
\end{tabular}


Section 5.1. Extended Surfaces--No Internal Heating.

\section{Case No. References}

Descr iption

\section{Solution}

5.1.40 83 Sheet fin for square array tubes with convection boundary, $h_{f} t_{f}$.

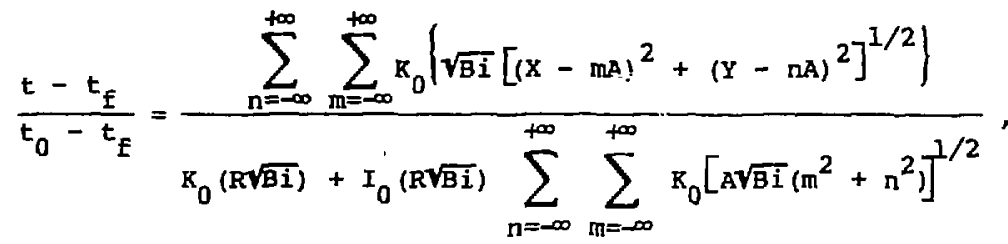

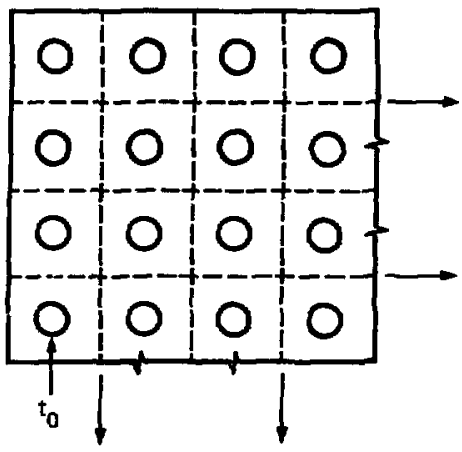
$\mathrm{n}, \mathrm{m} \neq \mathbf{0}$

$$
\phi=\frac{2 \pi}{R \sqrt{B i}\left[(A / R)^{2}-\pi\right]}
$$

$$
x \frac{R_{1}(R \sqrt{B i})-I_{1}(R \sqrt{B i}) \sum_{n=-\infty} \sum_{m=-\infty}^{+\infty} R_{0}\left[A \sqrt{B i}\left(m^{2}+n^{2}\right]^{1 / 2}\right.}{R_{0}(R \sqrt{B i})+I_{0}(R \sqrt{B i}) \sum_{n=-\infty}^{+\infty} \sum_{m=-\infty}^{+\infty} R_{0}\left[A \sqrt{B i}\left(m^{2}+n^{2}\right)\right]^{1 / 2}},
$$
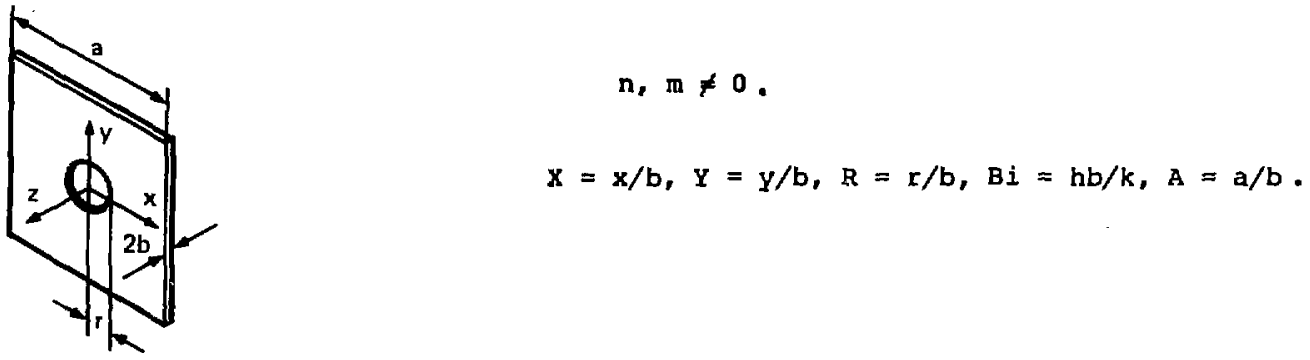
Section 5.1. Extended Surfaces--No Internal Heating.

Case No. References Description

Solution

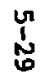


Section 5.1. Ex:tended Surfaces--No Internal Heating.

Case No. References Description Solution


Section 5.2. Extended Surfaces-With Internal Heating.

\section{Case No. References}

Description

Solution

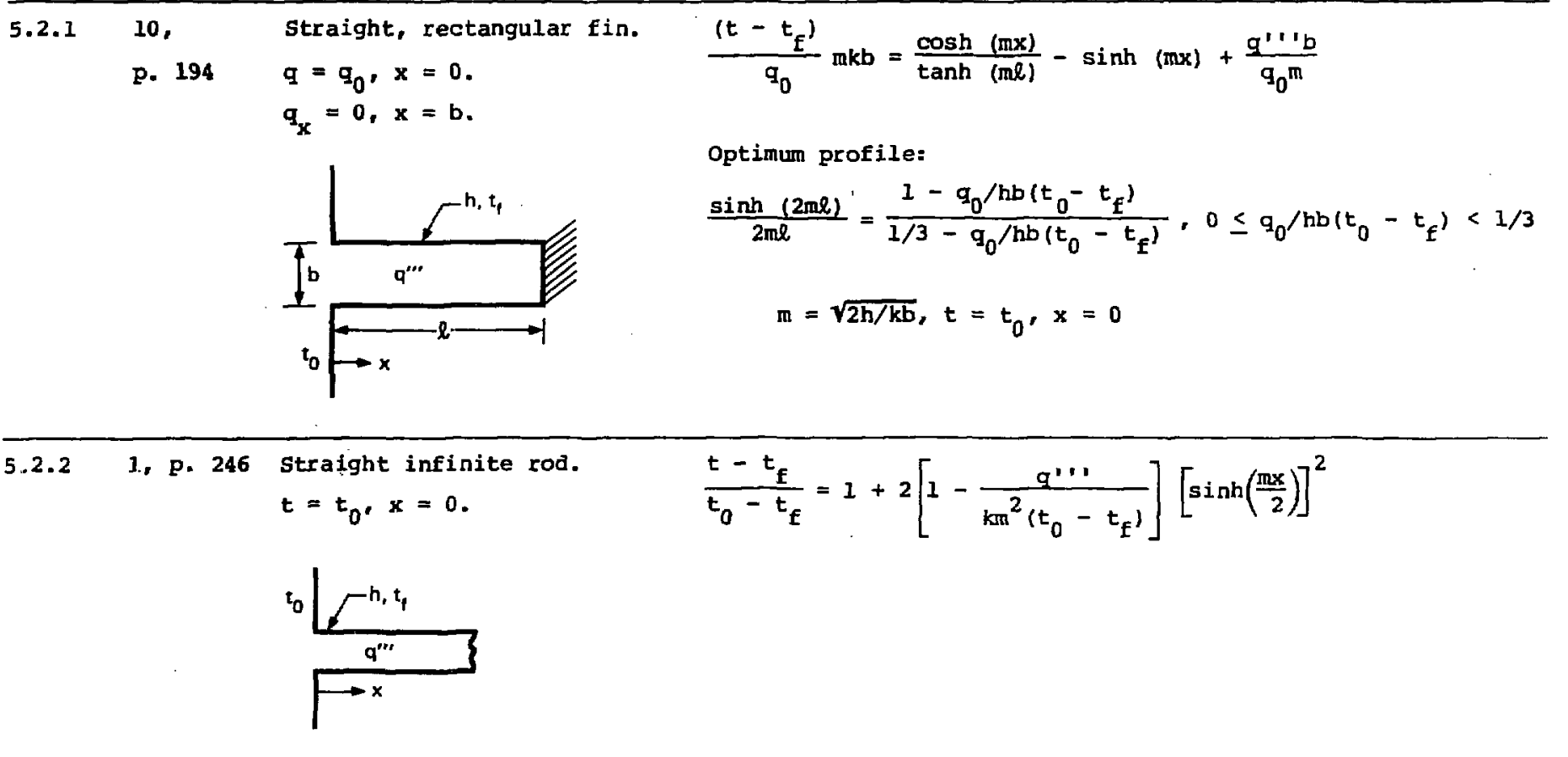


Section 5.2. Bxtended Surfaces-With Internal Eeating.

Case No. References Description Solution

$\varliminf_{\mathbf{N}}^{u}$ 
Section 5.2. Extended Surfaces-With Internal Heating.

Case No. References Description Solution

un

$\underset{\omega}{\omega}$ 


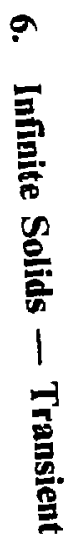

REPRODUSED FROM BEST AVAILABLE COPY 
Section 6.1. Infinite Solids-No Inte ii Heating.

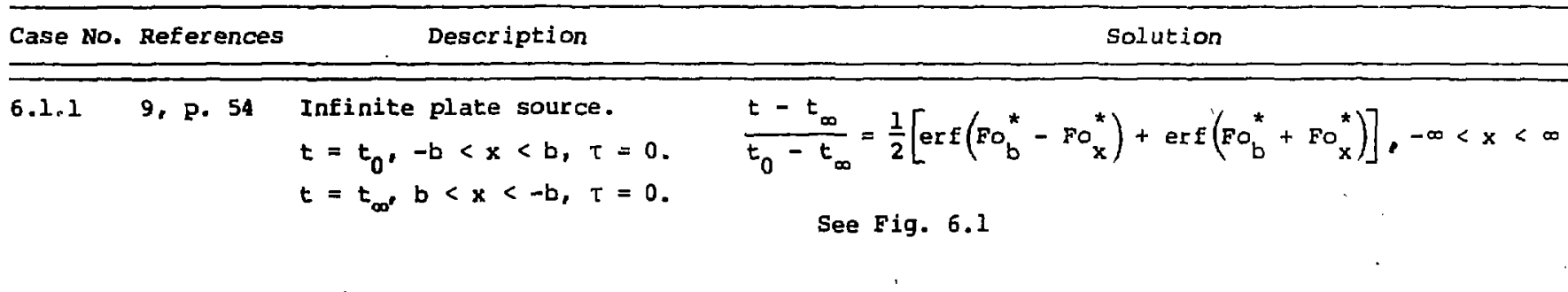


Section 6.1. Infinite Solids--No Internal Heating.

6.1.1.1 74, Case 6.1.1 with a plate of

p. 424 different properties.

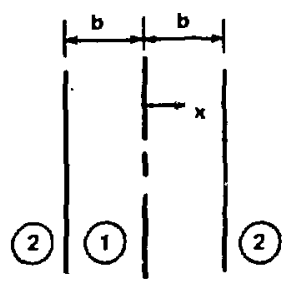

i $\frac{t-t_{\infty}}{t_{0}-t_{\infty}}=1-\frac{1}{1+k} \sum_{n=1}^{\infty}(-H)^{n-1} \operatorname{erfc}\left[\frac{(2 n-1)-x}{2 \sqrt{F o_{b 1}}}\right]$

$\left.+\operatorname{erfc}\left[\frac{(2 n-1)+x}{2 \sqrt{F_{b l}}}\right]\right\},-1<x<+1$

$\frac{t-t_{\infty}}{t_{0}-t_{\infty}}=\frac{k}{k+I} \operatorname{erfc}\left(\frac{x-1}{2 \sqrt{F O_{b 2}}}\right)-\frac{K(1+H)}{1+K} \sum_{n=1}^{\infty}(1 H)^{n-1}$ $x \operatorname{erfc}\left(\frac{x-i+2 n \sqrt{\alpha_{2} / \alpha_{1}}}{2 \sqrt{\mathrm{Fo}_{\mathrm{b} 2}}}\right), 1<x<-1$

$R=\frac{\rho_{1} c_{1} k_{1}}{\rho_{2} c_{2} k_{2}}, H=\frac{1-K}{1+k}$

Interface temp:

$\frac{t-t_{\infty}}{t_{0}-t_{\infty}}=\frac{R}{1+R}-\frac{2 K}{(1+k)^{2}} \sum_{n=1}^{\infty}(-H)^{n-1} \operatorname{erfc}\left(F o_{b l}^{*}\right), x=1$

For Fo $\mathrm{b}_{2}$ small:

$\frac{t_{2}-t_{\infty}}{t_{0}-t_{\infty}} \simeq \frac{k}{1+k} \operatorname{erfc}\left(\frac{x-1}{2 \sqrt{F o_{b 2}}}\right)-\frac{2 k}{(1+k)^{2}} \operatorname{erfc}\left(\frac{x-1+2 \sqrt{\alpha_{2} / \alpha_{1}}}{2 \sqrt{F o_{b 2}}}\right)$ 
Section 6.1. Infinite Solids--No Internal Heating.

6.1.1.2 74, Case 6.1.1 with a pulse source

p. 427

of heat of strength $Q \mathrm{~J} / \mathrm{m}^{2}$ at $x=0, T=0$.

$t=t_{0},-\infty<x<\infty, \tau=0$.

i

$\frac{\left(t-t_{i}\right) \rho_{1} c_{1} b}{Q}=\frac{1}{\pi \sqrt{F O_{1}}}\left(\exp \left(-\frac{x^{2}}{F O_{1}}\right)-B \sum_{n=1}^{\infty}(-H)^{n-1}\right.$

$\left.\times\left\{\exp \left[\frac{(2 n-Y)^{2}}{4 F o_{1}}\right]+\exp \left[-\frac{(2 n+x)^{2}}{4 F o_{1}}\right]\right\}\right)$ $-1<x<1$

$$
\frac{\left(t-t_{j}\right) \rho_{1} c_{1} b}{Q}=\frac{K}{\sqrt{\alpha_{1} / \alpha_{2}}(1+K) \sqrt{\pi F_{2}}} \sum_{n=1}^{\infty}(-H)^{n-1}
$$

$$
x \exp \left\{-\frac{\left[x_{1}-1+(2 n-1) \sqrt{\alpha_{2} / \alpha_{1}}\right]^{2}}{4 \mathrm{Fo}_{2}}\right\}, 1<x<-1
$$

$$
K=\frac{\rho_{1} c_{1} k_{1}}{\rho_{2} c_{2} k_{2}}, H=\frac{1-k}{1+k}
$$

\subsubsection{9, p. 54 Case 6.1.1 with:}

$t=t_{0}(b-x) / b,-b<x<b$,

$\frac{-t_{\infty}}{t_{0}-t_{\infty}}=\frac{1-x}{2} \operatorname{erf}\left(F o_{b}^{*}-F o_{x}^{\star}\right)+\frac{1+x}{2} \operatorname{erf}\left(F O_{b}^{*}+F o_{x}^{*}\right)-\lambda \operatorname{erf}\left(F o_{x}^{*}\right)$

$\tau=0$.

$t=t_{\infty}, b<x<-b, \tau=0$.

$$
+\sqrt{\frac{\alpha \tau}{b^{2} \pi}}\left\{\exp \left[\frac{-(x+b)^{2}}{\sigma^{2}}\right]+\exp \left[\frac{-(x-b)^{2}}{\sigma^{2}}\right]-2 \exp \left(\frac{-x^{2}}{\sigma^{2}}\right)\right\}
$$


Section 6.1. Infinite Solius-No Internal Eeating.

\subsubsection{9, p. 56 Infinite rod source.}

$t=t_{0},|x|<d,|y|<b$,

$\tau=0$.

$t=t_{\infty},|x|>d$,

$|y|>b, \tau=0$.

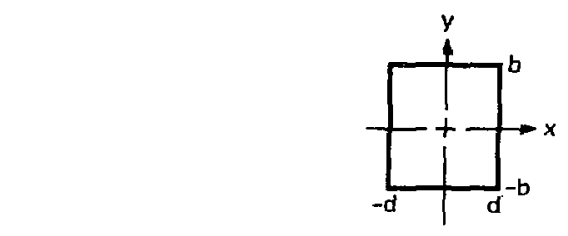

$$
\begin{aligned}
& \frac{t-t_{\infty}}{t_{0}-t_{\infty}}=\frac{1}{4}\left[\operatorname{erf}\left(\mathrm{Fo}_{d}^{\star}-F o_{x}^{\star}\right)+\operatorname{erf}\left(F o_{d}^{\star}+F o_{x}^{*}\right)\right] \\
& \times\left[\operatorname{erf}\left(\mathrm{Fo}_{b}^{*}-\mathrm{Fo}_{Y}^{\star}\right)+\operatorname{erF}\left(\mathrm{Fo}_{b}^{\star}+\mathrm{Fo}_{Y}^{*}\right)\right]
\end{aligned}
$$

6.1.4 74, P. 432 Infinite cylinder source.

$t=t_{0^{\prime}} r<I_{0^{\prime}} \tau=0$.

$t=t_{\infty}, r>r_{0}, \tau=0$.

$\frac{t-t_{\infty}}{t_{0}-t_{\infty}}=\frac{2}{\pi} \int_{0}^{\infty} \exp \left(-\lambda^{2} F_{0}\right)\left[\frac{J_{0}(\lambda R) J_{1}(\lambda)}{J_{1}(\lambda) Y_{0}(\lambda)-J_{0}(\lambda) Y_{1}(\lambda)}\right] \frac{d \lambda}{\lambda}, R>1$

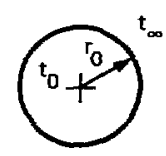

$\frac{t-t_{\infty}}{t_{0}-t_{\infty}}=\frac{4}{\pi^{2}} \int_{0}^{\infty} \exp \left(-\lambda^{2} F_{0}\right)\left[\frac{J_{0}(\lambda R) J_{1}(\lambda)}{\left[J_{1}(\lambda) Y_{0}(\lambda)-J_{0}(\lambda) Y_{1}(\lambda)\right]^{2}}\right] \frac{d \lambda}{\lambda^{2}}, R<1$

See Fig. 6.2 
Section 6.1. Infinite Solids-No Internal Heating.

Case No. References Description Solution

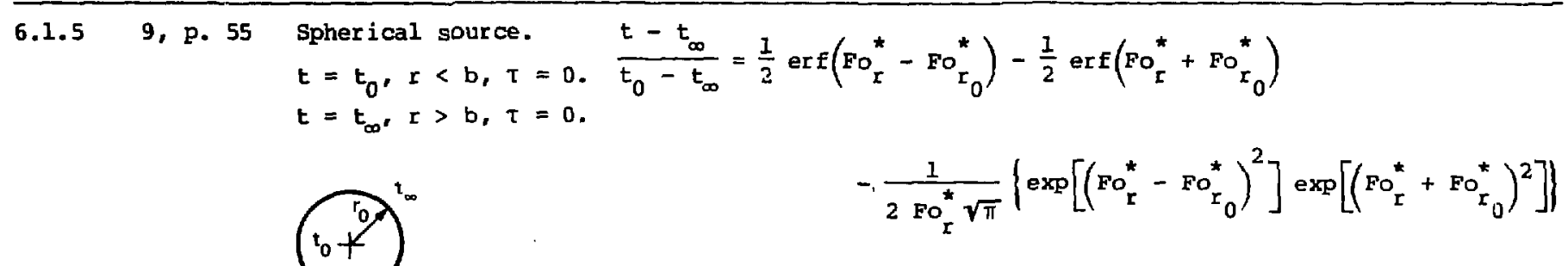

See Fig. 6.3 
Section 6.1. Infinite Solids-No Internal Heating.

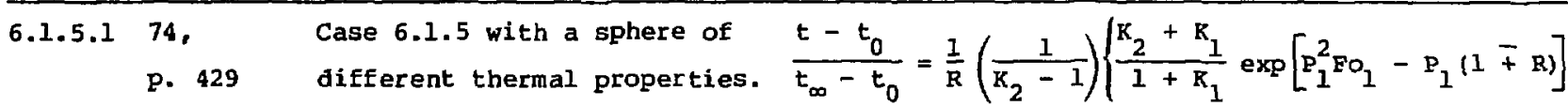

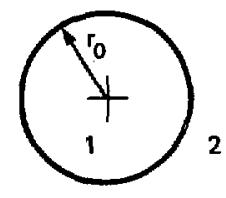

$$
\begin{aligned}
& \left.x \operatorname{erfc}\left(\frac{1 \mp \mathrm{R}}{2 \sqrt{\mathrm{FO}_{1}}}-P_{1} \sqrt{\mathrm{FO}_{1}}\right)-\operatorname{erf}\left(\frac{1 \mp \mathrm{R}}{2 \sqrt{\mathrm{FO}_{1}}}\right)\right\}, R \leq 1 . \\
& \frac{t-t_{\infty}}{t_{0}-t_{\infty}}=\frac{1}{R}\left\{\operatorname{erfc}\left(\frac{R-1}{2 \sqrt{F_{2}}}\right)+\frac{1}{K_{2}-1} \operatorname{erfc}\left(\frac{R-1}{2 \sqrt{F_{2}}}\right)\right. \\
& -\frac{K_{2}+K_{1}}{\left(K_{2}-1\right)\left(K_{1}+1\right)} \exp \left(P_{2}^{2} \mathrm{Fo}_{2}-P_{2} R-1\right) \operatorname{erfc}\left(\frac{R-1}{2 \sqrt{F O_{2}}}-P_{2} \sqrt{F O_{2}}\right) \\
& -\frac{1}{R_{2}-1} \operatorname{erfc}\left(\frac{R-1+2 \sqrt{\alpha_{2} / \alpha_{1}}}{2 \sqrt{F \sigma_{2}}}\right)+\frac{K_{2}+K_{1}}{\left(K_{2}-1\right)\left(K_{1}+1\right)} \\
& x \exp \left(\mathrm{P}_{2}^{2} \mathrm{Fo}_{2}-\mathrm{P}_{2} \mathrm{R}-1+2 \sqrt{\alpha_{2} / \alpha_{1}}\right) \operatorname{erfc}\left(\frac{\mathrm{R}-1+2 \sqrt{\alpha_{2} / \alpha_{1}}}{2 \sqrt{\mathrm{Fo}_{2}}}\right. \\
& \left.\left.-\mathrm{P}_{2} \sqrt{\mathrm{Fo}_{2}}\right)\right\}, \mathrm{R}>1 \\
& \mathrm{~K}_{1}=\frac{\rho_{1} c_{1} \mathrm{k}_{1}}{\rho_{2} c_{2} \mathrm{k}_{2}}, \mathrm{~K}_{2}=\frac{\mathrm{k}_{1}}{\mathrm{k}_{2}}, \mathrm{P}_{1}=\frac{\mathrm{K}_{2}-1}{\mathrm{~K}_{2}+\mathrm{K}_{2} / \mathrm{K}_{1}}, \mathrm{P}_{2}=\frac{\mathrm{K}_{2}-1}{1+\mathrm{K}_{1}} \\
& E(\overline{+} R)=f(-R)-f(+R)
\end{aligned}
$$


Section 6.1. Infinite Solids-No Internal Heating.

\section{Case No. References}

6.1.6 9, p. 61 Adjoined semi-infinite regions.

$t=t_{1}, x>0, t=0$.

$\frac{t-t_{2}}{t_{1}-t_{2}}=\frac{1}{2}\left[\operatorname{erf}\left(\right.\right.$ Fo $\left.\left._{x}^{\star}\right)+1\right],-\infty<x<\infty$

$t=t_{2}, x<0, T=0$.

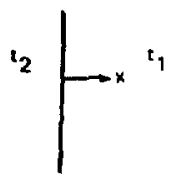

$\stackrel{0}{\vdots}$

$\begin{array}{ll}6.1 .7 \text { 7. p. } 89 \quad \begin{array}{l}\text { Specified temperature } \\ \text { distribution. } \\ \end{array} & t=f(x),-\infty<x<\infty, \tau=0 .\end{array} \int_{-\infty}^{\infty} f(x+\lambda \sigma) \exp \left(-\lambda^{2}\right) d \lambda$


Section 6.1. Infinite Solids-No Internal Heating.

6.1.8 9, p. B8 Adjoined semi-infinite regions with contact resistance.

$t=t_{1}, x>0, \tau=0$.

$t=t_{2}, x<0, \tau=0$.

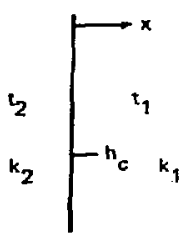

$\frac{t-t_{2}}{t_{1}-t_{2}}=\frac{1}{1+A}\left\{1+A\left[\operatorname{erf}\left(F O_{x}^{*}\right)+\exp \left(H_{1} x+H_{1}^{2}{ }_{1}^{2} / 4\right)\right.\right.$

$\left.\left.x \operatorname{erfc}\left(\mathrm{FO}_{\mathrm{x}}^{\star}+\mathrm{H}_{1} \sigma_{1} / 2\right)\right]\right\}, \mathrm{x}>0$

$\frac{t-t_{2}}{t_{1}-t_{2}}=\frac{1}{1+A}\left[\operatorname{erf} \mid F O_{x}^{\star}\right]-\exp \left(H_{2} x+H_{2}^{2} \sigma / 4\right)$

$\left.x \operatorname{erfc}\left(\left|\mathrm{Fo}_{\mathrm{x}}^{\star}\right|+\mathrm{H}_{2} \sigma_{2} / 2\right)\right], \quad \mathrm{x}<0$

$A=\frac{k_{2}}{k_{1}} \sqrt{\frac{\alpha_{1}}{\alpha_{2}}}, H_{1}=\frac{h(1+A)}{k_{1}^{A}}, H_{2}=\frac{h}{k_{2}}(1+A)$
6.1 .99 .9 .88 Adjoined semi-infinite regions--no contact

resistance.

$t=t_{1}, x>0, t=0$.

$t=t_{2}, x<0, t=0$.

$2 \longdiv { t }$

$$
\frac{t-t_{2}}{t_{1}-t_{2}}=\frac{1}{1+A}\left[1+A \operatorname{erf}\left(F O_{x}^{\star}\right)\right], x>0
$$

$\frac{t-t_{2}}{t_{1}-t_{2}}=\frac{1}{1+A} \operatorname{erf}\left(\left|\mathrm{Fo}_{x}^{\star}\right|\right), x<0$

$A=\frac{k_{2}}{k_{1}} \sqrt{\frac{\alpha_{1}}{\alpha_{2}}}$ 
Section 6.1. Infinite Solids--No Internal Heating.

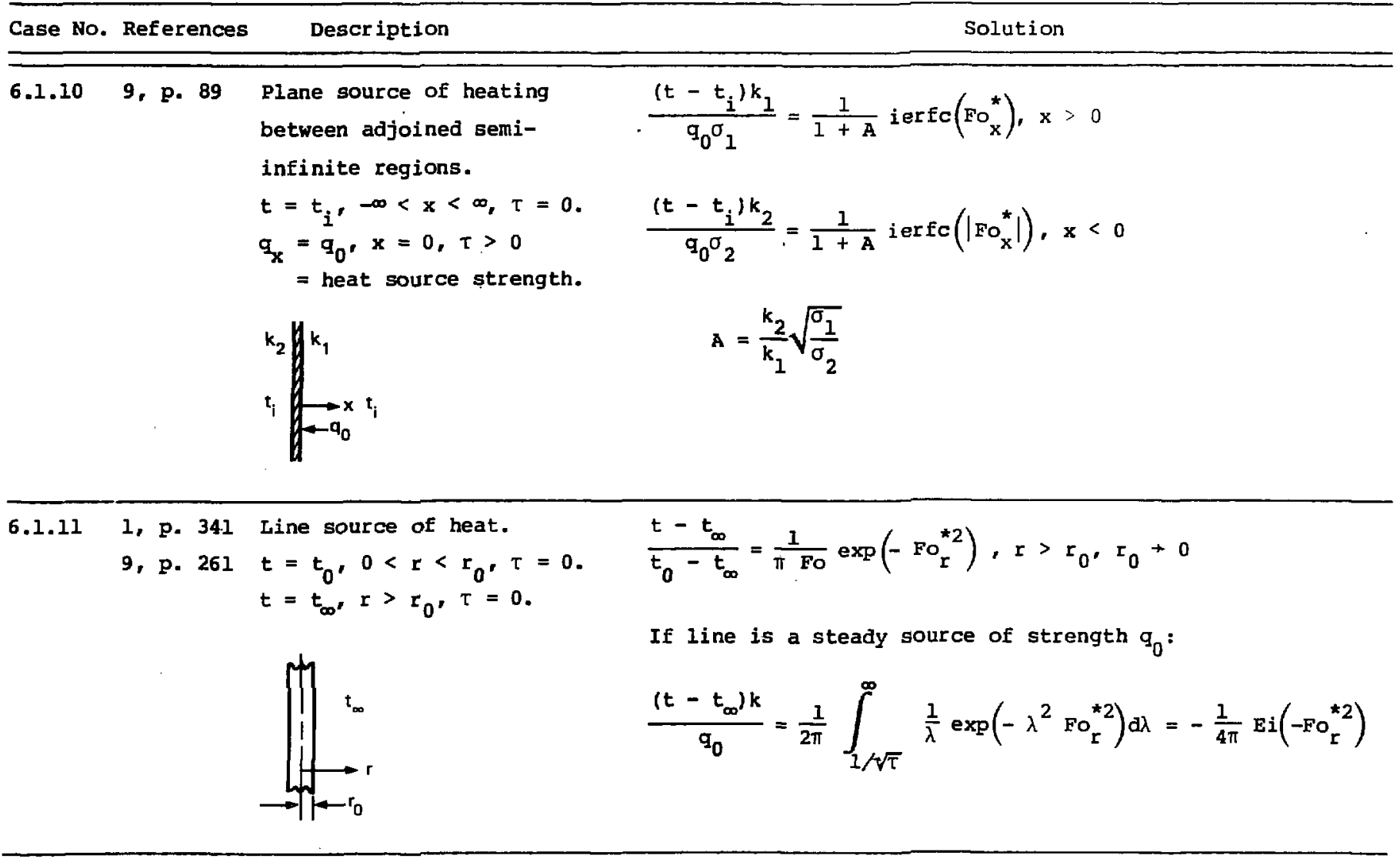


Section 6.1. Infinite Solids-No internal Heating.

Case No. References Description Solution

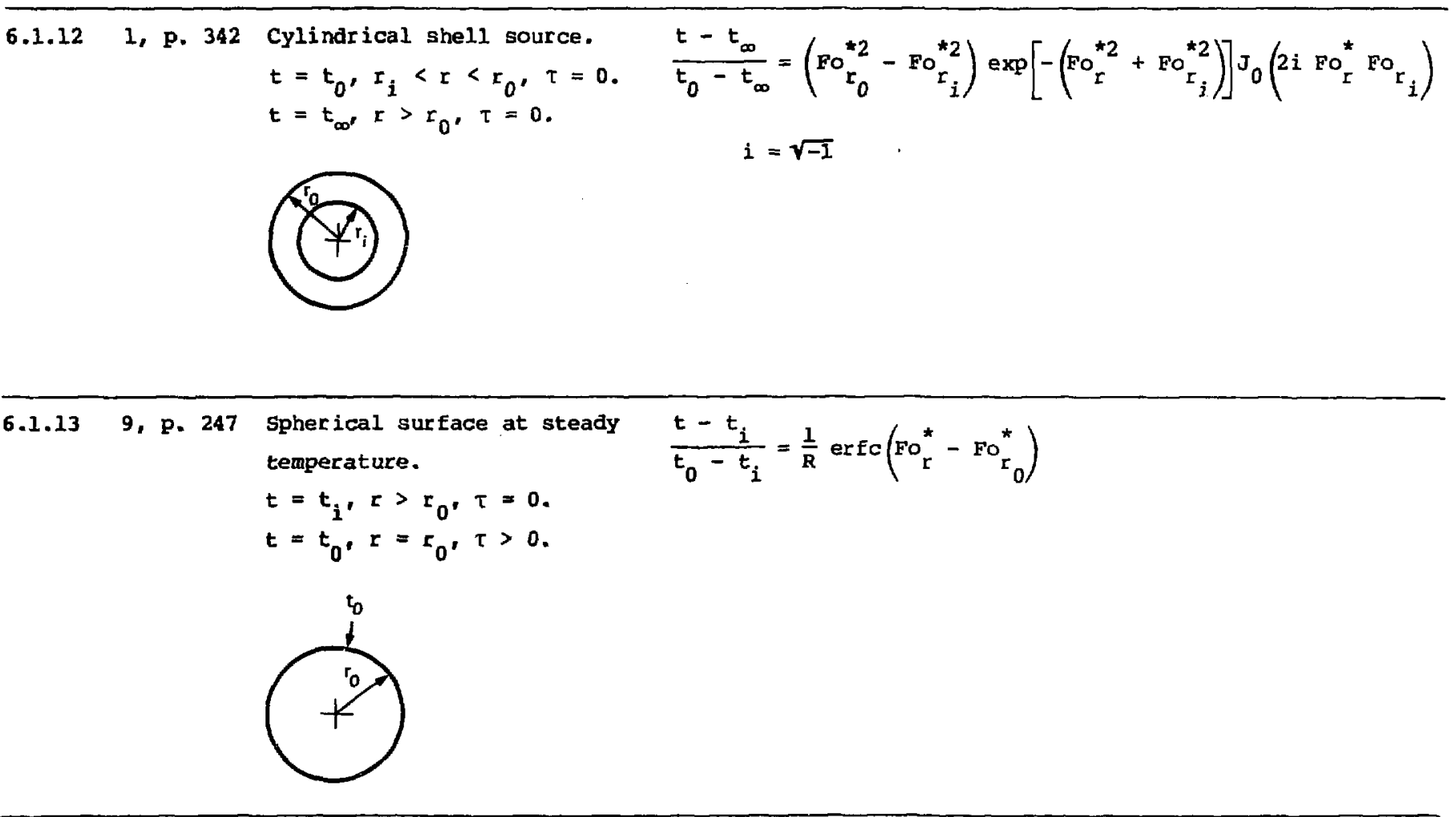


Section 6.1. Infinite Solids-No Internal Heating.

Case No. References Description Solution

6.1.14 9, p. 247 Spherical surface with convection boundary.

$t=t_{i}, r>r_{0}, \tau=0$.

$$
\frac{t-t_{i}}{t_{f}-t_{i}}=\frac{B i}{(B i+1) R}\left\{\operatorname{erfc}\left(F o_{c}^{\star}-F O_{r_{0}}^{*}\right)-\exp [(B i+1)(R-1)\right.
$$

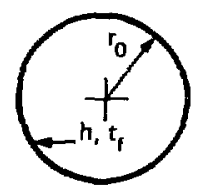

$\left.+(\mathrm{Bi}+1) \mathrm{Fo}] \operatorname{erfc}\left[\mathrm{Fo}_{\Gamma}^{\star}-\mathrm{Fo}_{\mathrm{F}_{0}}^{\star}+(\mathrm{Bi}+1) \sqrt{\mathrm{FO}}\right]\right\}$

$\stackrel{\stackrel{f}{5}}{6.1 .15}$ 9, p. 248 Spherical surface with steady heat flux.

$$
\begin{aligned}
\frac{\left(t-t_{i}\right) k}{q_{0} r_{0}}=\frac{I}{R}\left[\operatorname{erfc}\left(F O_{E}^{*}-F O_{F_{0}}^{*}\right)\right. & \\
& \left.\quad-\exp (R-1+F O) \operatorname{erfc}\left(F O_{r}^{*}-F O_{r_{0}}^{*}+\sqrt{F O}\right)\right]
\end{aligned}
$$$$
t=t_{i}, r=r_{0}, \tau=0 \text {. }
$$$$
q_{r}=q_{0}, r=r_{0} \text {. }
$$

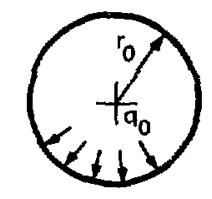


Section 6.1. Infinite Solids-No Internal Heating.

Case No. References Description Solution

$$
\begin{aligned}
& 6.1 .161 \text {, p. } 336 \text { Point source. } \\
& t=t_{i}, 0<I<I_{0^{\prime}} T=0 \text {. } \\
& t=t_{\infty}, r>r_{0^{\prime}} \tau=0 \text {. } \\
& \frac{t-t_{\infty}}{t_{i}-t_{\infty}}=\frac{r_{0}^{3}}{6 \sqrt{\pi \alpha^{3} \tau^{3}}} \exp \left(-F o_{r}^{\star 2}\right), r>r_{0}, r_{0}+0 \\
& \text { (P) } \\
& \text { If point is a steady source of strength } Q_{0} \text { : } \\
& \frac{(t-t) k}{Q_{0} / r_{0}}=\frac{r_{0}}{4 \pi^{3 / 2} \sqrt{\alpha}} \int_{1 / \sqrt{\tau}}^{\infty} \exp \left(-\lambda^{2} F \sigma_{r}^{\star 2}\right) d \lambda \\
& \text { For } \tau \rightarrow \infty \text { : } \\
& \frac{\left(t-t_{\infty}\right) k r}{Q_{0}}=\frac{1}{4 \pi}
\end{aligned}
$$$$
\text { 站 }
$$ 
Section 6.1. Infinite Solids--No Internal Heating.

\section{Case No. References}

$$
\begin{aligned}
& \begin{array}{llll}
6.1 .17 .1 & l, & \text { Spherical shell source. } & t-t_{\infty} \\
& \text { p. } 340 & t=t_{i}, r_{i}<r<r_{0^{\prime}} & \frac{R_{0}-1}{6 \sqrt{\pi} R_{i}-t_{\infty}}
\end{array} \\
& \tau=0 \text {. } \\
& t=t_{\infty}, r>r_{0}, \tau=0 \text {. } \\
& R=r / r_{i} \\
& \text { If the shell is a steady source of strength } Q_{0} \text { : } \\
& \frac{\left(t-t_{\infty}\right) k}{Q_{0} / r_{i}}=\frac{\sqrt{\alpha}}{4 \pi^{3 / 2} r} \int_{1 / \sqrt{\tau}}^{\infty} \frac{1}{\lambda^{2}}\left\{\exp \left[-\frac{\left(r-r_{i}\right)^{2} \lambda^{2}}{4 \alpha}\right]-\exp \left[-\frac{\left(r+r_{i}\right)^{2} \lambda^{2}}{4 \alpha}\right]\right\} \\
& \frac{\left(t-t_{\infty}\right) k r}{Q_{0}}=\frac{1}{4 \pi}
\end{aligned}
$$

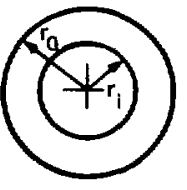

6.1.17.2 1, p. 342 Plane source.

9, p. 263

$t=t_{i},-l<x<+l, \tau=0$.

$t=t_{\infty}|x|>\ell, \tau=0$.

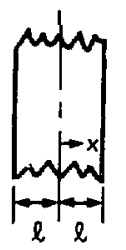

$\frac{t-t_{\infty}}{t_{i}-t_{\infty}}=\frac{1}{\pi F o} \exp \left(-F o_{x}^{* 2}\right),|x|>\ell, \ell+0$

If the plane is a steady source of strength $q_{0}$ :

$$
\begin{aligned}
\frac{\left(t-t_{\infty}\right) k}{q_{0} l} & =\frac{\sqrt{\alpha}}{l \sqrt{\pi}} \int_{1 / \sqrt{\tau}}^{\infty} \frac{1}{\lambda^{2}} \exp \left(-x^{2} \lambda^{2} / 4 \alpha\right) d \lambda \\
& =\sqrt{F o} \exp \left(-F o_{x}^{* 2}\right)-\frac{|x|}{2} \operatorname{erfc}\left(F o{ }^{*}|x|\right)
\end{aligned}
$$


Section 6.1. Infinite Solids--No Internal Heating.

\begin{tabular}{|c|c|c|c|c|}
\hline Case No. & References & Description & Solution & \\
\hline 6.1 .18 & 9, p. 335 & $\begin{array}{l}\text { Cylinder with steady } \\
\text { surface temp. } \\
t=t_{i}, r>r_{0}, \tau=0 . \\
t=t_{0}, r=r_{0}, \tau>0 .\end{array}$ & 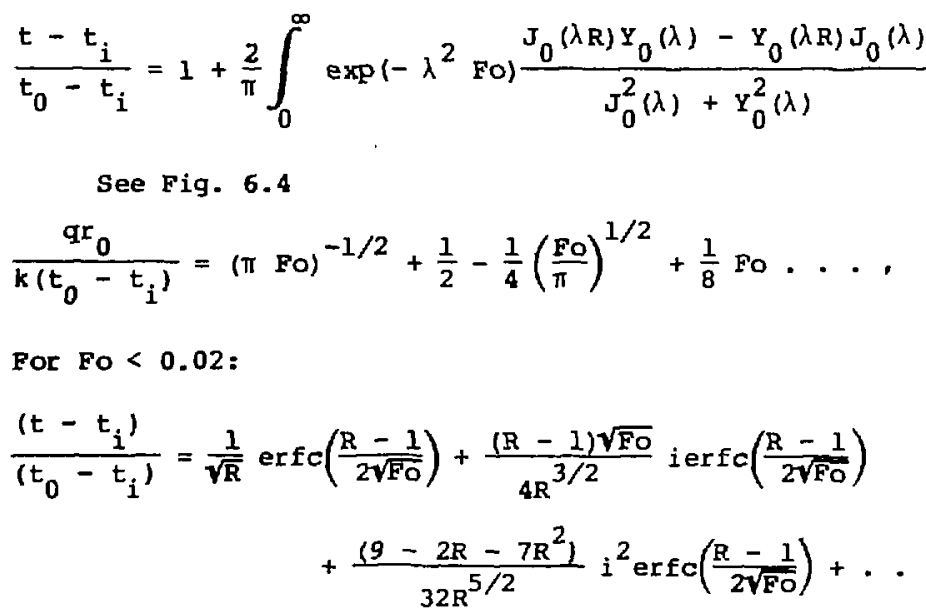 & $R=1$ \\
\hline
\end{tabular}

6.1 .19 9. p. 337 Cylindrical surface with
$\begin{aligned} & \text { convection boundary. } \\ & t=t_{i}, r>t_{0}, \tau=0 .\end{aligned}$


Section 6.1. Infinite Solids-No Internal Heating.

\section{Case No. References}

Description

Solution

6.1 .209 , p. 338 Cylindrical surface with steady heat flux.

$t=t_{i}, r>r_{0}, \tau=0$.

$q_{r}=q_{0}, r=r_{0}, \tau>0$.

号

$$
\frac{\left(t-t_{i}\right\} k}{q_{0} r_{0}}=-\frac{2}{\pi} \int_{0}^{\infty}\left[1-\exp \left(\lambda^{2} F O\right)\right] \frac{J_{0}(\lambda R) Y_{1}(\lambda)-Y_{0}(\lambda R) J_{I}(\lambda) d \lambda}{\left[J_{1}^{2}(\lambda)+Y_{1}^{2}(\lambda)\right] \lambda^{2}}
$$

For Fo $<0.02$ :

$\frac{\left(t-t_{i}\right) k}{g_{0} r_{0}}=2 \sqrt{\frac{F O}{R}} \operatorname{ierfc}\left(\frac{R-1}{2 \sqrt{F O}}\right)-\frac{3 R+1}{4 R} \sqrt{F O} i^{2} \operatorname{erfc}\left(\frac{R-1}{2 \sqrt{F O}}\right)+$

For Fo $\gg 1$ :

$\frac{\left(t-t_{i}\right) k}{q_{0}{ }^{5} j}=\ln \left(\frac{4 F O}{R^{2}}\right)-0.57722$ 
Section 6.1. Infinite s' ids--No Internal Heating.

6.1.20.1 74, Case 6.1.20 with two dif-

p. 434 erent thermal materials. $t=t_{i}, r>0, \tau=0$. $g_{\Sigma}=g_{0}, r=r_{0}, \tau>0$.

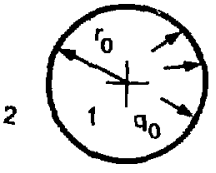

$$
\begin{aligned}
\frac{\left(t-t_{i}\right) k_{1}}{q_{0} r_{0}}=\frac{4}{\pi^{2}} \int_{0}^{\infty}\left[I-\exp \left(-\lambda^{2} F_{1}\right)\right] \frac{J_{0}\left(\lambda^{2} R\right) J_{1}(\lambda) d \lambda}{\lambda^{4}\left(\phi^{2}+\psi^{2}\right)}, R<1 \\
\frac{\left(t-t_{i}\right) k_{2}}{q_{0} r_{0}}=\frac{2}{\pi^{2}} \int_{0}^{\infty}\left[1-\exp \left(-\lambda^{2} F o_{1}\right)\right] \frac{J_{1}(\lambda)}{\lambda^{3}\left(\phi^{2}+\psi^{2}\right)} \\
\times\left[J_{0}\left(\sqrt{\frac{a_{1}}{\alpha_{2}}} \lambda R\right) \phi-Y_{0}\left(\sqrt{\frac{\alpha_{1}}{a_{2}}} \lambda R\right) \psi\right] d \lambda, R>1
\end{aligned}
$$$$
\phi=R J_{1}(\lambda) Y_{0}\left(\sqrt{\frac{\alpha_{1}}{\alpha_{2}}} \lambda\right)-\sqrt{\frac{\alpha_{1}}{\alpha_{2}}} J_{0}(\lambda) Y_{1}\left(\sqrt{\frac{\alpha_{1}}{\alpha_{2}}} \lambda\right)
$$$$
\psi=R J_{I}(\lambda) J_{0}\left(\sqrt{\frac{\alpha_{1}}{\alpha_{2}}} \lambda\right)-\sqrt{\frac{\alpha_{1}}{\alpha_{2}}} J_{0}(\lambda) J_{1}\left(\sqrt{\frac{\alpha_{1}}{\alpha_{2}}} \lambda\right)
$$

$K=\frac{k_{1}}{k_{2}}$ 
Section 6.1. Infinite Solids--No Internal Heating.

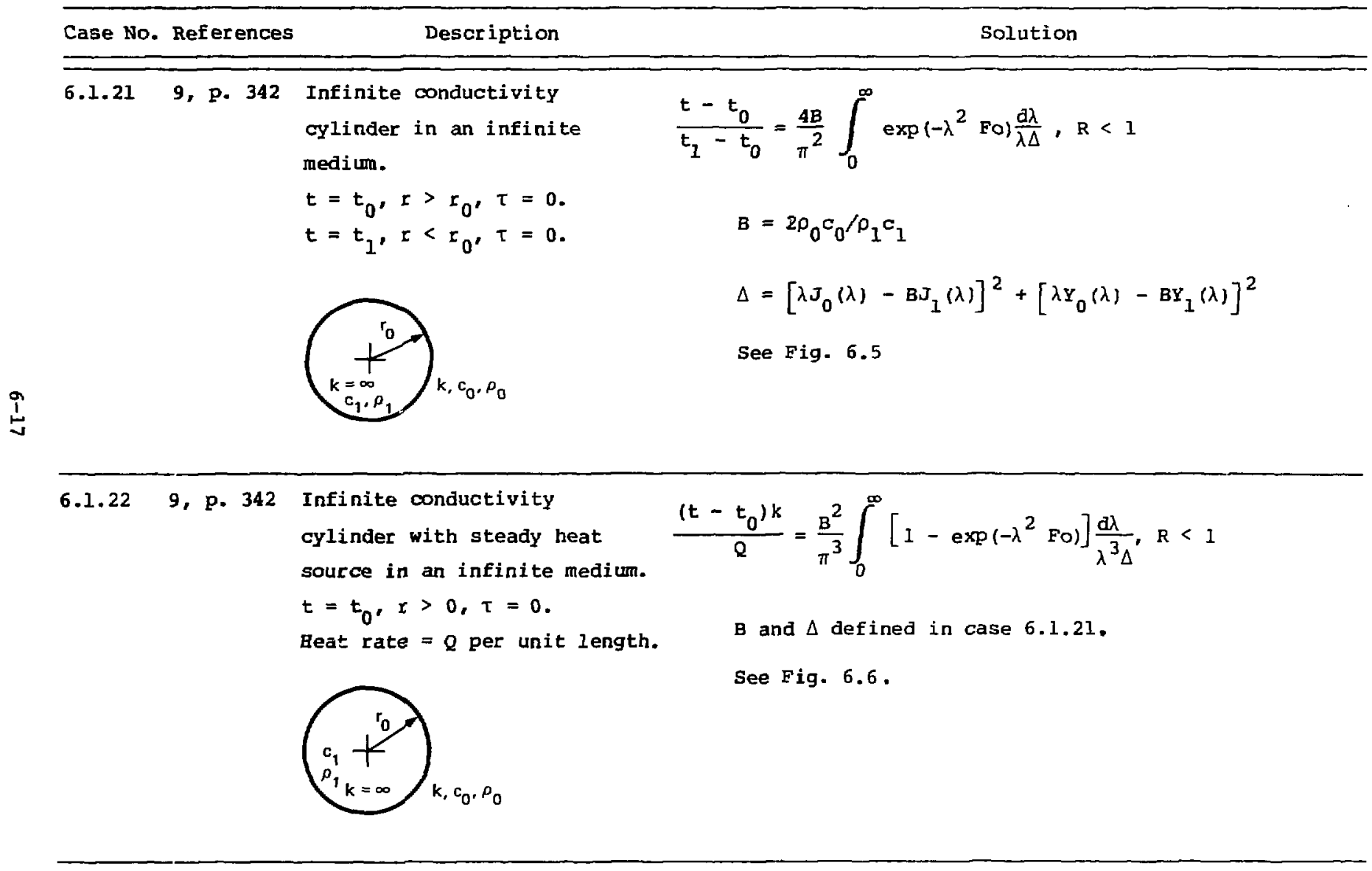


Section 6.1. Infinite Solids--No Internal Heating.

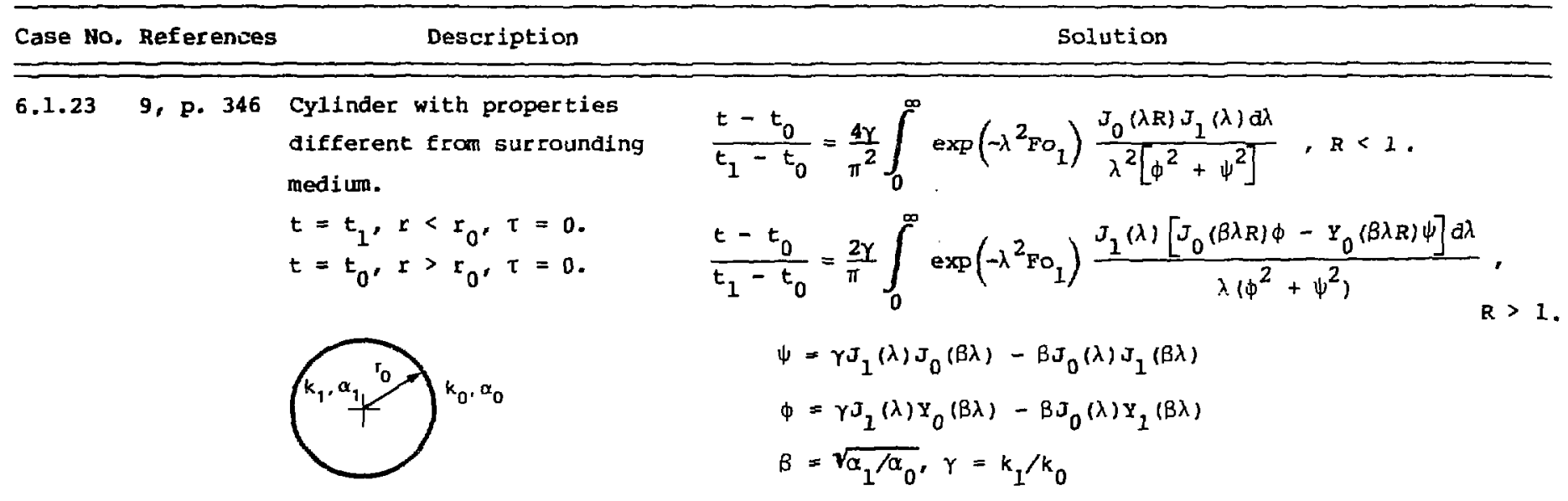

See case 6.1.4 for equal material properties.

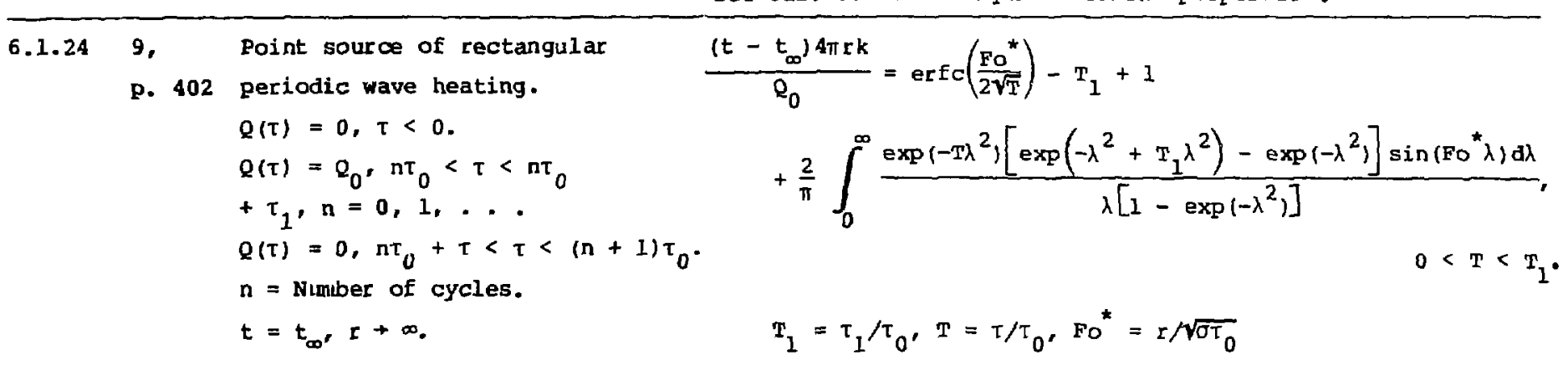


Section 8.2. Solids Bounded by Plare Surfaces--With Internal Heating.

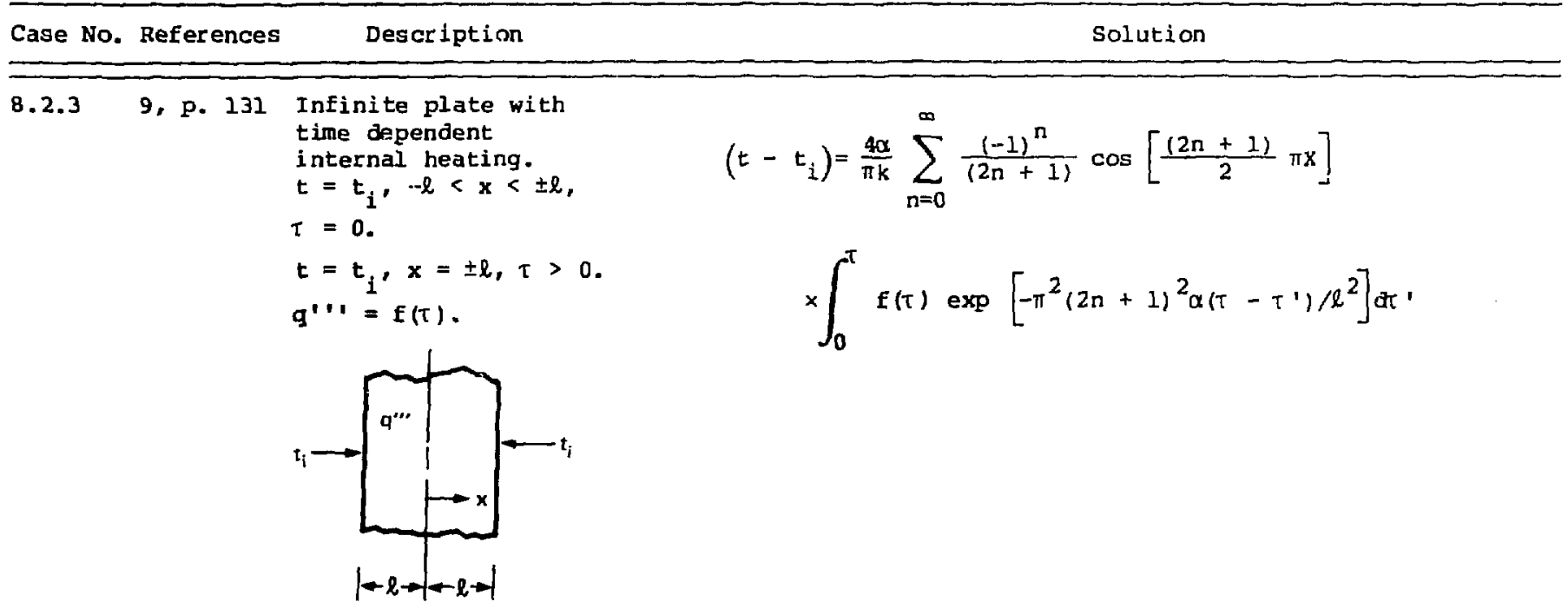

$\begin{aligned} & \text { 8.2.4 9. p. } 132 \text { Case } 8.2 .3 \text { with } \\ & f(\tau)=9_{0}^{11} \mathrm{e}^{-b \tau} .\end{aligned}$

$\frac{\left(t-t_{i} / k\right.}{q_{0}^{\prime \prime \prime} \ell^{2}}=\frac{1}{P d}\left[\frac{\cos (x \sqrt{P d})}{\cos (\sqrt{P d})}-1\right] \exp (-t \pi)$

$+\frac{2}{\pi P d} \sum_{n=0}^{\infty} \frac{(-1)^{n} \cos \left(\lambda_{n} x\right) \exp \left(-\lambda_{n}^{2} \text { Fo }\right)}{\lambda_{n}\left(1-\lambda_{n}^{2} / P d\right)}$

$\lambda_{n}=(2 n+1) \pi / 2$ 


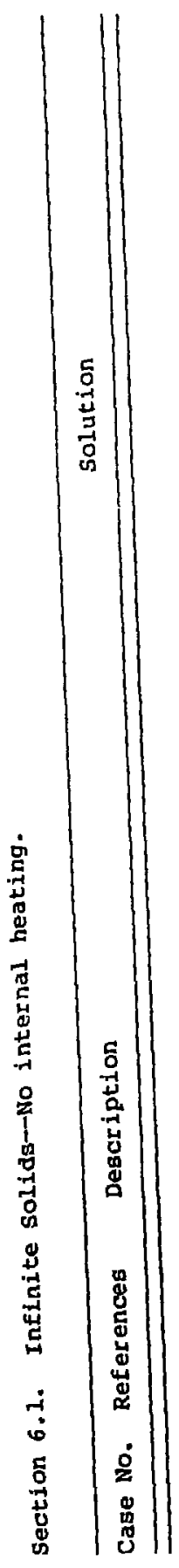

$6-20$ 
Section 6.1. Infinite Solids--Mo Internal Heating.

Case No. References 
Section 6.2. Infinite Solids--With Internal Heating.

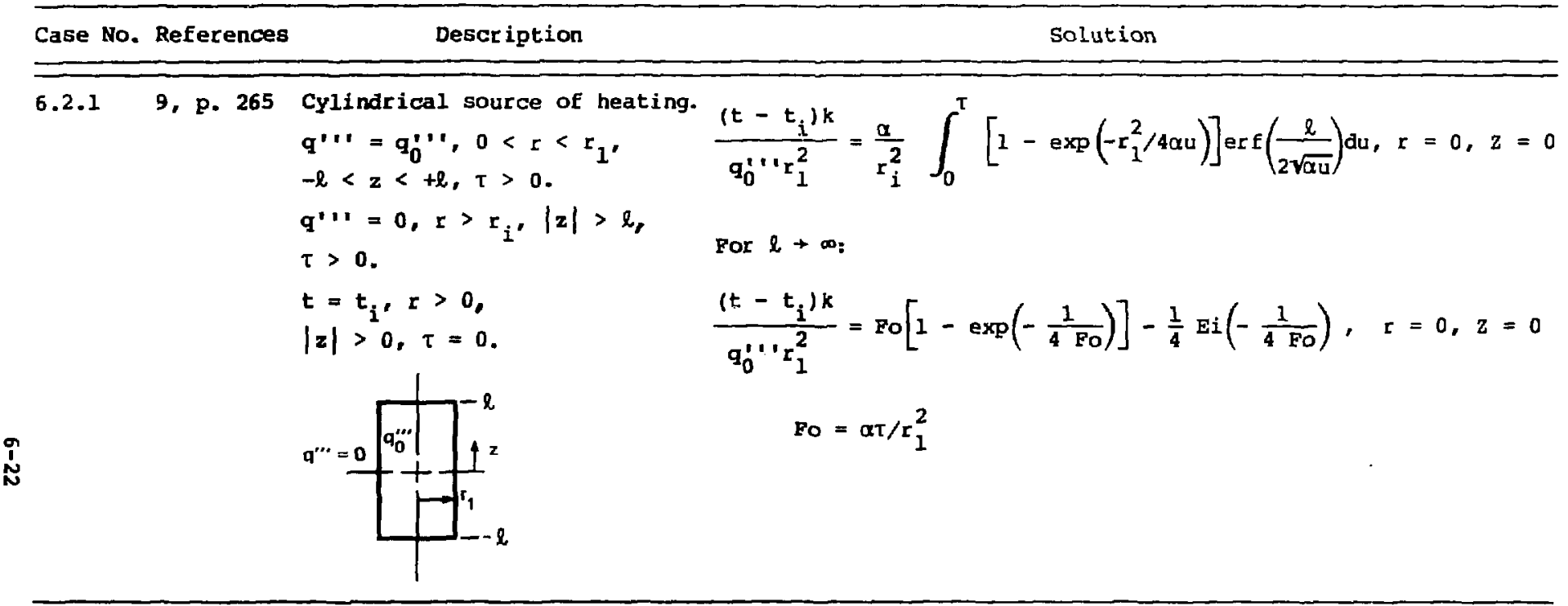

6.2.2 9, p. 347 Case 6.1.23 with a cylinder and internal heating.

$t=t_{0}, r>0, t=0$.

$\frac{\left(t-t_{0}\right) k_{0}}{q_{0}^{\prime} ' r_{0}^{2}}=\frac{4}{\pi^{2}} \int_{0}^{\infty} \frac{\left[1-\exp \left(-\lambda^{2} F_{0}\right)\right] J_{0}(\lambda R) J_{1}(\lambda) d \lambda}{\lambda^{4}\left[\phi^{2}+\psi^{2}\right]}, R<I$.

$q^{\prime \prime \prime}=q_{0}^{\prime \prime \prime}, 0<r<r_{0}$, $\mathbf{T}>0$.

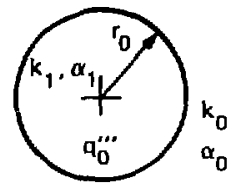

$\frac{\left(t-t_{0}\right) k_{0}}{q^{\prime \prime} x_{0}^{2}}=\frac{2}{\pi} \int_{0}^{\infty} \frac{\left[1-\exp \left(-\lambda F O_{1}\right)\right]\left[J_{0}(B \lambda R) \phi-Y_{0}(B \lambda R) \psi\right] d \lambda}{\lambda^{3}\left[\phi^{2}+\psi^{2}\right]}$,

$R>1$.

$B, \phi$, and $\psi$ defined in case 6.1 .23 . 
Section 6.2. Infinite Solids--With Internal Heating.

Case No. References

Description

Solution

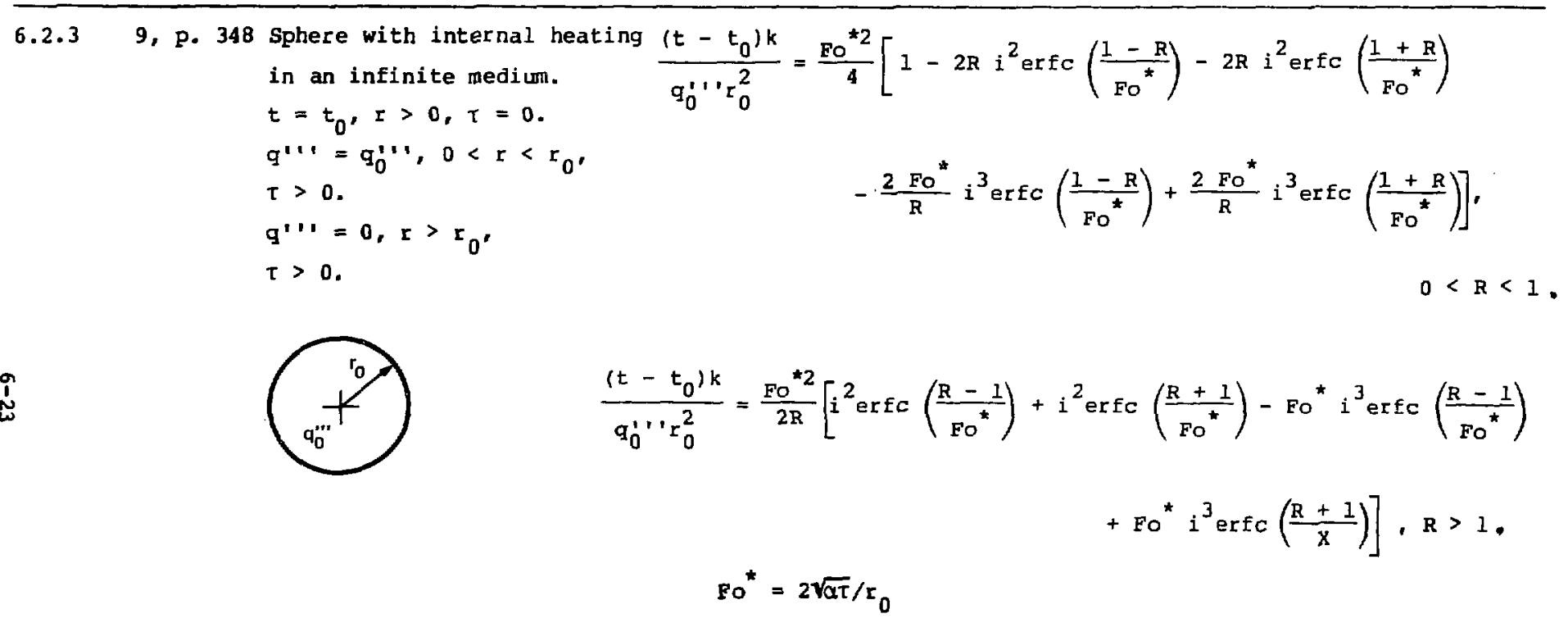


Section 6.2. Infinite Solids--With Internal Heating.

Case No. References Description Solution

6.2.4 9, p. 349 Infinite conductivity sphere in an infinite medium with contact resistance.

$t=t_{0}, r>0, \tau=0$. $\mathrm{q}^{\prime \prime \prime}=\mathrm{q}_{0}^{\prime \prime \prime}, 0<\mathrm{r}<\mathrm{r}_{0^{\prime}} \tau>0$. $q^{\prime \prime}=0, r>0, \tau>0$.

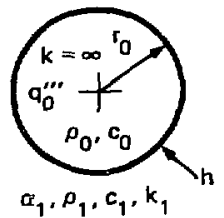

$$
\frac{\left(t-t_{0}\right) k}{g_{0}^{\prime} \cdot r_{0}^{2}}=\frac{1}{3}\left[\frac{1+B i}{B i}-\frac{6}{\pi} K^{2} B i^{2}\right.
$$$$
\left.\times \int_{0}^{\infty} \frac{\exp \left(\mathrm{Fo} u^{2}\right) d u}{\left[u^{2}(1+B i)-k B i\right]^{2}+\left[u^{3}-k B i u\right]^{2}}\right] .
$$

$0<R<1$.

For small values of $\tau$ :

$\frac{\left(t-t_{0}\right) k}{q_{0}^{\prime \prime} r_{0}^{2}}=\frac{K F O}{3}[1-($ K Bi Fo $) / 2+\ldots]$

For large values of $\tau$ :

$$
\begin{aligned}
& \frac{\left(t-t_{0}\right) k}{q_{0}^{\prime \prime}{ }^{2}}=\frac{1}{3}\left[\frac{1+B i}{B i}-\frac{1}{\pi F_{0}}-\frac{2+B i(2-k)}{2 B i k \pi \sqrt{F 0^{3}}}+\ldots\right] \\
& \quad k=\frac{3 p_{1} c_{1}}{p_{0} c_{0}}
\end{aligned}
$$


Section 6.2. Infinite Solids--With Internal Heating.

6.2.5 74, p. 426 Infinite plate with internal heating in an infinite medium.

$t=t_{0},-l<x<l, \tau=0$.

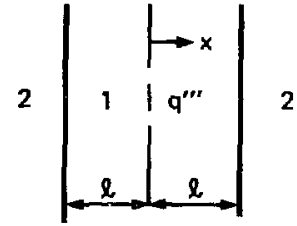

$\frac{t-t_{\infty}}{t_{0}-t_{\infty}}=I+\mathrm{POFO}_{1}-\frac{1}{1+k} \sum_{n=1}^{\infty}(-\mathrm{H})^{n-1}\left\{\operatorname{erfc}\left[\frac{(2 n-I) \mp x}{2 \sqrt{\mathrm{Fo}}{ }_{1}}\right]\right.$

$\left.+4 \mathrm{POFO}_{1} i^{2} \operatorname{erfc}\left[\frac{(2 \mathrm{n}-1) \mp \mathrm{x}}{2 \sqrt{\mathrm{FO}} \mathrm{O}_{1}}\right]\right\},-1<x<1$.

$\frac{t-t_{\infty}}{t_{0}-t_{\infty}}=\frac{1}{1+K}\left[\operatorname{erfc}\left(\frac{x+1}{2 \sqrt{\mathrm{FO}_{2}}}\right)+4 \mathrm{PO} \mathrm{FO}_{2} i^{2} \operatorname{erfc}\left(\frac{x-1}{2 \sqrt{\mathrm{FO}_{2}}}\right)\right]$

$-\frac{K(1+\mathrm{H})}{1+K} \sum_{n=1}^{\infty}(-\mathrm{H})^{\mathrm{n}-1}\left[\operatorname{erfc}\left(\frac{2 \mathrm{n} \sqrt{\alpha_{2} / \alpha_{1}}+\mathrm{X}-I}{2 \sqrt{\mathrm{Fo}}}\right)\right.$

$\left.+4 \mathrm{PO} \mathrm{FO}_{2} \mathrm{i}^{2} \operatorname{erfc}\left(\frac{2 \mathrm{n} \sqrt{\alpha_{2} / \alpha_{1}}+x-1}{2 \sqrt{\mathrm{Fo}_{2}}}\right)\right], 1<x<-1$

$K=\frac{\rho_{1} c_{1} k_{1}}{\rho_{2} c_{2} k_{2}}, H=\frac{1-K}{1+K}, f(\overline{+} X)=f(-X)-f(+X)$ 
Section 6.2. Infinite Solids- With internal heating.

Case No. References Description Solution

Case No. References Description

solution

Case No. References Den


Section 6.2. Infinite Solids--With Internal Heating. 
7. Semi-Infinite Solids - Transient

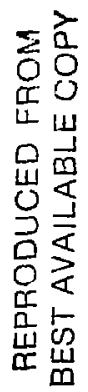


Section 7.1. Semi-Infinite Solids--No Internal Heating.

Case Wo. References

Description

Solution

7.1.1 9, p. 61 Constant surface temperature. $t-t$

$t=t_{0}+b x, x>0, \tau=0 . \quad \frac{t_{s}}{t_{0}-t_{s}}=\operatorname{erf}\left(F o_{x}^{*}\right)+\frac{b x}{t_{0}-t_{s}}$

$t=t_{s}, x=0, \tau>0$.

For $b=0$ :

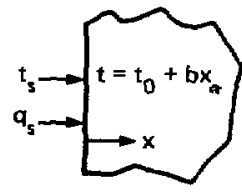

$\frac{q_{s} \sigma}{k\left(t_{s}-t_{0}\right)}=\frac{2}{\sqrt{\pi}}$

See Fig. 7.1

İ

7.1.2 7, p. 89 Variable initial temperature.

$t=E(x), x>0, \tau=0$.

$t=t_{s}, x=0, \tau>0$.

$t-t_{s}=\frac{1}{\sigma \sqrt{\pi}} \int_{0}^{\infty} f(\beta)\left\{\exp \left[-\left(\frac{x-\beta}{\sigma}\right)^{2}\right]-\exp \left[-\left(\frac{x+\beta}{\sigma}\right)^{2}\right]\right\} d B$

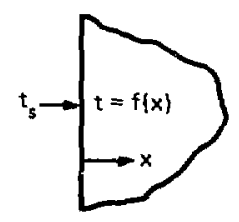

$f(B)=f(x)$ 
Section 7.1. Semi-Infinite Solids--No Internal Heating.

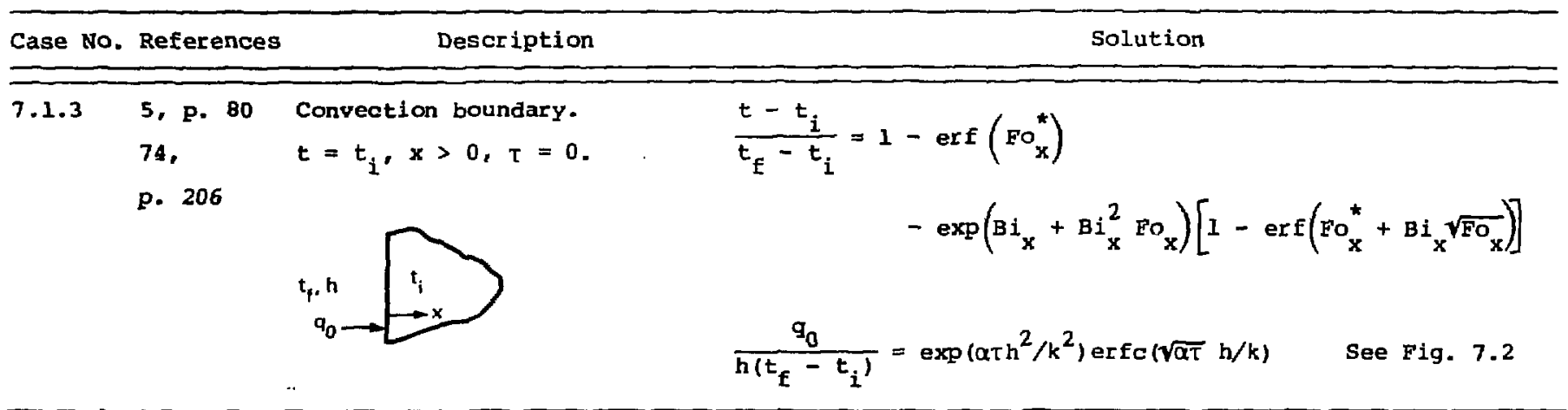

Ramp surface temperature.

$t=t_{i}, x>0, \tau=0$.

$\frac{t-t_{i}}{b \tau}=\left(1+2 F O_{x}^{\star 2}\right) \operatorname{erfc}\left(F O_{x}^{\star}\right)-\frac{2}{\sqrt{\pi}} \mathrm{Fo}_{x}^{*} \exp \left(-\mathrm{Fo}_{\mathrm{x}}^{\star 2}\right)$

$t=t_{i}+b \tau, x=0, \tau \geq 0$.

$t=t_{i}+b \tau \longrightarrow{ }_{i}^{t_{i}}$

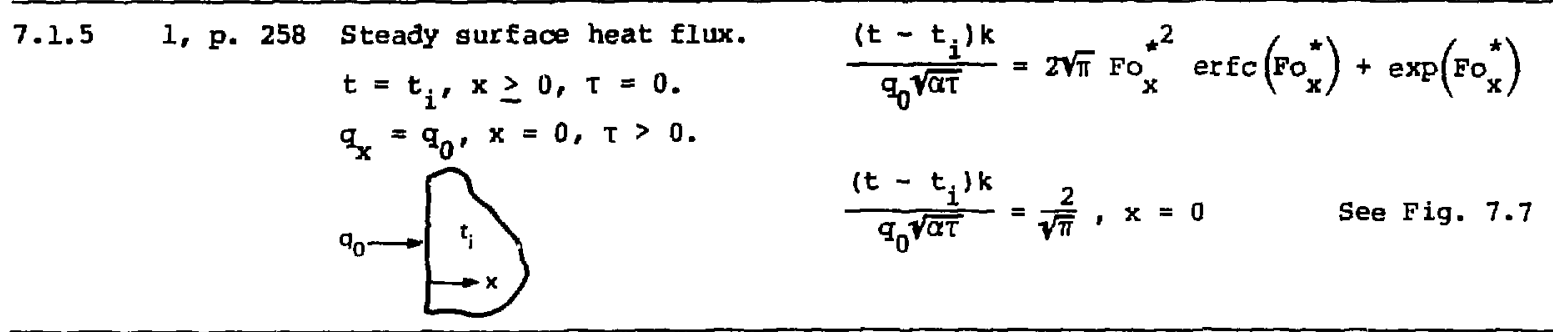


Section 7.1. Semi-Infinite Solids--No Internal Heating.

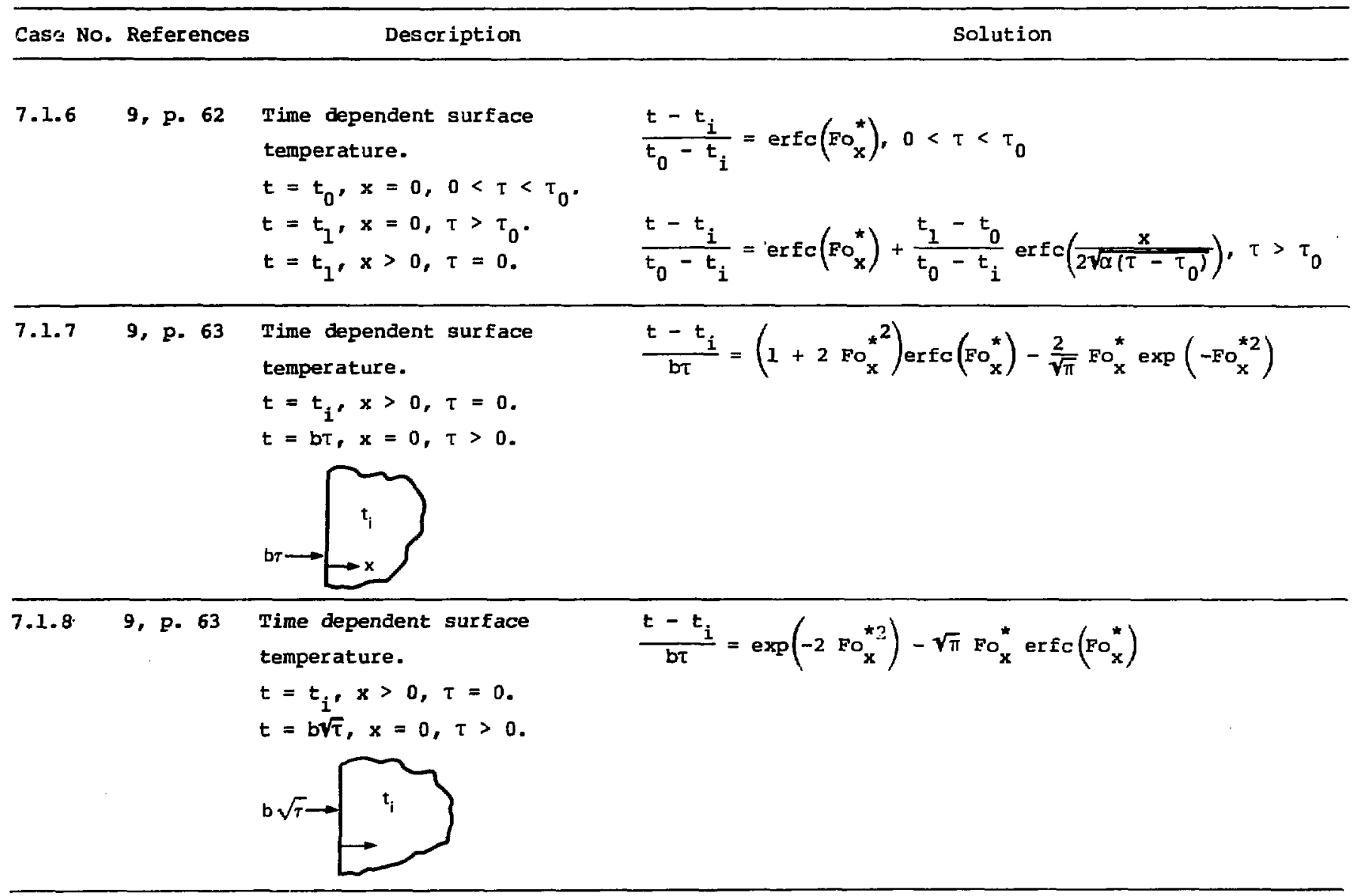


Section 7.1. Semi-Infinite Solids--No Internal Heating.

Case No. References Description

Solution

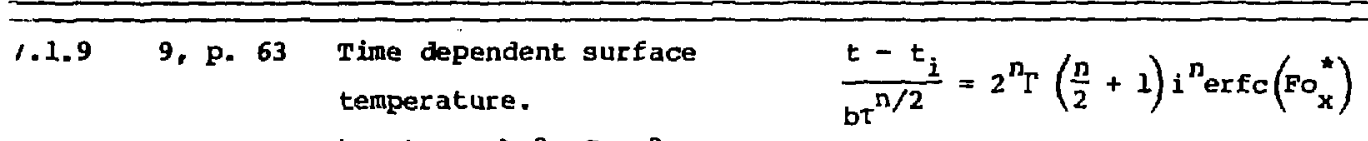

$$
\begin{aligned}
& t=t_{i}, x>0, \tau=0 . \\
& t=t_{i}+b \tau^{n / 2}, x=0, \tau>0, \text { see Fig. } 7.6
\end{aligned}
$$

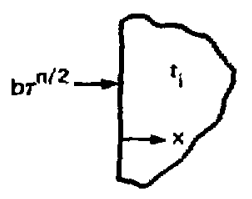

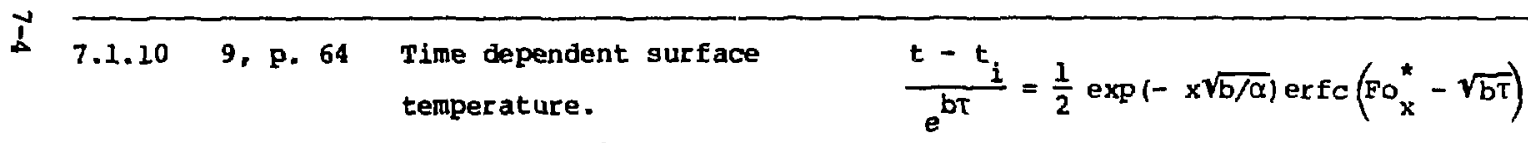

$$
\begin{aligned}
& t=t_{i}, x>0, \tau=0 . \\
& +\exp (x \sqrt{b / \alpha}) \operatorname{erfc}\left(\mathrm{Fo}_{x}^{*}+\sqrt{b \tau}\right)
\end{aligned}
$$

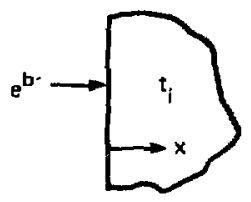


Section 7.I. Semi-Infinite Solids--No Internal Heating.

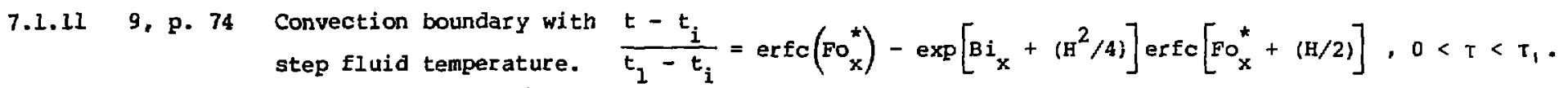
$t=t_{i}, x>0, \tau=0$.

$t_{E}=t_{1}, 0 \leq \tau \leq \tau_{1}$.

$t_{f}=t_{2}, \tau>\tau_{1}$.

$\frac{t-t_{i}}{t_{1}-t_{i}}=\operatorname{erfc}\left(\mathrm{FO}_{x}^{*}\right)-\exp \left[\mathrm{Bi}_{\mathrm{x}}+\left(\mathrm{H}^{2} / 4\right)\right] \operatorname{erfc}\left[\mathrm{FO}_{\mathrm{x}}^{*}+(\mathrm{H} / 2)\right]$

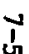

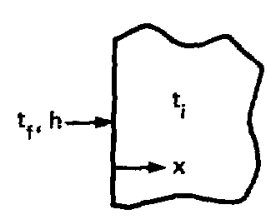

$+\frac{t_{2}-t_{1}}{t_{1}-t_{i}}\left\{\operatorname{erfc}\left[\frac{x}{2 \sqrt{\alpha\left(\tau-\tau_{0}\right)}}\right]-\exp \left[B i_{x}+\frac{h \alpha\left(\tau-\tau_{0}\right)}{k}\right]\right.$ $\left.x \operatorname{erfc}\left[\frac{x}{2 \sqrt{\alpha\left(\tau-\tau_{0}\right)}}+\frac{h \sqrt{a\left(\tau-\tau_{0}\right)}}{k}\right]\right\}, \tau>\tau_{0}$. $H=\frac{2 h \sqrt{\alpha \tau}}{k}$ 
Section 7.1. Semi-Infinite Solids--No Internal Heating.

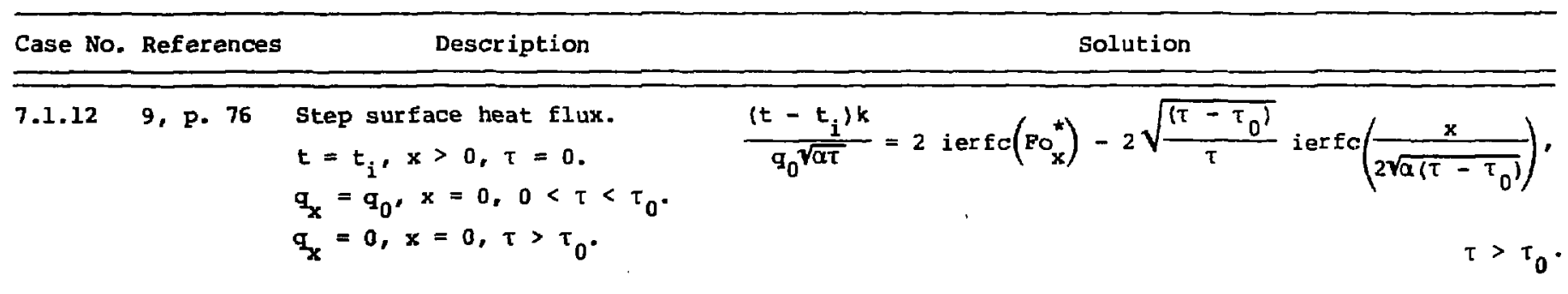

See case 7.1.5 for $0<\tau<\tau_{0}$.

\begin{tabular}{|c|c|c|c|}
\hline 7.1 .13 & 9, p. 76 & $\begin{array}{l}\text { Time dependent surface } \\
\text { heat flux. } \\
t=t_{1}, x>0, \tau=0 . \\
q_{x}=k / \sqrt{\sigma \tau \pi}, x=0, \\
0<\tau<\tau_{0} . \\
q_{x}=0, x=0, \tau>\tau_{0} .\end{array}$ & $\begin{array}{l}t-t_{i}=1, x=0,0<\tau<\tau_{0} \\
t-t_{i}=\frac{2}{\pi} \sin ^{-1}\left(\sqrt{\tau_{0} / \tau}\right), x=0, \tau>\tau_{0} \\
t-t_{i}=\frac{2}{\pi} \int_{0}^{\sqrt{\tau_{0} /\left(\tau-\tau_{0}\right)}} \frac{\exp \left[-F 0_{x}^{\star 2}\left(1+u^{2}\right)\right]}{1+u^{2}} d u, \tau>\tau_{0}\end{array}$ \\
\hline
\end{tabular}


Section 7.1. Semi-Infinite Solids--No Internal Heating.

\begin{tabular}{|c|c|c|c|}
\hline Case No. & References & Description & Solution \\
\hline 7.1 .14 & 9, p. 77 & $\begin{array}{l}\text { Time dependent surface } \\
\text { heat flux. } \\
t=t_{i}, x>0, \tau=0 . \\
q_{x}=q_{0} \tau^{n / 2}, x=0, \tau>0, \\
n=-1,0,1,2 \ldots\end{array}$ & $\begin{array}{l}\frac{\left(t-t_{i}\right) k}{q_{0} \sqrt{\alpha \tau} \tau^{n / 2}}=\frac{\Gamma(1+n / 2)}{\Gamma\left(\frac{3}{2}+\frac{n}{2}\right)}, x=0, \tau>0 \\
\frac{\left(t-t_{i}\right) k}{g_{0} \sqrt{\alpha \tau} \tau^{n / 2}}=2(4)^{n / 2} \Gamma(1+n / 2) i^{n+1} \operatorname{erfc}\end{array}$ \\
\hline
\end{tabular}

See Fig. 7.8 for $n=2$.

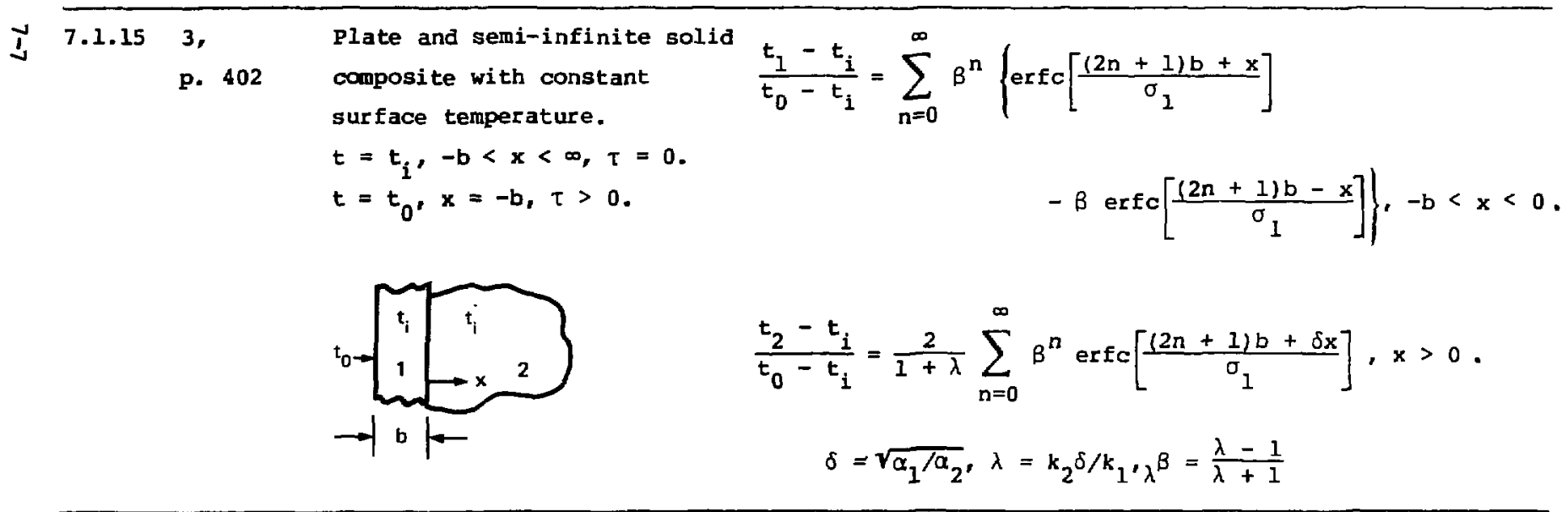


Section 7.1. Semi-Infinite Solids--No Internal Heating.

Case No. References Description Solution

\begin{tabular}{|c|c|c|}
\hline $\begin{array}{r}7.1 .15 .174, \\
\text { p. } 409\end{array}$ & $\begin{array}{l}\text { Case } 7.1 .15 \text { with: } \\
t=t_{1}, 0<x<b, \\
\tau=0 . \\
t=t_{2}, x>b, \tau=0 . \\
t=t_{0}, x=0, \tau>0 .\end{array}$ & 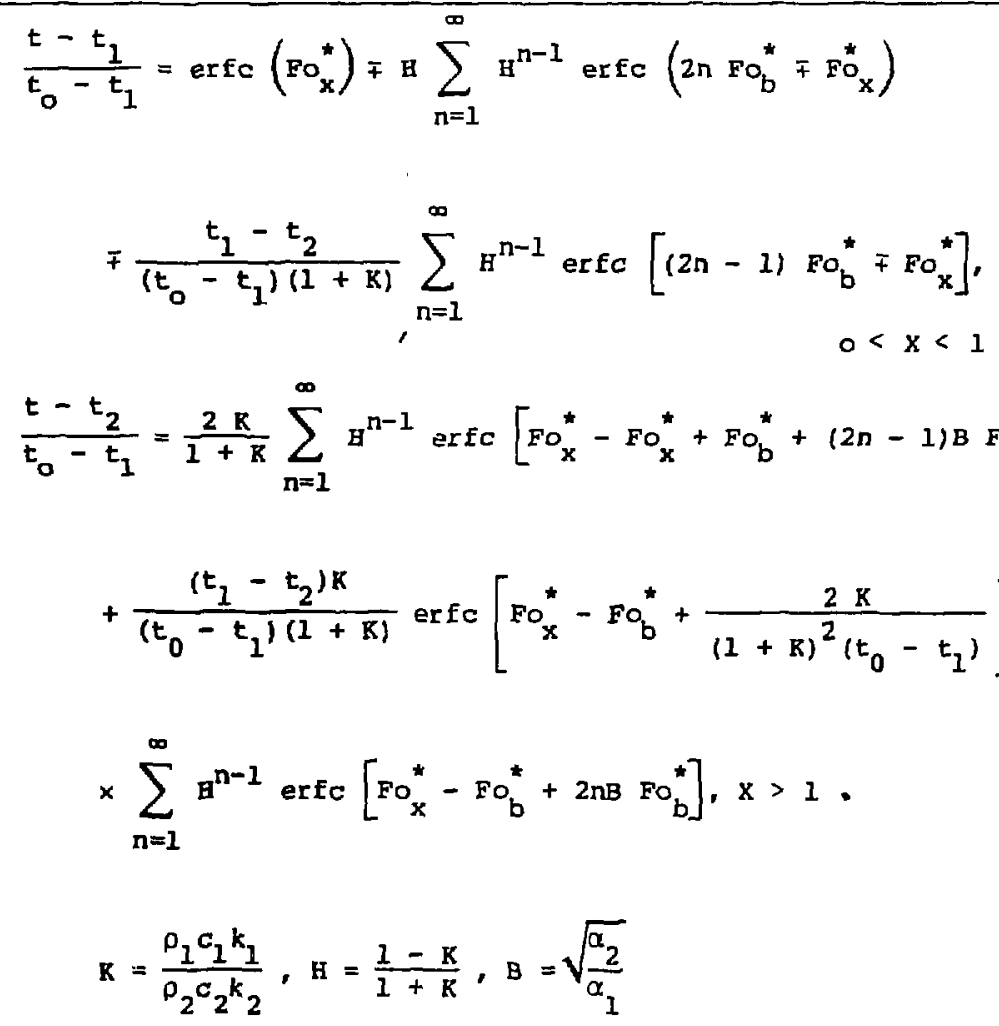 \\
\hline
\end{tabular}


Section 7.1. Semi-Infinite Solids--No Internal Beating.

\section{Case No. References}

Description

Solution

7.1.16 7, p. 99 Periodic surface temper ature.

$t=\left(t_{M}-t_{0}\right) \cos \left(\frac{2 \pi n \tau}{\tau_{0}}\right)$,

$x=0, \tau>0$.

$t=t_{0}, x>0, t=0$.

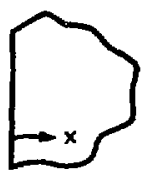

$$
\begin{aligned}
\frac{t-t_{0}}{t_{M}-t_{0}} & =\exp \left(-\sqrt{\frac{\omega}{2 \alpha}} x\right) \cos \left(\omega \tau-\sqrt{\frac{\omega}{2 \alpha}} x\right), \\
T_{m} & =\text { maximum surface temperature } \\
\tau_{0} & =\text { period of periodic temperature } \\
\omega & =2 \pi n / \tau_{0}
\end{aligned}
$$

Maximum temp:

$\frac{t_{\max }-t_{0}}{t_{M}-t_{0}}=\exp \left(-\sqrt{\frac{\omega}{2 \alpha}} x\right)$

Time for $t_{\max }$ to reach $x$ :

$\frac{t_{M}}{t_{0}}=\frac{m}{n}+\frac{x}{2 \sqrt{\alpha n \pi \tau_{0}}}, m=0,1,2, \ldots$.

Heat transferred into solid, $\tau_{1}<\tau<\tau_{2}$ :

$\frac{q \sqrt{a / \tau_{0}}}{k\left(t_{M}-t_{0}\right)}=\frac{1}{\sqrt{2 \pi}}\left[\cos \left(\omega \tau_{1}-\frac{\pi}{4}\right)-\cos \left(\omega \tau_{2}-\frac{\pi}{4}\right)\right]$ 
Section 7.1. Semi-In:inite Solids--No Internal Heating.

\begin{tabular}{|c|c|c|c|}
\hline Case No. & References & Description & Solution \\
\hline 7.1 .17 & 7, p. 106 & $\begin{array}{l}\text { Reriodic fluid temperature. } \\
t_{f}=\left(t_{f M}-t_{0}\right) \cos \left(\frac{2 \pi n \tau}{\tau_{0}}\right) \\
\tau>0 \\
t=t_{0}, x>0, \tau=0\end{array}$ & $\begin{array}{l}\frac{t-t_{0}}{t_{f M}-t_{0}}=\frac{\exp \left(-\sqrt{\frac{\omega}{2 \alpha}} x\right) \cos \left[\omega \tau-\sqrt{\frac{\omega}{2 \alpha}} x-\tan ^{-1}\left(\frac{1}{1+H}\right)\right]}{\left[1+(2 / H)+\left(2 / H^{2}\right)\right]^{1 / 2}} \\
\quad B=\frac{\alpha \tau_{0}^{h^{2}}}{n \pi k^{2}}, \omega=2 n \pi / \tau_{0} \\
t_{f M}=\max \text { fluid temperature } \\
\quad \tau_{0}=\text { period of periodic fluid temperature } \\
\text { Maximum temp: } \\
\frac{t_{M}-t_{0}}{\left(t_{f M}-t_{0}^{\prime}\right.}=\frac{\exp \left(-\sqrt{\frac{\omega}{2 \alpha}} x\right)}{\left[1+(2 / H)+\left(2 / H^{2}\right)\right]^{1 / 2}}\end{array}$ \\
\hline 7.1 .18 & 9, p. 68 & $\begin{array}{l}\text { Square wave surface } \\
\text { temperature. } \\
t=t_{m}+t_{D}, x=0 \\
2 m \tau_{0}<\tau<(2 m+1) \tau_{0} \\
m=0,1,2, \ldots \\
t=t_{m}-t_{D}, x=0 \\
(2 m+1) \tau_{0}<\tau< \\
(2 m+2) \tau_{0}\end{array}$ & $\begin{aligned} \frac{t-t_{D}}{t_{m}-t_{D}} & =\frac{4}{\pi} \sum_{n=0}^{\infty} \frac{1}{2 n+1} \exp \left(-x \sqrt{\frac{(2 n+1) \pi}{2 \alpha \pi}}\right) \sin \left[\frac{(2 n+1) \pi \pi}{t_{0}}\right. \\
\tau_{0} & =\text { period of per iodic temp } \\
t_{m} & =\text { mean surface temp } \\
t_{D} & =\text { deviation of surface temp from } t_{m}\end{aligned}$ \\
\hline $\begin{array}{l}7.1 .19 \\
7.1 .20 \\
7.1 .21\end{array}$ & $\begin{array}{l}2 \text {, p. } 248 \\
2 \text {, p. } 248 \\
2 \text {, p. } 248\end{array}$ & $\begin{array}{l}\text { Quarter-infinite solid. } \\
\text { Eighth-infinite solid. } \\
\text { Semi-infinite plate. }\end{array}$ & $\begin{array}{l}\text { Dimensionless temperatures equal product of solution for } \\
\text { semi-infinite solid (case } 7.1 .1 \text { or } 7.1 .3 \text { ) and solution for } \\
\text { infinite plate (case } 8.1 .6 \text { or } 8.1 .7 \text { ). See Figs. } 9.4 a \text { and } 9.4 b \text {. }\end{array}$ \\
\hline
\end{tabular}


Section 7.1. Semi-Infinite Solids-No Internal Heating.

\begin{tabular}{lll}
\hline Case No. References & Description & Solution
\end{tabular}

7.1.22 4, p. 84 Infinite cylinder in a semi-infinite solid.

Cylinder assumed massless.

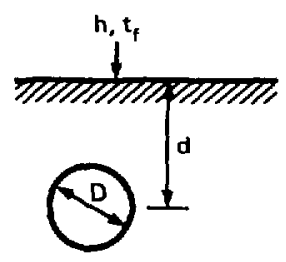

Time for soil to reach steady state:

$$
\frac{\alpha \tau}{D^{2}} \simeq C\left(d / D+1 / B i_{D}\right)^{1.44}
$$

$c=4.6$ for const. cylinder temperature.

$c=6: 0$ for const. heat flux from cylinder.

The heat transferred during the time given above:

$\frac{G \alpha}{R D^{2}\left(t_{C}-t_{f}\right)}=12\left(d / D+1 / B i_{D}\right) 1.25$.

Temperature of cylinder $=t_{c}$.

Initial temperature of semi-infinite solid $=t_{f}$. $t_{c}>t_{f}$

The time for the cylinder to return to temp $t$ after steady state is achieved and heating is stopped:

$$
\frac{\alpha T}{D^{2}}=\left(\alpha / D+1 / B i_{D}\right)^{2.3} f\left(\frac{t-t_{E}}{t_{C}-t_{E}}\right) \text {. }
$$

See Fig. 7.4 for values of $f$. 
Section 7.1. Semi-Infinite Solids--No Internal Heating.

\section{Case No. References}

Description

Solution

7.1.23 9, p. 264 Semi-infinite surface heating.

$\mathbf{t}=\mathbf{t}_{\mathrm{i}}, 0<\mathrm{x}<\infty$,

$-\infty<!<+\infty, \tau=0$.

$\mathrm{q}_{\mathrm{x}}=\mathrm{q}_{0}, \mathrm{x}=0$,

$-\infty<y<0$.

$\mathrm{q}_{\mathrm{x}}=0, \mathrm{x}=0$,

$0<y<+\infty$.

$\stackrel{\vec{N}}{\stackrel{N}{N}}$

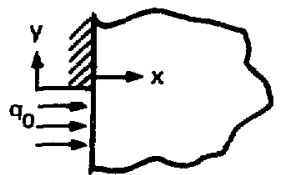

7.1.24 9, p. 264 Infinite strip heated surface.

$t=t_{i}, 0<x<\infty$,

$-\infty<y<+\infty, \tau=0$.

$g_{x}=q_{0}, x=0$,

$-a<y<+a$.

$\mathrm{q}_{\mathrm{x}}=0, x=0$,

$\frac{\left(t-t_{i}\right) k}{q_{0} \sqrt{\alpha \tau}}=\frac{1}{\sqrt{\pi}}\left[\operatorname{erfc}\left(F O_{Y}^{*}\right)+\frac{F O_{Y}^{\star}}{\sqrt{\pi}}\right.$ Ei $\left.\left(-F O_{Y}^{\star 2}\right)\right], x=0$

$y>a, y<-a$.

$$
\begin{aligned}
& \frac{\left(t-t_{i}\right) k}{q_{0} a}=\frac{\sqrt{F o}_{a}}{\sqrt{\pi}}\left\{\operatorname{erf}\left(\frac{1+Y}{2 \sqrt{F o}}\right)+\operatorname{erf}\left(\frac{1-Y}{2 \sqrt{F O}}\right)\right. \\
& \left.-\left(\frac{1+Y}{2 \sqrt{F_{0}} \pi}\right) \operatorname{Ei}\left[-\left(\frac{1+Y}{2 \sqrt{F O}_{\alpha}}\right)^{2}\right]-\left(\frac{1-Y}{2 \sqrt{F O}_{\alpha}}\right)^{2} \operatorname{Ei}\left[-\left(\frac{1-Y}{2 \sqrt{F_{\alpha}}}\right)^{2}\right]\right\}
\end{aligned}
$$

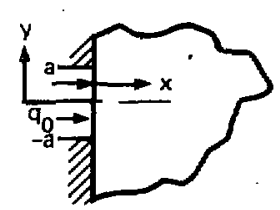

See Fig. 7.5 
Section 7.1. Semi-Infinite Solids--No Internal Heating.

Case No. References

Description

7.1.25 9, p. 264 Heating through a circular surface.

$q_{x}=q_{0}, 0<r<r_{c}$

$x=0, \tau>0$.

$q_{x}=0, r>r_{c}, x=0, \tau>0$.

$t=t_{i}, 0<r<\infty$,

$0<x<\infty, \tau=0$.

$\stackrel{J}{\stackrel{5}{\omega}}$
Solution

$\frac{\left(t-t_{i}\right) k}{g_{0} r_{c}}=2 \sqrt{{ }^{F O_{c}}}\left(\right.$ ierfc $\left(F O_{x}^{*}\right)$

$-\operatorname{ierfc}\left[\mathrm{Fo}_{x}^{*} \sqrt{1+\left(f_{c} / x\right)^{2}}\right], I=0$

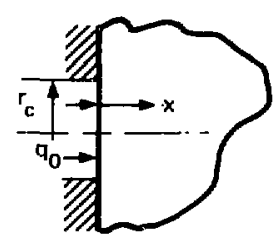


Section 7.1. Semi-Infinite Solids--No Internal Heating.

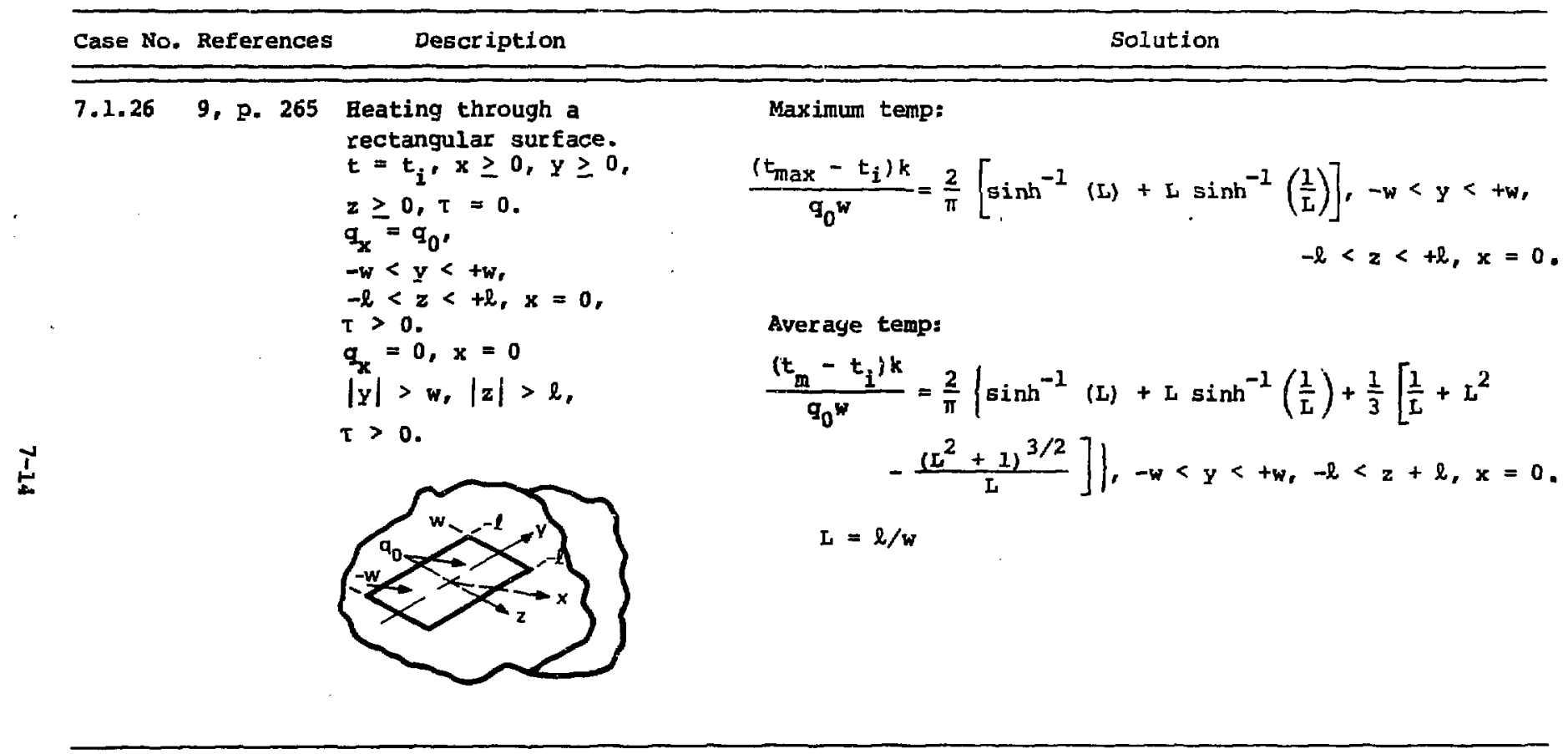


Section 7.1. Semi-Infinite Solids--No Internal Heating.

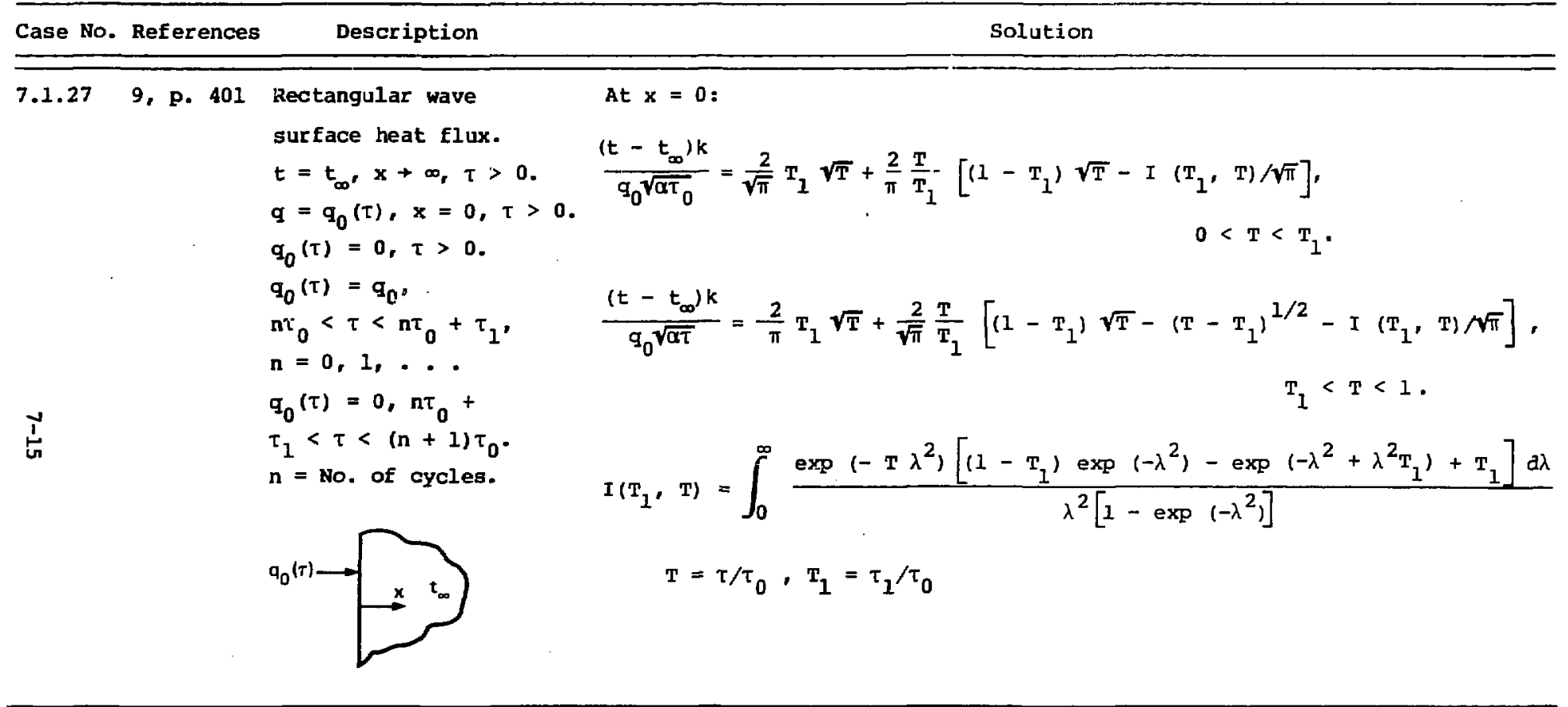


Section 7.1. Semi-Infinite Solids--No Internal Heating.

Case No. References Description ' Solution

7.1.28 9, p. 419 Semi-infinite cylinder See case 9.1.20

with convection boundary.

$t=t_{0}, 0<r_{0}<r_{.}, z>0, \tau=0$.

$t=t_{1}, 0<r<r_{0}, z=0, \tau>0$.

Convection boundary

of $h, t_{0}$ at $I=I_{0}, z>0, \tau>0$.

7.1.29 9, p. 419 Case 7.1.28 with:

See case 9.1.21

$t=t_{1}, r=r_{0}, z>0$,

T $>0$.

$t=t_{0}, 0<r<r_{0}, z=0, \tau>0$. 
Section 7.1. Semi-Infinite Solids--No Internal Heating.

\begin{tabular}{|c|c|c|c|}
\hline Case No. & References & Description & Solution \\
\hline 7.1 .30 & $\begin{array}{l}19, \\
\text { p. 3-83 }\end{array}$ & $\begin{array}{l}\text { Plate and semi-infi- } \\
\text { nite solid composite } \\
\text { with constant surface } \\
\text { heat flux. } \\
t=t_{0}, 0<x_{1}<\delta, 0 \\
<x_{2}<\infty, \tau=0 \text {. } \\
k_{1}+\infty .\end{array}$ & $\begin{array}{l}\frac{k_{2}\left(t_{1}-t_{0}\right)}{2 q^{n} \sqrt{c_{2}}}=\frac{1}{2 m \sqrt{F O_{2}}}\left[\exp \left(m^{2} \mathrm{Fo}_{2}\right) \text { erfc }\left(m \sqrt{F O_{2}}\right)-1\right] \\
\text { Heat flux at interface: } \\
q / q^{n}=1-\exp \left(m^{2} F_{2}\right) \text { erfc }\left(m \sqrt{\pi} o_{2}\right), x_{1}=\delta . \\
m=\left(\rho_{2} c_{2} / \rho_{1} c_{1}\right) \\
\text { See Fig. } 7.9\end{array}$ \\
\hline 7.1 .31 & $\begin{array}{l}74, \\
\text { p. } 211\end{array}$ & $\begin{array}{l}\text { Semi-infinite rod with } \\
\text { convection boundary. } \\
t=t_{i}, x>0, \tau=0 \\
t=t_{f^{\prime}} x=0, \tau>0\end{array}$ & 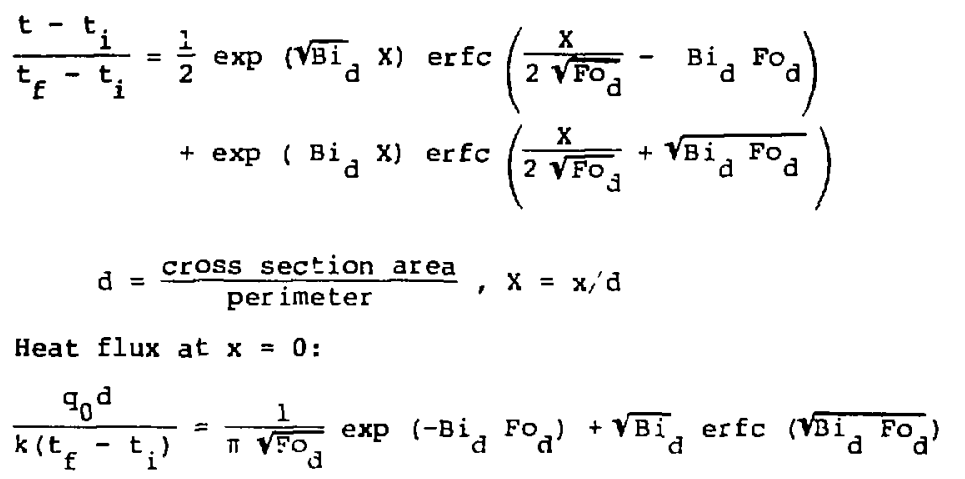 \\
\hline
\end{tabular}


Section 7.1. Semi-Infinite Solids--No Internal Heating.

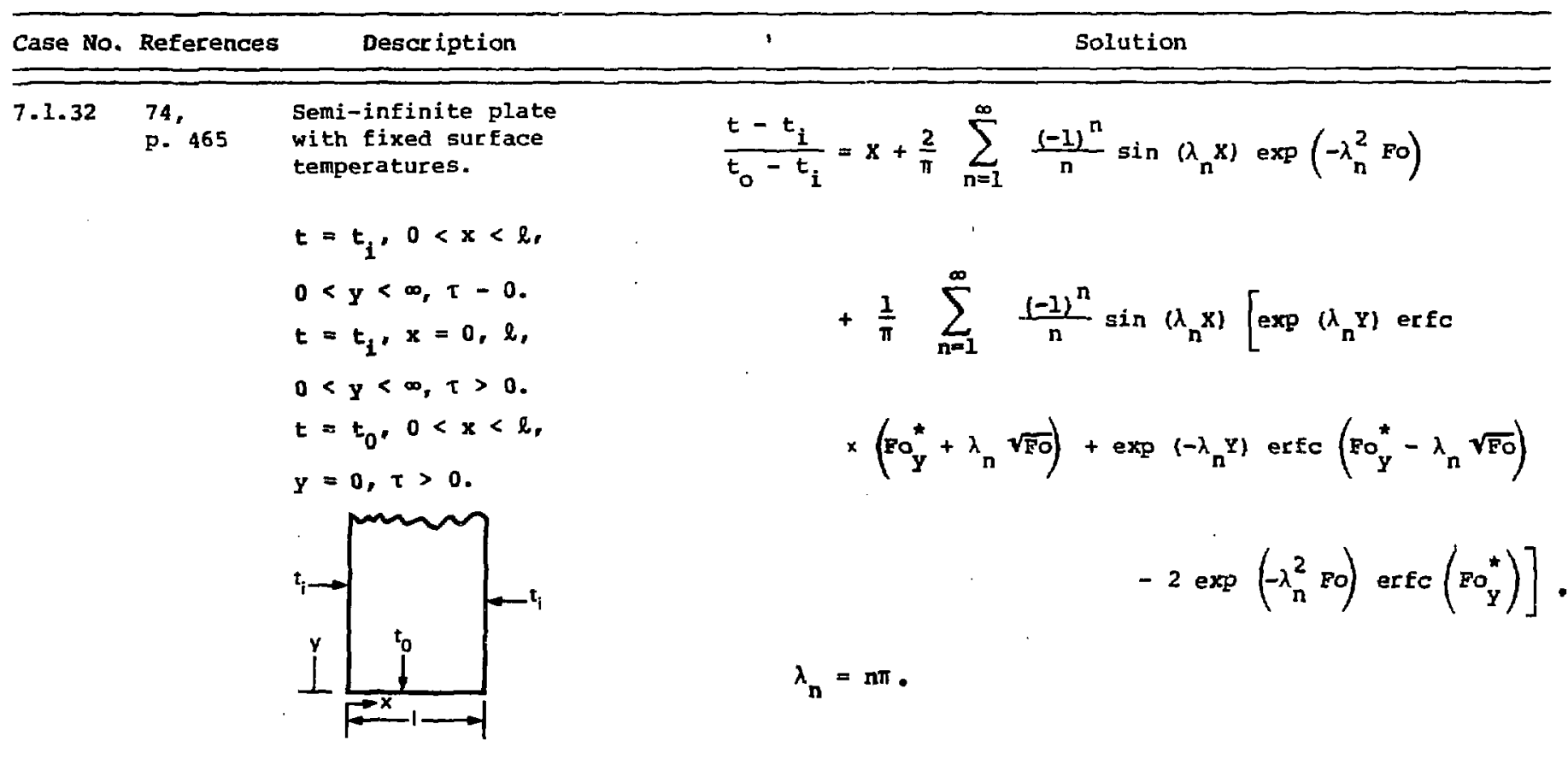


Section 7.1. Semi-Infinite Solids--No Internal Heating.

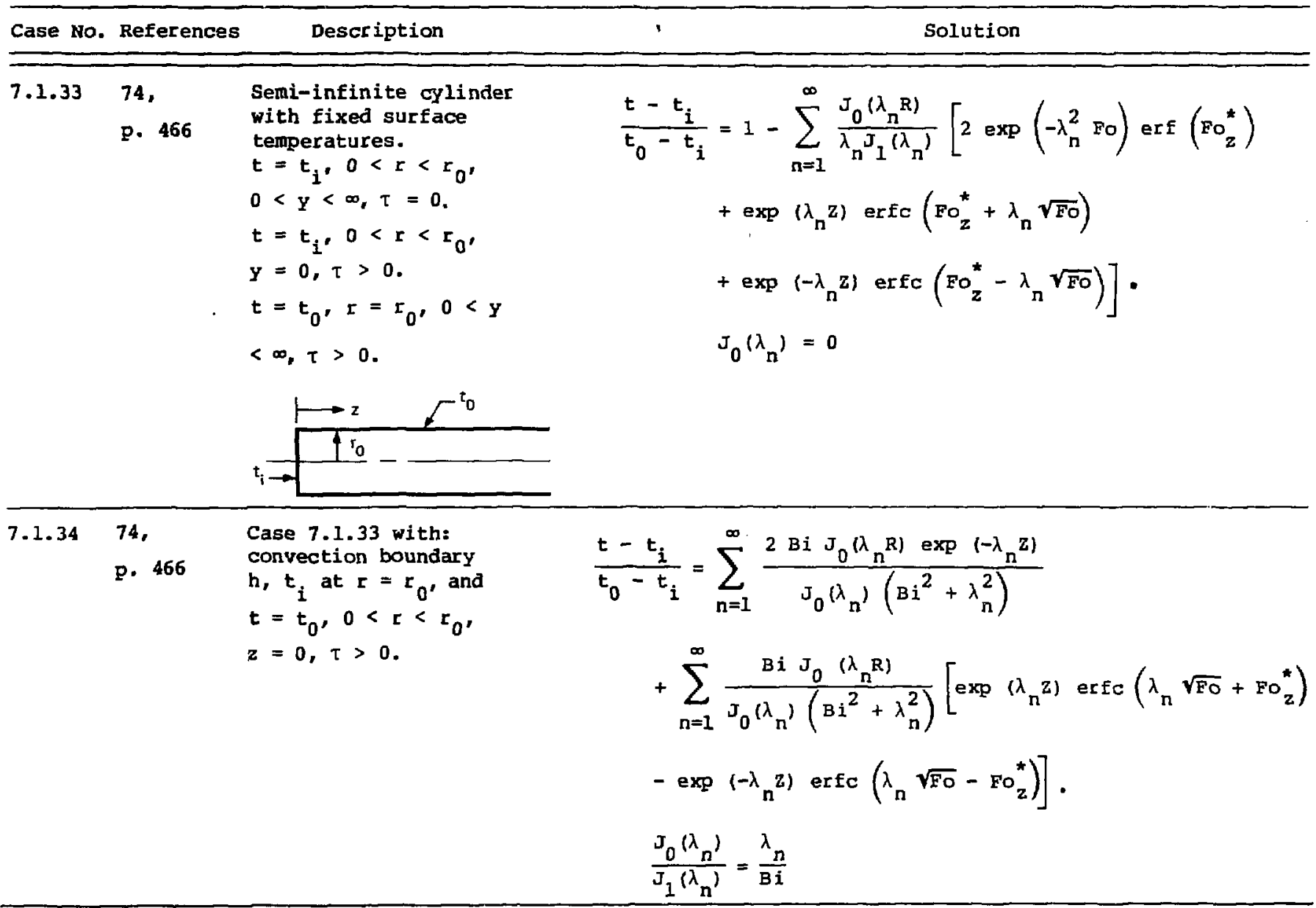


Section 7.1. Semi-infinite Solids-No Internal Heating.

Case No. References Description

Solution

j̀ 
Section 7.1. Semi-infinite Solids-No Internal Heating. 
Section 7.2. Semi-infinite Solids--With Internal Heating.

Case No. References Description

Solution

7.2.1 9, p. 79 steady surface temperature and initial temperature distribution.

$t=\mathrm{T}_{1}+\mathrm{bx}, \mathrm{x} \geq 0$,

$\tau=0$.

$t=t_{0}, x=0, \tau>0$.

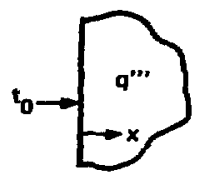

$\frac{t-t_{0}}{\mathrm{~T}_{1}}=\left(1+\mathrm{PO}^{\star}+\mathrm{PO}^{\star} \mathrm{FO}^{\star 2}\right)$ erf $\left(\mathrm{FO}_{\mathrm{x}}^{\star}\right)+\frac{2}{\sqrt{\pi}} \mathrm{PO}^{\star} \mathrm{FO}_{\mathrm{X}}^{\star} \exp \left(-\mathrm{FO}_{\mathrm{X}}^{\star 2}\right)$

$+\frac{\mathrm{bx}}{\mathrm{T}_{1}}-\mathrm{Po}^{*} \mathrm{Fo}_{\mathrm{x}}^{* 2}$

N

$$
\begin{aligned}
& \text { 7.2.2 9, p. } 79 \text { Case 7.2.1--variable } \\
& \text { heating. } \\
& t=T_{1}+b x, x \geq 0 \text {, } \\
& \tau=0 \text {. } \\
& \frac{\left(t-t_{0}\right) k B^{2}}{g_{0}^{\prime \prime}}=\frac{b x}{T_{1} P O^{\star}}+\left(\frac{1}{P O^{*}}-1\right) \text { rf }\left(F O^{*}\right)+1-e^{-B x} \\
& t=t_{0,} x=0, \tau>0 \text {. } \\
& +\frac{1}{2} e^{\lambda-\beta x} \text { eric }\left(\sqrt{\lambda}-F 0^{*}\right)-\frac{1}{2} e^{\lambda+B x} \operatorname{erfc}\left(\sqrt{\lambda}+F O^{*}\right) \\
& q^{\prime \prime \prime}=q_{0}^{\prime \prime \prime} \mathrm{e}^{-B x} \text {. } \\
& P_{0}^{*}=\frac{q_{0}^{\prime \prime \prime}}{k T_{1} \beta^{2}}, \lambda=\alpha \beta^{2} \tau
\end{aligned}
$$

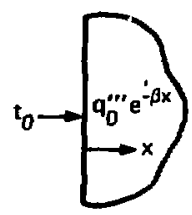


Section 7.2. Semi-infinite Solids -With Internal Heating.

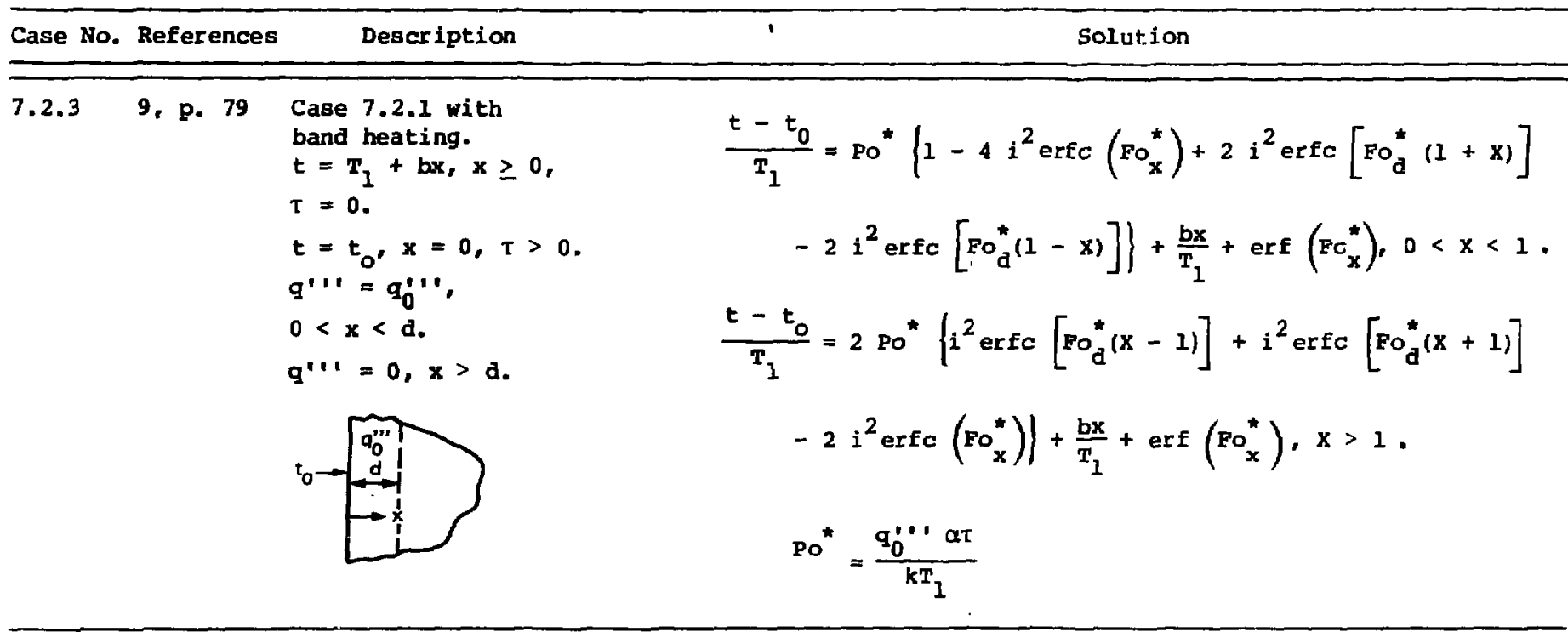


Section 7.2. Semi-infinite Solids--with Internal Heating.

Case No. References

Description

Solution

7.2.4 9, p. 80 Insulated boundary and band heating.

$t=t_{i}, x \geq 0, \tau=0$.

$q_{x}=0, x=0, \tau>0$.

$q^{\prime \prime \prime}=q_{0}^{\prime \prime \prime}, 0<x<a$,

$\tau>0$.

$q^{\prime \prime}=0, x>d$.

$$
\begin{aligned}
& \frac{\left(t-t_{i}\right) k}{q_{0}^{\prime} \cdot d \tau}=1-2 i^{2} \operatorname{erfc}\left[F o_{d}^{*}(1-x)\right]-2 i^{2} \operatorname{erfc}\left[F_{d}^{*}(1+x)\right] \text {, } \\
& 0<x<1 \text {. }
\end{aligned}
$$

$$
\frac{\left(t-t_{i}\right) k}{q_{0}^{\prime \prime} a \tau}=2\left\{i^{2} \operatorname{erfc}\left[F O_{d}^{*}(x-1)\right]-i^{2} \operatorname{erfc}\left[F O_{d}^{*}(x+1)\right]\right\} .
$$

In

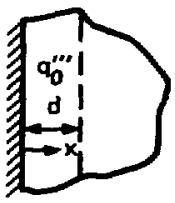

7.2.5 9, p. $80 \quad$ Insulated boundary
and variable heating

$t=t_{0}+b x, x>0$,

$\tau=0$.

$q_{x}=0, x=0, \tau>0$.

$q^{\prime \prime \prime}=q_{0}^{\prime \prime \prime} e^{-\beta x}$,

$\mathrm{x}>0, \mathrm{t}>0$.

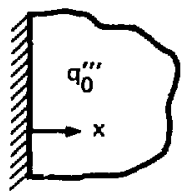

$$
\begin{aligned}
& \frac{\left(t-t_{0}\right) k B^{2}}{q_{0}^{\prime \prime \prime}}=\frac{\lambda^{2}}{\operatorname{Po}_{x}^{*}}-\exp (-\beta x)+\left(\frac{\lambda^{2}}{F O_{x}^{*} P O_{x}^{*}}+2 \lambda\right) \operatorname{erfc}\left(F O_{x}^{*}\right) \\
& \quad+\frac{1}{2} \exp \left(\lambda^{2}-\beta x\right) \operatorname{erfc}\left(\lambda-F O_{x}^{*}\right)+\frac{1}{2} \exp \left(\lambda^{2}+\beta x\right) \operatorname{erfc}\left(\lambda+F O_{x}^{*}\right)
\end{aligned}
$$

$\mathrm{Po}_{\mathrm{x}}^{\star}=\frac{\mathrm{q}_{0}^{\prime \prime}{ }^{\prime \prime} \alpha \tau}{\mathrm{kbx}}, \lambda=\beta \sqrt{\alpha \tau}$ 
Section 7.2. Semi-infinite Solids--With Internal Heating.

Case No. References Description

Solution

7.2.6 9, p. 323 Plate and semi-infinite solid composite.

$t=t_{0},-b<x<\infty$,

$\frac{\left(t_{I}-t_{0}\right) k_{I}}{q_{0}^{\prime \prime \prime \alpha}}=I-4 \sum_{n=0}^{\infty} \beta^{n}\left[i^{2} \operatorname{erfc}\left(\frac{(2 n+1)+x}{2 \sqrt{F_{0}}}\right)\right.$

$\tau=0$.

$t=t_{0}, x=-b, \tau>0$.

$-\beta i^{2} \operatorname{erfc}\left[\frac{(2 n+1)-x}{2 \sqrt{F O}}\right]+\frac{\lambda}{1+\lambda} \quad i^{2} \operatorname{erfc}\left(\frac{2 n+x}{2 \sqrt{F O}}\right)$

$q^{\prime \prime \prime}=q_{0}^{\prime \prime \prime}$

$-b<x<0$, $\tau>0$.

$q^{\prime \prime \prime}=0, x^{\circ}<0, \tau>0$.

$\left.-\frac{\lambda}{1+\lambda} i^{2} \operatorname{erfc}\left(\frac{(2 n+2)}{2 \sqrt{F O}}+x\right)\right],-1<x<0$.

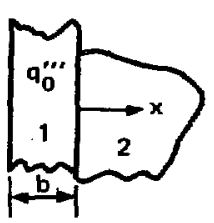

$\frac{\left(t_{2}-t_{0}\right) k_{1}}{q_{0}^{\prime \prime \prime a r}}=\frac{4}{(1+\lambda)} \sum_{n=0}^{\infty} \beta^{n}\left\{i^{2} \operatorname{erfc}\left(\frac{2 n+\delta x}{2 \sqrt{F O}}\right)\right.$

$\left.+i^{2} \operatorname{erfc}\left[\frac{(2 n+2)+\delta x}{2 \sqrt{F O}}\right]-2 i^{2} \operatorname{erfc}\left[\frac{(2 n+1)+\delta x}{2 \sqrt{F O}}\right]\right\}, x>0$.

$\delta=\sqrt{a_{1} / a_{2}}, \lambda=k_{2} \delta / k_{1}, \dot{\beta}=\frac{\lambda-1}{\lambda+1}$, Fo $=\frac{\alpha_{1} \tau}{b^{2}}, x=\frac{x}{b}$ 
Section 7.2. Seri-infinite Solids--With Internal Heating.

Case No. References Description Solution

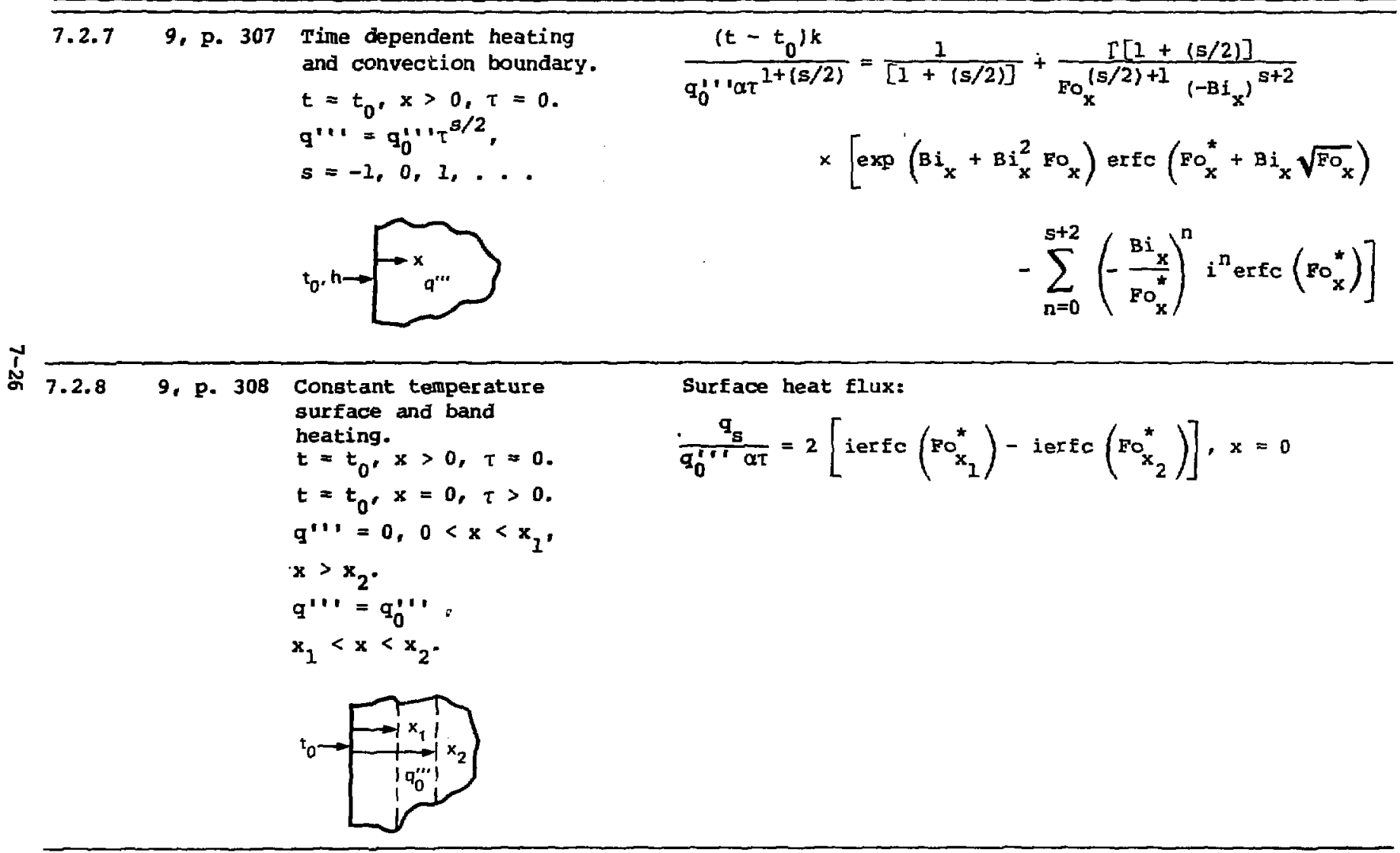


Section 7.2. Semi-infinite Solids--With Internal Heating.

\begin{tabular}{|c|c|c|c|}
\hline Case No. & References & Description & Solution \\
\hline 7.2 .9 & $\begin{array}{l}54 \\
19, \\
\text { p. } 384\end{array}$ & $\begin{array}{l}\text { Exponentiai heating. } \\
t=t_{0}, x>0, \tau=0 . \\
q^{\prime \prime}=q_{0}^{\prime \prime \prime} \exp (-\mu x) \text {. }\end{array}$ & 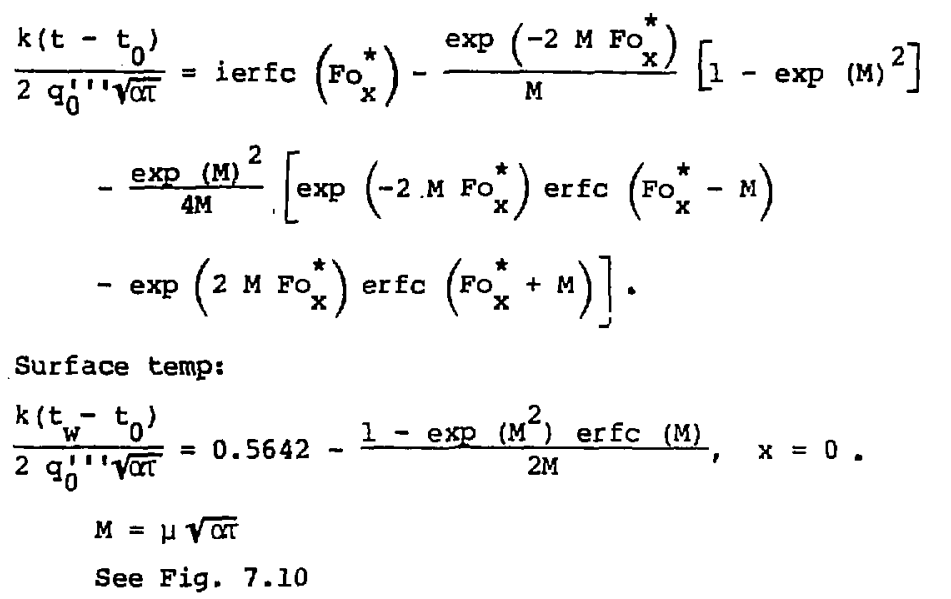 \\
\hline
\end{tabular}



Section 7.2. Semi-infinite Solids--With Internal Heating.

Case No. References Description

Solution 

Section 8.1. Solids Bounded by Plane Surfaces--No Internal Heating.

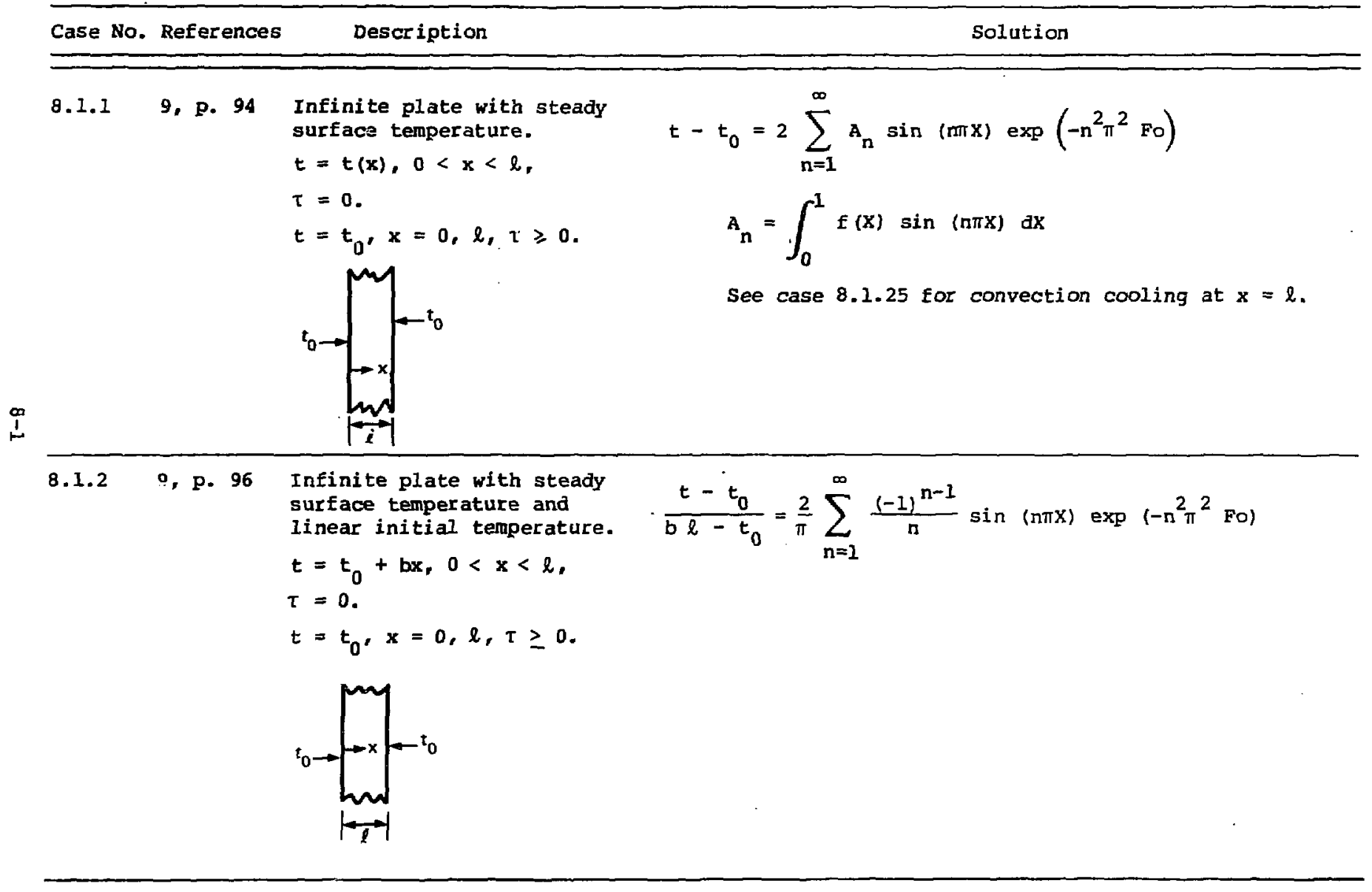


Section 8.1. Solids Bounded by Plane Surfaces-No Internal Heating.

\section{Case No. References}

Description

Solution

8.1.3 9, p. 97 Infinite plate with steady surface temperature and bilinear initial temperature.

$$
\frac{t-t_{0}}{t_{c}-t_{0}}=\frac{8}{\pi^{2}} \sum_{n=0}^{\infty} \frac{1}{(2 n+1)^{2}} \cos \left[\frac{(2 n+1)}{2} \pi x\right] \exp \left[-\frac{(2 n+1)^{2}}{4} \pi^{2} F o\right]
$$

$t=\left(t_{c}-t_{0}\right)(l-|x|) /$

$\left(l+t_{0}\right),-l<x<+l$,

$\tau=0$.

$=1-|x|-2 \sqrt{F_{0}} \sum_{n=0}^{\infty}(-1)^{n}\left\{i \operatorname{erfc}\left(\frac{2 n+|x|}{2 \sqrt{F_{0}}}\right)\right.$

$t=t_{0}, x= \pm \ell, \tau>0$.

$\left.-\operatorname{ierfc}\left[\frac{(2 n+2)-|x|}{2 \sqrt{E O}}\right]\right\}$

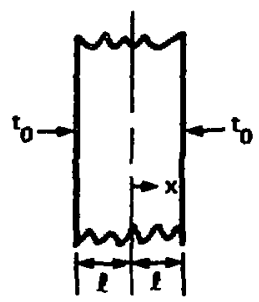


Section 8.1. Solids Bounded by Plane Surfaces--No Internal Beating.

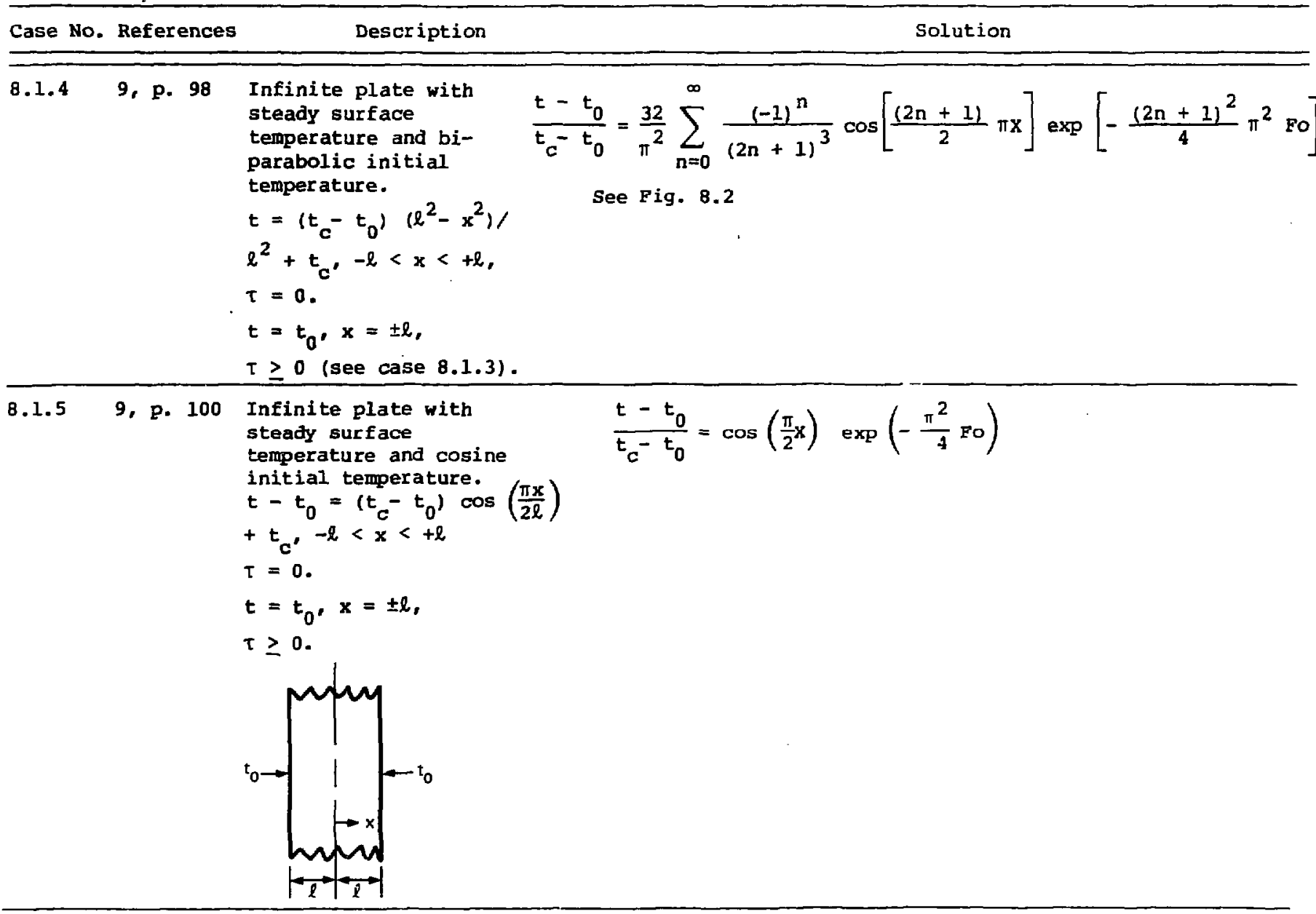


Section 8.1. Solids Bounded by Plane Surfaces -No Internal Heating.

Case No. References Description Solution

\begin{tabular}{|c|c|c|c|}
\hline 8.1 .6 & $\begin{array}{rll}9, & \text { p. } 97 \\
74, & \text { p. } 113\end{array}$ & $\begin{array}{l}\text { Infinite plate with } \\
\text { steady temperature and } \\
\text { constant initial } \\
\text { temperature. } \\
t=t_{i},-l<x<\ell, \\
\tau=0 . \\
t=t_{0}, x= \pm l \\
\tau \geq 0\end{array}$ & $\begin{array}{l}\frac{t-t_{0}}{t_{i}-t_{0}}=2 \sum_{n=0}^{\infty} \frac{(-1)^{n}}{\lambda_{n}} \cos \left(\lambda_{n} X\right) \exp \left(-\lambda_{n}^{2} \text { Fo }\right) \\
\text { Mean temp: } \\
\frac{t_{m}-t_{0}}{t_{i}-t_{0}}=2 \sum_{n=0}^{\infty} \frac{1}{\lambda_{n}^{2}} \exp \left(-\lambda_{n}^{2} \text { Fo }\right) \\
\frac{t_{\text {In }}-t_{0}}{t_{1}-t_{0}}=1-2 \sqrt{\frac{\text { Fo }}{\pi}}, \text { Fo }<0.1 \\
\text { Beat } 1 \text { oss: } \\
\frac{Q}{2 l_{\text {pc }}\left(t_{i}-t_{0}\right)}=2 \sum_{n=1}^{\infty} \frac{\sin \left(\lambda_{n}\left[1-\exp \left(-\lambda_{n}^{2} F\right.\right.\right.}{\lambda_{n}\left[\lambda_{n}+\sin \left(\lambda_{n}\right) \cos 1\right.} \\
\quad \lambda=(2 n+1) \pi / 2, \operatorname{see~Fig.~B.3~}\end{array}$ \\
\hline
\end{tabular}


Section 8.1. Solids Bounded by Plane Surfaces--No Internal Heating.

8.1.7.1 74, Infinite plate with

p. 220

convection boundary (general).

$t=f(x),-l<x<+l$, $\tau=0$.

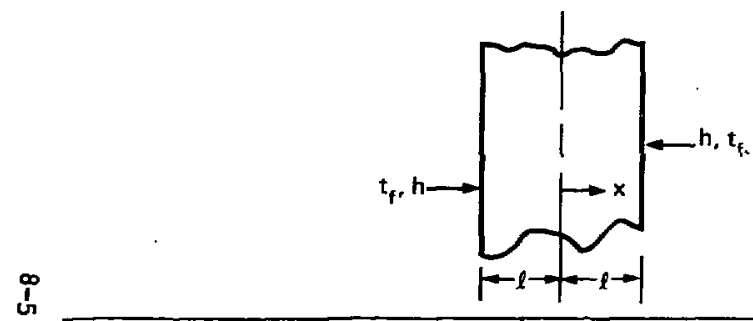

$$
t-t_{f}=2 \sum_{n=1}^{\infty} \frac{\lambda_{n} \cos \left(\lambda_{n} X\right) \exp \left(-\lambda_{n}^{2} F o_{\ell}\right)}{\lambda_{n}+\sin \left(\lambda_{n}\right) \cos \left(\lambda_{n}\right)} \int_{0}^{1}\left[E(X)-t_{f}\right] \cos \left(\lambda_{n} x\right) d x
$$$$
\cot \left(\lambda_{n}\right)=\lambda_{n} / B i_{l}
$$

$\lambda_{n}$ given in Table 14.1
8.1.7.2 3, p. 294 Infinite plate with 74 , p. 223 convection boundaryconstant initial termp. $t=t_{i},-l<x<+l$, $\tau=0$.

(see case 8.1 .7 .1 )

$$
\frac{t-t_{f}}{t_{i}-t_{f}}=2 \sum_{n=1}^{\infty} \frac{\sin \left(\lambda_{n}\right) \cos \left(\lambda_{n} x\right) \exp \left(-\lambda_{n}^{2} F 0\right)}{\lambda_{n}+\sin \left(\lambda_{n}\right) \cos \left(\lambda_{n}\right)}
$$$$
\cot \left(\lambda_{n}\right)=\lambda_{n} / \mathrm{Bi}
$$

$$
\frac{t-t_{f}}{t_{i}-t_{f}}=1-\cos (\sqrt{B i} x) \exp (-B i \text { Fo }), B i<0.1
$$$$
\frac{t-t_{f}}{t_{i}-t_{f}}=\operatorname{erf}\left(\frac{1-x}{2 \sqrt{F O}}\right)-\exp \left[B i(1-x)+B i^{2} F O\right] \text { erfc }\left(\frac{1-X}{2 \sqrt{F O}}+B i \sqrt{F O}\right)
$$$$
+\operatorname{erfc}\left(\frac{1+X}{2 \sqrt{F O}}\right)-\exp \left[B i(1+X)+B i^{2} F O\right] \operatorname{erfc}\left(\frac{1+x}{2 \sqrt{\mathrm{FO}}}+B i \sqrt{\mathrm{FO}}\right) \text {. }
$$

$$
\text { Fo }<0.1 \text {. }
$$


Section 8.1. Solids Bounded by Plane Surfaces--No Internal Heating.

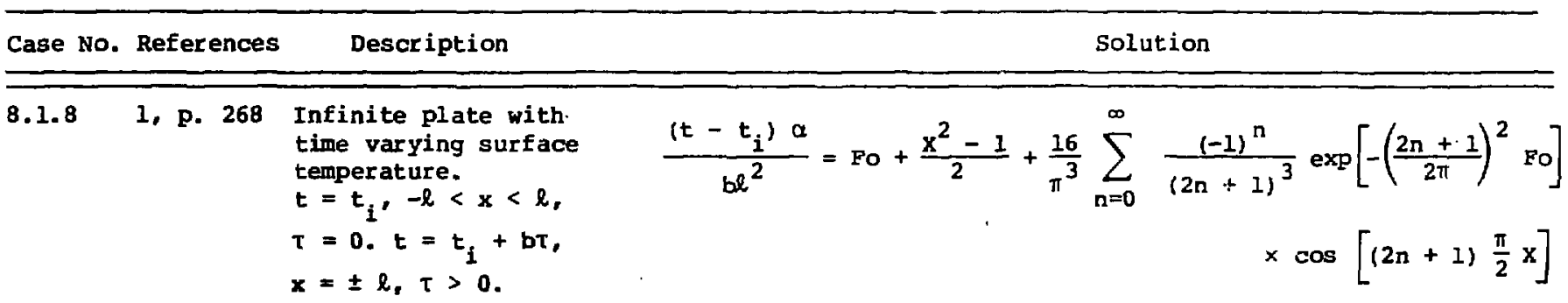

ơ⿱

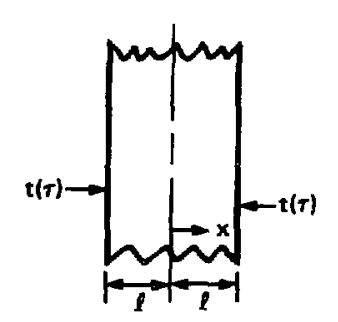

See Table B.1, Fig. 8.16 and Fig. 8.25.

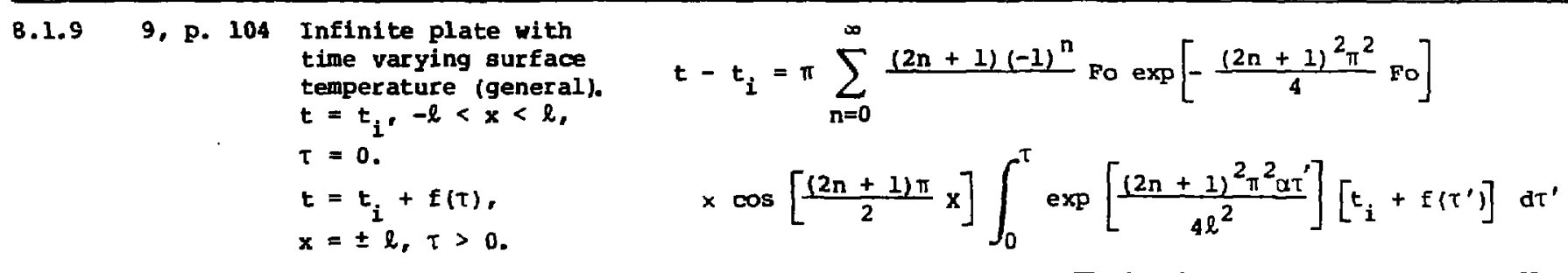


Section 8.1. Solids Bounded by Plane Surfaces--No Internal Heating.

Case No. References Description Solution

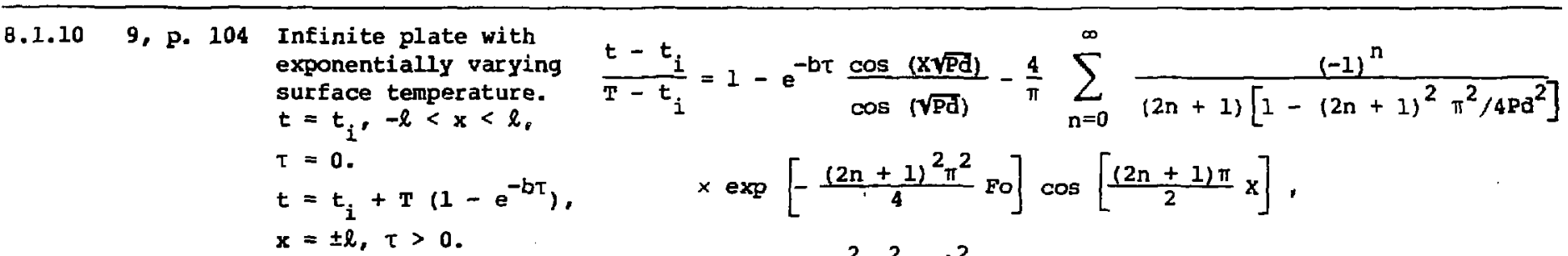

$b \neq(2 n+1)^{2} \pi^{2} \alpha / 4 l^{2}$

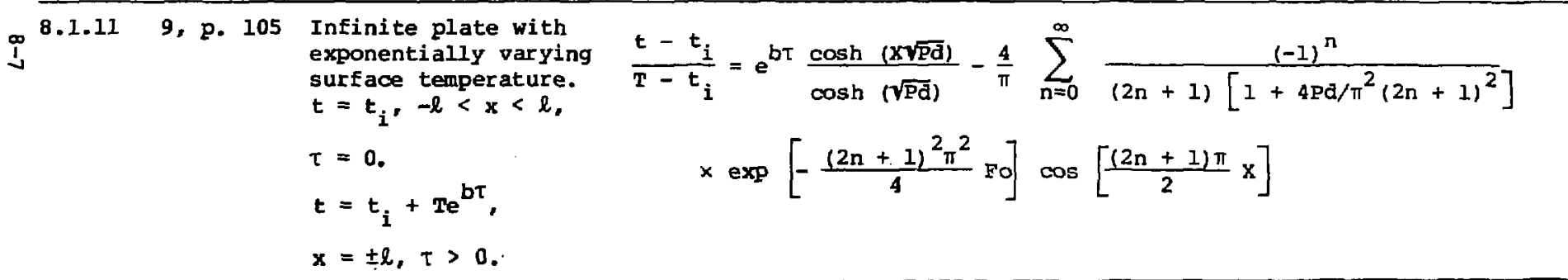


Section 8.1. Solids Bounded by Plane Surfaces--No Internal Heating.

Case No. References Description Solution

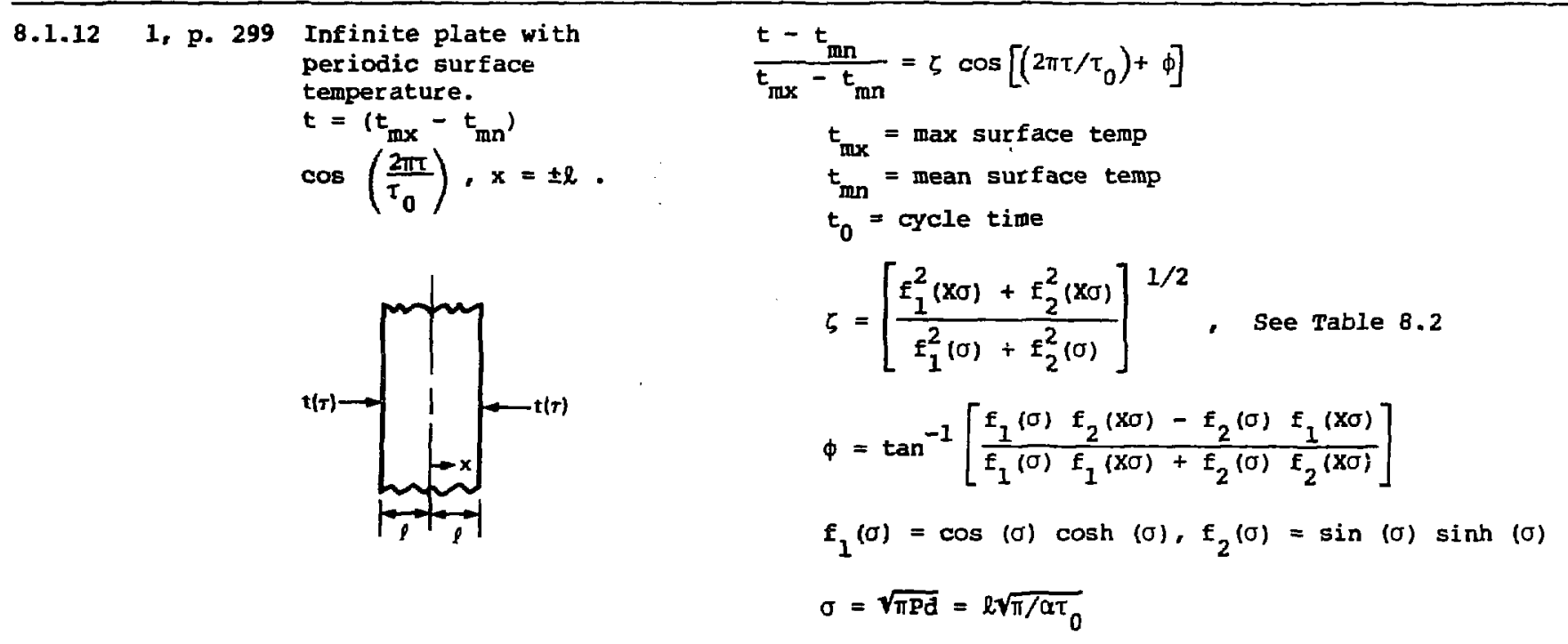

Heat stored during half cycle:

$Q=2 l p c\left(t_{\operatorname{mx}}-t_{\operatorname{mn}}\right) F(\sigma) / \sigma$

$F(\sigma)=\left[\frac{a f_{1}^{2}(\sigma)+d f_{2}^{2}(\sigma)}{f_{1}^{2}(\sigma)+f_{2}^{2}(\sigma)}\right]^{1 / 2}$ see Fig. 8.5

$\mathrm{df}_{1}(\sigma)=\cos (\sigma) \sinh (\sigma)-\sin (\sigma) \cosh (\sigma)$

$\mathrm{df}_{2}(\sigma)=\sin (\sigma) \cosh (\sigma)+\cos (\sigma) \sinh (\sigma)$ 
Section 8.1. Solids Bounded by Plane Surfaces-No Internal Heating.

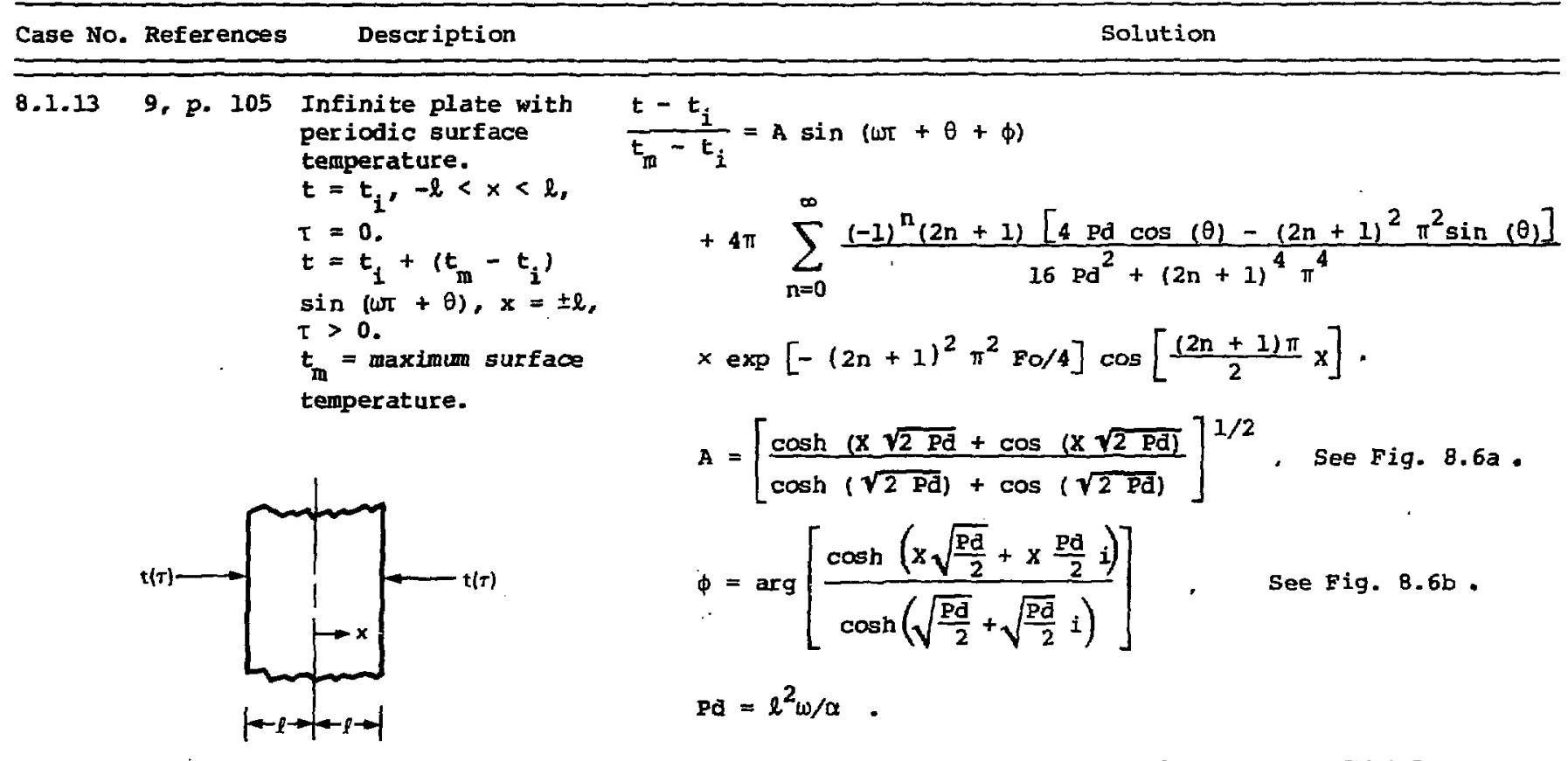


Section B.1. Solids Bounded by Plane Surfaces--No Internal Heating.

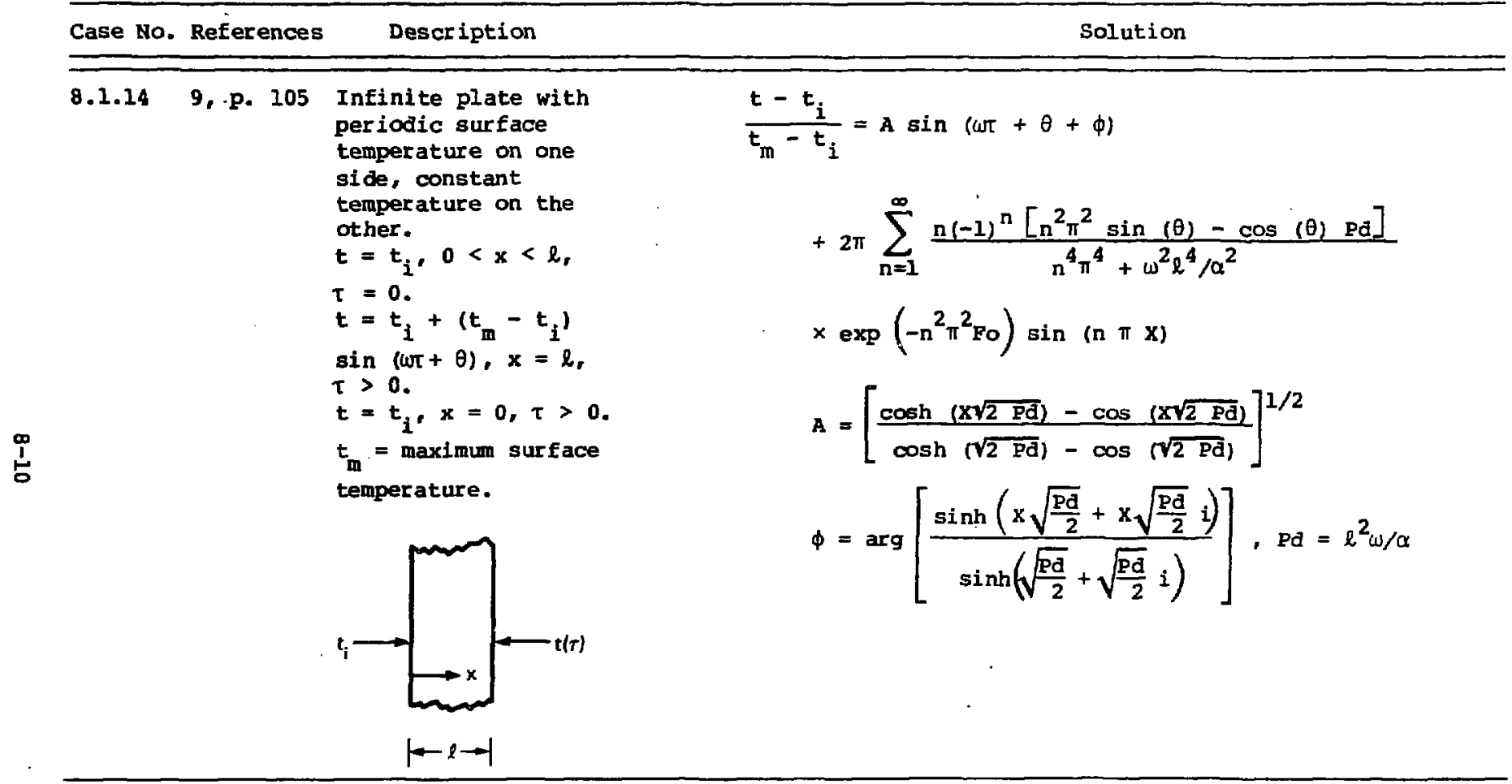


Section 8.1. Solids Bounded by Plane Surfaces--No Internal Heating.

Case No. References Description Solution

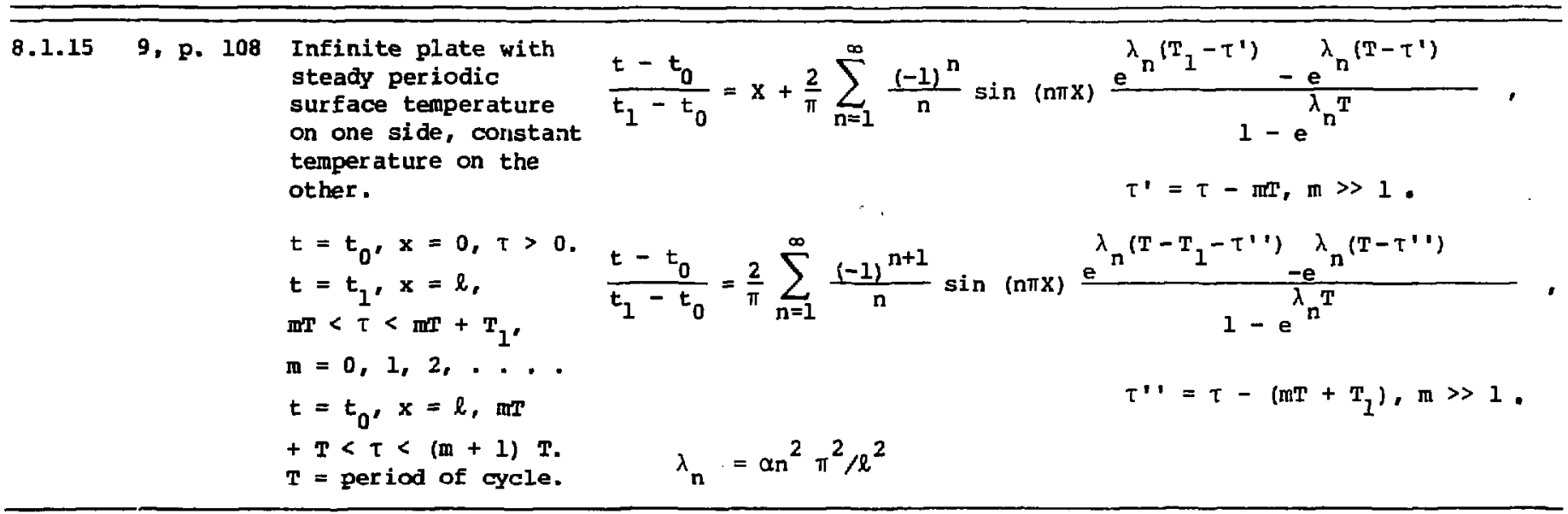


Section 8.1. Solids Bounded by Plane Surfaces--No Internal Heating.

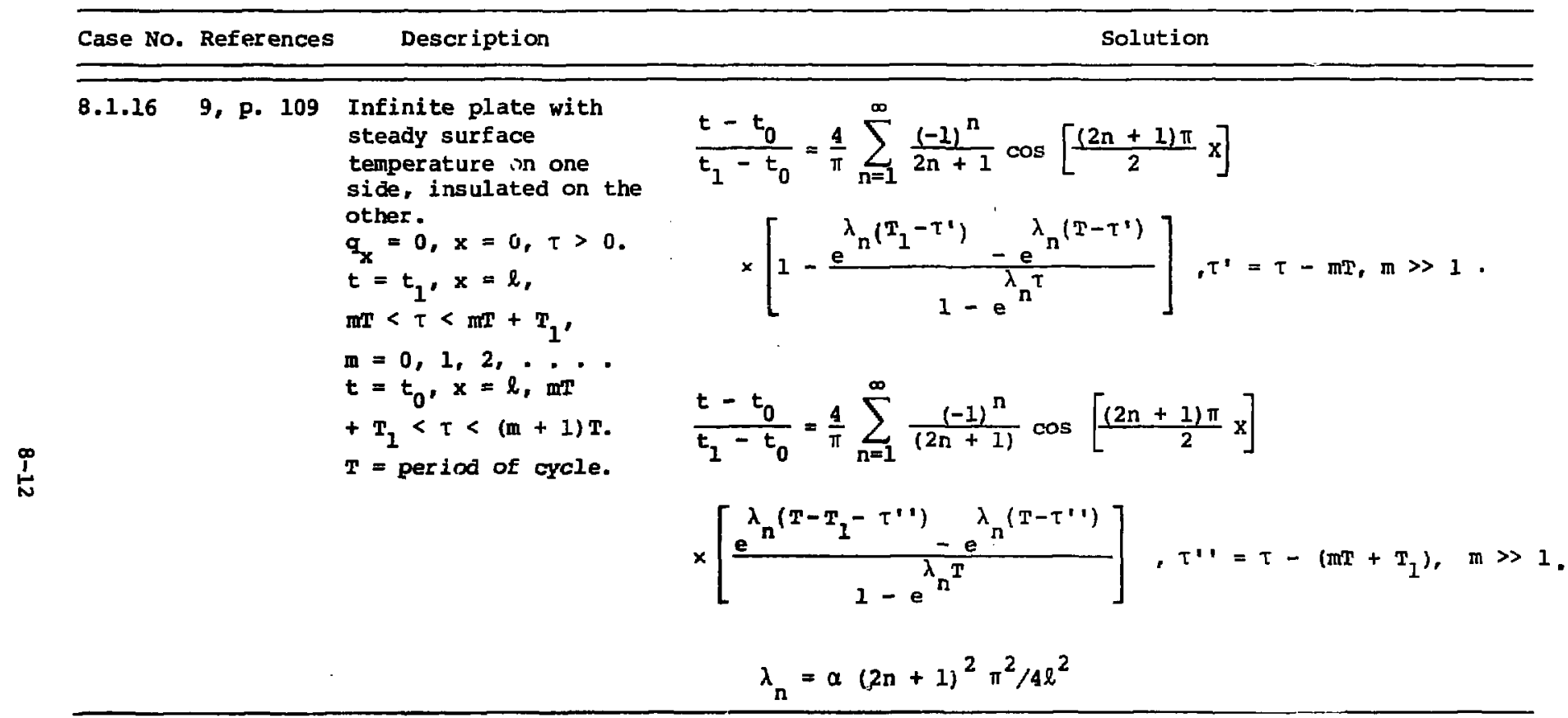


Section 8.1. Solids Bounded by Plane Surfaces--No Internal Heating.

\section{Case No. References}

8.1.17 4, p. 82 Infinite plate with 48

$\stackrel{\substack{1 \\ \omega}}{\omega}$ steady surface heat Elux and convection boundaries.

$t=t_{0}, 0<x<\ell, \tau=0$.

$q_{x}=q_{0}, x=l, \tau>0$.

$t_{f}=t_{0}, \tau>0$.

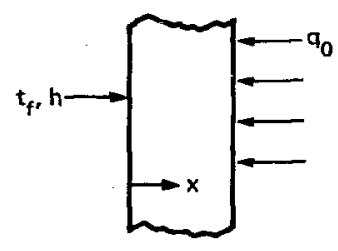

$1 \rightarrow-1 \rightarrow 1$

$$
\begin{aligned}
& \frac{\left(t-t_{0}\right) h}{q_{0}}=\frac{B i}{2} \sqrt{\frac{F O}{\pi}}\left\{\exp \left[-\frac{(x-1)^{2}}{4 F O}\right]+\exp \left[-\frac{(x+1)^{2}}{4 F O}\right]\right\} \\
& -B^{2} \text { Fo }\left[\exp \left(B i_{x}+B i+4 B i^{2} \text { Fo }\right) \operatorname{erfC}\left(\frac{x+1}{4 \sqrt{F O}}+B i \sqrt{F O_{0}}\right)\right] .
\end{aligned}
$$

See Fig. 8.18.

For $\mathrm{Bi} \rightarrow \infty$ :

$$
\frac{\left(t-t_{0}\right) k}{q_{0} l}=\frac{\sqrt{F_{0}}}{2 \sqrt{\pi}}\left\{\exp \left[-\frac{(x-1)^{2}}{4 \text { FO }}\right]+\exp \left[-\frac{(x+1)^{2}}{4 \text { FO }}\right]\right\} \text {. }
$$

See case 8.1.20 for special solutions. 
Section 8.1. Solids Bounded by Plane Surfaces--No Internal Heating.

\begin{tabular}{|c|c|c|c|}
\hline Case No. & References & Description & Solution \\
\hline 8.1 .18 & $\begin{array}{l}14 \\
47 \\
74, \text { p. } 174\end{array}$ & $\begin{array}{l}\text { Infinite plate with } \\
\text { steady surface heat } \\
\text { Elux and variable } \\
\text { thermal conductivity. } \\
t=t_{0},-l<x<+l \text {, } \\
\tau=0 . \quad k=k(t), \quad \alpha \text { is } \\
\text { constant. } \\
q_{x}=q_{0}, x=l,-l \text {. }\end{array}$ & $\begin{array}{l}\frac{q_{0} l}{k_{0} t_{0}}\left[\frac{1}{2}(x-1)^{2}+(x-1)+\frac{1}{3}+F o-\frac{2}{\pi^{2}} \sum_{n=1}^{\infty} \frac{1}{n^{2}} \cos [n \pi(x-1)]\right. \\
\left.\quad x \exp \left(-n^{2} \pi^{2} F_{0}\right)\right]=\frac{1}{k_{0}} \int_{0}^{T} k(T) d T \\
\quad T=\left(t-t_{0}\right) / t_{0}, k_{0}=k\left(t_{0}\right) \\
\text { For } k(t)=k_{0}\left[1+B\left(t-t_{0}\right)\right]: \\
\quad \frac{1}{k_{0}} \int_{0}^{T} k(T) \text { aT }=\frac{t-t_{0}}{t_{0}}+\frac{B t_{0}}{2}\left(\frac{t-t_{0}}{t_{0}}\right)^{2} \\
\text { For } B=0 \text {, see Fig. } 8.7 \text {. } \\
\text { For } \beta=0 \text { and } q_{0} \text { terminated at } T=D \text {, see Fig. 8.1;. }\end{array}$ \\
\hline
\end{tabular}


Section 8.1. Solids Bounded by Plane Surfaces--No Internal Heating.

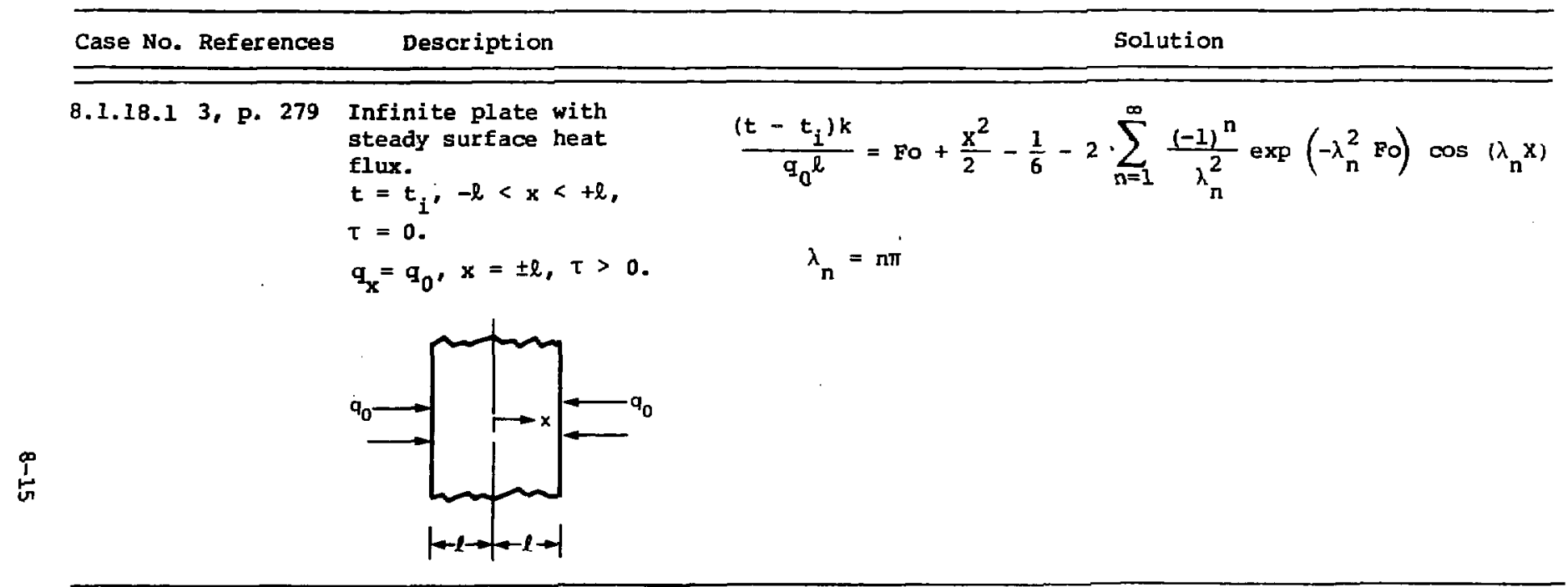


Section 8.1. Solids Bounded by Plane Surfaces--No Internal Beating.

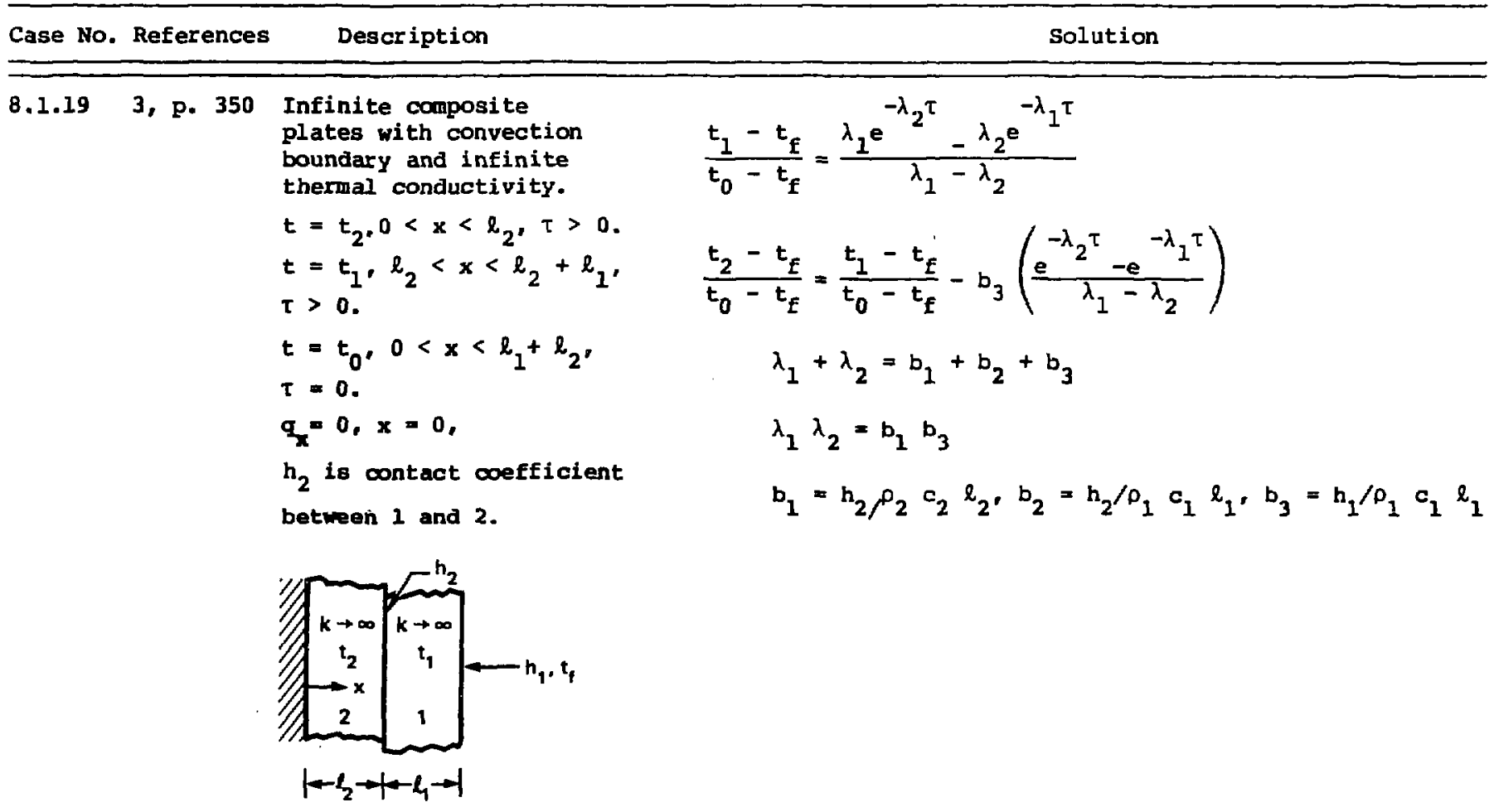


Section 8.1. Solids Boundē by Plane Surfaces--No Internal Heating.

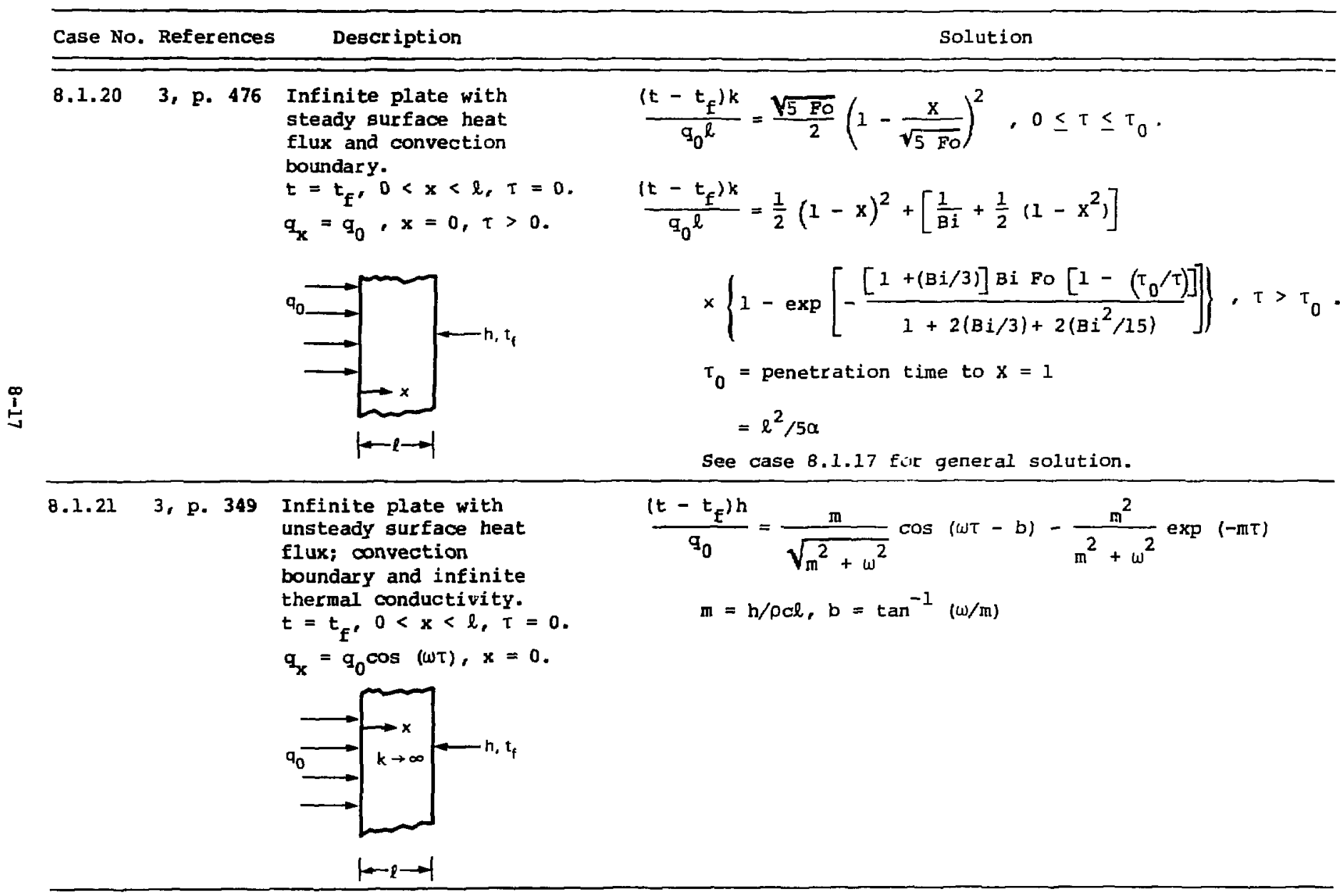


Section 8.1. Solids Bounded by Plane Surfaces--No Internal Heating.

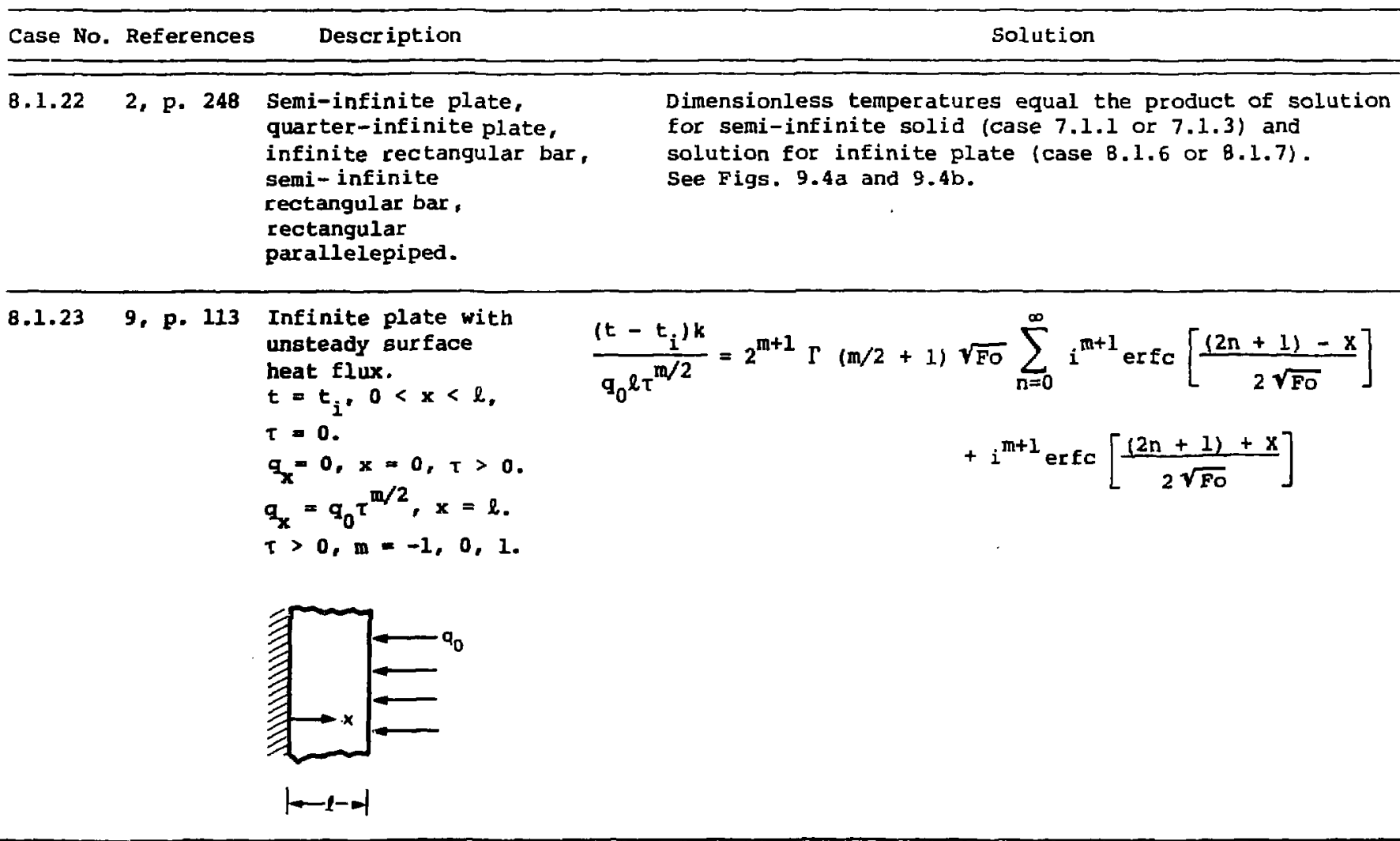


Section 8.1. Solids Bounded by Plane Surfaces--No Internal Heating.

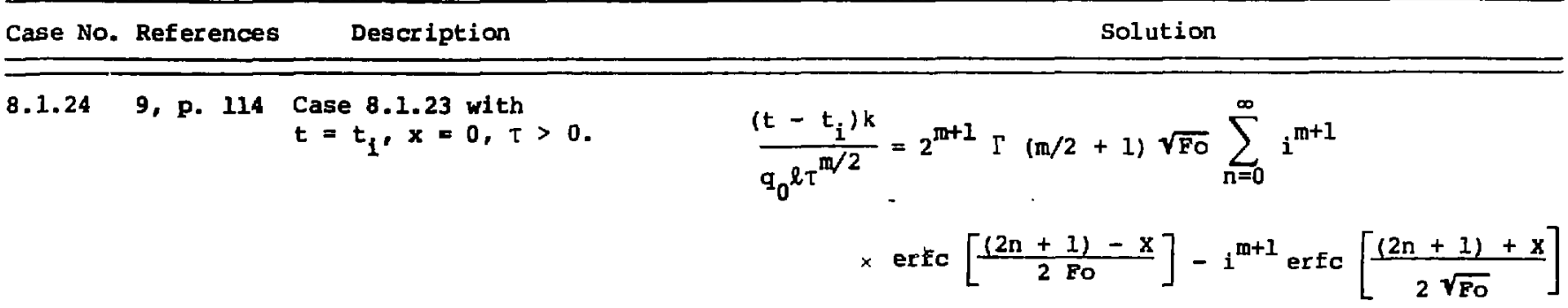

$$
\begin{aligned}
& \text { 8.1.25.1 9, P. } 120 \text { Infinite plate with } \\
& \text { steady surface } \\
& \text { temperature, convex- } \\
& \text { sion boundary and } \\
& \text { variable initial } \\
& \text { temper nature. } \\
& t=f(x), 0<x<\ell, \tau=0 \text {. } \\
& t=t_{0}, x=0, \tau<0 \text {. } \\
& \left(t-t_{0}\right)=2 \sum_{n=1}^{\infty} \exp \left(-\lambda_{n}^{2} \mathrm{Fo}\right) \frac{\left(B i^{2}+\lambda_{n}^{2}\right) \sin \left(\lambda_{n} x\right)}{B i^{2}+\lambda_{n}^{2}+B i}
\end{aligned}
$$

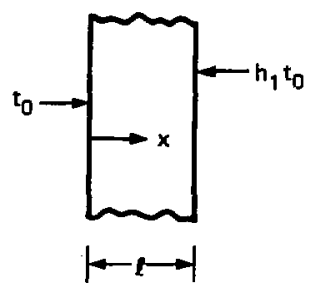

$$
\begin{aligned}
& x \int_{0}^{1}\left[t_{0}-f(x)\right] \sin \left(\lambda_{n} x\right) d x \\
& B i=h l / k, \lambda_{n} \cot \left(\lambda_{n}\right)+B i=0 \\
& \text { See case 8.1.1 for } t=t_{0}, x=\ell, \tau>0
\end{aligned}
$$


Section B.1. Solids Bounded by Plane Surfaces--No Internal Heating.

Case No. References Description

8.1.25.2 74, p. 239 Infinite plate with steady surface temp and convection boundaries.

$t=t_{0}, 0<x<l$, $\tau=0$.

$t=t_{0}, x=0, \tau>0$.

0
1
0
Solution

$$
\frac{t-t_{0}}{t_{E}-t_{0}}=2 \sum_{n=1}^{\infty} \frac{\left(B i^{2}+\lambda_{n}^{2}\right)\left[1-\cos \left(\lambda_{n}\right)\right]}{\left(B i^{2}+B i+\lambda_{n}^{2}\right)} \sin \left(\lambda_{n} x\right) \exp \left(-\lambda_{n}^{2} \text { Fo }\right)
$$

$$
\lambda_{n} \cot \left(\lambda_{n}\right)+B i=0
$$

See Fig. 8.24.

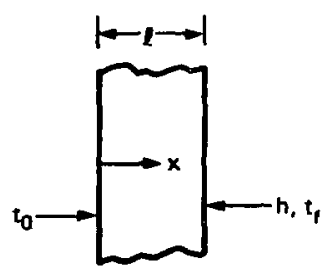


Section 8.1. Solids Bounded by Rlane Surfaces--No Internal Heating.

Case No. References Description Solution

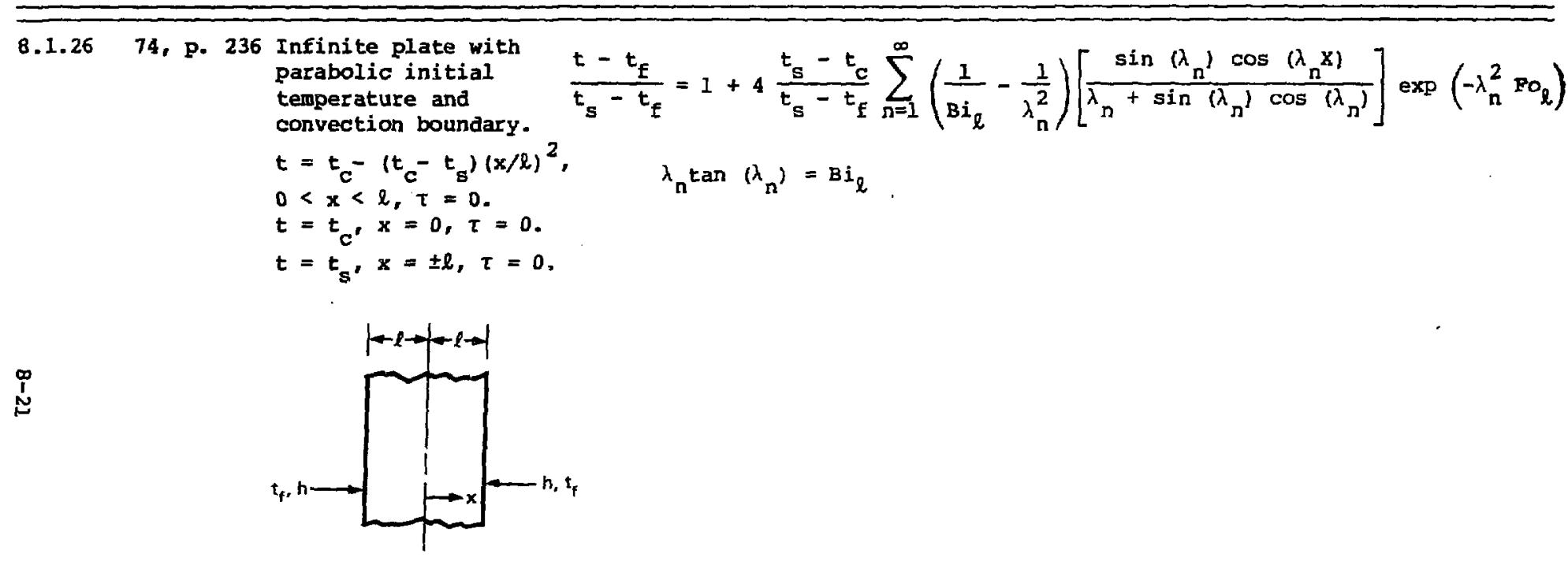


Section 8.1. Solids Bounded by Plane Surfaces-No in. . . . Heating.

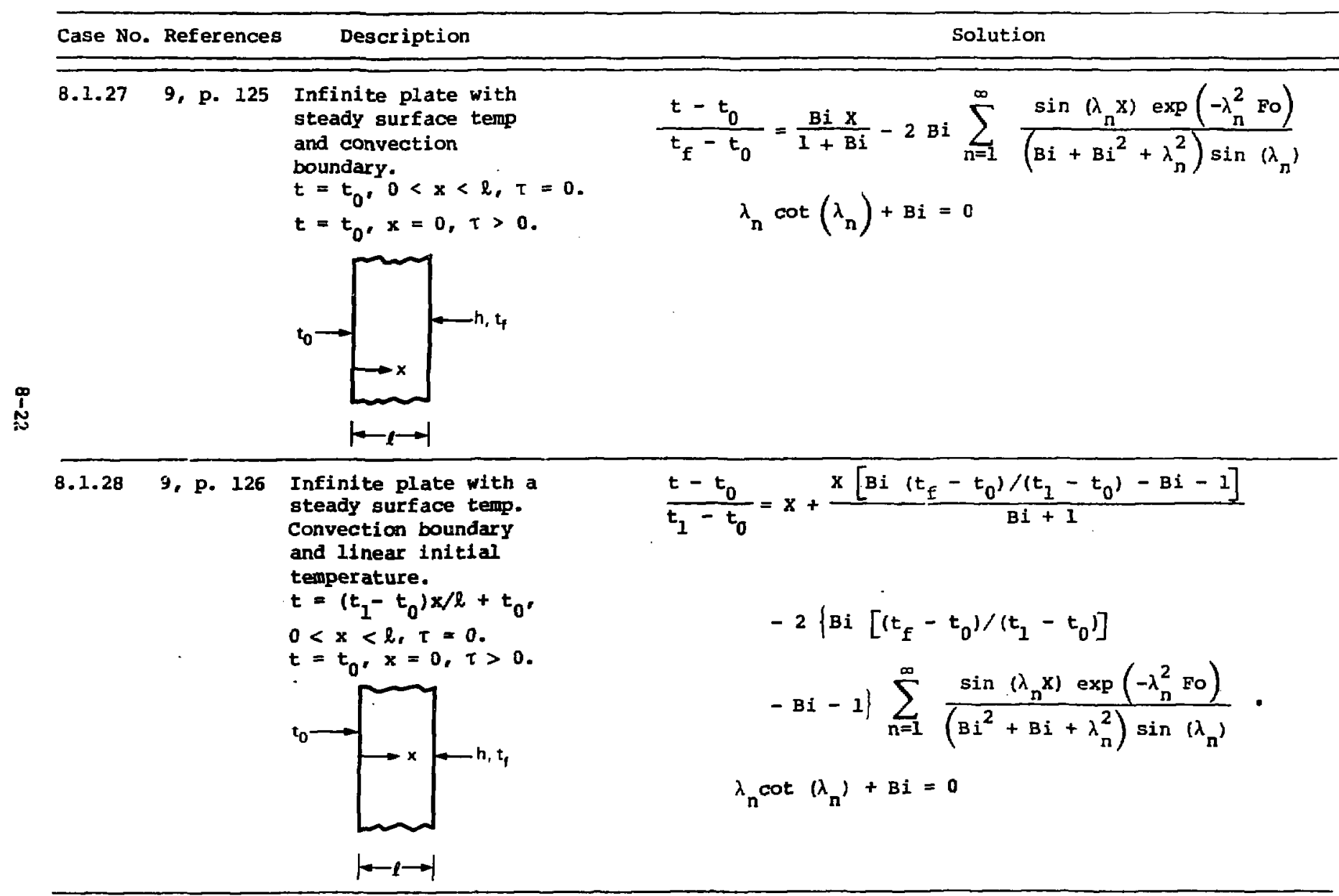


Section 8.1. Solids Bounded by Plane Surfaces--No Internal Heating.

\begin{tabular}{lll}
\hline Case No. References & Description & Solution \\
\hline
\end{tabular}

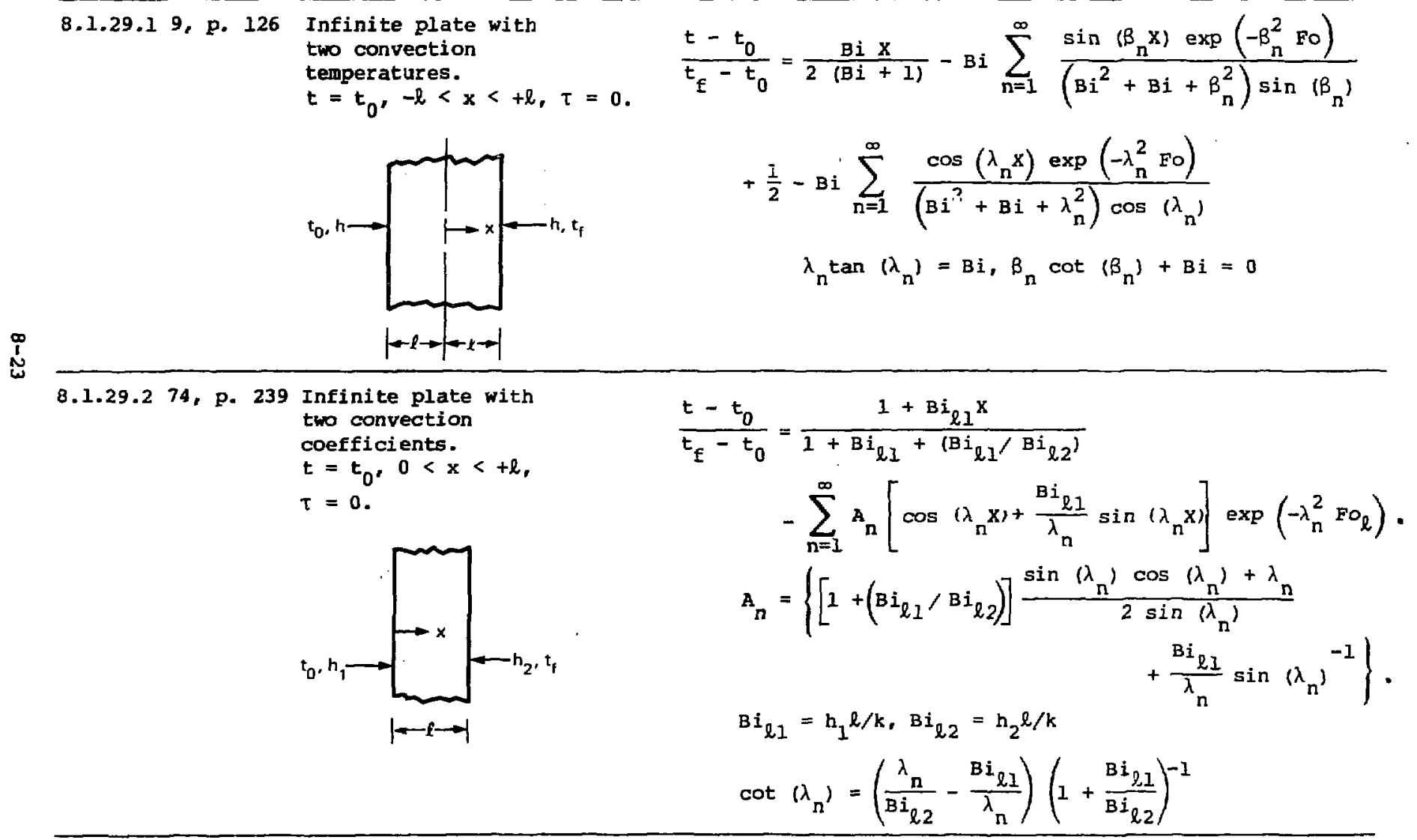


Section 8.1. Solids Bounded by Plane Surfaces--No Internal Heating.

Case No. References

Description

Solution

8.1.30 9, p. 127 Infinite plate with convection boundaries and time dependent

fluid temperature.

$t=t_{i},-l<x<+l, \tau=0$.

$t_{f}=\phi(\tau)$.

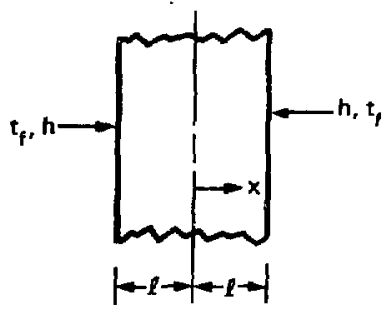

$t-t_{i}=2 B i \frac{\alpha}{\ell^{2}} \sum_{n=1}^{\infty} \frac{\lambda_{n}^{2} \cos \left(\lambda_{n} x\right) \exp \left(-\lambda_{n}^{2} \text { Fo }\right)}{\left(B i+\lambda_{n}^{2}+B i^{2}\right) \cos \left(\lambda_{n}\right)}$

$x \int_{0}^{\tau} \exp \left(\alpha \lambda_{n}^{2} \tau^{\prime} / l^{2}\right) \phi\left(\tau^{\prime}\right) d \tau$

$\stackrel{\infty}{1}$

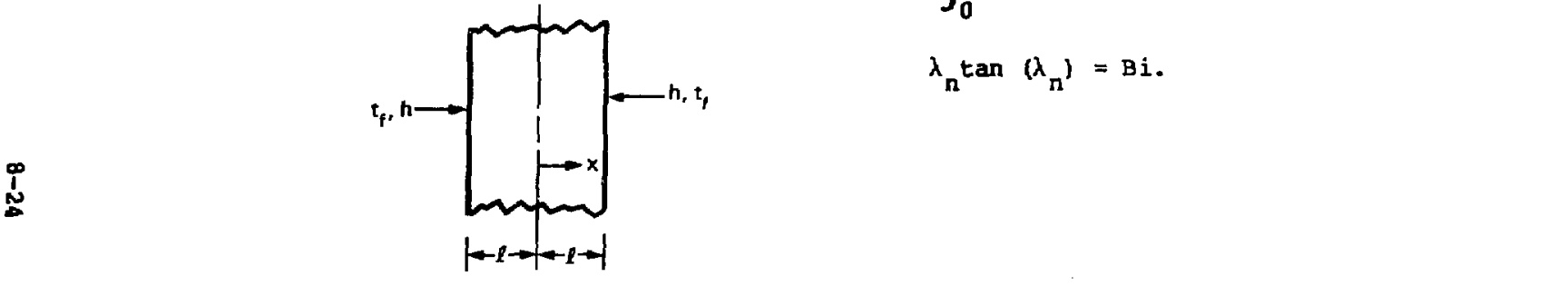


Section 8.1. Solids Bounded by Plane Surfaces--No Internal Heating.

8.1.30.1 74, p. 316 Case 8.1.30 with

$t_{f}=t_{f m}-\left(t_{f m}-t_{i}\right) e^{-b \tau}$

$$
\frac{t-t_{i}}{t_{f m}-c_{i}}=1-\frac{\cos (\sqrt{P d} x) \exp (-P d \quad F o)}{\cos (\sqrt{P d})-\frac{1}{B i} \sqrt{P d} \sin (\sqrt{P d})}
$$

芯

$$
\begin{aligned}
& -\sum_{n=1}^{\infty} \frac{A_{n} \cos \left(\lambda_{n} X\right) \exp \left(-\lambda_{n}^{2} \text { Fo }\right)}{1-\lambda_{n}^{2} / P d} \\
& A_{n}=\frac{(-1)^{n+1} 2 B i\left(B i^{2}+\lambda_{n}^{2}\right)^{1 / 2}}{\lambda_{n}\left(B i^{2}+B i+\lambda_{n}^{2}\right)}
\end{aligned}
$$

Mean Temp:

$$
\begin{aligned}
& \frac{t_{I I}-t_{i}}{t_{f I I}-t_{i}}=1-\frac{\exp (-P d \text { Fo })}{\sqrt{P d}\left[\cot (\sqrt{P d})-\frac{1}{B i} \sqrt{P d}\right]} \\
& -2 B^{2} \sum_{n=1}^{\infty} \frac{\exp \left(-\lambda_{n}^{2} F o\right)}{\lambda_{n}^{2}\left(B i^{2}+B i+\lambda_{n}^{2}\right)\left[1-\left(\lambda_{n}^{2} / P d\right)\right]}
\end{aligned}
$$


Section 8.1. Solids Bounded by Plane Surfaces--No Internal Heating.

8.1.31 9, p. 127 Case 8.1.30 with $t_{E}=\left\{\begin{array}{l}t_{0}, 0<\tau<\tau_{0} \\ t_{1,} \tau>\tau_{0} .\end{array}\right.$

$$
\begin{gathered}
\frac{t-t_{i}}{t_{0}-t_{i}}=1-2 B i \sum_{n=1}^{\infty} \frac{\cos \left(\lambda_{n} x\right) \exp \left(-\lambda_{n}^{2} \text { Fo }\right)}{\left(B i^{2}+\lambda_{n}^{2}+B i\right) \cos (\lambda n)}, 0<\tau<\tau_{0} \\
\frac{t-t_{i}}{t_{1}-t_{1}}=1-2 \operatorname{Bi} \sum_{n=1}^{\infty} \frac{\left[T+(1-T) \exp \left(\lambda_{n}^{2} F O_{0}\right)\right]}{\left(B i^{2}+\lambda_{n}^{2}+B i\right) \cos \left(\lambda_{n}\right)} \\
\times \cos \left(\lambda_{n} X\right) \exp \left(-\lambda_{n}^{2} F O\right)
\end{gathered}
$$

$$
T=\left(t_{0}-t_{i}\right) /\left(t_{I}-t_{i}\right), F O_{0}=\alpha \tau_{0} / l^{2}, \lambda_{n} \tan \left(\lambda_{n}\right)=B i
$$


Section 8.1. Solids Bounded by Plane Surfaces--No Internal Heating.

\section{Ref-}

Case No. erences

Description

Solution

8.1.32 9, Case 8.1.30 with

p. $127 \quad t_{f}=\left(t_{0}-t_{i}\right)$ sin

74,

$(\omega \tau+\varepsilon)+t_{i}$

p. $325 \tau>0$.

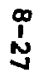

$\frac{t-t_{i}}{t_{0}-t_{i}}=\frac{B i M_{0}}{M_{1}} \sin \left(\omega \tau+E+B_{0}-B_{1}\right)$

$+2 B i \sum_{n=1}^{\infty} \frac{\left[\frac{2 \mathrm{FO}^{\star 2}}{\lambda_{n}^{2}} \cos (\varepsilon)-\sin (E)\right] \cos \left(\lambda_{n} \mathrm{X}\right) \exp \left(-\lambda_{n}^{2} \text { Fo }\right)}{\left(1+4 \mathrm{FO}^{\star 4} / \lambda_{n}^{4}\right)\left(\lambda_{n}^{2}+B i^{2}+B i\right) \cos \left(\lambda_{n}\right)}$

$M_{0} e^{i \beta_{0}}=\cosh \left(X \mathrm{FO}^{\star}\right) \cos \left(\mathrm{X} \mathrm{FO}{ }^{\star}\right)+i \sinh \left(\mathrm{X} \mathrm{FO}{ }^{*}\right)\left(\mathrm{X} \mathrm{FO}{ }^{*}\right) \sin \left(\mathrm{X} \mathrm{FO}{ }^{*}\right)$

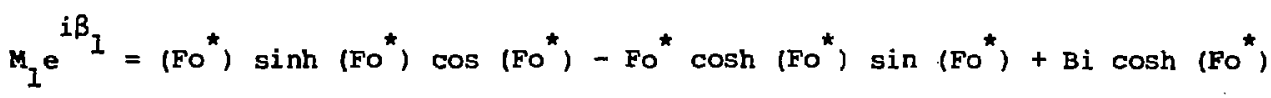

$\times \cos \left(\mathrm{FO}^{*}\right)+i\left[\mathrm{FO}^{\star} \sinh \left(\mathrm{FO}^{*}\right) \cos \left(\mathrm{FO}^{\star}\right)+\mathrm{FO}^{*} \cosh \left(\mathrm{FO}^{\star}\right) \sin \left(\mathrm{FO}^{*}\right)\right.$

$+B i \sinh (L) \sin (L)]$

$F 0^{\star}=\ell \sqrt{\omega / 2 \alpha}, \lambda_{n} \tan \left(\lambda_{n}\right)=B i$ 
Section 8.1. Solids Bounded by Plane Surfaces--No Internal Heating.

\begin{tabular}{|c|c|c|c|}
\hline Case No. & References & Description & Solution \\
\hline 8.1 .33 & $\begin{array}{l}9, \text { p. } 127 \\
89\end{array}$ & $\begin{array}{l}\text { Case } 8.1 .30 \text { with } \\
t_{f}=t_{i}+b \tau, \tau>0\end{array}$ & $\begin{array}{l}\frac{\left(t-t_{i}\right) \alpha}{b l^{2}}=F O+\frac{B i x^{2}-B i-2}{2 B i} \\
\quad+2 B i \sum_{n=1}^{\infty} \frac{\cos \left(\lambda_{n} x\right) \exp \left(-\lambda_{n}^{2} F O\right)}{\lambda_{n}^{2}\left(B i^{2}+\lambda_{n}^{2}+B i\right) \cos \left(\lambda_{n}\right)} \\
\lambda_{n} \tan \left(\lambda_{n}\right)=B i, \text { See Fig. 8.26. } \\
\text { (See Fig. B.1.25 for Bi }=\infty .)\end{array}$ \\
\hline 8.1 .34 & 9, p. 128 & $\begin{array}{l}\text { Infinite plate in } \\
\text { perfect contact with } \\
\text { an infinite plate of } \\
\text { infinite conductivity. } \\
t=t_{i}, 0<x<l_{1}+\ell_{2} \text {, } \\
\tau=0 . \\
q_{x}=0, x=0, \tau>0 . \\
-q_{x}=q_{0}, x=l_{1}+l_{2} \text {, }\end{array}$ & $\begin{array}{l}\frac{\left(t-t_{i}\right) k}{q_{0} l_{1}}=\frac{M}{1+M}\left[F O_{1}+\frac{x^{2}}{2}-\frac{3+M}{6(1+M)}\right] \\
-2 M^{2} \sum_{n=1}^{\infty} \frac{\cos \left(\lambda_{n} x\right) \exp \left(-\lambda_{n}^{2} F O_{1}\right)}{\lambda_{n}^{2}\left(\lambda_{n}^{2}+M^{2}+M\right) \cos \left(\lambda_{n}\right)} \\
\lambda_{n} \cot \left(\lambda_{n^{\prime}}\right)=-M \\
M=\frac{\rho_{1} C_{1} l_{1}}{\rho_{2} C_{2} l_{2}}, F O_{1}=\alpha_{1} \tau / l_{1}^{2}, x=x / l_{1}\end{array}$ \\
\hline
\end{tabular}


Section 8.1. Solids Bounded by Plane Surfaces--No Internal Heating.

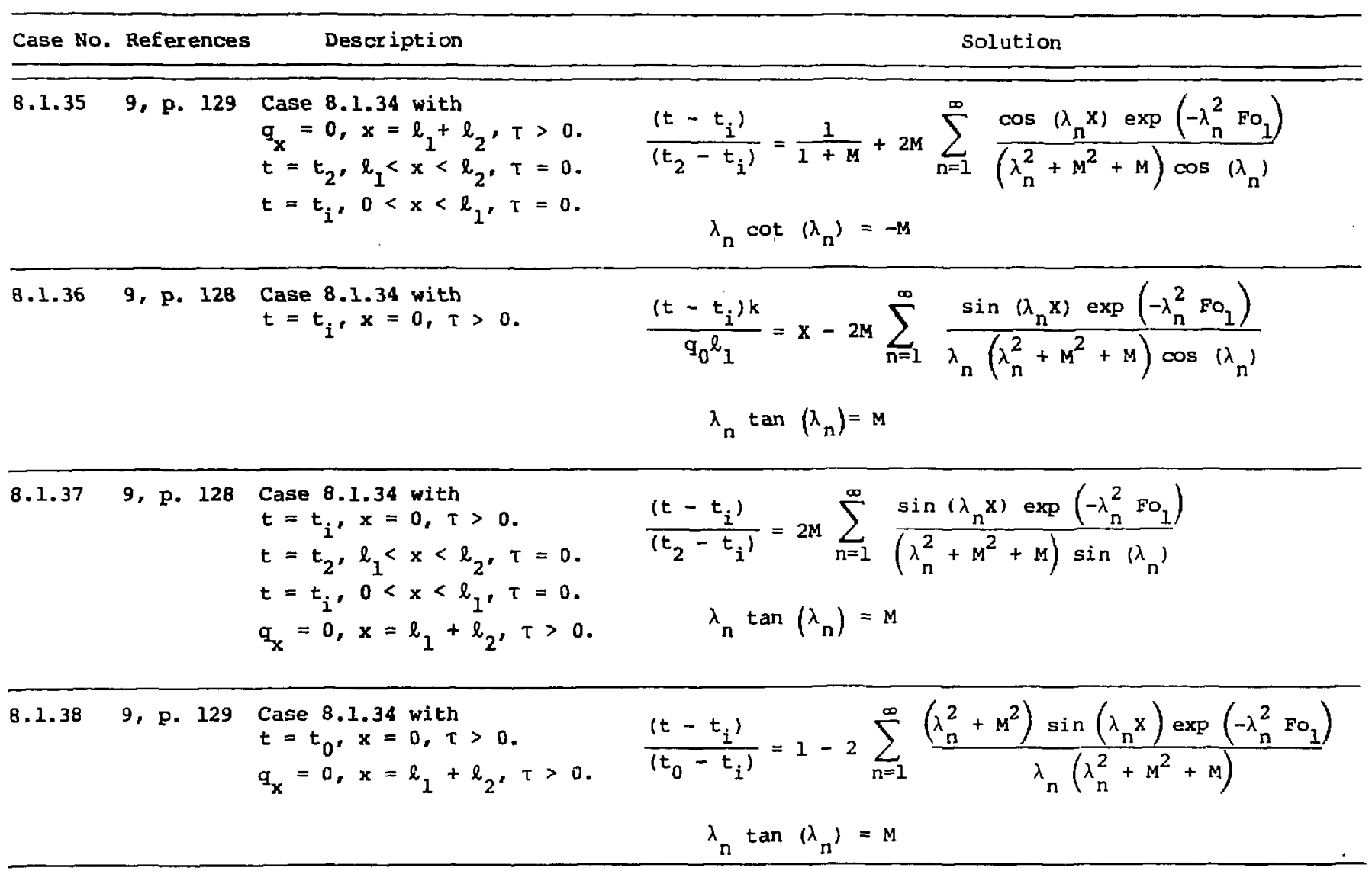


Section 8.1. Solids Bounded by Plane Surfaces-No Internal Heating.

\begin{tabular}{|c|c|c|c|}
\hline Case No. & References & Description & Solution \\
\hline 8.1 .39 & 9. p. 129 & $\begin{array}{l}\text { Case 8.1.34 with } \\
q_{x}=0, x=\ell_{1}+\ell_{2}, \tau>0 . \\
q_{x}=q_{0}, x=0, \tau>0 .\end{array}$ & $\begin{array}{l}\frac{\left(t-t_{i}\right) k(1+M)}{q_{0} l_{1}}=M \text { Fo }+1-X+\frac{M}{2}(1-X)^{2}-\frac{M(3+M)}{6(1+M)} \\
-2(1+M) \sum_{n=1}^{\infty} \frac{\left(M^{2}+\lambda_{n}^{2}\right) \cos \left(\lambda_{n} x\right) \exp \left(-\lambda_{n}^{2} F O_{1}\right)}{\lambda_{n}^{2}\left(\lambda_{n}^{2}+M^{2}+M\right)} . \\
\lambda_{n} \cot \left(\lambda_{n}\right)+M=0\end{array}$ \\
\hline 8.1 .40 & 9. p. 129 & $\begin{array}{l}\text { Case } 8.1 .34 \text { with } \\
\text { convection boundary } \\
h_{1} t_{f} \text { at } x=l_{1}+l_{2} \text {. } \\
t=t_{2}, l_{1}<x<l_{2} \\
\tau=0 \text {. }\end{array}$ & $\begin{array}{l}\frac{\left(t-t_{2}\right)}{\left(t_{i}-t_{2}\right)}=2 \sum_{n=1}^{\infty} \frac{\left(B i_{1}-\lambda_{n}^{2} / M\right) \cos \left(\lambda_{n} x\right) \exp \left(-\lambda_{n}^{2} F o_{1}\right)}{\left[\left(B I_{1}-\lambda_{n}^{2} / M\right)^{2}+\lambda_{n}^{2}+\lambda_{n}^{2} / M+B i_{1}\right] \cos \left(\lambda_{n}\right)} \\
\lambda_{n} \tan \left(\lambda_{n}\right)=B i_{1}-\lambda_{n}^{2} / M, B i=h l_{1} / k_{1}\end{array}$ \\
\hline
\end{tabular}

(See Ref. 82 for solution of material 1 of low conductivity and material 2 of high conductivity.) 
Section 8.1. Solids Bounded by Plane Surfaces--No Internal Heating.

\begin{tabular}{|c|c|c|c|}
\hline Case No. & References & Description & Solution \\
\hline 8.1 .41 & 9, P. 129 & $\begin{array}{l}\text { Case } 8.1 .40 \text { with } \\
-q_{x}=f(\tau), x=0, \\
\tau>0 .\end{array}$ & $\begin{aligned} &\left(t-t_{i}\right)=\frac{2 \alpha}{k l^{3}} \sum_{n=1}^{\infty} \frac{\lambda_{n}^{2} \cos \left(\lambda_{n} X\right) \exp \left(-\lambda_{n}^{2} F O_{1}\right)}{\left[\left(B i_{1}-\lambda_{n}^{2} / M\right)^{2}+(1+1 / M)^{\prime} \lambda_{n}^{2}+B i\right] \cos \left(\lambda_{n}\right)} \\
& \times \int_{0}^{T} f\left(\tau^{\prime}\right) \exp \left(\lambda_{n}^{2} \alpha \tau^{\prime} / l^{2}\right) d{ }^{\prime} . \\
& \lambda_{n} \tan \left(\lambda_{n}\right)=B i_{1}-\lambda_{n}^{2} / M .\end{aligned}$ \\
\hline 8.1 .42 & 9, p. 129 & $\begin{array}{l}\text { Case } 8.1 .34 \text { with a } \\
\text { contact resistance } h_{c} \\
\text { between the two } \\
\text { plates. }\end{array}$ & $\begin{array}{l}\frac{\left(t-t_{i}\right)(M+1) k}{G_{0} l_{1}^{M}}=F O_{1}+\frac{1}{2}(1-x)^{2}-\frac{3 B i_{c}+6+M B i_{c}}{6 B i_{c}(1+M)} \\
+2 B i_{c}(1+M) \sum_{n=1}^{\infty} \frac{\lambda_{n}^{2}-M B i_{c}}{P_{n} \cos \left(\lambda_{n}\right)} \cos \left(\lambda_{n}-\lambda_{n} x\right) \exp \left(-\lambda_{n}^{2} F o\right) \\
\left(\lambda_{n}^{2}-M B i_{c}\right) \tan \left(\lambda_{n}\right)=B i_{c} \lambda_{n} . \\
P_{n}=\lambda_{n}^{6}+\lambda_{n}^{4}\left(B i_{c}^{2}+B i_{c}-2 M B i_{c}\right)+M B i_{c}^{2}(1+M) \lambda_{n}^{2} . \\
B i_{c}=h_{c} l_{1} / k_{1} .\end{array}$ \\
\hline
\end{tabular}


Section 8.1. Solids Bounded by Plane Surfaces--No Internal Heating.

\begin{tabular}{|c|c|c|}
\hline Case No. References & Description & Solution \\
\hline 8.1.43.19, p. 324 & $\begin{array}{l}\text { Composite plates of } \\
\text { different materials. } \\
t=t_{0},-l_{1}<x<l_{2}, \\
\tau=0 . \\
t=t_{1}, \\
x=-l_{1}, \tau<0 . \\
t=t_{0}, x=l_{2}, \\
\tau>0 .\end{array}$ & 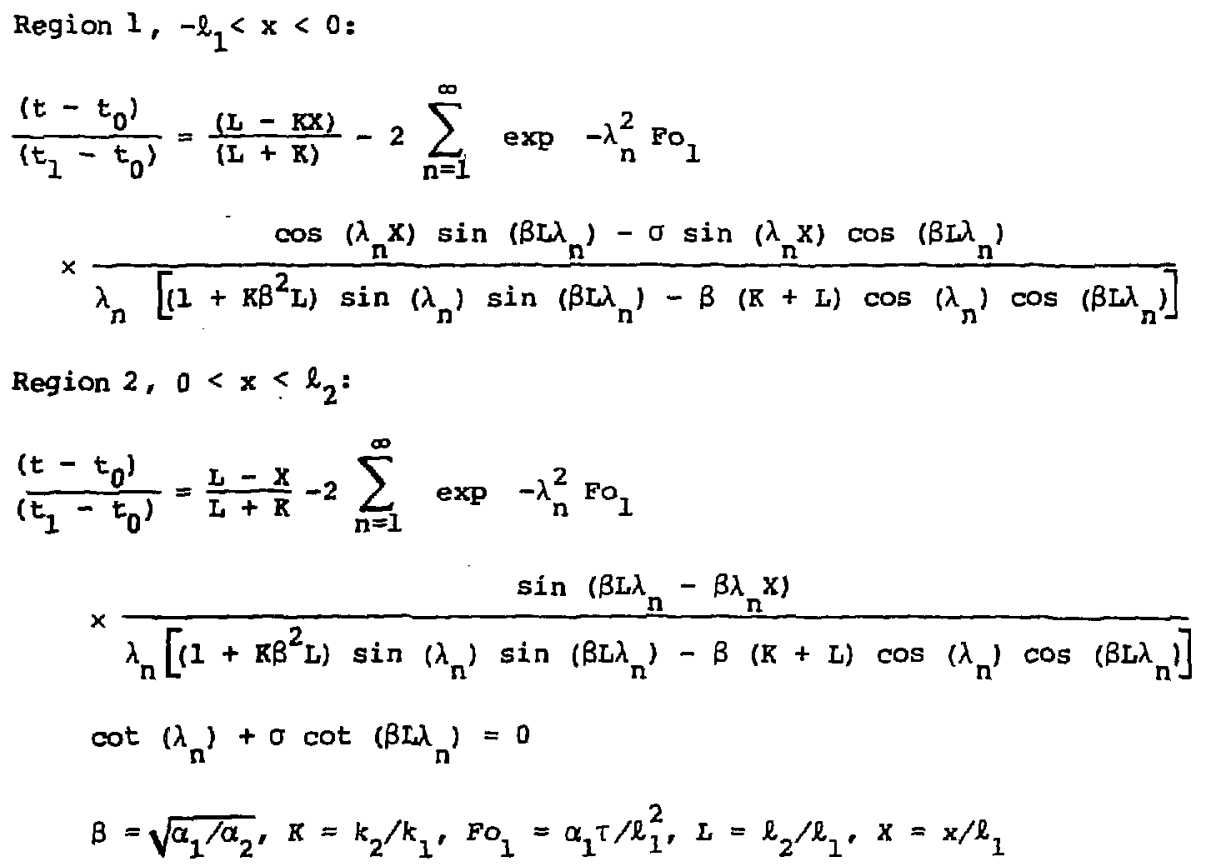 \\
\hline
\end{tabular}


Section 8.1. Solids Bounded by Plane Surfaces-No Internal Heating.

Case No. References Description Solution

$$
\frac{t-t_{i}}{t_{E}-t_{i}}=1-2 \sum_{n=1}^{\infty} \frac{1}{\lambda_{n} \psi} \cos \left(\lambda_{n} x / \sqrt{A}\right) \exp \left(-\lambda_{n}^{2} L^{2} \mathrm{Fo}_{1} / A\right), 0<x<L .
$$
with insulated surface and convectian boundary.

$t=t_{1}, 0<x<d_{1}+$ $l_{2}, \tau=0$.

$q_{x}=0$,

$x=0, \tau>0$.

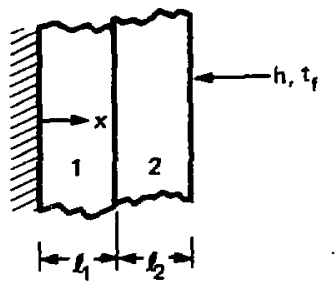

$\frac{t-t_{i}}{t_{f}-t_{i}}=1-2 \sum_{n=1}^{\infty} \frac{1}{\lambda_{n} \psi}\left\{\cos \left[\lambda_{n}(x-L)\right] \cos \left(\lambda_{n} L / \sqrt{A}\right)\right.$

$\left.-R \sin \left[\lambda_{n}(X-L)\right] \sin \left(\lambda_{n} L / \sqrt{A}\right)\right\} \exp \left(-\lambda_{n}^{2} L^{2} \mathrm{FO}_{1} / A\right), L<x<1$.

$\psi=\left[\left(1+k L / \sqrt{A}+\frac{1+L}{B i}\right) \sin \left(\lambda_{n}\right)+\lambda_{n}\left(\frac{1+L}{B i}\right)\right.$

$\left.x(1+K L / \sqrt{A}) \cos \left(\lambda_{m}\right)\right] \cos \left(\lambda_{n} L / \sqrt{A}\right)+\left[\left(1+k L / \sqrt{\AA}+\frac{1+L}{B i}\right) \cos \left(\lambda_{n}\right)\right.$ $\left.-\lambda_{n}\left(\frac{1+L}{B i}\right)\left(1+\frac{L}{K V / A}\right) \sin \left(\lambda_{n}\right)\right] K \sin \left(\lambda_{n} L / \sqrt{A}\right)$.

$\frac{L \lambda_{n}}{B i}(1+L) \tan \left(\lambda_{n} L / \sqrt{A}\right)=1-\frac{\lambda_{n}}{B i}(1+L) \tan \left(\lambda_{n}\right)$

$-K \tan \left(\lambda_{n}\right) \tan \left(\lambda_{n} L / \sqrt{A}\right)$. $B i=h l_{1} / K_{2}, L=l_{1} / l_{2}, P O_{1}=\alpha_{1} \tau / l_{1}^{2}, A=\alpha_{1} / \alpha_{2}, \quad k=\frac{\rho_{1} C_{1} k_{1}}{\rho_{2} C_{2} k_{2}}$ 
Section 8.1. Solids Bounded by Plane Surfaces--No Internal Heating.

Case No. References

Description

Solution

8.1.44.1 9, p. 416 Rectangular

parallelpiped.

$t=t_{i}, 0<x<w$,

$0<y<b, 0<z<a$,

$\tau=0$.

$t=t_{0}, x=0$,

$0<y<b, 0<z<a$,

$\tau>0$.

$t=t_{i}, a n$

$\underset{1}{\infty}$

other faces, $\tau>0$.

$\lambda_{m, n, l}=\ell^{2} \pi^{2}+\left(\bar{m} \pi w / b^{2}\right)+(\bar{n} \pi w / d)^{2}$ $\frac{t-t_{i}}{t_{0}-t_{i}}=\frac{16}{\pi^{2}} \sum_{m=0}^{\infty} \sum_{n=0}^{\infty} \frac{\sin (\bar{m} \pi x) \sin (\vec{n} \pi z) \sinh \left[(I-x) \sqrt{\lambda_{m, n, 0}}\right]}{\bar{m} \vec{n} \sinh \left(\lambda_{m, n, 0}\right)}$

$-\frac{32}{\pi} \sum_{\pi=0}^{\infty} \sum_{n=0}^{\infty} \sum_{\ell=0}^{\infty} \frac{\ell \sin (\bar{m} \pi Y) \sin (\bar{n} \pi \mathrm{Z}) \sin (\ell \pi X) \exp \left(-\lambda_{m, n, l} \text { Fo }\right)}{\bar{m} \bar{n} \lambda_{m, n, l}}$

$\bar{m}=2 m+1, \bar{n}=2 n+1, X=x / w, Y=y / b, z=z / d$

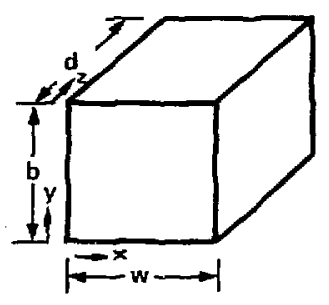


Section 8.1. Solids Bounded by Plane Surfaces--No Internal Heating.

\begin{tabular}{|c|c|c|c|}
\hline Case No. & References & Description & Solution \\
\hline 8.1 .44 .2 & 74, P. 287 & $\begin{array}{l}\text { Rectangular parallele- } \\
\text { piped with convection } \\
\text { boundary. } \\
t=t_{i^{\prime}}-\ell_{1}<x<+l_{1} \\
-\ell_{2}<y<+\ell_{2} \\
-\ell_{3}<z<+\ell_{3^{\prime}} t=0 .\end{array}$ & $\begin{array}{c}\frac{t-t_{0}}{t_{f}-t_{0}}=1-\sum_{n=1}^{\infty} \sum_{m=1}^{\infty} \sum_{s=1}^{\infty} A_{n 1} A_{m 2} A_{s 3} \cos \left(\lambda_{n 1} x\right) \cos \left(\lambda_{m 2} Y\right) \\
\times \cos \left(\lambda_{s 3} z\right) \exp \left(\lambda_{n 1}^{2} F o_{1}+\lambda_{m 2}^{2} F o_{2}+\lambda_{s 3}^{2} F o_{3}\right) \\
A_{n, m, s}=\frac{(-1)^{(n, m, s)+1} 2 B i_{i}\left(B i_{i}^{2}+\lambda_{n, m, s, i}^{2}\right)^{1 / 2}}{2}\end{array}$ \\
\hline
\end{tabular}


Section 8.1. Solids Bounded by Plane Surfaces--No Internal Beating.

Case No. References Description Solution

8.1.45 9, p. 417 Case 8.1 .44 .1 with
$t=t_{0} \sin (u \pi+E)$,
$x=0,0<y<b$,
$0<z<d, \tau>0$.

$\frac{t-t_{i}}{t_{0}-t_{i}}=\frac{16}{\pi^{2}} \sum_{m=0}^{\infty} \sum_{n=0}^{\infty} \frac{\sin (\bar{m} \pi) \sin (\bar{n} \pi z) M_{m, n} \sin \left(\omega \pi+\varepsilon+\phi_{m_{\ell} n}\right)}{\bar{m} \bar{n}}$

㭊

$-\frac{32}{\pi} \sum_{m=0}^{\infty} \sum_{n=0}^{\infty} \sum_{\ell=0}^{\infty} \frac{\ell \sin (\bar{m} \pi Y) \sin (\bar{n} \pi) \sin (\ell \pi X)\left(w \cos \varepsilon-\lambda_{m, n} n \sin \varepsilon\right) \exp \left(-\lambda \omega \pi / w^{2}\right)}{\left(w^{2}+\lambda_{m, n, l}^{2}\right) \bar{m} \bar{n}}$

$M_{m, n} \exp \left(i \phi_{m, n}\right)=\frac{\sinh \left\{(i-x)\left[(\bar{m} \pi w / b)^{2}+(\bar{n} \pi w / d)^{2}+i w\right]^{1 / 2}\right\}}{\sinh \left\{\left[\left([\pi m w / b)^{2}+(\bar{n} \pi w / d)^{2}+i w\right]^{1 / 2}\right\}\right.}$

$w=w^{2} \omega / \alpha$, See case 8.1.44.1 for $\lambda_{m, n, l}, x, y, z, \bar{m}, \bar{n}$ 
Section 8.1. Solids Bounded by Plane Surfaces--No Internal Heating.

Case No. References

8.1.46 9, p. 419 Wedge with steady surface temperature. $t=t_{i}, 0<\theta<\theta_{0}$, $\frac{t-t_{i}}{t_{0}-t_{i}}=\frac{\theta}{\theta_{0}}+\frac{2}{\theta_{0}} \sum_{n=1}^{\infty}(-1)^{n}\left[\begin{array}{ll}\sin (2 n+I) \pi \theta / \theta_{0}\end{array}\right]$

$\tau=0$.

$\times \int_{0}^{\infty} \frac{\exp \left(-\alpha u^{2} / \Sigma^{2}\right)}{u} J_{s}(u) d u$

$t=t_{0}, \theta=0, \tau>0$.

$t=t_{i}, \theta=\theta_{0}, \tau>0$.

$s=(2 n+1) \pi / \theta_{0}$

岕

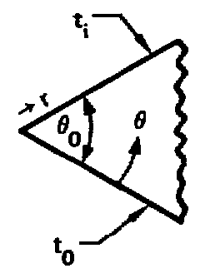

$$
\begin{aligned}
& \text { For } t=t_{0}, \theta=0, \theta_{0}, \tau>0: \\
& \frac{t-t_{i}}{t_{i}-t_{i}}=1-\frac{4}{\theta_{0}} \cdot \sum_{n=0}^{\infty} \sin (s \theta) \int_{0}^{\infty} \exp \left(-\infty \pi u^{2} / r^{2}\right) \frac{J_{s}(u) d u}{u}
\end{aligned}
$$


Section 8.1. Solids Bounded by Plane Surfaces--No Internal Beating.

\section{Case No. References}

Description

Solution

8.1.47 33 Composite slabs with ramp surface

temperature.

$t=t_{i}, 0 x<\ell_{1}+l_{2}$,

$\frac{t-t_{0}}{t_{i}-t_{0}}=2 \sum_{n=1}^{\infty} \frac{\operatorname{Pa}\left(\lambda_{n}^{2}+M^{2}\right) \exp \left(-\lambda_{n}^{2} F_{1}\right)\left[I-\exp \left(\lambda_{n}^{2} / P d\right)\right] \sin \left(\lambda_{n} X\right)}{\lambda_{n}^{3}\left(\lambda_{n}^{2}+M^{2}+M\right)}$

$\tau=0$.

$t=t_{i}+\left\langle t_{0}-t_{i}\right\rangle b \tau$,

$\lambda_{n} \tan \left(\lambda_{n}\right)=M, M=l_{1} \rho_{1} c_{1} / l_{2} \rho_{2} c_{2}, X=x / l_{1}$

$\mathbf{x}=0,0<\tau<1 / \mathrm{b}$.

See cases 8.1 .34 to 8.1 .42 for other conditions.

$\underset{\infty}{\stackrel{1}{W}}$

$t=t_{0}, x=0, \tau>1 / b$.

$q_{x}=0, x=l_{1}+l_{2}$.

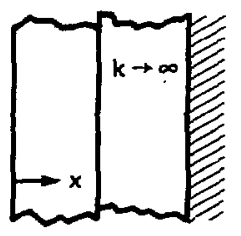

$\operatorname{lil}_{l_{1} \rightarrow+l_{2} \rightarrow 1}$ 
Section 11.1. Change of Phase--Piane Interface.

\begin{tabular}{|c|c|c|c|}
\hline Case No. & References & Description & Solution \\
\hline 11.1 .9 & 9, p. 292 & $\begin{array}{l}\text { Convection boundary at } \\
\text { original solid-liquid } \\
\text { boundary. } \\
t=t_{1}, x>0, t=0, t_{1}>t_{m} \\
t_{m}=m e l t i n g \text { temp. }\end{array}$ & $\begin{aligned} & \frac{t_{s}-t_{m}}{t_{m}-t_{F}}=B i_{x}-\frac{F B i_{x}^{2}}{2}\left(1+2 ! F O_{x}\right)-\frac{F B i_{x}^{3}}{3 !}\left(1+31 F O_{x}\right)+\ldots, 0<x<w \\
& w=F B i_{x} F O_{x}-\frac{F^{2} B i_{x}^{3}}{2}(1+F) F O_{x}^{2}+\ldots \\
& F=k_{s}\left(t_{m}-t_{f}\right) / \alpha_{s} P r, F O_{x}=\alpha_{s} \tau / x^{2}, B i_{x}=h x / k_{s}\end{aligned}$ \\
\hline
\end{tabular}


Section B.1. Solids Bounded by Plane Surfaces--No Internal Heating.

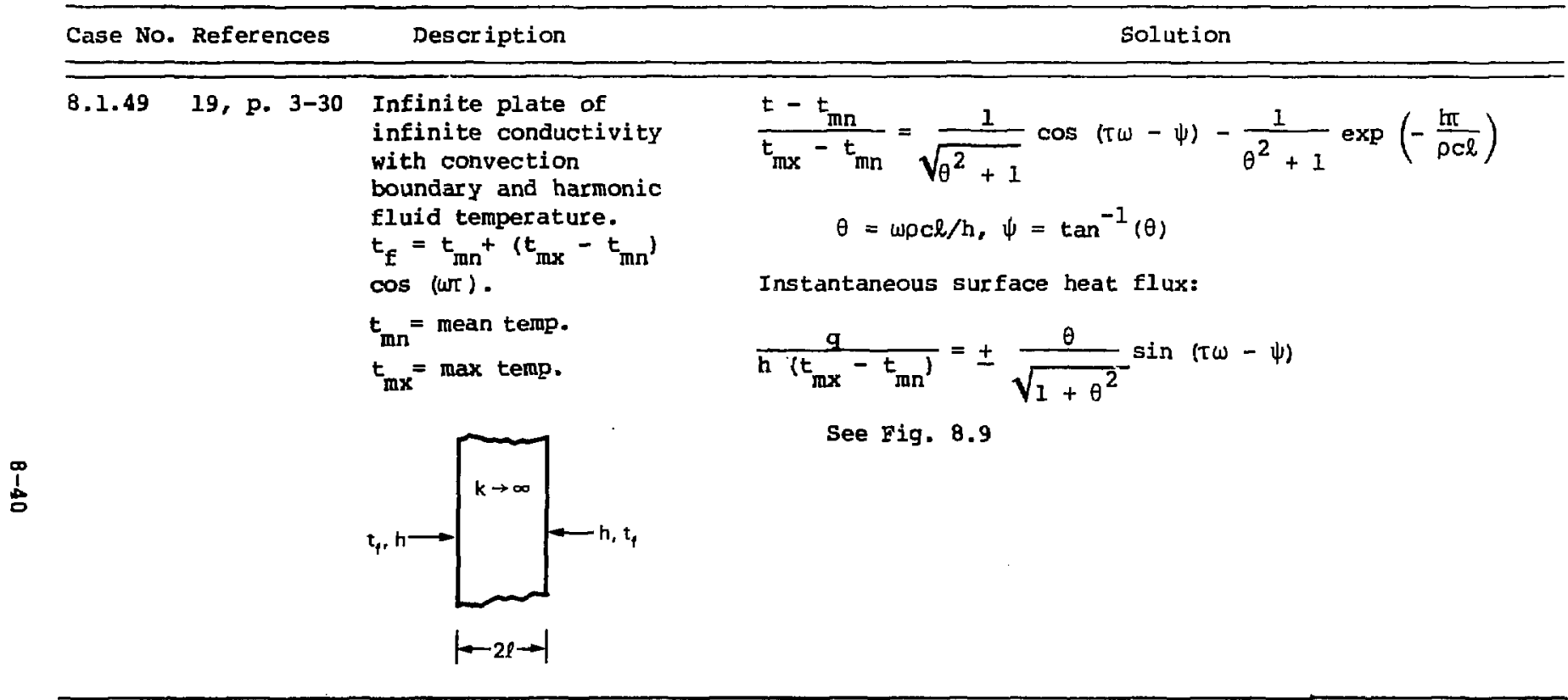


Section 8.1. Solids Bounded by Plane Surfaces--No Internal Heating.

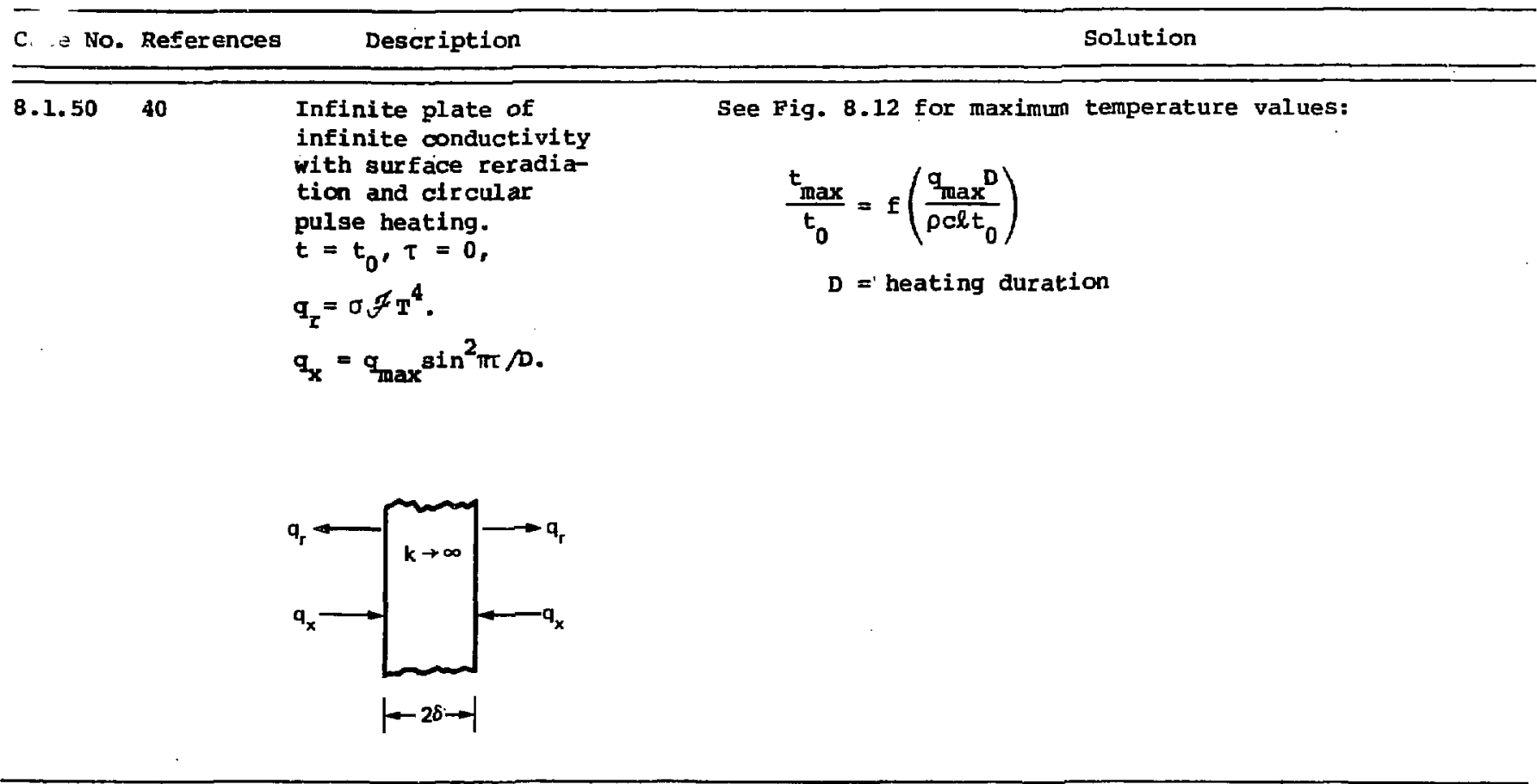


Section 8.1. Solids Bounded by Plane Surfaces--No Internal Heating.

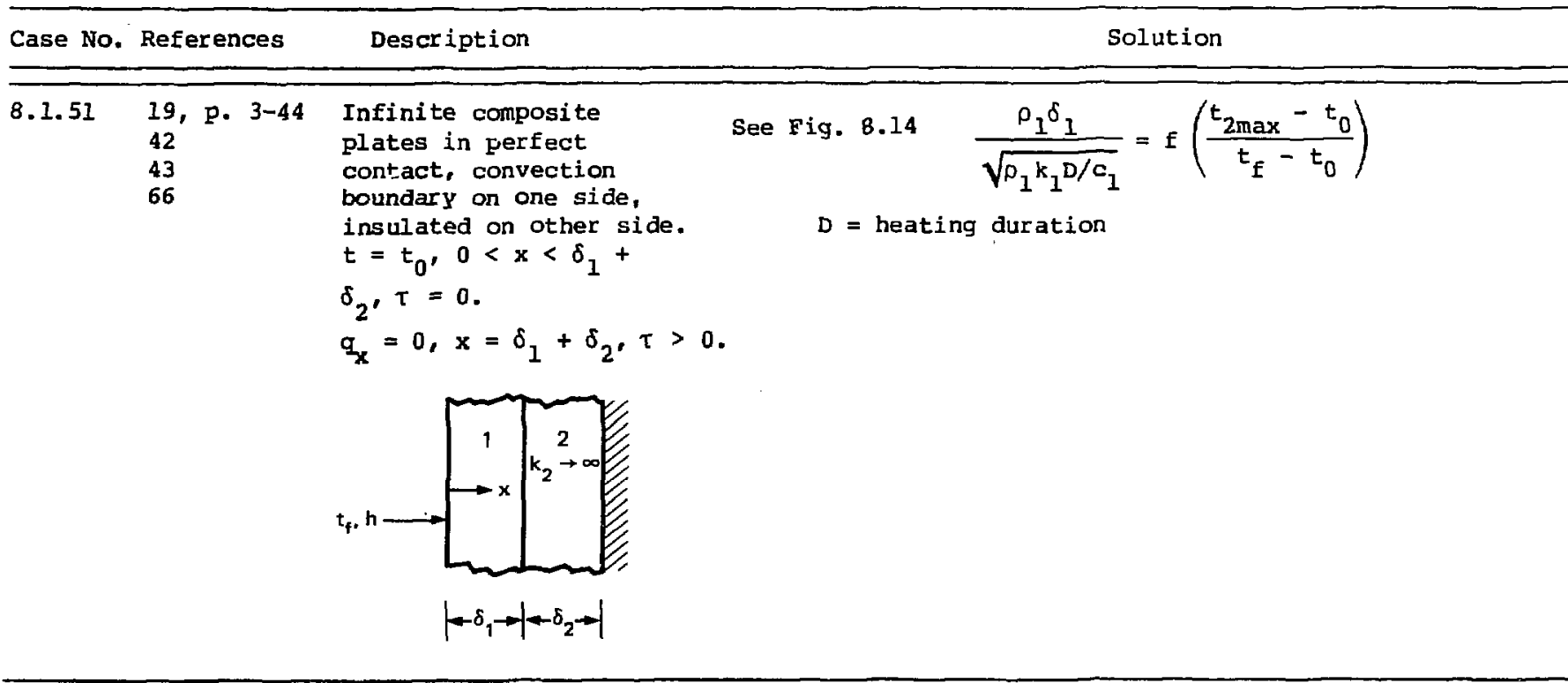



Section 8.1. Solids Bounded by Plane Surfaces--No Internal Heating.

Case No. References Description Solution

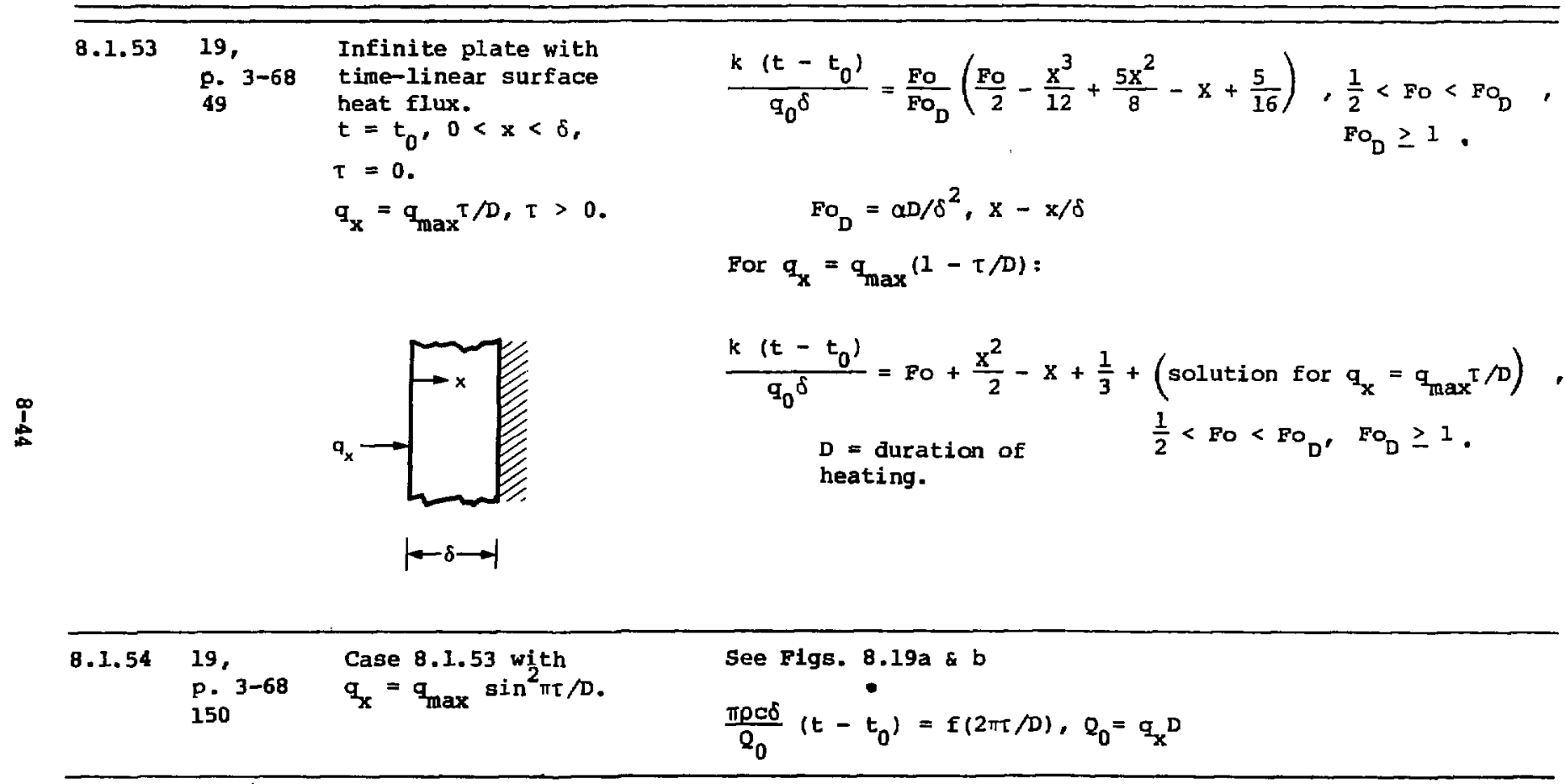


Section 8.1. Solids Bounded by Plane Surfaces--No Internal Heating.

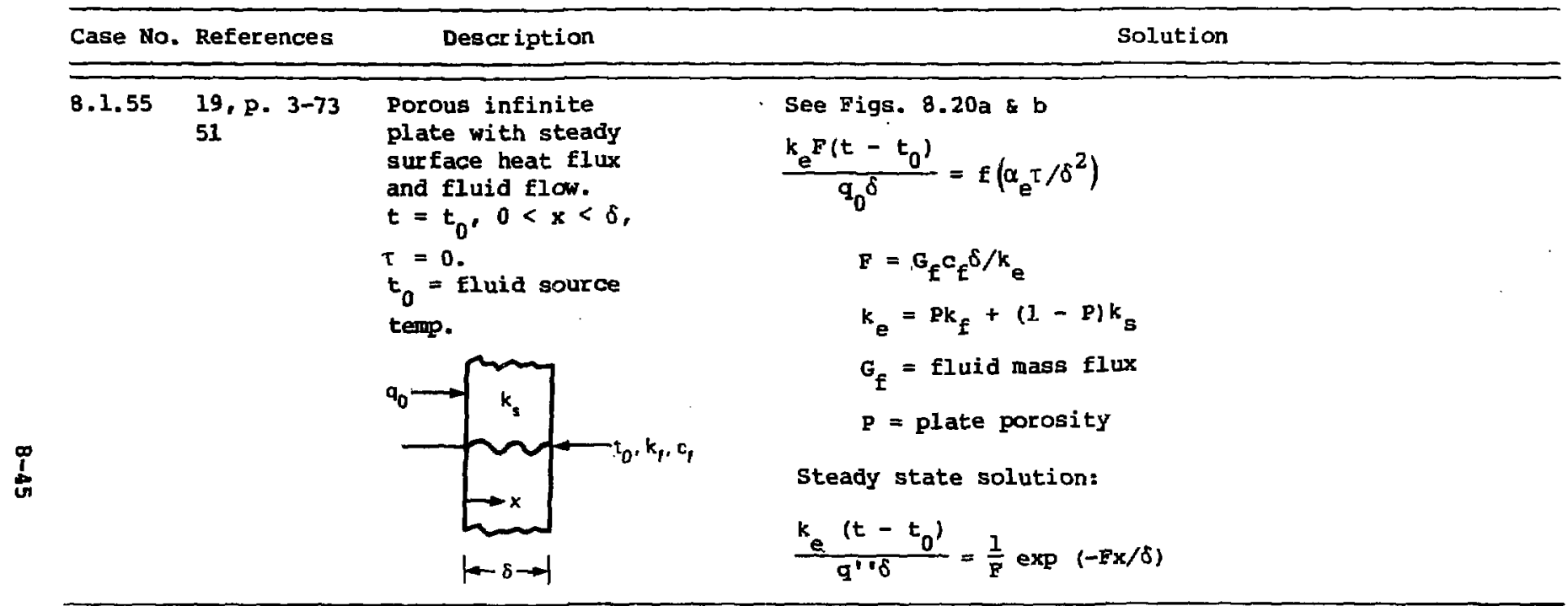


Section 8.1. Solids Bounded by Plane Surfaces--No Internal Heating.

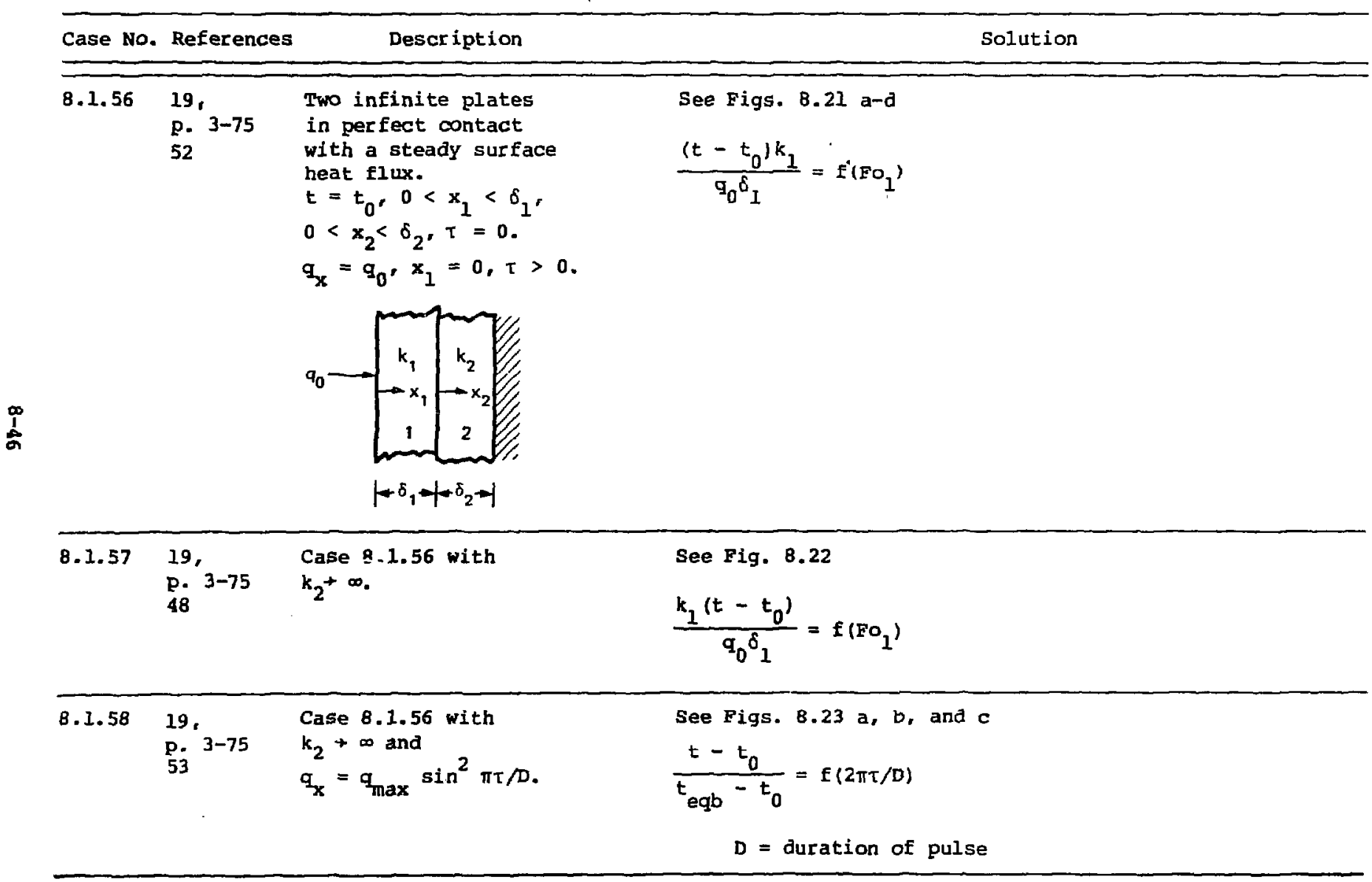


Section 8.1. Solids Bounded by Plane Surfaces-No Internal Heating.

\section{Case No. References}

\section{Description}

8.1. 59

74. p. 245 Thin plate with two convection conditions. $t=t_{0},-l<x<+l$, $\tau=0$.

$\stackrel{1}{1}$

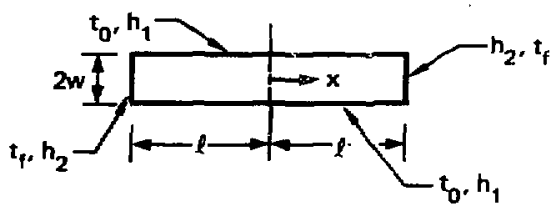

\section{Solution}

$$
\begin{aligned}
& \frac{t-t_{0}}{t_{f}-t_{0}}=\frac{\cosh \left(B i_{1 W}^{1 / 2} x\right)}{\cosh \left(B i_{l W}^{1 / 2} L\right)+\frac{L \sqrt{B i}_{1 W}}{B i_{2 W}} \sinh \left(B i_{l W}^{1 / 2}\right)_{L}} \\
& -2 \sum_{n=1}^{\infty} \frac{\lambda_{n}^{2} \sin \lambda_{n} \cos \left(\lambda_{n} x\right) \exp \left[-\left(\lambda_{n}^{2}+B i_{2 w}{ }^{2}\right) \text { Fo }{ }_{w}\right]}{\left(\lambda_{n}^{2}+B i_{L w} L^{2}\right)\left[\lambda_{n}+\sin \left(\lambda_{n}\right) \cos \left(\lambda_{n}\right)\right]} \\
& \lambda_{n} \tan \left(\lambda_{n}\right)=B i_{2 w}, B i_{1 w}=h_{1} w / k, B i_{2 w}=h_{2} w / k \\
& X=x / w, L=\ell / w
\end{aligned}
$$

Mean temp:

$$
\begin{aligned}
& \frac{t_{m}-t_{0}}{t_{f}-t_{0}}=\frac{\tanh \left(B i_{I W}^{1 / 2} L\right)}{B i_{1 W}^{1 / 2} L+\frac{B i_{I W}}{B i_{2 W}} I^{2} \tanh \left(B i_{I W}^{1 / 2} L\right)} \\
& -2 \sum_{n=1}^{\infty} \frac{\lambda_{n}^{2} B_{n} \exp \left[-\left(\lambda_{n}^{2}+B i_{I w} L^{2}\right) F O_{w}\right]}{\lambda_{n}^{2}+B i_{l w} L^{2}} \\
& B_{n}=\frac{B i_{1 w}}{\lambda_{n}^{2}\left(B i_{l w}^{2}+B i_{l w}+\lambda_{n}^{2}\right)}
\end{aligned}
$$


Section 8.1. Solids Bounded by Plane Surfaces--No Internal Heating.

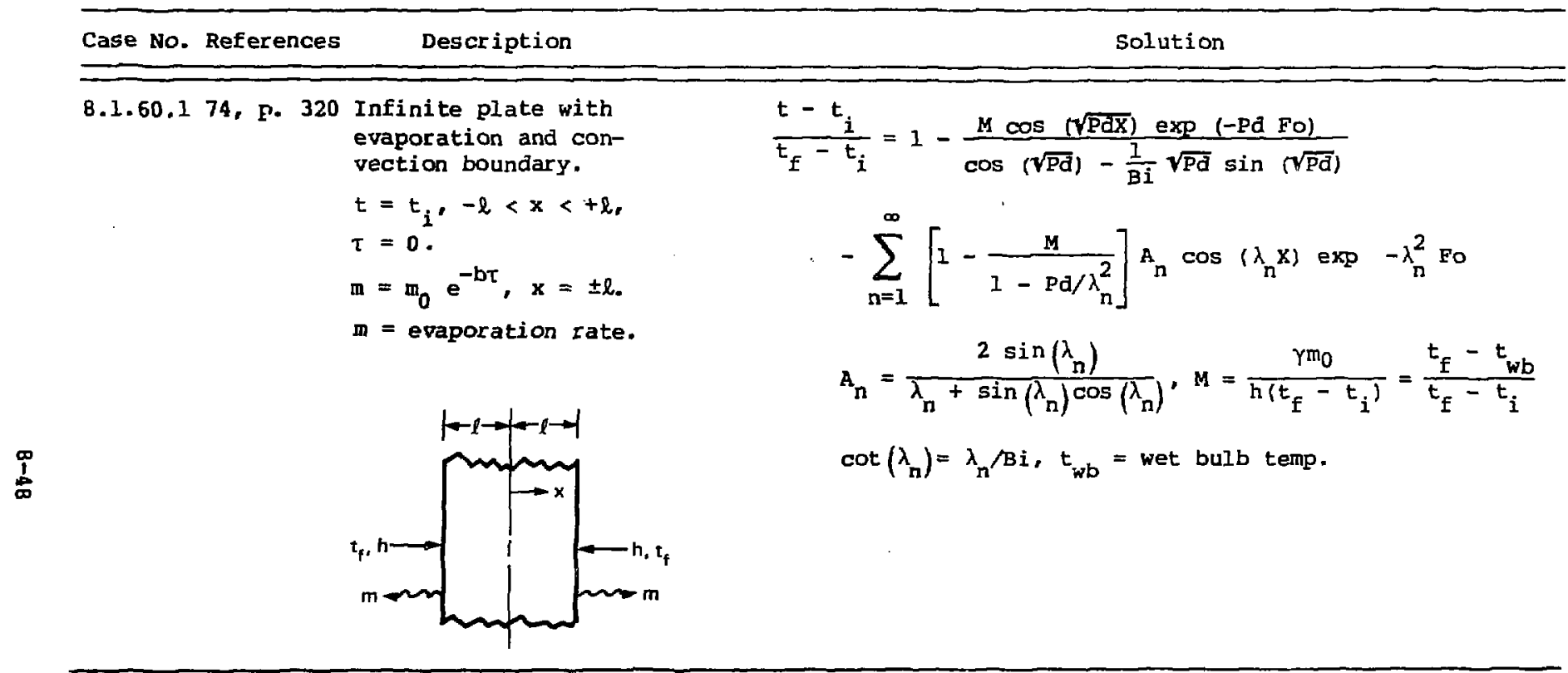


Section 8.1. Solids Bounded by Plane Surfaces--No Internal Heating.

\begin{tabular}{lll}
\hline Case No. References Sescription & Solution \\
\hline
\end{tabular}

8.1.60.2 74, p. 321 Case 8.1.60.1 with asymetrical boundary conditions.

$$
\frac{t-t_{i}}{t_{f}-t_{i}}=1-\frac{M_{1}+M_{2}}{2}-\sum_{n=1}^{\infty}\left(1-\frac{M_{1}+M_{2}}{2}\right)_{n} \cos \left(\lambda_{n} X\right) \exp \left(-\lambda_{n} F o\right)
$$

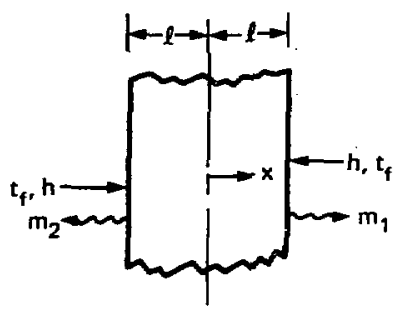

$$
\begin{aligned}
& -\frac{\left(M_{1}-M_{2}\right) B i x}{2(1+B i)}-\sum_{m=1}^{\infty} \frac{\left(M_{1}-M_{2}\right)}{2} A_{m} \sin \left(\lambda_{m} X\right) \exp \left(-\lambda_{m} F 0\right) \\
& A_{n}=\frac{2 \sin \left(\lambda_{n}\right)}{\lambda_{n}+\sin \left(\lambda_{n}\right) \cos \left(\lambda_{n}\right)}, A_{m}=\frac{2 \cos \left(\lambda_{m}\right)}{\lambda_{m}-\cos \left(\lambda_{m}\right) \sin \left(\lambda_{m}\right)} \\
& \cot \left(\lambda_{n}\right)=\lambda_{n} / B i, \tan \left(\lambda_{m}\right)=-\lambda_{m} / B i
\end{aligned}
$$

Mean temp:

$$
\frac{t_{m}-t_{i}}{t_{f}-t_{i}}=1-\frac{M_{1}+M_{2}}{2}-2 \sum_{n=1}^{\infty}\left(1-\frac{M_{1}+M_{2}}{2}\right) \frac{B i^{2} \exp \left(-\lambda_{n}^{2} \text { Fo }\right)}{\lambda_{n}^{2}\left(B i^{2}+B i+\lambda_{n}^{2}\right)}
$$


Section 8.1. Solids Bounded by Plane Surfaces-No Internal Heating.

Case No. References 
Section 8.1. Solids Bounded by Plane Surfaces-No Internal Heating.

Case No. References Description

Solution 
Section 8.2. Solids Bounded by Plane Surfaces--With Internal Heating.

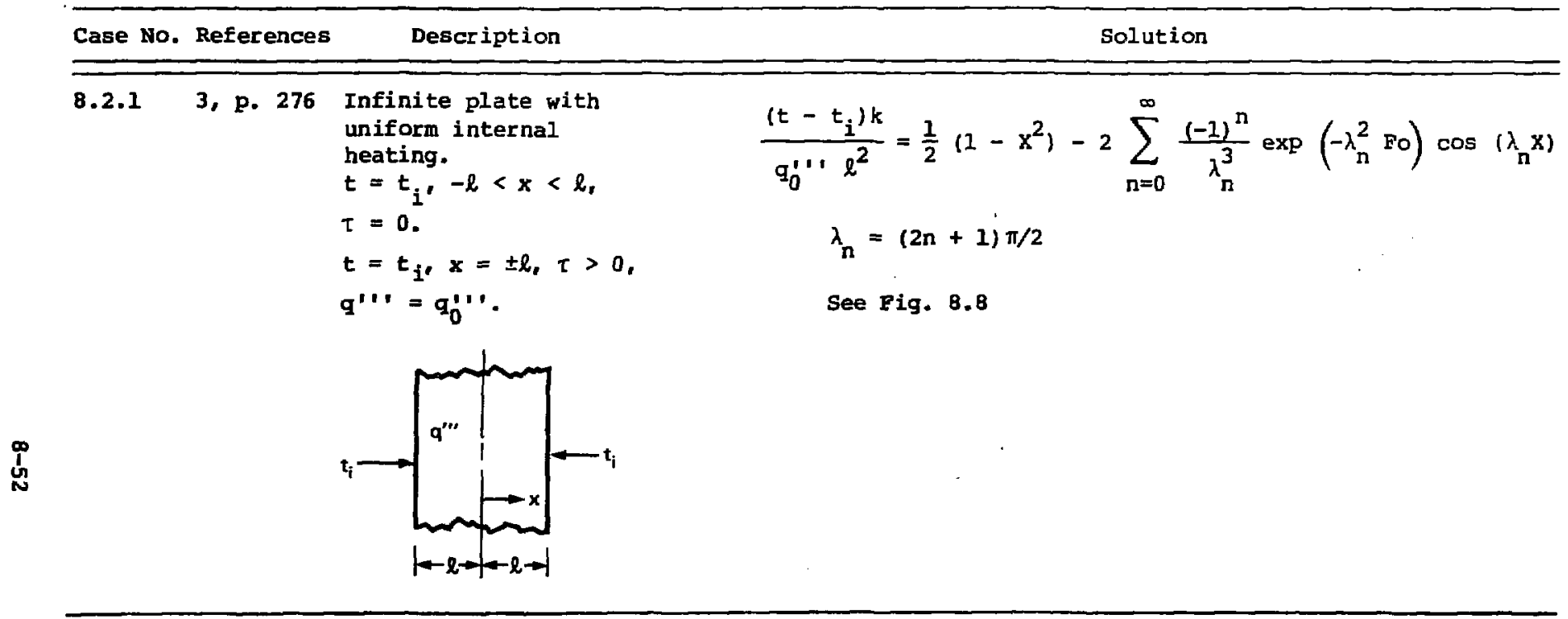


Section 8.2. Solids Bounded by Plane Surfaces--With Internal Heating.

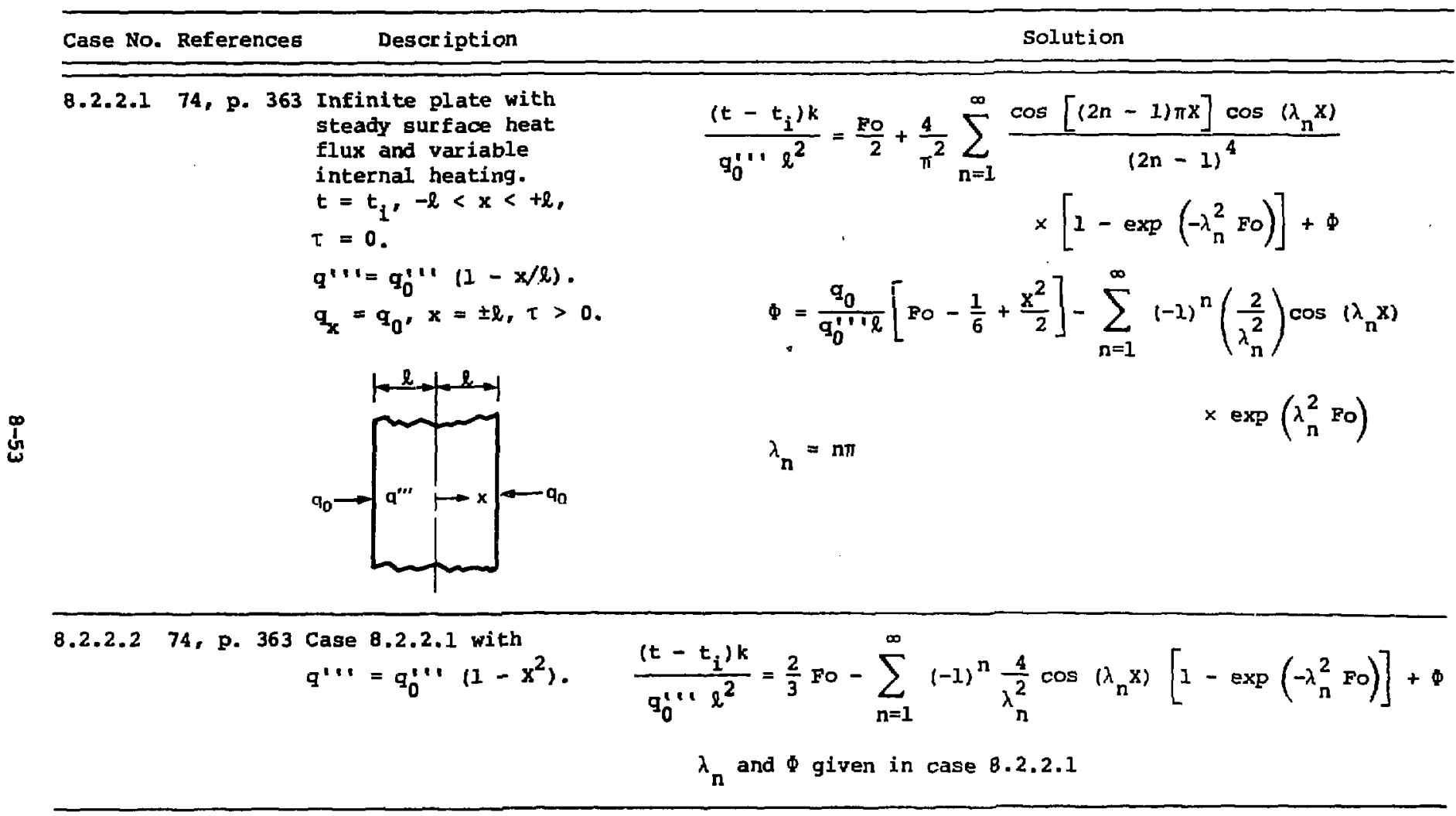


Section 8.2. Solids Bul ld by Plane Surfaces-With Internal Heating.

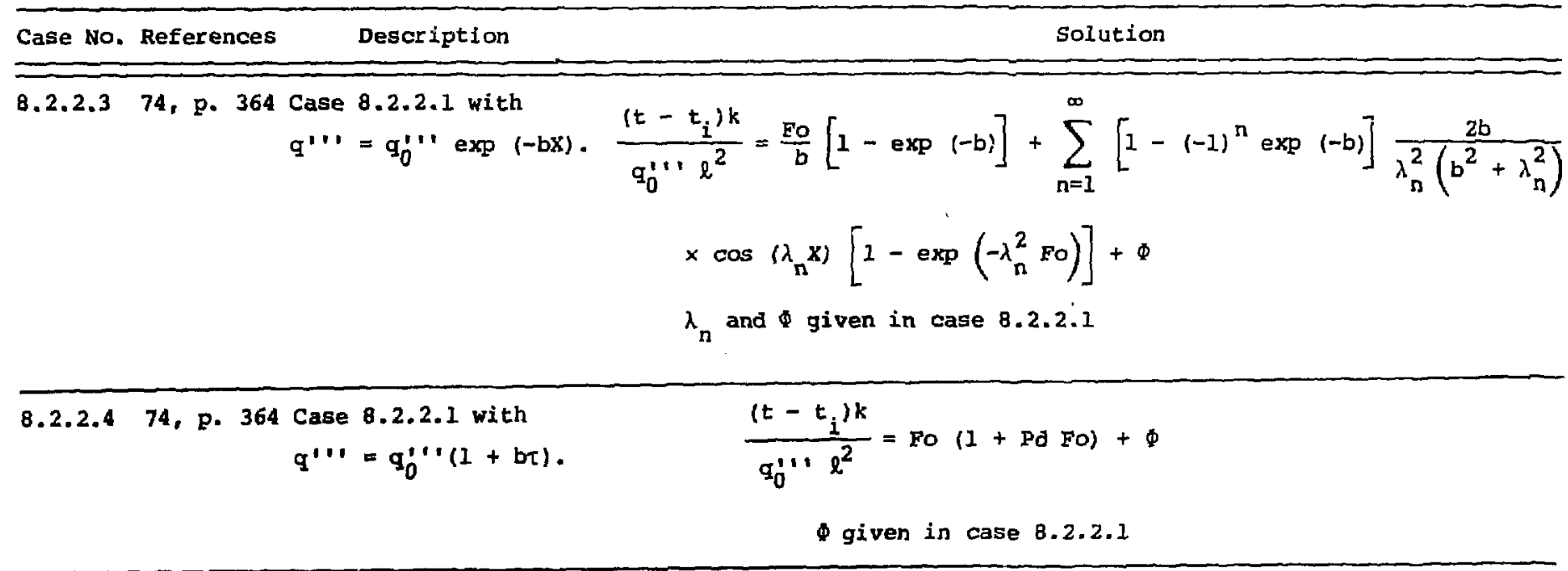


Section 6.1. Infinite Solids--No Internal Heating.

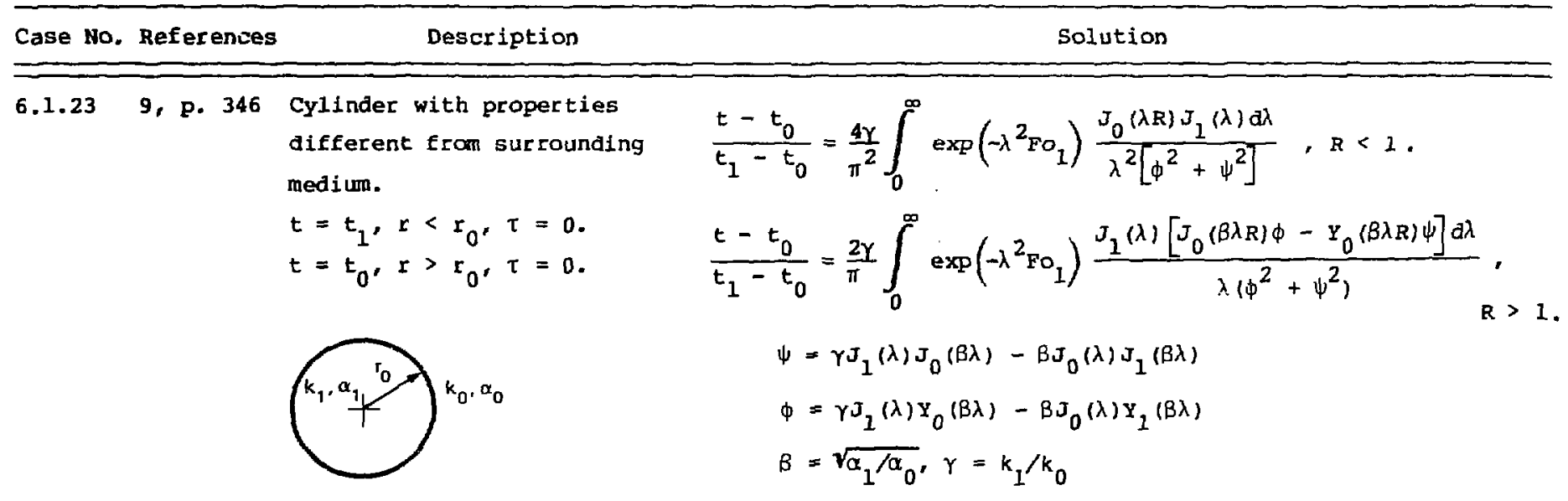

See case 6.1.4 for equal material properties.

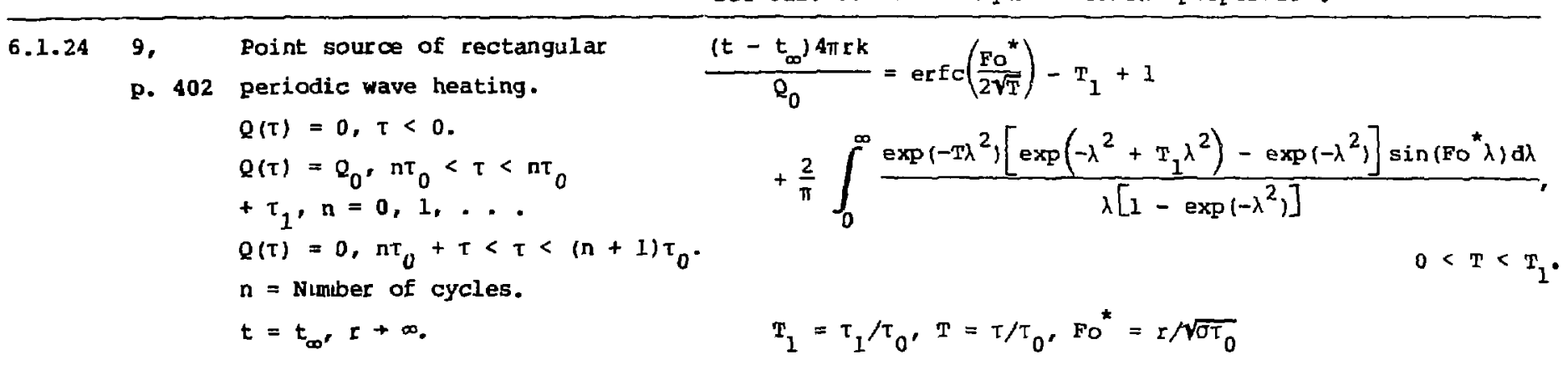


Section 8.2. Solids Bounded by Plare Surfaces--With Internal Heating.

\section{Case No. References}

Description

Solution

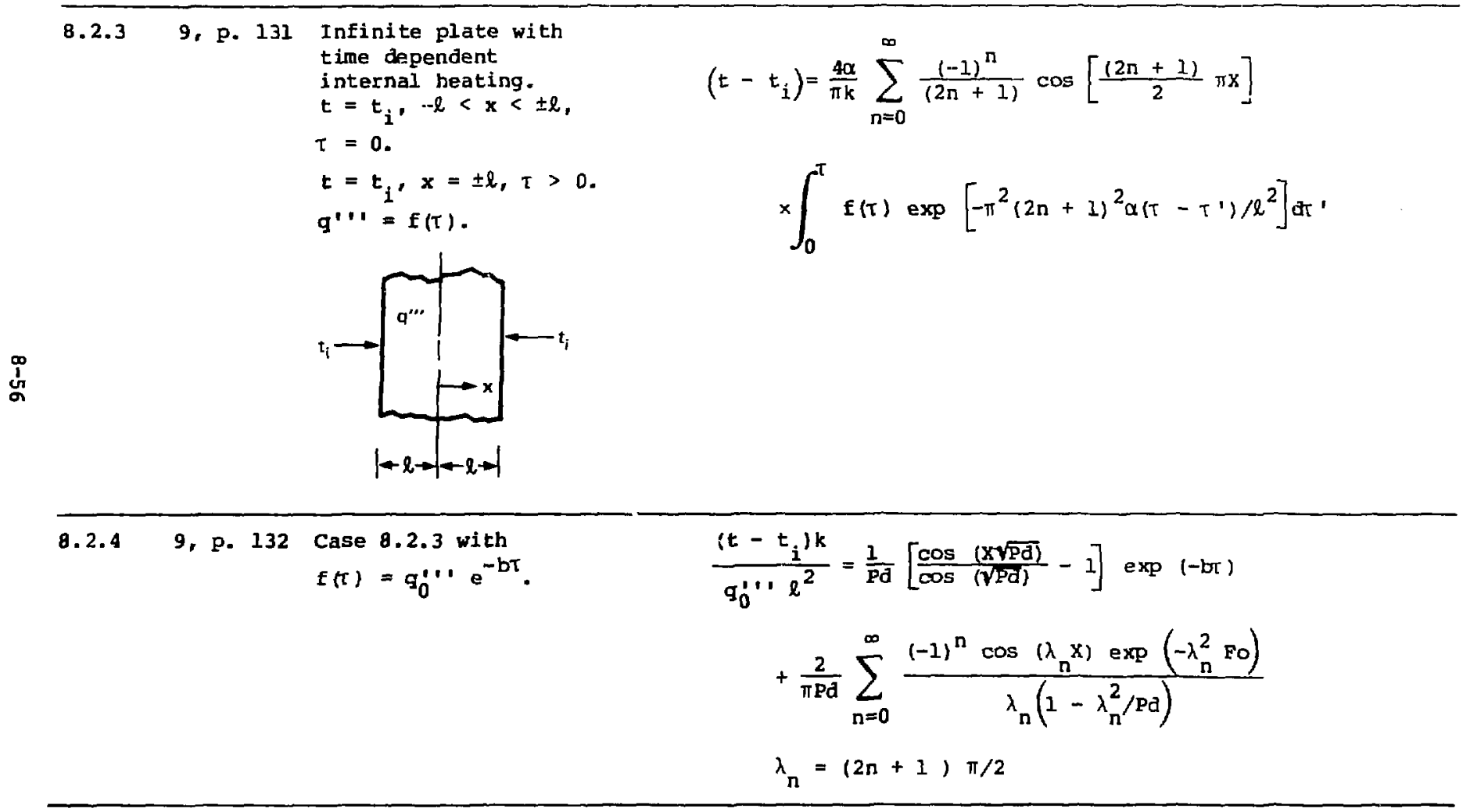


Section 8.2. Sollds Bounded by Plane Surfaces -With Internal Heating.

Case No. References

$9.2 .5 \quad 9$, p. 132

Case 8.2 .1 with

$q \cdots=E(x)$.
Solution

$$
\begin{aligned}
& \left(t-t_{1}\right)=\frac{4 \ell^{2}}{\pi^{2} k} \sum_{n=1}^{\infty} \frac{1}{n^{2}} \cos \left(\frac{n \pi}{2} x\right)\left[1-\exp \left(-\frac{n^{2} \pi^{2}}{4} 80\right)\right] \\
& \quad \times \int_{-1}^{1} f(x) \cos \left(\frac{n \pi}{2} x\right) d x
\end{aligned}
$$


Section 8.2. Solids Bounded by Plane Surfaces--With Internal Heating.

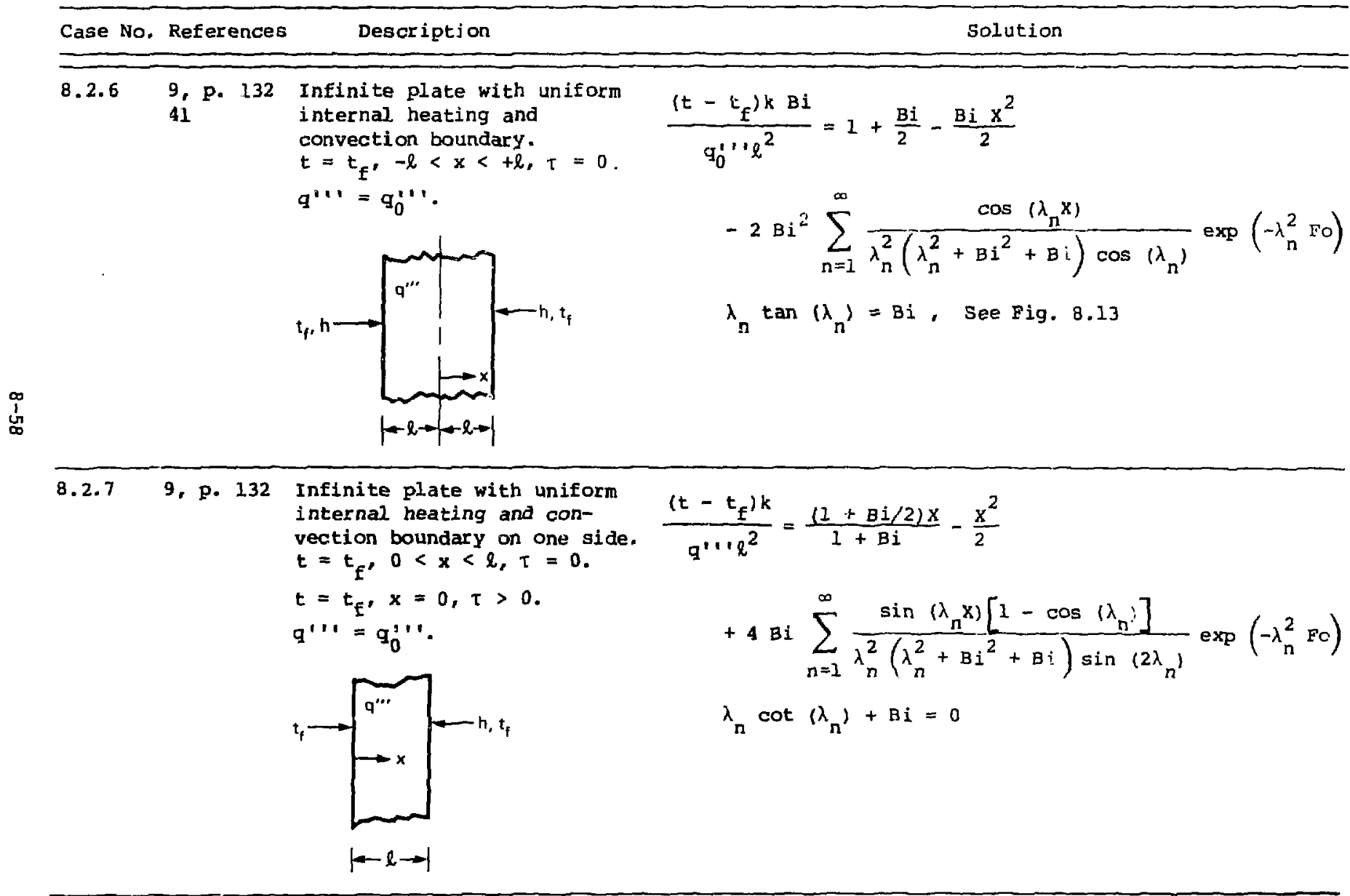


Section 8.2. Solids Bounded by Plane Surfaces-With Internal Heating.

\begin{tabular}{|c|c|c|c|}
\hline Case No. & References & Description & Solution \\
\hline 8.2 .8 & & $\begin{array}{l}\text { Infinite plate with } \\
\text { time-dependent heating. } \\
t=t_{0}, 0<x<l, \tau=0 . \\
t=t_{0}, x=0, l, \tau>0 \\
q^{\prime \prime \prime}=\beta r^{s / 2}, \\
s=-1,0,1,2, \ldots\end{array}$ & $\begin{array}{l}\frac{\left(t-t_{0}\right) k}{B a \pi^{1+B / 2}}=\frac{1}{(1+s / 2)}\left\{1-\Gamma(2+s / 2) 2^{s+2}\right. \\
\left.\quad \times \sum_{n=0}^{\infty}(-1)^{n}\left[1^{s+2} \operatorname{erfc}\left(\frac{n+x}{2 \sqrt{F o}}\right)+i^{s+2} \operatorname{erfc}\left(\frac{n+1-x}{2 \sqrt{F O}}\right)\right]\right\}\end{array}$ \\
\hline 8.2 .9 & 9. p. 404 & 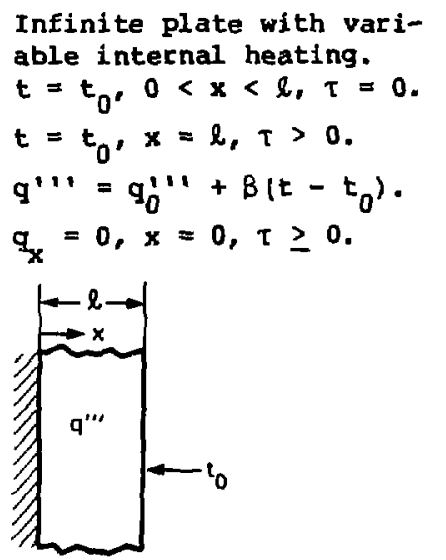 & $\begin{array}{l}\frac{\left(t-t_{0}\right) k}{q_{0}^{\prime \prime} \cdot l^{2}}=\frac{1}{B}\left\{\frac{\cos (\sqrt{B} x)}{\cos (\sqrt{B})}-1\right\} \\
\quad+\frac{16}{\pi} \sum_{n=0}^{\infty} \frac{(-1)^{n} \exp \left\{\left[-(2 n+1)^{2} \pi^{2}+4 B\right] \text { Fo/4 }\right\} \cos [(2 n+1) \pi x / 2]}{\left[4 B-(2 n+1)^{2} \pi^{2}\right](2 n+1)} \\
\text { E }=B l^{2} / k\end{array}$ \\
\hline
\end{tabular}


Section 8.2. Solids Bounded by Plane Surfaces-With Internal Heating.

Case No. References' Description Solution

8.2.10 9, p. 405 Case 8.2.9 with convection boundary

$h, t_{0}$ at $x=l$.

$$
\begin{aligned}
& \frac{\left(t-t_{0}\right) k}{q_{0}^{\prime \prime} l^{2}}=\frac{B i \cos (\sqrt{B X}\rangle}{B[B i \cos (\sqrt{B})-\sqrt{B} \sin (\sqrt{B})}-\frac{1}{B} \\
& \quad+2 \operatorname{Bi} \sum_{n=0}^{\infty} \frac{\cos \left(\lambda_{n} X\right) \exp \left[\left(B-\lambda_{n}^{2}\right) \text { Fo }\right]}{\left(B-\lambda_{n}^{2}\right)\left[\lambda_{n}^{2}+B i(B i+1)\right] \cos \left(\lambda_{n}\right)} \\
& \lambda_{n} \tan \left(\lambda_{n}\right)=B i, B=B l^{2} / k
\end{aligned}
$$

我
8.2 .11
19.
p. $3-29$
Infinite plate of infinite
$\bar{G} \ln [I-(\theta / G)]+\theta=L H / \bar{C}$
conductivity, variable
specific heat, convection
boundaries and steady heating.
$\bar{G}=\left(1+q_{0}^{\prime \prime}, \ell\right) / h L\left(t-t_{f}\right), \bar{c}=\left(1-c_{f}\right) / c_{O}, H=m / \rho c \ell$
$t=t_{0}, 0<x<2 l, \tau=0$.
$c=c_{0}+B\left(t-t_{0}\right)$.
$q^{\prime \prime \prime}=q_{0}^{\prime \prime \prime}$.
H evaluated at $t_{0}, c_{E}$ evaluated at $t_{E}$

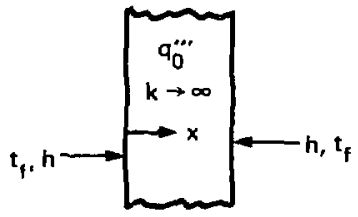
$L=\ell \times($ surface area $/$ volume $)=1$
$\theta=\left(t-t_{0}\right) /\left(t_{f}-t_{0}\right)$
$\mid-2 \ell-1$ 
Section 8.2. Solids Bounded by Plane Surfaces--With Internal Heating.

\begin{tabular}{|c|c|c|c|}
\hline Case No. & References & Description & Solution \\
\hline 8.2 .12 & $\begin{array}{l}\text { 19. } \\
\text { p. } 3-29\end{array}$ & $\begin{array}{l}\text { Case } 8.2 .11 \text { for infinite } \\
\text { square rod and cube. }\end{array}$ & $\begin{array}{ll}\text { See case } 8.2 .11, \text { set: } & L=2 \text { for square rod } \\
& L=3 \text { for cube }\end{array}$ \\
\hline 8.2 .13 & $\begin{array}{l}\text { 19, } \\
\text { p. } 3-32\end{array}$ & $\begin{array}{l}\text { Infinite plate of infinite } \\
\text { conductivity with surface } \\
\text { radiation and steady heating. } \\
t=t_{0}, 0<x<2 l, \tau=0 \text {. } \\
q_{r}=\sigma S\left(r_{s}^{4}-T^{4}\right) \text {. } \\
T_{s}=\text { source temp. } \\
q_{0}^{\prime \prime \prime}=q_{0}^{\prime \prime \prime} \text {. }\end{array}$ & $\begin{array}{c}\ln \left[\frac{(\theta+N)(1-N)}{(\theta+N)(1+N)}\right]+2 \tan ^{-1}(\theta / N)=2 \tan ^{-1}(1 / N)+4 M^{3} \\
\theta=T / T_{0}, N=\left(q_{0}^{\prime \prime} \cdot \ell / \sigma S T_{0}^{4}+\theta_{5}^{4}\right)^{1 / 4}, M=\sigma S T_{0}^{3} \tau / \rho c l\end{array}$ \\
\hline
\end{tabular}


Section 8.2. Solids Bounded by Plane Surfaces--With Internal Heating.

\section{Case No. References}

Description

Solution

8.2.14 19, Case 8.2.13 with simulta-

p. $3-33$

neous surface convection

and radiation.

$q_{x}=h\left(t_{f}-t\right)+\sigma \mathscr{F}\left(T_{s}^{4}-T^{4}\right)$,

$x=0,2 l, \tau>0$.

$M=A_{1} \ln \left(\frac{\theta-R_{1}}{\theta+R_{1}}\right)+A_{2} \ln \left(\frac{\theta-R_{2}}{\theta+R_{2}}\right)+\frac{A_{3}}{2} \ln \left[\frac{(\theta-\eta)^{2}+\phi^{2}}{(1-n)^{2}+\phi^{2}}\right]$

怘

$$
\begin{aligned}
& +\frac{A_{4}}{\phi}\left[\tan ^{-1}\left(\frac{\theta-\eta}{\phi}\right)-\tan ^{-1}\left(\frac{1-n}{\phi}\right)\right] \\
& A_{1}=\left\{8 \sqrt{\nu-\eta^{2}}\left[\eta \sqrt{\nu-\eta^{2}}+\left(\nu / 2+n^{2}\right)\right]\right\}^{-1} \\
& A_{2}=\left\{8 \sqrt{v-n^{2}}\left[n \sqrt{v-n^{2}}-\left(v / 2+n^{2}\right)\right]\right\}^{-1} \\
& A_{3}=v\left(v^{2}+8 \eta^{4}\right) \\
& A_{4}=\left(v / 2-v^{2}\right) /\left(v^{2}+8 n^{4}\right) \\
& R_{1}=-\eta-\sqrt{\nu-\eta^{2}}, R_{2}=-\eta+\sqrt{v-\eta^{2}} \\
& \phi=\sqrt{\eta+\beta / 4}, \eta=\sqrt{\beta / 2} \\
& \beta=\left(\frac{s^{2}}{2} \sqrt{\frac{s^{4}}{4}}+\frac{64}{27} N^{12}\right)^{1 / 3}+\left(\frac{s^{2}}{4}-\sqrt{\frac{s^{4}}{4}+\frac{64}{27} N^{12}}\right)^{1 / 3} \\
& v=\sqrt{N_{2}^{4}+\beta^{2}, 4} \\
& \mathbf{s}=\mathbf{h} / \sigma \mathscr{F} \mathrm{T}_{0}^{3} \\
& N=\left[q^{\prime \prime \prime \ell / \sigma S P} T_{0}^{4}+\theta_{s}^{4}+s \theta_{f}\right]^{1 / 4}
\end{aligned}
$$


Section 8.2. Solids Bounded by Plane Surfaces-with Internal Heating.

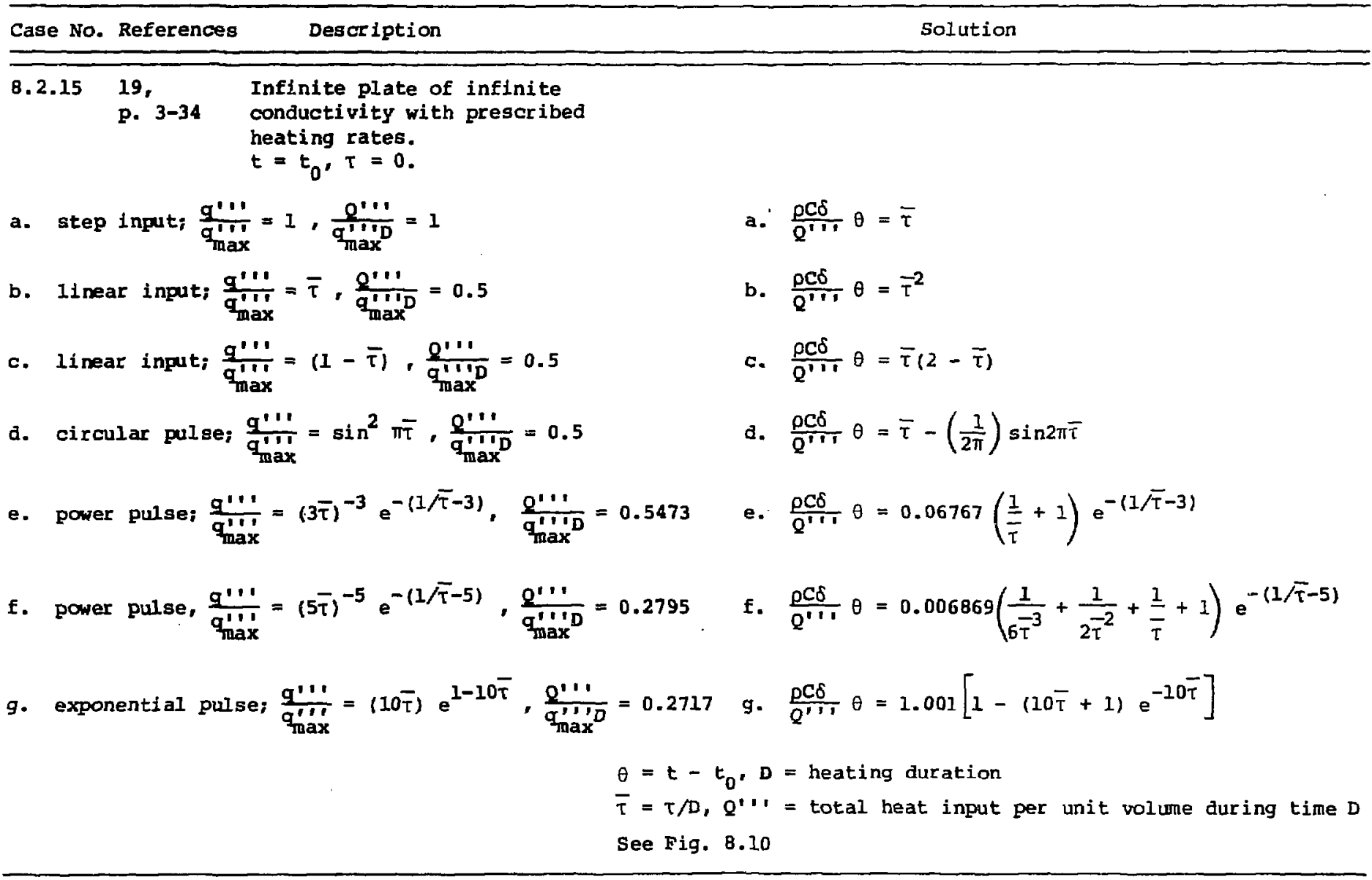


Section 8.2. Solids Bounded by Plane Surfaces--With Internal Heating.

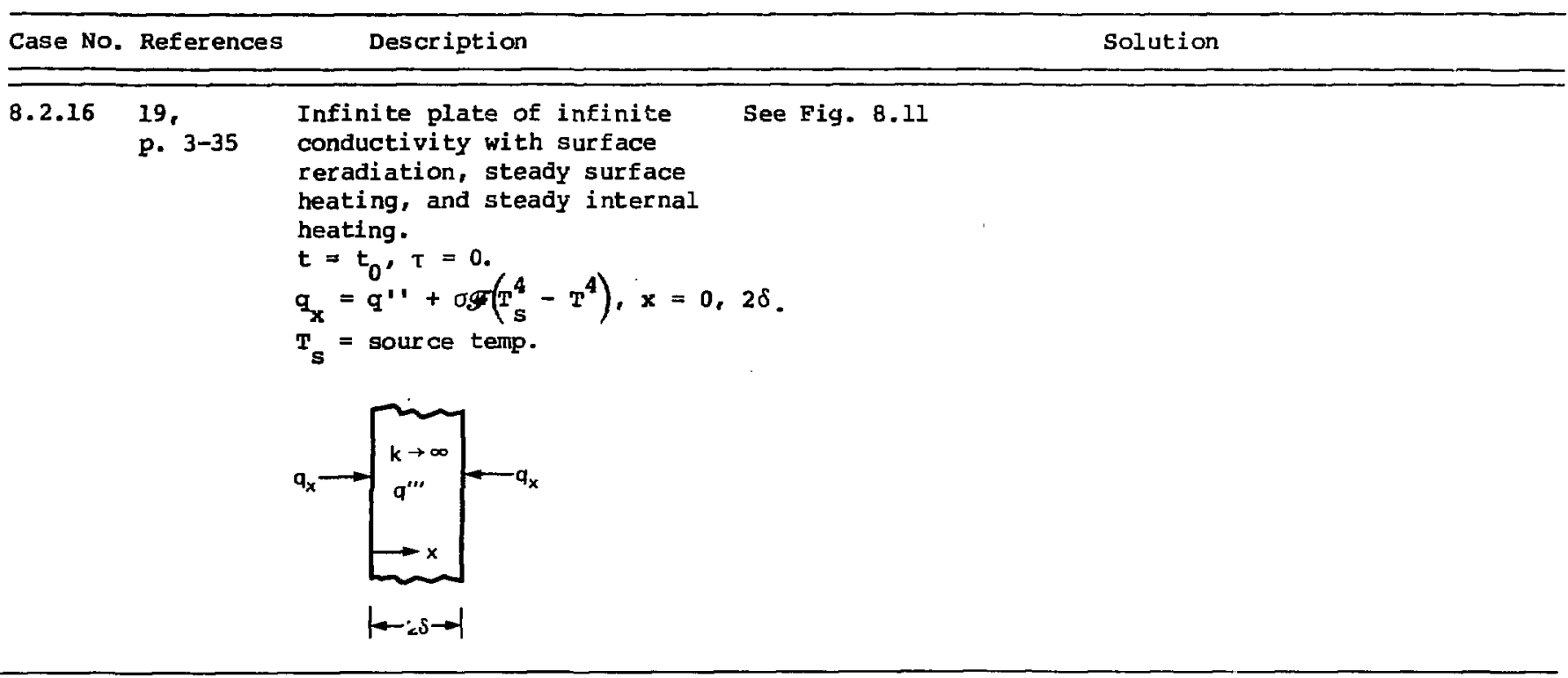


Section 8.2. Solids Bounded by Plane Surfaces--With Internal Heating.

8.2.17 74, p. 386 Infinite plate with two syumetrical planar heat pulses and convection boundaries.

$t=t_{F},-l<x<+l, \tau=0$.

Instantaneous pulse occurs at $\tau=0, x= \pm x$, with

strength $Q\left(J / \mathrm{m}^{2}\right)$.

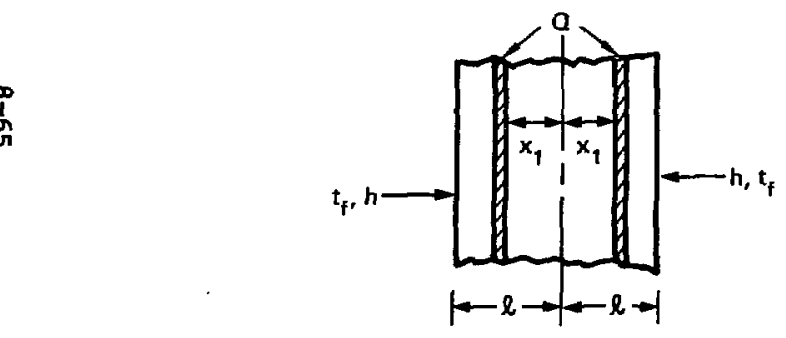

罗

Mean temp:

$\frac{\left(t_{m}-t_{f}\right) k \ell}{Q \alpha}=2 \sum_{n=1}^{\infty} \frac{B i^{2} \cos \left(\lambda_{n} x_{1}\right) \exp \left(-\lambda_{n}^{2} \text { Fo }\right)}{\sin \left(\lambda_{n}\right) \lambda_{n}\left(B i^{2}+B i+\lambda_{n}^{2}\right)}$.

For $\mathrm{Bi}=\infty$ :

$$
\begin{aligned}
& \frac{\left(t-t_{f}\right) k \ell}{Q \alpha}=2 \sum_{n=1}^{\infty}(-1)^{n+1} \cos \left[(2 n-1) \pi x_{1}\right] \cos [(2 n-1) \pi x] \\
& \quad x \exp \left[-(2 n-1)^{2} \pi^{2} F \circ / 4\right] .
\end{aligned}
$$

$$
\begin{aligned}
& \frac{\left(t-t_{f}\right) k \ell}{Q d}=2 \sum_{n=1}^{\infty} \frac{\lambda_{n}}{\lambda_{n}+\sin \left(\lambda_{n}\right) \cos \left(\lambda_{n}\right)} \cos \left(\lambda_{n} x\right) \exp \left(-\lambda_{n}^{2} \text { Fo }\right) \\
& \quad \times \int_{0}^{1}\left[f(x)-t_{f}\right] \cos \left(\lambda_{n} x\right) d x .
\end{aligned}
$$$$
x \exp \left(-\lambda_{n}^{2} \text { Fo }\right)
$$ 
Section 8.2. Solids Bounded by Plane Surfaces--With Internal Heating.

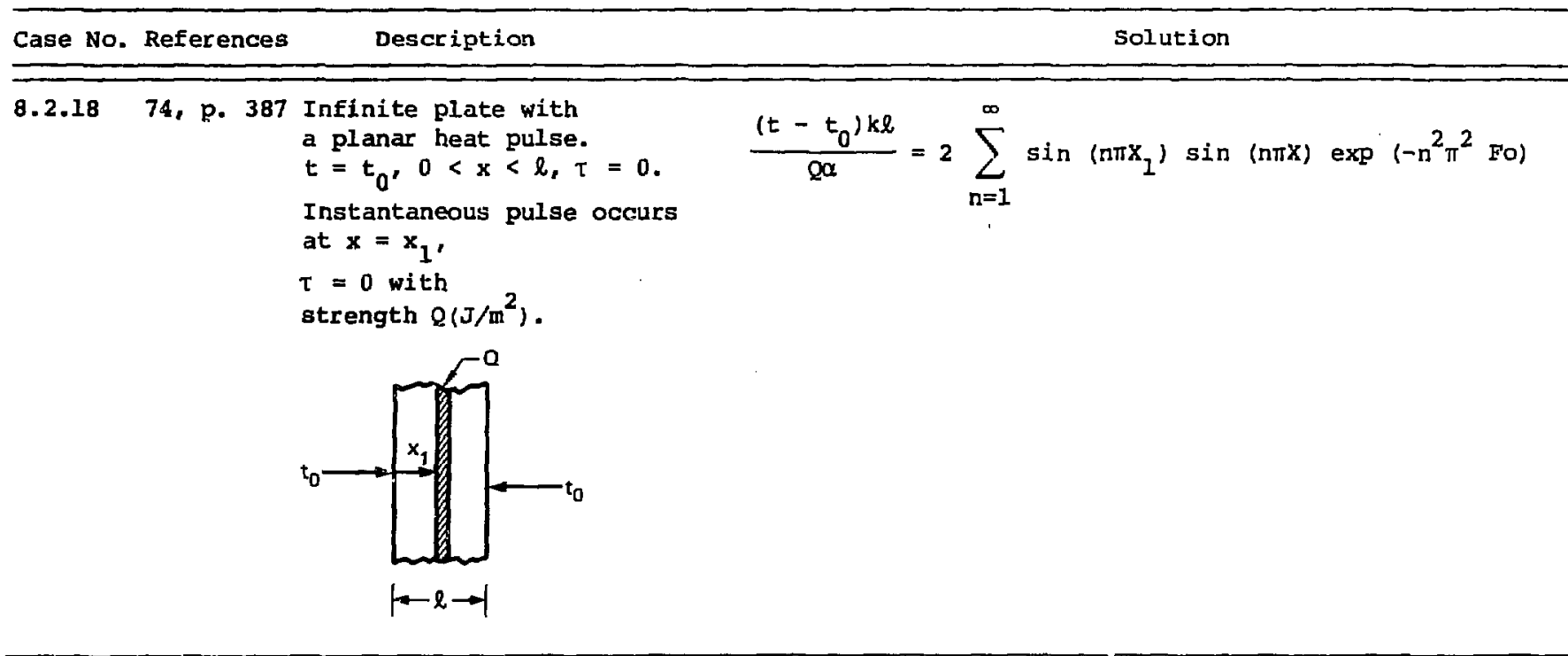


Section 8.2. Solids Bounded by Plane Surfaceg--With Internal Heating.

Case No. References Description Solution

$\stackrel{\infty}{1}$ 
Section 8.2. Solids Bounded by Plane Surfaces-With Internal Heating.

Case No. References Description Solution

䀡 


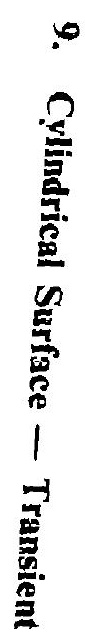


Section 9.1. Solids Bounded by Cylindrical Surfaces--No Internal Heating.

\begin{tabular}{lll}
\hline Case No. References & Description & Solution \\
\hline
\end{tabular}

\begin{tabular}{|c|c|c|c|}
\hline 9.1 .1 & $\begin{array}{l}2, \text { p. } 240 \\
74, \text { p. } 131\end{array}$ & $\begin{array}{l}\text { Infinite cylinder with } \\
\text { steady surface temp. } \\
t=t_{i}, 0<\tau<r_{0}, \tau=0 \text {. } \\
t=t_{0}, r=r_{0}, \tau>0 \text {. } \\
t_{0}\end{array}$ & $\begin{array}{l}\frac{t-t_{0}}{t_{i}-t_{0}}=2 \sum_{n=1}^{\infty} \frac{1}{\lambda_{n}} \exp \left(-\lambda_{n}^{2} \text { Fo }\right) \frac{J_{0}\left(\lambda_{n} R\right)}{J_{1}\left(\lambda_{n}\right)} \\
\quad J_{0}\left(\lambda_{n}\right)=0 \text {, see Fig. } 9.2 \\
\text { Cumulative heating: } \\
\frac{Q}{Q_{0}}=4 R \sum_{n=1}^{\infty} \frac{1}{\lambda_{n}^{2}}\left[1-\exp \left(-\lambda_{n}^{2} \text { EO }\right) \frac{J_{1}\left(\lambda_{n} R\right)}{J_{0}\left(\lambda_{n}\right)}\right] \\
\qquad Q_{0}=\pi r_{0}^{2} \rho c\left(t_{D}-t_{i}\right)\end{array}$ \\
\hline 9.1 .2 & $\begin{array}{l}\text { 1, p. } 269 \\
9, \text { p. } 328\end{array}$ & $\begin{array}{l}\text { Infinite cylinder with time } \\
\text { dependent surface temp. } \\
t=t_{i}, 0<\tau<r_{0}, \tau=0 . \\
t_{s}=t_{i}+c \tau, r=r_{0}, \tau>0 .\end{array}$ & $\begin{array}{l}\frac{t-t_{1}}{\operatorname{cr}_{0}^{2} / \alpha}=F o+\frac{1}{4}\left(R^{2}-1\right)+2 \sum_{n=1}^{\infty} \exp \left(-\lambda_{n}^{2} F o\right) \frac{J_{0}\left(\lambda_{n} R\right)}{\lambda_{n}^{3} J_{1}\left(\lambda_{n}\right)} \\
\quad J_{0}\left(\lambda_{n}\right)=0 \\
\text { See Table } 9.1 \text { and Fig. } 9.8\end{array}$ \\
\hline
\end{tabular}


Section 9.1. Solids Bounded by Cylindrical Surfaces--No Internal Heating.

Case No. References

Description

Solution

9.1 .3 .174$, p. 270 Infinite cylinder with convection boundary and variable initial temp.

$t=E(r), 0<r<r_{0}, \tau=0$.

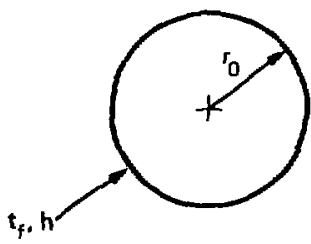

$$
\begin{aligned}
& t-t_{E}=2 \sum_{n=I}^{\infty} \frac{J_{0}\left(\lambda_{n} R\right) \exp \left(-\lambda_{n}^{2} F O\right)}{J_{0}^{2}\left(\lambda_{n}\right)+J_{I}^{2}\left(\lambda_{n}\right)} \int_{0}^{l} R\left[f(r)-t_{E}\right] J_{0}\left(\lambda_{n} R\right) d R \\
& \text { Bi } J_{0}\left(\lambda_{n}\right)=\lambda_{n} J_{I}\left(\lambda_{m}\right)
\end{aligned}
$$

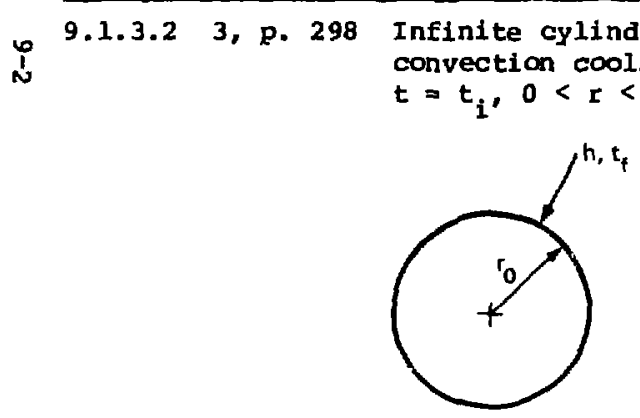

$$
\begin{gathered}
\frac{t-t_{F}}{t_{i}-t_{E}}=2 \sum_{n=1}^{\infty} \frac{B i J_{0}\left(\lambda_{n} R\right) \exp \left(-\lambda_{n}^{2} \text { Fo }\right)}{\left(\lambda_{n}^{2}+B i^{2}\right) J_{0}\left(\lambda_{n}\right)} \\
\lambda_{n} J_{I}\left(\lambda_{n}\right)+B i J_{0}\left(\lambda_{n}\right)=0
\end{gathered}
$$

See Figs. 9.1 arc

Cumulative heating:

$\frac{Q}{Q_{0}}=4 \sum_{n=1}^{\infty} \frac{B i^{2}\left[1-\exp \left(-\lambda_{n}^{2} F O\right)\right]}{\lambda_{n}^{2}\left(\lambda_{n}^{2}+B i^{2}\right)}, Q_{0}=\pi r_{0}^{2} \rho c\left(t_{i}-t_{F}\right)$

For Fo $<0.02, R \gg 0$ :

$$
\begin{gathered}
\frac{\left(t-t_{i}\right)}{\left(t_{f}-t_{i}\right)}=2 B i \sqrt{\frac{F O}{R}} \text { ierfc }\left(\frac{1-R}{2 \sqrt{F O}}\right)+4 B i \frac{F O}{\sqrt{R}} \\
\quad \times\left(\frac{2}{8 R}+\frac{3}{8}-B i\right) i^{2} \operatorname{erfc}\left(\frac{1-R}{2 \sqrt{F O}}\right)+\ldots
\end{gathered}
$$


Section 9.1. Solids Bounded by Cylindrical Surfaces--No Internal Heating.

Case No. References Description Solution

9.1.4 9, p. 199 Infinite cylinder with initial temp varying with radius.

$$
\begin{aligned}
& t=t_{i}-b r^{2}, \\
& 0<c<r_{0}, \tau=0 .
\end{aligned}
$$$$
\frac{t-t_{0}}{b r_{0}^{2}}=2 \sum_{n=1}^{\infty} \frac{\exp \left(-\lambda_{n}^{2} \text { Fo }\right) J_{0}\left(R \lambda_{n}\right)}{\lambda_{n}^{2} J_{1}^{2}\left(\lambda_{n}\right)}
$$

$$
\begin{aligned}
& x\left[\lambda_{n}\left(\frac{t_{1}-t_{0}}{b r_{0}^{2}}-1\right) J_{1}\left(\lambda_{n}\right)+2 J_{2}\left(\lambda_{n}\right)\right] \\
& J_{0}\left(\lambda_{n}\right)=0
\end{aligned}
$$

is

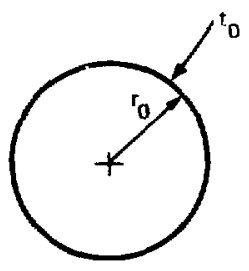

9.1.5 9, p. 201 Infinite cylinder with peiodic surface temperature. $t=t_{i}, 0<I<r_{0^{\prime}} \tau=0$. $t=\left(t_{m}-t_{i}\right) \sin (w t+E)$, $r=r_{0}, \tau>0$. $t_{m}=\max$ fluid temp.

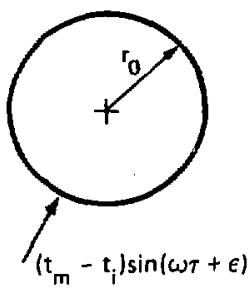

$$
\begin{aligned}
& \frac{t-t_{i}}{t_{m}-t_{i}}=\operatorname{real}\left\{\frac{I_{0}(R \sqrt{i P d})}{i I_{0}(\sqrt{i P d})} \exp [i(\omega t+\varepsilon)]\right\} \\
& +2 \sum_{n=1}^{\infty} \frac{\exp \left(-\lambda_{n}^{2} F o\right) \lambda_{n}\left[P d \cos (\varepsilon)-\lambda_{n}^{2} \sin (\varepsilon)\right] J_{0}\left(R \lambda_{n}\right)}{\left(\lambda_{n}^{4}+P d^{2}\right) J_{1}\left(\lambda_{n}\right)} \\
& J_{0}\left(\lambda_{n}\right)=0, \text { Pd }=r_{0}^{2} \omega / \alpha
\end{aligned}
$$


Section 9.1. Solids Bounded by Cylindrical Surfaces- No Internal Heating.

Case No. References Description Solution

9.1.6.1 9, p. 202 Infinite cylinder with convection cooling and linear time-dependent fluid temp.

$t=t_{i}, 0<r<r_{0}, \tau=0$.

$t_{E}=b \tau+t_{i}$

$$
\begin{aligned}
& \frac{\left(t-t_{i}\right) \alpha}{b r_{0}^{2}}=\text { Fo }-\frac{1}{4}\left(1-R^{2}+\frac{2}{B i}\right)+2 B i \sum_{n=1}^{\infty} \frac{\exp \left(-\lambda_{n}^{2} F_{0}\right) J_{0}\left(\lambda_{n} R\right)}{\lambda_{n}^{2}\left(B i^{2}+\lambda_{n}^{2}\right) J_{0}\left(\lambda_{n}\right)} \\
& \lambda_{n} J_{1}\left(\lambda_{n}\right)=\text { Bi } J_{0}\left(\lambda_{n}\right)
\end{aligned}
$$

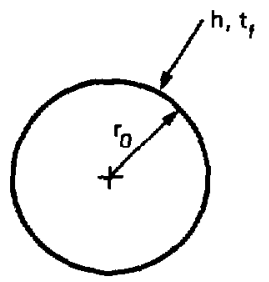

Mean temp:

$$
\frac{t_{m}-t_{i}}{b \tau}=F O-\frac{1}{8}\left(1+\frac{4}{B i}\right)+4 B i^{2} \sum_{n=1}^{\infty} \frac{\exp \left(-\lambda_{n}^{2} \text { Fo }\right)}{\lambda_{n}^{4}\left(\lambda_{n}^{2}+B i^{2}\right)}
$$

See Fig. 9.9 
Section 9.1. Solids Bounded by Cylindrical Surfaces--No Internal Heating.

\section{Case No. References}

Description

Solution

9.1.6.2 74, p. 317 Case 9.1.6.1 with

$t_{E}=t_{E m}-\left(t_{E m}-t_{i}\right) e^{-b \tau}$

$$
\begin{aligned}
& \frac{t-t_{i}}{t_{f m}-t_{i}}=1-\frac{J_{0}(\sqrt{P d} R) \exp (-P d F O)}{\sqrt{P d} J_{0}(\sqrt{P d})-\frac{1}{B i} P d J_{1}(\sqrt{P d})} \\
& -2 \text { Bi } \sum_{n=1}^{\infty} \frac{J_{0}\left(\lambda_{n}^{R}\right) \exp \left(-\lambda_{n}^{2} F o\right)}{J_{0}\left(\lambda_{n}\right)\left(\lambda_{n}^{2}+B i^{2}\right)\left[1-\left(\lambda_{n}^{2} / P d\right)\right]}
\end{aligned}
$$

Mean term :

$$
\begin{aligned}
\frac{t_{\mathrm{m}}-t_{i}}{t_{f_{\mathrm{m}}}-t_{i}} & =1-\frac{2 J_{I}(\sqrt{P d}) \exp (-P d \mathrm{FO})}{\sqrt{P d} J_{0}(\sqrt{P d})-\frac{1}{B i} P d J_{1} \sqrt{P d}} \\
-4 B i^{2} & \sum_{n=1}^{\infty} \frac{\exp \left(-\lambda_{n}^{2} F O\right)}{\lambda_{n}^{2}\left(\lambda_{n}^{2}+B i^{2}\right)\left[I-\left(\lambda_{n}^{2} / P d\right)\right]}
\end{aligned}
$$


Section 9.1. Solids Bounded by Cylindrical Surfaces--No Internal Heating.

Case No. References Description Solution

9.1.6.3 74, p. 320 Infinite cylinder with convection and evaporation boundary.

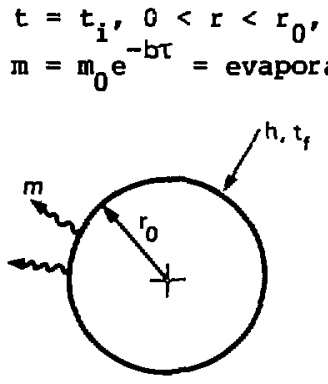

$$
\frac{t-t_{i}}{t_{E}-t_{i}}=1-\frac{M J_{0}(\sqrt{P d} R) \exp (-P d F O)}{J_{0}(\sqrt{P d})-(\sqrt{P d} / B i) J_{1}(\sqrt{P d})}
$$

$-2 \sum_{n=1}^{\infty}\left\{1-\frac{M}{\left[1-\left(P d / \lambda_{n}^{2}\right)\right]}\right\}_{n} \cos \left(\lambda_{n} R\right) \exp \left(-\lambda_{n}^{2} F_{0}\right)$

$A_{n}=\frac{B i}{J_{0}\left(\lambda_{n}\right)\left(\lambda_{n}^{2}+B i^{2}\right)}, \quad M=\frac{Y\left(m_{0}\right)}{h\left(t_{f}-t_{i}\right)}$,

$J_{0}\left(\lambda_{n}\right) / J_{1}\left(\lambda_{n}\right)=\lambda_{n} / B i$ 
Section 9.1. Solids Bounded by Cylindrical Surfaces--No Internal Heating.

\section{Case No. References}

Description

Solution

9.1 .79$, p. 202 Infinite cylinder with periodic convection boundary. $t=t_{i}, 0<r<r_{0}, \tau=0$.

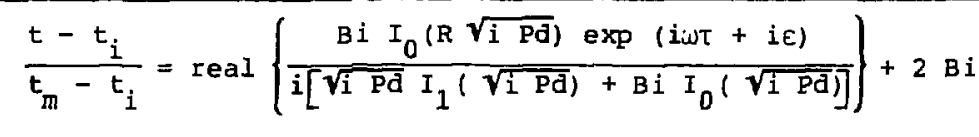$$
t_{f}=\left(t_{m}-t_{i}\right) \sin (\omega \tau+\varepsilon) \text {. }
$$

$t_{m}=\max$ fluid temp.

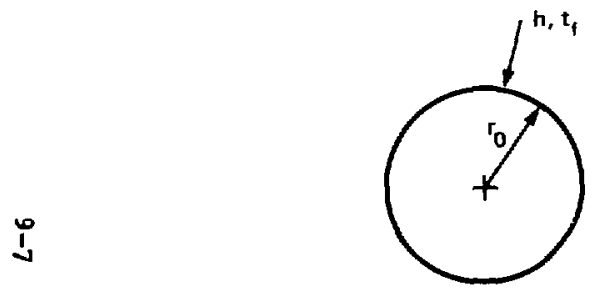

$\times \sum_{n=1}^{\infty} \exp \left(-\lambda_{n}^{2}\right.$ Fo $) \frac{\lambda_{n}^{2}[P d \cos (\varepsilon)-\sin (\varepsilon)] J_{0}\left(\lambda_{n} R\right)}{\left(\lambda_{n}^{4}+P d^{2}\right)\left(\lambda_{n}^{2}+B i^{2}\right) J_{0}\left(\lambda_{n}\right)}$

$\lambda_{n} J_{l}\left(\lambda_{n}\right)=B i J_{0}\left(\lambda_{m}\right)$, Pd $=r_{0}^{2} w / \alpha$

9.1 .89 9. p. 203 Infinite cylinder with steady surface heat flux. $t=t_{i}, 0<r<r_{0}, \tau=0$. $q_{r}=q_{0}, r=r_{0}, \tau>0$.

$$
\frac{\left(t-t_{i}\right) k}{q_{0} r_{0}}=2 \text { Fo }+\left(R^{2} / 2\right)-(1 / 4)-2 \sum_{n=1}^{\infty} \exp \left(-\lambda_{n}^{2} F_{0}\right) \frac{J_{0}\left(\lambda_{n} R\right)}{\lambda_{n}^{2} J_{0}\left(\lambda_{n}\right)}
$$

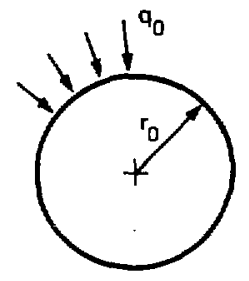

$J_{1}\left(\lambda_{n}\right)=0$, See Fig. 9.3

For Fo $<0.02, R \gg 0$ :

$\frac{\left(t-t_{i}\right) k}{q_{0}{ }^{r} 0}=2 \sqrt{\frac{F O}{R}}$ ierfc $\left(\frac{1-R}{2 \sqrt{F O}}\right)+\frac{F O\left(1+\frac{3 R}{R}\right)}{2 R^{3 / 2}} i^{2} \operatorname{erfc}\left(\frac{1-R}{2 \sqrt{F O}}\right)+\ldots$ 
Section 9.1. Solids Bounded by Cylindrical surfaces--No Internal Heating.

9.1.9 9, p. 207 Infinite hollow cylinder with steady surface temp.

$t=t_{i}, r_{i}<r<r_{0}, \tau=0$.

$\frac{t-t_{0}}{t_{i}-t_{0}}=\pi \sum_{n=1}^{\infty} \exp \left(-\lambda_{n}^{2} F_{0}\right)$

$t \Rightarrow t_{0}, r=r_{i}, r_{0}, \tau>0$.

$\times \frac{J_{0}\left(R_{i} \lambda_{n}\right)\left[J_{0}\left(\lambda_{n} R\right) Y_{0}\left(\lambda_{n}\right)-J_{0}\left(\lambda_{n}\right) Y_{0}\left(\lambda_{n} R\right)\right]}{J_{0}\left(\lambda_{n} R_{i}\right)+J_{0}\left(\lambda_{n}\right)}$

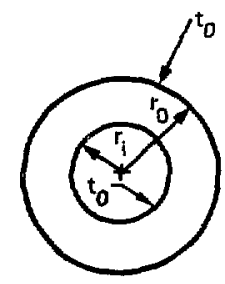

$J_{0}\left(\lambda_{n} R_{i}\right) Y_{0}\left(\lambda_{n}\right)-J_{0}\left(\lambda_{n}\right) Y_{0}\left(\lambda_{n} R_{i}\right)=0$

$R=r / r_{0}$, Fo $=\alpha \pi / r_{0}^{2}$ 
Section 9.1. Solids Bounded by Cylindrical Surfaces--No Internal Heating.

\begin{tabular}{lll}
\hline Case No. References & Description & Solution \\
\hline
\end{tabular}

9.1.10.1 9, P. 207 Infinite hollow cylinder with different surface temp. $t=t_{i}, r_{i}<r<r_{0^{\prime}} \tau=0$.

$t=t_{0}, r=r_{0}, \tau>0$.

$t=t_{i}, r=r_{i}, \tau>0$.

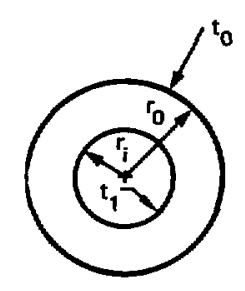

$$
\begin{aligned}
\frac{T}{T_{i}}= & \pi \sum_{n=1}^{\infty} \exp \left(-\lambda_{n}^{2} F o\right) \frac{J_{0}\left(a_{n}\right) U_{0}\left(R \lambda_{n}\right)}{J_{0}\left(a_{n}\right)+J_{0}\left(b_{n}\right)}-\pi \frac{T_{0}}{T_{i}} \\
& \times \sum_{n=1}^{\infty} \frac{\left[J_{0}\left(a_{n}\right)-\left(T_{1} / T_{0}\right) J_{0}\left(b_{n}\right)\right] J_{0}\left(a_{n}\right) U_{0}\left(R \lambda_{n}\right)}{J_{0}^{2}\left(a_{n}\right)-J_{0}^{2}\left(b_{n}\right)} \exp \left(-\lambda_{n}^{2} F o\right) \\
& +\frac{T_{0}\left[\left(T_{1} / T_{0}\right) \ln (1 / R)+\ell n\left(R / R_{i}\right)\right]}{T_{i} \ln \left(1 / R_{i}\right)} \\
& a_{n}=R_{i} \lambda_{n}, b_{n}=\lambda_{n}, U_{0}\left(R \lambda_{n}\right)=J_{0}\left(\lambda_{n} R\right) Y_{0}\left(b_{n}\right)-J_{0}\left(b_{n}\right) Y_{0}\left(\lambda_{n} R\right) \\
& J_{0}\left(\lambda_{n} R_{i}\right) Y_{0}\left(\lambda_{n}\right)-J_{0}\left(\lambda_{n}\right) Y_{0}\left(\lambda_{n}\right) Y_{0}\left(\lambda_{n} R_{i}\right)=0 \\
& R=r / r_{0}, \text { Fo }=\alpha \tau / r_{0}^{2} \\
& \text { See Fig. } 9.7 a \text { for } t_{I}=t_{i}, \text { and Fig. } 9.7 b \text { for } t_{0}=t_{i} \cdot
\end{aligned}
$$


Section 9.1. Solids Bounded by Cylindrical Surfaces--No Internal Heating.

\section{Case No. References}

Desaription

Solution

9.1.10.2 74, p. 282 Infinite hollow cylinder with two convection boundaries and variable initial temperaturegeneral case.

$t=f(R), r_{i}<r<r_{0}, \tau=0$.

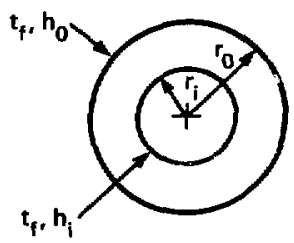

$t-t_{f}=\sum_{n=1}^{\infty} E_{n} \exp \left(-\lambda_{n}^{2} F o\right) w\left(\lambda_{n}, R\right)$

$$
\begin{gathered}
w\left(\lambda_{n} R\right)=-\left[B i_{i} Y_{0}\left(\lambda_{n}\right)+\lambda_{n} Y_{1}\left(\lambda_{n}\right)\right] J_{0}\left(\lambda_{n}^{R}\right) \\
+\left[\lambda_{n} J_{1}\left(\lambda_{n}\right)+B i_{i} J_{0}\left(\lambda_{n}\right)\right] Y_{0}\left(\lambda_{n}^{R}\right) .
\end{gathered}
$$$$
E_{n}=\frac{\pi^{2}}{2} \lambda_{n}^{2}\left[B i_{0} J_{0}\left(\lambda_{n} R_{0}\right)-\lambda_{n} \bar{F}_{1}\left(\lambda_{n} R_{n}\right)\right]^{2}
$$$$
\times \int_{1}^{R_{0}} R\left[f(R)-t_{f}\right] W\left(\lambda_{n}, R\right) d R\left(( \lambda _ { n } ^ { 2 } + B i _ { 0 } ^ { 2 } ) \left[B i_{i} J_{0}\left(\lambda_{n}\right)\right.\right.
$$$$
\left.\left.+\lambda_{n} J_{1}\left(\lambda_{n}\right)\right]^{2}-\left(\lambda_{n}^{2}+B i_{i}^{2}\right)\left[{ }^{B i_{i}} J_{0}\left(\lambda_{n} R_{0}\right)-\lambda_{n} J_{1}\left(\lambda_{n} R_{0}\right)\right]^{2}\right)^{-1}
$$

$\left[B i_{1} J_{0}\left(\lambda_{n}\right)+\lambda_{n} J_{1}\left(\lambda_{n}\right)\right]\left[B i_{0} Y_{0}\left(\lambda_{n} R_{0}\right)-\lambda_{n} Y_{1}\left(\lambda_{n} R_{0}\right)\right]$

$-\left[B i_{0} J_{0}\left(\lambda_{n} R_{0}\right)-\lambda_{n} J_{1}\left(\lambda_{n} R_{0}\right)\right]\left[{ }^{B i}{ }_{i} Y_{0}\left(\lambda_{n}\right)-\lambda_{n} Y_{1}\left(\lambda_{n}\right)\right]=0$.

$$
B i_{i}=n_{i} r_{i} / k, B i_{0}=h_{0} r_{i} / k, R=r / r_{n}
$$


Section 9.1. Solids Bounded by Cylindrical Surfaces--No Internal Heating.

Case No. References Description Solution

\begin{tabular}{|c|c|c|c|}
\hline 9.1 .11 & 3. p. 409 & $\begin{array}{l}\text { Infinite hollow cyliader } \\
\text { with steady inside } \\
\text { surface temperature. } \\
t=t_{i}, r<r<r_{0}, \tau=0 \text {. } \\
t=t_{1}, r=r_{i}, \tau>0 . \\
q_{r}=0, r=r_{0}, \tau>0 .\end{array}$ & $\begin{array}{l}\frac{t-t_{i}}{t_{1}-t_{i}}=1-2 \sum_{n=1}^{\infty} \exp \left(-\lambda_{n}^{2} F 0\right) \frac{Y_{1}\left(\lambda_{n}\right) J_{0}\left(\lambda_{n} R\right)-J_{1}\left(\lambda_{n}\right) Y_{0}\left(\lambda_{n} R\right)}{\lambda_{n} \Delta_{0}\left(R_{1}\right)-\lambda_{n} R_{i} \Delta_{1}\left(R_{i}\right)} \\
\Delta_{0}\left(R_{i}\right)=Y_{0}\left(\lambda_{n}\right) J_{0}\left(\lambda_{n} R_{i}\right)-J_{0}\left(\lambda_{n}\right) Y_{0}\left(\lambda_{n} R_{i}\right) \\
\Delta_{1}\left(R_{i}\right)=Y_{I}\left(\lambda_{n}\right) J_{1}\left(\lambda_{n} R_{i}\right)-J_{1}\left(\lambda_{n}\right) Y_{1}\left(\lambda_{n} R_{i}\right) \\
Y_{1}\left(\lambda_{n}\right) J_{0}\left(\lambda_{n} R_{i}\right)-J_{I}\left(\lambda_{n}\right) Y_{0}\left(\lambda_{n} R_{i}\right)=0 \\
F_{0}=\alpha / r_{0}^{2}, R=r / r_{0}\end{array}$ \\
\hline 9.1 .12 & 74, p. 200 & $\begin{array}{l}\text { Infinite hollow cylirder with } \\
\text { steady surface heat flux. } \\
t=t_{i}, r_{i}<r<r_{0}, \tau=0 \text {. } \\
-q_{r}=q_{0}, r=r_{0}, \tau>0 \\
g_{r}=0, r=r_{1}, \tau>0 \text {. }\end{array}$ & 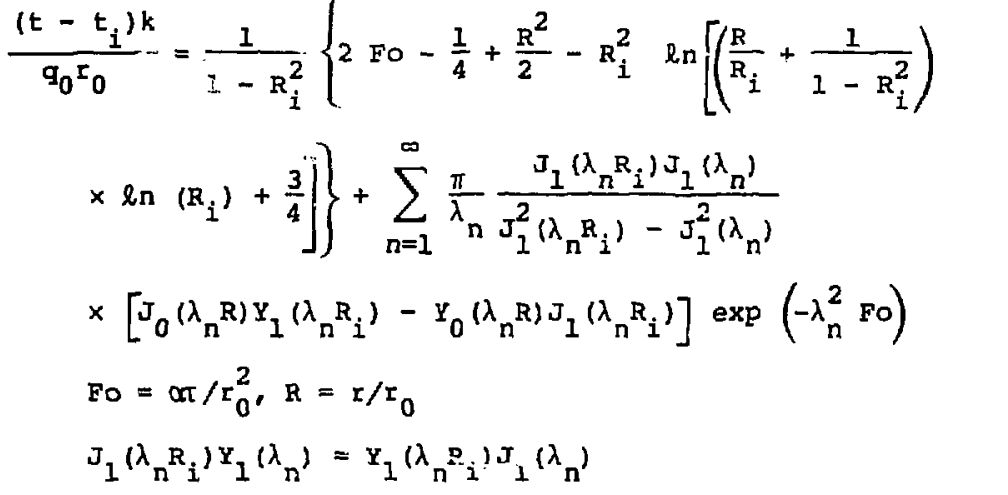 \\
\hline
\end{tabular}


Section 9.1. Solids Bounded by Cylindrical Surfaces--No Internal Heating.

Case No. References Description Solution

9.1 .139 , p. 210 Infinite cylinder with variable initial temperature and constant surface temp.

$t=f(r, \theta), 0<r<r_{0}, \tau=0$.

$t-t_{0}=\sum_{m=1}^{\infty} \sum_{n=0}^{\infty}\left[A_{n, m} \cos (n \theta)+B_{n, m} \sin (n \theta)\right]$

$t=t_{0}, r=r_{0}, \tau>0$.

$\times J_{n}\left(\lambda_{m} R\right) \exp \left(-\lambda_{m}^{2} F O^{2}\right)$

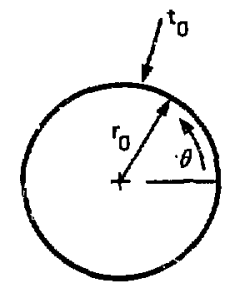

$A_{0, m}=\frac{1}{\pi\left[J_{0}^{\prime}\left(\lambda_{m}\right)\right]^{2}} \int_{0}^{1} \int_{-\pi}^{\pi} R\left[f(R, \theta)-t_{0}\right] J_{0}\left(\lambda_{m} R\right) d R d \theta$

$A_{n, m}=\frac{2}{\pi\left[J_{n}^{\prime}\left(\lambda_{m}\right)\right]^{2}} \int_{0}^{l} \int_{-\pi}^{\pi} R\left[E(R, \theta)-t_{0}\right] \cos (n \theta) J_{n}\left(\lambda_{m} r\right) d R d \theta$

$B_{n, m}=\frac{2}{\pi\left[J_{n}^{\prime}\left(\lambda_{m}\right)\right]^{2}} \int_{0}^{l} \int_{-\pi}^{\pi} R\left[E(R, \theta)-t_{0}\right] \sin (n \theta) J_{n}\left(\lambda_{m} R\right) d R d \theta$

$J_{n}\left(\lambda_{m}\right)=0$ 
Section 9.1. Solids Bounded by Cylindrical Surfaces--No Internal Heating.

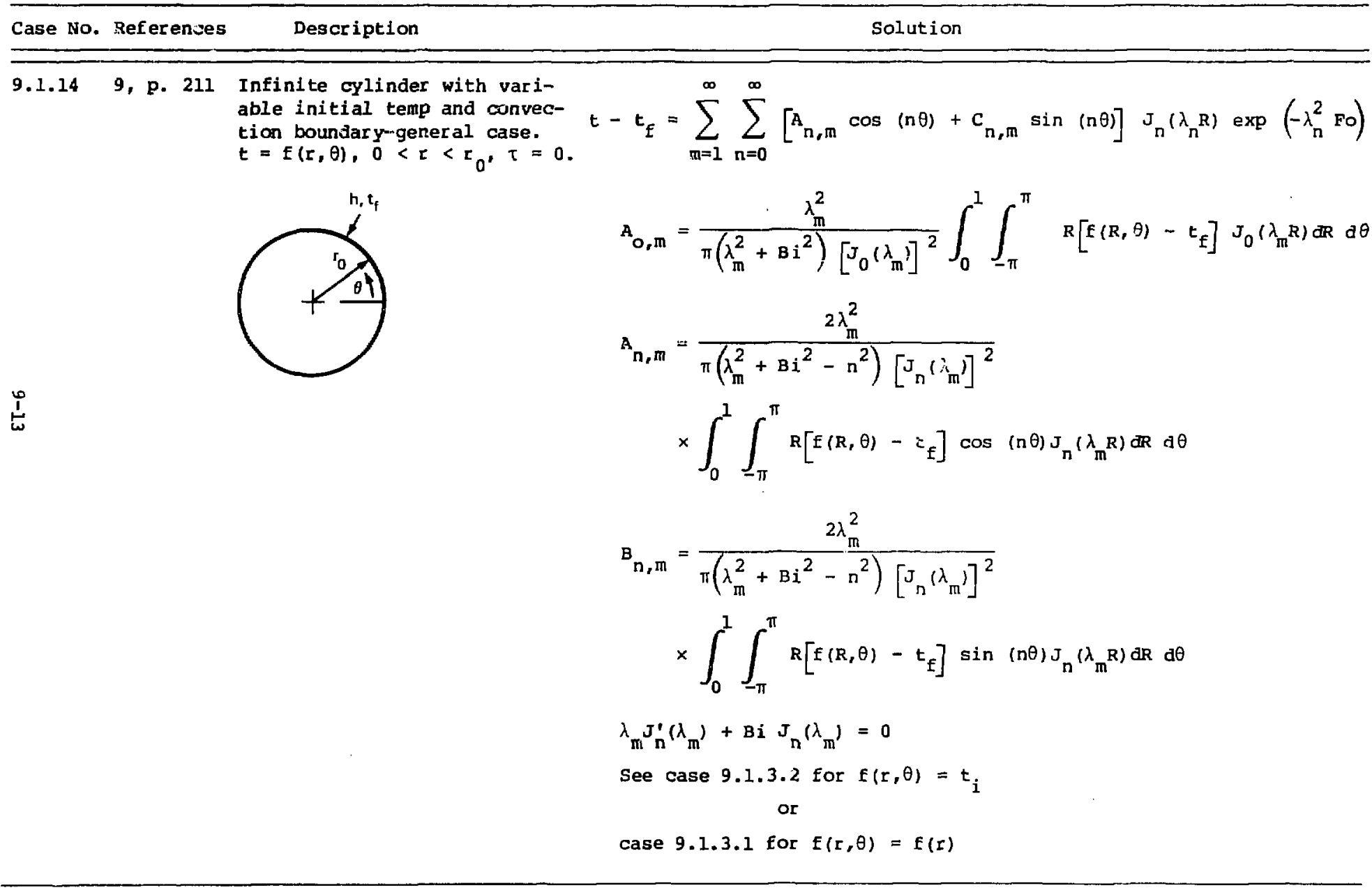


Section 9.1. Solids Bounded by Cylindrical Surfaces--No Internal Heating.

Case No. References Description Solution

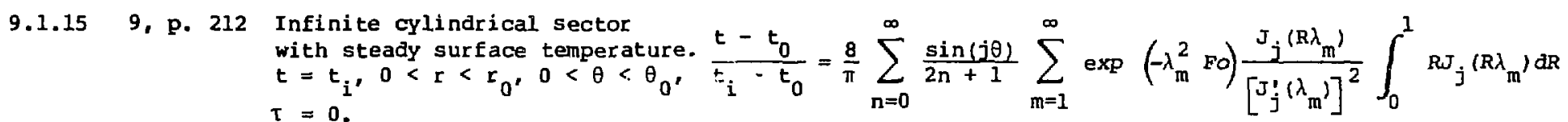

$$
\begin{aligned}
& t=t_{0}, r=r_{0}, \theta=0, \tau>0 . \quad j=(2 n+1) \pi / \theta_{0}, J_{j}\left(\lambda_{m}\right)=0
\end{aligned}
$$

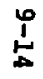

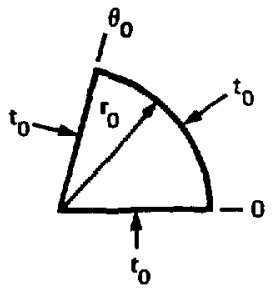

\subsubsection{2, p. 248 Semi-infinite cylinder.}

Dimensionless temperature equals product of solution for semi-infinite solid (case 7.1 .1 or 7.1 .3 ) and solution for infinite cylinder (case 9.1 .1 or 9.1 .3 ). See Figs. $9.4 \mathrm{a}$ and $9.4 \mathrm{~b}$.

\section{1 .172 , p. 248 Finite cylinder.}

Dimensionless temperature equals product of solution for infinite plate (case 8.1 .6 or 8.1 .7 ) and solution for infinite cylinder (case 9.1 .1 or 9.1 .3 ). See Figs. 9.4a and 9.4b.

$\begin{array}{ll}9.1 .18 & \begin{array}{l}\text { Infinite cylinder in a see case } 7.1 .22 \\ \text { semi-infinite soijd. }\end{array}\end{array}$


Section 9.1. Solids Bounded by Cylindrical Surfaces--No Internal Beating.

\section{Case No. Keferences}

Description

Solution

9.1 .199 , p. 418 Finite cylinder.

$t=t_{0}, 0<r<x_{0}$,

$0<z<\ell, T=0$.

$t=t_{1}, 0<r<x_{0}$

$z=0, \tau>0$.

$\mathrm{t}=\mathrm{t}_{0}$

$0<r<r_{0}, z=l, \tau>0$.

convection boundary $h, t_{0}$

at $r=r_{0} r>0$. $\frac{t-t_{0}}{t_{1}-t_{0}}=\sum_{n=1}^{\infty} \frac{B i J_{0}\left(R \lambda_{n}\right) \sinh \left[(1-z) \lambda_{n} L\right]}{\left(B i^{2}+\lambda_{n}^{2}\right) J_{0}\left(\lambda_{n}\right) \sinh \left(\lambda_{n} L\right)}-4 \pi B i$

$\times \sum_{n=1}^{\infty} \sum_{m=1}^{\infty} \frac{m_{0}\left(R \lambda_{n}\right) \sin (m \pi) \exp \left\{-\left[\lambda_{n}^{2}+(m \pi / L)^{2}\right] F o\right\}}{\left[\left(\lambda_{n} L\right)^{2}+(m \pi)^{2}\right]\left[B i^{2}+\lambda_{n}^{2}\right] J_{0}\left(\lambda_{n}\right)}$

$\lambda_{n} J_{1}\left(\lambda_{n}\right)=B i J_{0}\left(\lambda_{n}\right)$

$\mathrm{Bi}=\mathrm{hr}_{0} / \mathrm{k}, \mathrm{R}=\mathrm{r} / \mathrm{r}_{0}, \mathrm{z}=\mathrm{z} / \mathrm{l}, \mathrm{FO}_{\mathrm{O}}=\alpha \pi / \mathrm{r}_{0}^{2}, \mathrm{~L}=\ell / \mathrm{r}_{\mathrm{O}}$

茎

9.1.20 9, p. 418 Case 9.1.1B with convection boundary $h, t_{0}$ at $z=\ell, \tau>0$. $\frac{t-t_{0}}{t_{1}-t_{0}}=2 B i \sum_{n=1}^{\infty} \frac{J_{0}\left(R \lambda_{n}\right)\left(\lambda_{n} \cosh \left[\lambda_{n} L(1-z)\right]+B i \sinh \left[\lambda_{n} L(1-z)\right]\right.}{\left(B i^{2}+\lambda_{n}^{2}\right) J_{0}\left(\lambda_{n}\right)\left[\lambda_{n} \cosh \left(L \lambda_{n}\right)+B i \sinh \left(L \lambda_{m}\right)\right]}$

$-4 B i \sum_{n=1}^{\infty} \sum_{m=1}^{\infty} \frac{\beta_{m}\left(B i^{2} L^{2}+\beta_{m}^{2}\right) J_{0}\left(R \lambda_{n}\right) \sin \left(z \beta_{m}\right) \exp \left[-\left(\lambda_{n}^{2}+\beta_{m}^{2} / L^{2}\right) F o\right]}{\left(B i^{2}+\lambda_{n}^{2}\right)\left(B i^{2} L^{2}+\beta_{m}^{2}+B i L\right)\left(L^{2} \lambda_{n}^{2}+\beta_{m}^{2}\right) J_{0}\left(\lambda_{n}\right)}$

$\beta_{\mathrm{m}} \cot \beta_{\mathrm{m}}=-\mathrm{Bi} \mathrm{L}, \mathrm{L}=\ell / \mathrm{r}_{0}$

See case 9.1 .19 for $\lambda_{n^{\prime}} B i, R, z$, and Fo 
Section 9.1. Solids Bounded by Cylindrical Surfaces--No Internal Heating.

9.1.21 9, p. 419 Semi-infinite cylinder with convection boundary.

$t=t_{0}, 0<r<r_{0}, z>0$,

$\tau=0$.

$t=t_{1}, 0<r<r_{0^{\prime}}$

$z=0, \tau>0$.

Convection boundary $h, t_{0}$

at $r=r_{0}, z>0, \tau>0$.

$$
\begin{aligned}
& \frac{t-t_{0}}{t_{1}-t_{0}}=B i \sum_{n=1}^{\infty} \frac{J_{0}\left(R \lambda_{n}\right)}{\left(B i^{2}+\lambda_{n}^{2}\right) J_{0}\left(\lambda_{n}\right)}\left\{2 \exp \left(-\lambda_{n} z\right)+\exp \left(\lambda_{n} z\right)\right. \\
& \left.\quad x \operatorname{erfc}\left(\frac{\lambda_{n}}{2 F O^{*}}+z F^{*}\right)-\exp \left(-\lambda_{n} z\right) \operatorname{erfc}\left(\frac{\lambda_{n}}{2 F_{0}^{*}}-F^{*}\right)\right\} \\
& \lambda_{n} J_{1}\left(\lambda_{n}\right)=B i J_{0}\left(\lambda_{n}\right) \\
& B i=h r_{0} / k, R=r / r_{0}, z=z / r_{0}, \mathrm{Fo}^{\star}=r_{0} / 2 \sqrt{\alpha T}
\end{aligned}
$$

9.1.22 9, p. 419 Semi-infinite cylinder with steady surface temperature. $t=t_{0}, 0<r<r_{0}, z>0$, $\tau=0$.

$t=t_{1}, r=r_{0}, z>0$,

$\tau>0$.

$$
\frac{t-t_{0}}{t_{1}-t_{0}}=1-\sum_{n=1}^{\infty} \frac{J_{0}\left(R \lambda_{n}\right)}{\lambda_{n} J_{1}\left(\lambda_{n}\right)}\left\{2 \exp \left(-\lambda_{n}^{2} \text { Fo }\right) \operatorname{erf}\left(Z F_{0}^{*}\right)+\exp \left(\lambda_{n} z\right)\right.
$$$$
t=t_{0^{\prime}}, 0<r<r_{0^{\prime}}
$$$$
z=0, \tau>0 \text {. }
$$

See case 9.1 .20 for $\lambda_{n^{\prime}} F 0^{*}, R_{\text {r and }} z$ 
Section 9.1. Solids Bounded by Cylindrical Surfaces-No Internal Heating.

9.1.23 9, P. 420 Cone with steady surface temperature.

$t=t_{i}, 0<\theta<\theta_{0}, \tau=0$. $t=t_{0}, \theta=\theta_{0}, \tau>0$.

$$
\begin{gathered}
\frac{t-t_{i}}{t_{0}-t_{i}}=1+\sqrt{2} \sum_{n=0}^{\infty} \frac{(2 n+1) \Gamma[(n+1) / 2] p_{n}(\lambda)}{\left.n \Gamma(n / 2)\left[d p_{n}(\lambda) / d n\right]\right]_{\lambda=\lambda_{0}}} \\
\quad \times \int_{0}^{\infty} \frac{\operatorname{exp~}\left(-\alpha \tau / r^{2}\right) J_{n+1 / 2}(u) d u}{u^{3 / 2}} \\
p_{n}\left(\lambda_{0}\right)=0 \text { (Legendre polynomial) }
\end{gathered}
$$

Infinite composite cylinder.

$t=t_{i}, I_{3}<t<r_{1}, \tau=0$.

$t=t_{0}, r=r_{3}, \tau>0$.

$q_{r}=0, r=r_{1}$.

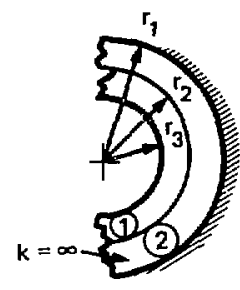

$\frac{t-t_{0}}{t_{i}-t_{0}}=\pi \sum_{n=1}^{\infty} \exp \left(-\lambda_{n}^{2}\right.$ Fo $)$ FG $\left[\lambda_{n}^{2} J_{0}\left(R_{2} \lambda_{n}\right)-N \lambda_{n} J_{1}\left(R_{2} \lambda_{n}\right)\right]$

$F=\frac{J_{0}\left(\lambda_{n} R_{3}\right)}{\left[J_{0}\left(\lambda_{n} R_{3}\right)\right]^{2}\left[\lambda_{n}^{2}-N\left(N-2 / R_{2}\right) \lambda_{n}^{2}\right]-\left[\lambda_{n}^{2} J_{0}\left(R_{2} \lambda_{n}\right)-N \lambda_{n} J_{1}\left(R_{2} \lambda_{n}\right)\right]^{2}}$

$G=J_{0}\left(R \lambda_{n}\right)\left[\lambda_{n}^{2} Y_{0}\left(R \lambda_{n}\right)-N Y_{1}\left(R_{2} \lambda_{n}\right)\right]-Y_{0}\left(R \lambda_{n}\right)$

$\times\left[\lambda_{n}^{2} J_{0}\left(R_{2} \lambda_{n}\right)-N \lambda_{n} J_{1}\left(R_{2} \lambda_{n}\right)\right]$

$R=I / r_{1}, N=\frac{\left(R_{2}-1\right)^{2} \rho_{1} c_{1}}{\left(R_{3}-1\right)^{3} \rho_{2} c_{2}}, J_{0}\left(R_{3} \lambda_{n}\right)=0$ 
Section 9.1. Solids Bounded by Cylindrical Surfaces--No Internal Heating.

Case No. References

Description

Solution

9.1 .2533

Finite cylinder with convection

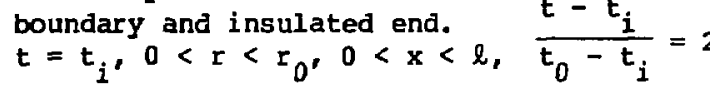

$\tau=0, t=t_{0}, 0<r<r_{0}$,

$x=0, \tau>0 . q_{x}=0$,

$0<r<r_{0}, x=l, \tau>0$.

Convection boundary $h$,

$h_{2}, t_{0}$ at $r=r_{0}$.

占

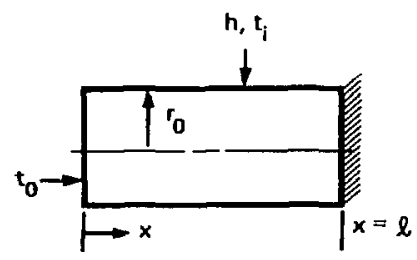

$\times \sum_{m=0}^{\infty} \sum_{n=1}^{\infty} \frac{(2 m+1) B i J_{0}\left(\lambda_{n} R\right) \cos \left[\left(\frac{2 m-1}{2}\right)(1-x) \pi\right]}{L^{2}\left[\lambda_{n}^{2}+(2 m+1)^{2} \pi^{2} / 4 L^{2}\right]\left(\lambda_{n}^{2}+B i^{2}\right) J_{0}\left(\lambda_{n}\right)}$.

$$
\times \exp \left\{-\mathrm{Fo}\left[\lambda_{n}^{2}+\frac{(2 m+1)^{2} \pi^{2}}{4 L^{2}}\right]\right\}
$$

$$
\lambda_{n} J_{1}\left(\lambda_{n}\right)=B i J_{0}\left(\lambda_{n}\right)
$$

$\mathrm{Bi}=\mathrm{hr} \mathrm{r}_{0} / \mathrm{k}, \mathrm{L}=\mathrm{l} / \mathrm{r}_{0}, \mathrm{R}=\mathrm{r} / \mathrm{r}_{0}, \mathrm{X}=\mathrm{x} / \mathrm{l}, \mathrm{Fo}=\alpha \pi / \mathrm{r}_{0}^{2}$ 
Section 9.1. Solids Bounded by Cylindrical Surfaces--No Internal Heating.

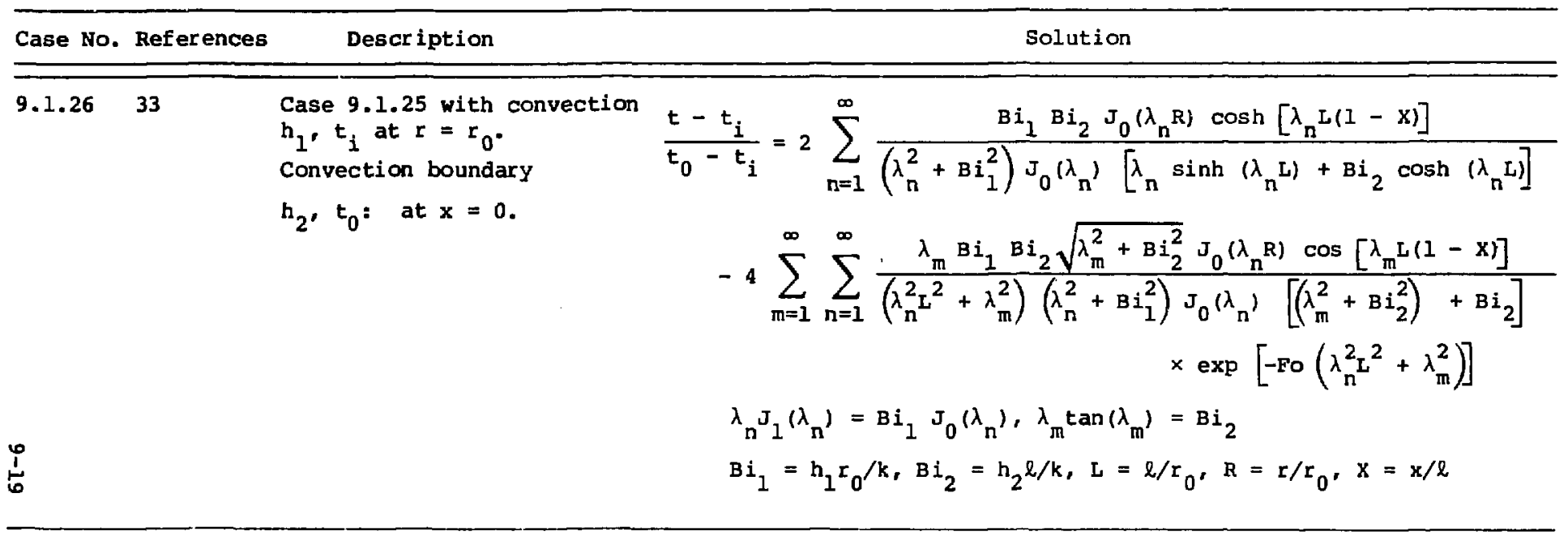


Section 9.1. Solids Bounded by Cylindrical Surfaces--No Internal Heating.

\begin{tabular}{|c|c|c|c|}
\hline Case No. & References & Description & Solutior, \\
\hline 9.1 .28 & 33 & $\begin{array}{l}\text { Finite cylinder with a } \\
\text { linear time dependent } \\
\text { temperature on one end. } \\
t=t_{i}, 0<\tau<r_{0^{\prime}} \\
0<x<\ell, \tau=0 . \\
t=t_{i}+b\left(t_{0}-t_{i}\right) \tau, \\
x=0,0<\tau<b . \\
t=t_{0}, x=0, \tau>b . \\
q_{x}=0,0<r<r_{0}, x=l, \\
\tau>0 . \text { Convection boundary } \\
h, t_{i}: \text { at } r=r_{0}, \\
t=f(\tau) \rightarrow t_{0}\end{array}$ & 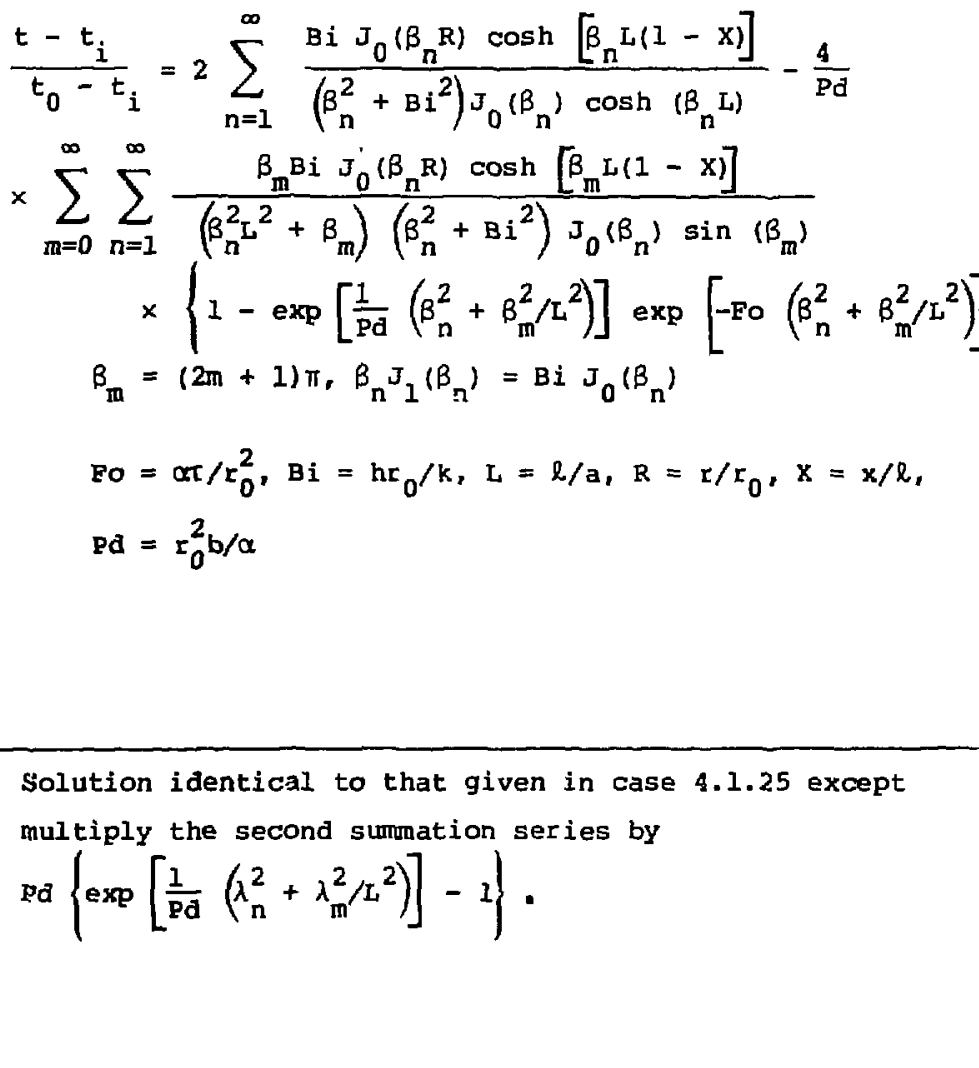 \\
\hline
\end{tabular}


Seation 9.1. Solids Bounded by Cylindrical Surfaces--No Internal Heating.

\section{Case No. References}

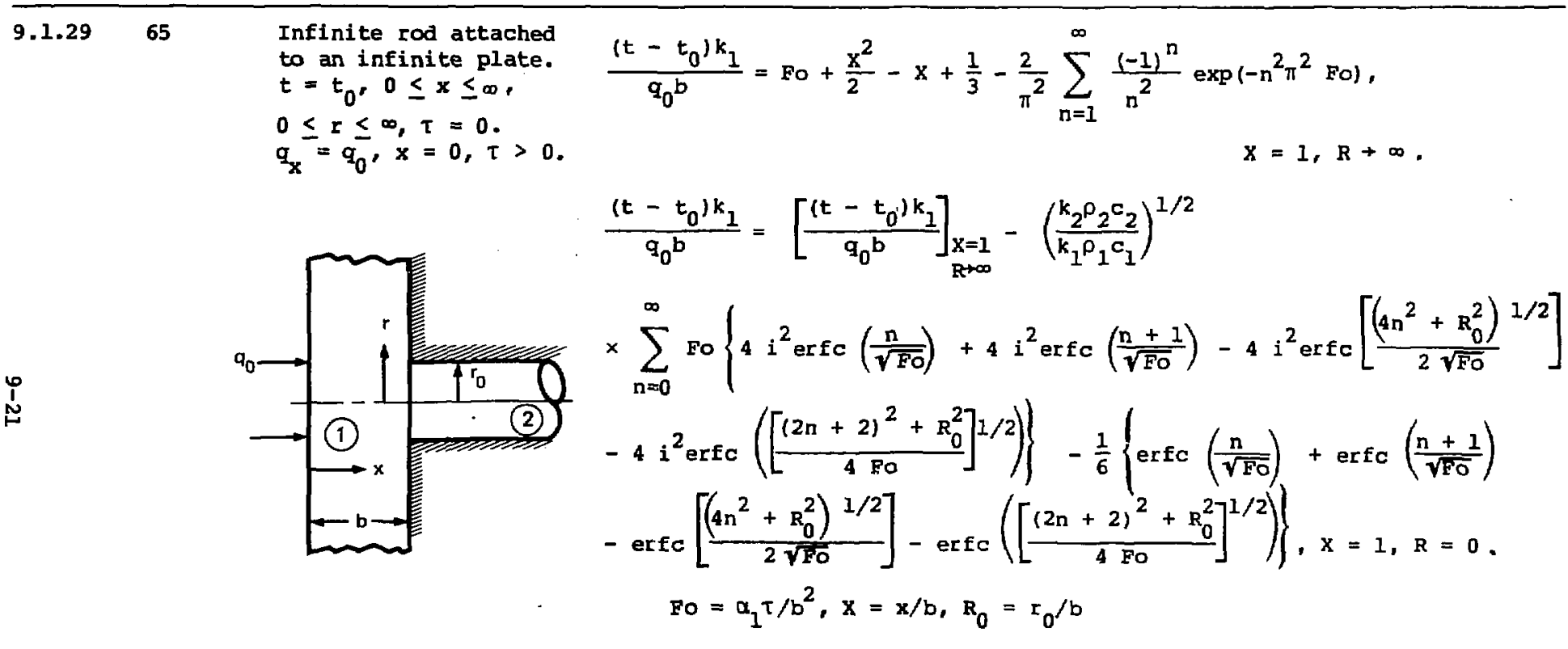


Section 9.1. Solids Bounded by Cylindrical Surfaces--No Internal teating. 
Section 9.1. Solids Bounded by Cylindrical Surfaces--No Internal Heating.

Case No. References Description Solution


Section 9.2. Solids Bounded by Cylindrical Surfaces--With Internal Heating.

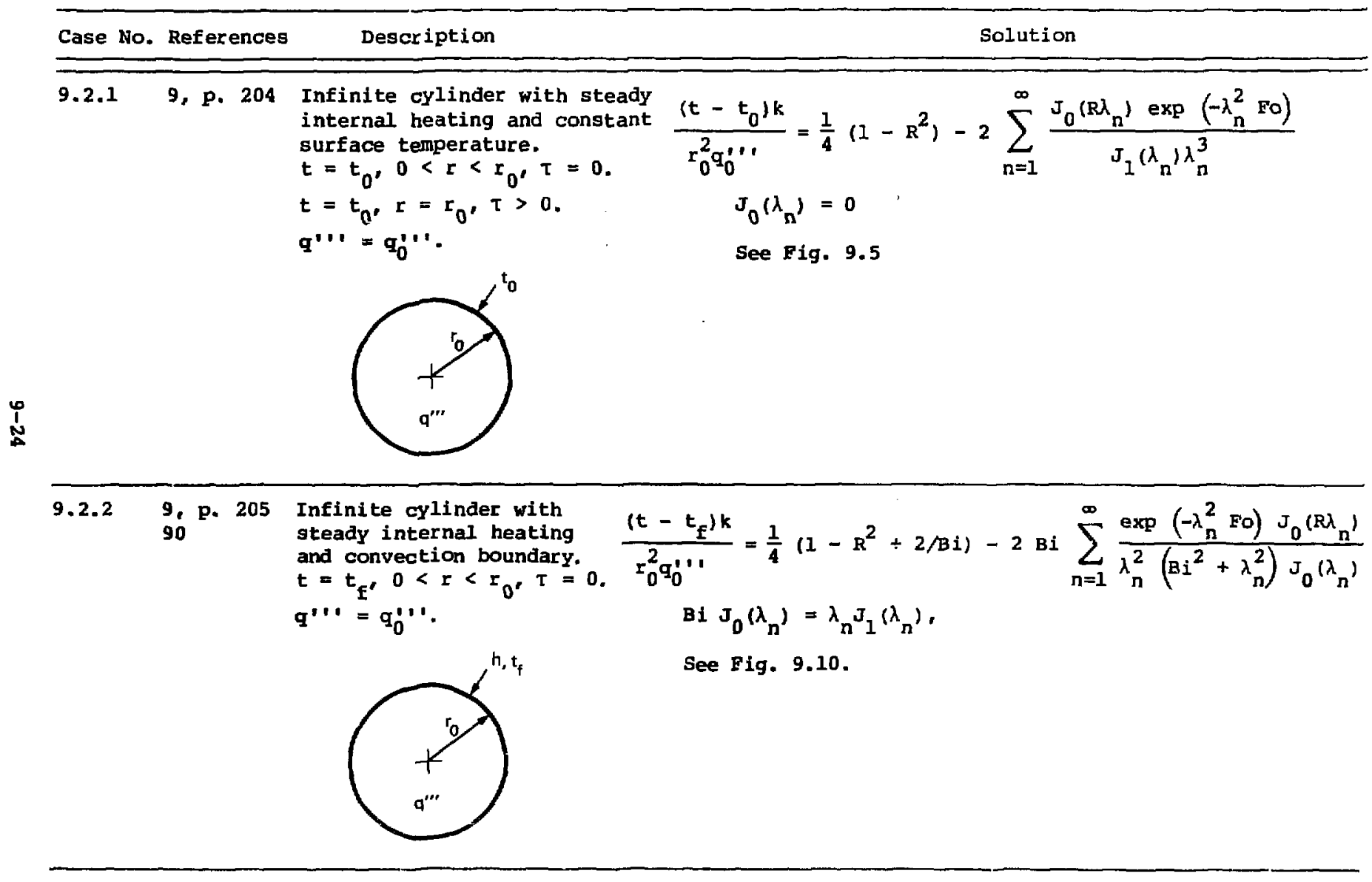


Section 9.2. Solids Bounded by Cylindrical Surfaces--With Internal Heating.

Case No. References Description Solution

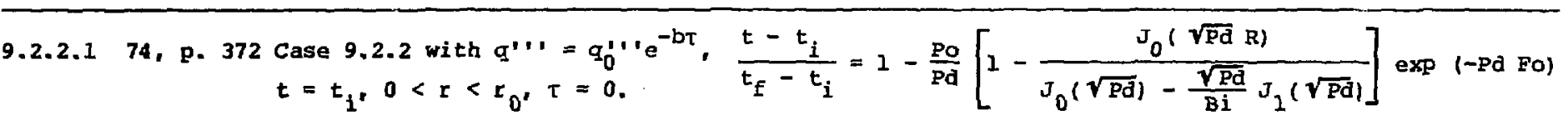

$-\sum_{n=1}^{\infty}\left(1-\frac{P O}{P d-\lambda_{n}^{2}}\right) A_{n} J_{0}\left(\lambda_{n}^{R}\right) \exp \left(-\lambda_{n}^{2}\right.$ Fo $)$

$$
A_{n}=\frac{2 B i}{J_{0}\left(\lambda_{n}\right)\left(\lambda_{n}^{2}+B i^{2}\right)} \cdot \frac{J_{0}\left(\lambda_{n}\right)}{J_{1}\left(\lambda_{n}\right)}=\frac{\lambda_{n}}{B i}
$$

ì

\subsubsection{9, p. 204 Infinite cylinder with} exponential time-dependent internal heating.

$t=t_{0}, 0<r<r_{0} r=0$.

$$
\begin{aligned}
& \frac{\left(t-t_{0}\right) k}{r_{0}^{2} q_{0}^{\prime \prime \prime}}=\frac{\exp (-b c)}{P d}\left\{\frac{J_{0}(R \sqrt{P d})}{J_{0}(\sqrt{P d})}-1\right\} \\
& -\frac{2}{\operatorname{Pd}} \sum_{n=1}^{\infty} \frac{\exp \left(-F o \lambda_{n}^{2}\right) J_{0}\left(R \lambda_{n}\right)}{\lambda_{n}\left(\lambda_{n}^{2} / P d-1\right) J_{1}\left(\lambda_{n}\right)}
\end{aligned}
$$$$
t=t_{0}, r=t_{0}, \tau>0 \text {. }
$$$$
q^{\prime \prime \prime}=q_{0}^{\prime}{ }^{\prime} e^{-b \tau} \text {. }
$$

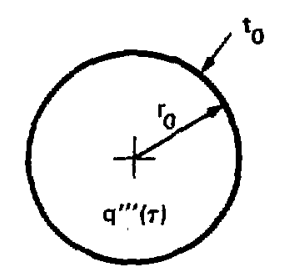


Section 9.2. Solids Bounded by Cylindrical Surfaces--With Internal Heating.

\section{Case No. References}

Description

9.2.4 3, p. 363 Infinite pipe with steady fluid flow and sudden heat generation in the wall. Fluid: $t=t_{1}, \tau=0$. Pipe: $t=t_{i}, \tau=0$. Fluid velocity $=\mathrm{v}$. Wall heating: $q^{\prime \prime \prime}=q_{0}^{\prime \prime} '$, $\tau>0$.

No axial conduction.

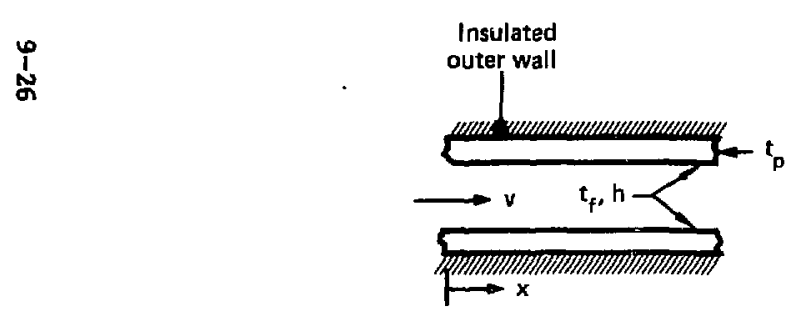

$\xi=b_{f} x / v, \lambda=1+b_{f} / b_{p}, \tau \hbar=b_{p}(\tau-x / v)$,

$b_{f}=h P_{i} / \rho_{f} c_{f} A_{f}, b_{p}=h p_{i} / \rho_{p} c_{p}{ }_{p}, \tau^{+}=b_{p} \tau$

$A_{f}=$ flow area, $A_{p}=$ pipe section area,

$P_{i}=$ pipe inside perimeter.

See Figs. 9.6a, 9.6b, and 9.6c.
Pipe temp:

$$
\begin{aligned}
& \frac{\left(t_{p}-t_{i}\right) h P_{i}}{q_{0}^{\prime \prime \prime}{ }_{p}(\lambda-1) / \lambda^{2}}=\lambda \tau^{+}+\left(\frac{1}{\lambda-1}\right)\left(1-e^{\lambda \tau^{*}}\right)-e^{-\xi}\left[\left(\lambda \tau^{\star}-1\right)\right. \\
& \quad \times \Psi\left(\xi, \tau^{*}\right)-\lambda \phi\left(\xi, \tau^{*}\right)+\left(\frac{1}{\lambda-1}\right) e^{\left.-\lambda \tau^{*} \Lambda\left(\xi, \tau^{*}, \lambda\right)\right], \tau^{+} \geq(\lambda-1) \xi .}
\end{aligned}
$$

Fluid temp:

$$
\begin{aligned}
& \frac{\left(t_{f}-t_{i}\right) h P_{i}}{q_{0}^{\prime \prime \prime} A_{p}(\lambda-1) / \lambda^{2}}=\lambda \tau^{+}-\left(1-e^{\left.-\lambda \tau^{+}\right)}-e^{-\xi}\right. \\
& \quad \times\left[\left(\lambda \tau^{*}+\lambda-1\right) \psi\left(\xi, \tau^{*}\right)-\lambda \phi\left(u, \tau^{*}\right)-e^{-\lambda \tau^{*}} \Lambda\left(\xi, \tau^{\star}, \lambda\right)\right], \tau^{+} \geq(\lambda-1) \xi . \\
& \Psi\left(\xi, \tau^{*}\right)=\sum_{n=0}^{\infty} \frac{\xi^{n}}{(n !)^{2}} \int_{0}^{\tau^{*}} \delta e^{-\delta} d \delta
\end{aligned}
$$$$
\phi\left(\xi, \tau^{*}\right)=\sum_{n=0}^{\infty} \frac{\xi^{n}}{(n !)^{2}} \int_{0}^{\tau^{*}} \delta^{n+1} e^{-\delta} d \delta
$$$$
\Lambda\left(\xi, \tau^{*}, \lambda\right)=\sum_{n=0}^{\infty} \frac{[\xi /(\lambda-1)]^{n}}{(n !)^{2}} \int_{0}^{(\lambda-1) \tau^{*}} \delta^{n} e^{\delta} d \delta
$$ 
Section 9.2. Solids Bounded by Cylindrical Surfaces--With Internal Heating.

Case No. References Description Solution

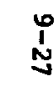

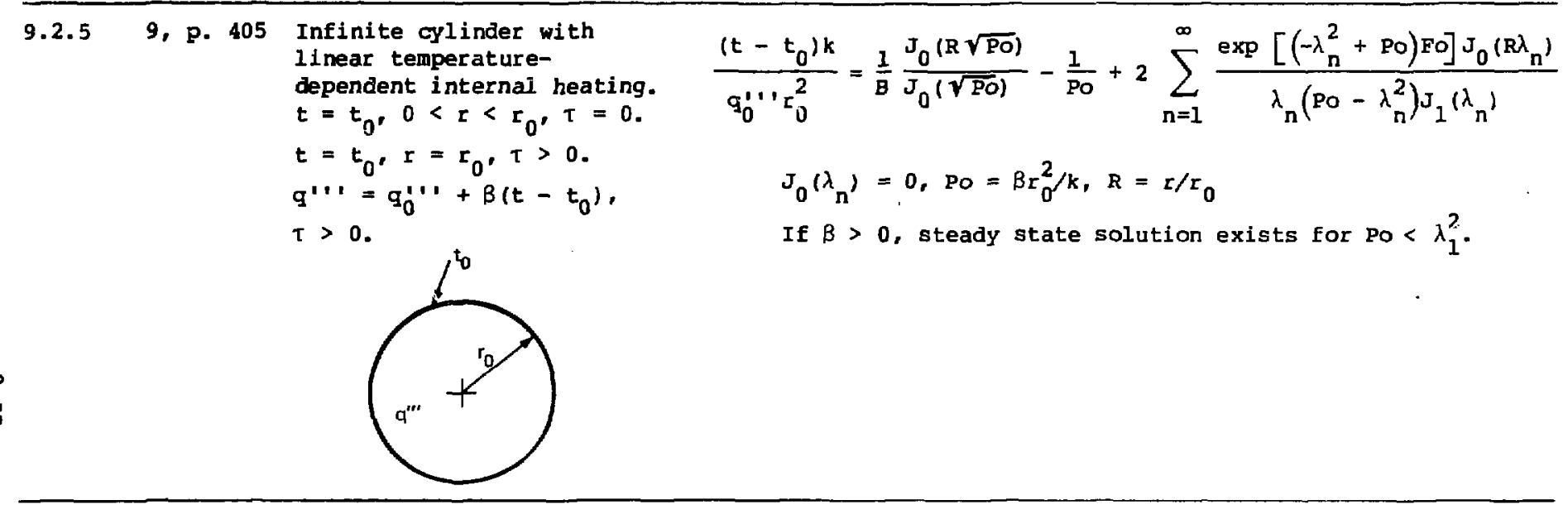

\begin{tabular}{lll}
\hline 9.2 .6 & 19, & Infinite cylinder of \\
p. 3-29 & $\begin{array}{l}\text { infinite conductivity, } \\
\text { variable specific heat, } \\
\text { convection boundary, and } \\
\text { unifom heating. }\end{array}$
\end{tabular}

See case B.2.11, set $L=2$, radius $=\ell$.

uniform heating.

$\begin{array}{lll}9.2 .7 & 19 . & \text { Case } 9.2 .6 \text { with a short } \\ & \text { p. } 3-29 & \text { cylinder of length } 2 l .\end{array}$

See case 8.2 .11 , set $L=3$, radius $=\ell$. cylinder of length $2 l$. 
Section 9.2. Solids Bounded by Cylindrical Surfaces--With Internal Heating.

\begin{tabular}{|c|c|c|c|}
\hline Case No. & References & Description & Solution \\
\hline 9.2 .8 & 63 & $\begin{array}{l}\text { Hollow infinite cylinder } \\
\text { with exponentially space } \\
\text { varying heat generation. } \\
t=t_{i}, r_{i}<r<r_{0}, \tau=0 \\
t=t_{1}(\tau), r=r_{1}, \tau>0 \\
t=t_{2}(\tau), r=r_{2}, \tau>0 \\
q^{\prime \prime}=\operatorname{mi} \exp \left[-\beta\left(r-r_{1}\right)\right]\end{array}$ & 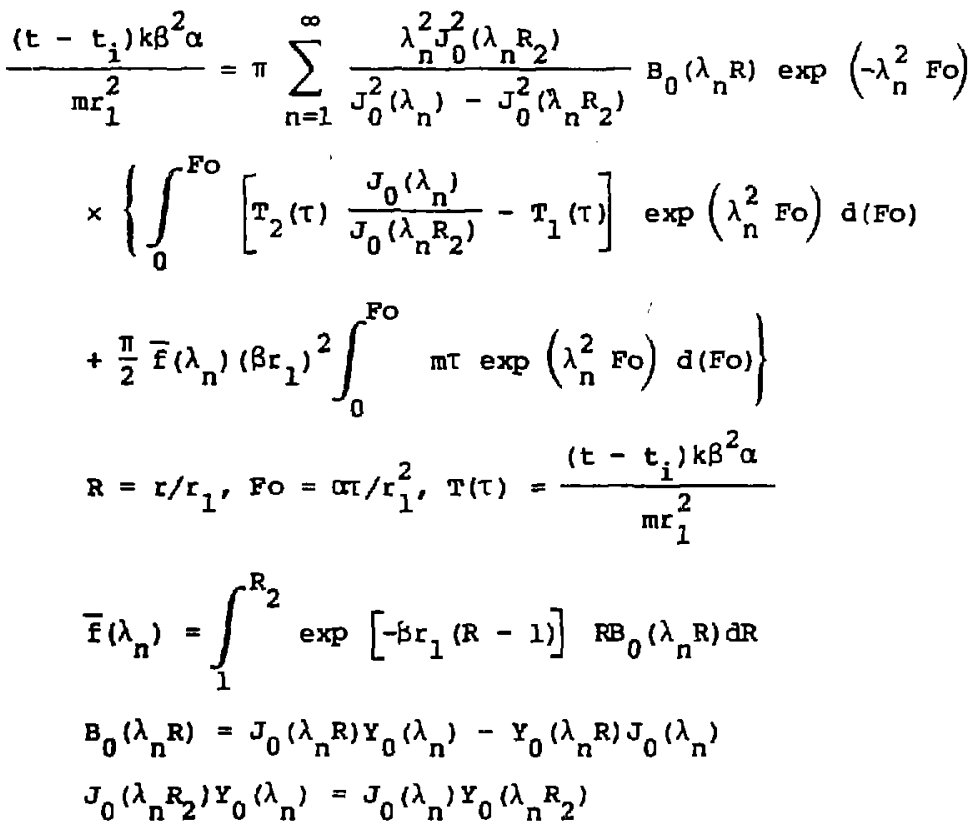 \\
\hline
\end{tabular}


Section 9.2. Solids Bounded by Cylindrical Surfaces-With Internal Heating.

9.2.9 74, p. 375 Infinite cylinder with a steady surface heat flux and steady internal heating. $t=t_{i}, 0<r<r_{0}, \tau=0$. $q^{\prime \prime \prime}=q_{0}^{\prime \prime \prime} 0<r<r_{0^{\prime}} \tau>0$. $q_{r}=q_{0}, r=r_{0}, \tau>0$.

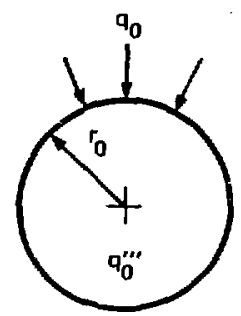

$$
\frac{\left(t-t_{i}\right) k}{q_{0}^{\prime} ' r_{0}^{2}}=F_{0}+\phi
$$

$$
\begin{aligned}
& \phi=\frac{K i}{F_{0}}\left[2 \text { Fo }-\frac{1}{4}\left(I-2 R^{2}\right)-2 \sum_{n=1}^{\infty} \frac{J_{0}\left(\lambda_{n} R\right) \exp \left(-\lambda_{n}^{2} F o\right)}{\lambda_{n}^{2} J_{0}\left(\lambda_{n}\right)}\right] \\
& J_{0}^{\prime}\left(\lambda_{n}\right)=0
\end{aligned}
$$

9.2.10 74, p. 375 Case 9.2.9 with

$q^{\prime \prime \prime}=q_{0}^{\prime \prime}\left(1-R^{2}\right)$

$$
\begin{aligned}
& \frac{\left(t-t_{i}\right) k}{q_{0}^{\prime \prime \prime} r_{0}^{2}}=\frac{1}{3} \text { Fo }+\pi \sum_{n=1}^{\infty} \frac{\left[2 J_{i}^{\prime}\left(\lambda_{n}\right)-J_{0}\left(\lambda_{n}\right)\right]}{\lambda_{n}^{3} J_{0}^{2}\left(\lambda_{n}\right)} \\
& \quad \times J_{0}\left(\lambda_{n} R\right)\left[1-\exp \left(-\lambda_{n}^{2} F_{0}\right)\right]+\phi \\
& \phi \text { given in case } 9.2 .9
\end{aligned}
$$


Section 9.2. Solids Bounded by Cylindrical Surfaces--With Internal Heating.

\begin{tabular}{|c|c|c|c|}
\hline Case No. & Réferences & Description & Solution \\
\hline 9.2 .11 & 74, p. 375 & $\begin{array}{l}9.2 .9 \text { with } \\
=q_{0}^{\prime \prime \prime}(1+b \tau) .\end{array}$ & $\frac{\left(t-t_{i}\right) k}{q_{0}^{\prime \prime \prime} r_{0}^{2}}=\mathrm{FO}\left(1+\frac{1}{2}\right.$ Pd Fo $)+\phi$ \\
\hline
\end{tabular}

$\begin{aligned} & 9.2 .1274, \text { p. } 376 \text { case } 9.2 .9 \text { with } \frac{\left(t-t_{i}\right) k}{q^{\prime \prime \prime}=q_{0}^{\prime \prime \prime} \exp (-b \tau) .} \quad \frac{1}{q_{0}^{\prime \prime} r_{0}^{2}}[1-\exp (-P d F o)]+\phi \\ & \phi \text { given in case } 9.2 .9\end{aligned}$

西

$\stackrel{0}{\vdots}$

$$
\frac{\left(t-t_{i}\right) k}{q^{\prime \prime} r_{0}^{2}}=\frac{1}{P d} \sin (P d F O)+\phi
$$

given in case 9.2 .9

9.2 .1474$, p. 376 Case 9.2 .9 with

$q^{\prime \prime \prime}=q_{0}^{\prime \prime}{ }^{\prime \prime}{ }^{n}$.

$\frac{\left(t-t_{1}\right) k}{q{ }^{\prime \prime} r_{0}^{2}}=\frac{(P d \text { Fo })^{n}}{n+1} \mathrm{Fo}+\phi$

$\emptyset$ given in case 9.2 .9 
Section 9.2. Solids Bounded by Cylindrical Surfaces--With Internal Heating.

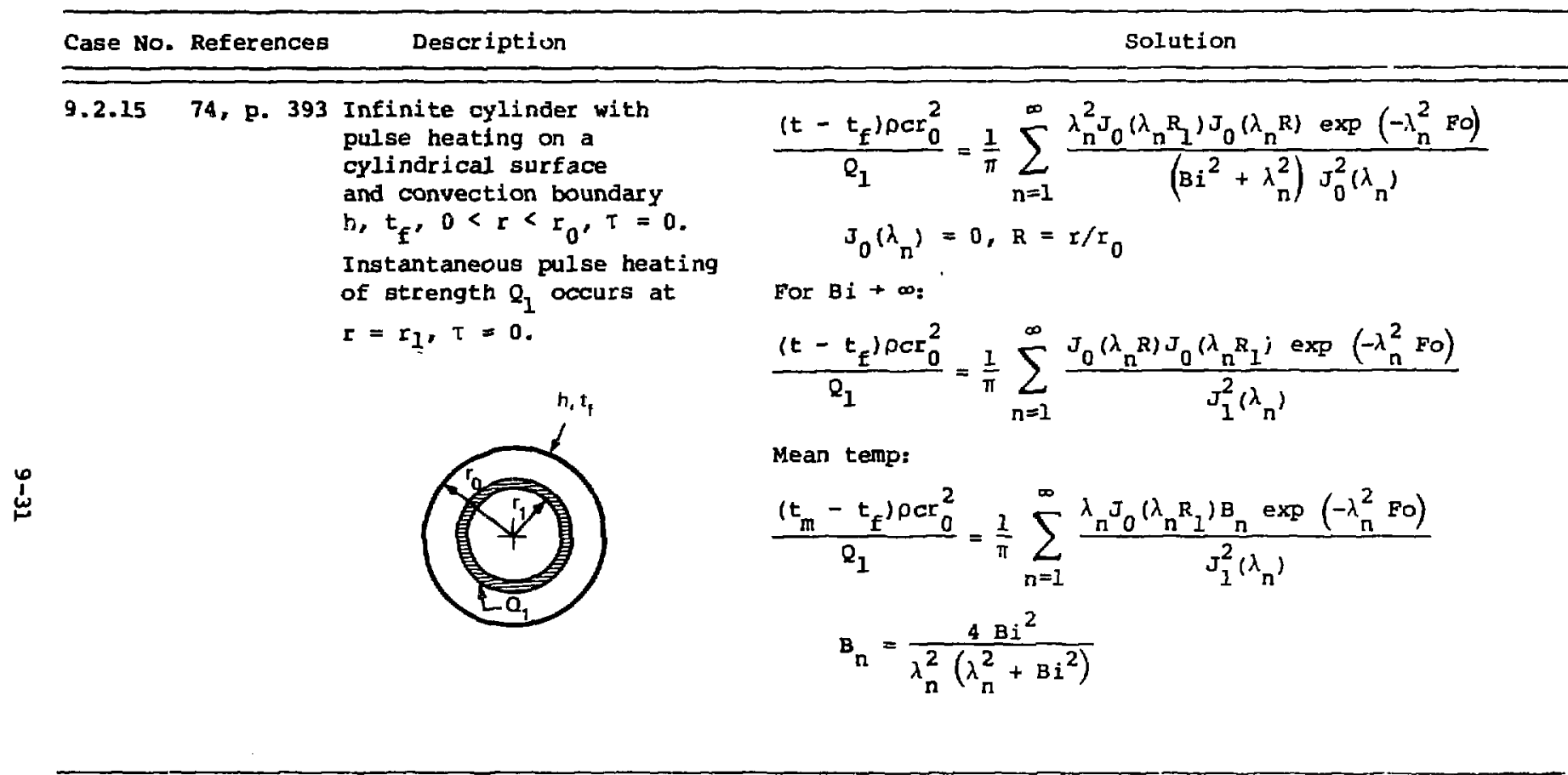


Section 9.2. Solids Bounded by Cylindrical Surfaces--With Internal Heating.

Case No. References Description Solution


Section 9.2. Solids Bounded by Cylindrical Surfaces--With Internal Heating. 


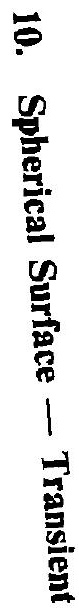

REPRODUCED FROM BEST AVAILABLE COPY 
Section 10.1. Solids Bounded by Spherical Surfaces--No Internal Heating.

\section{Case No. References}

10.1.1 2, p. 239 Sphere with steady

74, p. 126 surface temperature.

$t=t_{i}, 0<r<r_{0}, \tau=0$.

$t=t_{0}, r=\varepsilon_{0}, \tau>0$.

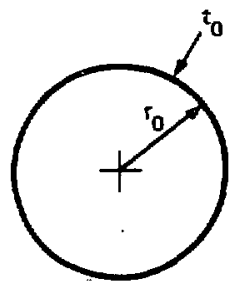

$$
\begin{aligned}
& \frac{t-t_{0}}{t_{i}-t_{0}}=\frac{2}{\pi} \sum_{n=1}^{\infty} \frac{(-1)^{n+1}}{n} \exp \left(-n^{2} \pi^{2} \text { Fo) } \sin (n \pi R)\right. \\
& \quad=1-\frac{1}{R} \sum_{n=1}^{\infty}\left[\operatorname{erfc}\left(\frac{2 n-1-R}{2 \sqrt{F O}}\right)-\operatorname{erfc}\left(\frac{2 n-1+R}{2 \sqrt{F O}}\right)\right], \text { Fo }<1 .
\end{aligned}
$$

Mean temp:

$\frac{t_{m}-t_{0}}{t_{1}-t_{0}}=\frac{6}{\pi^{2}} \sum_{n=1}^{\infty} \frac{1}{n^{2}} \exp \left(-n^{2} \pi^{2} \mathrm{Fo}\right)$.

Cumulative heat rate:

$$
\frac{Q}{Q_{0}}=\frac{6}{\pi^{3}} \sum_{n=1}^{\infty} \frac{(-1)^{n+1}}{n^{3}}\left[1-\exp \left(-n^{2} \pi^{2} F o\right)\right], Q_{0}=\frac{4}{3} \pi r_{0}^{3} \rho c\left(t_{0}-t_{i}\right) \text {. }
$$

See Fig. 10.1 
Section 10.1. Solids Bounded by Spherical Surfaces--No Internal Heating.

Case No. References Description Solution

10.1.2.1 74, Sphere with convection

p. 254 boundary-general case

$t=f(r), 0<r<r_{0}$

$\tau=0$.

$$
\begin{array}{r}
t-t_{f}=2 \sum_{n=1}^{\infty} \frac{\lambda_{n} \sin \left(\lambda_{n} R\right) \exp \left(-\lambda_{n}^{2} \text { Fo }\right)}{\left[\lambda_{n}-\sin \left(\lambda_{n}\right) \cos \left(\lambda_{n}\right)\right] R} \int_{0}^{1} R\left[f(r)-t_{f}\right] \\
x \sin \left(\lambda_{n} R\right) d R .
\end{array}
$$

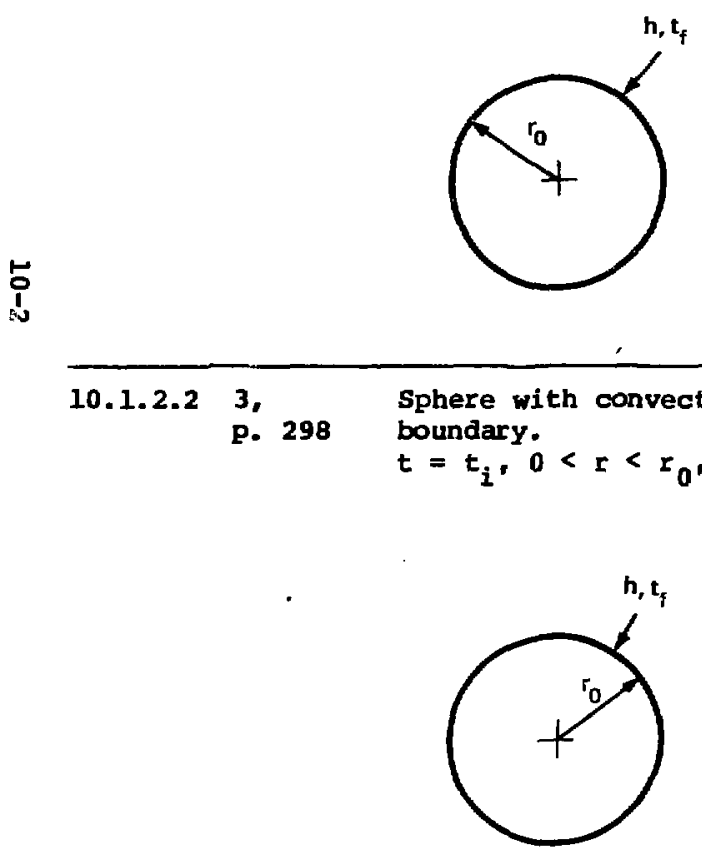

$$
\tan \left(\lambda_{n}\right)=\frac{\lambda_{n}}{(1-B i)}
$$

See Table 14.2 of roots

Sphere with convection

indary.

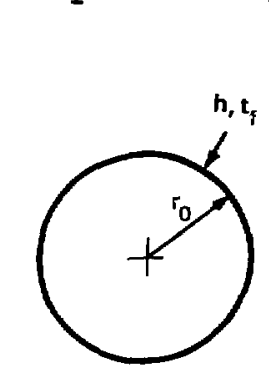

$\frac{t-t_{f}}{t_{i}-t_{f}}=2 \sum_{n=1}^{\infty} \frac{B i \sin \left(\lambda_{n}\right) \exp \left(-\lambda_{n}^{2} \text { Fo }\right) \sin \left(\lambda_{n} R\right)}{\lambda_{n}-\sin \left(\lambda_{n}\right) \cos \left(\lambda_{n}\right) \lambda_{n} R}$

$\lambda_{n} \cos \left(\lambda_{n}\right)=(1-\theta i) \sin \left(\lambda_{n}\right)$

Cumulative heat rate:

$$
\begin{aligned}
\frac{Q}{Q_{0}}= & 6 \sum_{n=1}^{\infty} \frac{\left[\sin \left(\lambda_{n}\right)-\lambda_{n} \cos \left(\lambda_{n}\right)\right]\left[1-\exp \left(-\lambda_{n}^{2} F\right)\right]}{\lambda_{n}^{3}\left[\lambda_{n}-\sin \left(\lambda_{n}\right) \cos \left(\lambda_{n}\right)\right]} \\
& Q_{0}=\frac{4}{3} \pi r_{0}^{3} \rho c\left(t_{i}-t_{f}\right) \\
& \text { See Figs. } 10.2 a, 10.2 b, \text { and } 10.2 c
\end{aligned}
$$


Section 10.1. Solids Bounded by Spherical Surfaces--No Internal Heating.

Case No. References Description Solution

10.1.3 9, p. 233 Sphere with initial term $f(r)$ and surface temp $\phi(\tau)$, general case.

\section{$\phi(\tau)$}

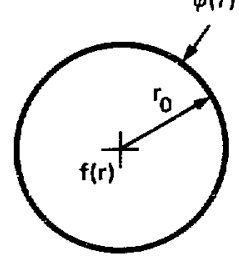

$T=\frac{2}{R} \sum_{n=1}^{\infty} \exp \left(-n^{2} \pi^{2}\right.$ FO $\sin (n \pi R)$

$\times\left\{\int_{0}^{1} \bar{R} f(\bar{R}) \sin (n \pi \bar{R}) d \bar{R}-n \pi(-1)^{n}\right.$ Fo $\left.\int_{0}^{1} \exp \left(n^{2} \pi^{2} F_{0} \lambda\right) \phi(\lambda) d \lambda\right\}$
占 $10.1 .4 \quad 9$. p. 235
Sphere with surface temp proportional to time. $t=t_{i}, 0<r<t_{0}, \tau=0$. $t_{0}=b \tau+t_{i}, I=I_{0}, \tau>0$.

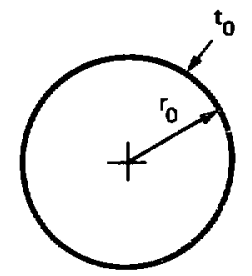

$$
\frac{t-t_{i}}{t_{0}-t_{i}}=\left(I+\frac{R^{2}-1}{6 \text { Fo }}\right)-\frac{2}{\pi^{3} \text { Fo } R} \sum_{n=I}^{\infty} \frac{(-1)^{n}}{n^{3}} \operatorname{exg}\left(-n^{2} \pi^{2} \text { Fo }\right) \sin (\pi \pi R)
$$

See Table 10.1 and Fig. 10.8 
Section 10.1. Solids Bounded by Spherical Surfaces--No Internal Heating.

Case No. References

Description

Solution

10.1.5 9, p. 235 Sphere with variable Sphere with variable
initial temp.
$t=\left(t_{c}-t_{0}\right)\left(r_{0}-r\right) /\left(r_{0}+t_{0}\right) \cdot \frac{t-t_{0}}{t_{c}-t_{0}}=\frac{8}{\pi^{3}} \sum_{n=0}^{\infty} \frac{1}{(2 n+1)^{3}} \exp \left[-\pi^{2}(2 n+1)^{2}\right.$ Fo $] \sin [(2 n+1) \pi R]$
$0<r<r_{0} \tau=0$.

$0<r<r_{0}, \tau=0$.

$t=t_{0}, r=r_{0}, \tau>0$.

$\stackrel{5}{1}$

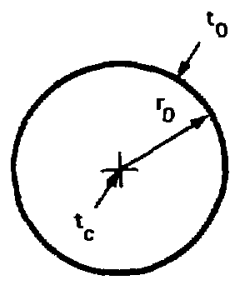

10.1.6 9, p. 236 Sphere with variable initial temp (parabolic).

$$
\begin{aligned}
& t=\left(t_{c}-t_{0}\right)\left(r_{0}^{2}-r^{2}\right) /\left(r_{0}^{2}+t_{0}\right), \\
& 0<r<r_{0}, \tau=0 . \\
& t=t_{0}, r=r_{0}, \tau>0 .
\end{aligned}
$$$$
\frac{t-t_{0}}{t_{c}-t_{0}}=\frac{12}{\pi^{3}} \sum_{n=1}^{\infty} \frac{(-1)^{n-1}}{n^{3}}
$$$$
\sin (n \pi R) \exp \left(-n^{2} \pi^{2} \mathrm{FO}\right)
$$

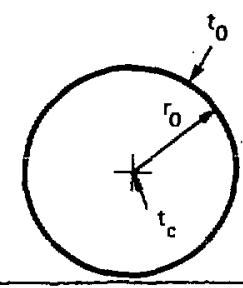


Section 10.1. Solids Bounded by Spherical Surfaces--No Internal Heating.

Case No. References Description Solution

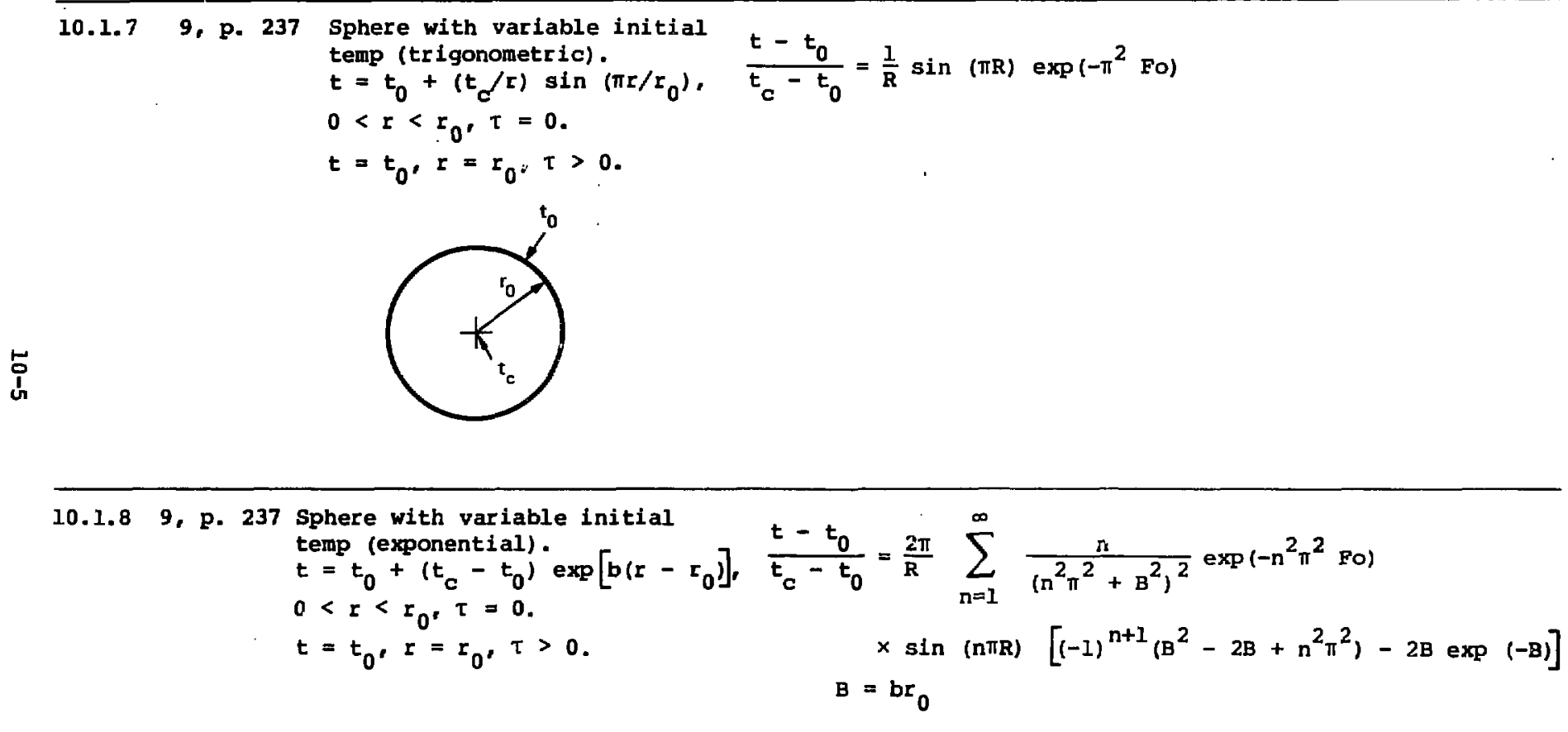


Section 10.1. Solids Bounded by Spherical Surfaces -No Internal Heating.

\section{Case No. References}

Description

Solution

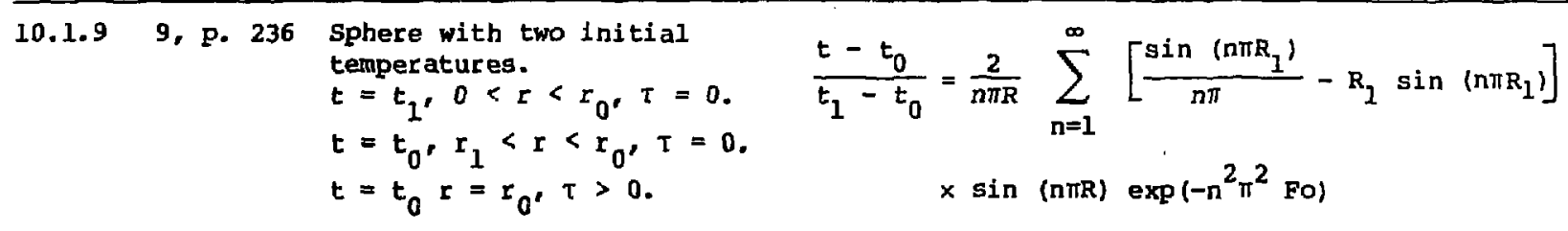

5.

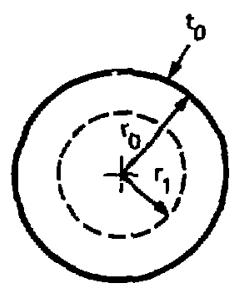

10.1.10 9, p. 237 Sphere with polynaminal

initial temperature.

$t=t_{1}-t_{0}+b_{1} r+b_{2} r^{2}+b_{3} r^{3}$

$\frac{t-t_{0}}{t_{1}-t_{0}}=2 \sum_{n=1}^{\infty} \sin (n \pi R)\left\{\frac{1}{n \pi R}(-1)^{n \cdot \dot{r} I}+\frac{b_{1}^{*}}{n^{3} \pi^{3}}\left[\left(n^{2} \pi^{2}-2\right)\right.\right.$

$+\ldots, 0<r<r_{0}, \tau=0$.

$t=t_{0}, r=r_{0}, \tau>0$.

$$
\begin{aligned}
& \left.\times(-1)^{n+1}-2\right]+\frac{b_{2}^{*}}{n^{4} \pi^{4}}\left(n^{3} \pi^{3}-6 n \pi\right)(-1)^{n+1}+\frac{b_{3}^{*}}{n^{5} \pi^{5}} \\
& \times\left[24-\left(n^{4} \pi^{4}-12 n^{2} \pi^{2}+24\right\}(-1)^{n}+\ldots . .\right] \exp \left(-n^{2} \pi^{2} F O\right) \\
b_{1}^{*} & =b_{1} r_{0} / R, b_{2}^{*}=b_{2} r_{0}^{2} / R_{2} b_{3}^{*}=b_{3} r_{0}^{3} / R, \ldots \ldots
\end{aligned}
$$


Section 10.1. Solids Bounded by Spherical Surfaces--No Internal Heating.

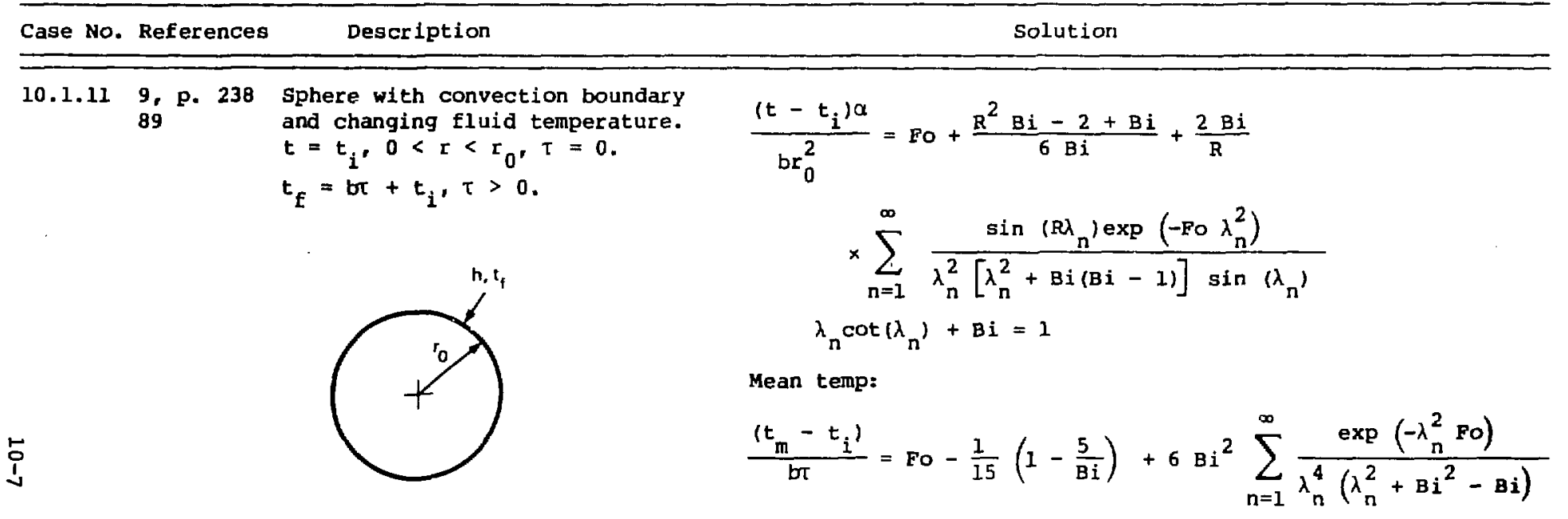

See Fig. 10.9 
Section 10.1. Solids Bounded by Spherical Surfaces--No Internal Beating.

Case No. References Description Solution

$\begin{aligned} \text { 10.1.11.1 74, } & \text { Case 10.1.11 with } \\ \text { P. } 317 & t_{f}=t_{f-}-\left(t_{1}-t_{j}\right) e^{-b \tau} .\end{aligned} \frac{t-t_{i}}{t_{f m}-t_{i}}=1-\frac{R B i \sin (\sqrt{P d} R) \exp (-P d F O)}{(B i-1) \sin (\sqrt{P d}+\sqrt{P d} \cos (\sqrt{P d})}$

p. 317

$$
\begin{aligned}
t_{f}=t_{f m}-\left(t_{f m}-t_{i}\right) e^{-b \tau} \cdot \quad \frac{t-t_{i}}{t_{f m}-t_{i}} & =1-\frac{R B i \sin (\sqrt{P d} R) \exp (-P d}{(B i-1) \sin (\sqrt{P d})+\sqrt{P d} \cos } \\
& -2 B i \sum_{n=1}^{\infty} \frac{A_{n} R \sin \left(\lambda_{n}^{R}\right) \exp \left(-\lambda_{n}^{2} \text { Fo }\right)}{\left(1-\lambda_{n}^{2} / P d\right) \lambda_{n}} \\
A_{n} & =\frac{(-1)^{n+1}\left[\lambda_{n}^{2}+(B i-1)^{27}\right] / 2}{\left(\lambda_{n}^{2}+B i^{2}-B i\right)}
\end{aligned}
$$

\section{Mean temp:}

$$
\begin{aligned}
\frac{t_{m}-t_{i}}{t_{f m}-t_{i}} & =1-\frac{3 B i[\tan (\sqrt{P d})-\sqrt{P d}] \exp (-P Q F O)}{\operatorname{Pd}[(B i-1) \tan (\sqrt{P d})+\sqrt{P d}]} \\
& -6 B^{2} \sum_{n=1}^{\infty} \frac{\exp \left(-\lambda_{n}^{2} F O\right)}{\lambda_{n}^{2}\left(\lambda_{n}^{2}+B i^{2}-B i\right)\left(1-\lambda_{n}^{2} / P d\right)}
\end{aligned}
$$


Section 10.1. Solids Bounded by Spherical Surfaces--No Internal Heating.

10.1.12 9, p. 238 Sphere with convection boundary and changing fluid temp.

$t=t_{i}, 0<r<r_{0}, \tau=0$.

$t_{f}=\left(t_{m}-t_{i}\right) \sin (\omega \tau+\varepsilon), \tau>0$.

$\frac{t-t_{i}}{t_{m}-t_{i}}=\frac{2 B i}{\operatorname{PdR}}$

$t_{m}=\max$ Eluid temp.

$\times\left\{\sum_{n=1}^{\infty} \frac{\frac{\lambda_{n}}{P d}\left[\frac{\lambda_{n}^{2}}{P d} \sin (\varepsilon)-\cos (\varepsilon)\right](B i-1) \sin \left(R \lambda_{n}\right)}{\left[\left(\lambda_{n}^{4} / P d\right)+1\right]\left(\lambda_{n}^{2}+B i^{2}-B i\right) \cos \left(\lambda_{n}\right)}\right.$

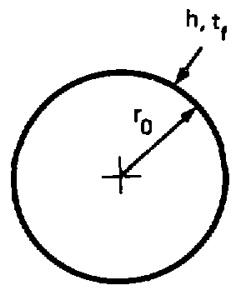

$\left.x \exp \left(-F \circ \lambda_{n}^{2}\right)+\frac{A_{1}}{2 A_{2}} \sin \left(\omega \tau+\varepsilon+\phi_{1}+\phi_{2}\right)\right\}$

$\mathrm{Pd}=\omega \mathrm{r}_{0}^{2} / \alpha$

$\lambda_{n} \cot \left(\lambda_{n}\right)+B i=1$

$A_{1} e^{i \phi_{1}}=\sinh \left(\omega_{1}\right) \cos \left(\omega_{1}\right)+i \cosh \left(\omega_{1}\right) \sin \left(\omega_{1}\right)$

$A_{2} e^{i \phi_{2}}=\omega_{2} \cosh \left(\omega_{2}\right)+(B i-1) \sinh \left(\omega_{2}\right)$

$\omega_{1}=R \sqrt{P d / 2}, \omega_{2}=(1+i) \sqrt{P d / 2}$ 
Section 10.1. Solids Bounded by Spherical Surfaces--No Internal Heating.

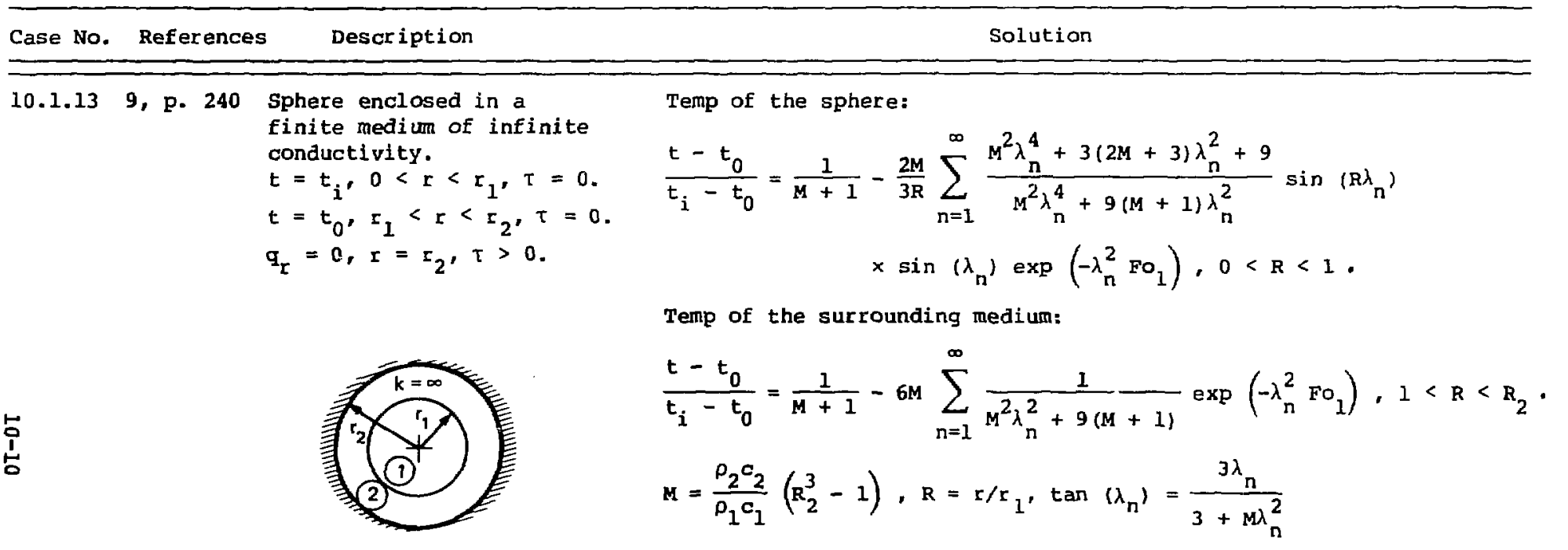

See Fig. 10.3 
Section 10.1. Solids Bounded by Spherical Surfaces--No Internal Heating.

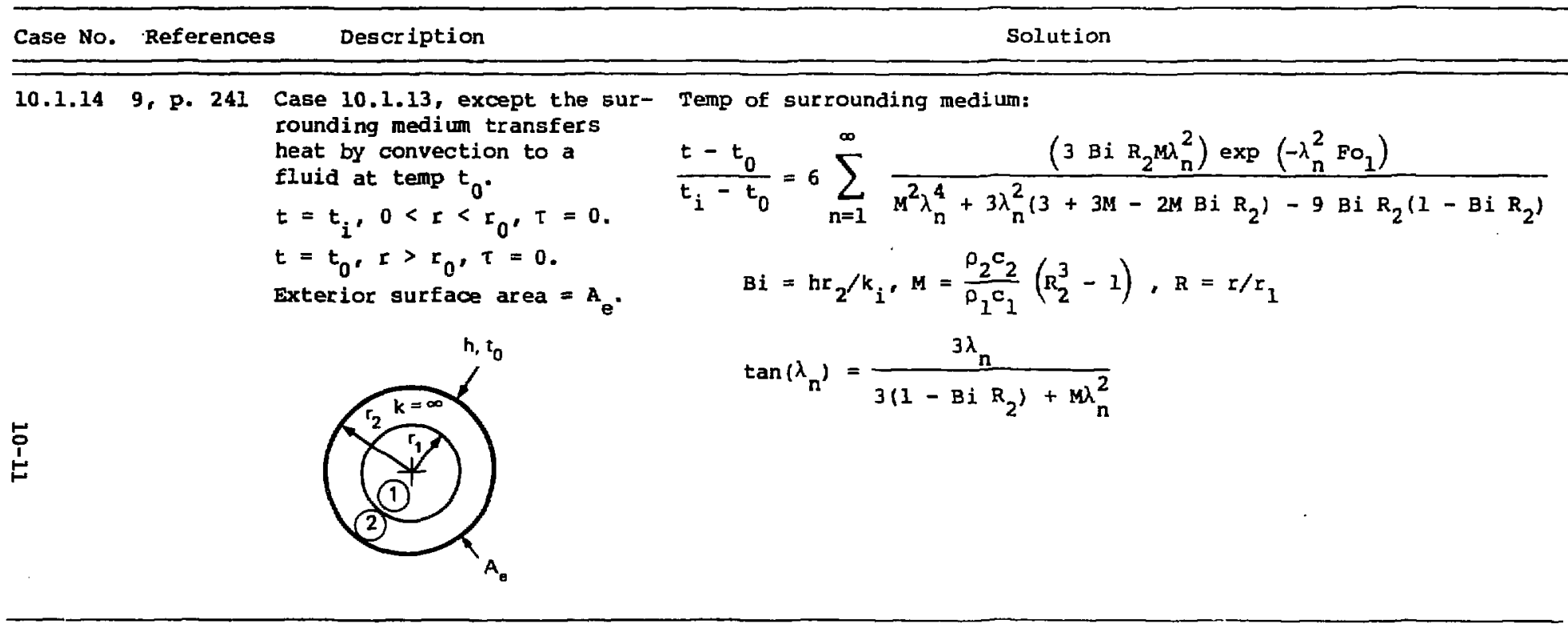


Section 10.1. Solids Bounded by Spherical Surfaces--No Internal Heating.

10.1 .159$, p. 242 sphere with steady surface heat flux.

$t=t_{i}, 0<\varepsilon<r_{0}, \tau=0$.

$q_{r}=q_{0}, r=r_{0}, \tau>0$.

$$
\begin{aligned}
& \frac{\left(t-t_{i}\right) k}{g_{0} r_{0}}=3 \text { Fo }+\frac{5 R^{2}-3}{10}-\frac{2}{R} \sum_{n=1}^{\infty} \frac{\sin \left(R_{2} \lambda_{n}\right) \exp \left(-\lambda_{n}^{2} F_{0}\right)}{\lambda_{n}^{2} \sin \left(\lambda_{n}\right)} \\
& \quad \tan \left(\lambda_{n}\right)=\lambda_{n}
\end{aligned}
$$

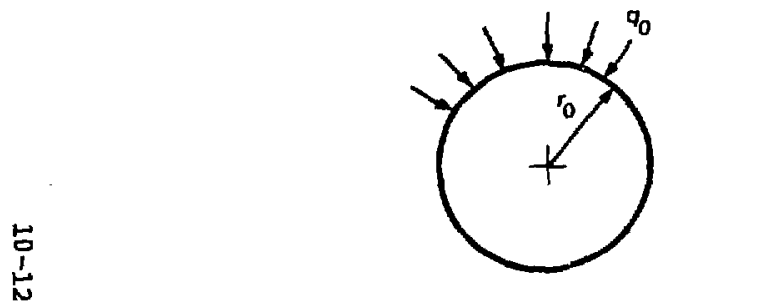

See Eig. 10.4

10.1.16 9, p. 246. Hollow sphere with two surface temperatures. $t=E(r), r_{i}<r<r_{0}$,
$t=t_{i}, r=r_{i}, \tau>0$.

$t=t_{i}, r=r_{1}, \tau>0$.
$t=t_{0}, r=r_{0}, \tau>0$. $\frac{T^{2}}{T_{i}}=\frac{R_{i}}{R}+\frac{\left(T_{0} / T_{i}-R_{i}\right) R^{*}}{R}+\frac{2}{R \pi} \sum_{n=1}^{\infty} \frac{T_{0} / T_{i} \cos (n \pi)-R_{i}}{n}$

$x \sin \left(n \pi R^{*}\right) \exp \left(-n^{2} \pi^{2} F o\right)+\frac{2\left(1-R_{1}\right)}{R} \sum_{n=1}^{\infty} \sin \left(n \pi R^{*}\right)$

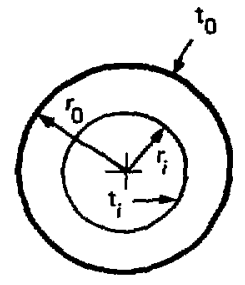
$x \exp \left(-n^{2} \pi^{2}\right.$ FO) $\int_{0}^{I}\left(R^{*}+R\right) E\left(R^{*}\right) \sin \left(n \pi R^{*}\right) d R^{*}$ $R^{*}=\left(r-r_{i}\right) /\left(r_{0}-r_{i}\right), R=r / r_{0}, F_{0}=\alpha T /\left(r_{0}-r_{i}\right)^{2}$ 
Section 10.1. Solids Bounded by Spherical Surfaces--No Internal Heating.

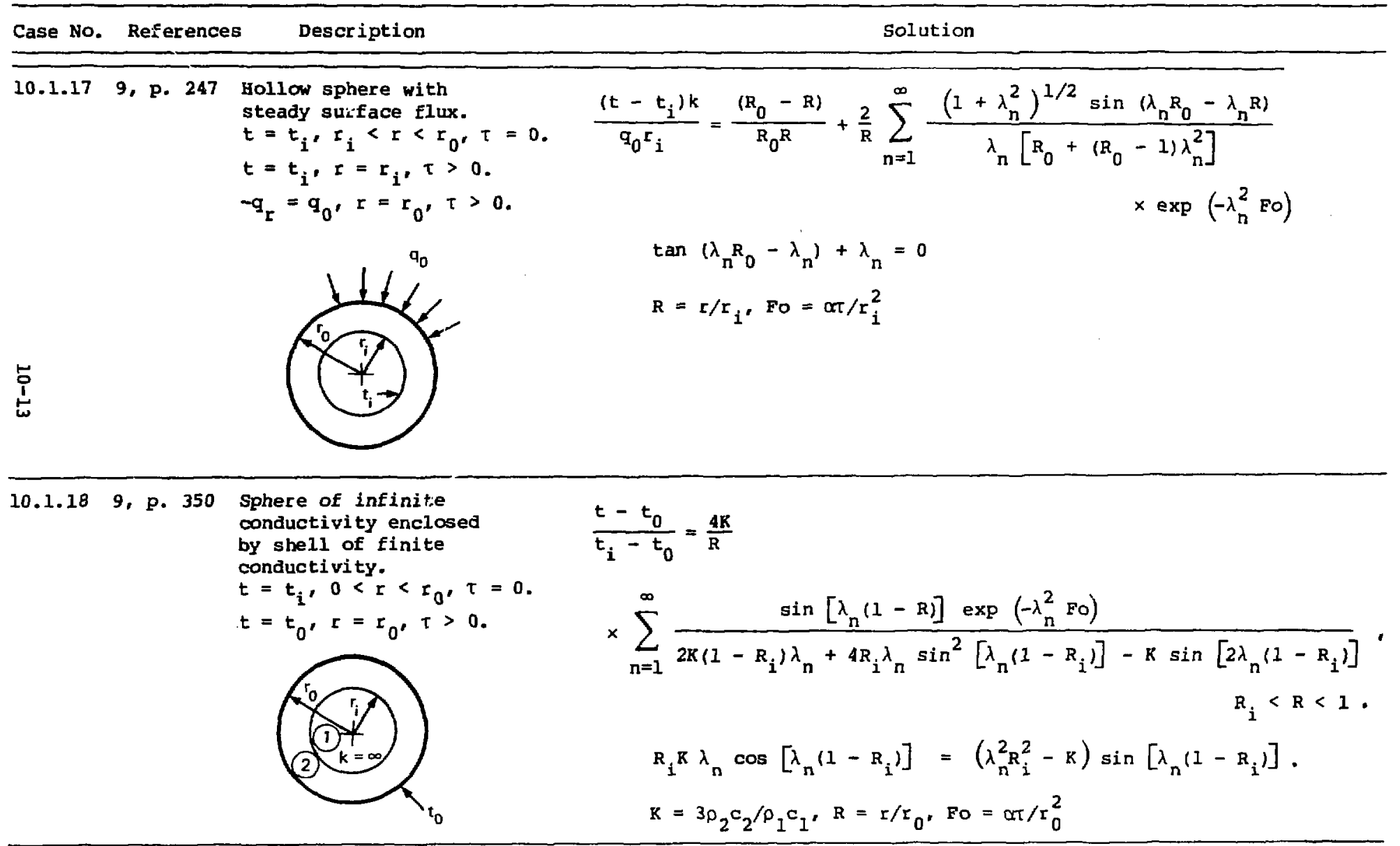


Section 10.1. Solids Bounded by Spherical Surfaces-No Internal Heating.

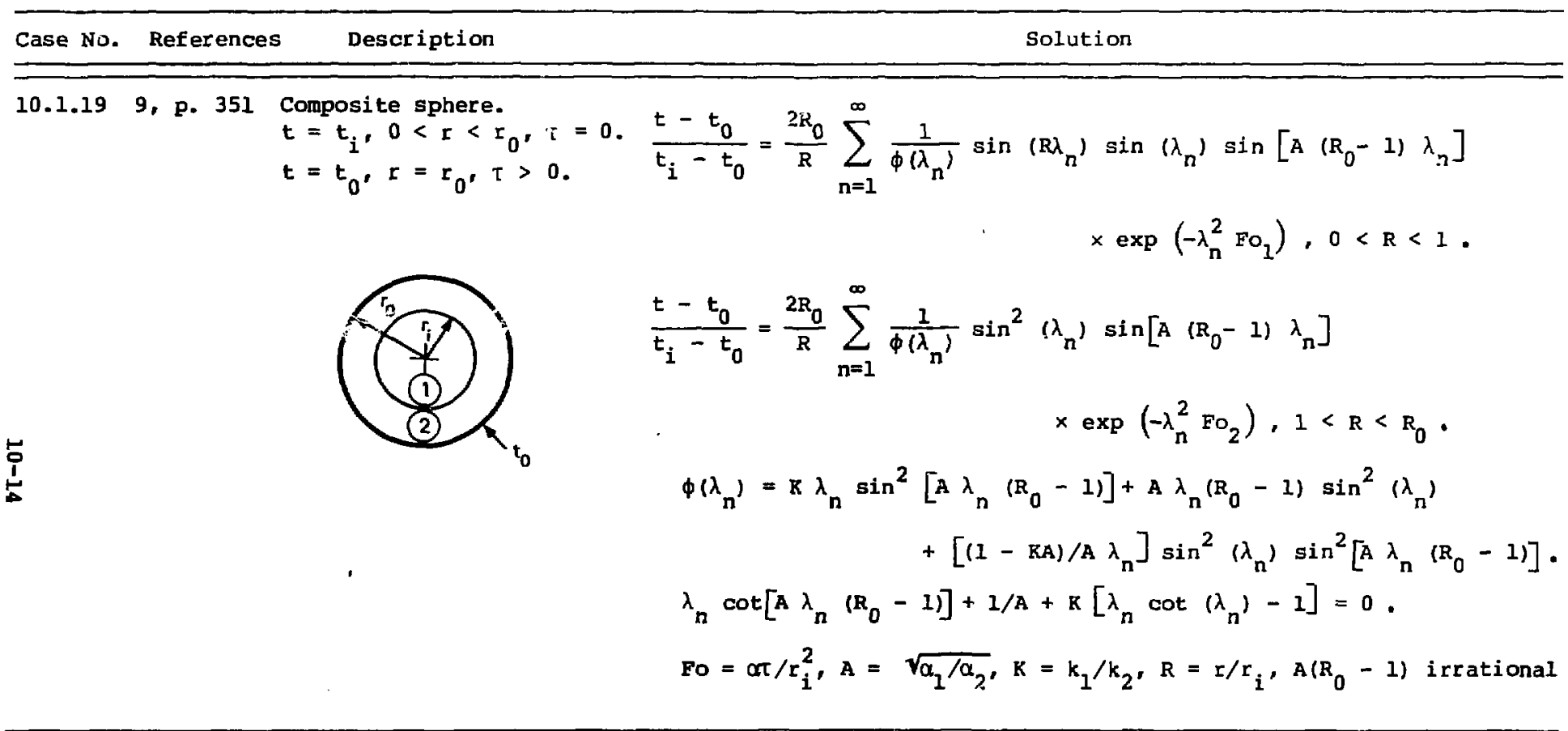


Section 10.1. Solids Bounded by Spherical Surfaces--iNo Internal Heating.

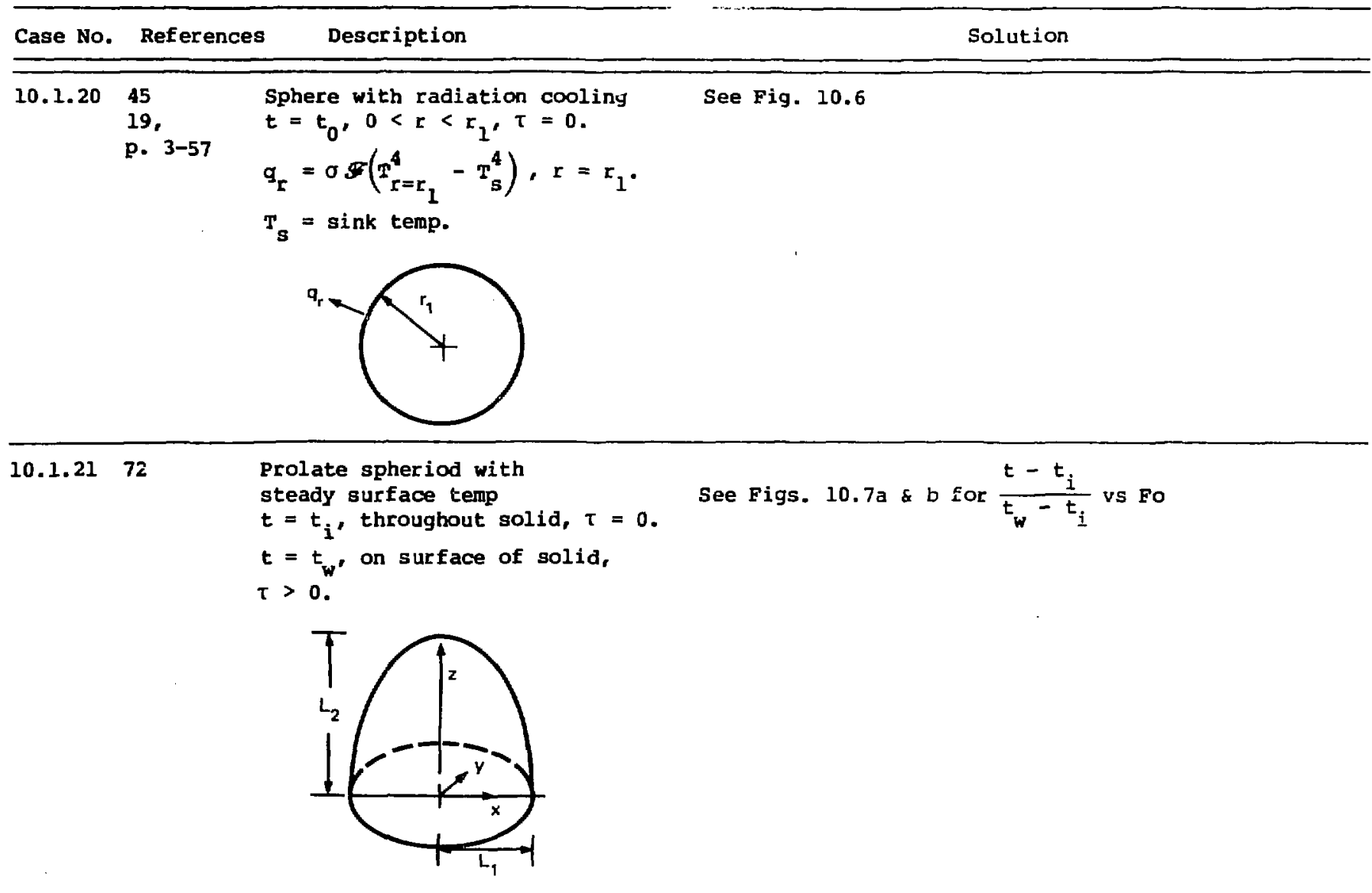


Section 10.1. Solids Bounded by Spherical Surfaces--No Internal Heating.

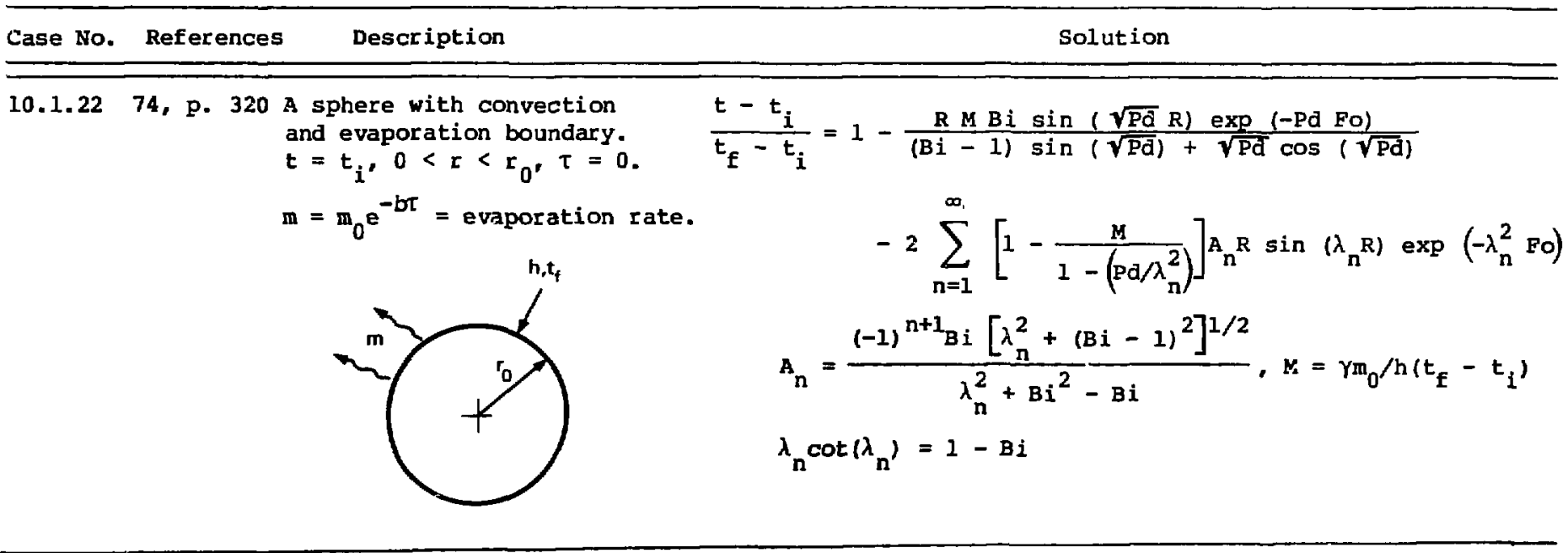


Section 10.1. Solids Bcunded by Spherical Surfaces--No Internal Heating.
Case No. References 
Section 10.1. Solids Bounded by Spherical Surfaces--No Internal Heating. 
Section 10.2. Solids Bounded by Spherical Surfaces--With Internal Heating.

Case No. References Description Solution

10.2.1 1, p. 307 Sphere with steady surface temp and internal heating.

$t=t_{i}, 0<r<r_{0}, T=0$.

$t=t_{0}, r=r_{0}, \tau>0$.

$q^{\prime \prime \prime}=q_{0}^{\prime \prime}, 0>r>r_{0}, \tau>0$.

$$
\begin{aligned}
\frac{\left(t-t_{0}\right) k}{q_{0}^{\prime \prime} r_{0}^{2}}=\frac{1}{6}\left(1-R^{2}\right) & +\frac{2}{\pi^{3} R} \sum_{n=1}^{\infty}\left(\frac{1}{n^{3}}-\frac{\pi^{2}}{n P_{0}}\right)(-1)^{n} \\
& \times \sin (n \pi R) \exp \left(-n^{2} \pi^{2} \text { Fo }\right) .
\end{aligned}
$$

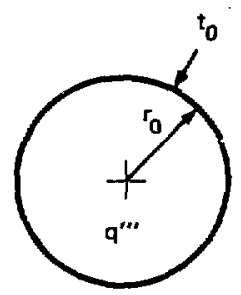

See Fig. 10.5 for condition $t_{i}=t_{0}$ 
Section 10.2. Solids Bounded by Spherlcal Surfaces-with Internal Heating.

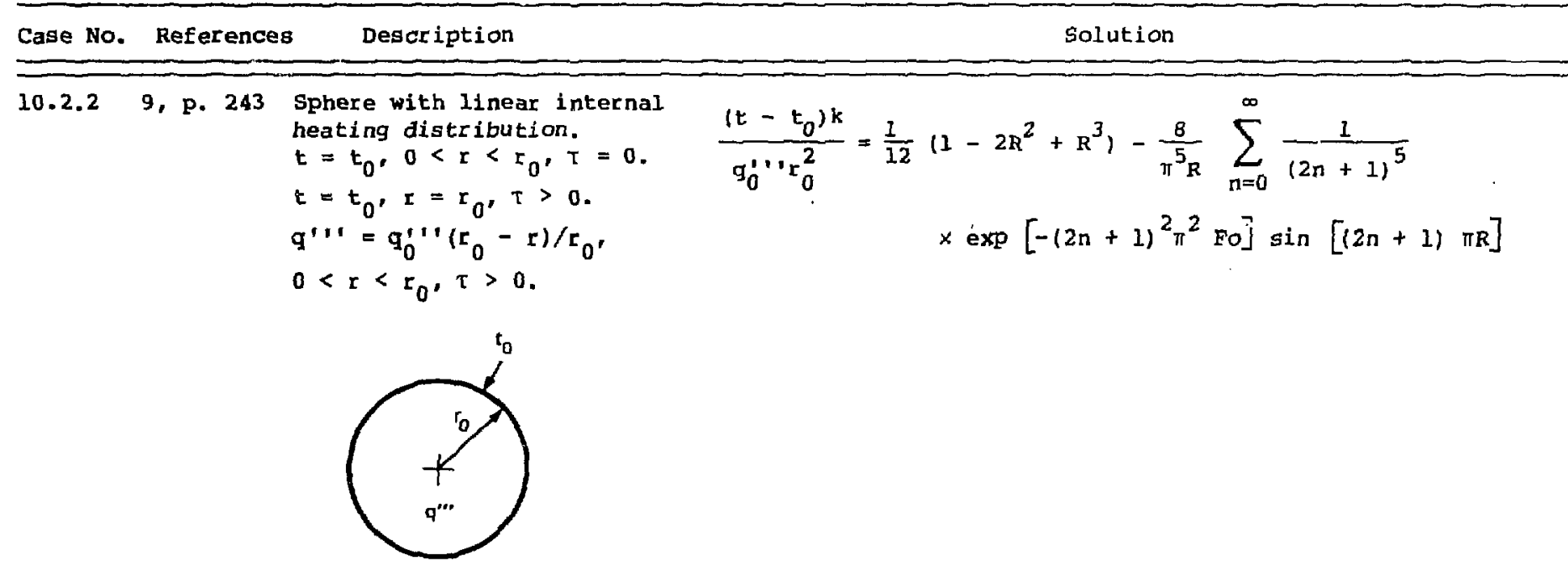

10.2.2.2 74,

P. 366

\section{Case 10.2.2 with}

$q^{\prime \prime \prime}=q_{0}^{\prime \prime}{ }^{p}{ }^{b / 2}$,

$b=1,0,1,2, \ldots$

$t=t_{i}, 0<r<r_{0}, \tau=0$.

$t=t_{0}, r=I_{0^{\prime}} \tau>0$.

$$
\begin{aligned}
& \frac{t-t_{i}}{t_{0}-t_{i}}=\sum_{n=1}^{\infty} \frac{1}{R}\left[\operatorname{erfc}\left(\frac{2 n-1-R}{2 \sqrt{F O}}\right)-\operatorname{erfc}\left(\frac{2 n_{i}-1+R}{2 \sqrt{F O}}\right)\right] \\
&+ \frac{P O F O}{1+(b / 2)}\left\{1-\Gamma\left(2+\frac{b}{2}\right) 2^{b+2}\right. \\
&\left.\times \sum_{n=1}^{\infty} \frac{1}{R}\left[i^{b+2} \operatorname{erfc}\left(\frac{2 n-1-R}{2 \sqrt{F O}}\right)-i^{b+2} \operatorname{erfc}\left(\frac{2 n-1+R}{2 \sqrt{E O}}\right)\right]\right\} \\
& \text { PO }=q_{0}^{\prime \prime} \tau^{b / 2} r_{0}^{2} / b\left(t_{0}-t_{i}\right)
\end{aligned}
$$


Section 10.2. Solids Bounded by Spherical Surfaces--With Internal Heating.

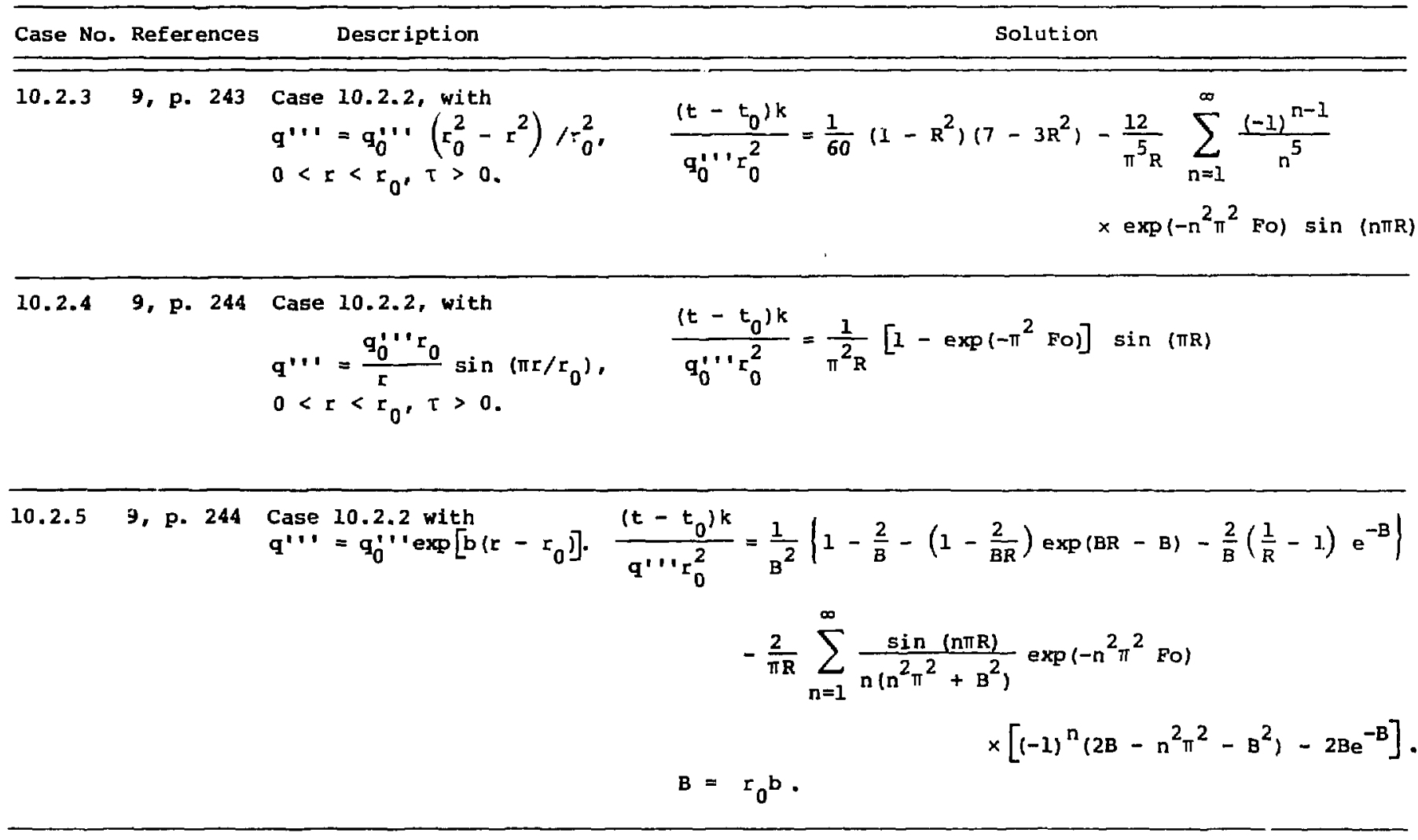


Section 10.2. Solids Bounded by Spherical Surfaces--with Internal Heating.

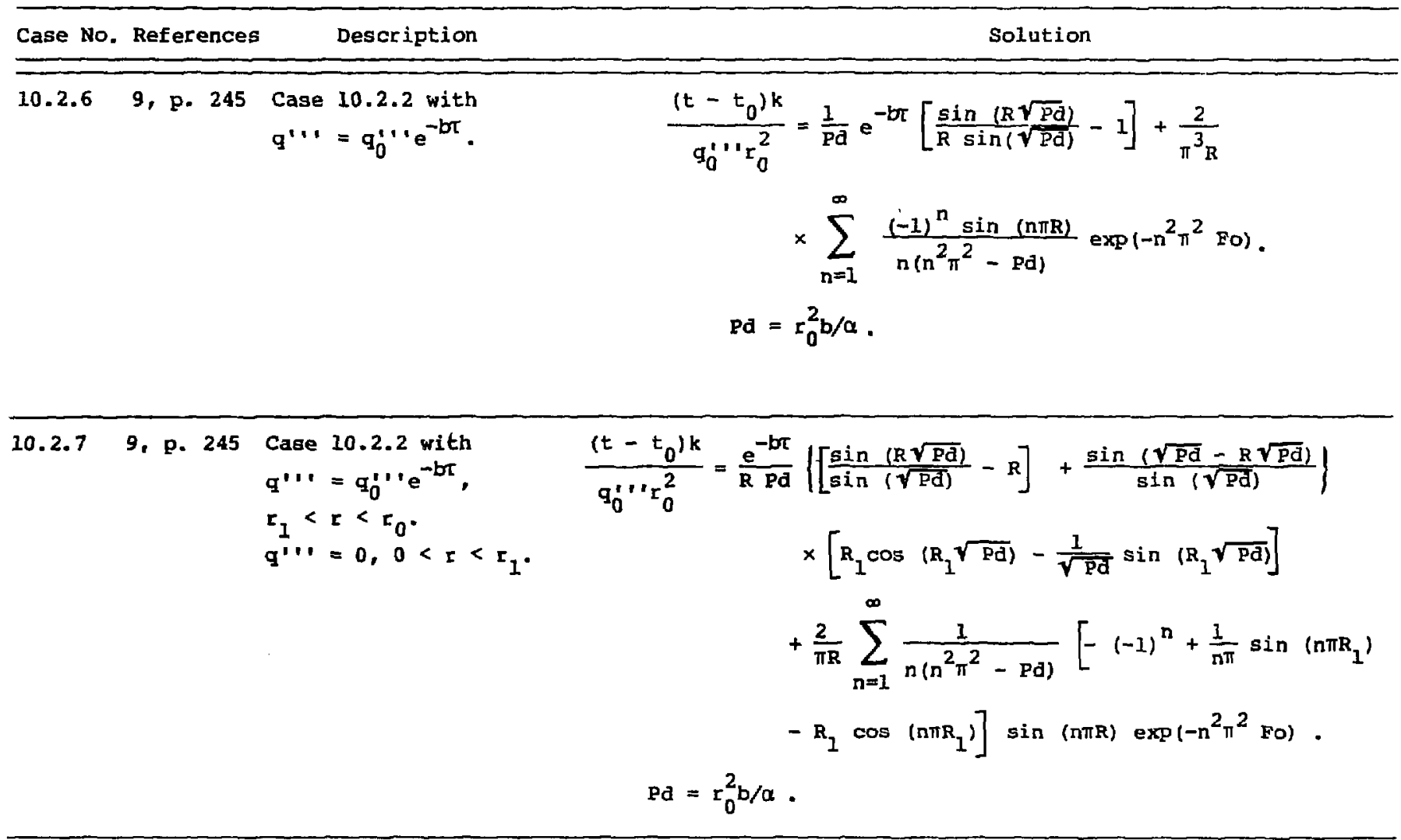


Section 10.2. Solids Bounded by Spherical Surfaces--With Internal Heating.

\section{Case No. References}

Description

Solution

10.2.8 9, p. 245 Case 10.2.2 with

$q^{\prime \prime \prime}=f(r), 0<r<r_{0}$,

$\frac{\left(t-t_{0}\right) k}{r_{0}^{2}}=\frac{2}{\pi^{2} R} \sum_{n=1}^{\infty} \frac{1}{n^{2}} \exp \left(-n^{2} \pi^{2} F o\right) \sin (n \pi R) \int_{0}^{1} R^{\prime} f^{\prime}\left(R^{\prime}\right) \sin \left(n R^{\prime}\right) d R^{\prime}$

$\tau>0$.

$+\frac{1}{R} \int_{0}^{R} R^{\prime 2} E\left(R^{\prime}\right) d R^{\prime}+\int_{R}^{l} R^{\prime} E\left(R^{\prime}\right) d R^{\prime}-\int_{0}^{1} R^{\prime 2} E\left(R^{\prime}\right) d R^{\prime}$

\begin{tabular}{ll}
10.2 .9 & 9, p. 245 \\
\hline & 90
\end{tabular}

Sphere with steady internal

heating and convection

boundary.

$t=t_{f^{\prime}} 0<r<r_{0}, \tau=0$.

$q^{\prime \prime \prime}=q_{0}^{\prime \prime}{ }^{\prime}, 0<r<r_{0^{\prime}}$

$\tau>0$.

$$
\begin{aligned}
& \frac{\left(t-t_{f}\right) k}{q_{0}^{\prime \prime \prime} r_{0}^{2}}=\frac{1}{6}\left(1-R^{2}+2 / B i\right)-2 \frac{B i}{R} \sum_{n=1}^{\infty} \frac{\sin \left(\lambda_{n} R\right) \exp \left(-\lambda_{n}^{2} F O\right)}{\lambda_{n}^{2}\left(\lambda_{n}^{2}+B i^{2}-B i\right) \sin \left(\lambda_{n}\right)} \\
& \lambda_{n} \cot \left(\lambda_{n}\right)=1-B i
\end{aligned}
$$

See Fig. 10.10

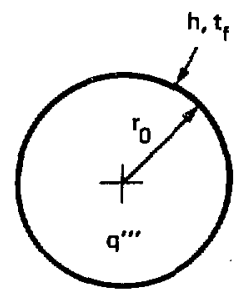


Section 10.2. Solids Bounded by Spherical Surfaces--With Internal Heating.

\section{Case No. References}

10.2.9.1 74, p. 366 Case 8.2.9 with $q^{\prime \prime \prime}=q_{0}^{\prime \prime \prime} e^{-b t}$

$$
\begin{aligned}
& \left.\frac{t-t_{i}}{t_{f}-t_{i}}=I-\frac{P o}{P d}\left[1-\frac{B i \sin (\sqrt{P d} R)}{R[(B i-1) \sin (\sqrt{P d})+\sqrt{P d} \cos (\sqrt{P d})}\right]\right] \\
& \times \exp (-P d \text { FO })-\sum_{n=1}^{\infty}\left(1-\frac{P O}{P d-\lambda_{n}^{2}}\right)^{A_{n} \sin \left(\lambda_{n} R\right) \exp \left(-\lambda_{n}^{2} \text { Fo }\right)} \\
& { }_{n}=\frac{2\left[\sin \left(\lambda_{n}\right)-\lambda_{n} \cos \left(\lambda_{n}\right)\right]}{\lambda_{n}-\sin \left(\lambda_{n}\right) \cos \left(\lambda_{n}\right)}, \tan \left(\lambda_{n}\right)=\frac{\lambda_{n}}{1-B i}
\end{aligned}
$$

\subsubsection{9, p. 246}

占
Sphere with parabolic initial temp and convection boundary. $t=q_{0}^{\prime \prime}\left(r_{0}^{2}-r^{2}\right) / 6 k+t_{1}$, $0<r<r_{0}, r=0$ (temp dist. for steady state with const. surface temp $=t_{1}$ ). $q^{\prime} ' \prime=q_{0}^{\prime}{ }^{\prime}, 0<\tau<r_{0^{\prime}} \tau>0$.

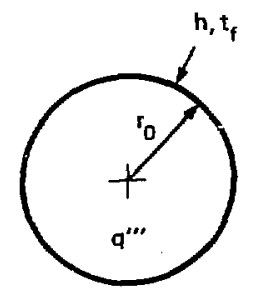

$$
\frac{\left(t-t_{1}\right) k}{q_{0}^{\prime}{ }^{\prime} r_{0}^{2}}=\frac{1}{6}\left(1-R^{2}\right)+\left[\frac{1}{P_{0}}+\frac{1}{3 B i}\right]\left[1-\frac{2 B i}{R}\right.
$$

$\left.\times \sum_{n=1}^{\infty} \frac{\sin \left(\lambda_{n} R\right) \exp \left(-\lambda_{n}^{2} \text { Fo }\right)}{\left(\lambda_{n}^{2}+B i^{2}-B i\right) \sin \left(\lambda_{n}\right)}\right]$.

$\lambda_{n} \cot \left(\lambda_{n}\right)=1-B i$, PO $=\frac{g_{0}^{\prime}+' r_{0}^{2}}{k\left(t_{f}-t_{1}\right)}$ 
Section 10.2. Solids Bounded by Spherical Surfaces--With Internal Heating.

\section{Case No. References}

Description

Solution

10.2.11 9, p. 350 Sphere of infinite conductivity enclosed by a shell of finite conductivity.

$t=t_{0}, 0<I<r_{0}, \tau=0$.

$q^{\prime \prime \prime}=q_{0}^{\prime \prime \prime}, 0<r<r_{0}$,

$\tau>0$.

$$
\frac{\left(t-t_{0}\right) k}{q_{0}^{\prime} ' r_{0}^{2}}=\frac{1}{3}\left(\frac{1}{R}-\frac{1}{R_{0}}\right)-\frac{4 R}{R}
$$

$\times \sum_{n=1}^{\infty} \frac{\sin \left[\lambda_{n}\left(R_{0}-1\right)\right] \sin \left[\lambda_{n}\left(R_{0}-R\right)\right] \exp \left(-\lambda_{n}^{2} F_{0}\right)}{\lambda_{n}\left\{2 \pi\left(R_{0}-1\right) \lambda_{n}+4 \lambda_{n} \sin ^{2}\left[\lambda_{n}\left(R_{0}-1\right)\right]-K \sin \left[2 \lambda_{n}\left(R_{0}-1\right)\right]\right\}}$.

$\mathbf{q}^{\prime} \cdot{ }^{\prime}=0, \mathrm{r}_{\mathrm{i}}<\mathrm{r}<\mathrm{r}_{0^{\prime}}$

$\tau>0$.

$K \lambda_{n} \cos \left[\lambda_{n}\left(R_{0}-1\right)\right]=\left(\lambda_{n}^{2}-k\right) \sin \left[\lambda_{n}\left(R_{0}-1\right)\right], 1<R<R_{0}$.

占

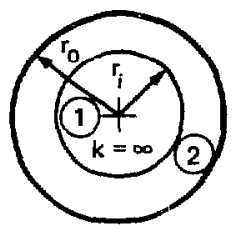

$R=3 \rho_{2} c_{2} / \rho_{1} c_{1}, R=r / r_{i}$, FO $=\alpha_{2} \tau / r_{i}^{2}$ ductivity, variable

See case 8.2.11, set $L=3$, radius $=\ell$

specific heat, convective

boundary, and steady

heating. 
Section 10.2. Solids Bounded by Spherical Surfäces--With Internal Heating.

Case No. References

Description

Solution

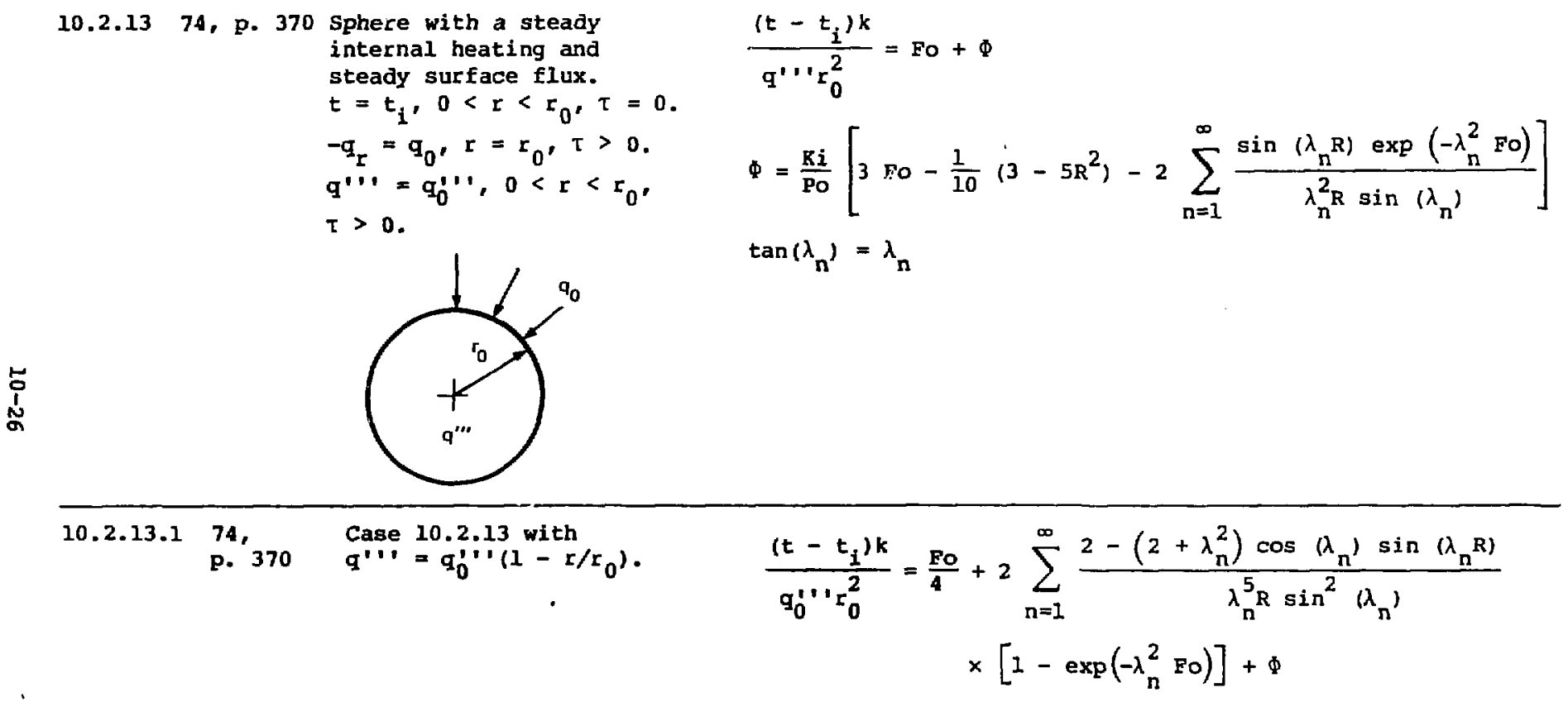

$\Phi$ given in case 10.2.13. 
Section 10.2. Solids Bounded by Spherical Surfaces--With Internal Heating.

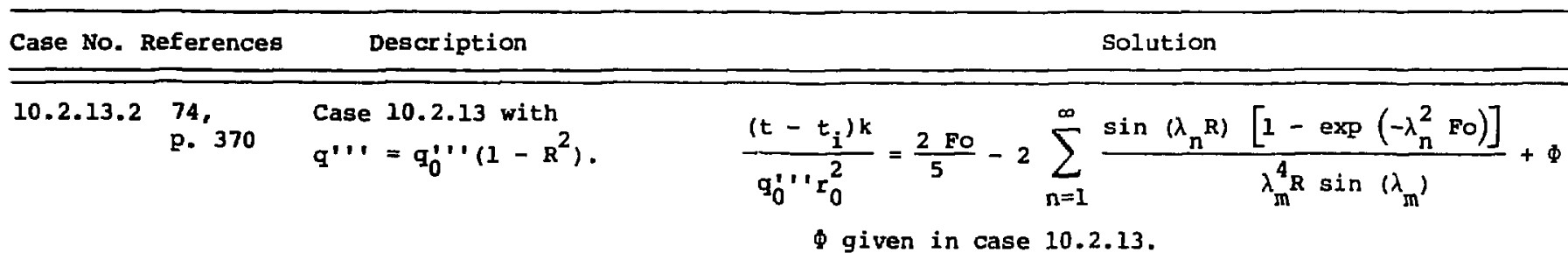

$\begin{aligned} \text { 10.2.13.3 74. } & \text { Case 10.2.13 with } \\ \text { p. } 370 & \text { q' }{ }^{\prime \prime}=q_{0}^{\prime \prime} \exp (-b R) .\end{aligned}$

$\underset{1}{1}$

$$
\begin{aligned}
& \frac{\left(t-t_{i}\right) k}{q_{0}^{\prime} '{ }^{\prime} r_{0}^{2}}=\frac{3 \text { Fo }\left[2-\exp (-b)\left(b^{2}+2 b+2\right)\right]}{b^{3}} \\
& +2 b \sum_{n=1}^{\infty} \frac{2 \lambda_{n}-\exp (-b)\left(2+b^{2}+2 b+\lambda_{n}^{2}\right) \sin \left(\lambda_{n}\right) \sin \left(\lambda_{n} R\right)}{\lambda_{n}^{2} \sin ^{2}\left(\lambda_{n}\right)\left(\lambda_{n}^{2}+b^{2}\right)^{2} R} \\
& \times\left[1-\exp \left(-\lambda_{n}^{2} \text { Fo }\right)\right]+\Phi
\end{aligned}
$$

Tiven in case 10.2.13.
10.2 .13 .574 , p. 371
Case 10.2 .13 with

$q^{\prime \prime \prime}=q_{0}^{\prime}{ }^{\prime} \exp (-b \pi)$.

$$
\begin{gathered}
\frac{\left(t-t_{i}\right) k}{q_{0}^{\prime} ' r_{0}^{2}}=F O\left(1+\frac{1}{2} \operatorname{Pd} \text { FO }\right)+\Phi \\
\Phi \text { given in case } 10.2 .13 .
\end{gathered}
$$

$$
\frac{\left(t-t_{i}\right) k}{q_{0}^{\prime \prime}{ }^{2} r_{0}^{2}}=\frac{1}{P d}[1-\exp (-P d F O)]+\Phi
$$

$\Phi$ given in case 10.2.13. 
Section 10.2. Solids Bounded by Spherical Surfaces--with Internal Heating.

\begin{tabular}{|c|c|c|c|}
\hline Case No. & References & Description & Solution \\
\hline 10.2 .13 .6 & $\begin{array}{l}\text { 74, } \\
\text { p. } 371\end{array}$ & $\begin{array}{l}\text { Case } 10.2 .13 \text { with } \\
q^{\prime \prime \prime}=q_{0}^{\prime \prime} \cos (b \pi) .\end{array}$ & $\begin{array}{c}\frac{\left(t-t_{i}\right) k}{q^{\prime} \cdot r_{0}^{2}}=\frac{1}{P d} \text { sin }(P d F o)+\Phi \\
\Phi \text { given in case } 10.2 .13\end{array}$ \\
\hline 10.2 .13 .7 & $\begin{array}{l}74, \\
\text { p. } 371\end{array}$ & $\begin{array}{l}\text { Case } 10.2 .13 \text { with } \\
q^{\prime \prime}=q_{0}^{\prime} " b t^{n} \text {. }\end{array}$ & $\begin{array}{l}\frac{\left(t-t_{i}\right) k}{q^{\prime}+r_{0}^{2}}=\frac{(P d F o)^{n}}{n+1} \text { Fo }+\Phi \\
\Phi \text { given in case } 10.2 .13 .\end{array}$ \\
\hline
\end{tabular}


Section 10.2. Solids Bounded by Spherical Surfaces--With Internal Heating.

10.2.14 74, p. 390 Sphere with pulse heating on a spherical surface and a convection boundary. $t=t_{E}, 0<\tau<\tau_{0}, \tau=0$. Instantaneous pulse of strength $Q_{1}$ occurs at $r=r_{1}, \tau=0$.

占

$$
\begin{aligned}
& \frac{\left(t-t_{f}\right) \rho c r_{0}^{3}}{Q_{1}}=\frac{1}{4 \pi} \sum_{n=1}^{\infty} \frac{\left[(B i-1)^{2}+\lambda_{n}^{2}\right]^{1 / 2}{ }_{n}^{A} \sin \left(\lambda_{n} R_{1}\right) \sin \left(\lambda_{n} R\right)}{B i R_{i} R} \times \exp \left(-\lambda_{n}^{2} F o\right) . \\
& A_{n}=(-1)^{n+1} \frac{2 B i\left[\lambda_{n}^{2}+(B i-1)^{2}\right]^{1 / 2}}{\lambda_{n}^{2}+B i^{2}-B i} . \\
& \tan \left(\lambda_{n}\right)=\frac{\lambda_{n}}{1-B i}, R=r / r_{0}
\end{aligned}
$$

For $B i+\infty$ :

$\frac{\left(t-t_{E}\right\rangle P C r_{0}^{3}}{Q_{1}}=\frac{1}{2 \pi} \sum_{n=1}^{\infty} \frac{1}{R_{1} R} \sin \left(n \pi R_{1}\right) \sin (n \pi R) \exp \left(-n^{2} \pi^{2} F O\right)$.

For $r_{1}=0$ :

$\frac{\left(t-t_{f}\right) \operatorname{ser}{ }_{i}^{3}}{Q_{1}}=\frac{1}{2 \pi R} \sum_{n=1}^{\infty} \frac{\lambda_{n}^{2} \sin \left(\lambda_{n} R\right) \exp \left(-\lambda_{n}^{2} F o\right)}{\lambda_{n}-\sin \left(\lambda_{n}\right) \cos \left(\lambda_{n}\right)}$.

Mean temp:

$$
\begin{aligned}
& \frac{\left(t_{m}-t_{f}\right) \rho \sigma r_{0}^{3}}{Q_{1}}=\frac{1}{4 \pi R_{I} B i} \sum_{n=1}^{\infty}\left[(B i-1)^{2}+\lambda_{n}^{2}\right]^{1 / 2} \lambda_{n} B_{n} \sin \left(\lambda_{n} R_{1}\right) \\
& B_{n}=\frac{6 B i^{2}}{\lambda_{n}^{2}\left(\lambda_{n}^{2}+B i^{2}-B i\right)}
\end{aligned}
$$


Section 10.2. Solids Bounded by Spherical Surfaces-with Internal Beating.

Case No. References Description Solution


Section 10.2. Solids Bounded by Spherical Surfaces--With Internal Heating. 
Section 11.1. Change of Phase--Plane Interface.

Case No. References

Description

Solution

11.1.1 9, p. 285 Initial surface maintained at Solid temp: constant temp: freezing liguid.

$t=t_{0}, x=0, \tau>0$.

$t=t_{1}, x>0, t=0$.

$\frac{t_{s}-t_{0}}{t_{m}-t_{0}}=\operatorname{erE}\left(F_{x s}^{*}\right) / \operatorname{erf}(\lambda), 0<x<\omega$.

$t_{m}=$ meliing temp.

Liquid temp;

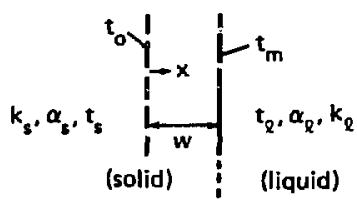

$$
\begin{aligned}
& \frac{t_{l}-t_{1}}{t_{m}-t_{1}}=\operatorname{erfc}\left(F o_{x l}^{*}\right) / \operatorname{erfc}\left\langle\lambda \sqrt{\alpha_{s}} \overline{\alpha_{l}}\right), x>w . \\
& \frac{\lambda r \sqrt{\pi}}{c_{s}\left(t_{m}-t_{0}\right)}=\frac{\exp \left(-\lambda^{2}\right)}{\operatorname{erf}(\lambda)}+\frac{\left(t_{m}-t_{l}\right)}{\left(t_{m}-t_{0}\right)} \frac{k_{l}}{k_{s}} \frac{\exp \left(-\lambda^{2} \alpha_{s} / \alpha_{l}\right)}{\operatorname{erfc}(\lambda)} \\
& w=2 \lambda \sqrt{\alpha_{s}}
\end{aligned}
$$

11.1.2 9, p. 287 Solid region maintained at melting temp: freezing liquid. $t=t_{m}, x<w, \tau>0$.

$t=t_{n}, x>0, \tau=0$.

$t_{1}<t_{m} \cdot t_{m}=$ melting temp.
Liquid temp:

$\frac{\left(t_{\ell}-t_{1}\right) c_{\ell_{l}}}{r}=\lambda \sqrt{\pi} \operatorname{erfc}\left(\mathrm{Fo}_{\mathrm{x} \ell}^{*}\right) / \exp \left(-\lambda^{2}\right), x>w$.

$w=2 \lambda \sqrt{\alpha_{l} \tau}$

$\lambda \exp \left(\lambda^{2}\right) \operatorname{erfc}(\lambda)=\left(t_{m}-t_{1}\right) c_{\ell} / \gamma \sqrt{\pi}$ 
Section 11.1. Change of Phase--Plane Interface.

Lase No. References Description Solution

11.1.3 9, p. 287 Melting of a solid in contact solid temp:

with another solid.

$t=t_{0}, x>0, \tau=0$.

$t=t_{1}>t_{m}, x=0, \tau>0$.

$t_{m}=$ melting temp.

$\frac{t_{s}-t_{0}}{t_{m}-t_{0}}=\operatorname{erfc}\left(\mathrm{Fo}_{x s}^{*}\right) / \operatorname{erfc}\left(\lambda \sqrt{\alpha_{l} / \alpha_{g}}\right), x>w$.

Liquid temp:

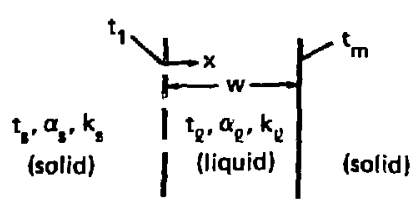

$$
\begin{aligned}
& \frac{t_{l}-t_{1}}{t_{m}-t_{1}}=\operatorname{erf}\left(F o_{x l}^{*}\right) / \operatorname{erf}(\lambda), 0<x<w . \\
& \frac{\exp \left(-\lambda^{2}\right)}{\operatorname{erf}(\lambda)}+\frac{k_{s} \sqrt{\alpha_{l}}\left(t_{l}-t_{m}\right) \exp \left(-\lambda^{2} \alpha_{l} / \alpha_{s}\right)}{k_{l} \sqrt{\alpha_{s}}\left(t_{l}-t_{m}\right) \operatorname{erfc}\left(\lambda \sqrt{\alpha_{\ell} / \alpha_{s}}\right)}=\frac{\lambda \gamma \sqrt{\pi}}{c_{\ell}\left(t_{1}-t_{m}\right)} \\
& w=2 \lambda \sqrt{\alpha_{l} \tau}
\end{aligned}
$$


Section 11.1. Change of Phase--Plane Interface.

Case No. References

Description

11.1.4 9, p. 288 Liquid freezing on solld-properties of solidified liquid different from original solid.

$t=t_{0}, x<0, \tau=0$.

$t=t_{1}, x>0, \tau=0$.

$t_{m}=$ melting terp.

卢

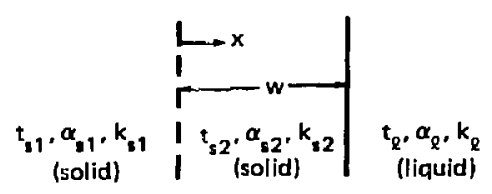

Original solid:

$\frac{t_{s I}-t_{0}}{t_{m}-t_{0}}=\frac{\sqrt{A}}{\sqrt{A}+K \operatorname{erf}(\lambda)}\left[1+\operatorname{erf}\left(F 0_{x s I}^{\star}\right)\right], x<0$.

Solidified liquid:

$\frac{t_{s 2}-t_{0}}{t_{m}-t_{0}}=\frac{\sqrt{\bar{A}}+K \operatorname{erf}\left(F_{\mathrm{xs}}^{*}\right)}{\sqrt{\mathrm{A}}+k \operatorname{erf}(\lambda)}, 0<x<w$.

Liquid:

$\frac{t_{l}-t_{1}}{t_{m}-t_{1}}=\operatorname{erfc}\left(F 0_{x \ell}^{*}\right) / \operatorname{erfc}\left(\lambda \sqrt{\alpha_{s 2} / \alpha_{l}}\right), x>w$.

$\frac{\lambda y \sqrt{\pi}}{c_{s 2}\left(t_{m}-t_{0}\right)}=\frac{R \exp \left(-\lambda^{2}\right)}{\sqrt{A}+x \operatorname{erf}(\lambda)}+\frac{k_{l} \sqrt{\alpha_{s 2}}\left(t_{m}-t_{1}\right) \exp \left(-\lambda^{2} \alpha_{s 2} / \alpha_{\ell}\right)}{k_{s 2} \sqrt{x_{l}}\left(t_{m}-t_{0}\right) \operatorname{erfc}\left(\lambda \sqrt{\alpha_{s 2} / \alpha_{l}}\right)}$

$w=2 \lambda \sqrt{\alpha_{s 2}{ }^{\top}}, K=k_{s 1} / k_{s 2}, A=\alpha_{s 1} / \alpha_{s 2}$ 
Section 11.1. Change of Phase--Plane Interface.

\begin{tabular}{lll}
\hline Case No. References & Description & Solution
\end{tabular}

11.1 .59$, p. 289 Melting of a solid in contact

with another solid--properties

of liquid different than

original solid.

$w=2 \lambda \sqrt{\alpha_{\ell}{ }^{\top}}$

$t=t_{1}>t_{m^{\prime}} x<0, \tau=0$.

$\frac{\lambda_{Y} \sqrt{\pi}}{c_{\ell}\left(t_{1}-t_{m}\right)}=\frac{k_{s 1} \sqrt{\alpha_{l}} \exp \left(-\lambda^{2}\right)}{k_{l} \sqrt{\alpha_{s l}}+k_{s 1} \sqrt{\alpha_{l}} \operatorname{erf}(\lambda)}$

$t=t_{0}, x>0, \tau=0$.

$+\frac{k_{s 2}{\sqrt{\alpha_{l}}}\left(t_{0}-t_{m} \exp \left(-\lambda^{2} \alpha_{\ell} / \alpha_{s 2}\right)\right.}{k_{\ell} \sqrt{\alpha_{s 2}}\left(t_{1}-t_{m}\right) \operatorname{erfc}\left(\lambda \sqrt{\alpha_{\ell} / \alpha_{s 1}}\right)}$.

See case 11.1 .4 for temperatures

壬

$t_{\mathbf{m}}=$ melting temperature.

11.1.6 9, p. 289 Solid melts over temp range of $t_{m l}$ to $t_{m 2}$. $t=t_{m 2}, x>0, \tau=0$.

$c_{l}^{*}=c_{l}+Y /\left(t_{m 2}-t_{m l}\right)$.
For case 11.1.1, change equation for $\lambda$ to:

$\frac{\exp \left[\left(\alpha_{s}-\alpha_{\ell}\right) \lambda^{2} / \alpha_{\ell}\right] \operatorname{erfc}\left(\lambda \sqrt{\alpha_{s} / \alpha_{\ell}}\right)}{\operatorname{erf}(\lambda)}=\frac{\left(t_{m 2}-t_{m 1}\right) k_{\ell} \sqrt{\alpha_{s}}}{\left(t_{m l}-t_{0}\right) k_{s} \sqrt{\alpha_{l}}}$.

For case 11.1.4, change equation for $\lambda$ to:

$\frac{R \exp \left[\left(\alpha_{s 2}-\alpha_{\ell}\right) \lambda^{2} / \alpha_{\ell}\right] \operatorname{erfc}\left(\lambda \sqrt{\alpha_{s l} / \alpha_{\ell}}\right)}{\sqrt{A}+k \operatorname{erf}(\lambda)}=\frac{\left(t_{m 2}-t_{m l}\right) k_{\ell} \sqrt{\alpha_{s 2}}}{\left(t_{m l}-t_{0}\right) \sqrt{\alpha_{\ell}}}$.

Use $c_{\ell}^{\star}$ for $c_{\ell}$ in the above equations and solutions given in

cases 11.1 .1 and 11.1.4. 
Section 11.1. Change of Phase--Plane Interface.

Case so. References Description Solution

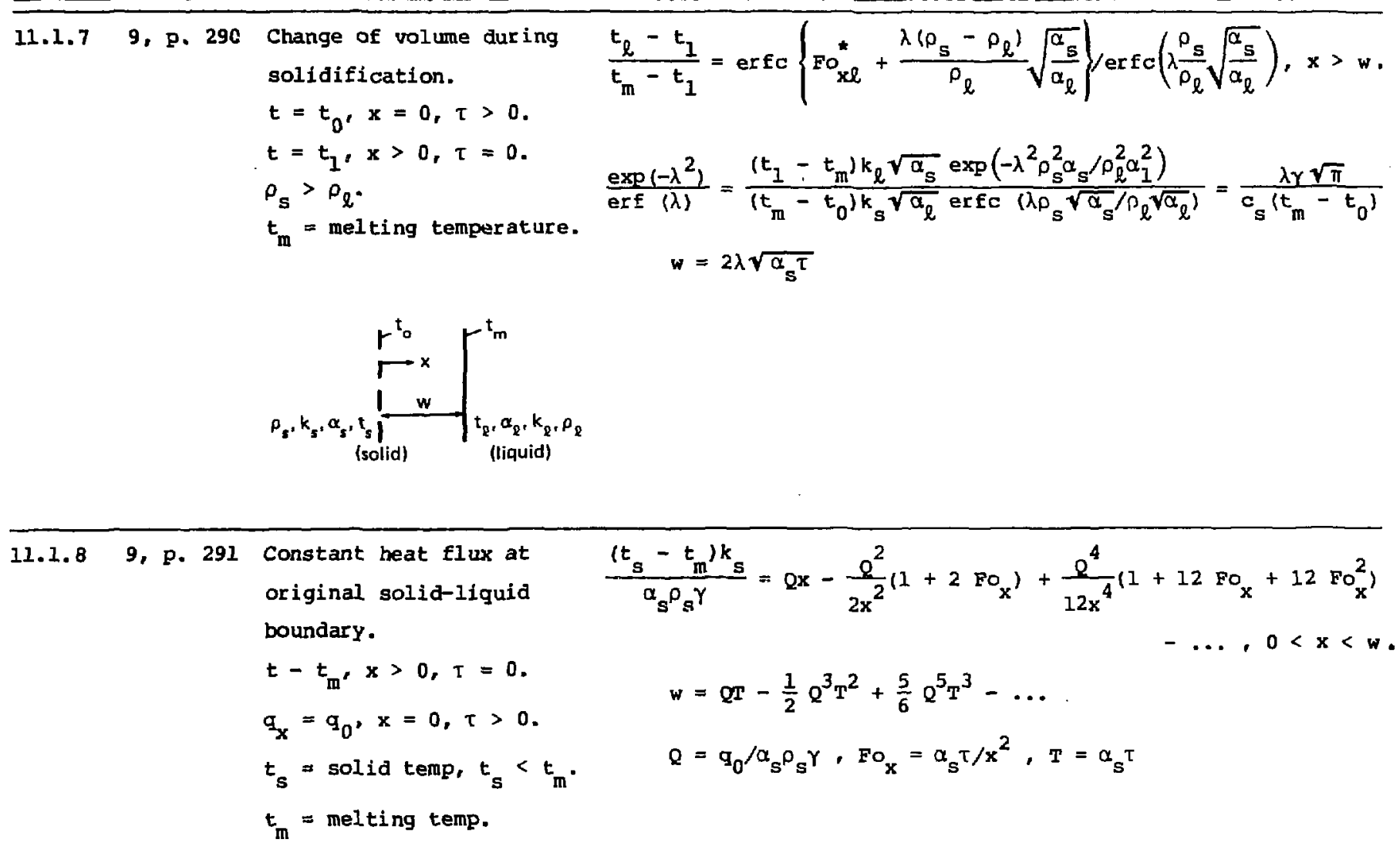


Section 11.1. Change of Phase--Piane Interface.

\begin{tabular}{|c|c|c|c|}
\hline Case No. & References & Description & Solution \\
\hline 11.1 .9 & 9, p. 292 & $\begin{array}{l}\text { Convection boundary at } \\
\text { original solid-liquid } \\
\text { boundary. } \\
t=t_{1}, x>0, t=0, t_{1}>t_{m} \\
t_{m}=m e l t i n g \text { temp. }\end{array}$ & $\begin{aligned} & \frac{t_{s}-t_{m}}{t_{m}-t_{F}}=B i_{x}-\frac{F B i_{x}^{2}}{2}\left(1+2 ! F O_{x}\right)-\frac{F B i_{x}^{3}}{3 !}\left(1+31 F O_{x}\right)+\ldots, 0<x<w \\
& w=F B i_{x} F O_{x}-\frac{F^{2} B i_{x}^{3}}{2}(1+F) F O_{x}^{2}+\ldots \\
& F=k_{s}\left(t_{m}-t_{f}\right) / \alpha_{s} P r, F O_{x}=\alpha_{s} \tau / x^{2}, B i_{x}=h x / k_{s}\end{aligned}$ \\
\hline
\end{tabular}


Section 11.1. Change of Phase--Plane Interface.

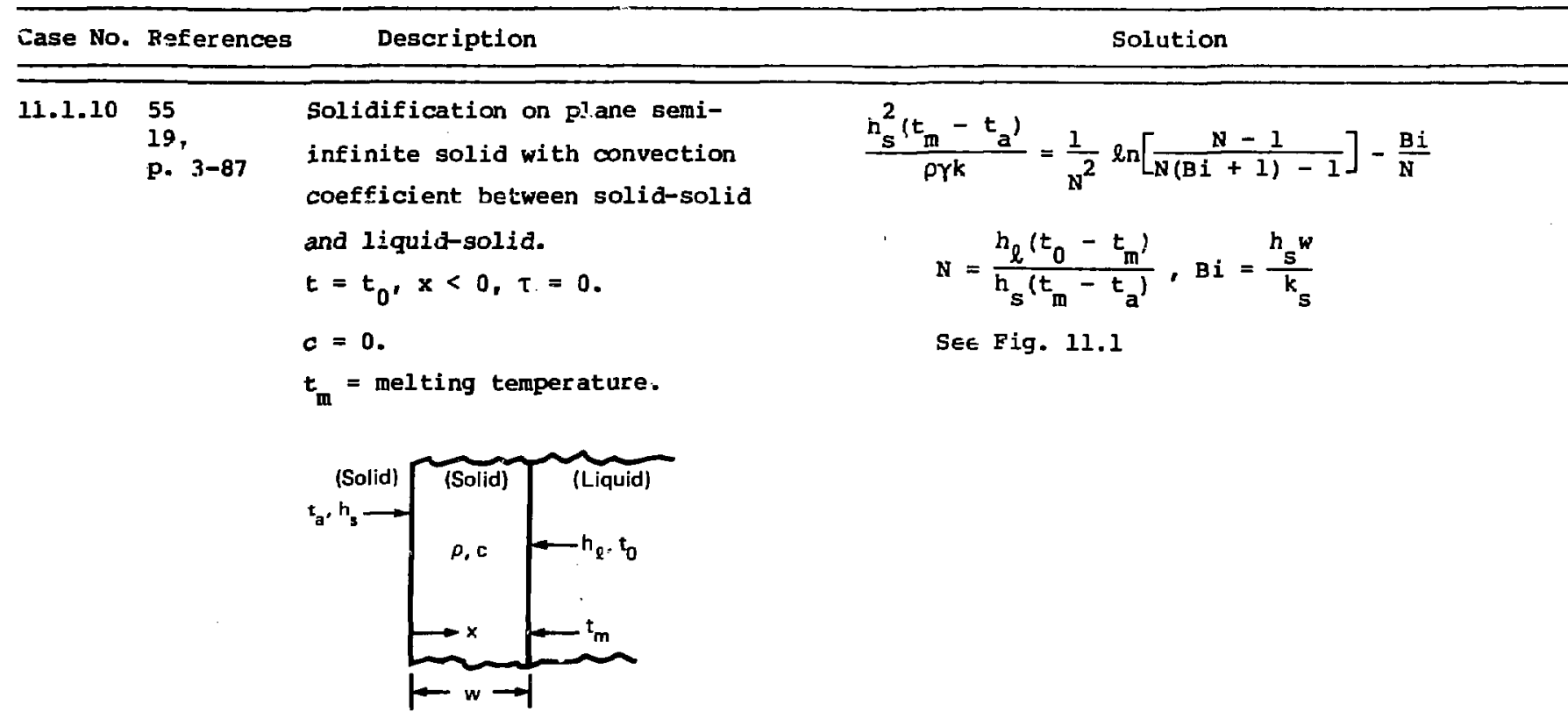


Section 11.1. Change of Phase--Plane Interface.

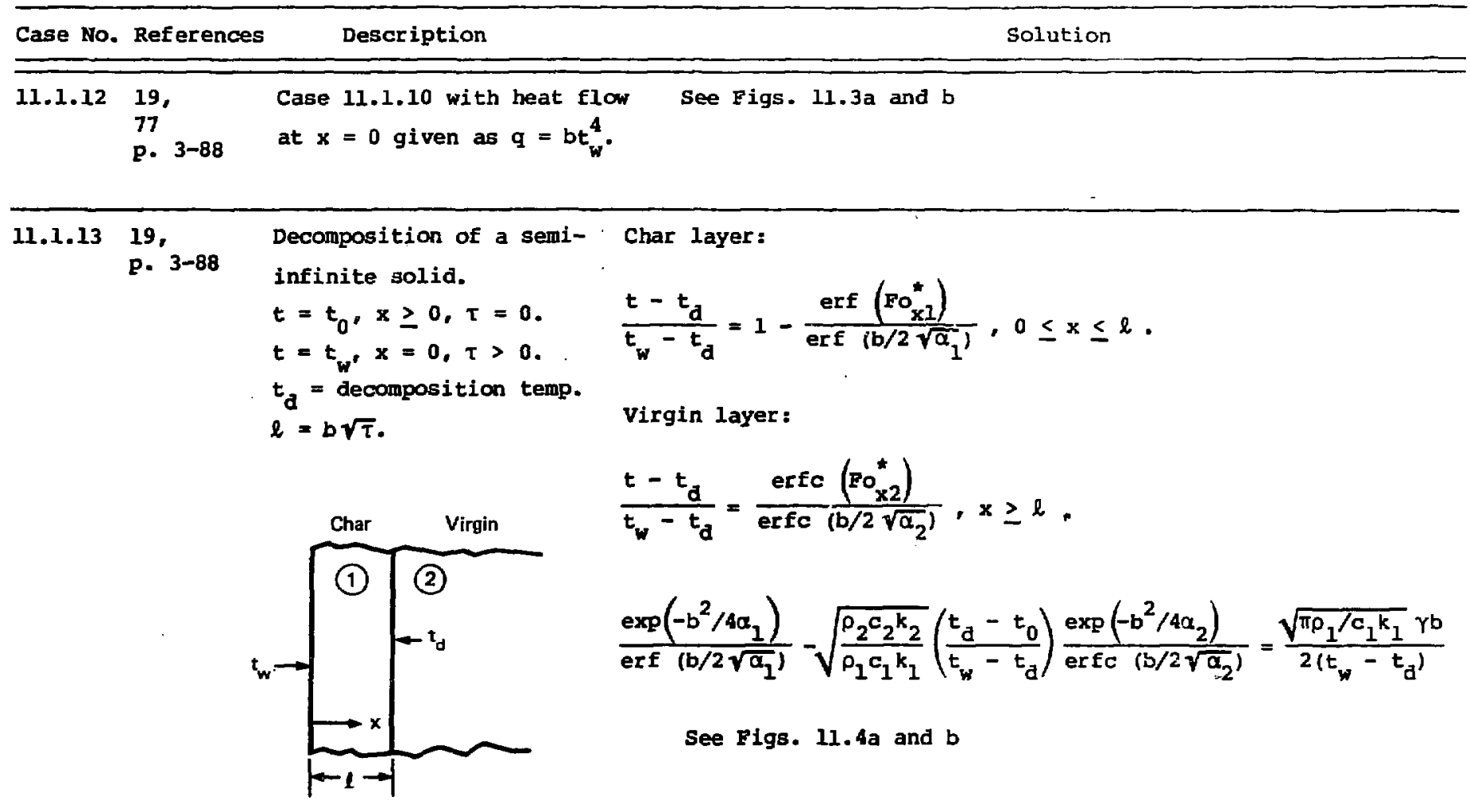


Section 11.1. Change of Phase--Plane Interface.

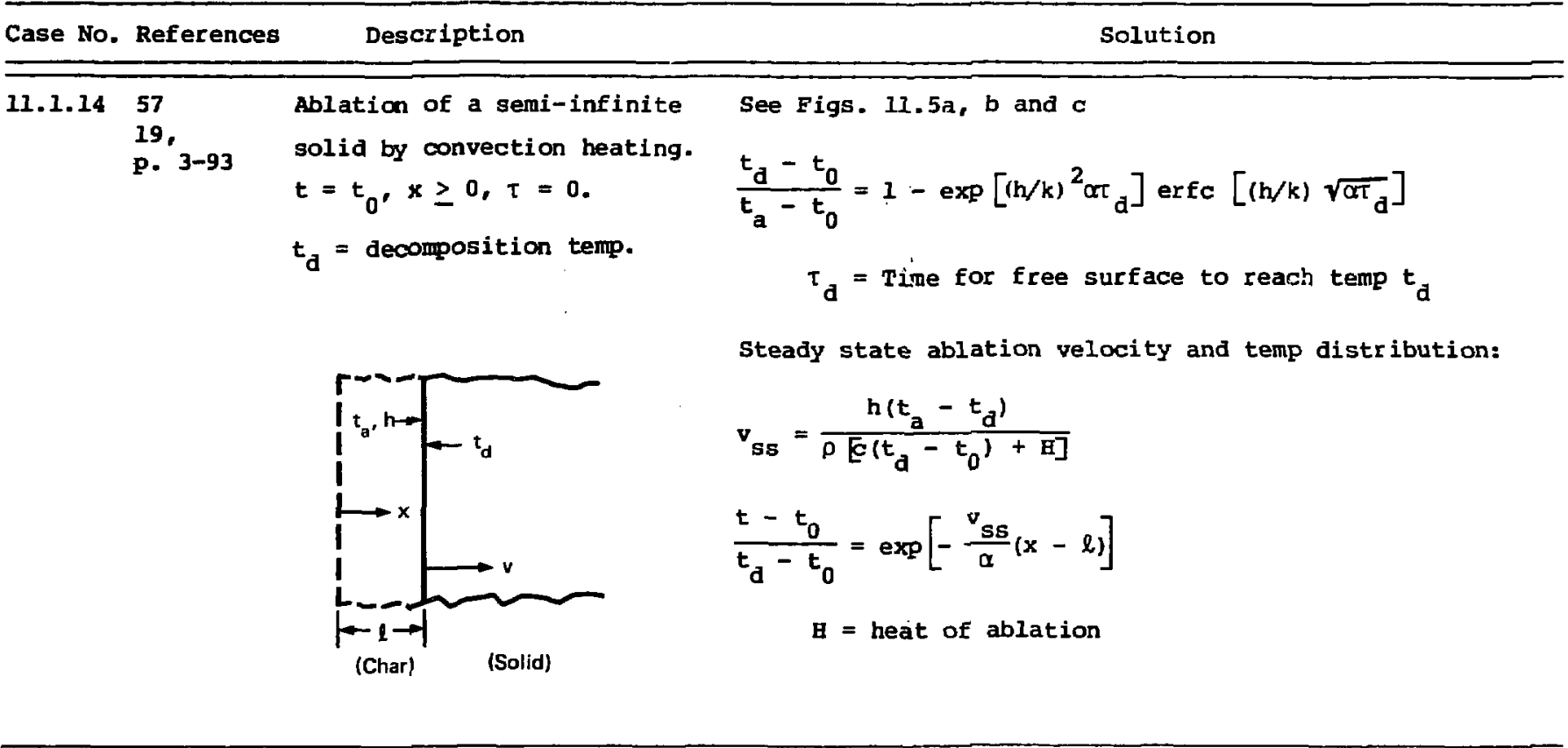


Section 11.1. Change of Phase--Plane Interface.

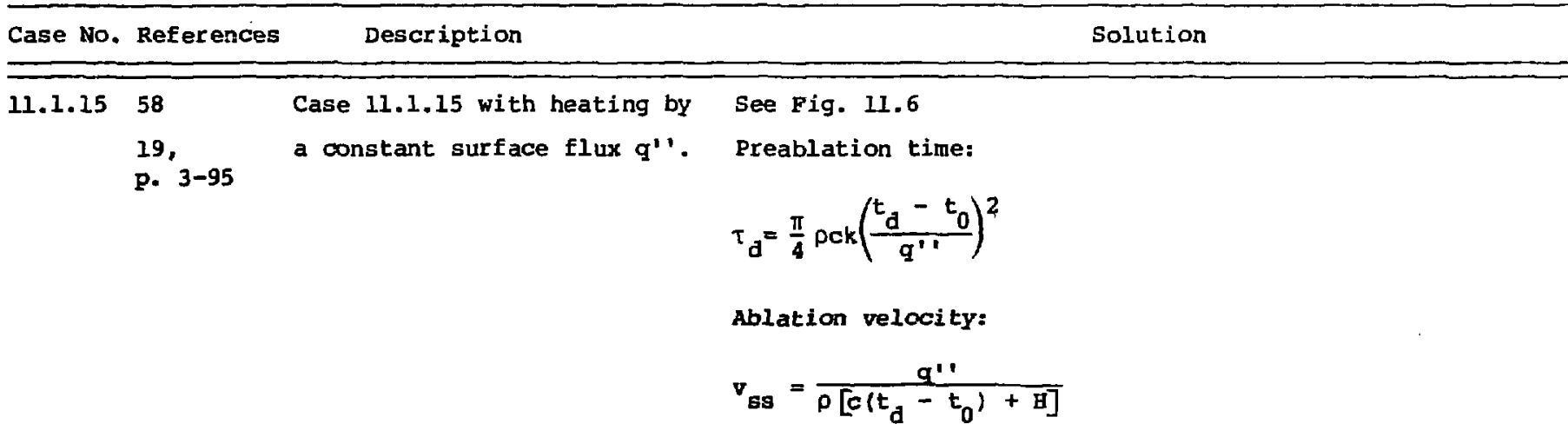


Section 11.1. Change of Phase-Plane interface.

Case No. References Description Solution


Section 11.1. Change of Phase--Plane interface.

Case No. References

Description

Solution 
Section 11.2. Change of Phase-Nonplanar Interface.

Case No. References Description Solution

11.2.1 9, p. 29 Liquid freezing on

a cylinder.

$t=t_{m}, 0<r<R$.

$t=t_{1}, r+\infty, t_{1}<t_{m}$.

$t_{m}=$ melting temp.

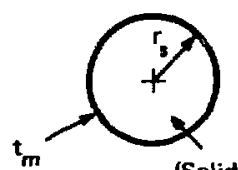

(Solid) (Liquid)

\section{Liquid temp:}

$$
\begin{aligned}
\frac{t_{l}-t_{1}}{t_{m}-t_{1}} & =E i\left(-F 0_{r l}^{ \pm 2}\right) / E i\left(-\lambda^{2}\right), r>r_{s} . \\
\lambda^{2} & \exp \left(\lambda^{2}\right) E i\left(-\lambda^{2}\right)+c_{l}\left(t_{m}-t_{1}\right) / \gamma=0 . \\
r_{s} & =2 \lambda \sqrt{a_{l} \tau}
\end{aligned}
$$

11.2.2 9, P. 295 Liquid freezing

on a sphere.

$t=t_{m}, 0<r<R$.

$t=t_{1}, r+\infty, t_{1}<t_{m}$.

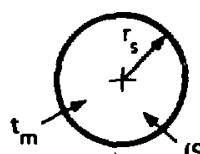

(Solid) (Liquid)

\section{Liquid temp:}

$$
\begin{aligned}
& \frac{t_{l}-t_{1}}{t_{\mathrm{m}}-t_{1}}=\frac{2 \lambda}{\exp \left(-\lambda^{2}\right) \cdots \sqrt{\pi} \operatorname{erfc}(\lambda)}\left\{\frac{1}{2 \mathrm{Fo}_{\mathrm{rl}}^{*}} \exp \left(-\mathrm{Fo}_{\mathrm{rl}}^{* 2}\right)\right. \\
&\left.-\frac{\sqrt{\pi}}{2} \operatorname{erfc}\left(\mathrm{Fo}_{\mathrm{rl}}^{*}\right)\right\}, r>r_{s} \\
& \lambda^{2} \exp \left(\lambda^{2}\right)\left\{\exp \left(-\lambda^{2}\right)-\lambda \pi \operatorname{erfc}(\lambda)\right\}=\frac{c_{\ell}}{2 \gamma}\left(t_{\mathrm{m}}-t_{1}\right) . \\
& r_{s}=2 \lambda \sqrt{\alpha_{\ell} \tau}
\end{aligned}
$$


Section 11.2. Change of Phase-Nonplanar Interface.

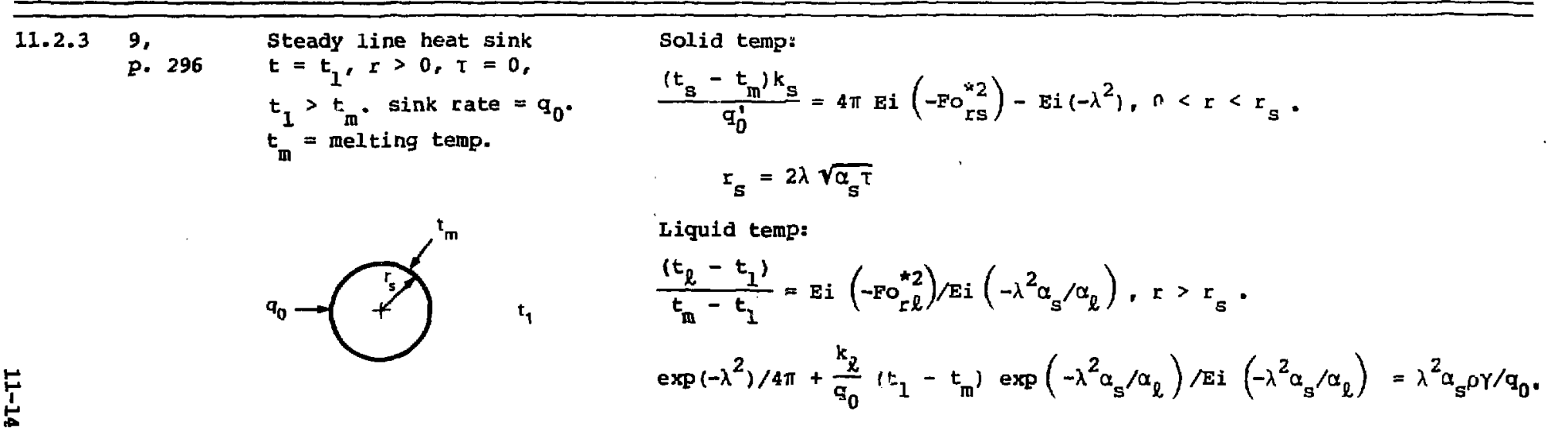

11.2.4 9, p. 296 Freezing on steady temp cylinder.

$t=t_{0}, r=t_{0}, \tau>0$.

$t_{0}<t_{\text {m. }}$

$t=t_{m}, r>r_{0}, t=0$.

$t_{\mathrm{m}}=$ melting terp.

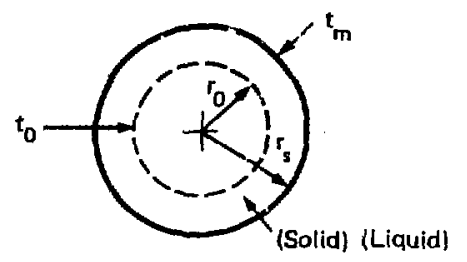

\section{Solid temp:}

$$
\begin{aligned}
& \frac{t_{B}-t_{0}}{t_{m}-t_{0}}=\ln \left(r / r_{a}\right) / \ln \left(r_{s} / r_{0}\right), r_{0}<r<r_{s} \\
& 2 r_{s}^{2} \ln \left(r_{s} / r_{0}\right)-r_{s}^{2}+a^{2}=4 k_{s}\left(t_{m}-t_{0}\right) \tau / \gamma F
\end{aligned}
$$


Section 11.2, Change $0:$ Phase-Nonplanar Inter face.

Case No. References

1. zription

Solution

\begin{tabular}{lll}
\hline 11.2 .5 & 19, & $\begin{array}{l}\text { Freezing inside a tube, } \\
\text { tube resistance neglected, } \\
\text { convection cooling at } r=r_{0}\end{array}$ \\
& $\begin{array}{l}t=t_{m} r=r_{i} . \\
t_{m}=\text { melting temp. }\end{array}$
\end{tabular}

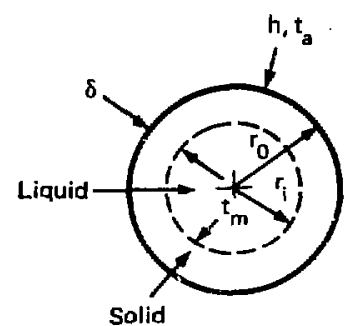

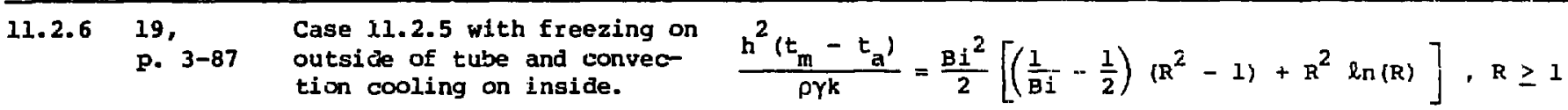

11.2.7 19, Case 11.2.5 with freezing

p. 3-88 inside a sphere.

$$
\frac{h^{2}\left(t_{m}-t_{a}\right)}{\rho \gamma k}=\frac{B i^{2}}{3}\left[\left(\frac{1}{B i}-1\right)\left(1-R^{3}\right)+\frac{3}{2}\left(1-R^{2}\right)\right], R \leq 1
$$


Section 11.2. Change of Phase-Nonplanar Interface.

$\frac{\text { Case No. References }}{\text { Description }}$


Section 11.2. Change of Phabe-Nonplanar interface. 
Section 11.2. Change of Phase--Nonplanar Interface.

Case No. References Description Solution


REPRODUCED FROM EEST AVAILABLE COPY 
Section 12.1. Traveling Boundaries.

12.1.1 9, p. 389 Semi-infinite solid with linear initial temp, time dependent surface temp and internal heating. $t=t_{0}+d x, x>0, \tau=0$. $\frac{t-t_{0}}{t_{1}-t_{0}}=D(1-v)+G+\frac{1}{2} \operatorname{erfc}[x(1-u)]$ $+\frac{1}{2} \exp \left(4 U x^{2}\right) \operatorname{erfc}[x(1+U)]+\frac{1}{2}\left(\frac{B}{U}+D+\frac{G}{U}\right)$ $t=t_{1}+b \tau, x=0, \tau>0$. Solid increasing at velocity u.

$\times\left\{(1+U) \exp \left(40 x^{2}\right) \operatorname{erfc}[x(1+U)]-(1-U) \operatorname{erfc}[x(1-0)]\right\}$.

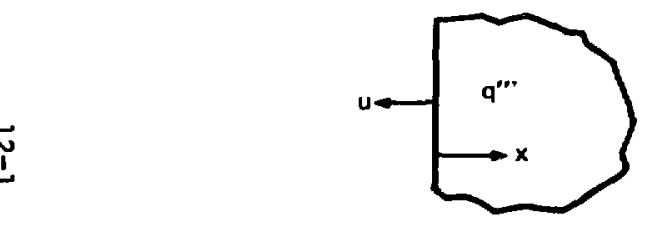

$B=b \tau /\left(t_{1}-t_{0}\right), D=d x /\left(t_{1}-t_{0}\right), G=q^{\prime} \cdot ' \alpha \tau / k\left(t_{1}-t_{0}\right)$

$U=u \pi / x, X=x / 2 \sqrt{\alpha \pi}$

12.1.2 9, p. 389 Semi-infinite solid with convection boundary. $t=t_{0}, x>0, \tau=0$. Solid increasing at velocity $u$.

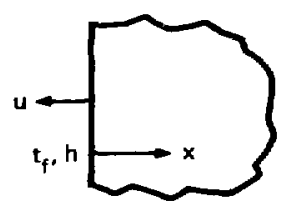

$\frac{t-t_{0}}{t_{f}-t_{0}}=\frac{1}{2}\left\{\operatorname{erfc}[X(1-0)]+\frac{F O B i}{\text { Fo Bi }-U} \exp (U / F O) \operatorname{erfc}[X(1+U)]\right.$ $+\frac{2 \text { Fo Bi }-U}{\text { Fo Bi-U }} \exp [\mathrm{Bi}(1-U+F O B i)] \operatorname{erfc}[\mathrm{X}(2 \mathrm{FO} B i+1-\mathrm{D})]$. $B i=h x / k, F O=\alpha \pi / x^{2}, x=x / 2 \sqrt{\sigma \pi}, U=u \pi / x$ 
Section 12.1. Traveling Boundaries.

\section{Case No. References}

Description

12.1.3 9, P. 389 Semi-infinite solid with steady periodic temp.

$t=\left(t_{0}-t_{m}\right) \cos (\omega \tau+\theta)$.

$x=0, \tau>0$.

Solid increasing at

velocity $u$.

$$
\begin{gathered}
\frac{t-t_{m}}{t_{0}-t_{m}}=\exp [u x / 2 \alpha-x \sqrt{b} \cos (\phi / 2)] \cos [\omega \tau-x \sqrt{b} \sin (\phi / 2)+\theta] \\
\frac{u^{2}}{4 \alpha^{2}}+\frac{i \omega}{\alpha}=b \exp (i \phi)
\end{gathered}
$$

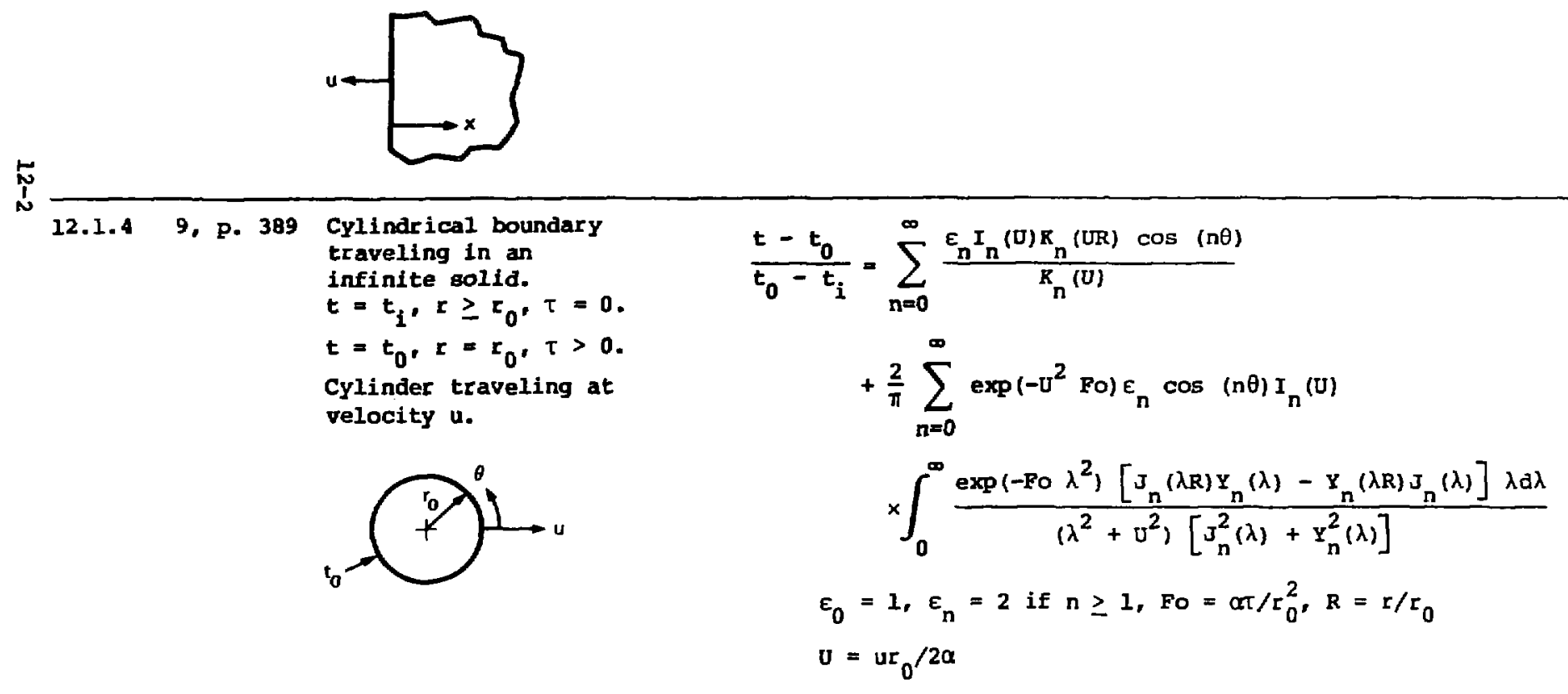


Section 12.1. Traveling Boundaries.

\section{Case No. Referenceg}

Description

Solution

12.1.5 9, p. 391 Thin rod with fixed end temps and convection cooling.

$t=t_{0}, 0<x<\ell, \tau=0$.

$t=t_{1}, x=l_{r} \tau>0$.

$t=t_{0}, x=0, \tau>0$.

The end boundaries traveling

at velocity $u$.

p = rod perimeter.

A $=$ rod section area.

$$
\frac{t-t_{0}}{t_{1}-t_{0}}=\frac{\sinh \left[\sqrt{U^{2} x^{2}+B i x^{2}}\right] \exp (U x-U)}{\sinh \left[\sqrt{U^{2}+B i}\right]}
$$

$+2 \pi \exp (u X-v) \sum_{n=1}^{\infty} \frac{(-1)^{n} n \sin (n \pi x)}{B i+u^{2}+n^{2} \pi^{2}}$

$x \exp \left[-\mathrm{Fo}\left(\mathrm{Bi}+\mathrm{v}^{2}+\mathrm{n}^{2} \pi^{2}\right)\right]$

$B i=h p \ell^{2} / k A, F O=\alpha / \ell^{2}, U=u \ell / 2 \alpha, x=x / \ell$

$\stackrel{\sim}{\omega}$

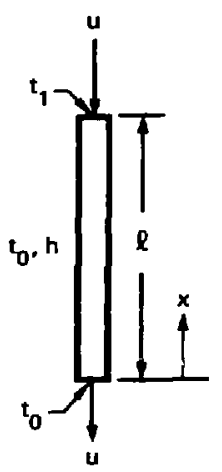


Section 12.1. Traveling Boundaries.

Case No. References Description

Solution 
Section 12.1. Traveling Boundaries.

\section{Case No. References Description}




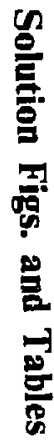

REPRODUCED FROM BEST AVAILABLE COPY 


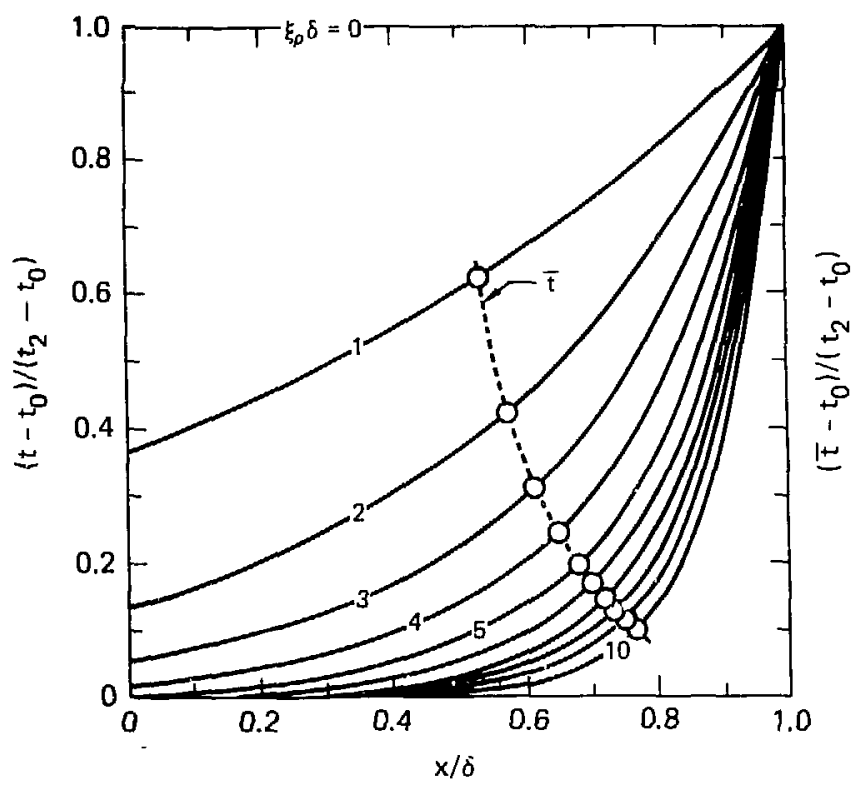

FIG. 1.1. Temperature distribution and mean temperature in a porous plate (case 1.1.4, source: Ref. 2, p. 221, Fig. 9.2).

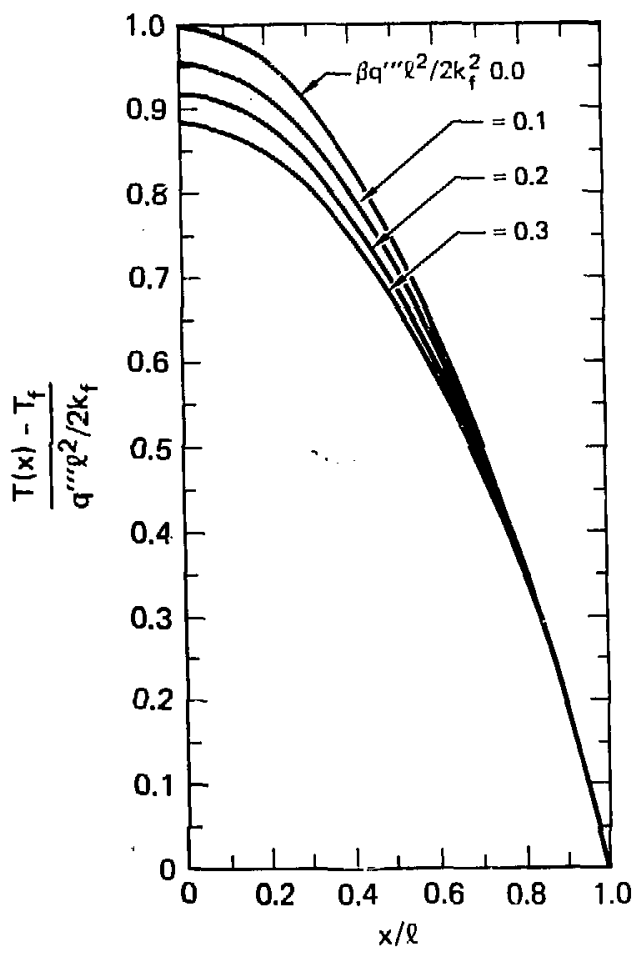

FIG. 1.2. Temperature distribution in an infinite plate with internal heating and temperature dependent conductivity (case 1.2.3, source: Ref. 3, p. 132, Fig. 3.23). 


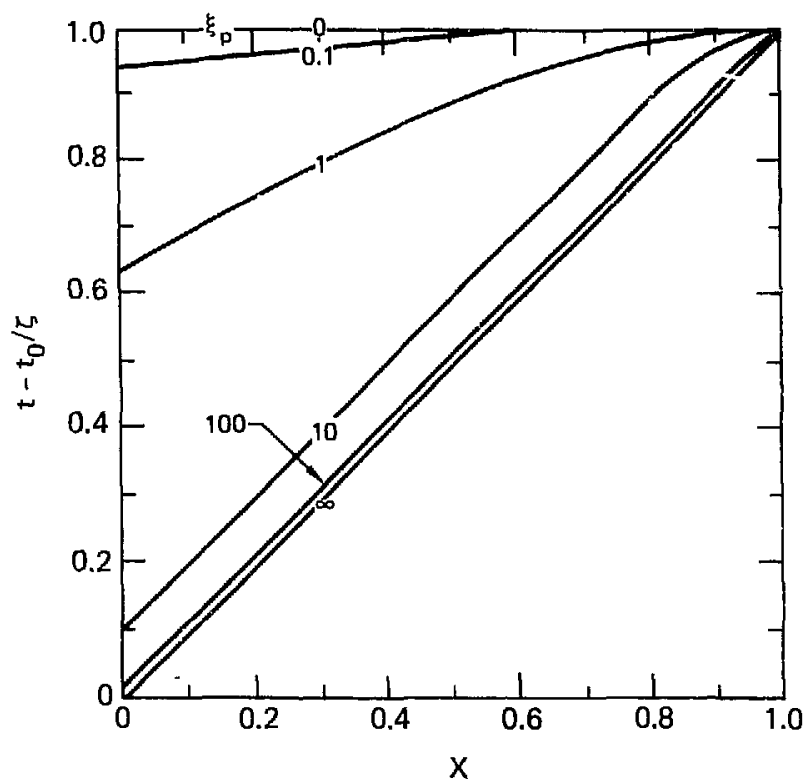

FIG. 1.3. Temperature distribution in a heat generating porous plate (case 1.2.6, source: Ref. 2, p. 223, Fig. 9.3).

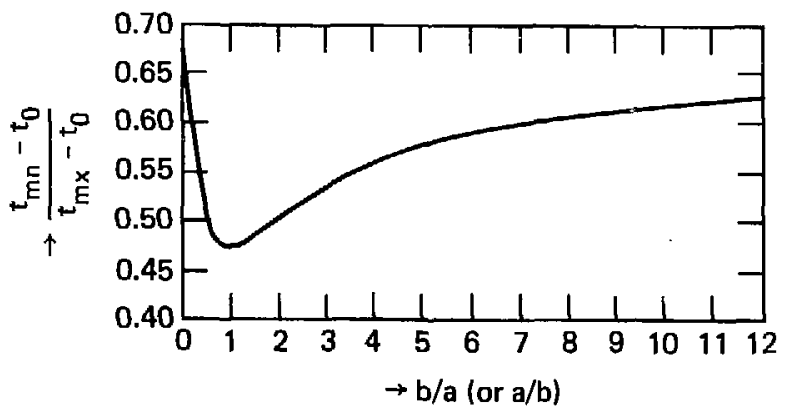

FIG. 1.4. Ratio of mean and maximum temperature excesses in an electrical coil of rectangular cross section (case 1.2.8, source: Ref. 2., p. 180, Fig. 10.5) . 


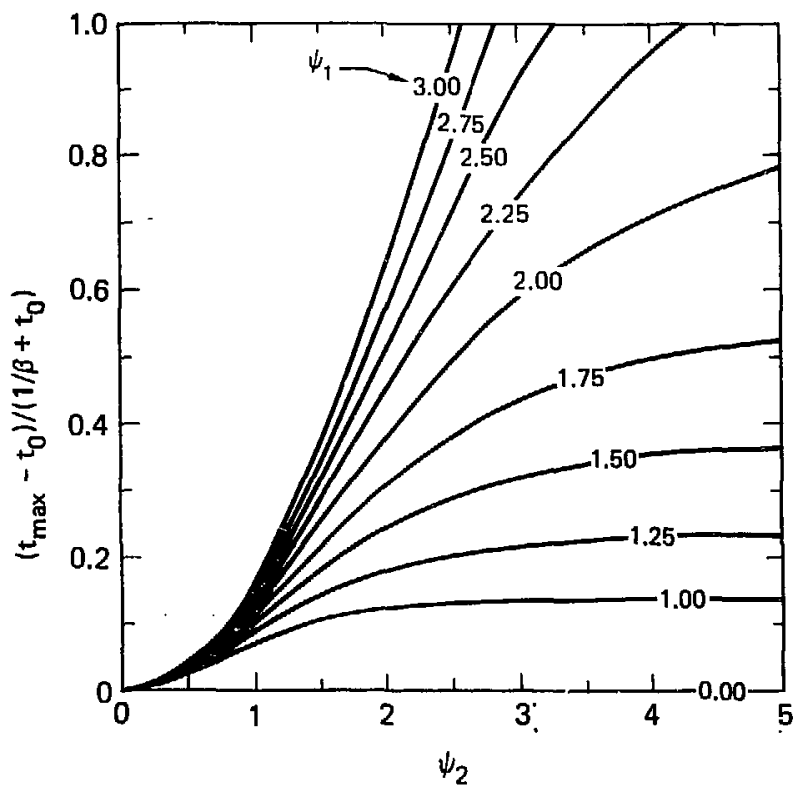

FIG. 1.5. Firgt-term approximation to the maximum temperature in a solid rectangular rod with internal heating, $\psi_{1}=\sqrt{q_{0} T \cdot / \beta k} 2 b, \psi_{2}=\sqrt{q_{0}^{1} 1 / \beta k} 2 a$ (case 1.2.10, source: Ref. 2, p. 198, Fig. B.16). 


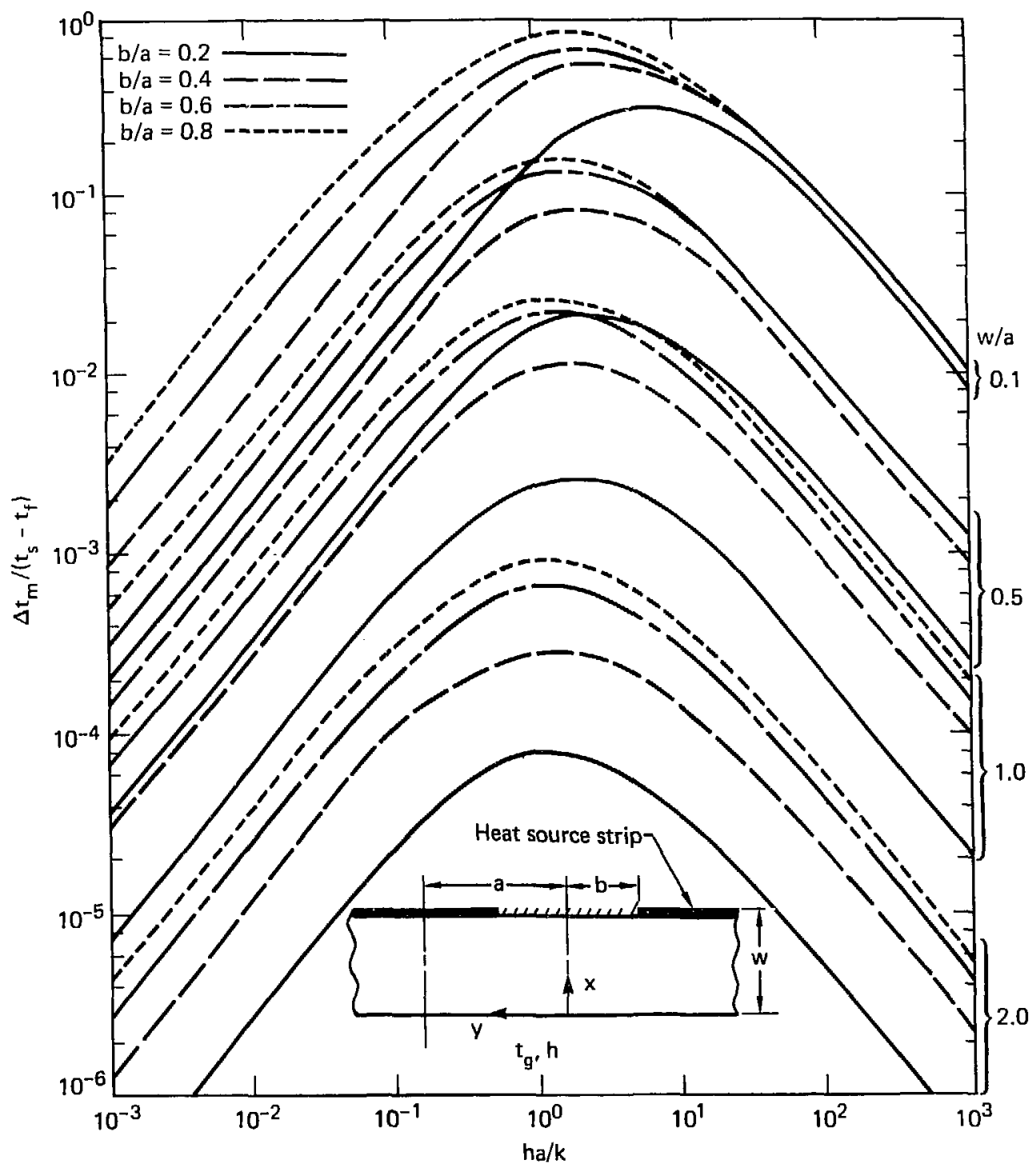

FIG. 1.6a. Maximum temperature variations on the cooled surface of a flat plate having equally spaced adiabatic and constant temperature strips on the opposite surface (case 1.1.30, source: Ref. 27). 


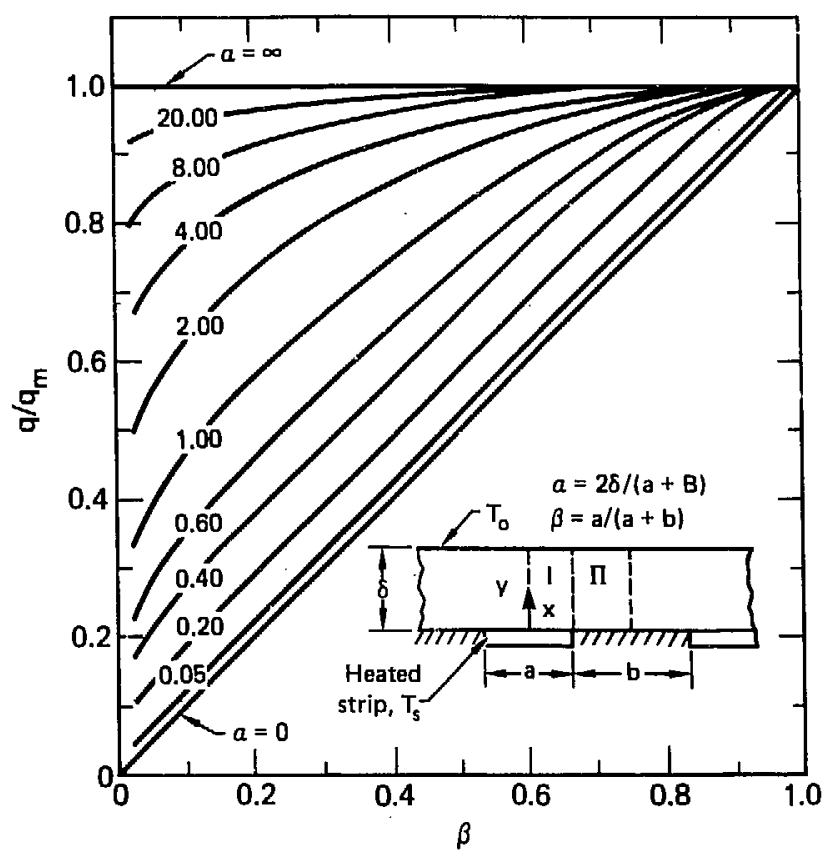

FIG. 1.6b. Heat flux through slabs held at a uniform temperature on one surface and having equally spaced constant temperature strips on the other (case 1.1.30, source: Ref. 88). 


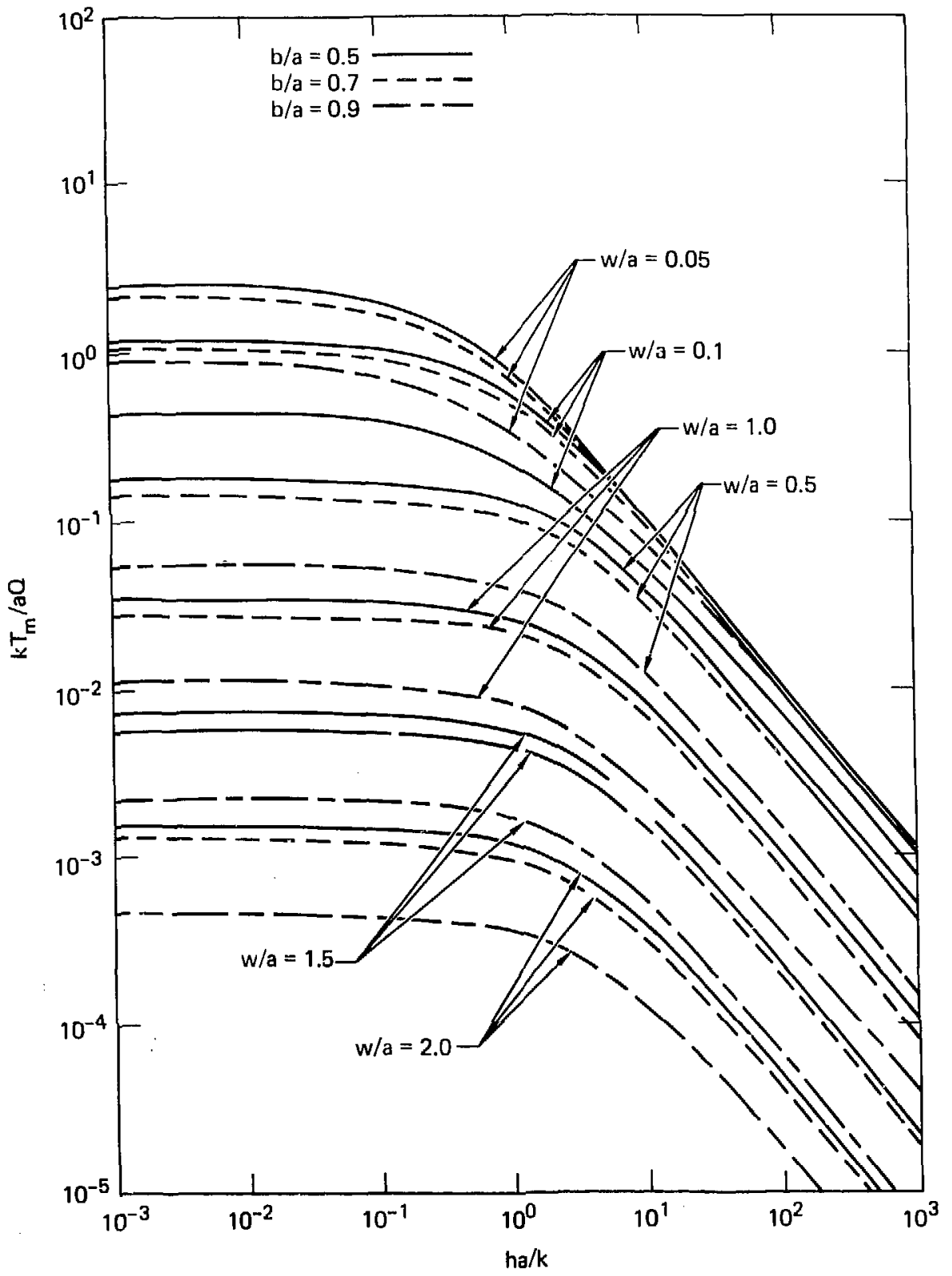

FIG. ‥7. Maximum temperature variation of the cooled surface of a flat plate havi alternating adiabatic and constant heat-flux strips on the opposite surface (case 1.1.31, source: Ref. 28). 


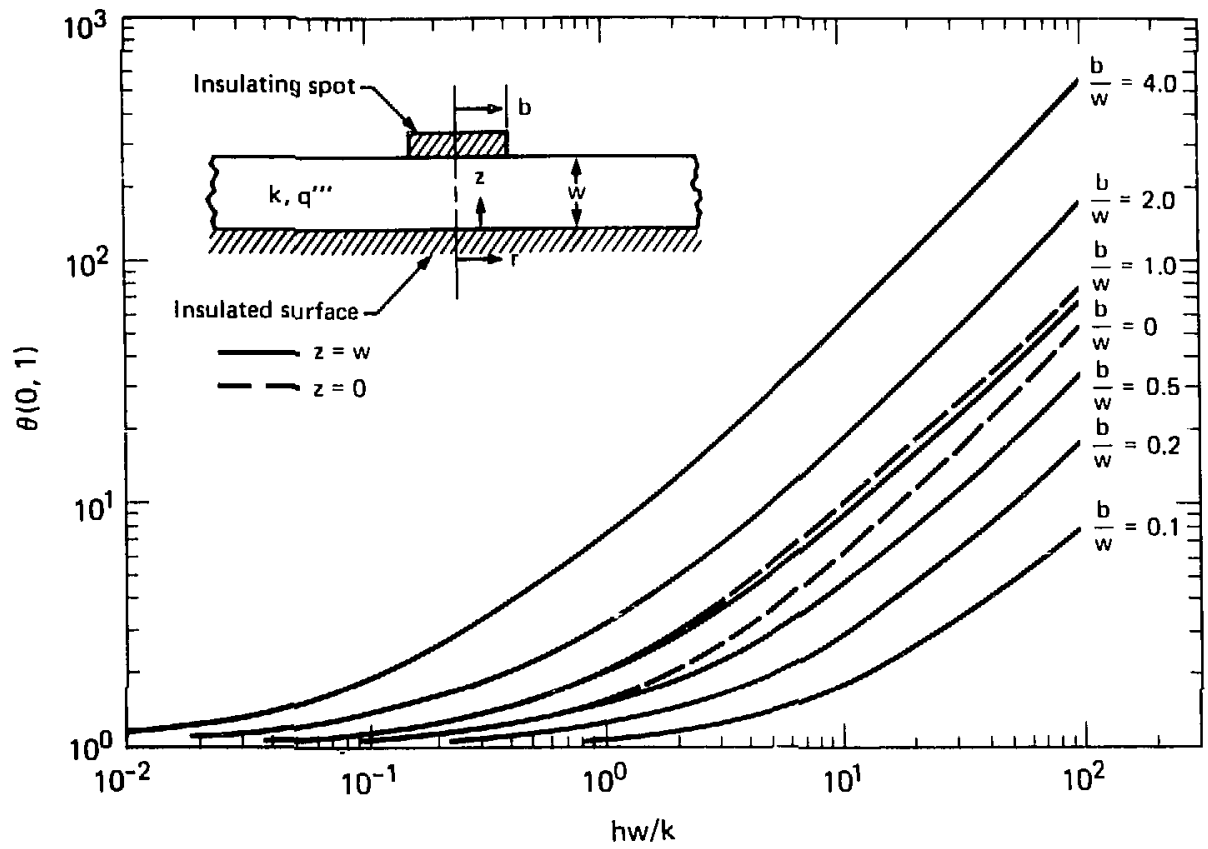

FIG. 1.8. Temperatures at the spot center of a spot-insulated plate having a uniform internal heat source (case 1.2.13, source: Ref. 29:.

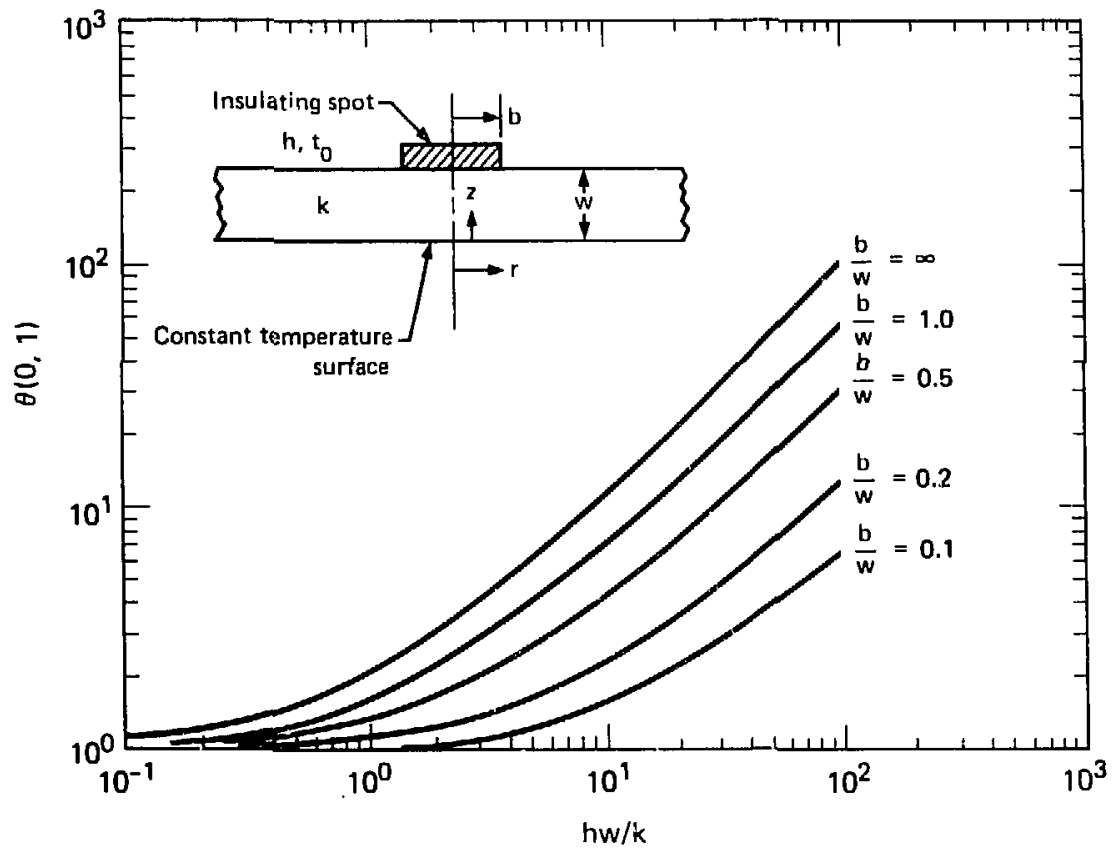

FIG. 1.9. Temperatures on the cooled surface and at the spot center of a spot-insulated plate having a constant temperature heat source on one face (case 1.1.32, source: Ref. 29). 


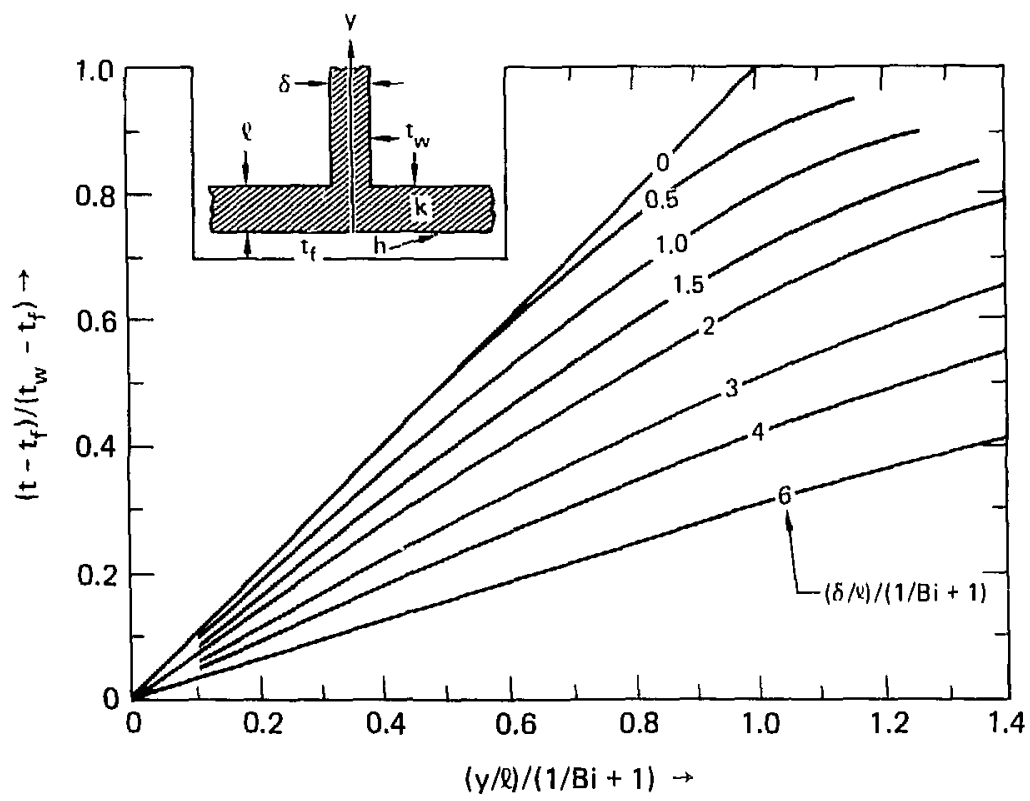

FIG. 1.10. Hotspot temperatures along plate/rib centerline $(B i=h l / k)$ (case 1.1.39, source: Ref. 19, p. 3-126, Fig. 77).
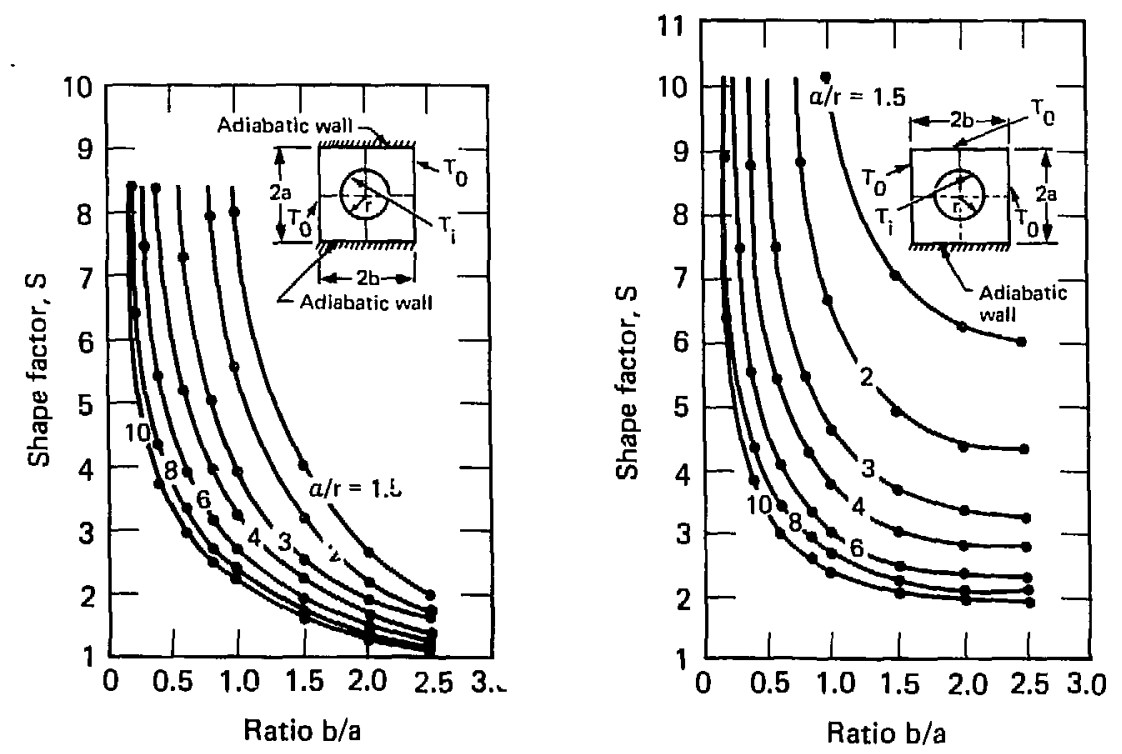

FIG. 1.11. Conductive shape factors for a rectangular section containing a constant temperature tube (case 1.1.44, source: Ref. 87). 


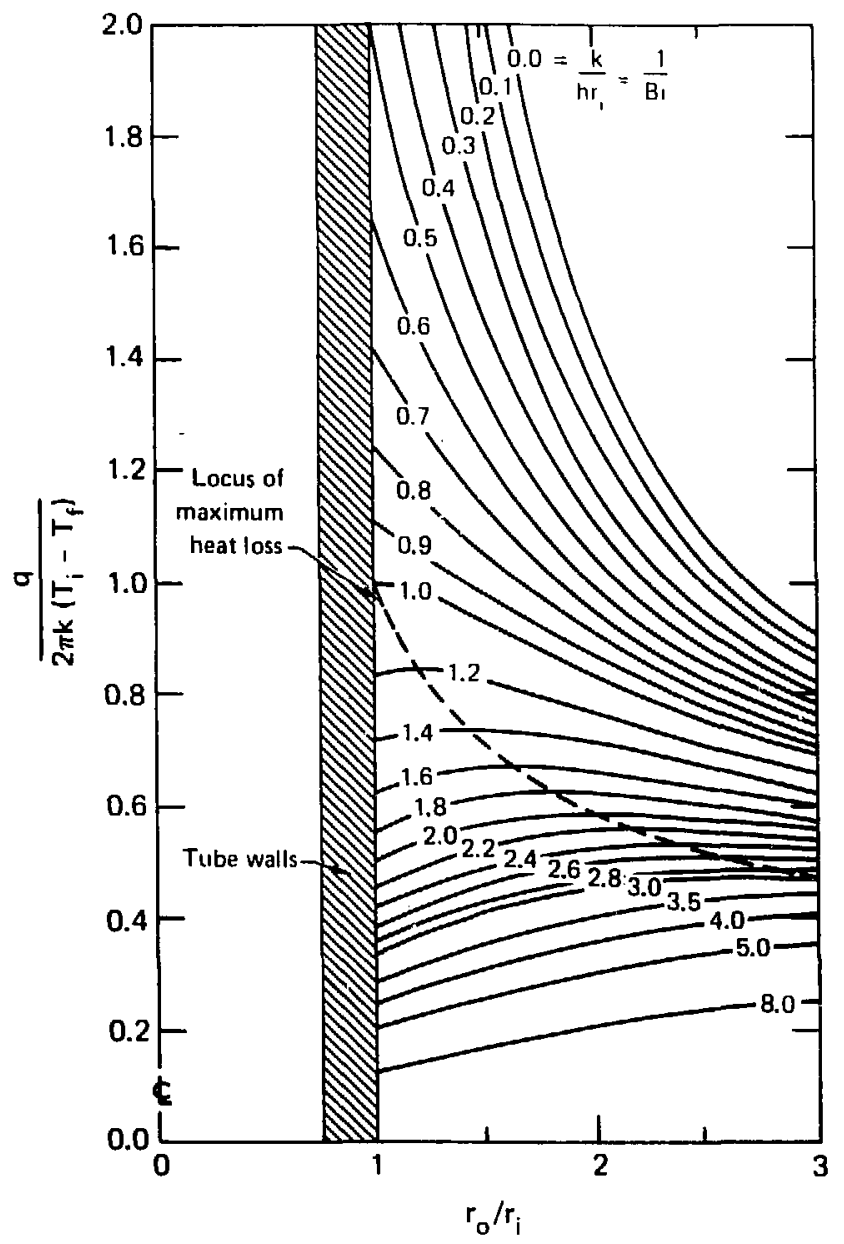

FIG. 2.1. Heat loss from insulated tubes (case 2.1.3, source: Ref. 3, p. 123, Fig. 3.18). 


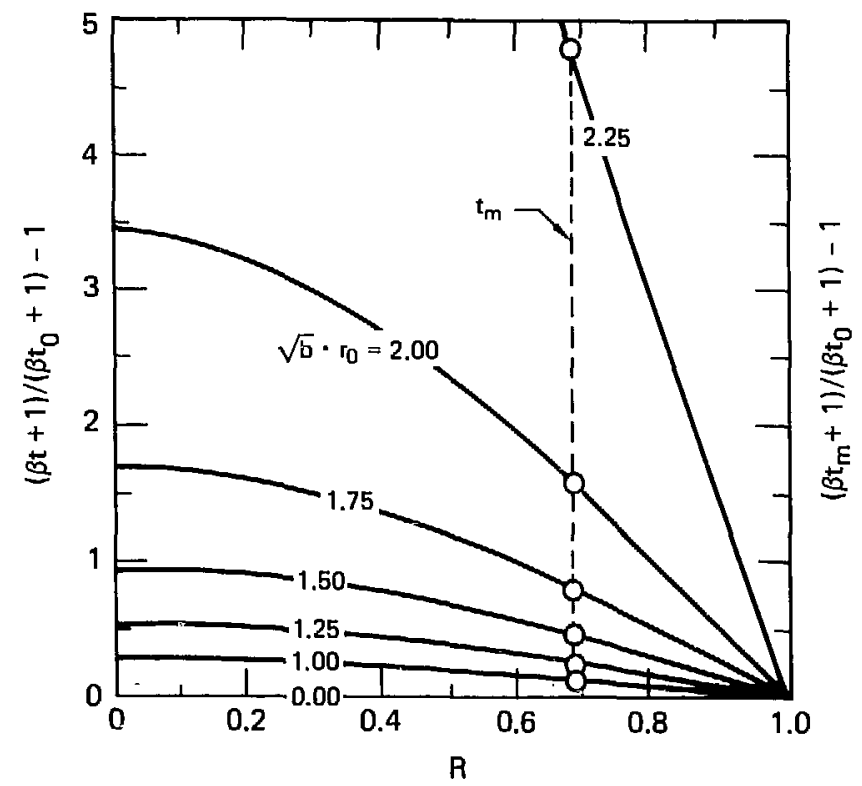

FIG. 2.2. Temperature distribution and mean temperature in a cylinder with temperature dependent heat source (case 2.2 .4 , source: Ref. 2, p. 189, Fig. 8.11).

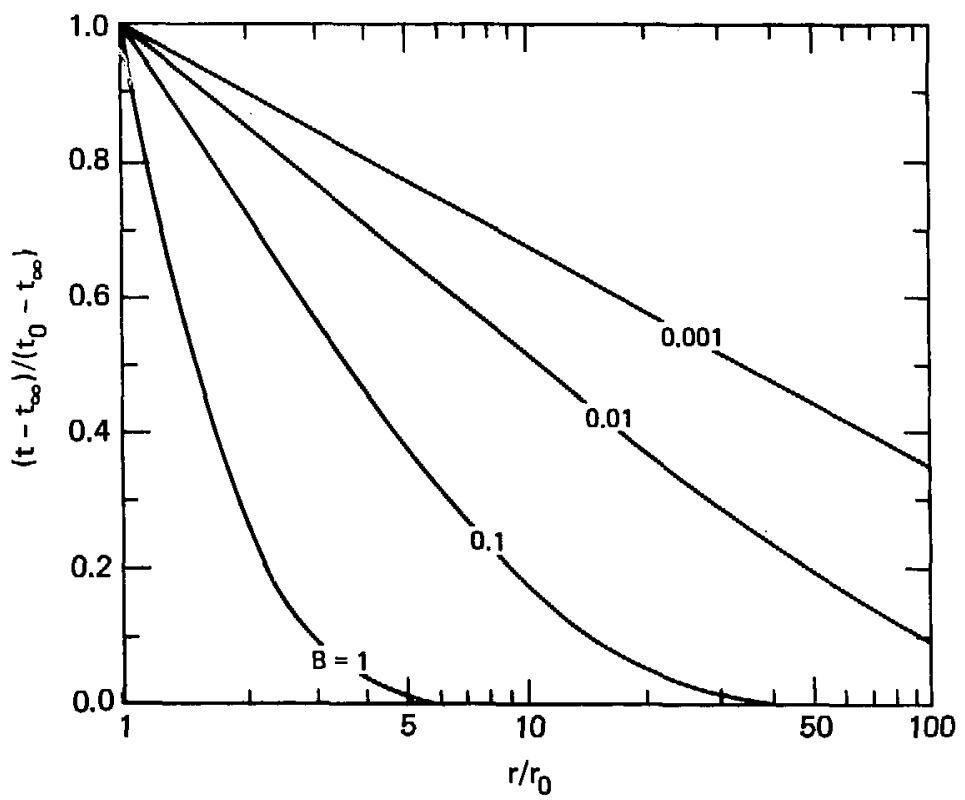

FIG. 2.3. Temperature distribution in an infinite plate with a cylindrical heat source, $B=\sqrt{\left(h_{1}+h_{2}\right) / k w} r_{0}, t_{0}=t$ at $r_{0}, t_{\infty}=t$ at $r=\infty$ (case 2.2 .1 source: Ref. 2, p. 175, Fig. 8.2). 


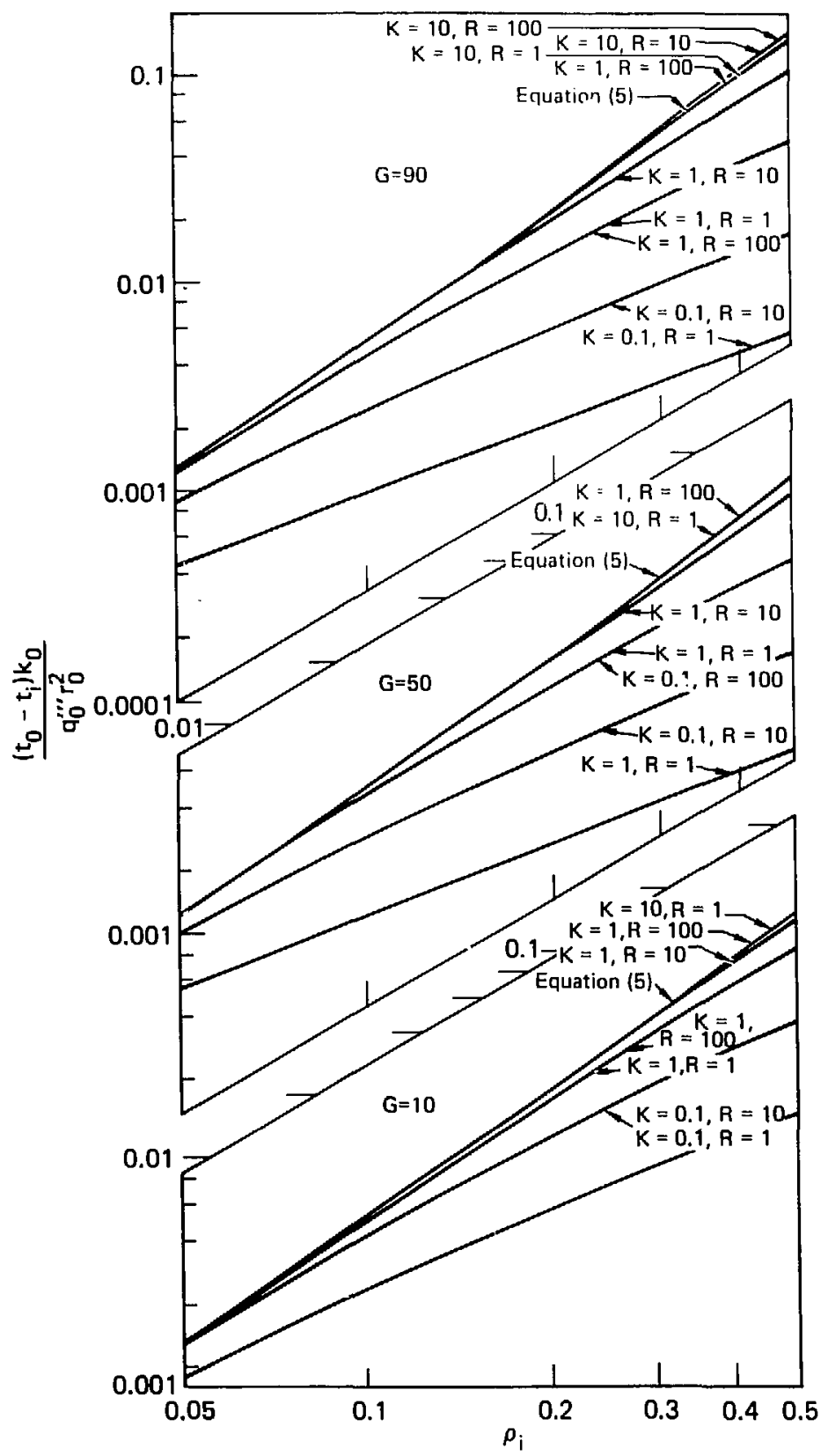

FIG. 2.4. The inside surface temperature of an infinite tube with temperature dependent conductivity and heating (case 2.2.17, source: Ref. 16, Fig. 1). Equation (5) given in case solution. 


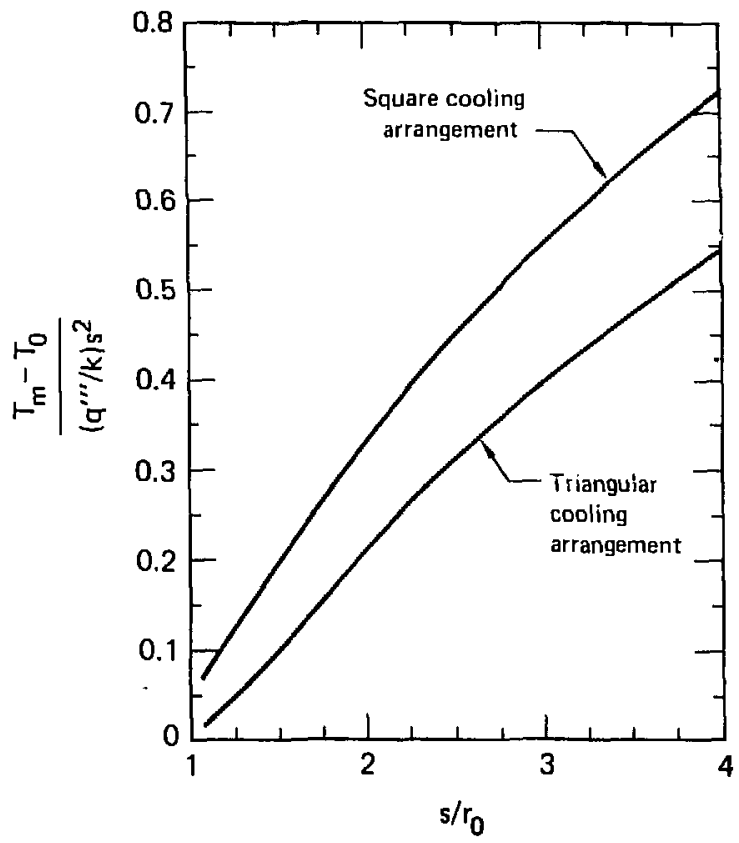

FIG. 2.5. Maximum (hot spot) temperatures in the cross section of a heat-generating solid (cases 2.2.18 and 2.2.19, source: Ref. 64, Fig. 3 ).

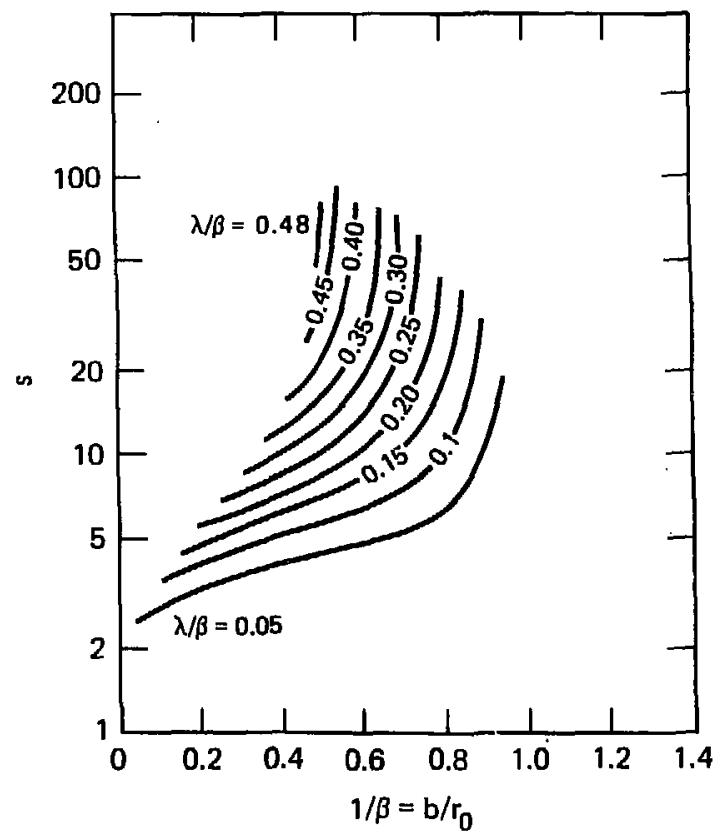

FIG. 2.6. Shape factor for a cylinder with two longitudinal holes (case 2.1.44, source: Ref. 73, Fig. 6). 


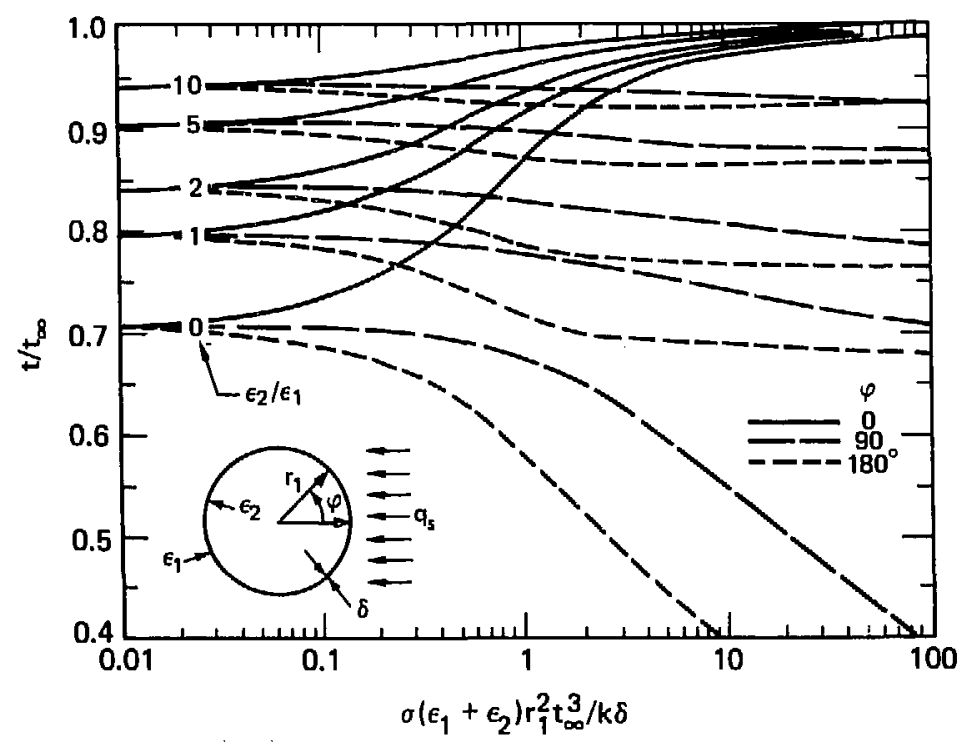

FIG. 3.1. Steady temperature of thin, nonrotating spherical shell in uniform radiation field (case 3.1.10, source: Ref. 19, pp. 3-112, Fig. 68). 


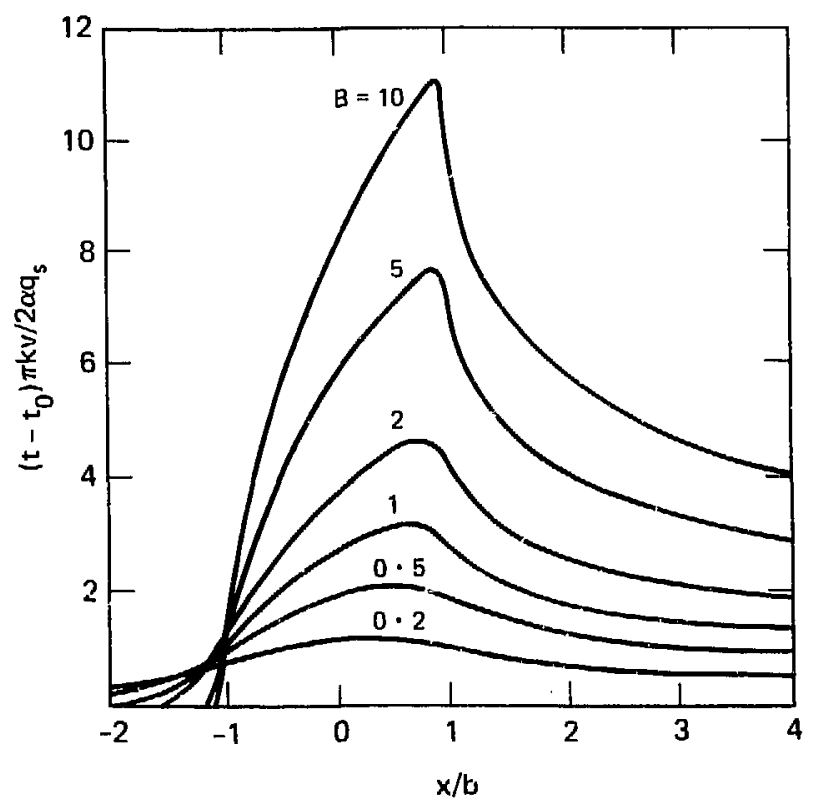

FIG. 4.1. Surface temperature of a semi-infinite solid with heating on the surface over width $2 b$, which moves at velocity u (case 4.1.8, source: Ref. 9, p. 270, Fig, 34).

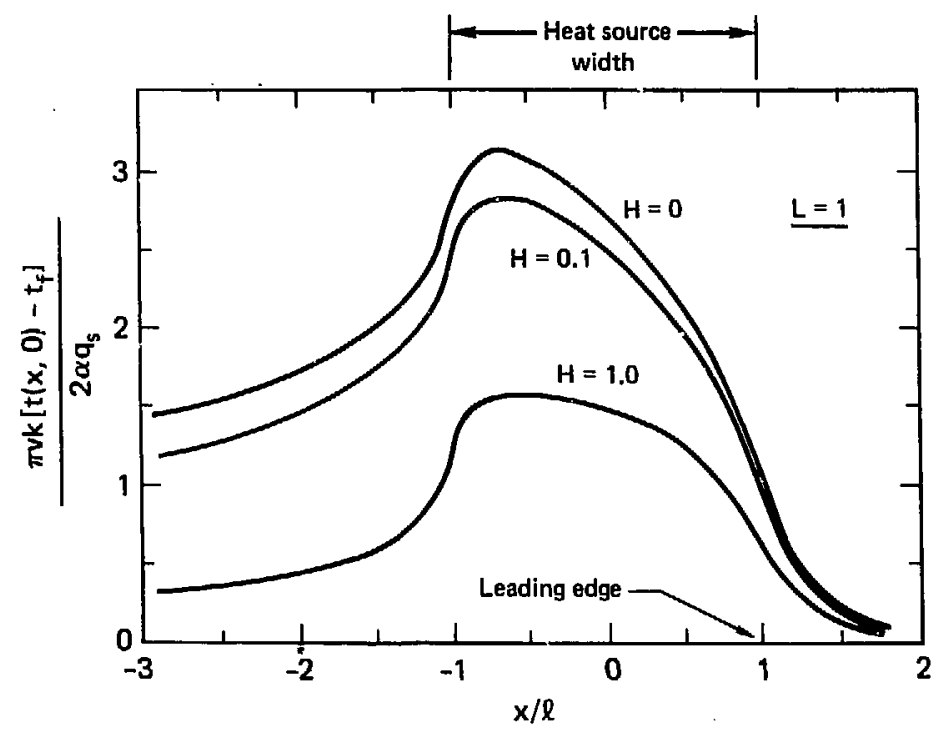

FIG. 4.2. Surface temperature of a convectively cooled semi-infinite solid with a traveling strip heat source (case 4.1.9, source: Ref. 30). 


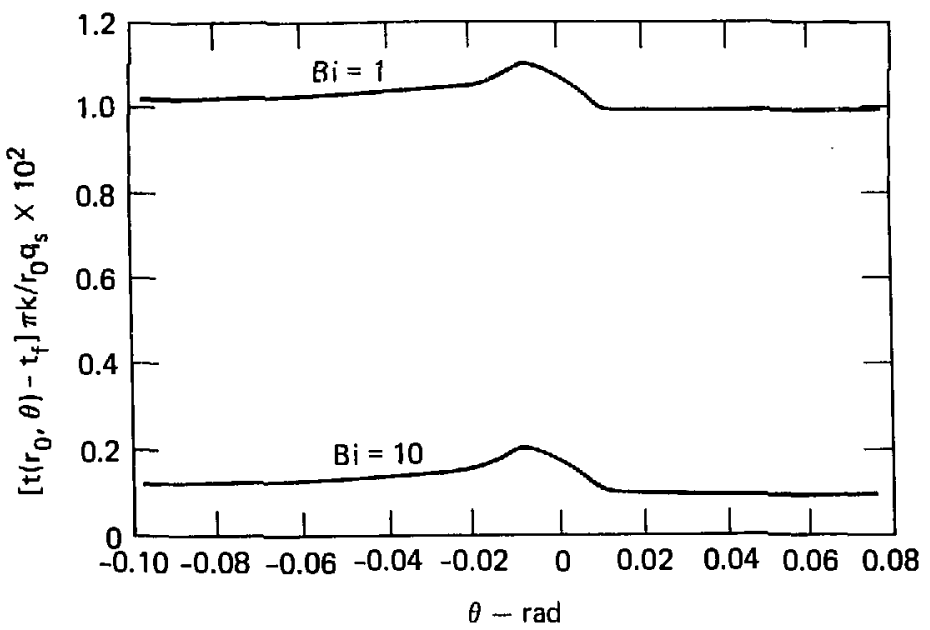

FIG. 4.3a. Surface temperature of an infinite cylinder with a rotating surface band source, $\phi=0.01$ (case 4.1.10, source: Ref. 30).

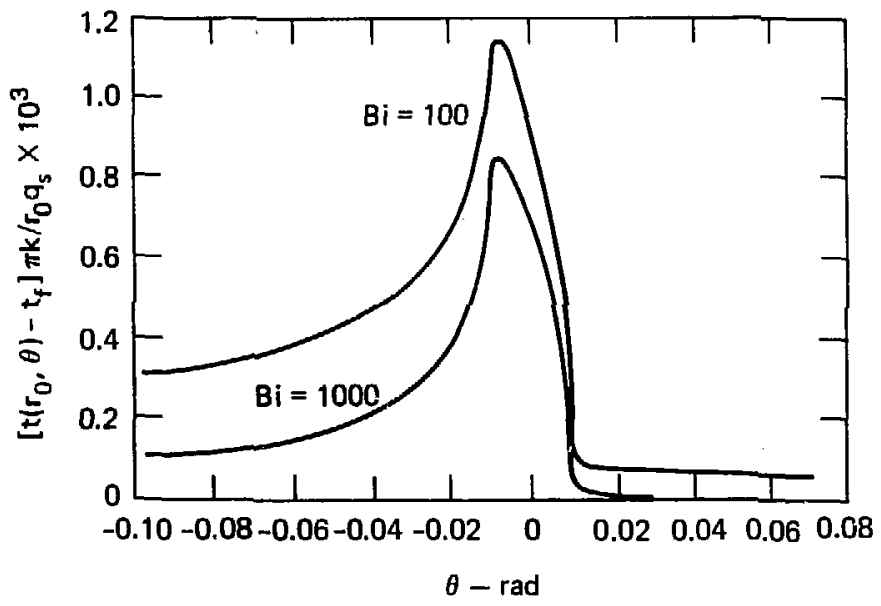

FIG. 4.3b. (Same conditions as for Fig. 4.3a). 

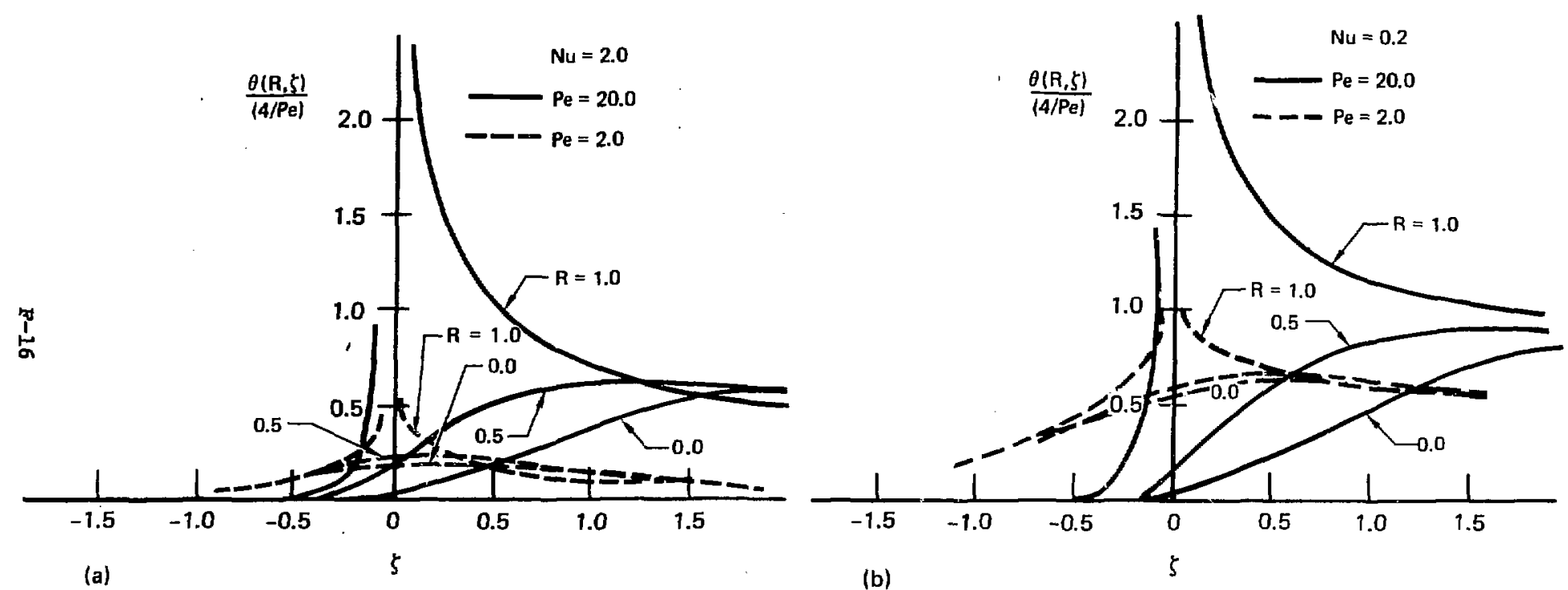

FIG. 4.4. Temperature distribution in a crlinder with a ring heat source (case 4.1.11, source: Ref. 34). $\theta=\left(t-t_{f}\right) 2 \pi r_{0} k / Q_{0}$. 

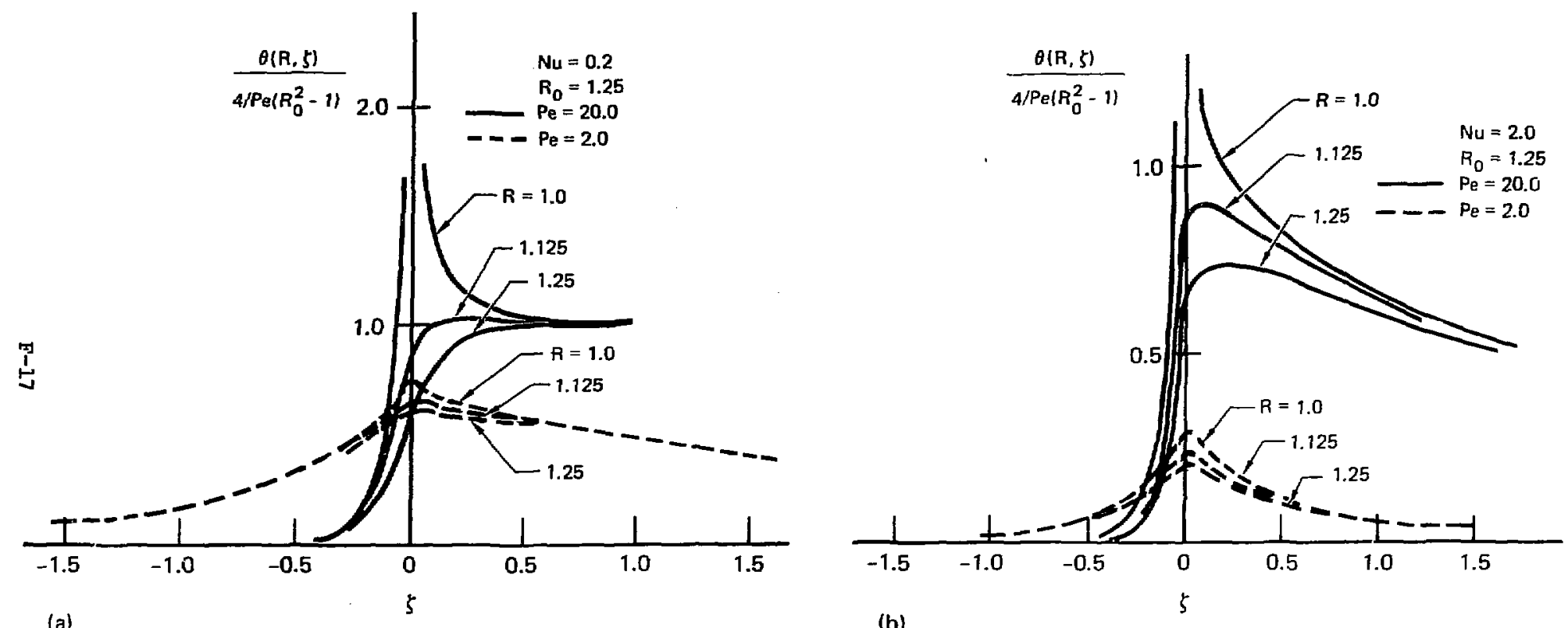

(b)

FIG. 4.5. Temperature distributions in a hollow cylinder with an inside ring heat source (case 4.1.12, source: Ref. 34). $\theta$ defined in Fig. 4.4. 


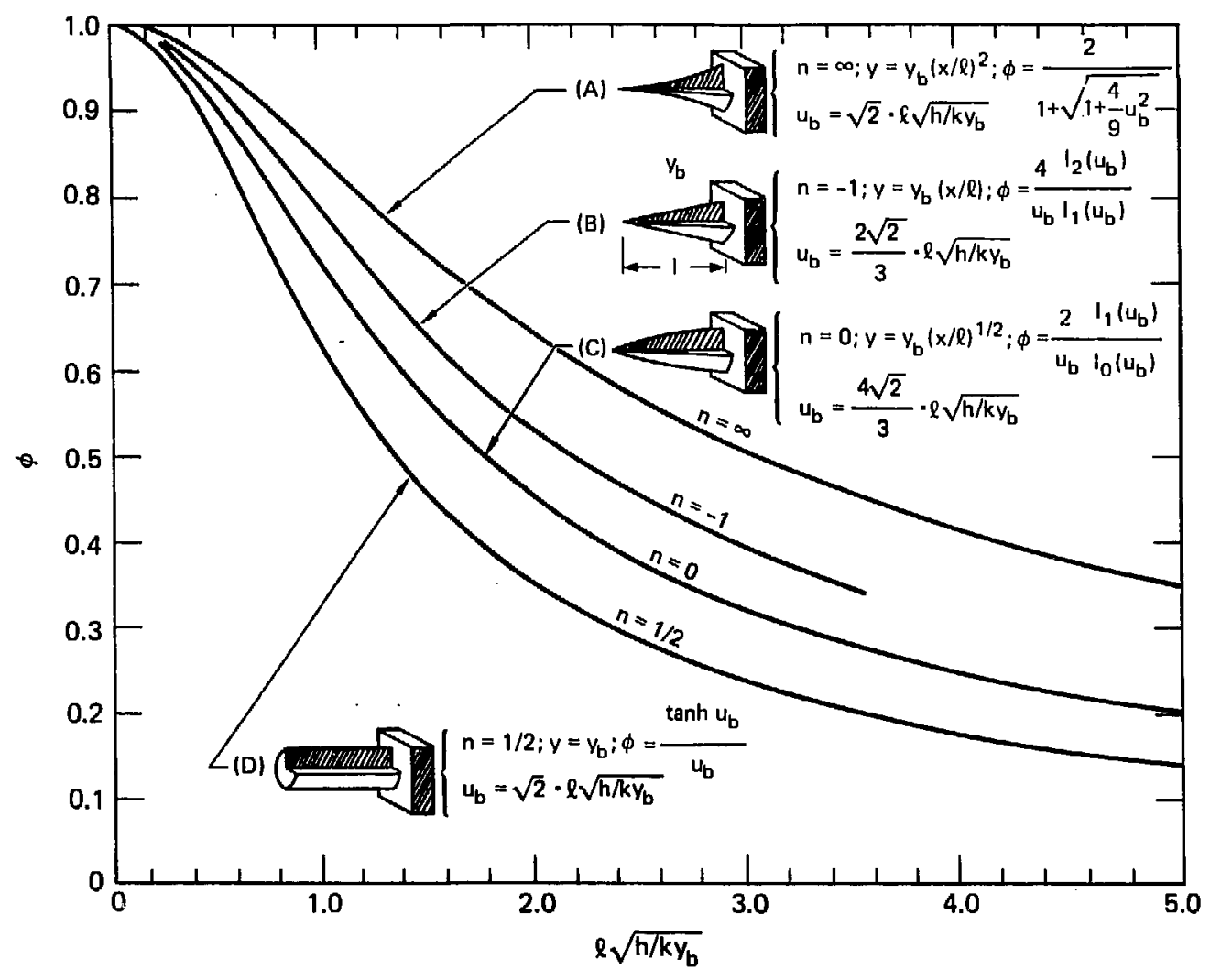

FIG. 5.1. Performance of pin fins lcases 5.1.2, 5.1.14, and 5.1.16 through 5.1.18, source: Ref. 8 and Ref. 7, p. 55, Fig. 3.14. 


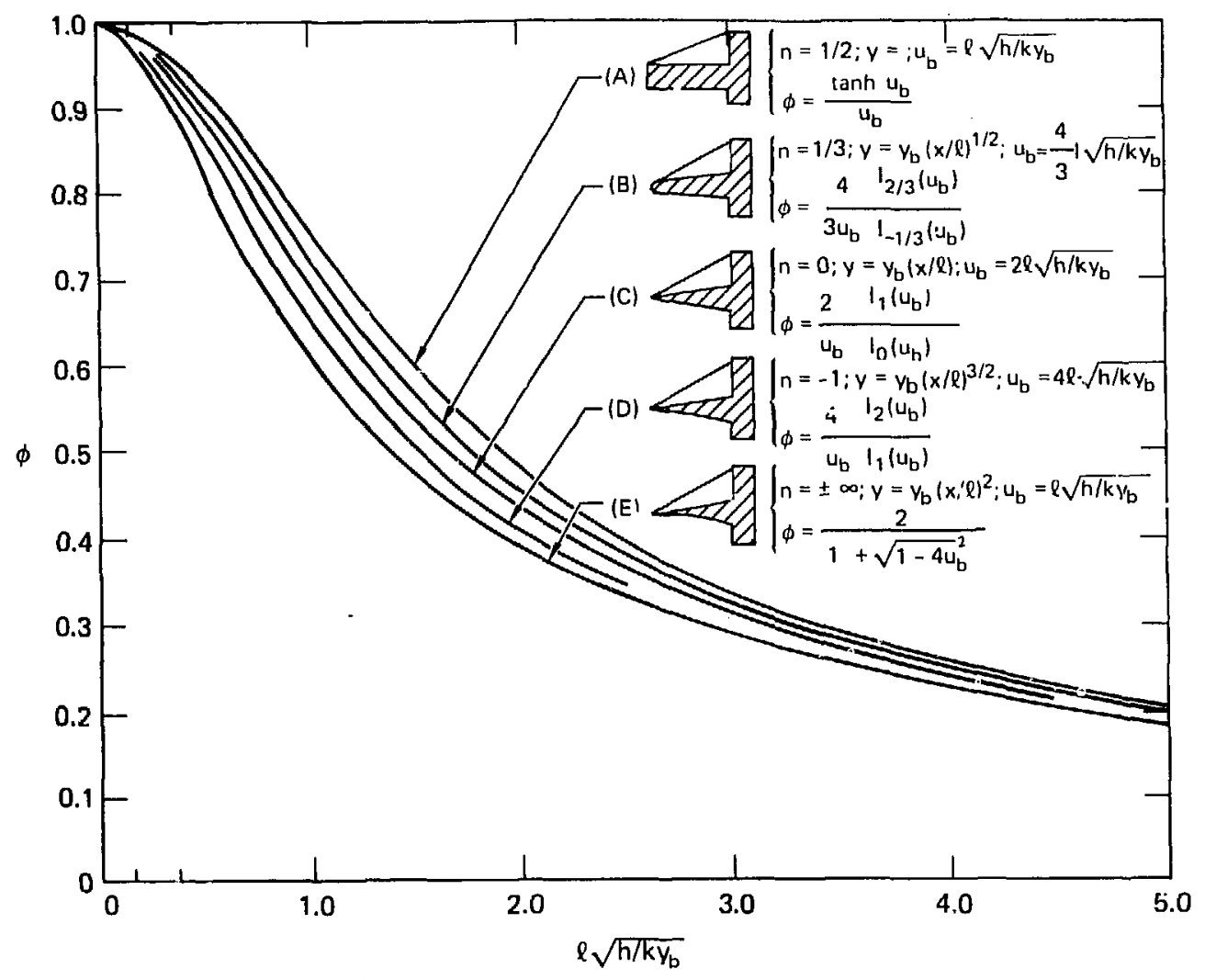

FIG. 5.2. Performance of straight fins (cases 5.1.4, 5.1.5, 5.1.7, 5.1.8, source: Ref. 8 and Ref. 7, p. 56, Fig. 3.15). 


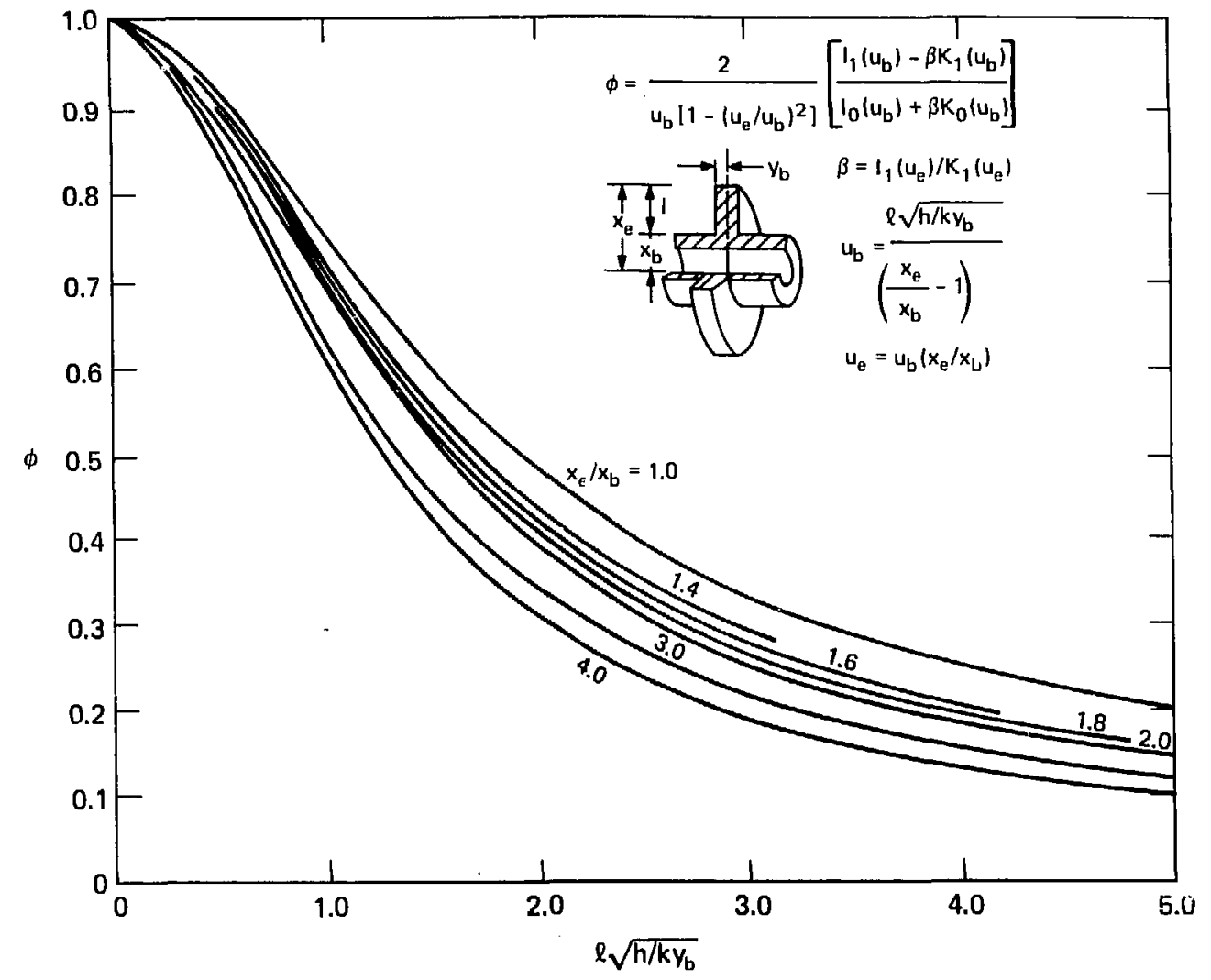

FIG. 5.3. Performance of circumferential fins of rectangular cross section (case 5.1.10, source: Ref. 8 and Ref. 7, p. 57, Fig. 3.16). 


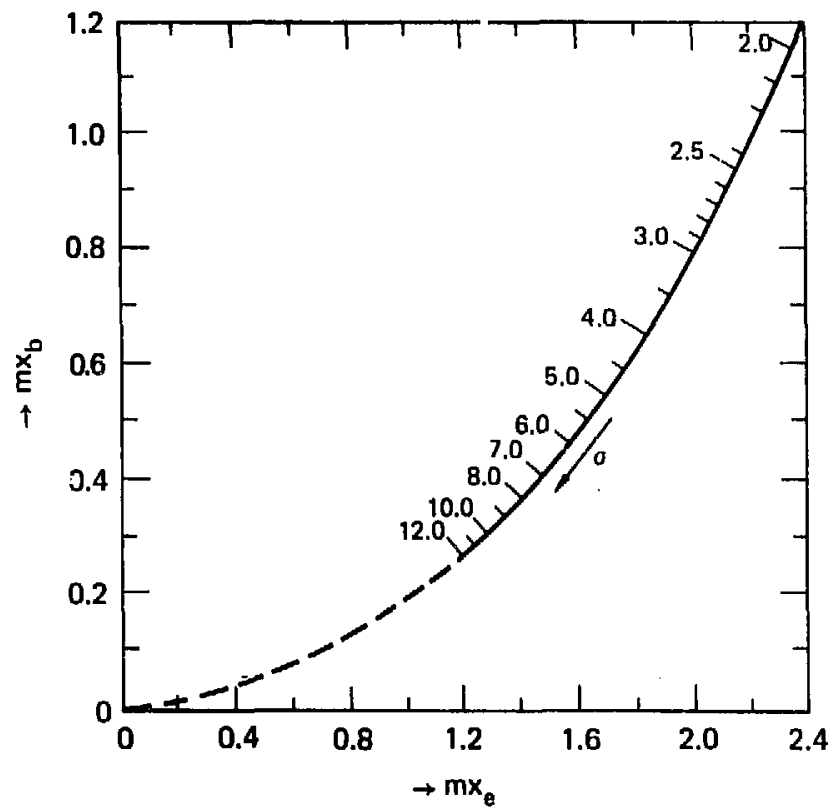

1G. 5.4. Curve for calculating dimensions of circular fin of rectangular profile requiring least material lcase 5.1.10, source: Ref. 1, p. 234, Fig. 11.11). $\sigma=0 / \pi h x_{b}^{2}\left(t_{0}-t_{f}\right)$. 


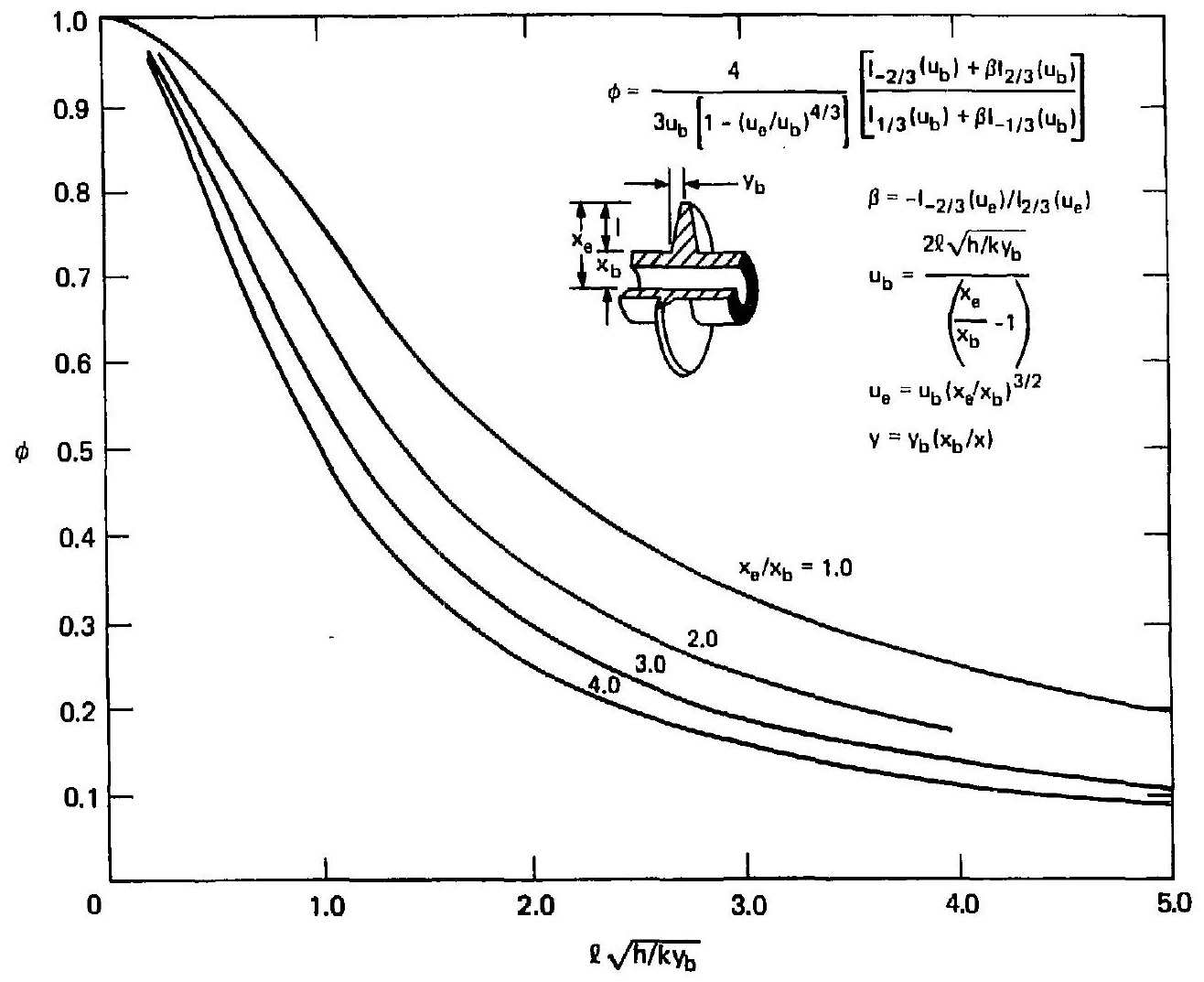

FIG. 5.5. Performance of cylindrical fins of triangular profile (case 5.1.11, source: Ref. 8 and Ref. 7, p. 58, Fig. 3.17). 


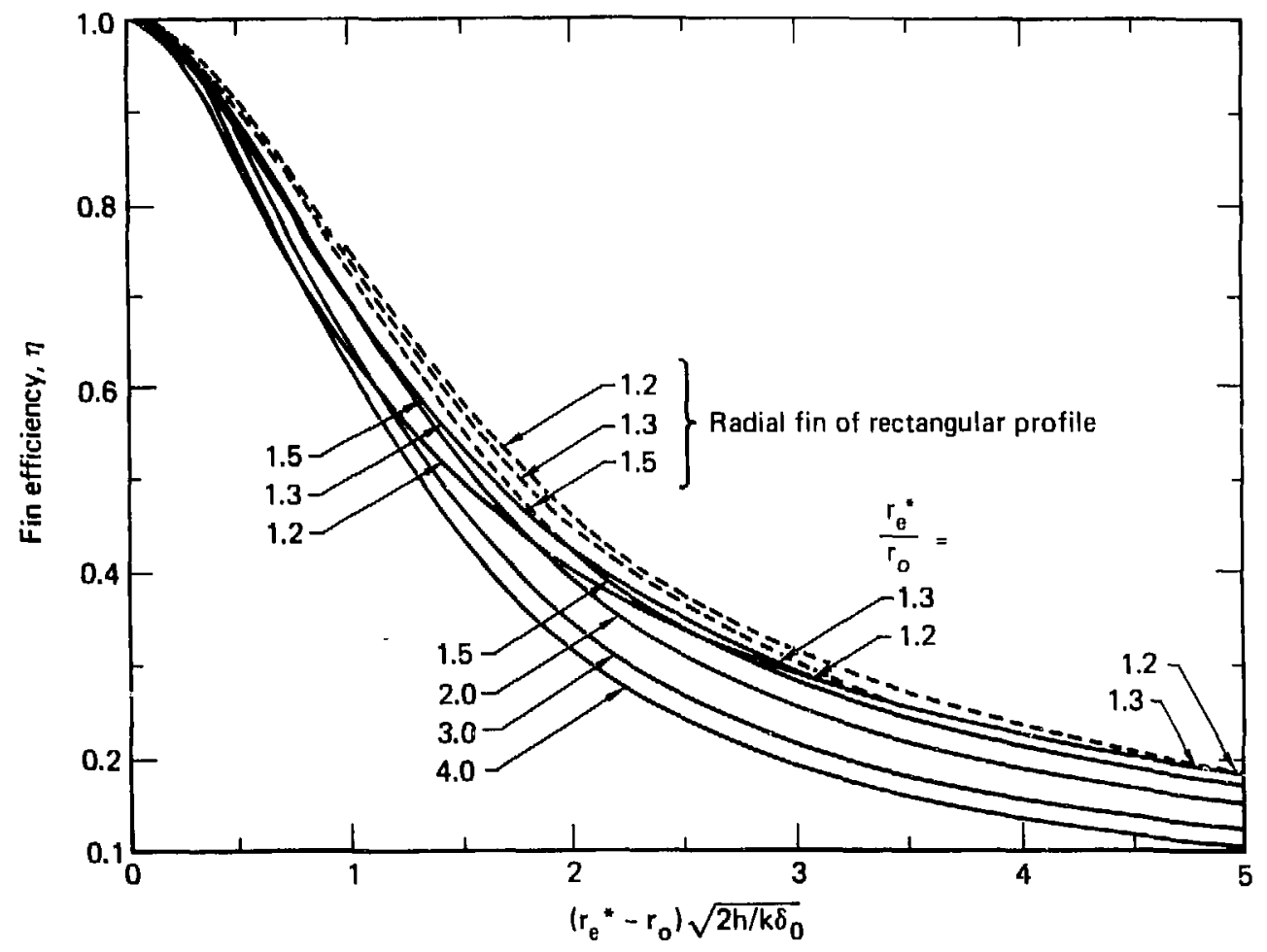

FIG. 5,6. Efficiency of an infinite fin heated by square arrayed round rods (case 5.1.19, source: Ref. 11 and Ref, 10, p. 135, Fig. 2.22). $\mathrm{r}_{\mathrm{e}}^{*}=(2 / \sqrt{\pi}) \mathrm{s}$. 


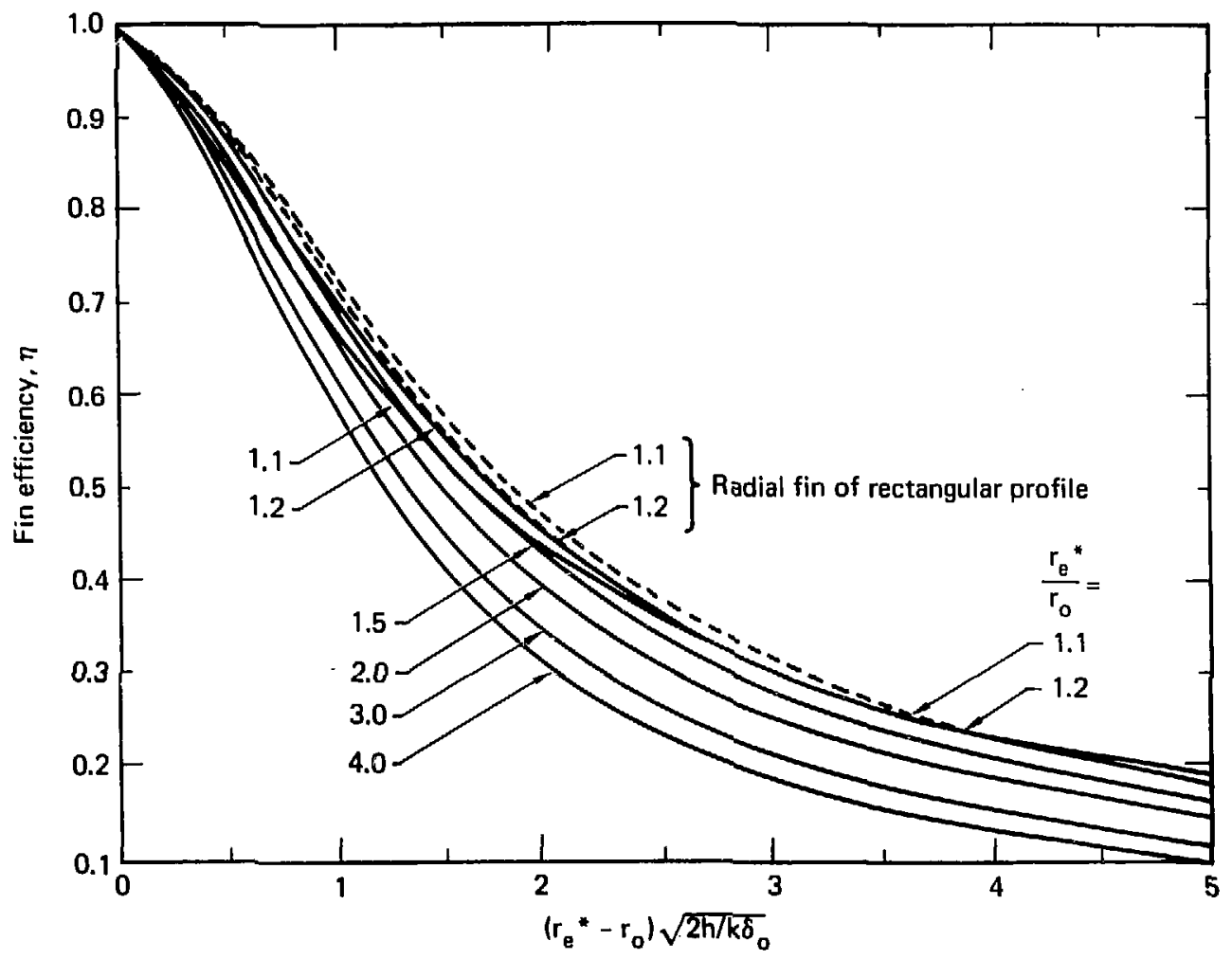

FIG. 5.7. Efficiency of an infinite fin heated by equilateral triangular arrayed round rods (case 5.1 .20 , source: Ref. 11 and Ref. 10, p. 136, Fig. 2.23). $\mathrm{r}_{\mathrm{e}}^{\star}=(2 \sqrt{3} / \pi)^{1 / 2} \mathrm{~s}$. 


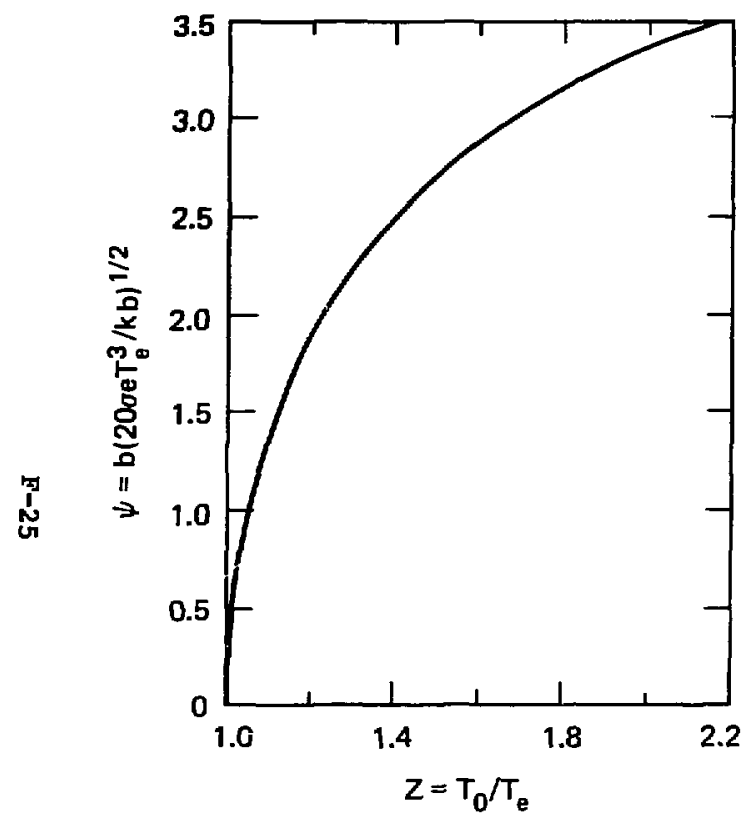

FIG. 5.8, Fin parameter as a function of $z$ for a straight fin radiating to free space (case 5.1.24, source: Ref. 10, p. 208, Fig. 4.31.

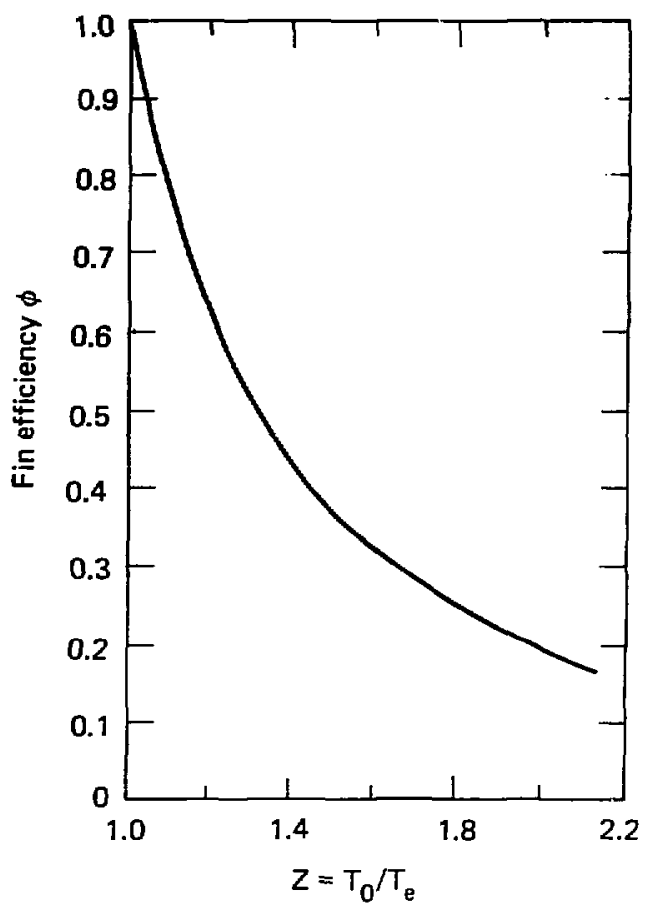

FIG. 5.9. Fin efficiency for a straight fin radiation to free space (case 5.1.24, source: Ref. 10, p. 209, Fig. 4.4). 


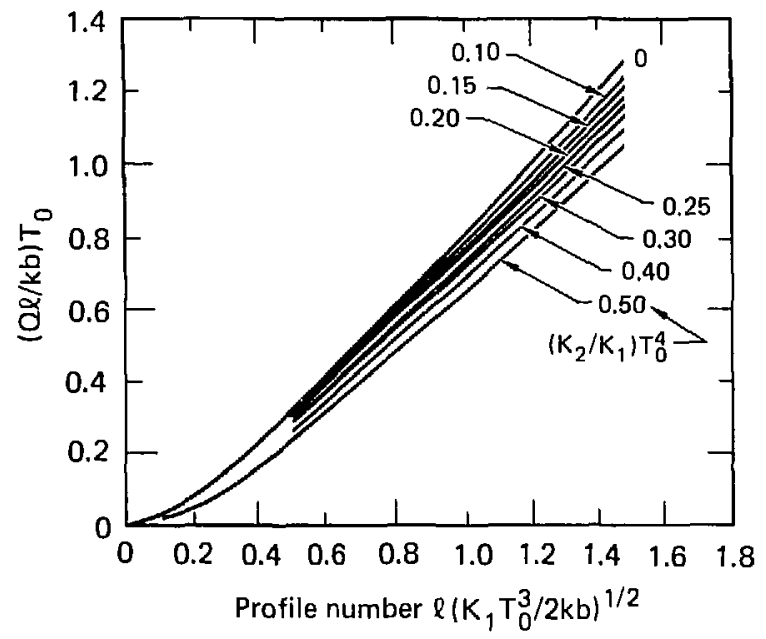

FIG. 5.10. Heat flow relationship for a straight fin of rectangular profile radiating to nonfree space (case 5.1.25, source: Ref. 10, p. 216, Fig. 4.8).

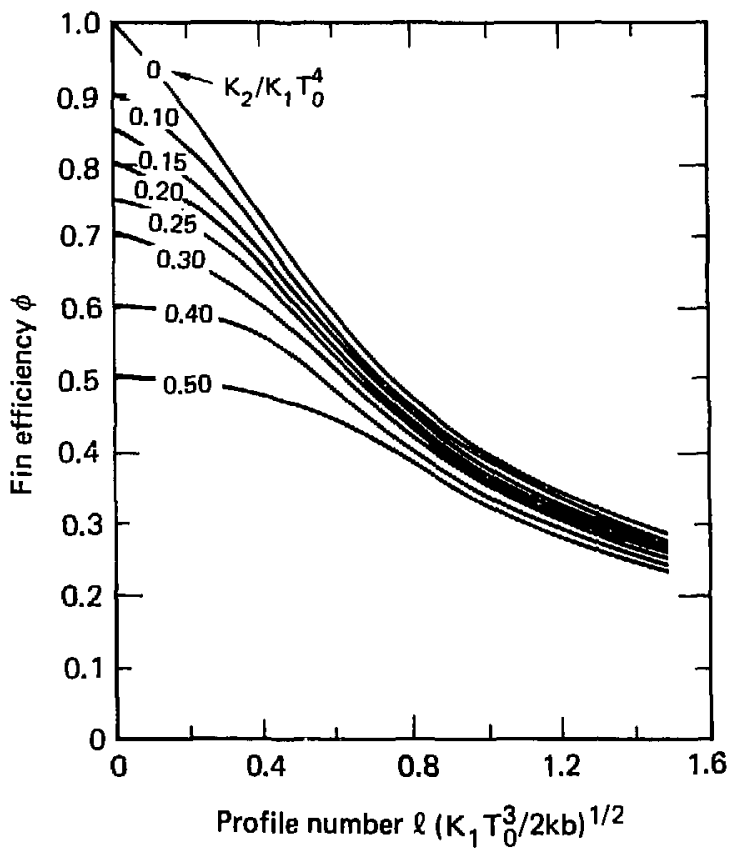

FIG. 5.11. Efficiency of a straight fin of rectangular profile radiating to nonfree space (case 5.1.25, source: Ref. 10, p. 216, Fig. 4.9). 


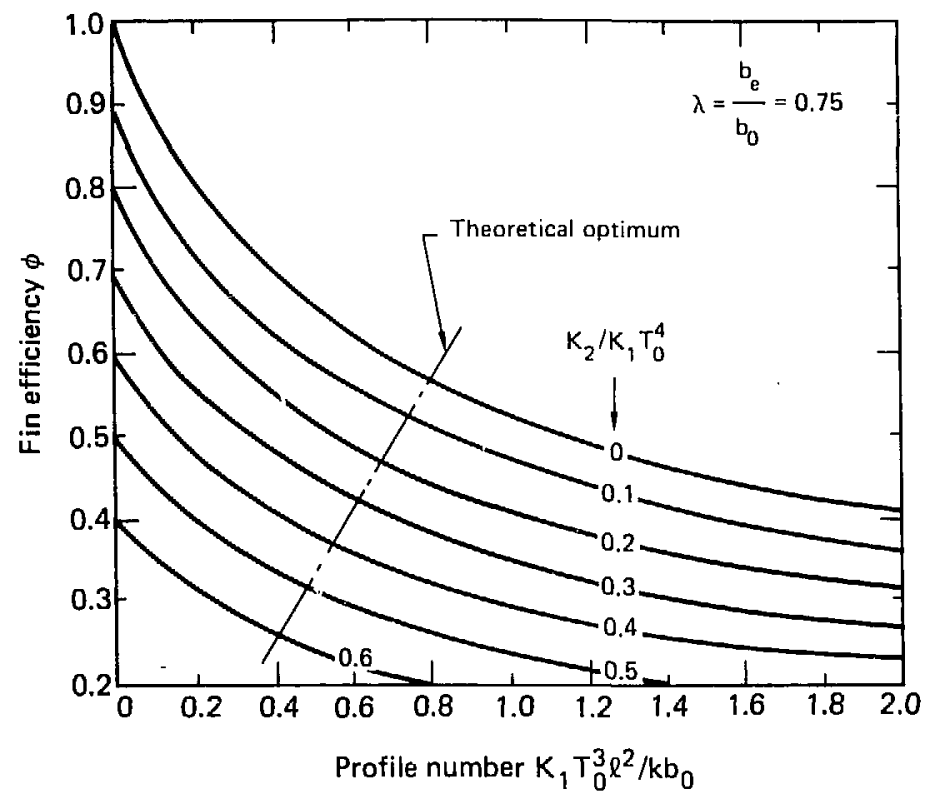

FIG. 5.12a. Fin efficiency for the longitudinal radiating fin of trapezoidal profile with a taper ratio of 0.75 (case 5.1 .26 , source: Ref. 10, p. 223, Fig. 4.12).

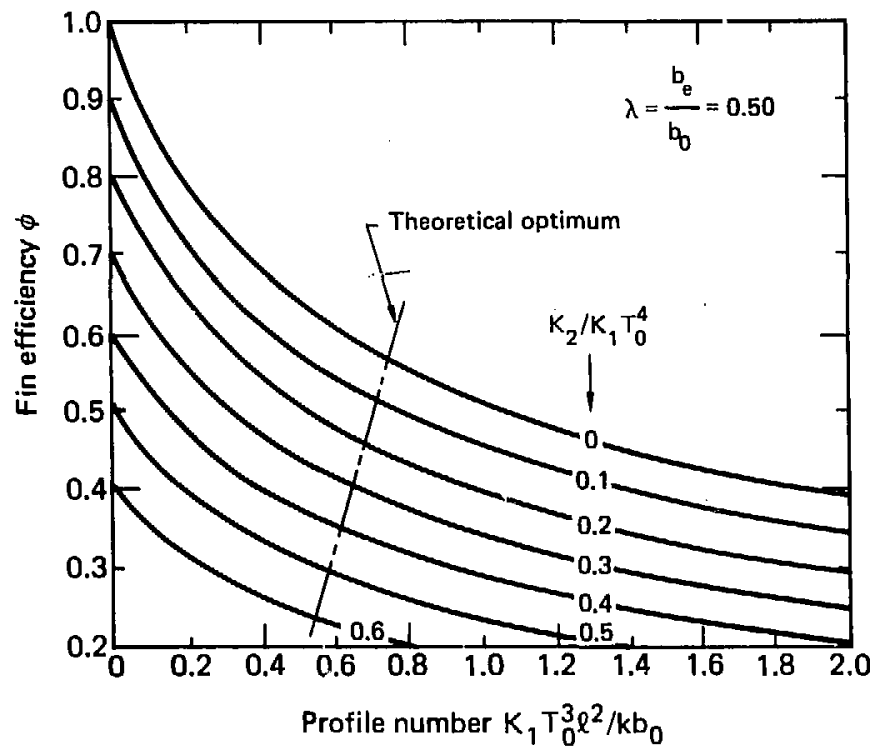

FIG. 5.12b. Fin efficiency for the longitudinal radiating $f$ in of trapezoidal profile with a taper ratio of 0.50 (case 5.1.26, source: Ref. 10, p. 224, Fig. 4.13). 


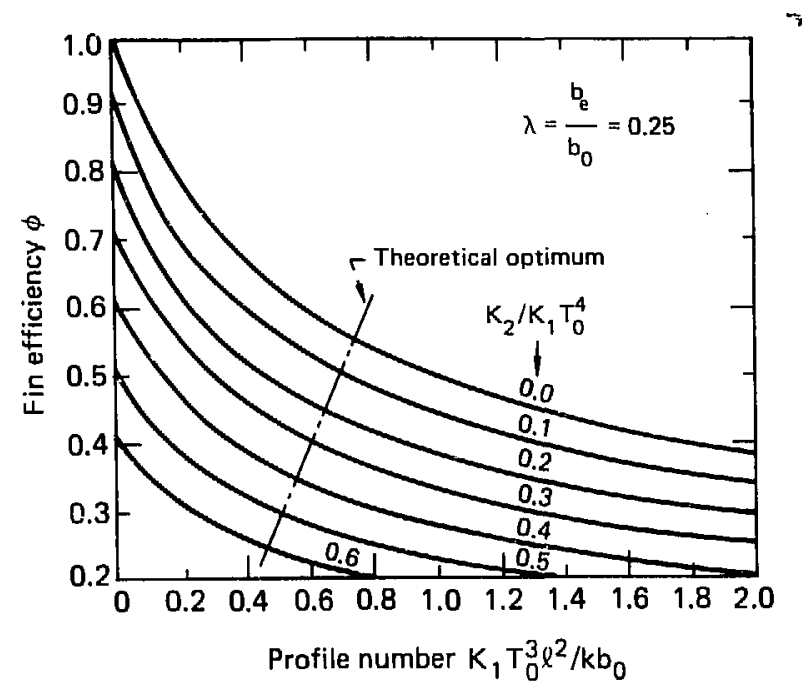

FIG. 5.12c. Fin efficiency for the longitudinal radiatiny fin of trapezoidal profile with a taper ratio of 0.25 (case 5.1 .26 , source: Ref. 10, p. 224, Fig. 4.14).

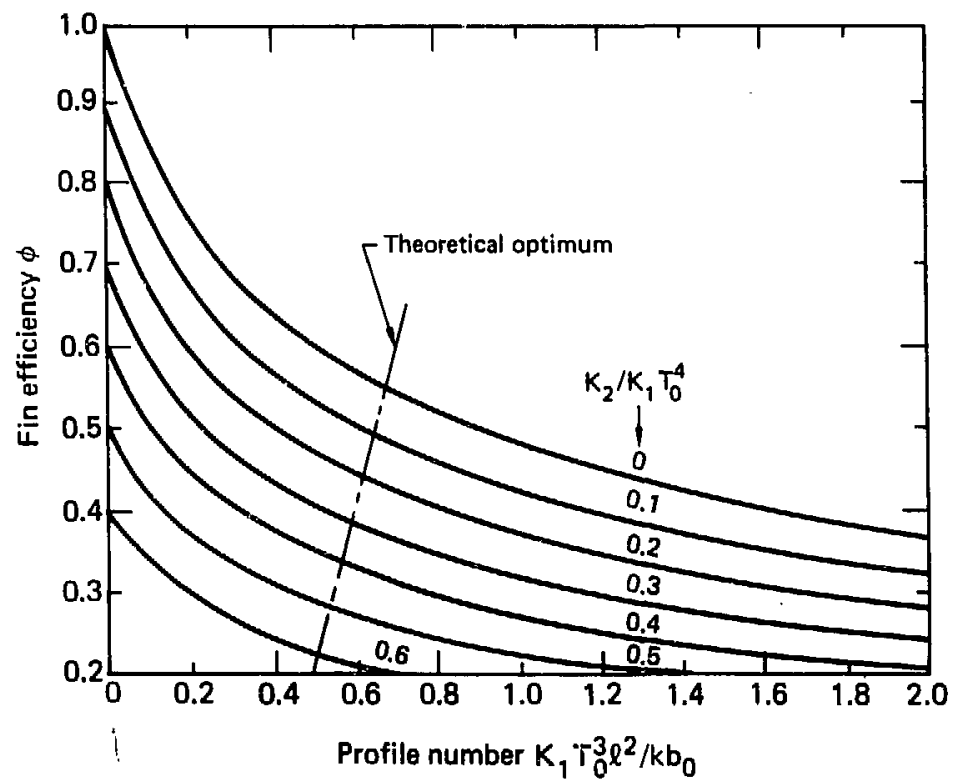

FIG. 5.12d. Fin efficlency for the longitudinal radiating fin of triangular profile (case 5.1.26, source: Ref. 10, p. 225, Fig. 4.15). 


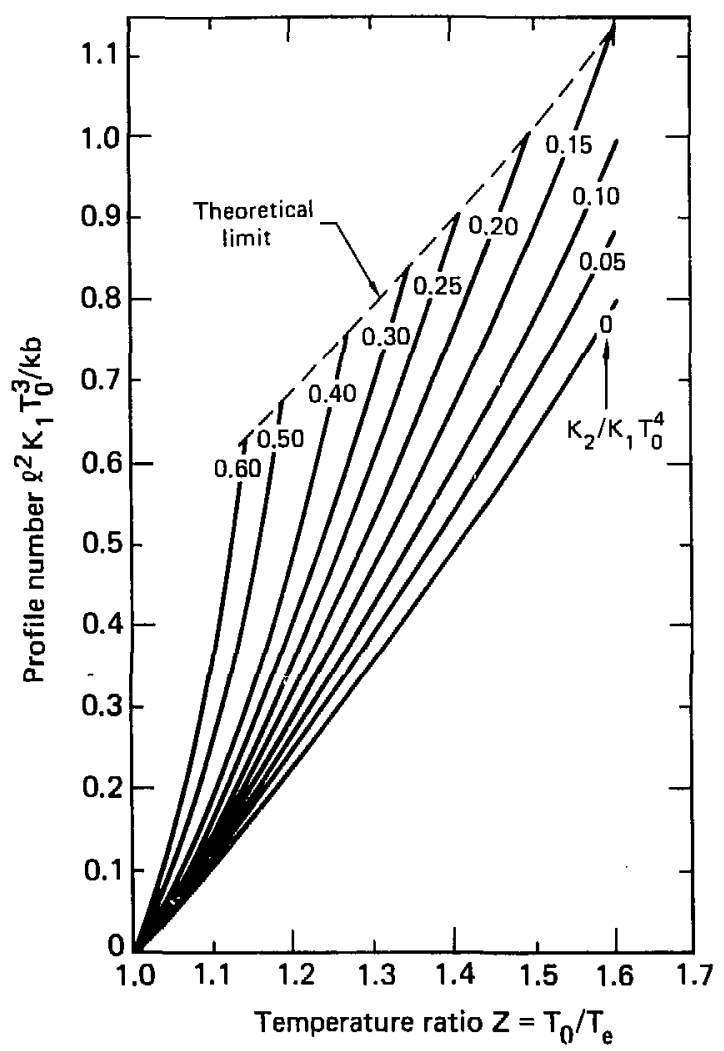

FIG. 5.13. Profile number of constant-temperature-gradient longitudinal radiating fin as a function of base-to-tip temperature ratio (case 5.1.28, source: Ref. 10, p. 233, Fig. 4.18). 


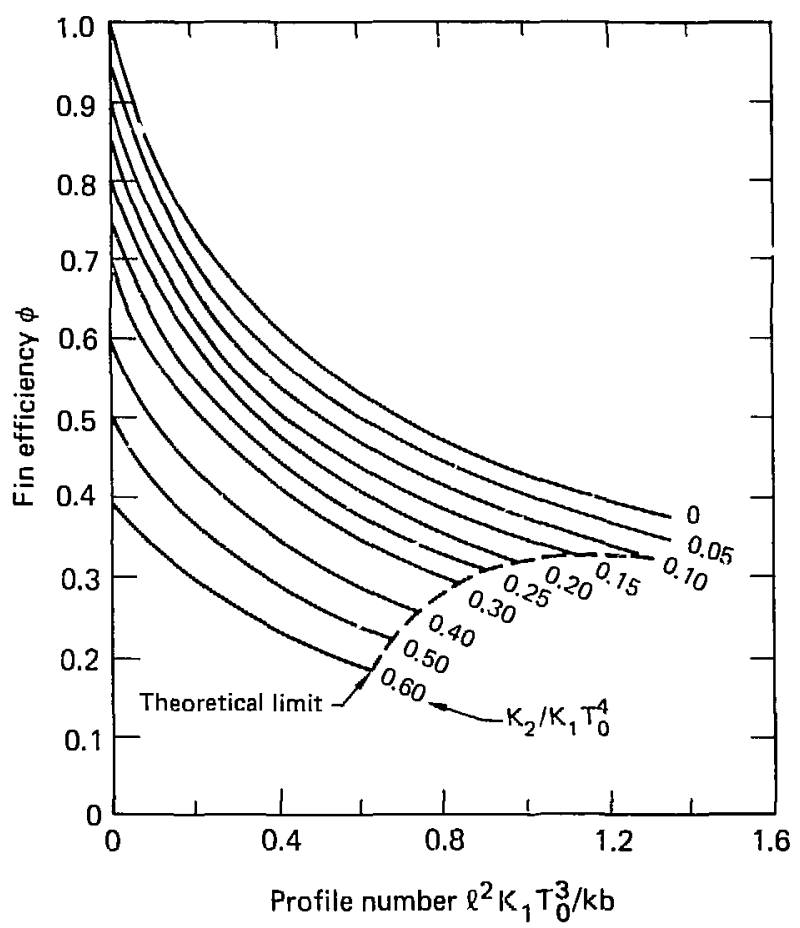

FIG. 5.14. Efficiency of constant-temperature-gradient longitudinal radiating fin as a function of profile number (case 5.1 .28 , source: Ref. 10, p. 232, Fig. 4.17).

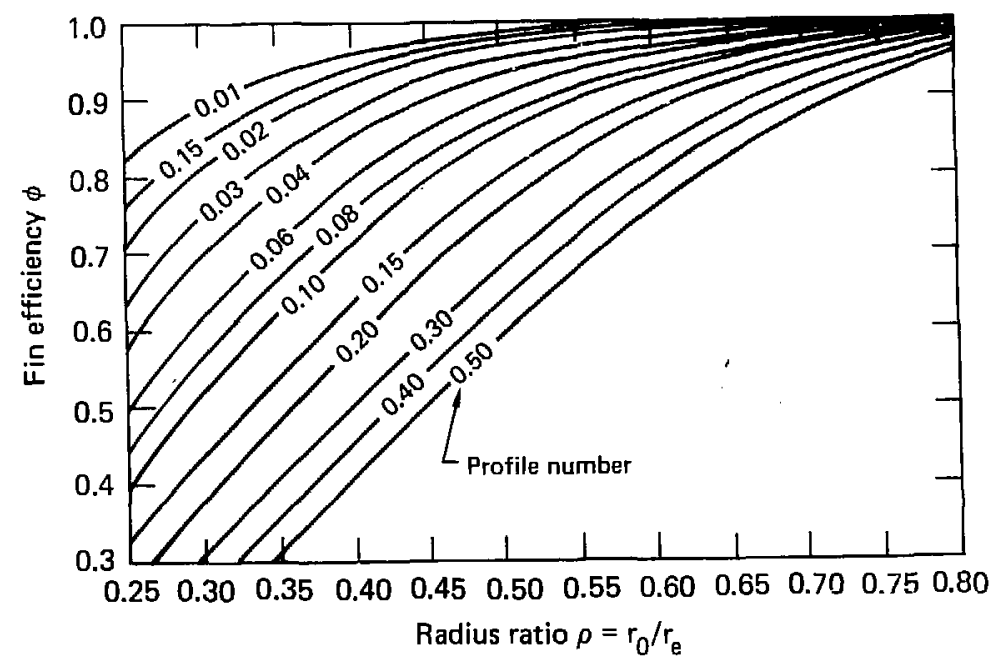

FIG. 5.15a. Radiation fin efficiency of radial fin of rectangular profile. Taper ratio, $\lambda=1.00$; environmental factor, $\mathrm{K}_{2} / \mathrm{K}_{1} \mathrm{~T}_{0}^{4}=0.00$ (case 5.1.29, source: Ref. 10, p. 250, Fig. 4.24). 


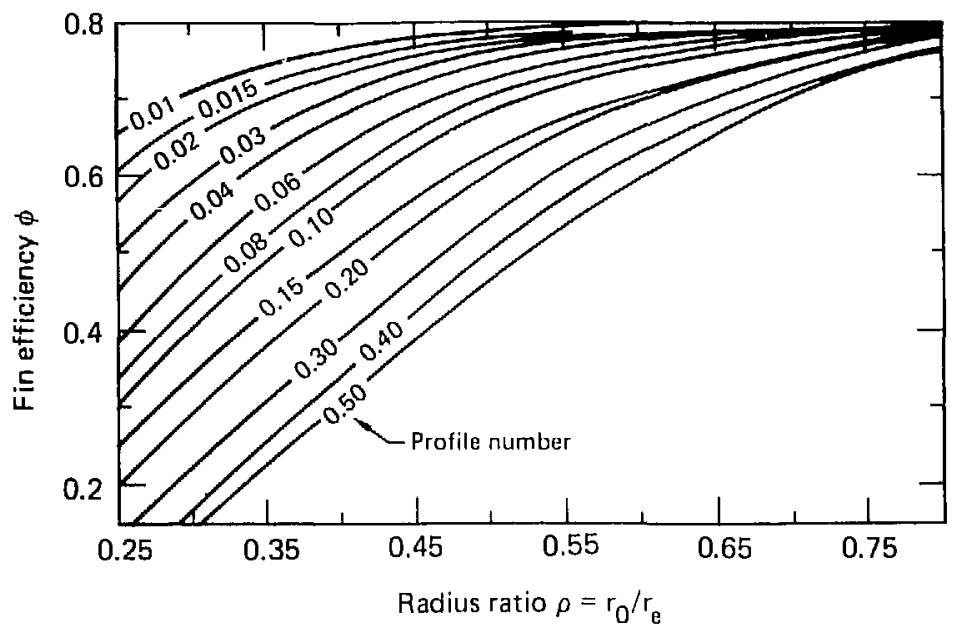

FIG. 5.15b. Radiation fin efficiency of radial fin of rectangular profile. Taper ratio, $\lambda=1.00$; environmental factor, $\mathrm{K}_{2} / \mathrm{K}_{1} \mathrm{~T}_{0}^{4}=0.20$ (case 5.1 .29 , source: Ref. 10, p. 251, Fig. 4.25).

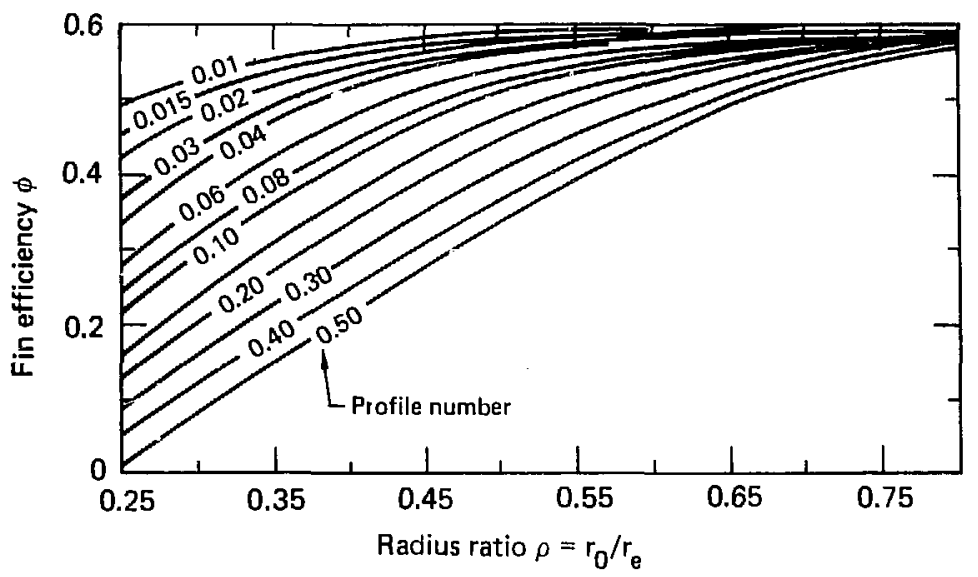

FIG. 5.15c. Radiation fin efficiency of radial fin of rectangular profile. Taper ratio, $\lambda=1.00$; environmental factor, $k_{2} / K_{1} T_{0}^{4}=0.40$ (case 5.1.29, source: Ref. 10, p. 251, Fig. 4.26). 


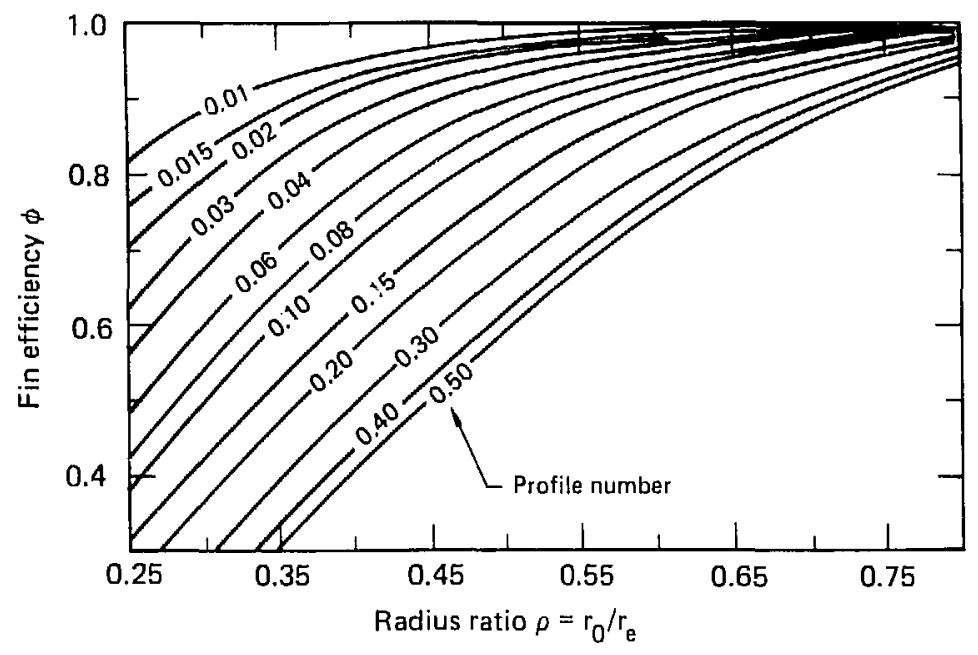

FIG. 5.15d. Radiation fin efficiency of radial fin of trapezoidal profile. Taper ratio, $\lambda=0.75$; environmental factor, $K_{2} / K_{1} T_{0}^{4}=0.00$ (case 5.1.29, source: Ref. 10, P. 252, Fig. 4.27).

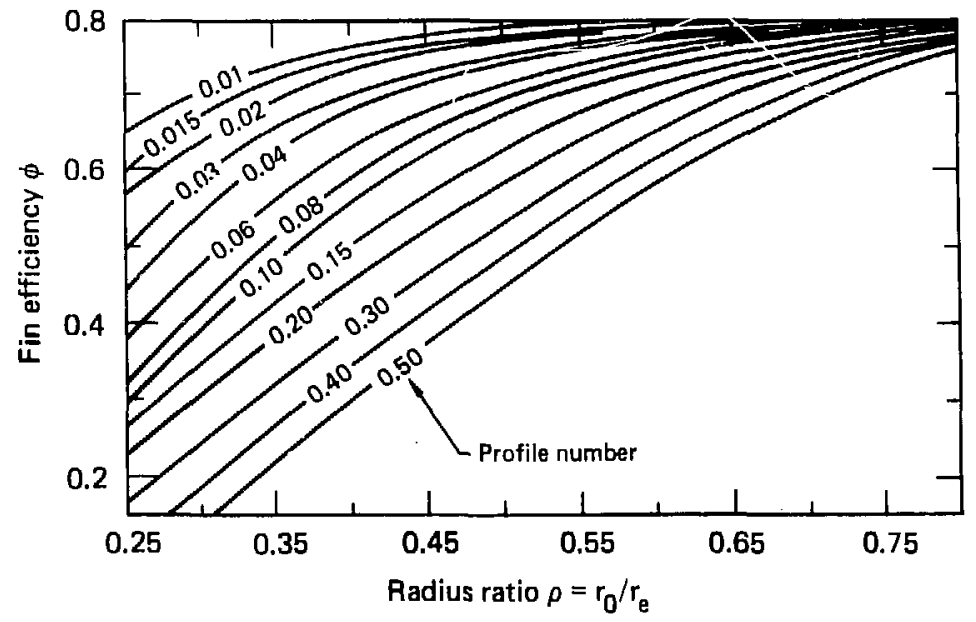

FIG. 5.15e. Radiation fin efficiency of radial fin of trapezoldal profile. Taper ratio, $\lambda=0.75$; environmental factor, $K_{2} / K_{1} T_{0}^{4}=0.20$ (case 5.1.29, source: Ref. 10, p. 252, Fig. 4.28). 


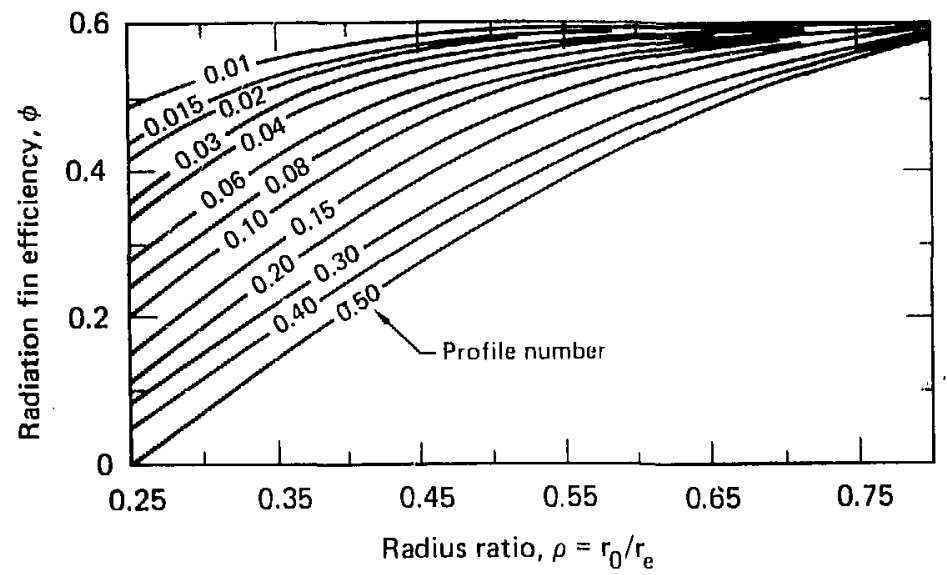

FIG. 5.15f. Radiation fin efficiency of radial fin of trapezoidal profile. Taper ratio, $\lambda=0.75$; environmental Eactor, $\mathrm{K}_{2} / \mathrm{K}_{1} \mathrm{~T}_{0}^{4}=0.40$ (case 5.1 .29 , source: Ref, 10, p. 253, Fig. 4.29).

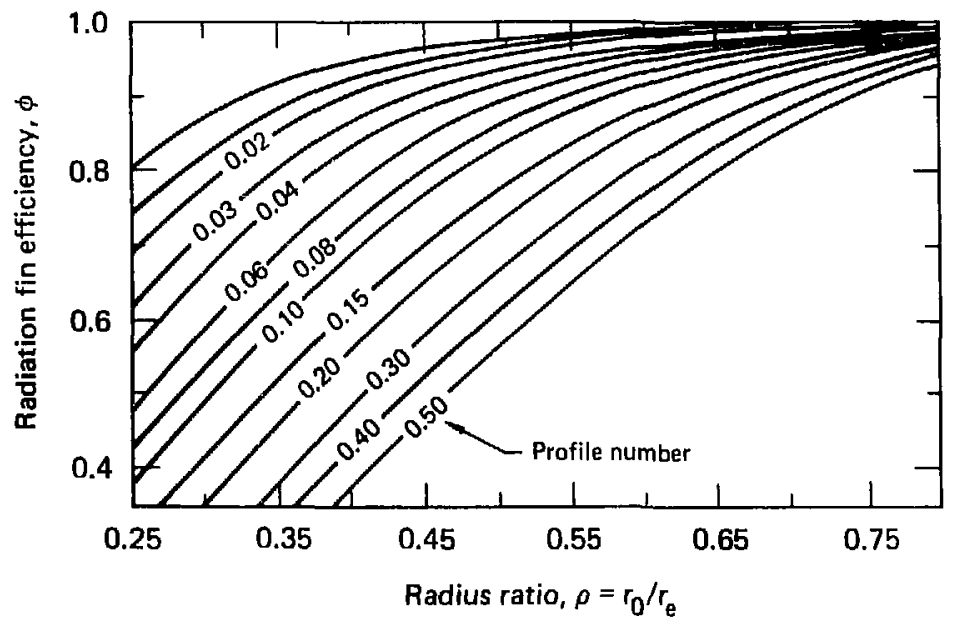

FIG. 5.15g. Radiation fin efficiency of radial fin of trapezoidal profile. Taper ratio, $\lambda=0.50$; envi ronmental factor, $K_{2} / K_{1} T_{0}^{4}=0.00$ (case 5.1.29, source: Ref. 10, p. 253, Fig. 4.30). 


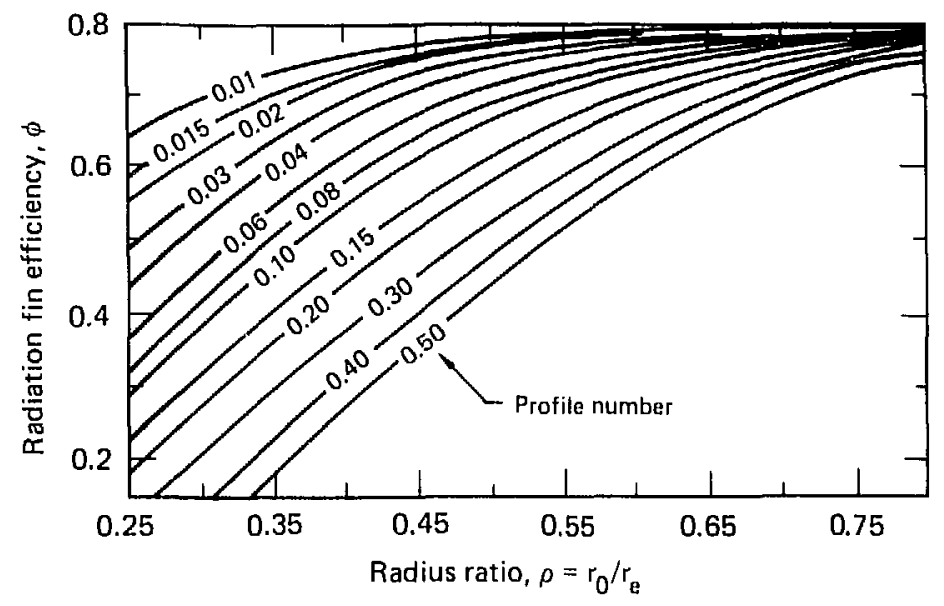

FIG. 5.15h. Radiation fin efficiency of radial fin of trapezoidal profile. Taper ratio, $\lambda=0.50$; environmental factor, $K_{2} / K_{1} T_{0}^{4}=0.20$ (case 5.1.29, source: Ref. 10, p. 254, Fig. 4.31).

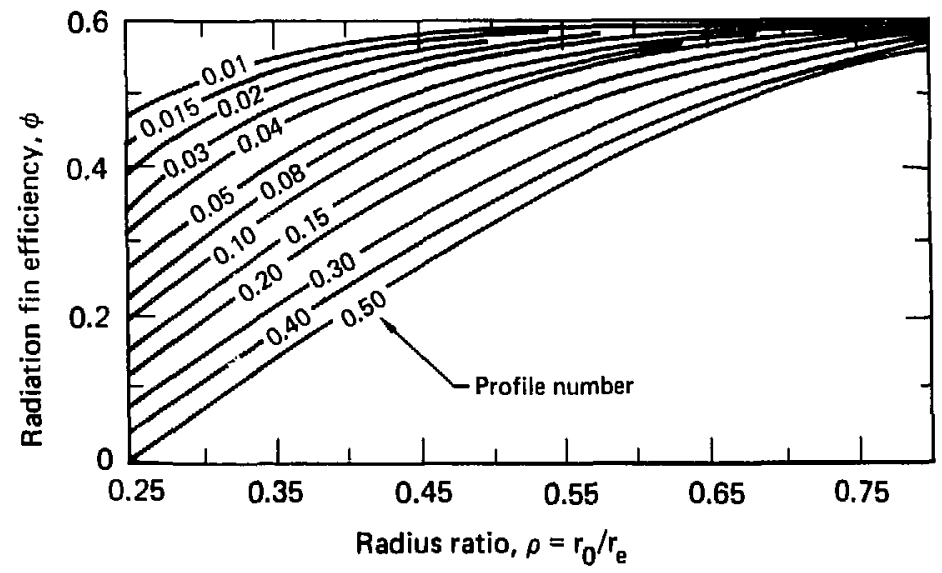

FIG. 5.15i. Radiation fin efficiency of radial fin of trapezoidal profile. Taper ratio, $\lambda=0.50$; environmental Eactor, $\mathrm{K}_{2} / \mathrm{K}_{1} \mathrm{~T}_{0}^{4}=0.40$ (case 5.1 .29 , source: Ref. 10, p. 254, Fig. 4.32). 


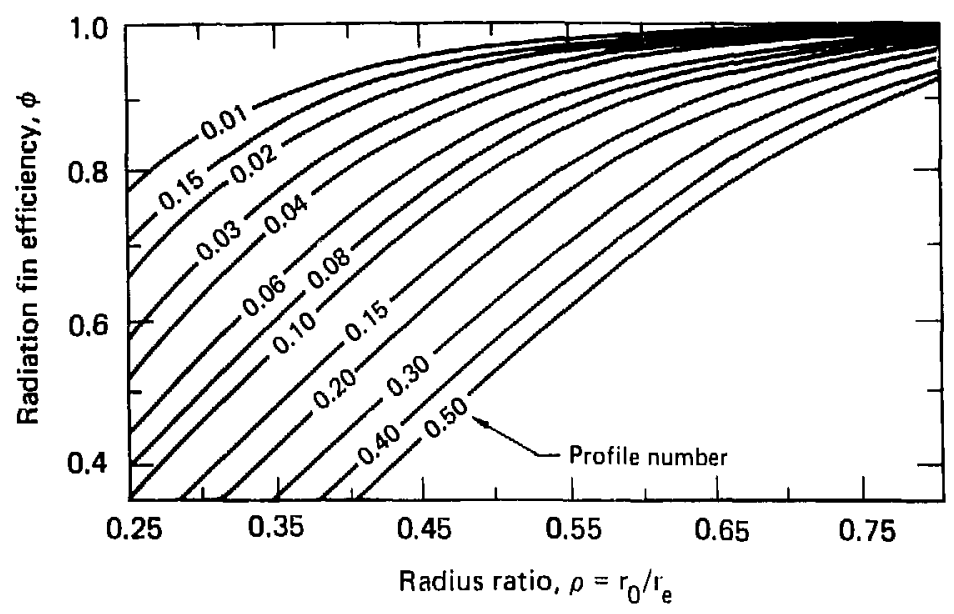

FIG. 5.15j. Radiation fin efficiency of radial fin of triangular profile. Taper ratio, $\lambda=0.00$; environmental factor, $K_{2} / k_{1} T_{0}^{4}=0.00$ (case 5.1.29, source: Ref. 10, p. 255, Fig. 4.33).

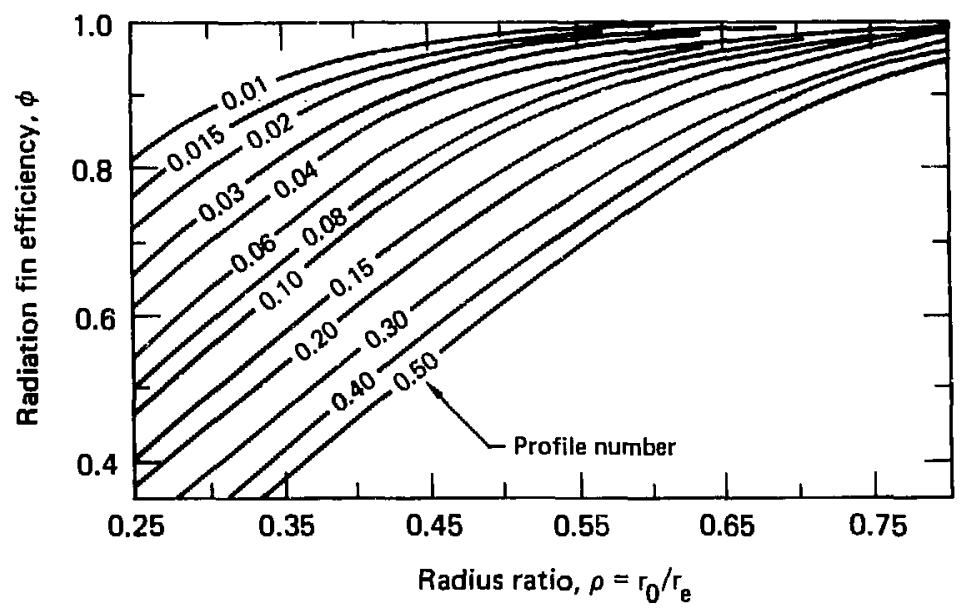

FIG. 5.15k. Radiation fin efficiency of radial fin of triangular profile. Taper ratio, $\lambda=0.00$; environmental factor, $K_{2} / K_{1} T_{0}^{4}=0.20$ (case 5.1.29, source: Ref. 10, p. 255, Fig. 4.34). 


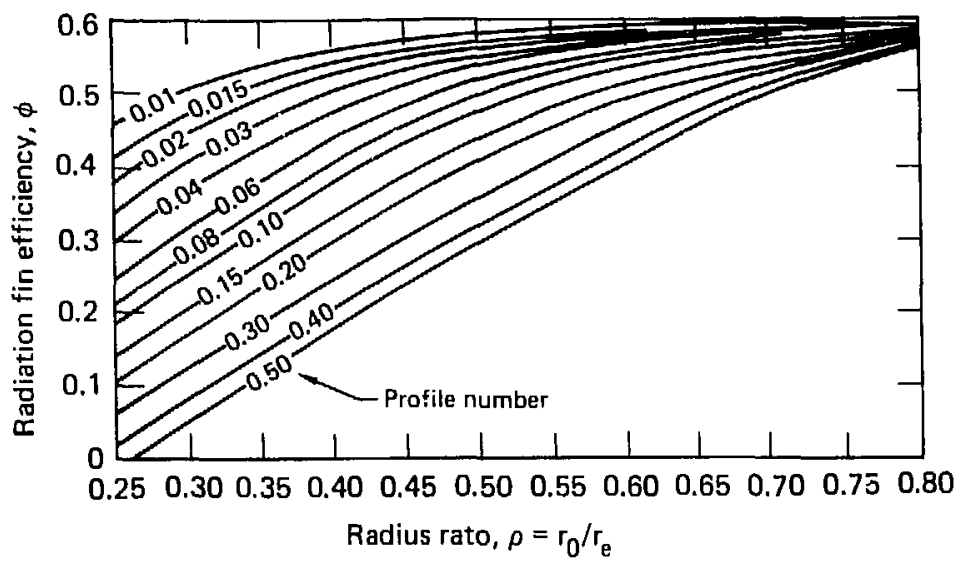

FIG. 5.15l. Radiation fin efficiency of radial fin of triangular profile. Taper ratio, $\lambda=0.00$; environmental factor, $K_{2} / K_{1} T_{0}^{4}=0.40$ (case 5.1.29, source: Ref. 10, p. 256 , Fig. 4.35).

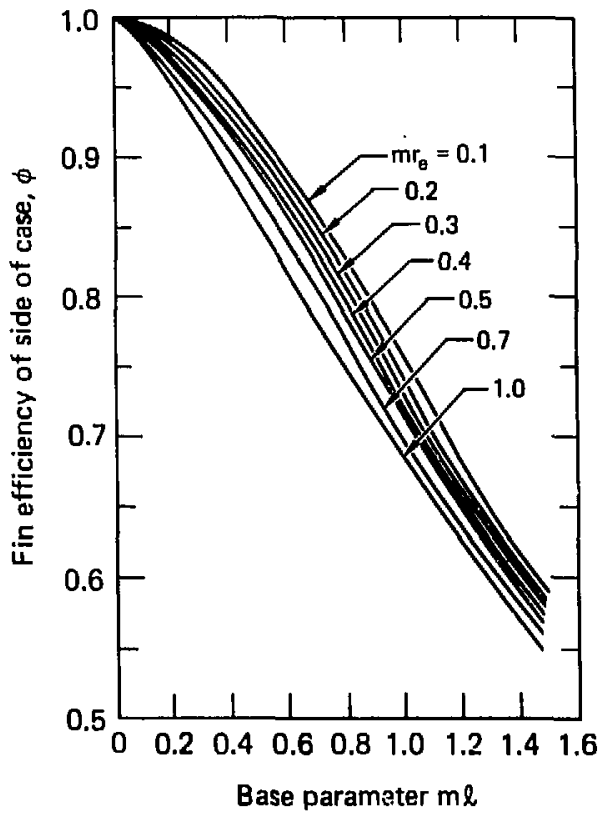

FIG. 5.16a. Efficiency of the side of a capped cylinder fin (case 5.1.30, source: Ref. 10, p. 276, Fig. 5.6). 


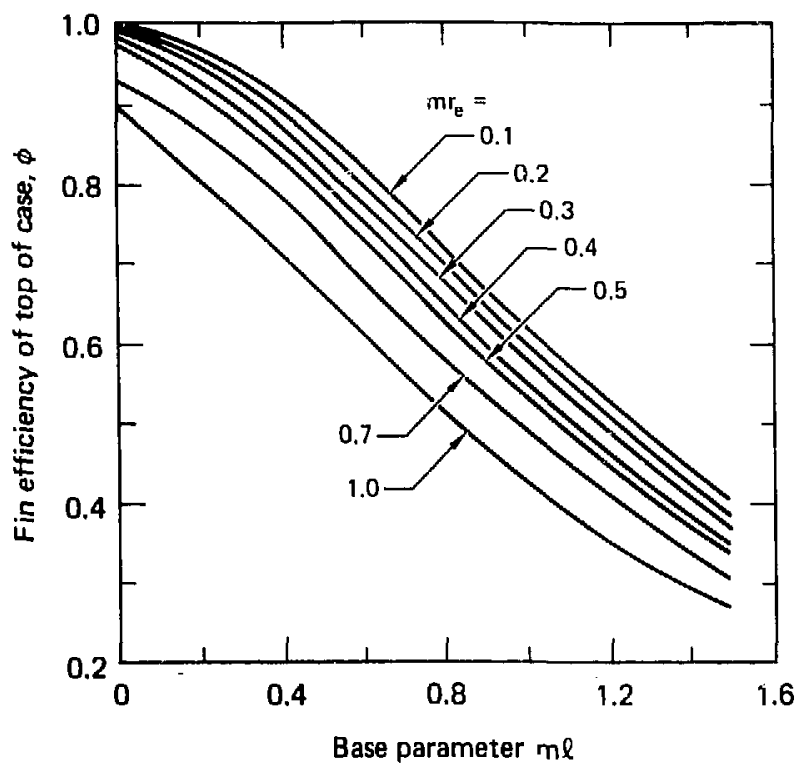

FIG. 5.16b. Efflctency of the top of a capped cylinder fin (case 5.1.30, source: Ref. 10, p. 277, Fig. 5.7). 


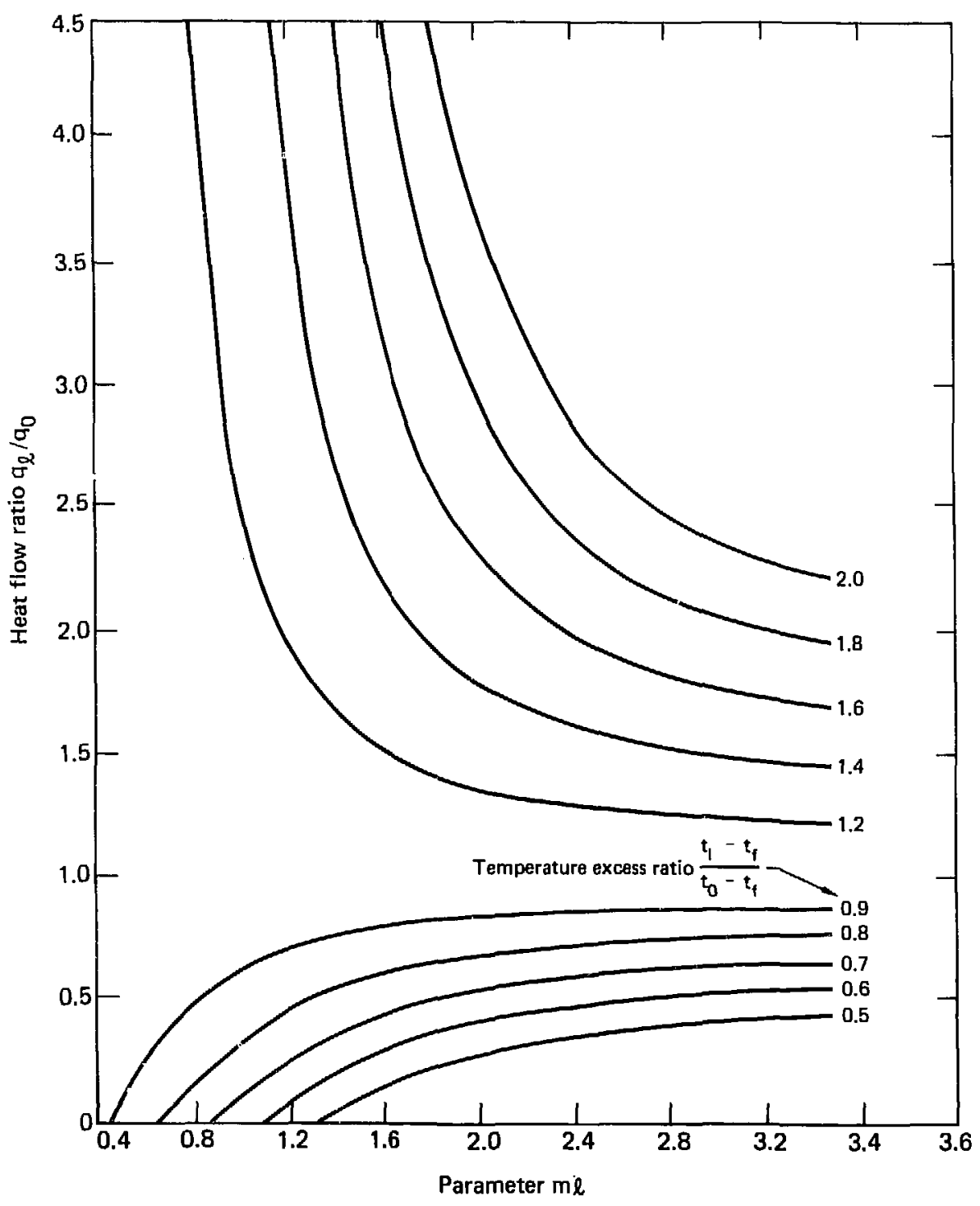

FIG. 5.17. Heat flow ratio $q_{\ell} / q_{0}$ as a function of $\mathrm{mb}$ and temperature excess ratio $\left(t_{1}-t_{f}\right) /\left(t_{0}-t_{f}\right)$ for the doubly heated rectangular fin (case 5.1.32, source: Ref. 10, p. 410, Fig. 8.10). 


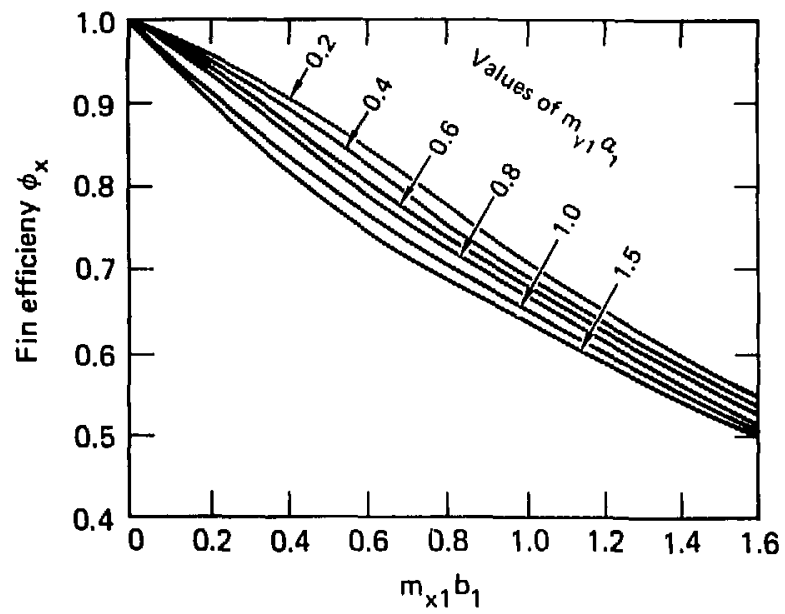

FIG. 5.18a. Efficiency of the vertical section of a straight, single Tee fin for $u=v$ (case 5.1.32, source: Ref. 10, p. 398, Fig. 8.4).

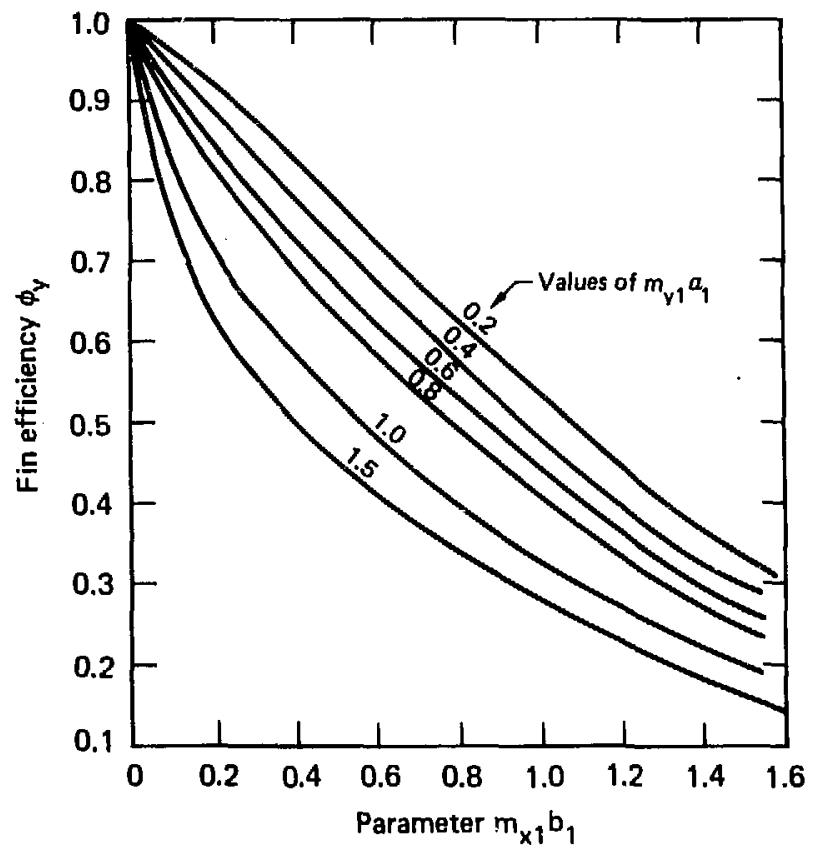

FIG. 5.18b. Efficiency of the horizontal section of a straight, single Tee fin for u $-v$ (case 5.1.32, source: Ref. 10, p. 399, Fig. 8.5). 


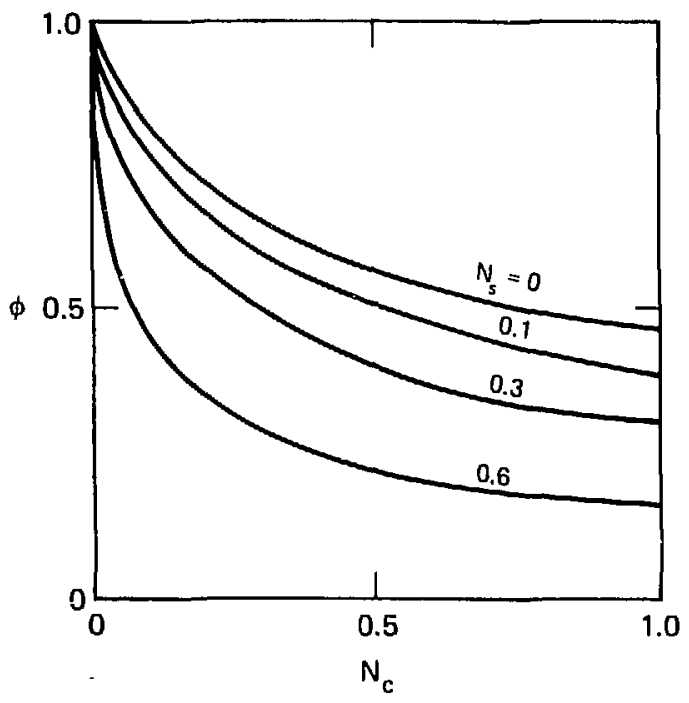

FIG. 5.19. Effectiveness of the concave parabolic fin radiating to non-free space (case 5.1.37, Bource: Ref. 31).

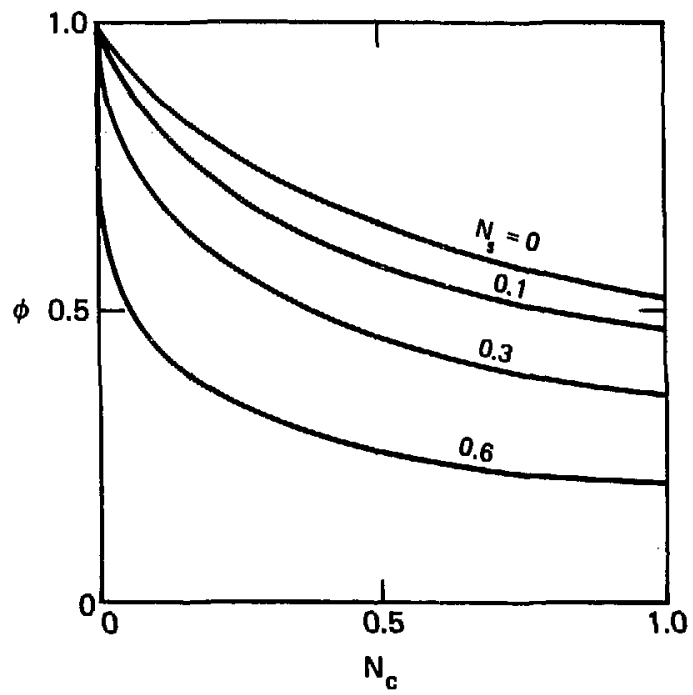

EIG. 5.20, Effectiveness of the convex parabolic fin radiating to non-free space (case 5.1.38, source: Ref. 31). 


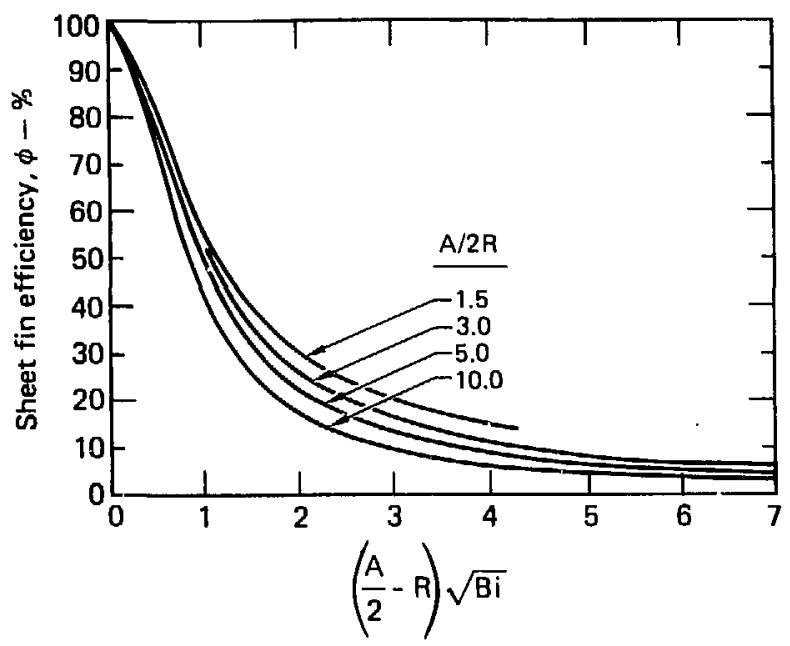

FIG. 5.21. Effectiveness of sheet fin with square array tubes (case 5.1.40, source: Ref. 83, p. 294, Fig. 2).

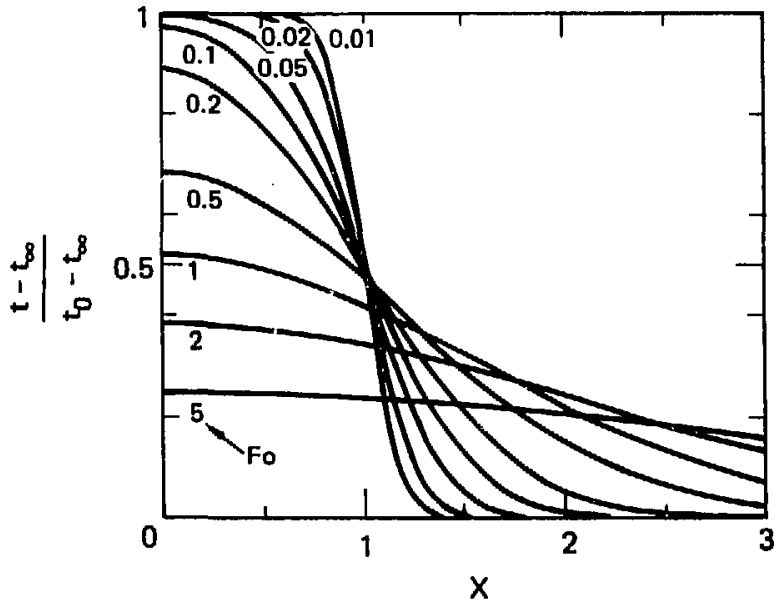

EIG. 6.1. Temperatures in an infinite region of which the region $|x|<b$ is inttially at temperature $t_{0}$ (case 6.1.1, source: Ref. 9, p. 55, Fig. 4a). 


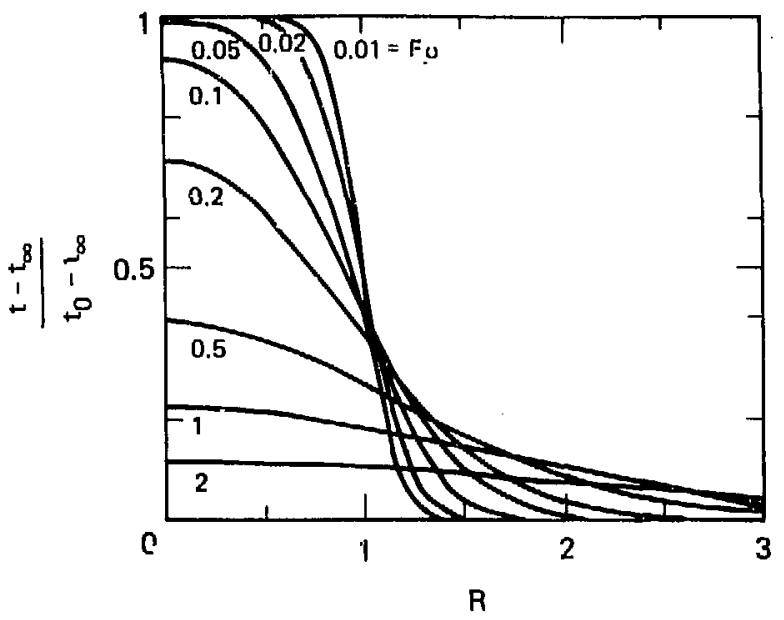

FIG. 6.2. Temperatures in an infinite region of which the region $r<r_{0}$ is initially at temperäture $t_{0}$ (case 6.1.4, source: Ref. 9, p. 55, Fig. 4b).

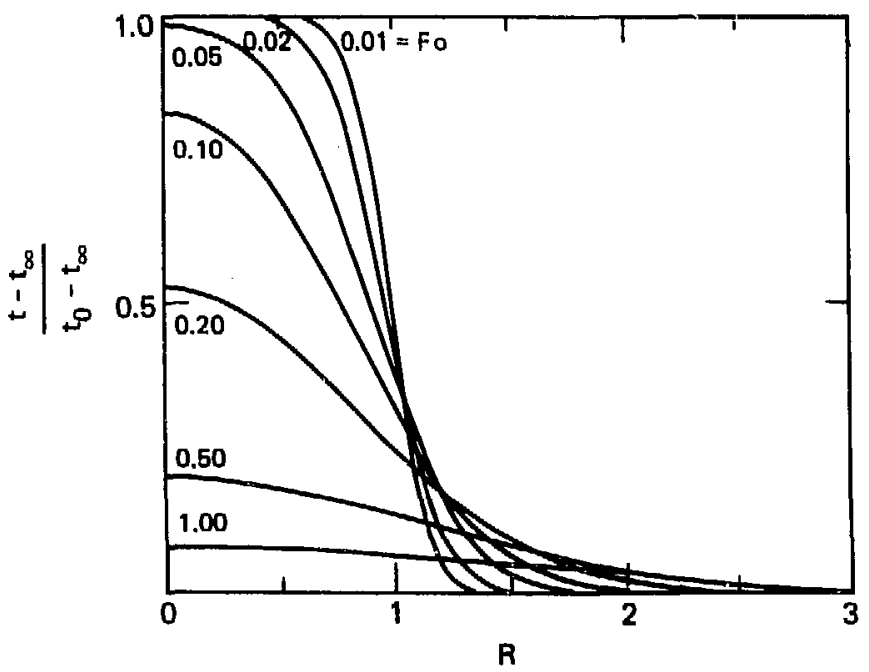

FIG. 6.3. Temperatures in an infinite region of which the region $r<r_{0}$ is initially at temperature $t_{0}$ (case 6.1.5, source: Ref. 9, p. 55, Fig. 4c). 


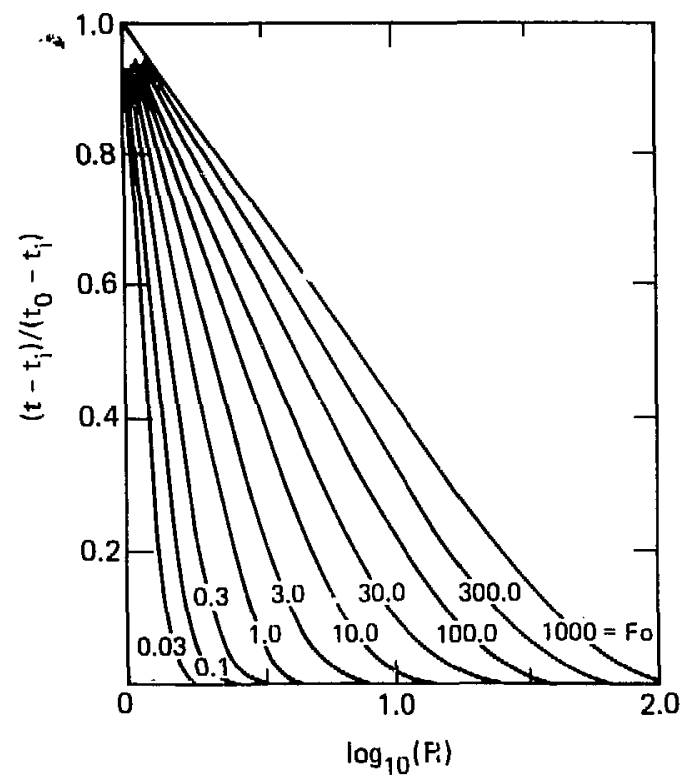

FIG. 6.4. Temperatures in an infinite region with steady temperature $t_{0}$ on the surface $r=r_{0}$ (case 6.1.18, source: Ref. 9, p. 337, Fig. 4l).

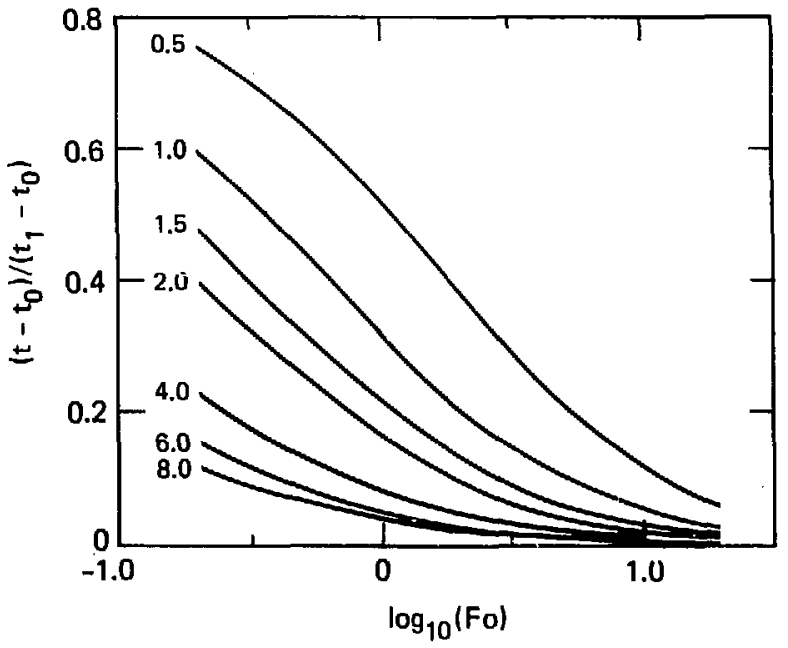

FIG. 6.5. Temperature in a cylinder of inf:nite conductivity, initially at temperature $t_{1}$, in an infinite medium inditially at $t_{0}$. Numbers on the curves are values of $2 \rho_{0} c_{0} / \rho_{1} c_{1}$ (case 6.1 .21 , source: Ref. 9, p. 342, Fig. 45). 


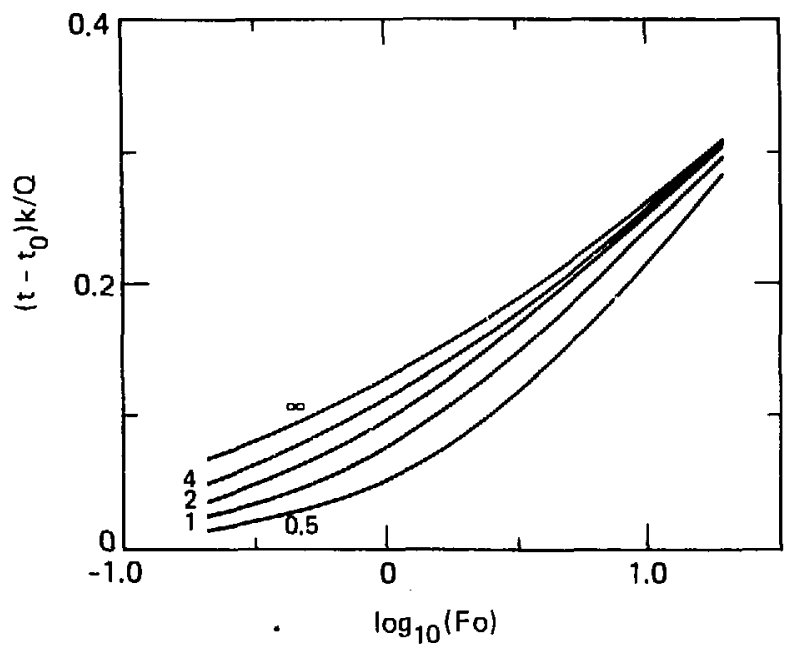

FIG. 6.6. Temperature in a cylinder of infinite conductivity, initially at temperature $t_{0}$, in an infinite medium. Numbers on the curves are values of $2 \rho_{0} c_{0} / \rho_{1} c_{1}$ (case 6.1 .22 , source: ref. 9, p. 343, Fig. 46).

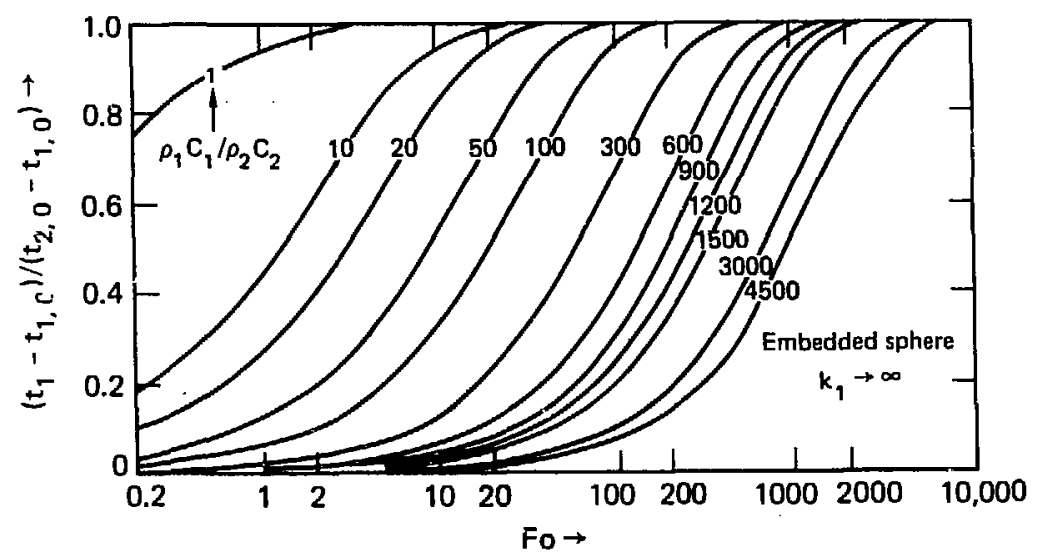

FIG. 6.7. Temperature response of solid sphere $0 \leq r \leq r_{1}$, with $k_{1}+\infty$ and initially at $t_{1}, 0$, embedded in an infinite solid $\left(\bar{r}>\bar{r}_{1}\right)$ initially at $t_{2,0}$ (case 6.1.26, source: Ref. 19, pp. 3-64, Fig. 37). 


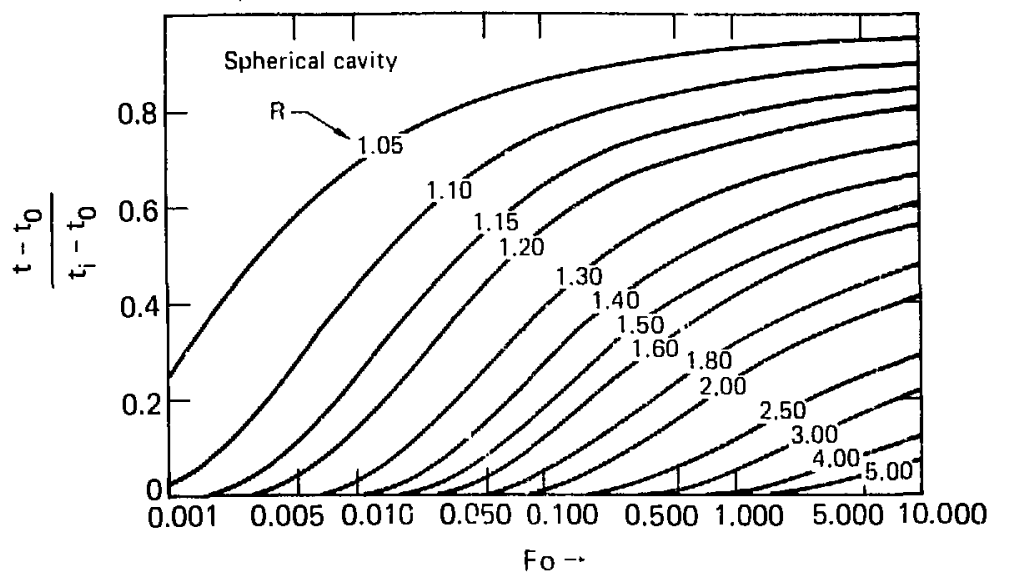

FIG. 6.7. Temperature response of an infinite solid with a constant spherical surface temperature of $t_{0}$ (case 6.1.13, source: Ref 74, p. 430, Fig. 10.9).

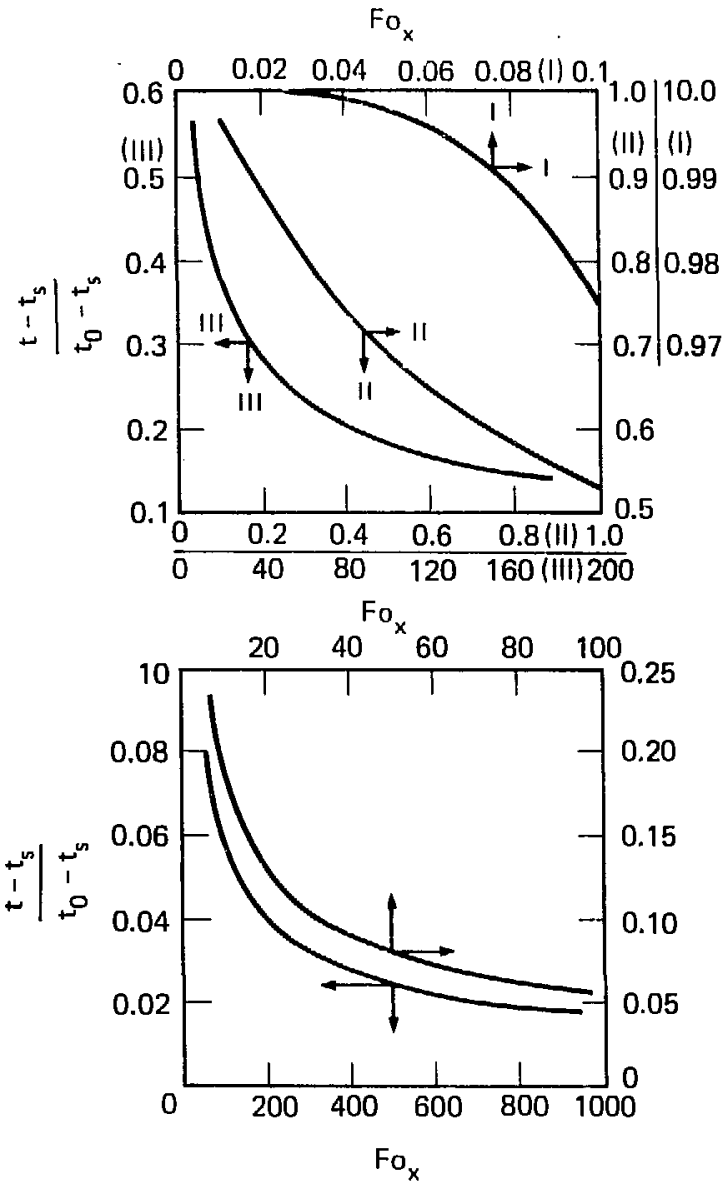

FIG. 7.1. Temperature distribution in the semi-infinite solid having a steady surface temperature (case $7.1 .1 \mathrm{~b}=0$, source: Ref. 74, p. 95, Figs. 4.4 and 4.5). 


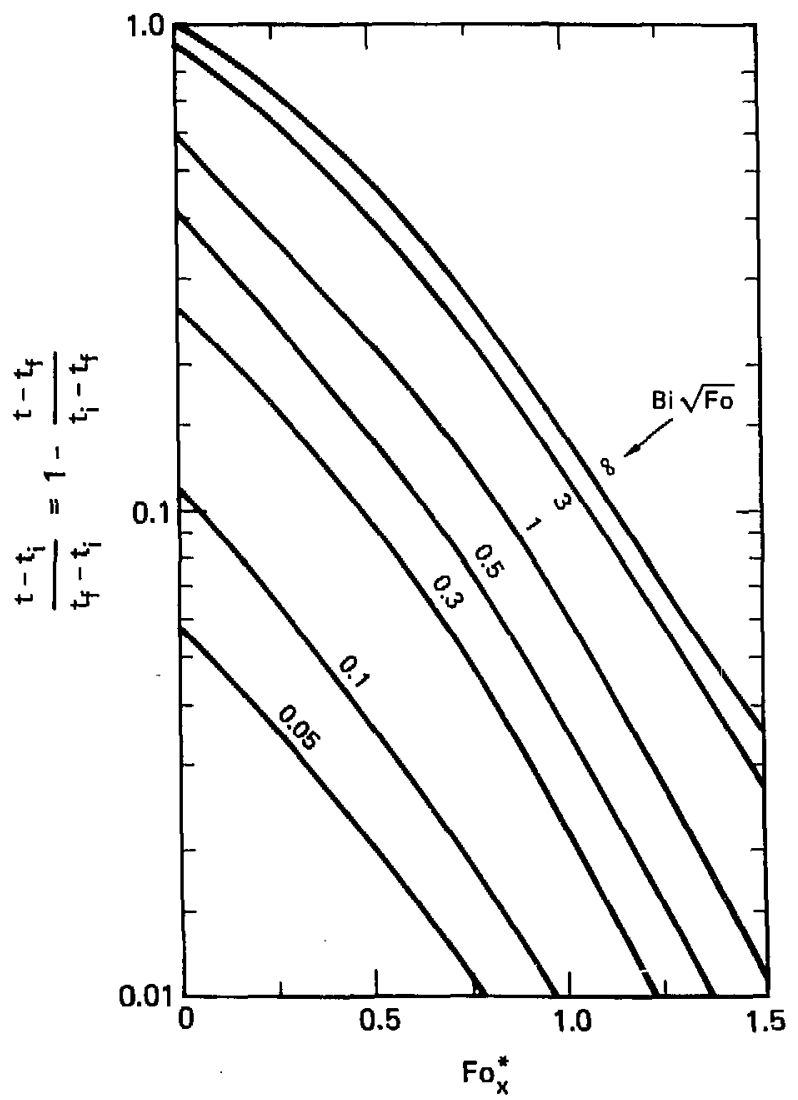

FIG. 7.2a. Temperature distribution in the semi-infinite solid with convection boundary condition (case 7.1.3, source: Ref. 5, p. 82, Fig. 4.6). 


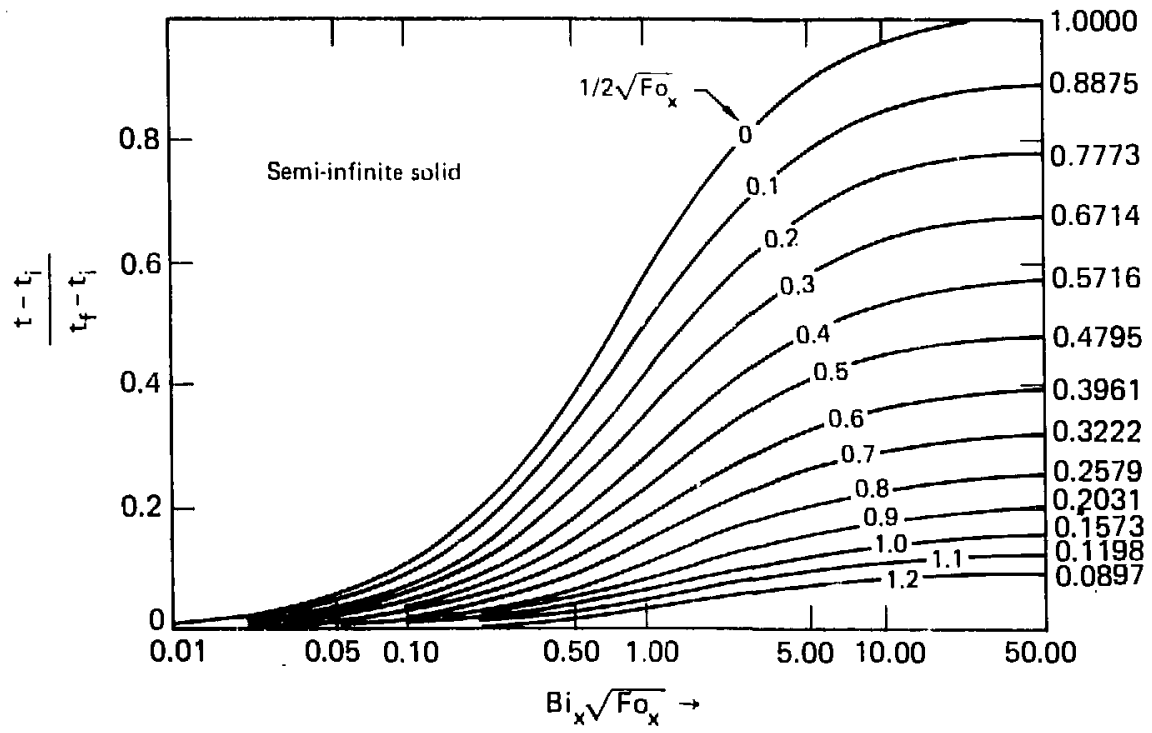

FIG. 7.2b. The dimensionless excess temperature vs the number $\mathrm{Bi}\left(\mathrm{Fo}_{\mathrm{x}}\right) \mathrm{l} / 2$ and various Fourier numbers for semi-infinite solid with a convection boundary (case 7.1.3, source: Ref. 74, p. 207, Fig. 6.2).

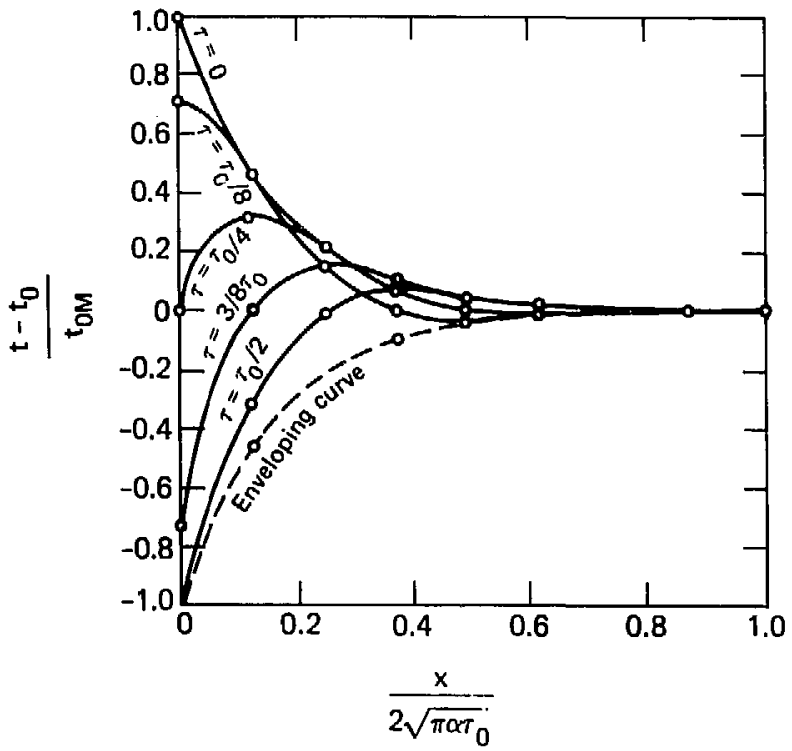

FIG. 7.3. Temperature distribution in a semi-infinite region when the surface temperature is harmonic (case 7.1.16, source: Ref. 7, p. 99, Fig. 4-19). 


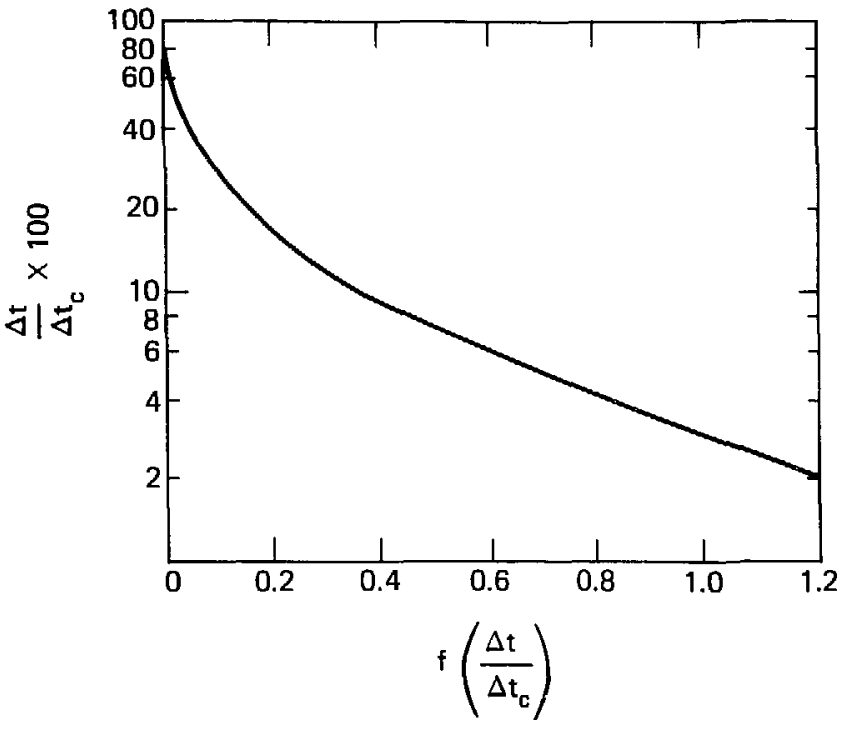

FIG. 7.4. Function $f\left[\left(t-t_{f}\right) /\left(t_{c}-t_{f}\right)\right]$ (case 7.1 .22 , source: Ref. 4, p.84, Fig. 4.15).

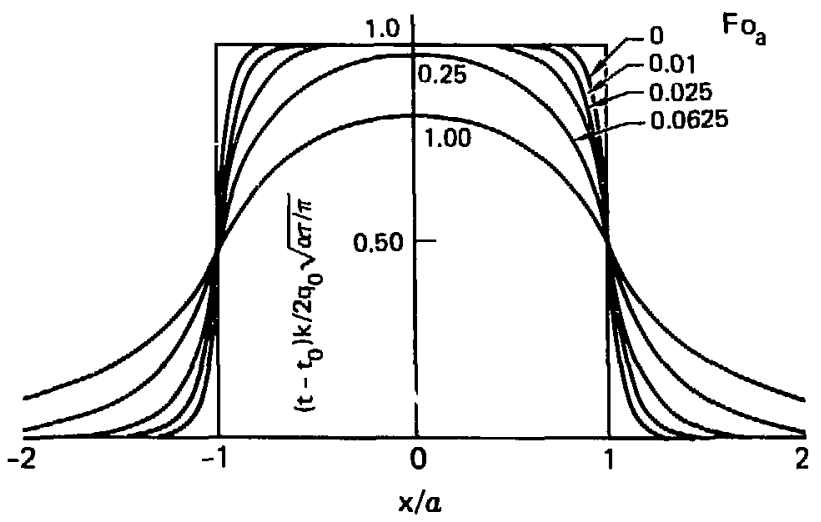

FIG. 7.5. Temperature distribution across a heated strip of width $2 a$ on the surface of a semi-infinite solid (case 7.1 .24 , source: Ref. 9, p. 265, Fig. 33). 


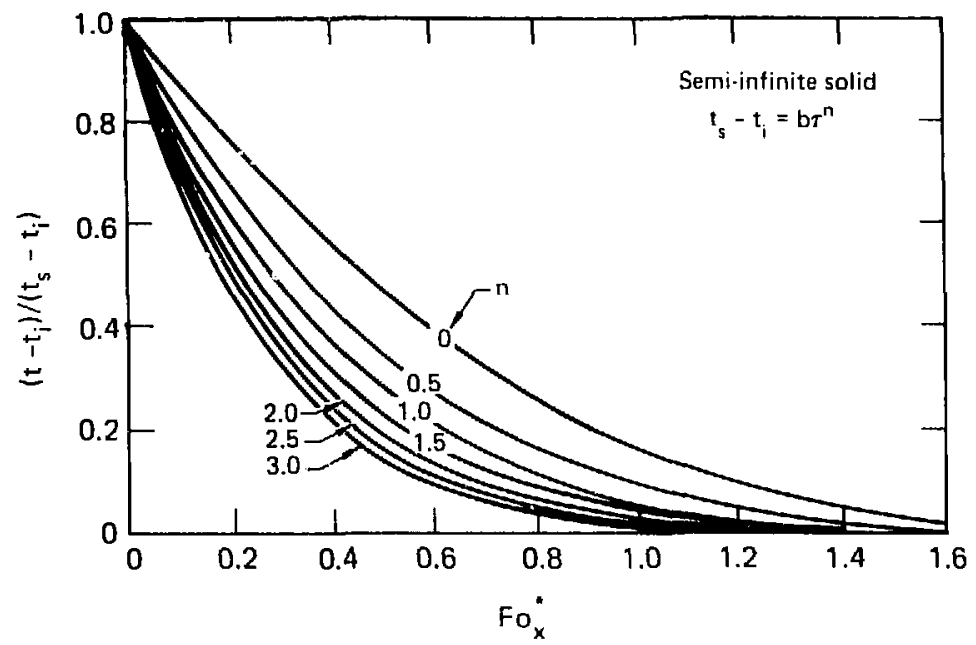

FIG. 7.6. Temperature response of semi-infinite solid ( $x \geq 0)$ with surface temperature $t_{s}$ suddenly increasing as power function of time, $t_{s}=t_{i}+b \tau^{n}$, (case 7.1.9, source: Ref. 19, Pp. 3-66, Fig. 39).

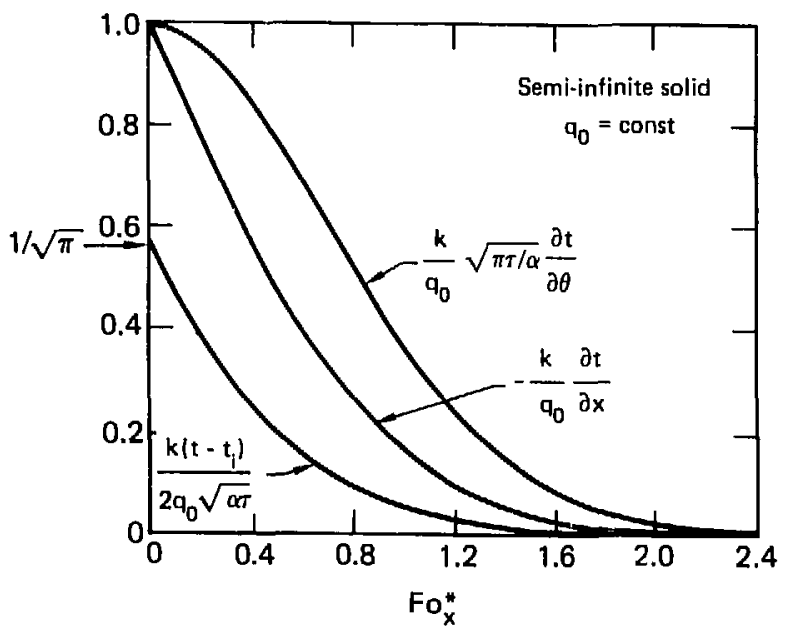

FIG. 7.7. Temperature response, temperature gradient, and heating rate in a semi-infinite solid after exposure to a constant surface heat flux $q_{0}$ (case 7.15, source: Ref. 19, pp. 3-81, Fig. 49). 


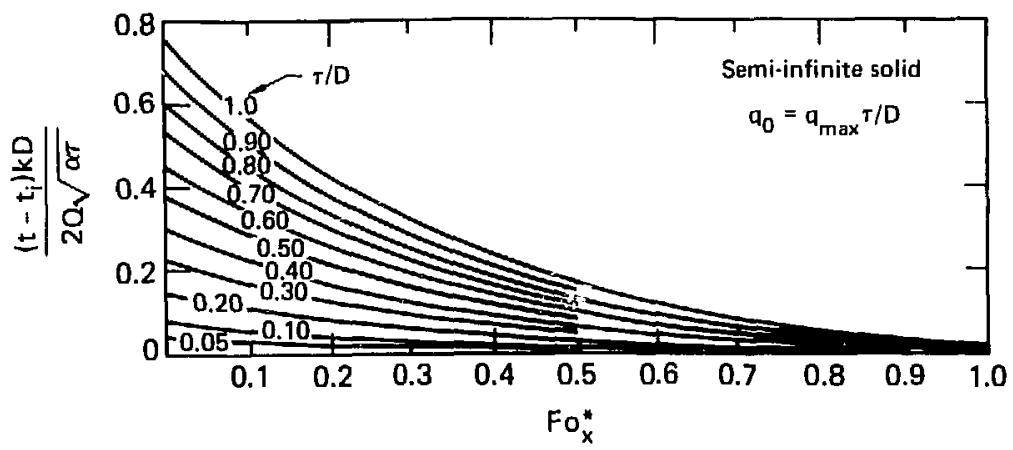

FIG. 7.8. Temperature response of a semi-infinite solid after sudden exposure to a linearly increasing surface heat flux for a duration $D, Q=D q_{m a x} / 2$, $q_{0}=q_{\max } \tau / D$ (case 7.1.14, source: Ref. 19, pp. 3-82, Fig. 50).

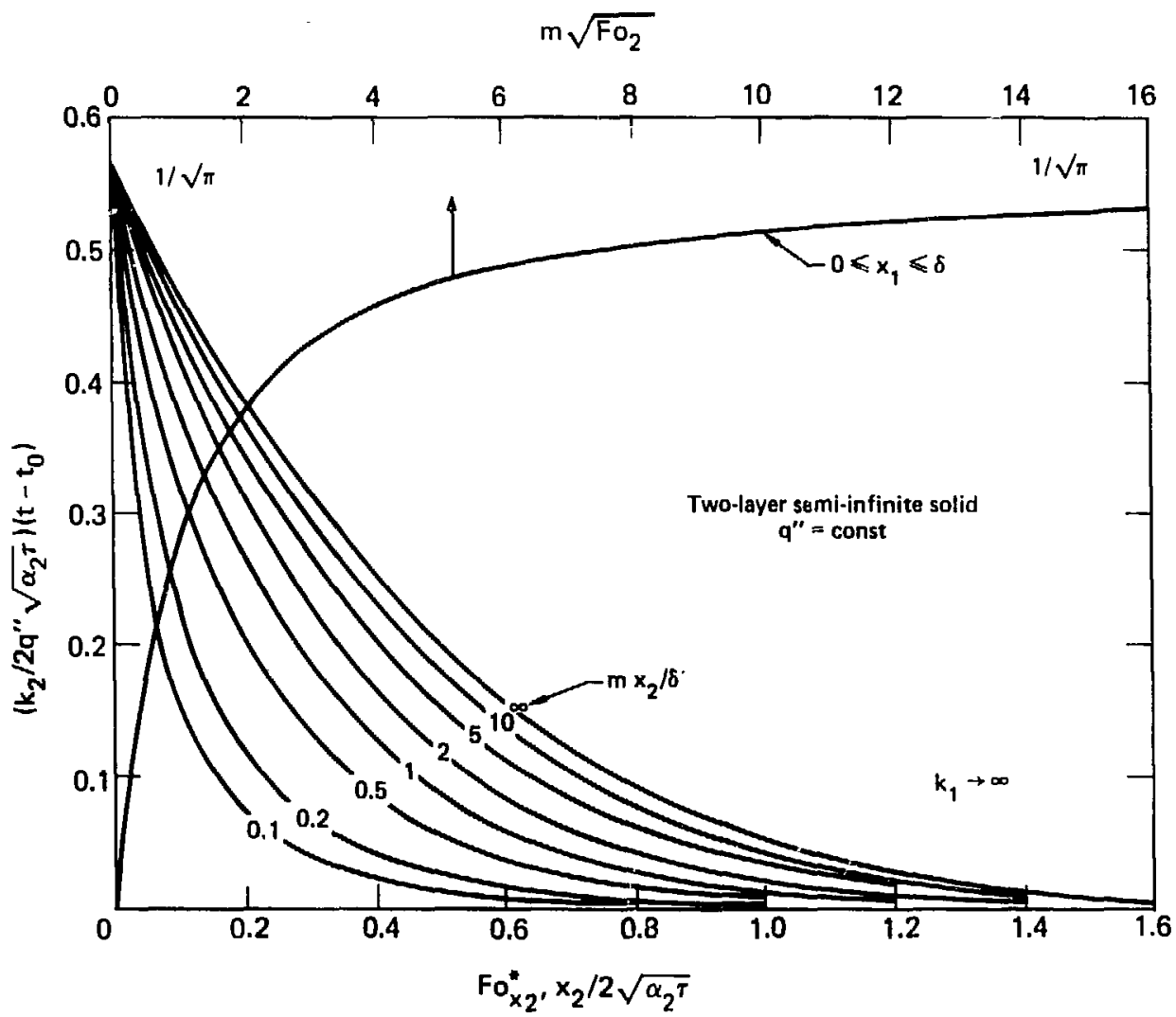

FIG. 7.9. Temperature response of an infinite conductivity plate and semiInfinite solid composite with a constant heat flux at $x_{1}=0$ (case 7.1 .30 , source: Ref. 19, pp. 3-84, Fig. 51). 


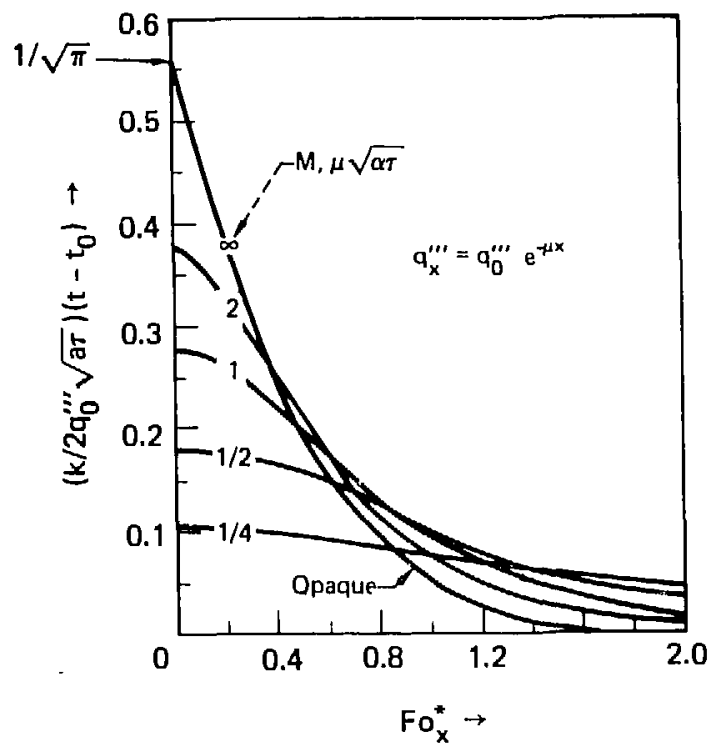

FIG. 7.10. Temperature distributions in a semi-infinite solid with exponential heating (case 7.2.9, source: Ref. 19, pp. 3-85, Fig. 52).

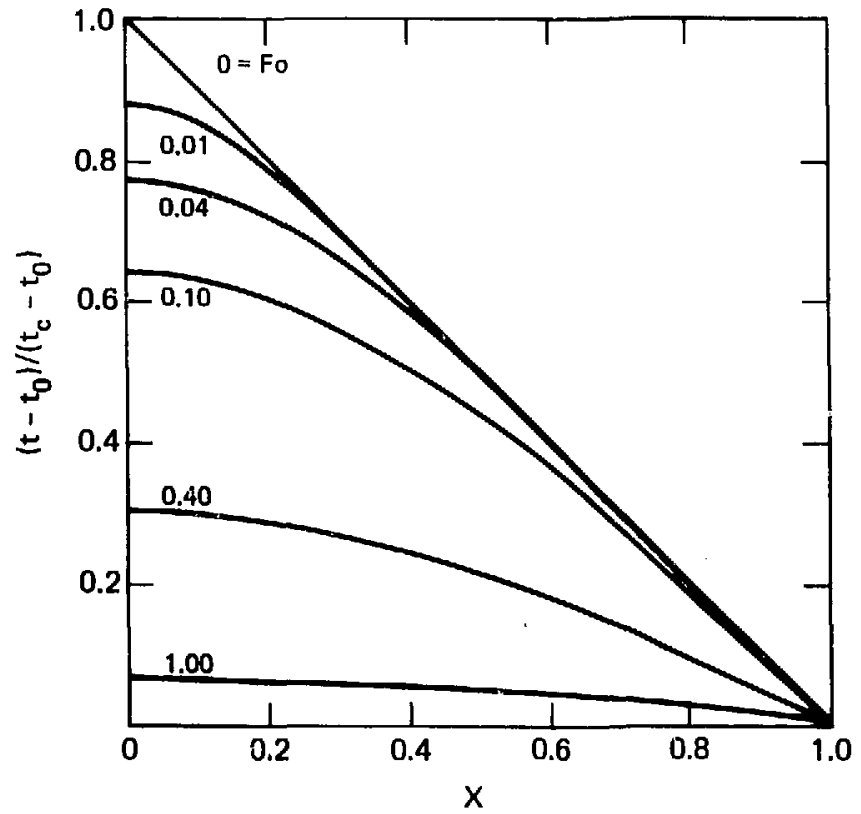

FIG. 8.1. Temperatures in a slab with a linear initial temperature distribution (case 8.1.3, source: Ref. 9, p. 97, Fig. 10). 


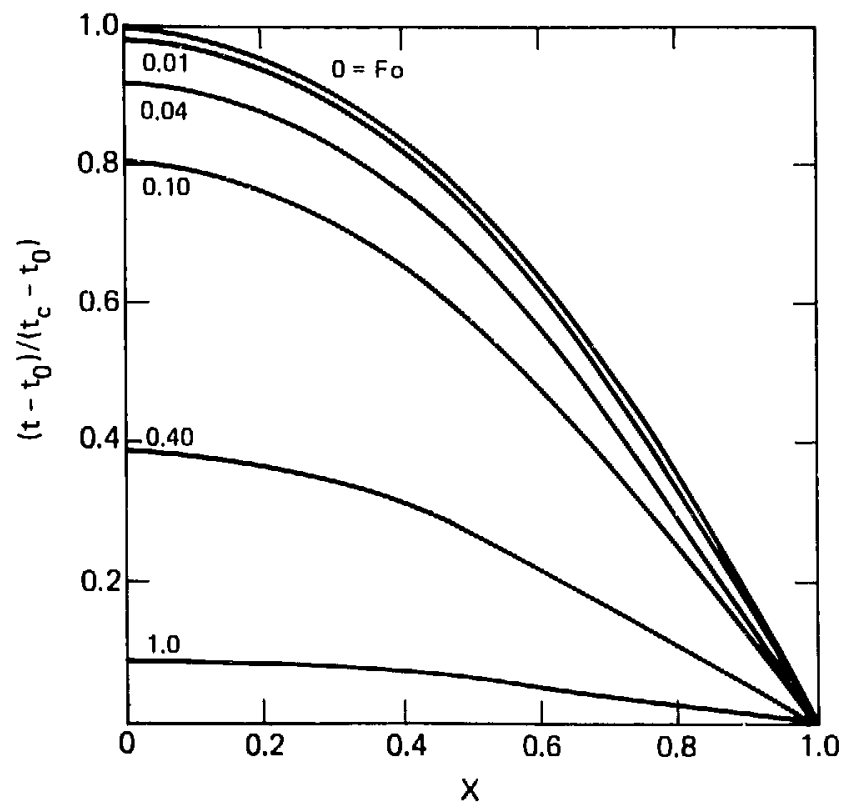

FIG. 8.2. Temperatures in a slab with a parabolic initial temperature distribution (case 8.1.4, source: Ref. 9, p. 98, Fig. 10).

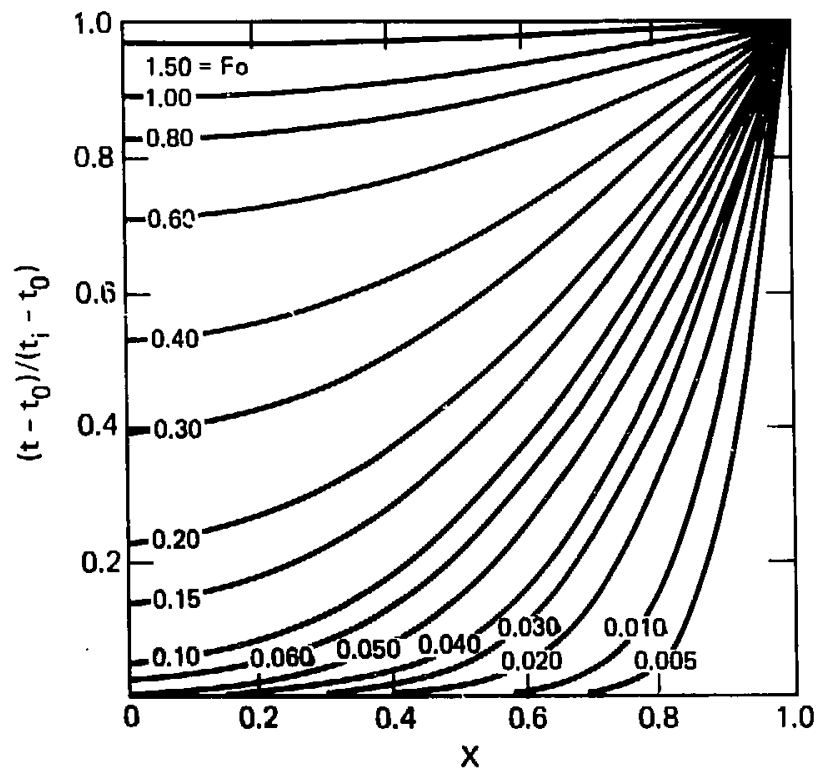

FIG. 8.3. Temperatures in the infinite plate with a constant intial temperature and steady surface temperature (case 8.1.6, source: Ref. 9, p. 101, Fig. 11). 


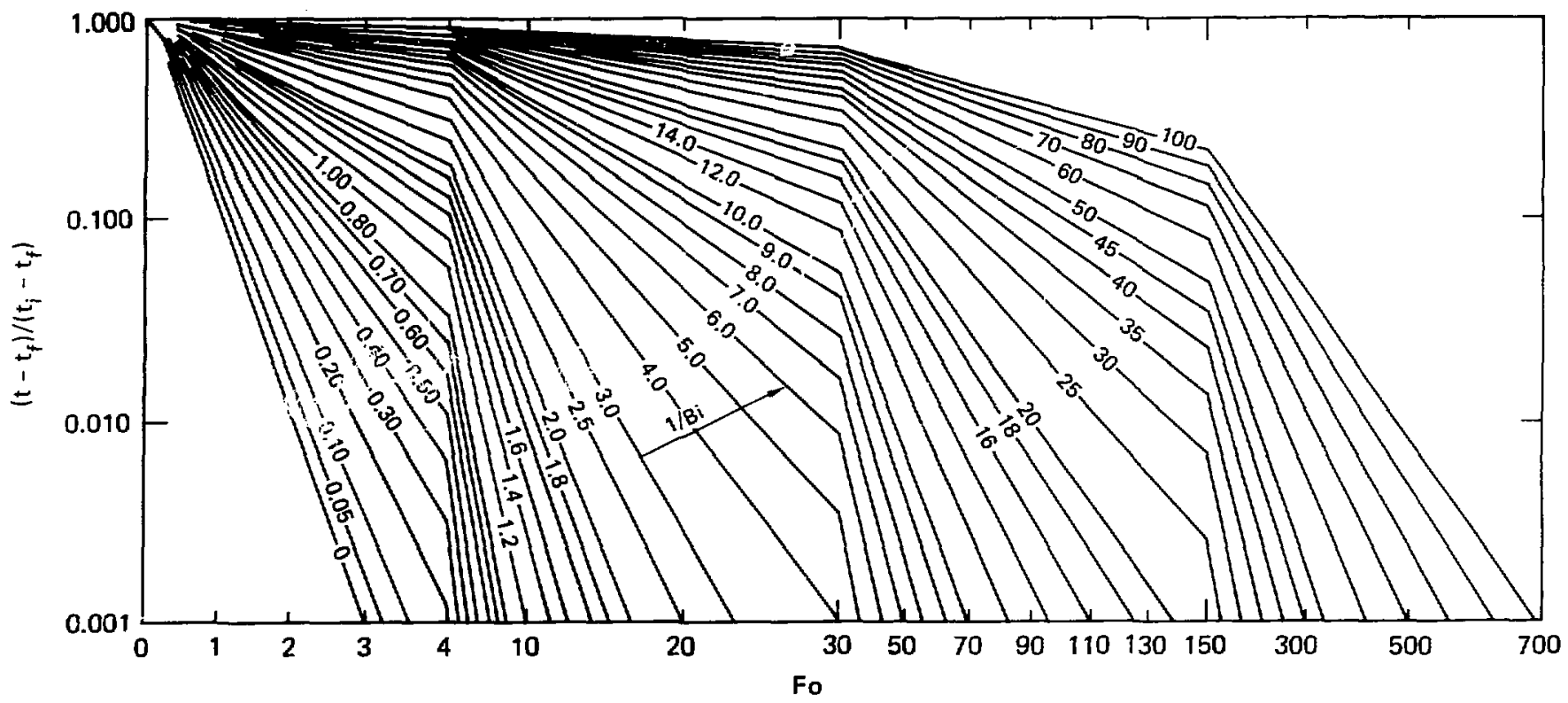

FIG. 8.4a. Midplane temperature of an infinite plate of thickness $2 l$ and convectively cooled. (case 8.1.7, source: Ref. 12, p. 227 and source: Ref. 5, p. 83, Fig. 4-7). 


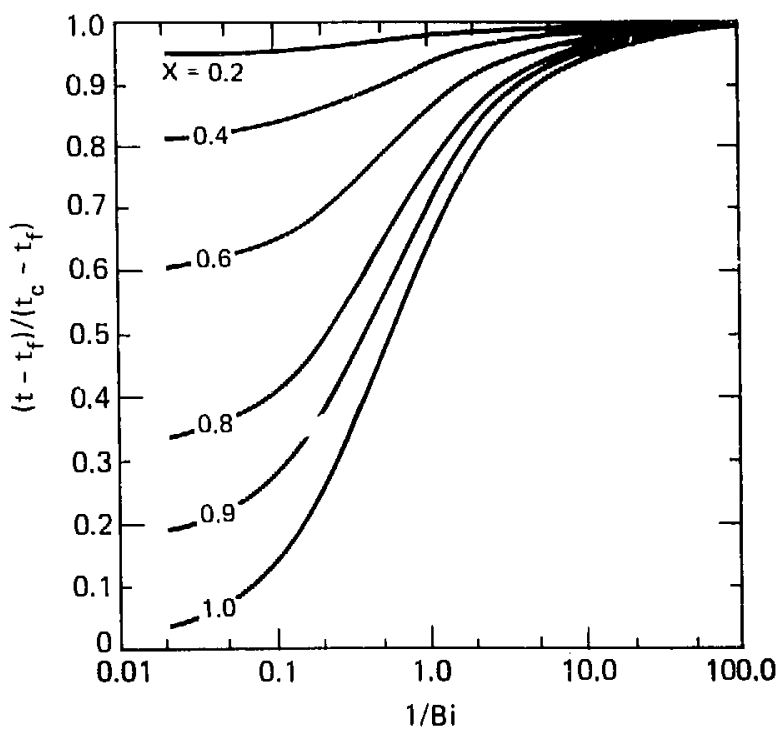

FIG. 8.4d. Temperature as a function of midplane temperature for an infinite plate of thickness $2 l$ and convectively cooled (case 8.1.7, source: Ref. 5, p. 86, Fig. 4-10).

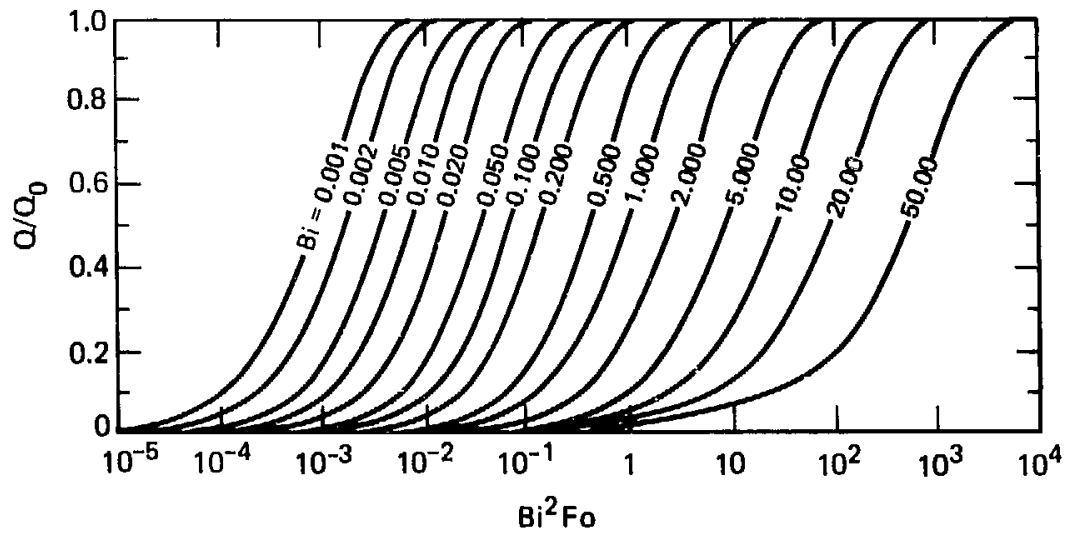

FIG. 8.4e, Relative heat loss from an infinite plate of thickness $2 l$ and convectively cooled (case 8.1.7, source: Ref. 5, p. 90, Fig. 4-14 and Ref. 13). 


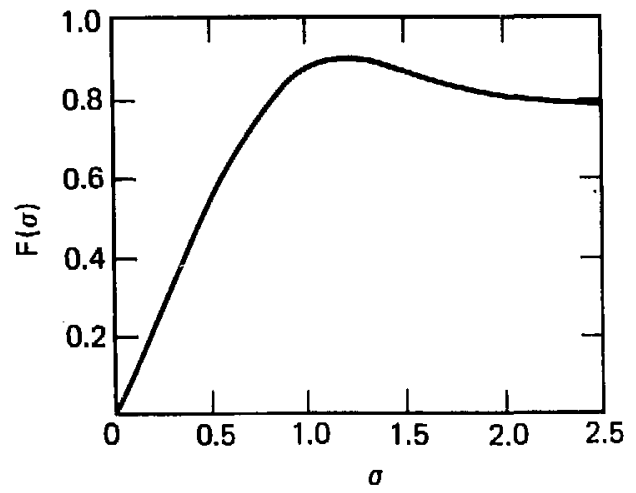

FIG. 8.5. The function $F(\sigma)$ (case 8.1.12, source: Ref. 1. p. 301, Fig. 14-3).

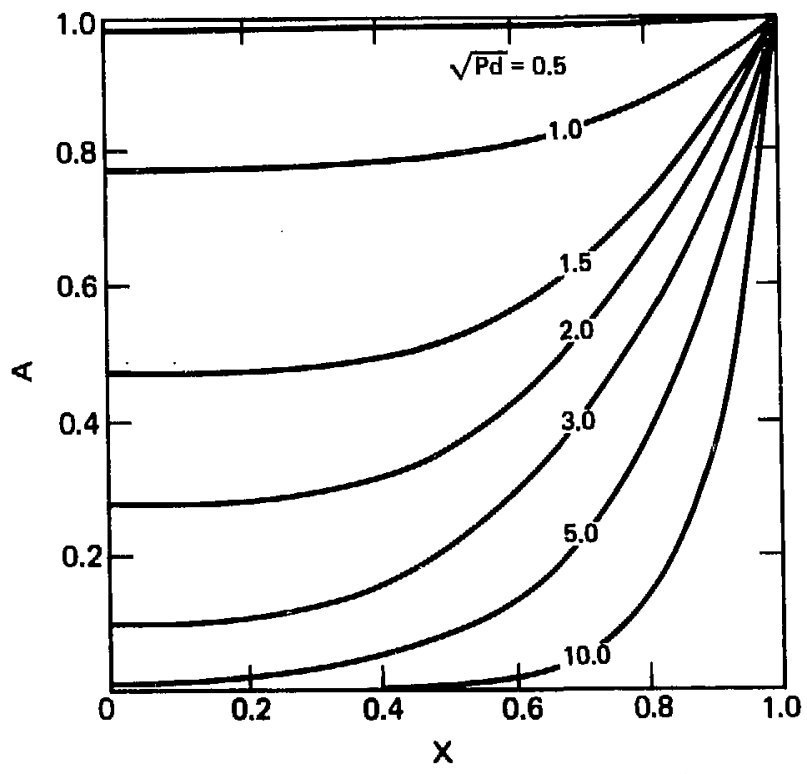

FIG. 8.6a. Varlation of amplitude of the steady oscillation of temperature in an Infinite plate caused by harmonlc surface temperature (case 8.1.13, source: Ref. 9, p. 106, Fig. 13). 


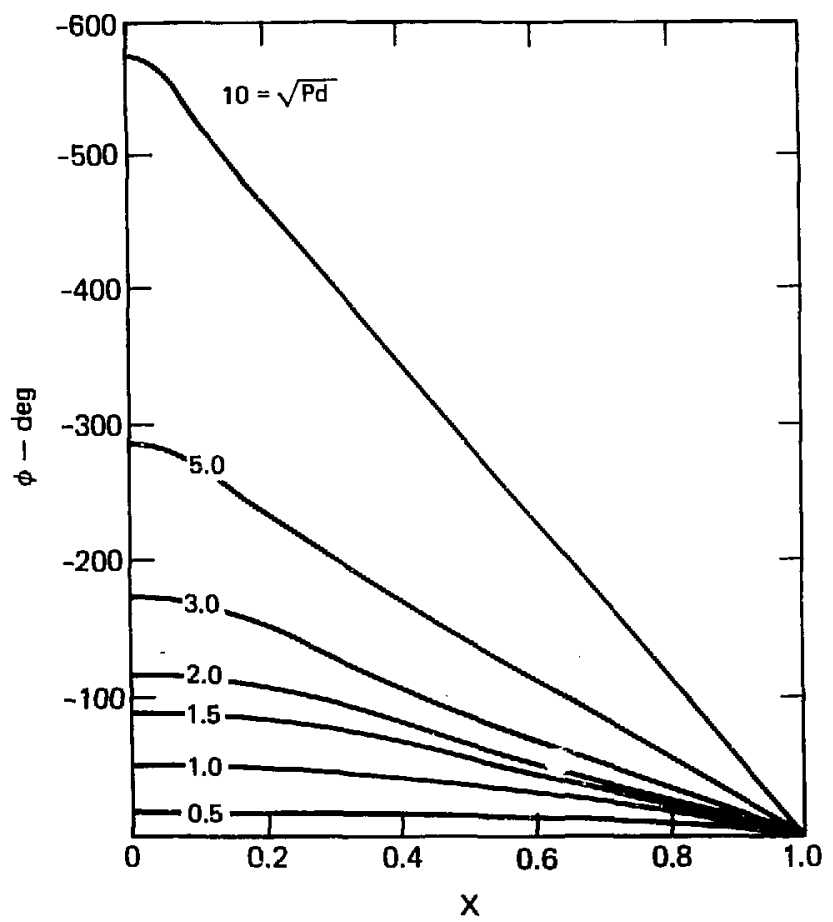

FIG. 8.6b. Tariation in phase of the steady oscillation of temperature in an infinite place caused by harmonic surface temperature (case 8.1 .13 , source: Ref. 9, p. 107, Fiy. 14). 

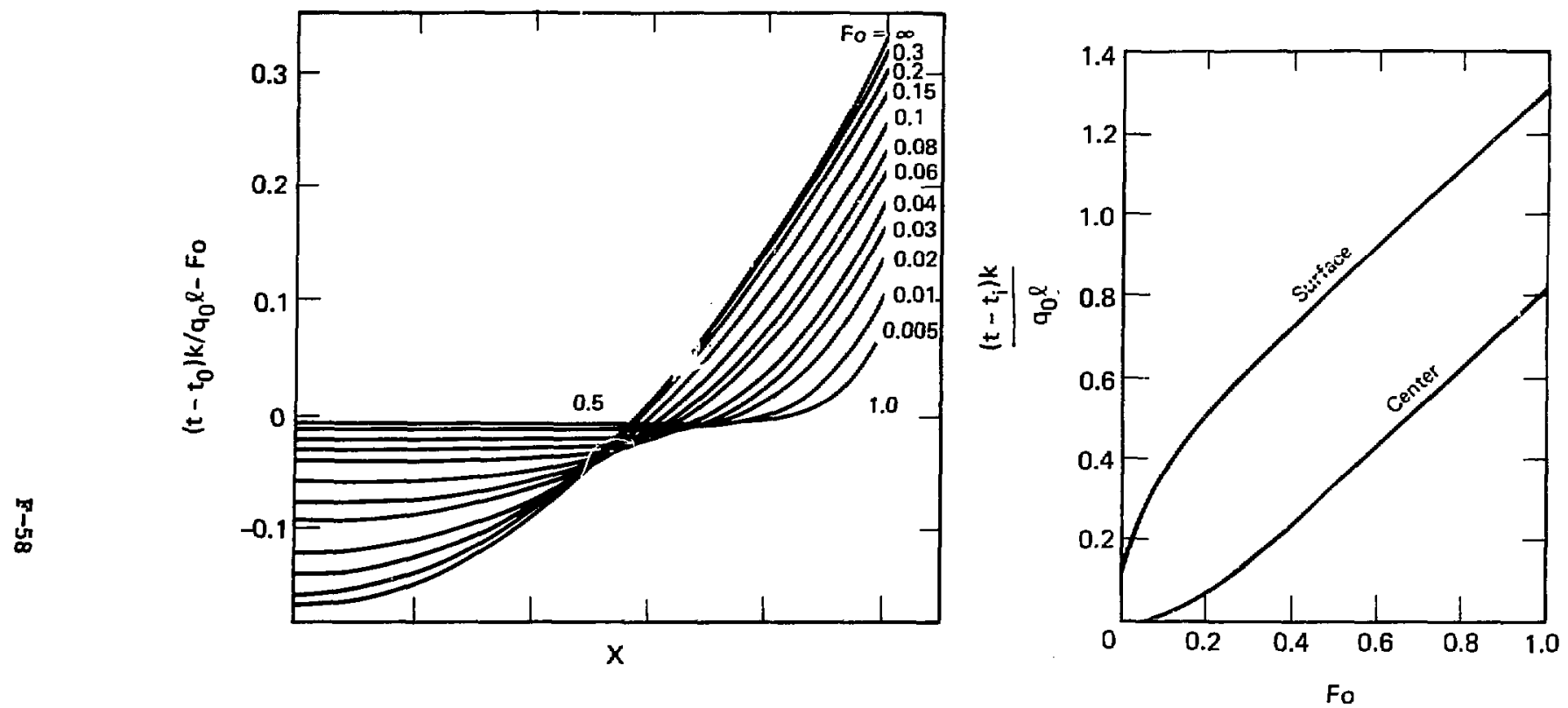

FIG. 8.7. Temperature distribution in an infinite plate with no heat flow at $x=0$ and constent heat flux $q_{0}$ at $x=\ell$ (case 8.1.18, source: Ref. 9, p. 113, Fig. 15). 


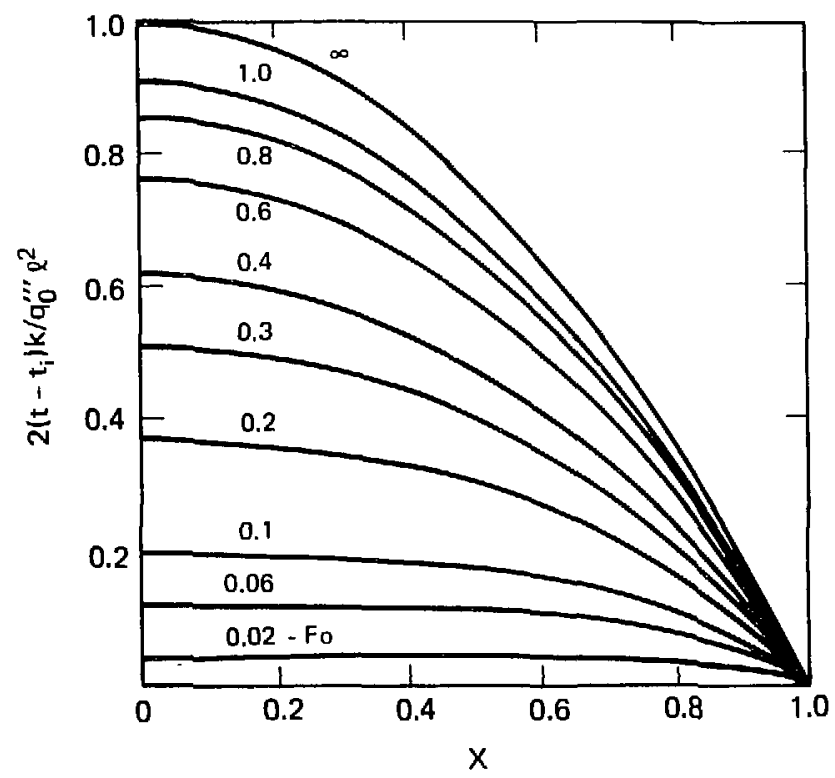

FIG. 8.8. Temperatures in an infinite plate with constant internal heating $\mathrm{q}^{\prime \prime}$ and surface temperature $t_{i}$ (case 8.2.1, source: Ref. 9, p. 131, Fig. 20).

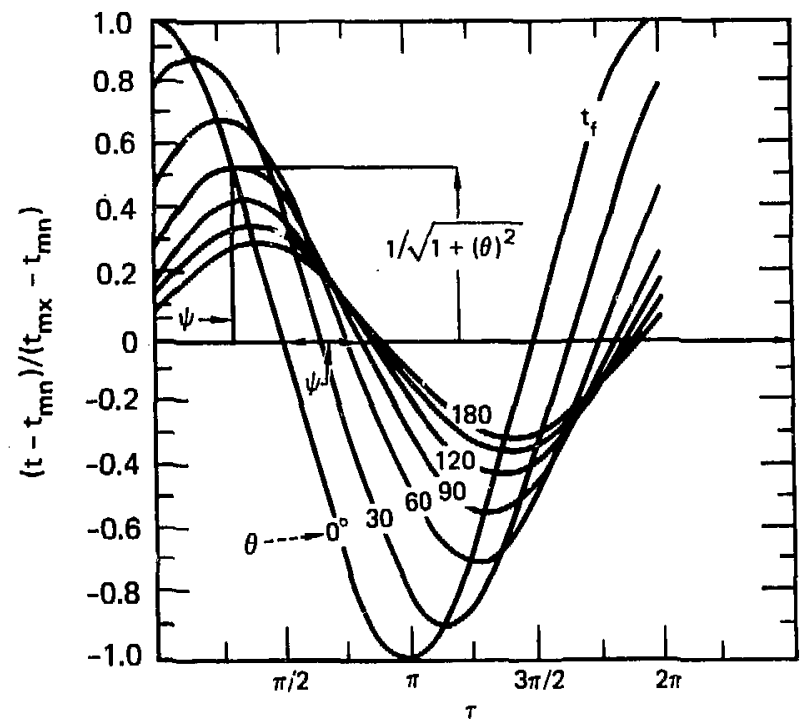

FIG. 8.9. Quasi-steady state temperature of an infinite conductivity plate with a harmonic fluid temperature (case 8.1.49, source: Ref. 19, Fig. 17b, p. $3-31$ ) 


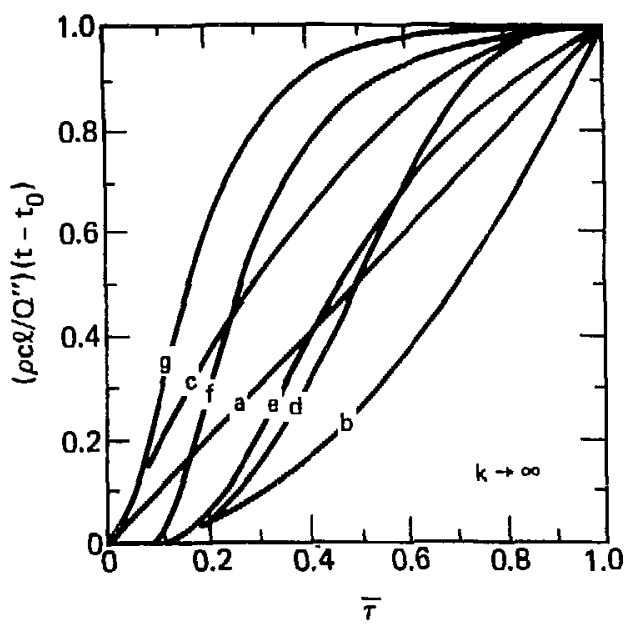

FIG. 8.10. Plate temperature response for prescribed heat inputs (case 8.2.15, source: Ref. 19, p. 3-35, FIg. 18). 

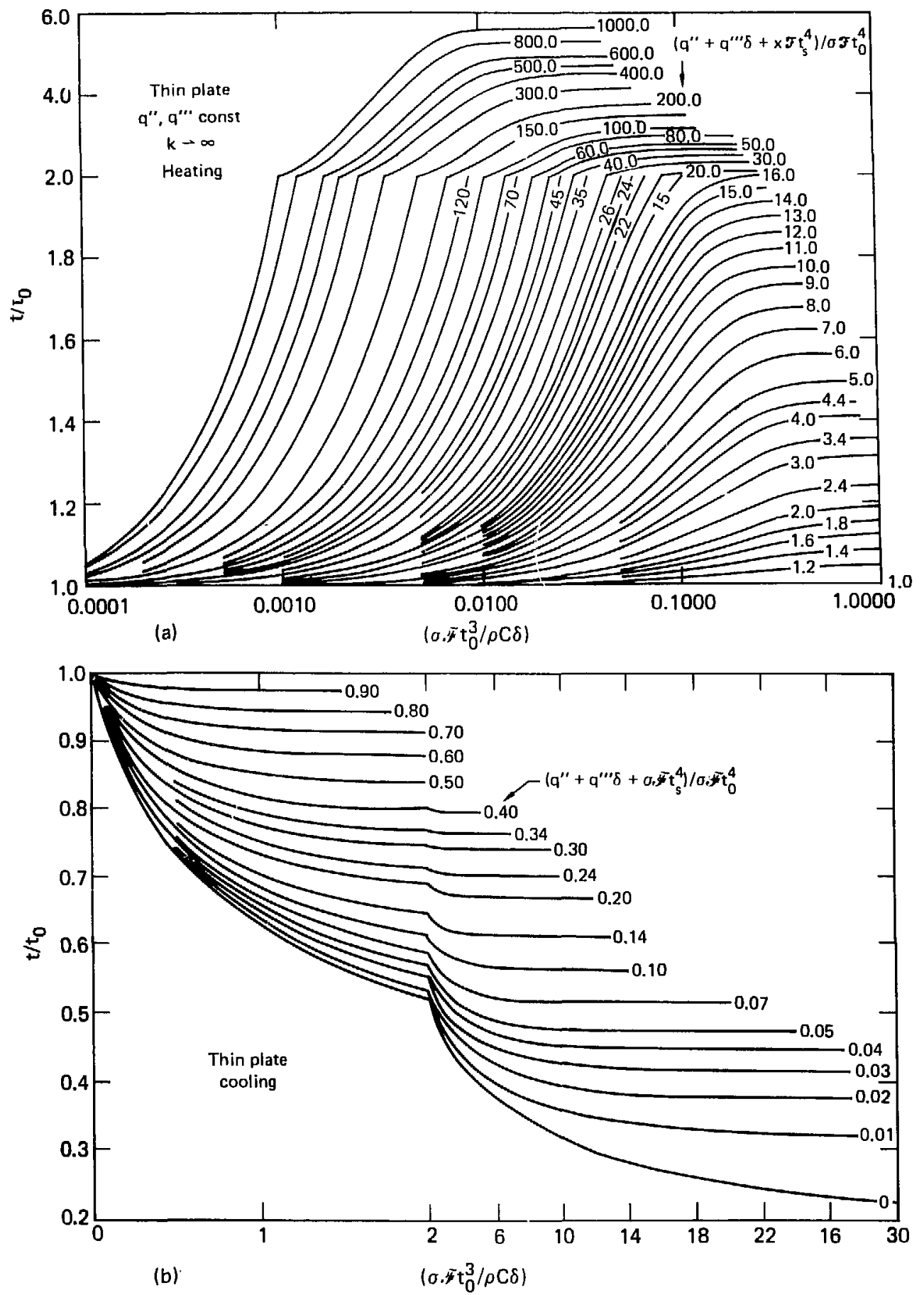

FIG. 8.11. Temperature response of thin generating plate suddenly exposed to constant heat input with surface reradiation: (a) heating, (b) cooling (case B.2.16, source: Ref. 19, p. 3-36, Fig. 19). 


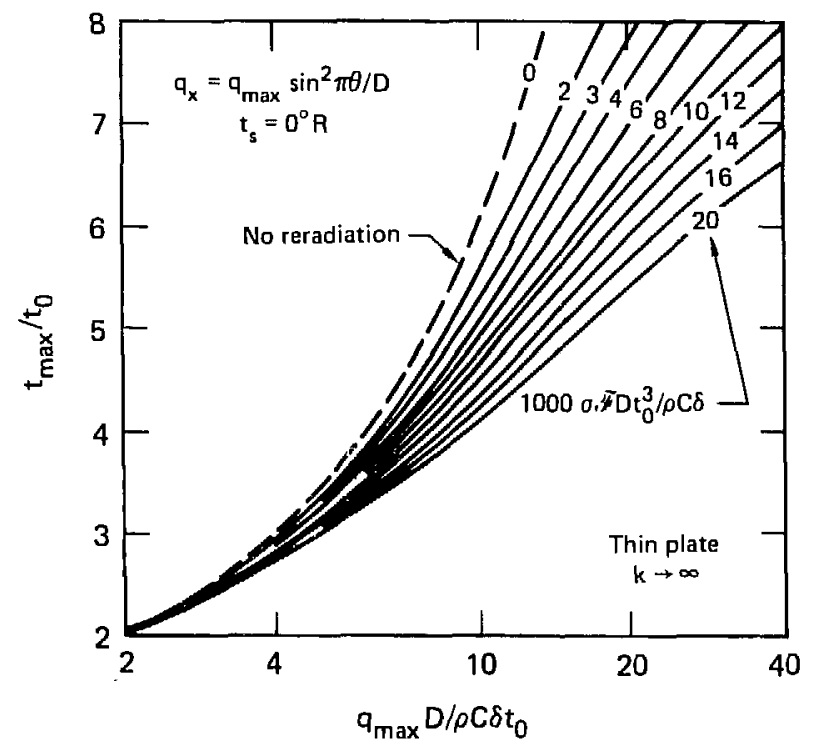

(a)

FIG. 8.12. Maximum temperature of thin insulated plate suddenly exposed to circular heat pulse with surface reradiation: (case 8.1 .50 , source: Ref. 19, p. 3-38, Fig. 20).

$$
\leftarrow \text { Fo }
$$

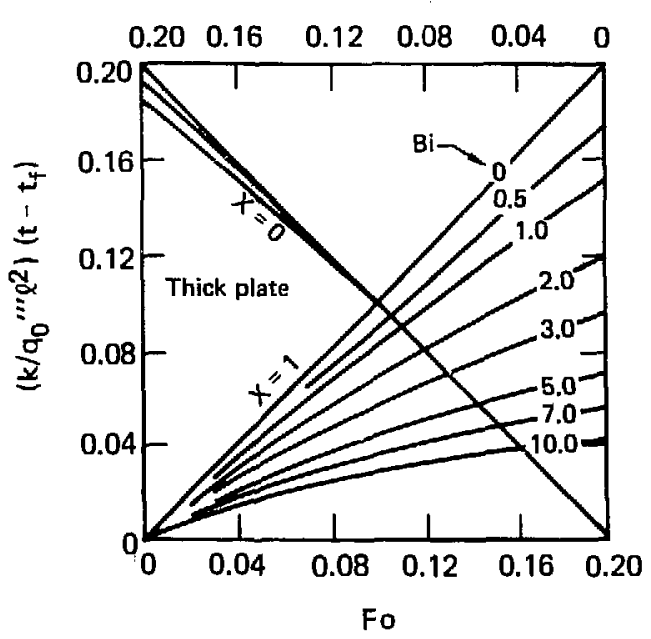

(a)

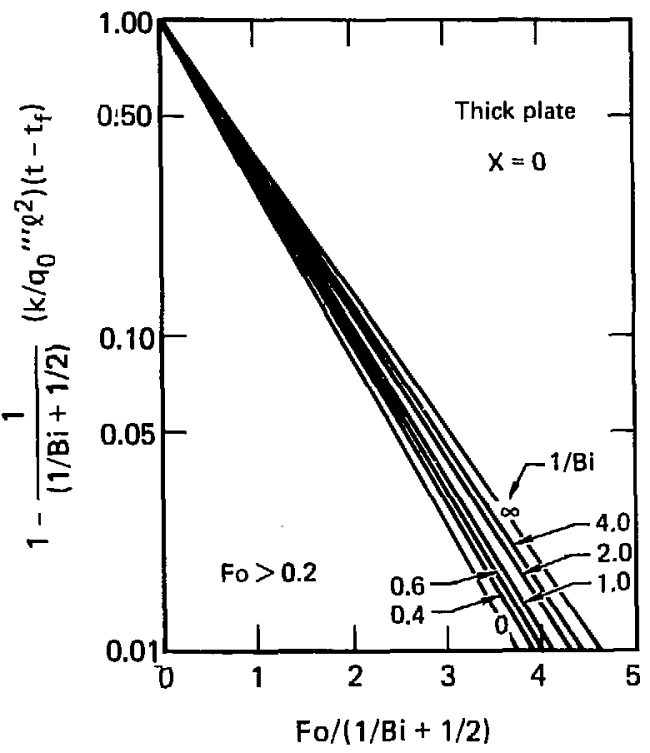

(b)

FIG. B.13. Temperature response of an infinite plate with uniform internal heating, $\ell=$ half thickness (case 8.2.6, source: Ref. 19, p. 3-43, Fig. 24 ). 


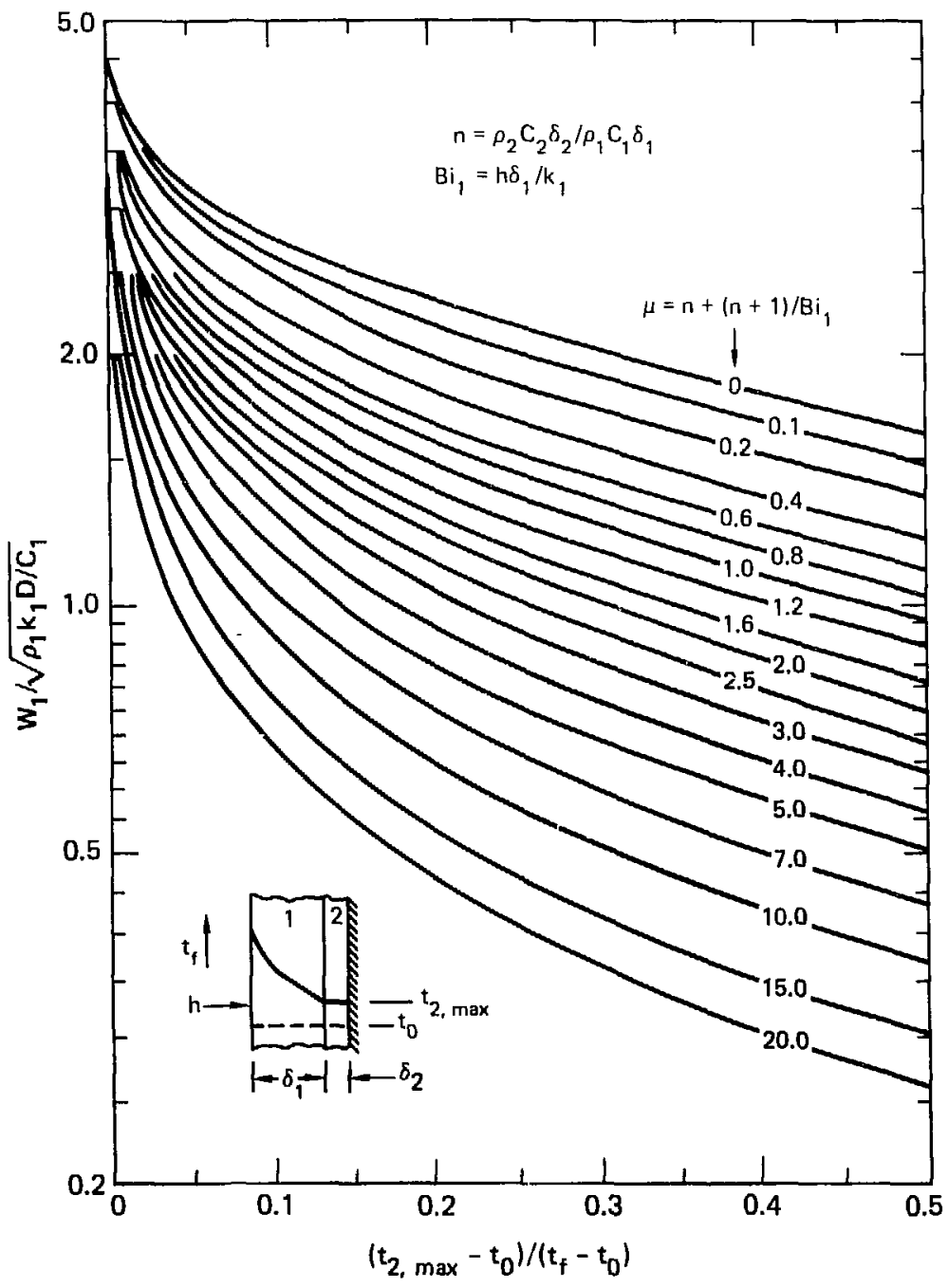

FIG. 8.14a. Insulation weight for substructure protection to $t_{2}$ max in heating duration $D, W_{1}=\rho_{1} \delta_{1}$ (case 8.1 .51 , source: Ref. 19 p. $3-48$, Fig. 27). 


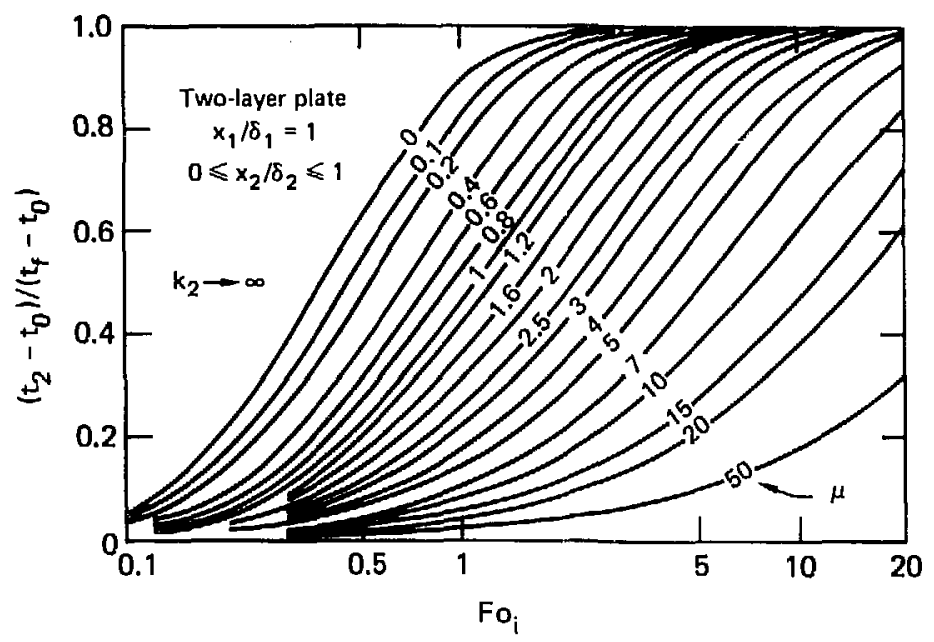

(a)

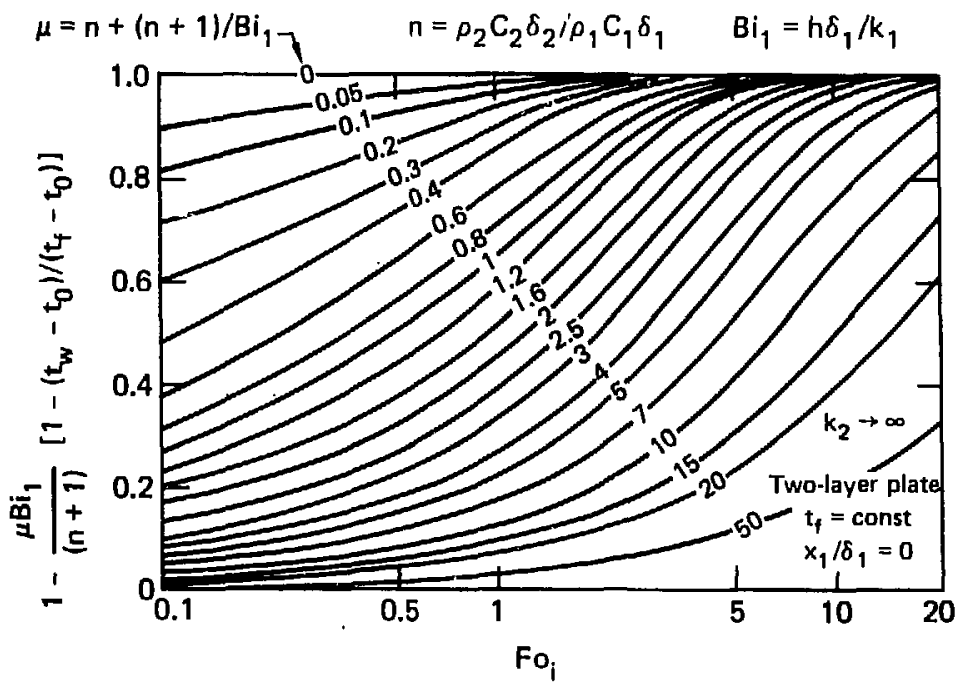

(b)

FIG. 8.14b. Tenperature response of thick plate $\left(0 \leq x_{1} \leq \delta_{1}\right)$ convectively heated at $x_{1}=0$ and in perfect contact at its rear face $x_{1}=\delta_{1}$ with a thin plate $\left(0 \leq x_{2} \leq \delta_{2}\right)$ insulated at $x_{2}=\delta_{2}$ : (a) $x_{1} / \delta_{1}=0$.

(b) $x_{1} / \delta_{1}=1$ (case 8.1.51, source: Ref. 19, p. 3-46, Fig. 26). 

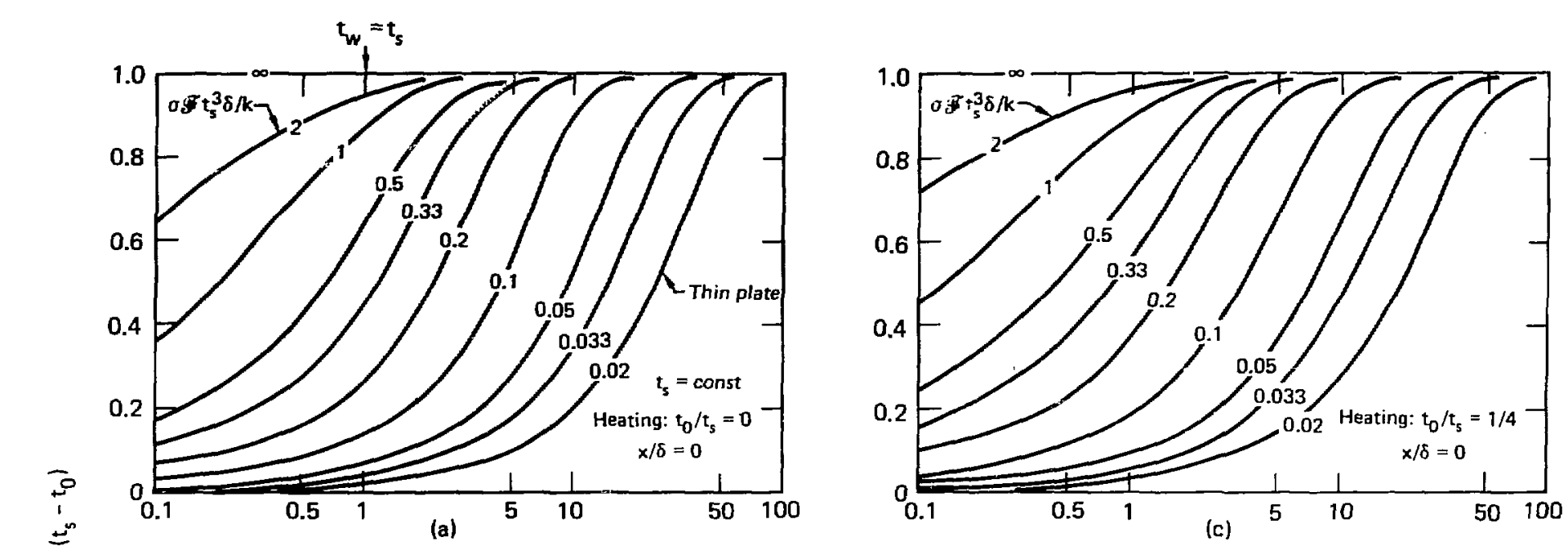

劉亡

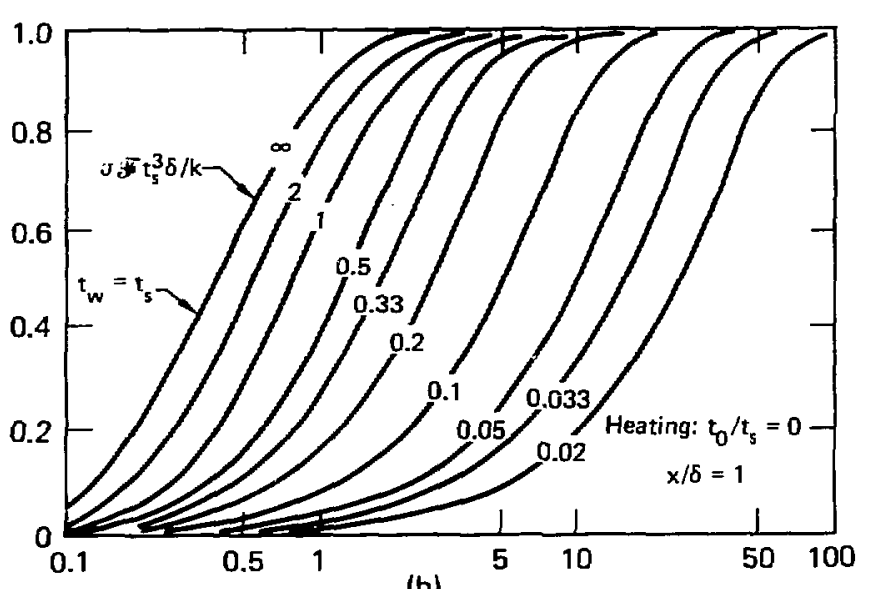

(b) Fo

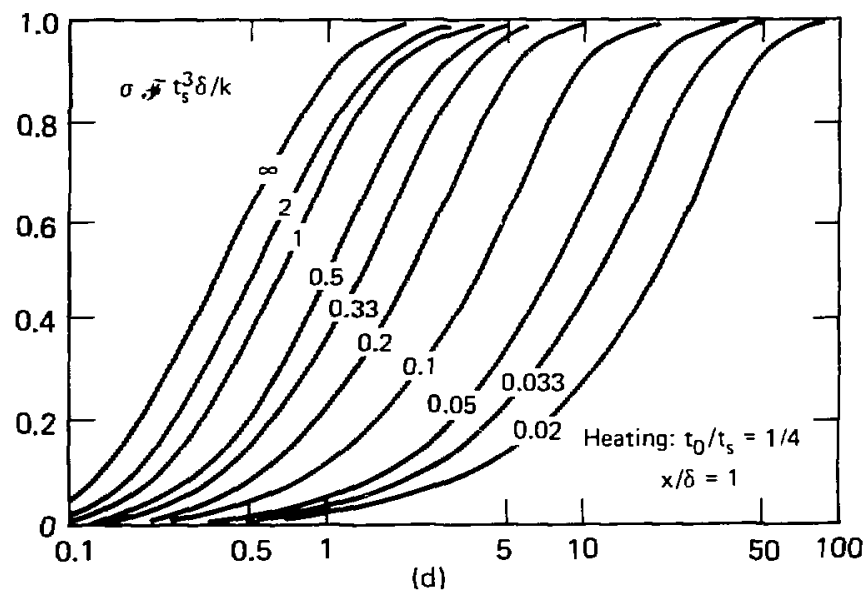

FIG, 8.15. Temperature response of thick plate $(0<\varepsilon: \delta)$ with insulated rear face $\mathbf{x}=\delta$ after sudden exposure to uniform radiative environment $t_{s}$ at $x={ }^{\prime}$ : " (a) heating, $t_{0} / t_{s}=0, x / \delta=0$, (b) heating, $t_{0} / t_{s}=0, x / \delta=1$, (c) heating, $t_{0} / t_{s}=1 / 4, x / \delta=:$, (d) heating, $t_{0} / t_{s}=1 / 4, x / \delta=1$ (case 8.1.52, source: Ref. 19, Pp. 3-50, Fig. 29). 


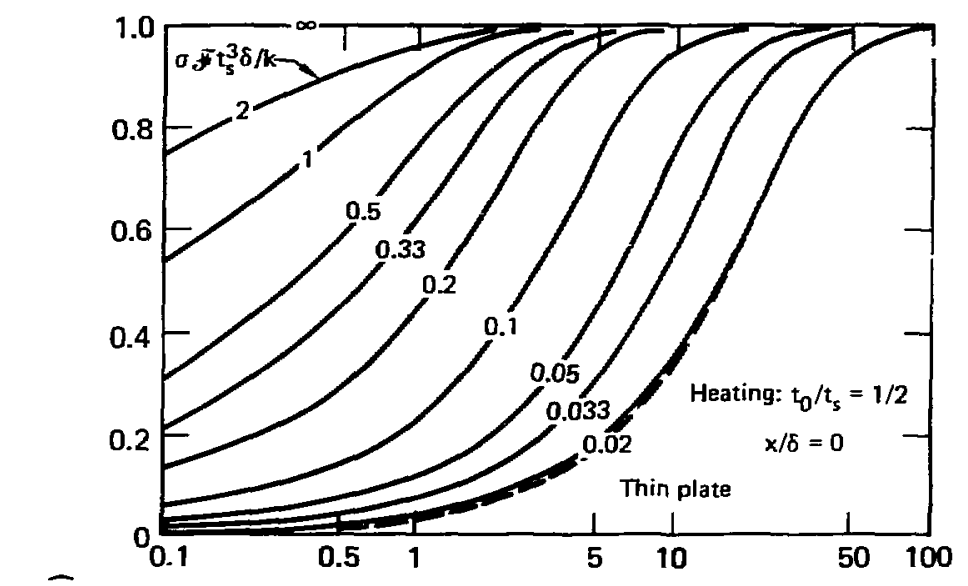

(e)

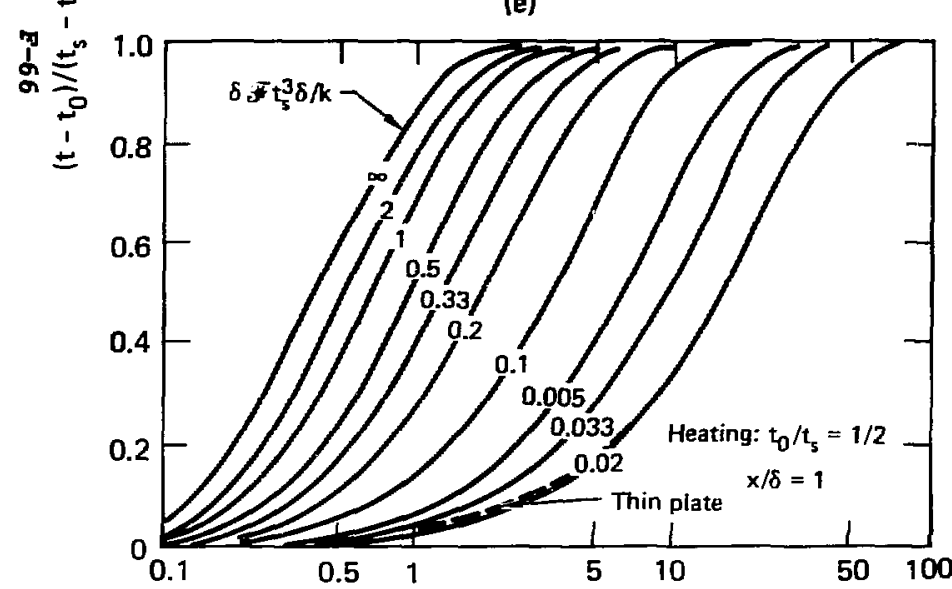

(f)

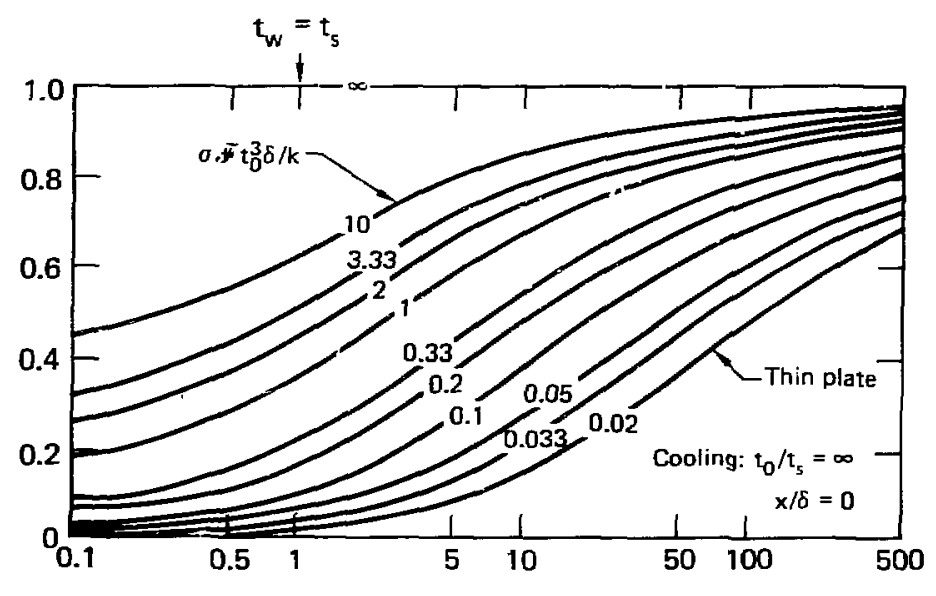

(g)

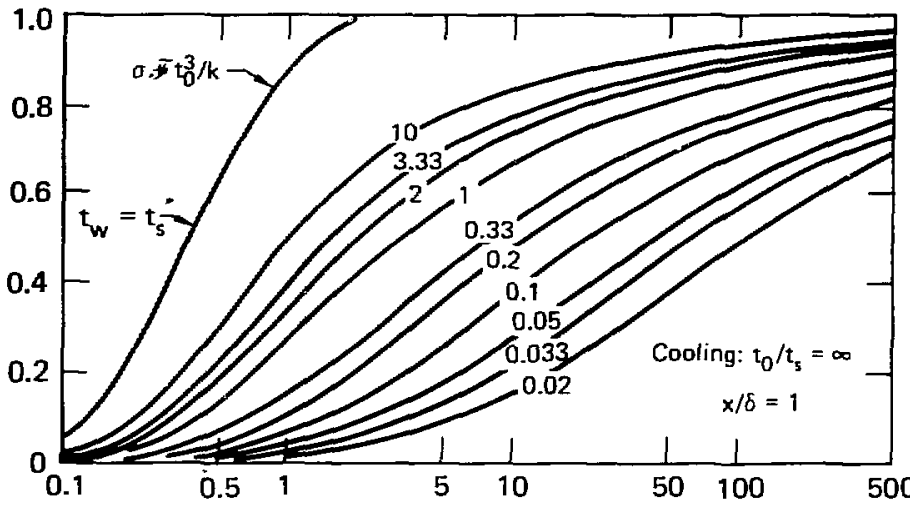

(h)

FIG. 8.15 (Cont.). Temperature response of thick plate $(0 \leq x \leq \delta$ ) with insulated rear face $x=\delta$ after sudden exposure to uniform radiative environment $t_{s}$ at $x=\overline{0}$ : (e) heating, $t_{0} / t_{s}=1 / 2, x / \delta=0,(f)$ heating, $t_{0} / t_{s}=1 / 2, x / \delta=1,(g)$ cooling, $t_{0} / t_{s}=\delta, x / \delta=0$, (h) cooling, $t_{0} / t_{s}=\delta, x /=1$. 


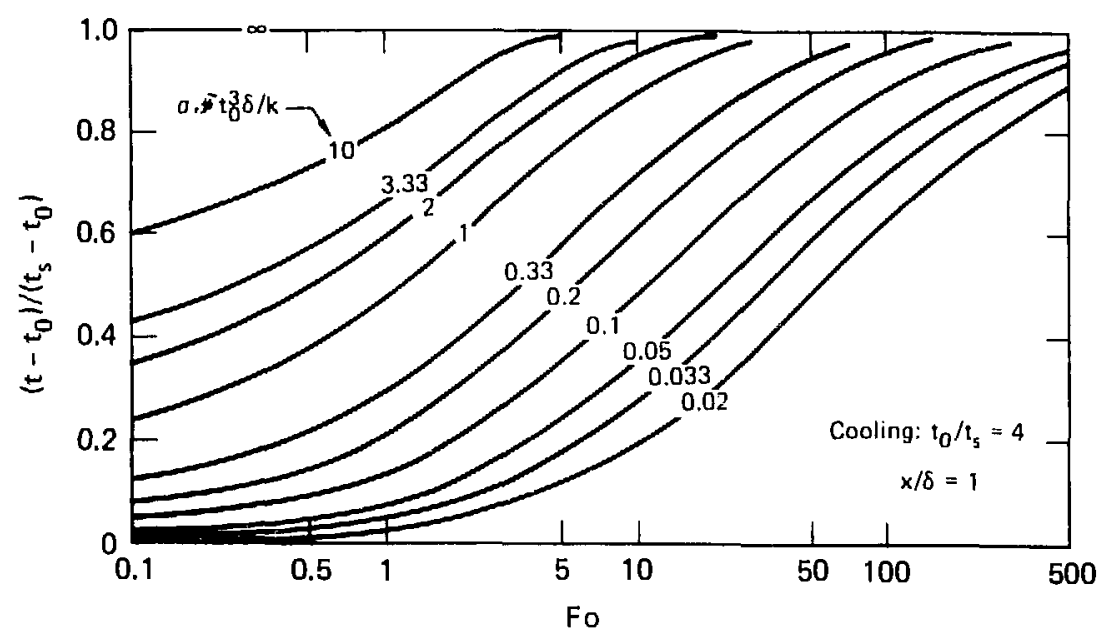

(i)

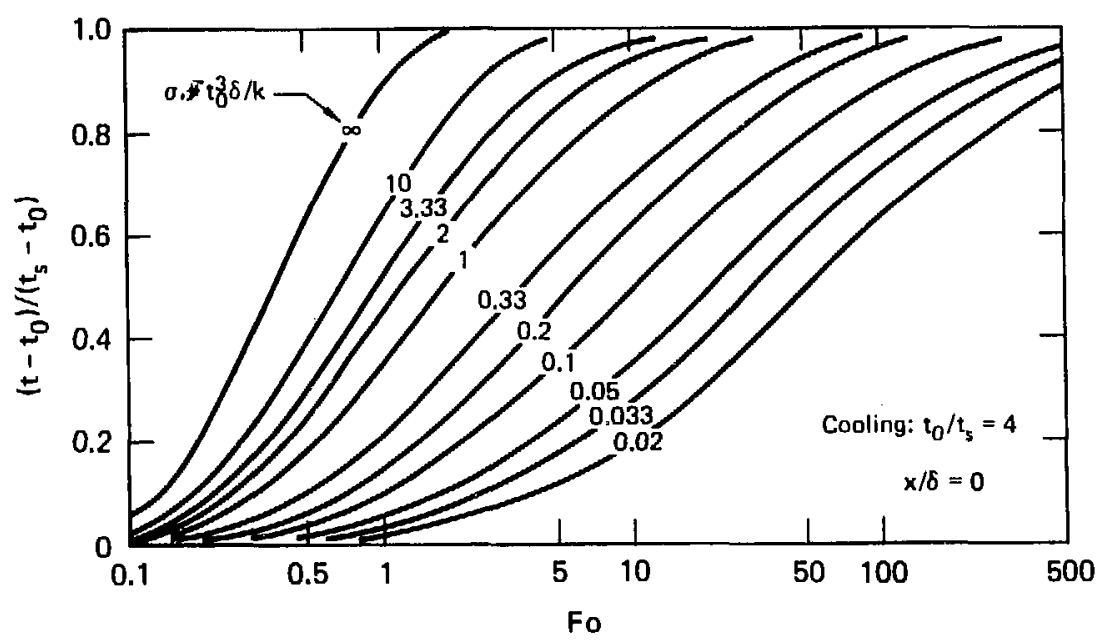

(j)

FIG. 8.15 (Cont.). Temperature response of thick plate $(0 \leq x \leq \delta)$ with insulated rear face $x=\delta$ after sudden exposure to uniform radiative envi ronment $t_{g}$ at $x=0:$ (k) cooling, $t_{0} / t_{g}=2, x / \delta=0$, (1) cooling, $t_{0} / t_{B}=2, x / 8=1$. 


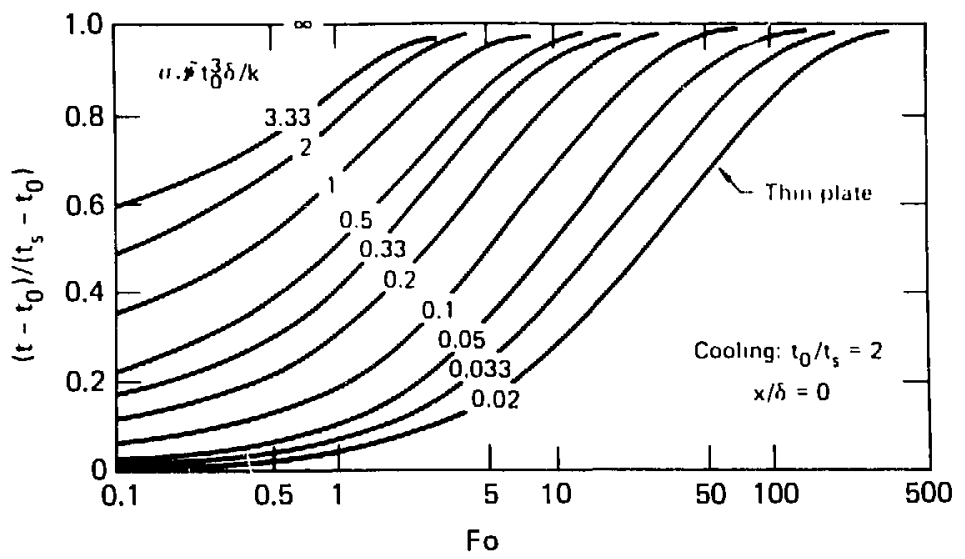

(k)

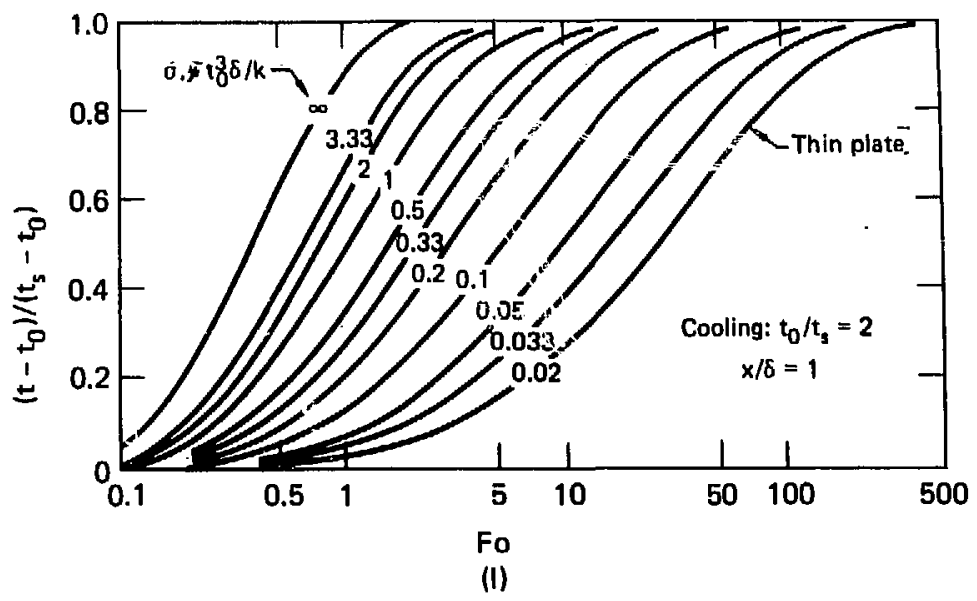

FIG. 8.15 (Cont.). Temperature response of thick plate $(0 \leq x \leq \delta)$ with insulated rear face $x=\delta$ after sudden exposure to uniform radiative environment $t_{8}$ at $x=0$ : (i) cooling, $t_{0} / t_{g}=4, x / \delta=0,(j)$ cooling, $t_{0} / t_{g}=4, x / \delta=1$. 


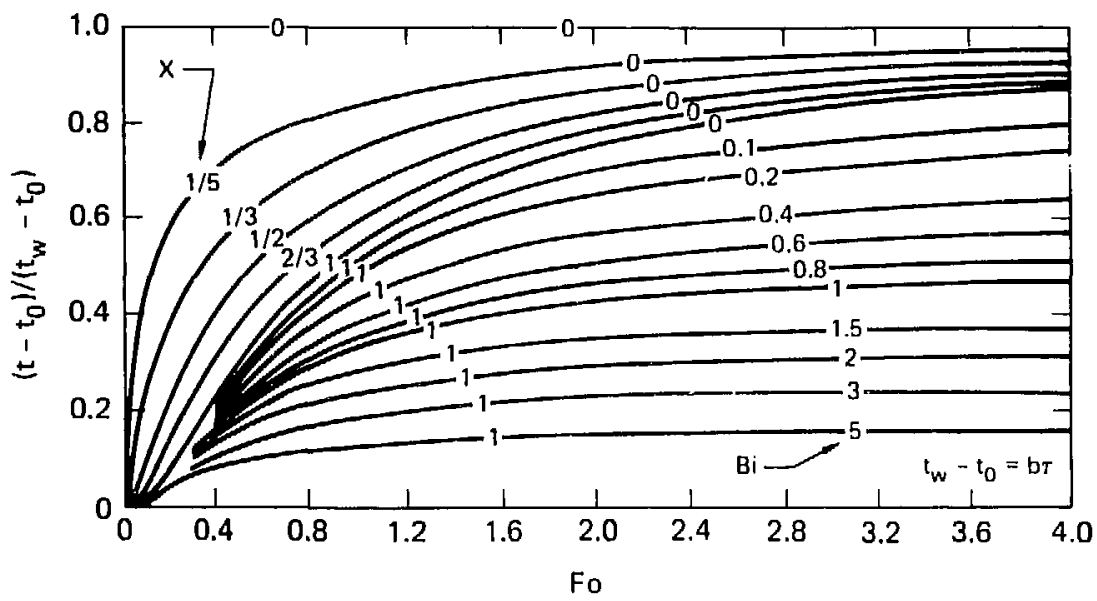

FIG. 8.16. Temperature response of thick plate $(0 \leq x \leq \delta)$ with surface temperature $t_{w}$ at $x=0$, increasing linearly with time and rear face $x=\delta$ insulated or exposed to uniform convective environment $t_{0}$ (case 8.1.8, sour ce: Ref. 19, pp. 3-60, Fig. 33).

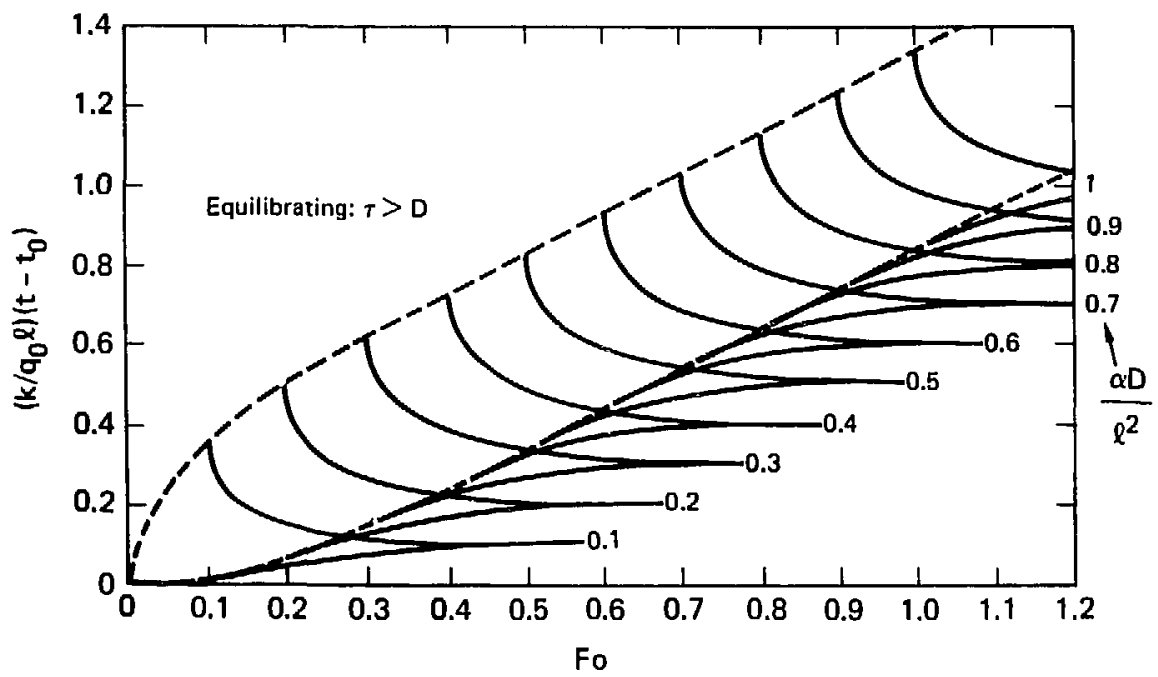

FIG. 8.17. Surface temperature response of a plate of thickness $2 l$ exposed to a steady heat flux $q_{0}$ on both sides for a time duration $D$ (case 8.1.18, source: Ref. 19, pp. 3-67, Fig. 40). 


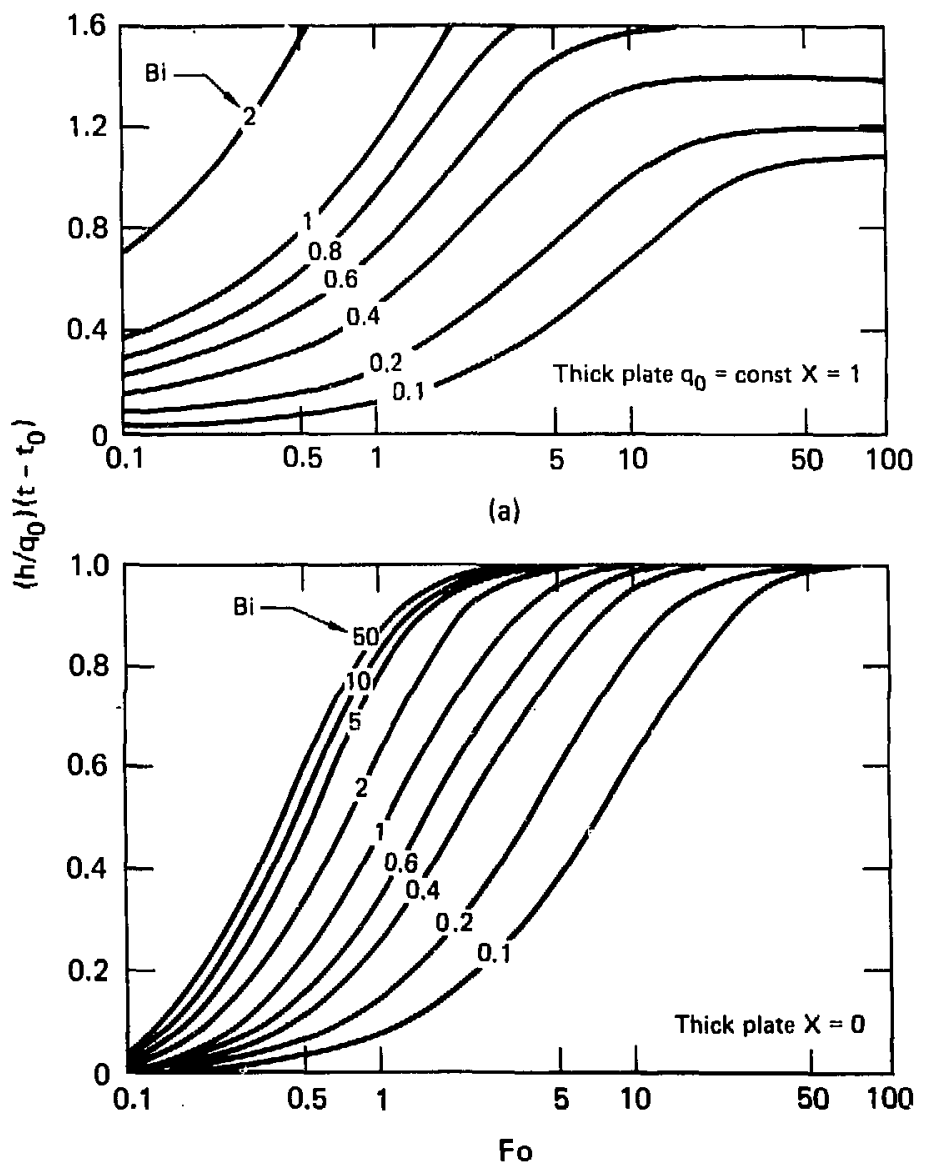

(b)

FIG. 8.18. Temperature response of a plate with steady heating $q_{0}$ at $x=\ell$ and convection boundary at $x=0$ to $t_{0}$ (a) $x / l=1$, (b) $x / l=0$ (case 8.1 .17 source: Ref. 19, PP. 3-69, Fig. 41). 


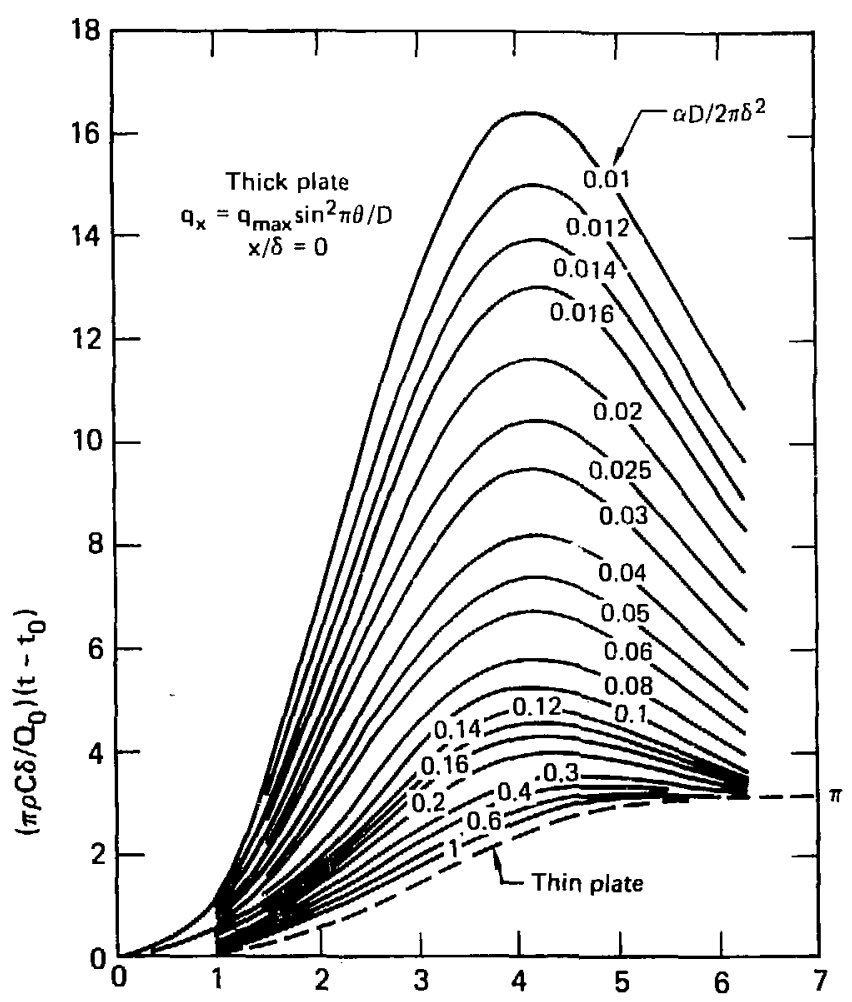

(a)

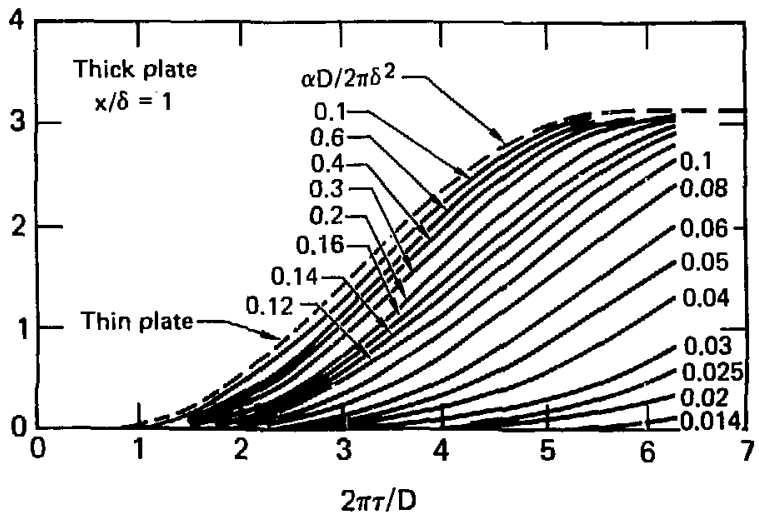

(b)

EIG. 8.15. Temperature response of an infinite plate exposed to a circular heat pulse $q_{\max } \sin ^{2}(\pi \tau / D)$ at $x=0$ and insulated at $x=\delta$ (a) $x / \delta=0$, (b) $x / \delta=1$ (case 8.1.54, source: Ref. 19, pp. 3-70, Fig. 42). 


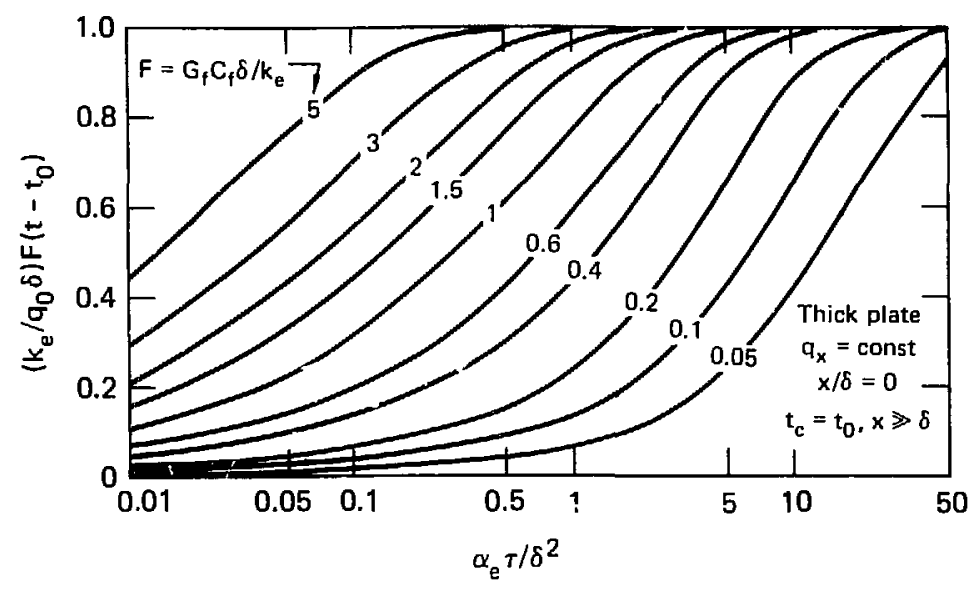

(a)

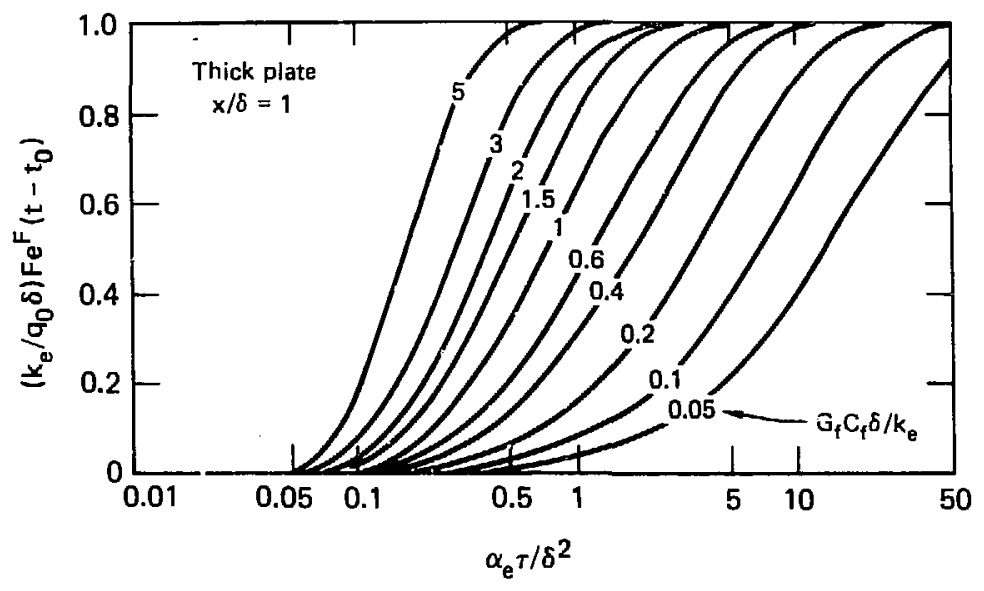

(b)

FIG. 8.20. Temperature response of an infinite porous plate after sudden exposure to a constant heat flux at $x=0$ and cooled by steady flow through plate from $x=\delta$ of a fluid initially at $t_{0}$ (a) $x / \delta=0$, (b) $x / \delta=1$ (case B.1.55, source: Ref. 19, pg. 3-74, Fig. 44). 


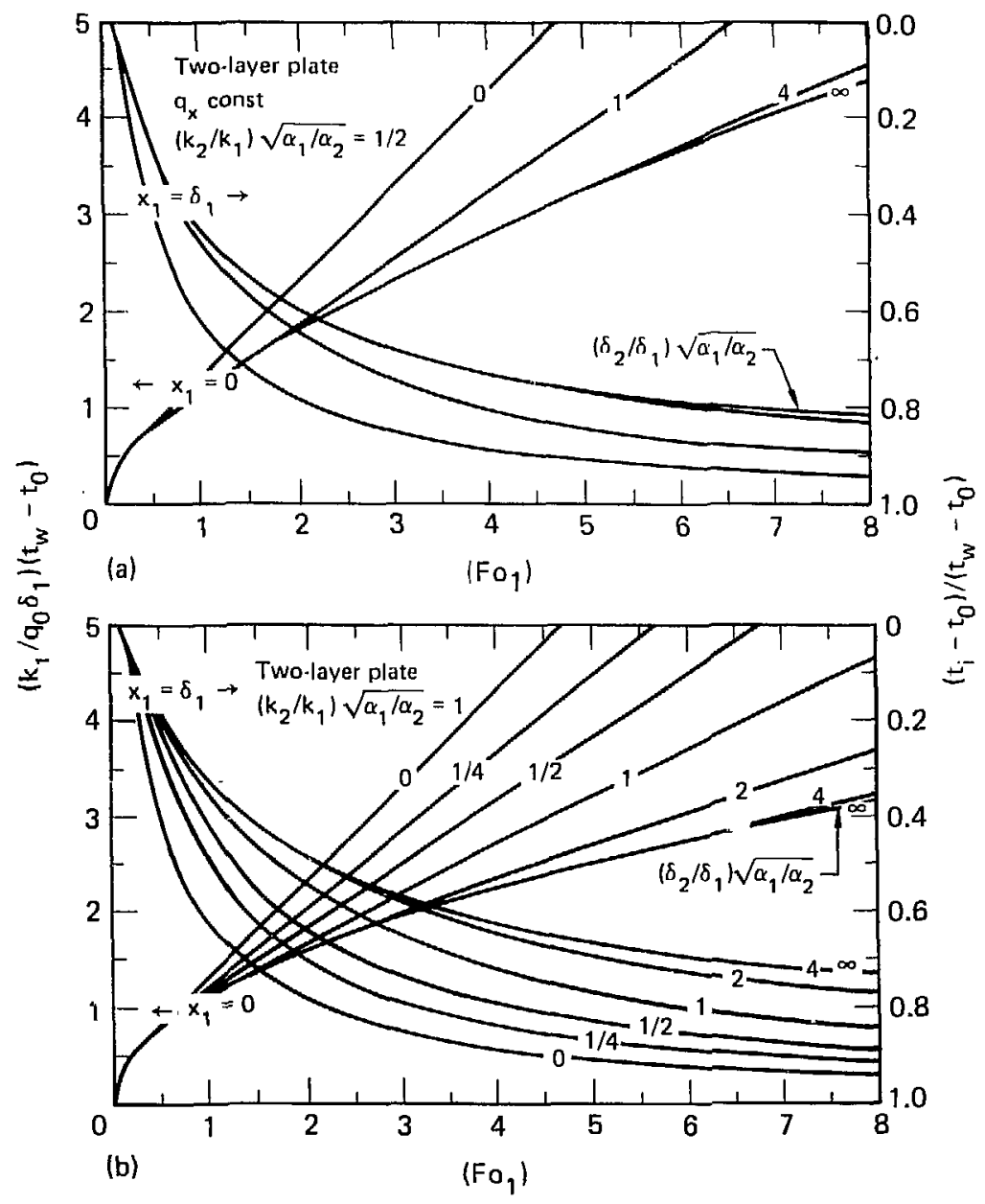

FIG. 8.21. Temperature cesponse of an infinite plate with a constant heat flux $q_{0}$ at $x_{1}=0$, in contact with a plate at $x_{1}=\delta_{1}$ and insulated on exposed surface of second plate $x_{2}=\delta_{2}$ (case 8.1.56, source: Ref. 19, pp. 3-76, Fig. 45). 


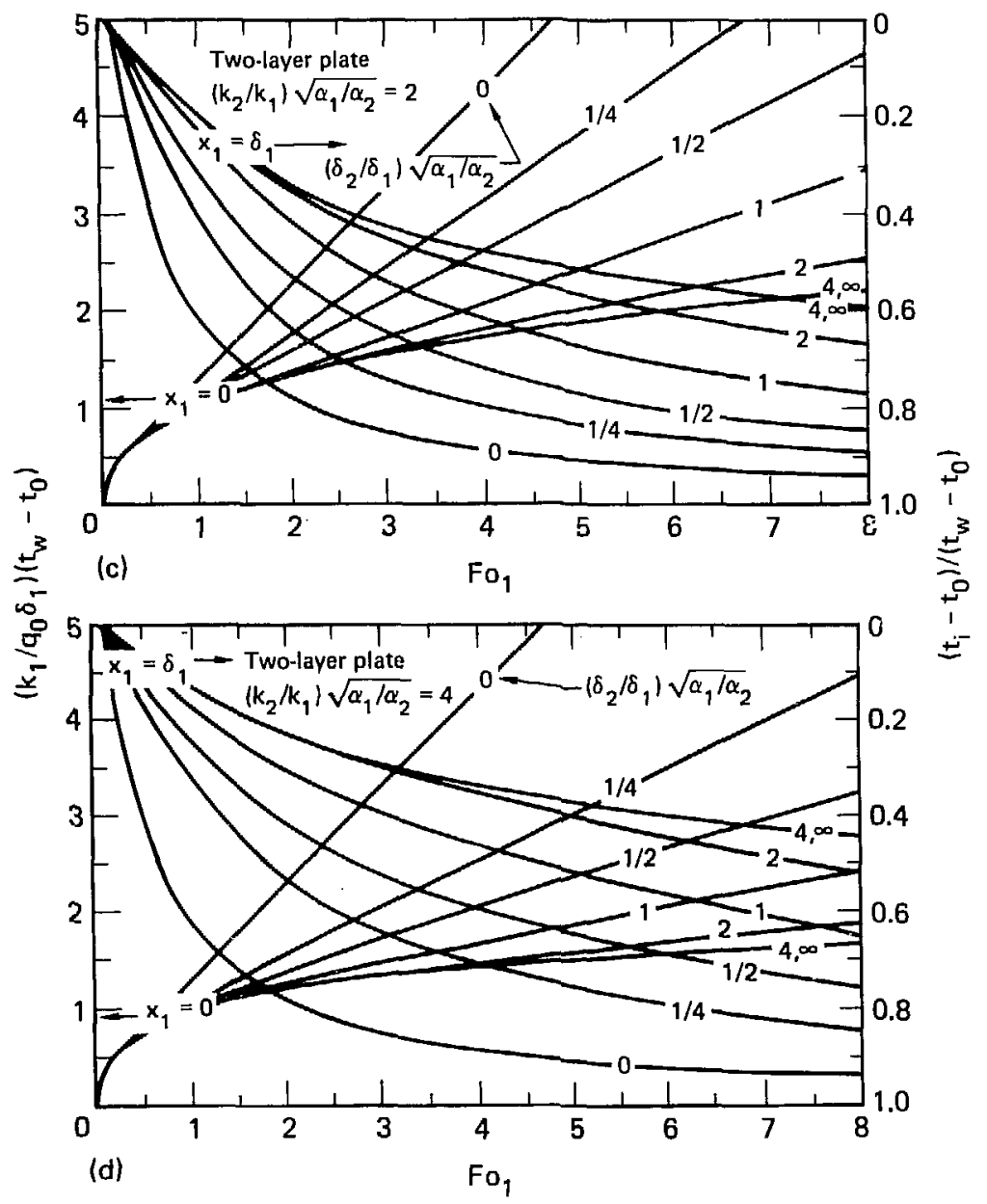

FIG. 8.21. (continued). 


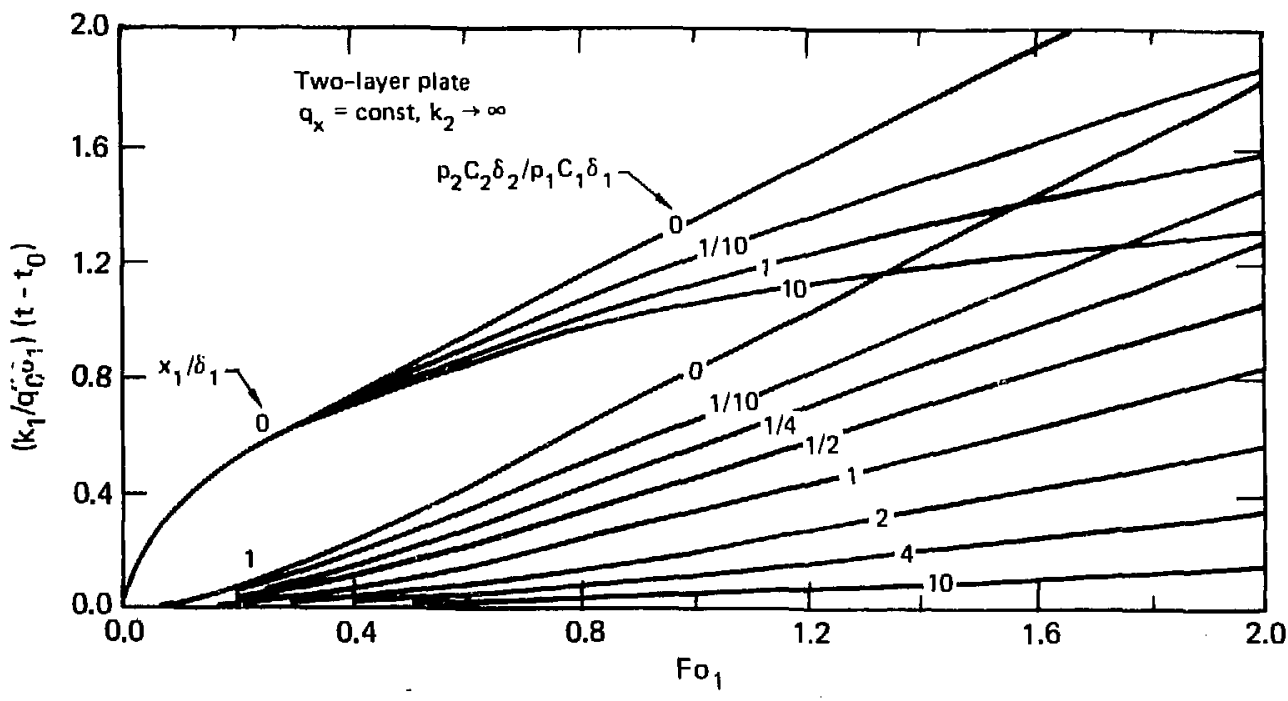

FIG. B.22. Temperature response of an infinite plate exposed to a constant heat flux $q^{\prime \prime}$ at $x_{1}=0$ and in perfect contact at $x_{1}=\delta_{1}$ with a plate of thickness $\delta_{2}$ insulated at $x_{2}=\delta_{2}$ (case 8.1.57, source: Ref. 19, Pp. 3-78, Fig. 46). 


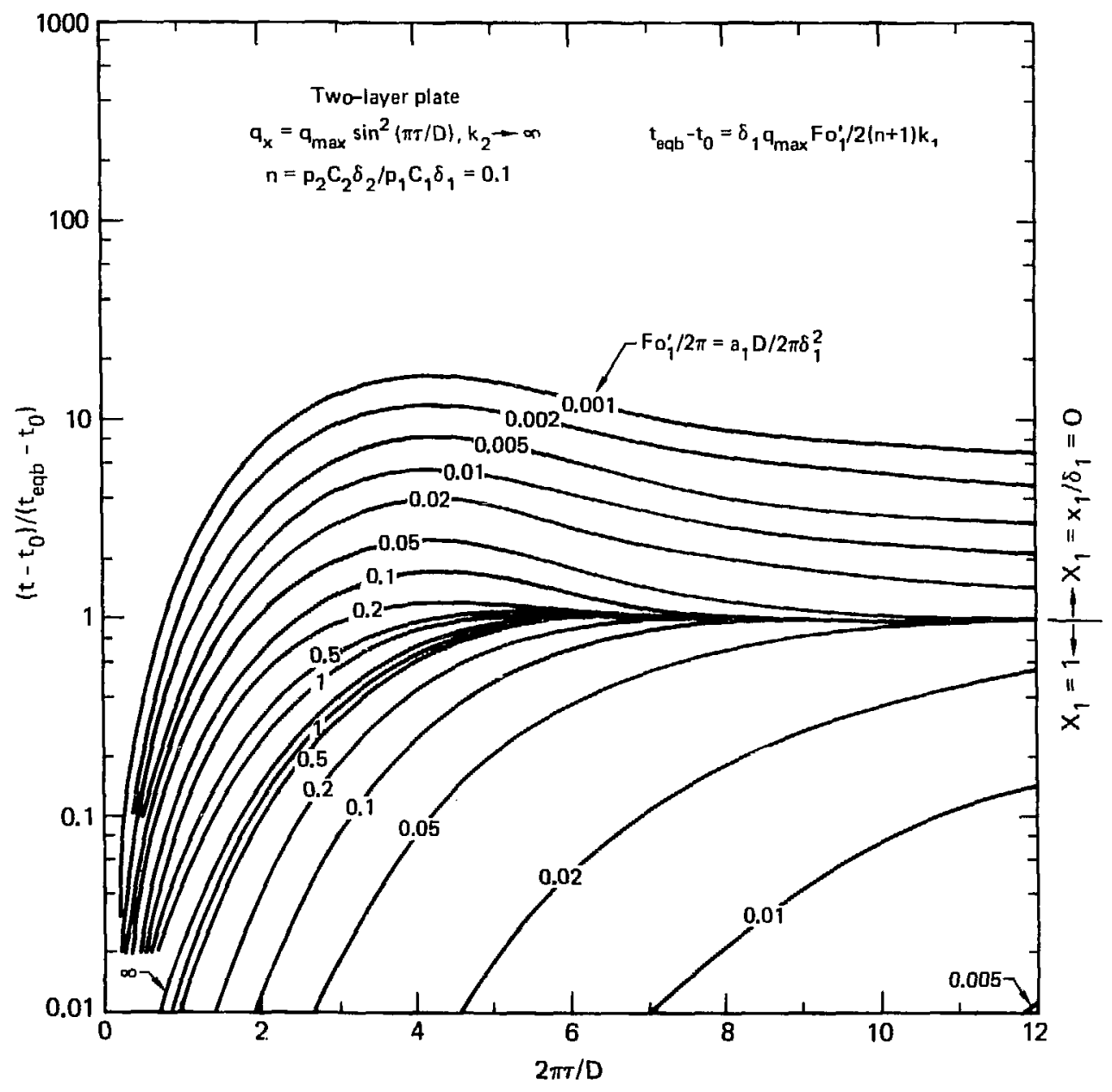

(a)

FIG. 8.23. Temperature response of an infinite plate exposed to a circular heat pulse at $x^{1}=0$ and in perfect contact with an infinite oonductivity plate of thickness $\delta_{2}$ (case 8.1.58, source: Ref. 19, pp. 3-78, Fig. 47). 


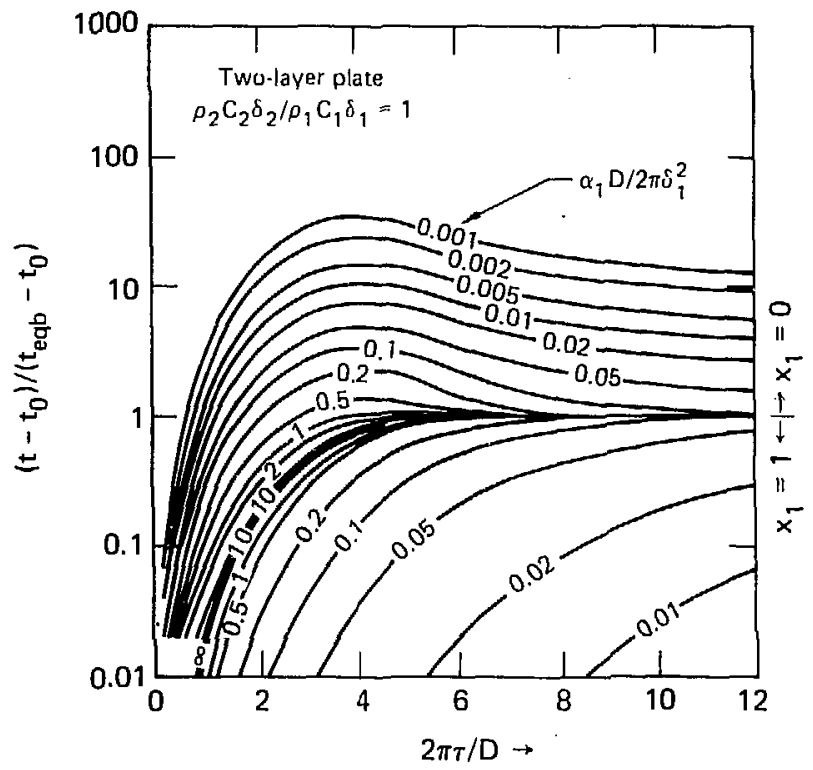

(b)

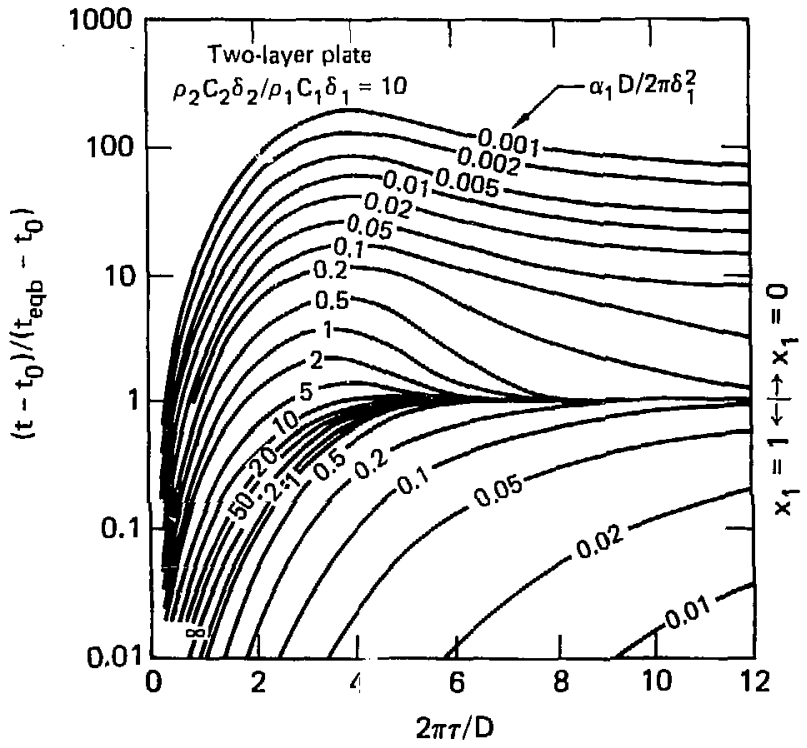

(c)

FIG. 8.23. (continued). 


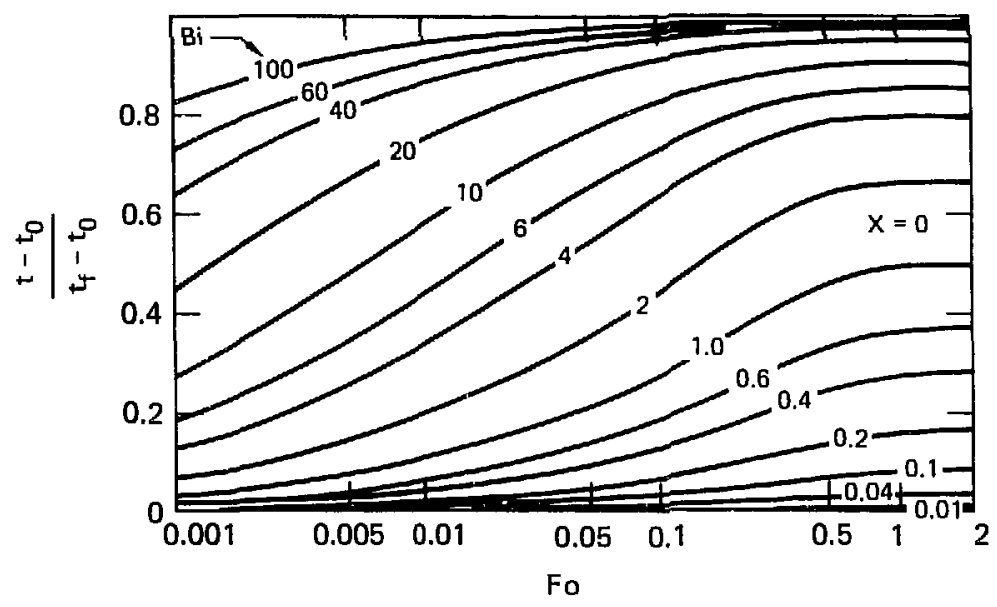

FIG. 8.24. Temperature response of an infinite plate surface having steady temperature and convection boundarles (case 8.1.25.2, source: Ref. 74, p. 240, Fig. 6.12).

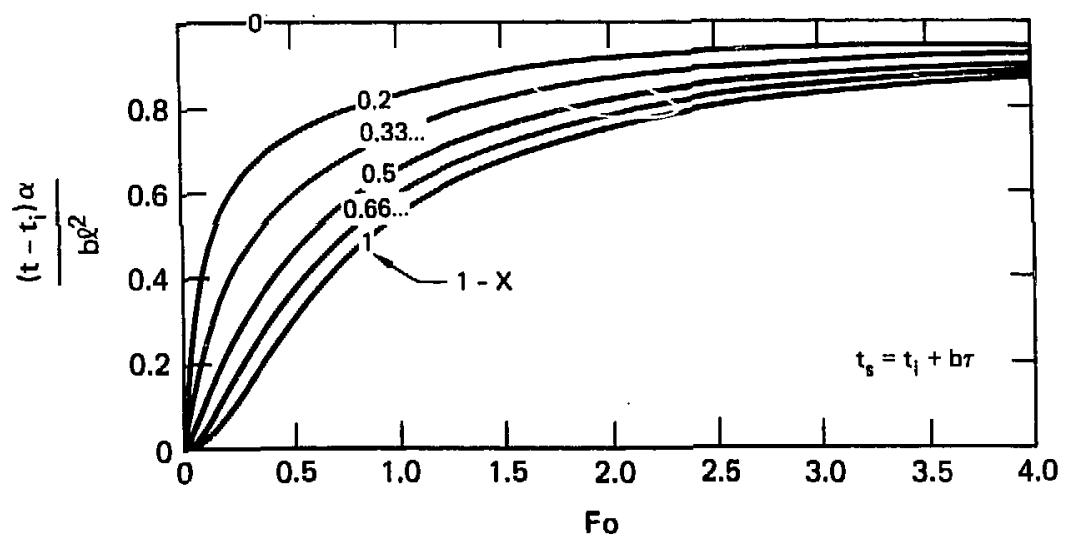

FIG. 8.25. Temperature of a flat plate having a ramp surface temperature equal to $t_{B}=t_{1}+$ bi (case 8.1.33, source: Ref. 74, p. 305, Fig. 7.1). 


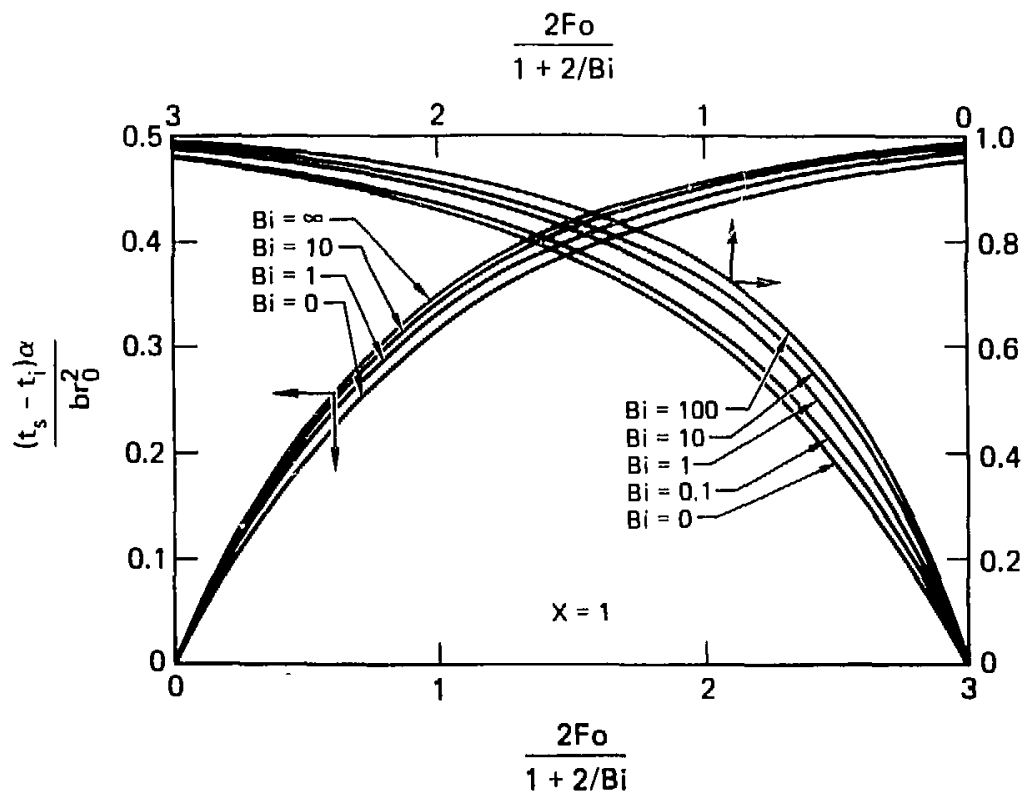

FIG. 8.26. Transient surface temperature of a slab convectively coupled to a linearly changing environment temperature equal to $t_{E}=t_{1}+b \tau$ (case 8.1.33, sour ce: Ref. 89). 
1

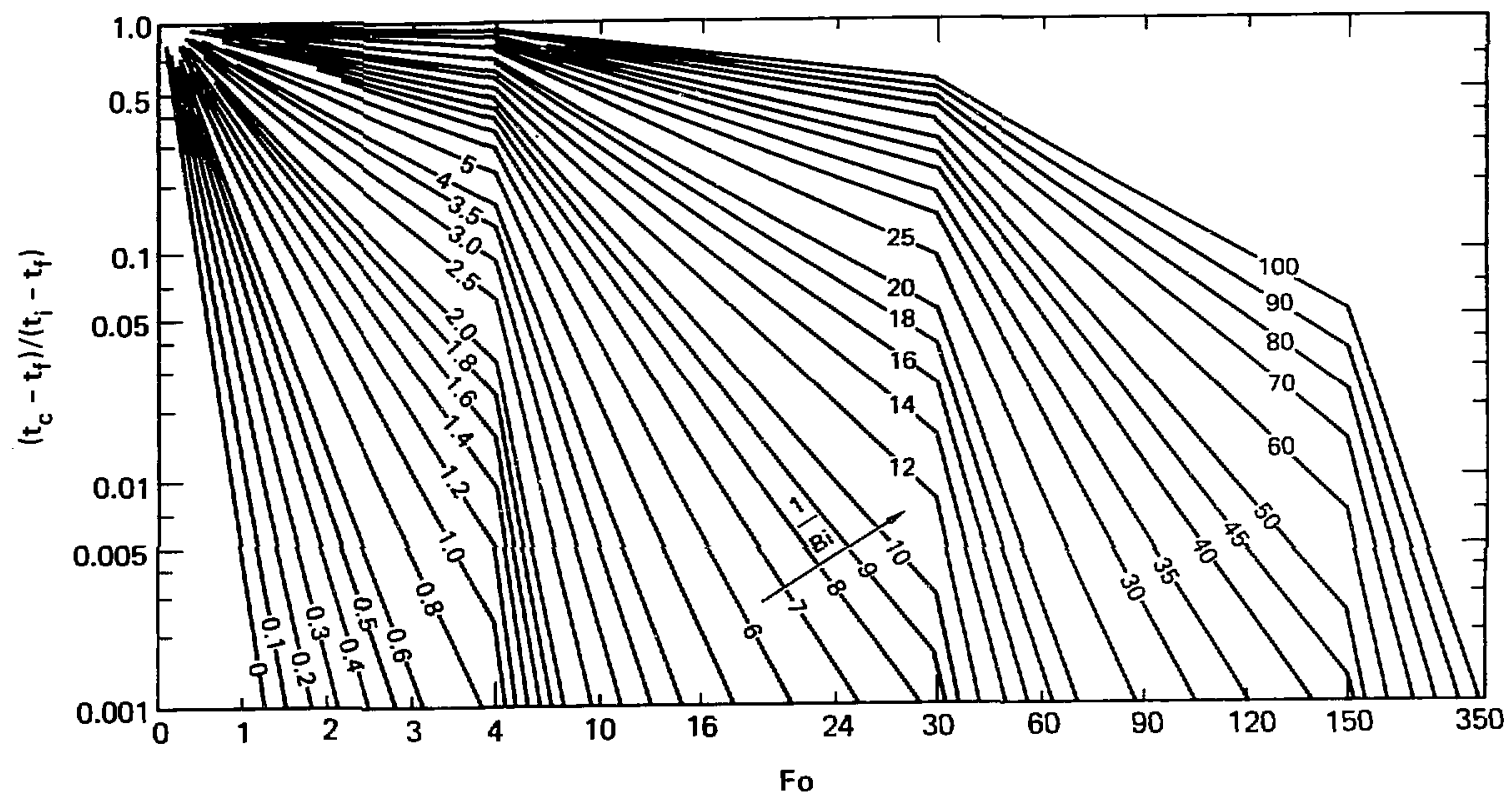

FIG. 9.1a. Axis temperature for an infinite cylinder of radius $r_{0}$ (case 9.1.3, source: Ref. 5, p. 84, Fig. 4-8 and Ref. 12). 


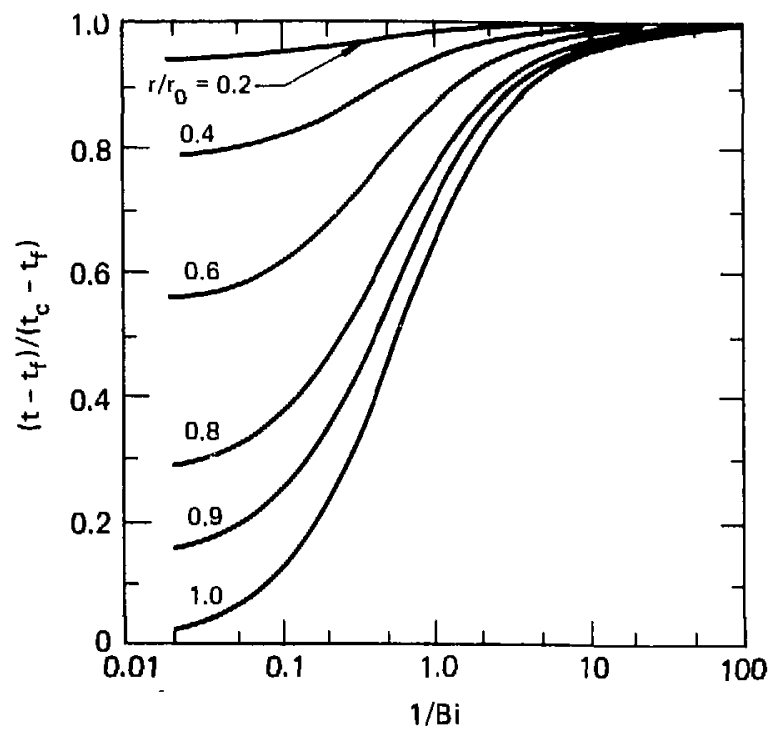

FIG. 9.1b. Temperature as a finction of axis temperature in an infinite cylinder of radius $r_{0}$ (case 9.1.3, source: Ref. 5, p. 87 and Ref. 12).

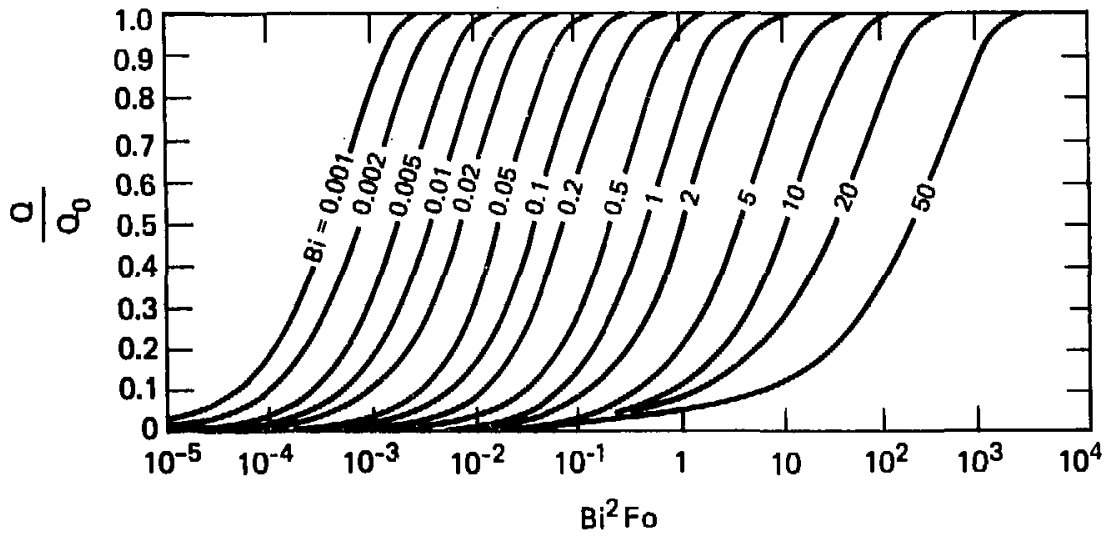

FIG. 9.1c. Dimensionless heat $1098, Q / Q_{0}$ of an infinite cylinder of radius $r_{0}$ with time (case 9.1.3, source: Ref, 5, p. 90, Fig. 4-15 and Ref. 13). 


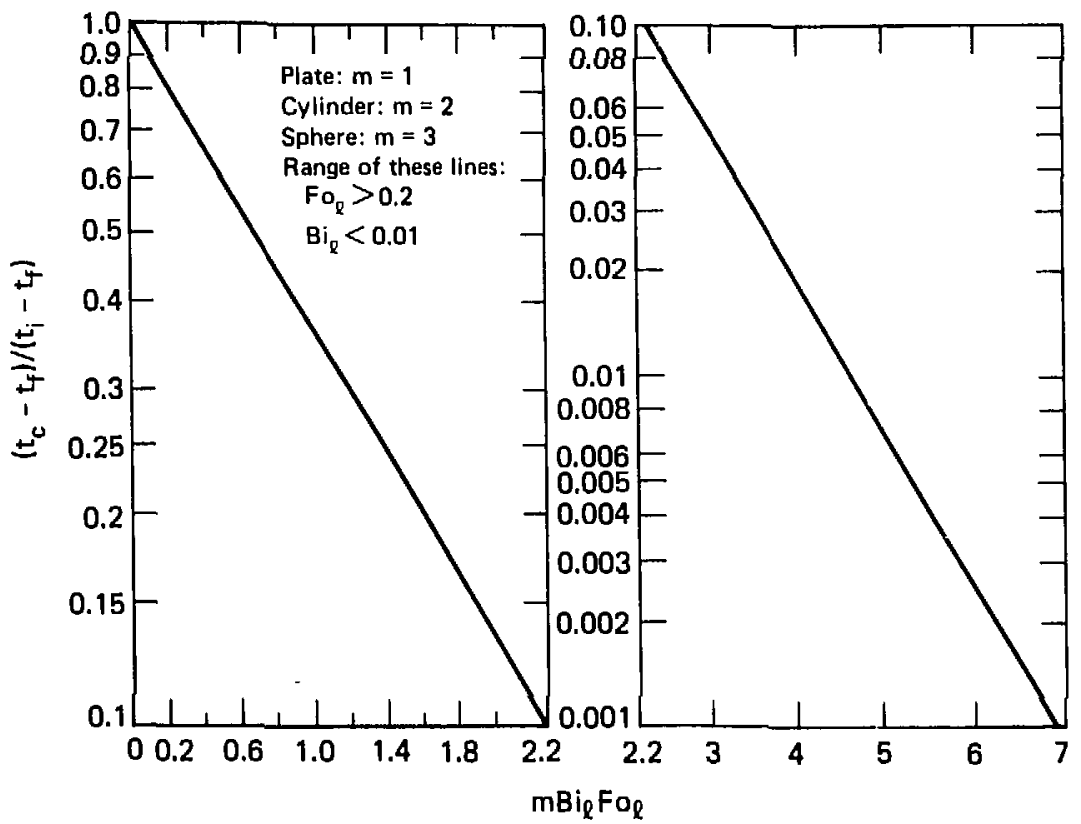

FIG. 9.1d. Center temperatures for plates, cylinders, and spheres for small values of $h$ lcase 9.1.3, 8.1.7 and 10.1., source: Ref. 5, p. 89, Fig. 4-13 and Ref. 12), $\ell$ is half thickness of radius.

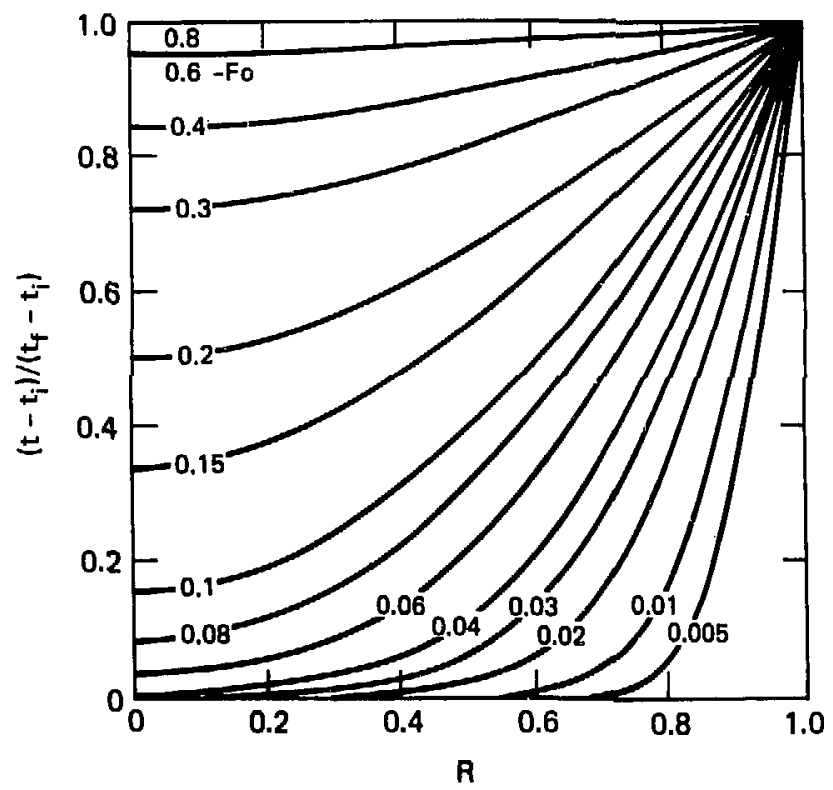

FIG. 9.2. Temperature distribution in an infinite cylinder with initial temperature $t_{1}$ and ateady surface temperature $t_{0}$ (case 9.1.1, source: Ref. 9, p. 200, Fig. 24). 


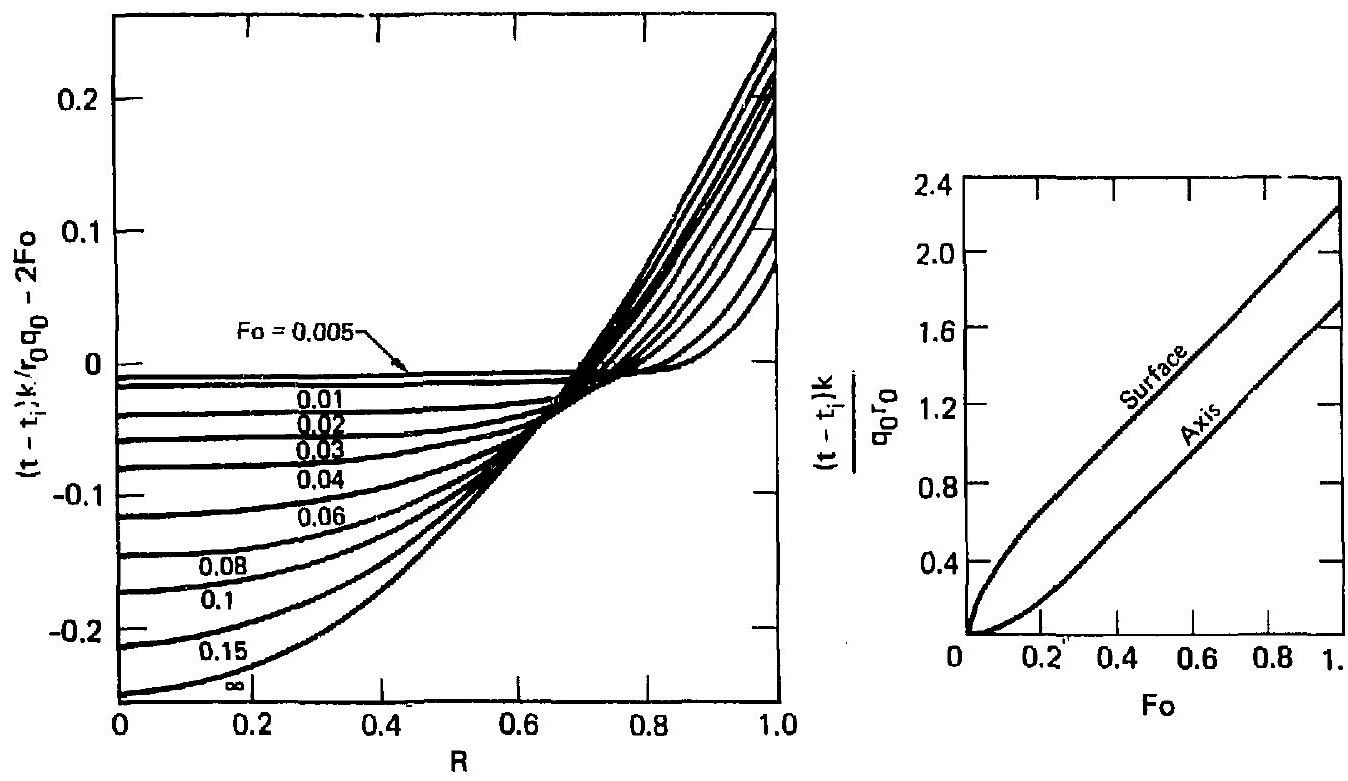

FIG. 9.3. Temperature in an Infinite cylinder with constant heat flux at the surface (case 9.1.8, source: Ref. 9, p. 203, Fig. 25). 


\begin{tabular}{|c|c|c|}
\hline sisfly & Quarter-infinite solid & Eighth-infinite solid \\
\hline Infinite plate & Semi-infinite plate & Quarter-infinite plate \\
\hline Infinite rectangular bar & Semi-infinite rectangular bar & Rectangular parallelepiped \\
\hline Infinite cylinder & Semi-infinite cylinder & Short cylinder \\
\hline \multicolumn{3}{|c|}{$\begin{array}{l}\mathrm{Fo}_{1}=a \tau / \delta_{1}^{2}=a \tau / \mathrm{r}_{1}^{2} \\
\mathrm{X}_{1}=\mathrm{x}_{1} / 2 \sqrt{a \tau}=\mathrm{Fo}_{\mathrm{x}}^{*} \\
\mathrm{~T}=\left(\mathrm{t}-\mathrm{t}_{\mathrm{w}}\right) /\left(\mathrm{t}_{0}-\mathrm{t}_{\mathrm{w}}\right)\end{array}$} \\
\hline
\end{tabular}

FIG. 9.4a. Product solutions for internal and central temperatures in solids with step change in surface temperature. $S(X)$ given in Fig. $7.2, P(F o)$ given in Fig. 8.4, C(FO) given in Fig. 9.1 (case 7.1.19, 7.1.20, 8.1.22, 9.1.16, and 9.1.17, source: Ref. 74, pp. 3-65, Fig. 38). 


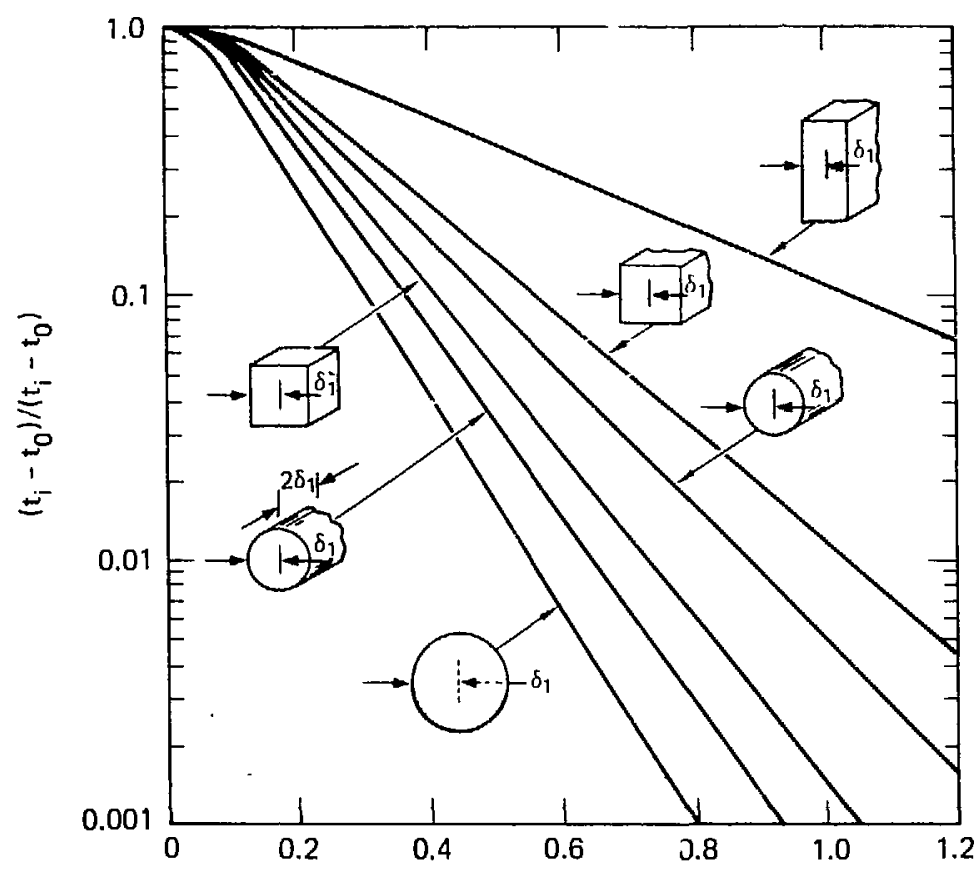

Fo, $\alpha \tau / \delta_{1}^{2}$

FIG. 9.4.b. Central temperaturss in an infinite plate, an infinite rod, an infinite cylinder, a cube, a sphere, and a finite cylinder of length equal to its diameter, with all surfaces at temperature $t_{0}$ (case 7.1.29, 7.1.20, 9.1.16, 9.1.17, and 8.1.22, source: Ref. 2, p. 248, Fig. 10-8).

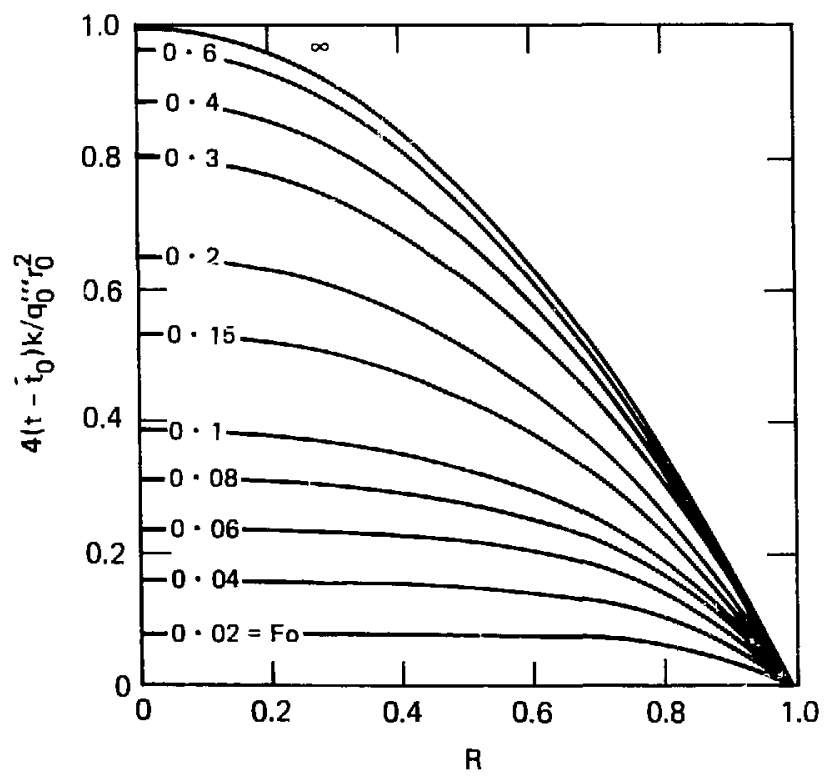

FIG. 9.5. Temperature distribution in an infinite cylinder with steady internal heating and a surface temperature $t_{0}$ (case 9.2.1, source: Ref. 9, p. 205, Fig. 26). 


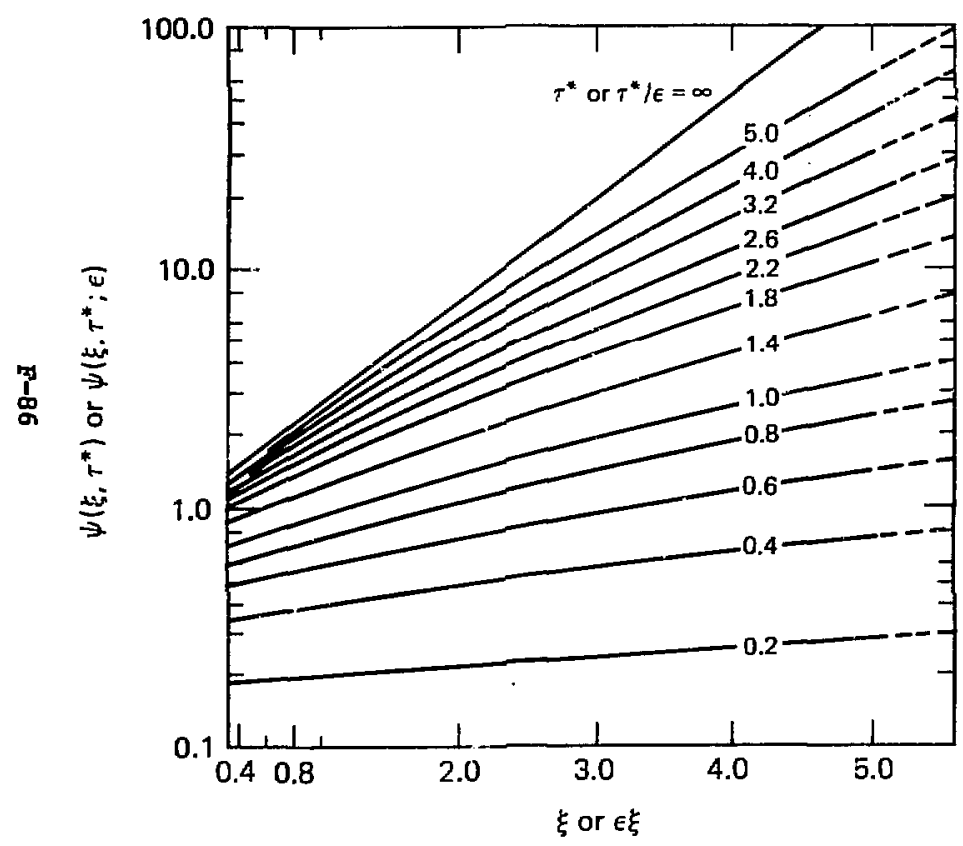

FIG. 9.6a. (case 9.2.4, source: Ref. 3, p. 356 , Fig. 7-5) .

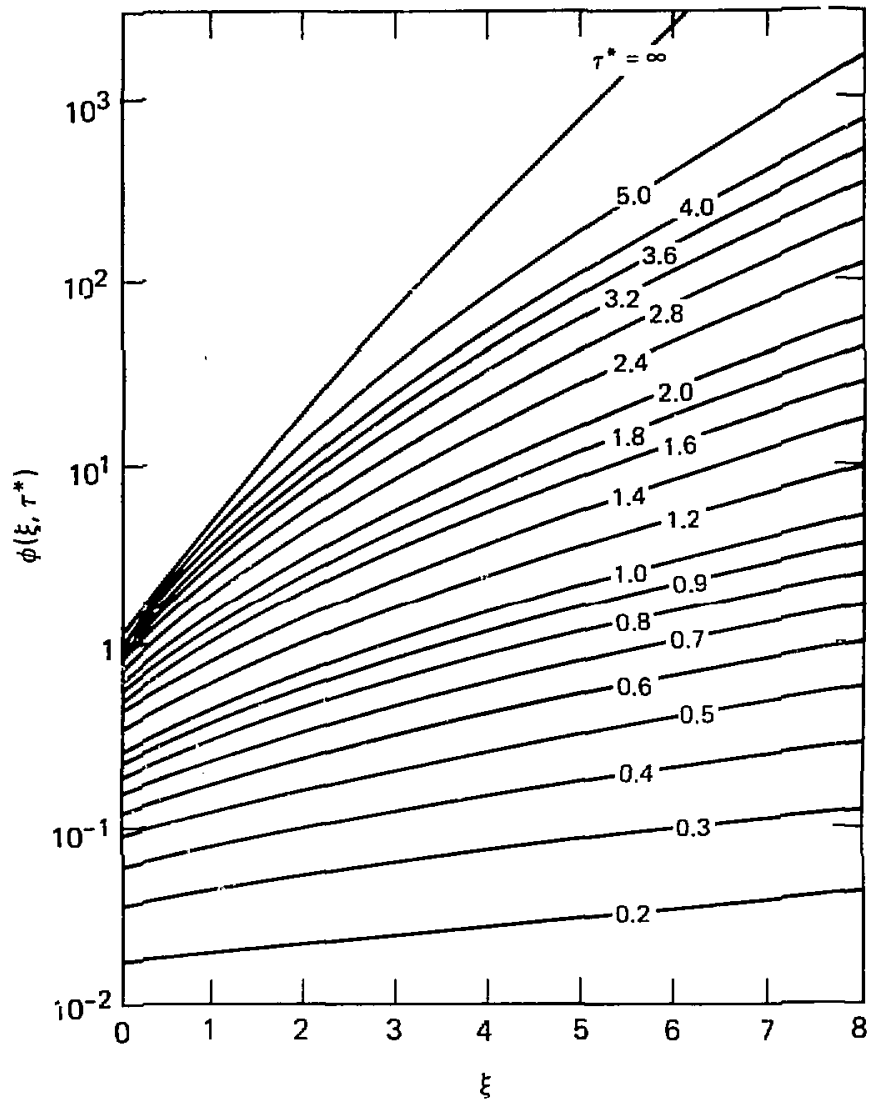

FIG. 9.6b. (case 9.2.4, source: Ref. 3, p. 364, Fig. $7-8)$. 


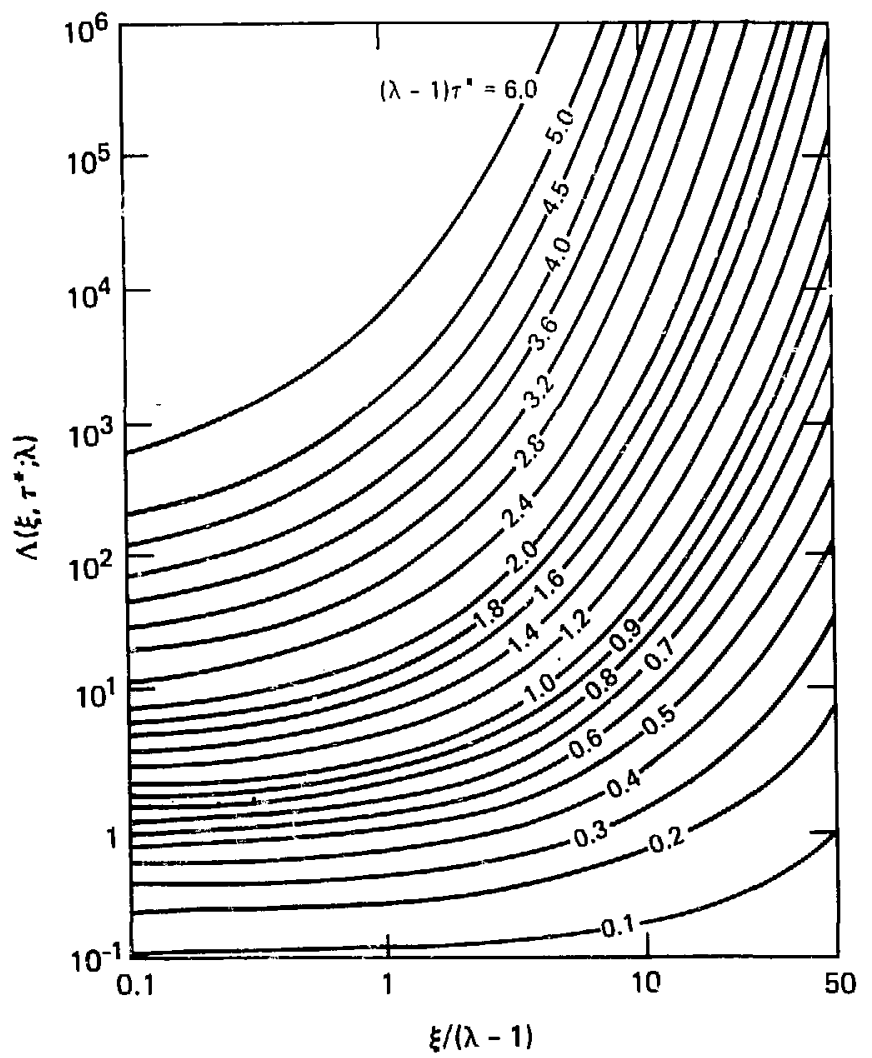

FIG. S.6c. (case 9.2.4, source: Ref. 3, p. 365, Fig. 7-9). 


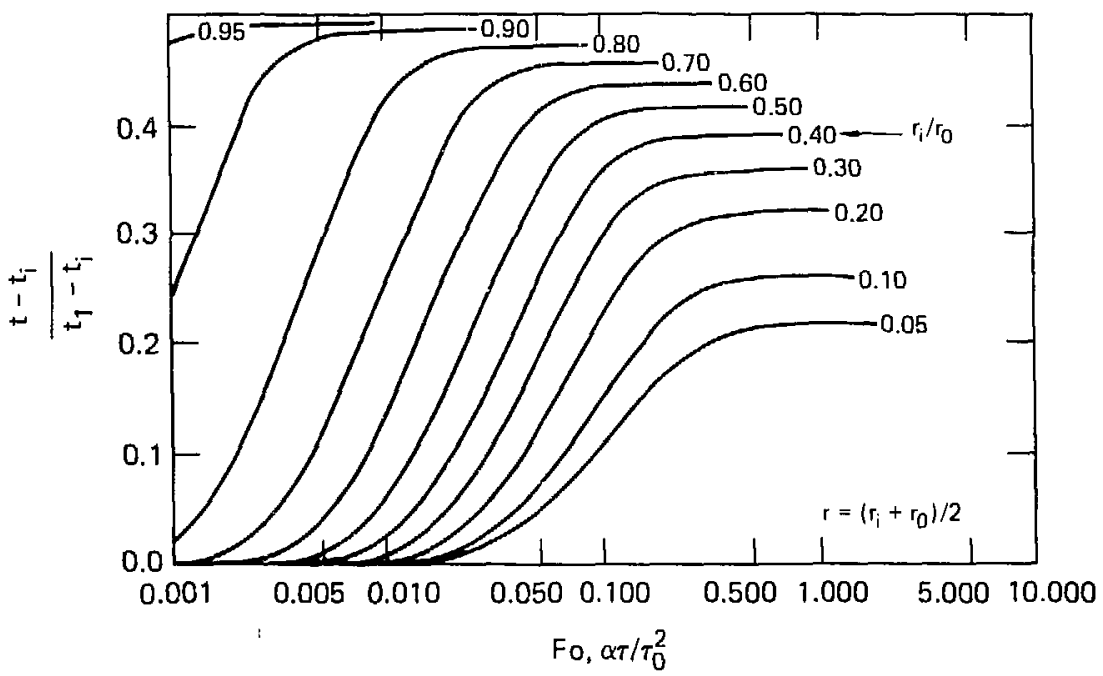

FIG. 9.7a. Temperature response of a hollow cylinder with a steady inside surface temperature equal to the initial temperature, $t_{i}$ (case 9.1.10, source: Ref. 74, p. 156, Fig. 4.24).

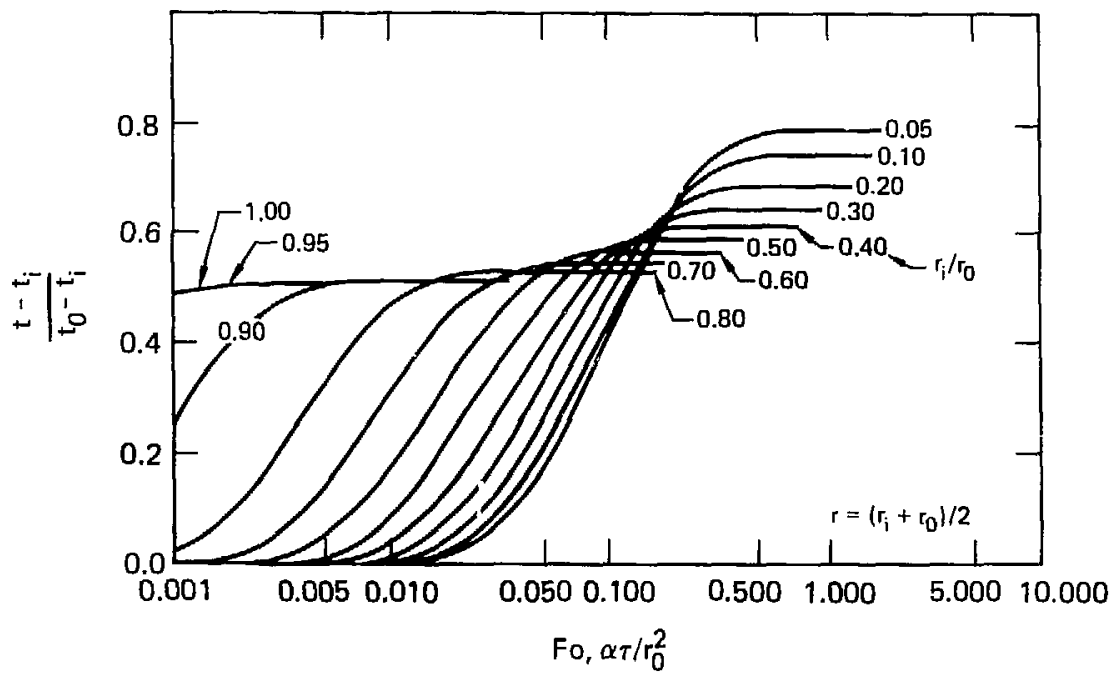

FIG. 9.7b. Temperature response of a hollow cylinder with a steady outside surface equal to the initial temperature, $t_{i}$ (case 9.1.10, source: Ref. 74, p. 157, Fig. 4.25). 


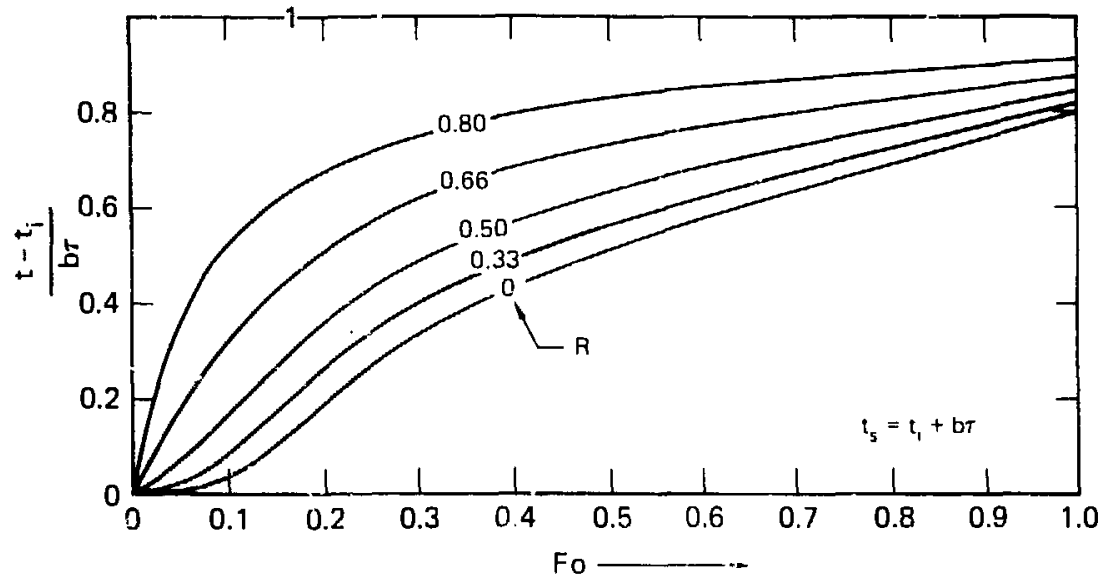

FIG. 9.8. Temperature of an infinite oylinder having a ramp surface temperature equal to $t_{s}=t_{i}+b \pi$ icase 9.1.2, source: Ref. 74, p. 312, Fig. 7.3).

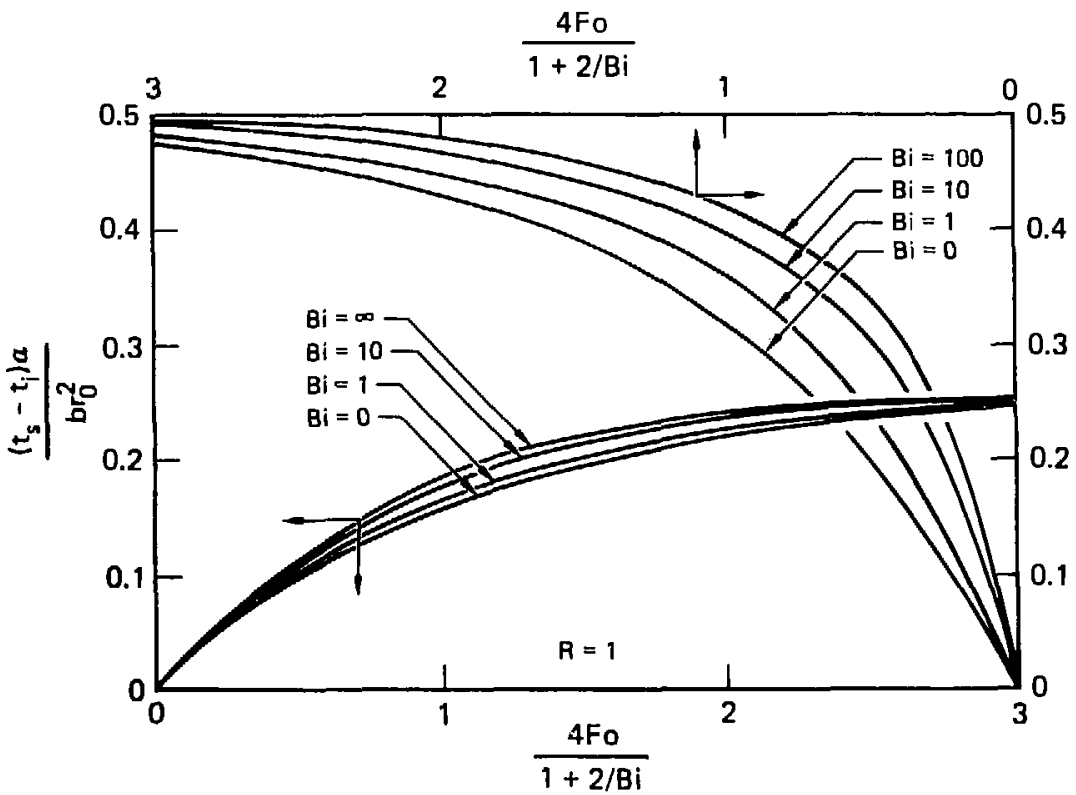

FIG. 9.9. Transient surface temperature of a cylinder convectively coupled to a linearly changing envi ronment temperature equal to $t_{f}=t_{i}+b \pi$ (case 9.1.6, source: Ref. 89). 


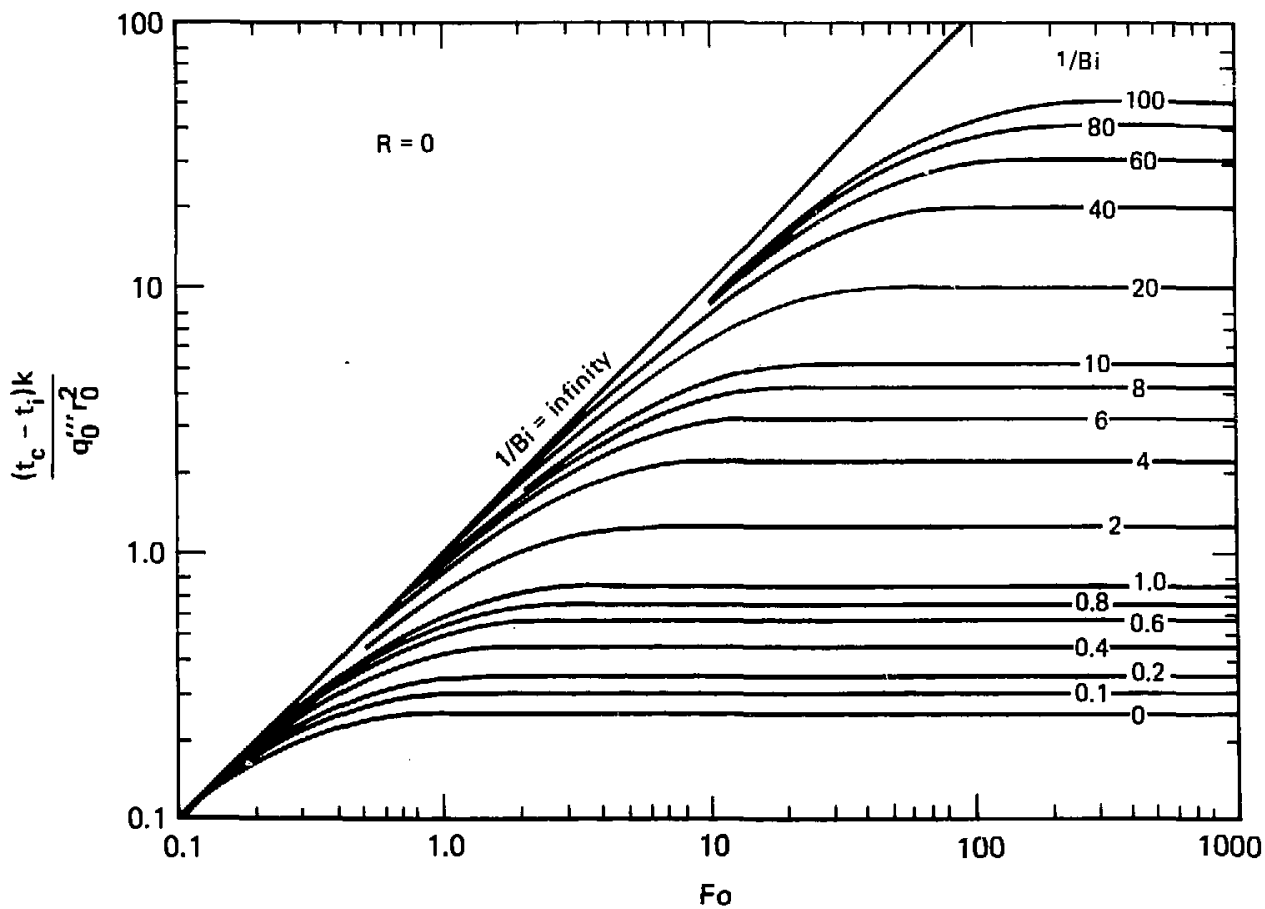

FIG. 9.10. Central temperature of an infinite cylinder with uniform internal heating and convection boundary (case 9.2.2, source: Ref. 90). 

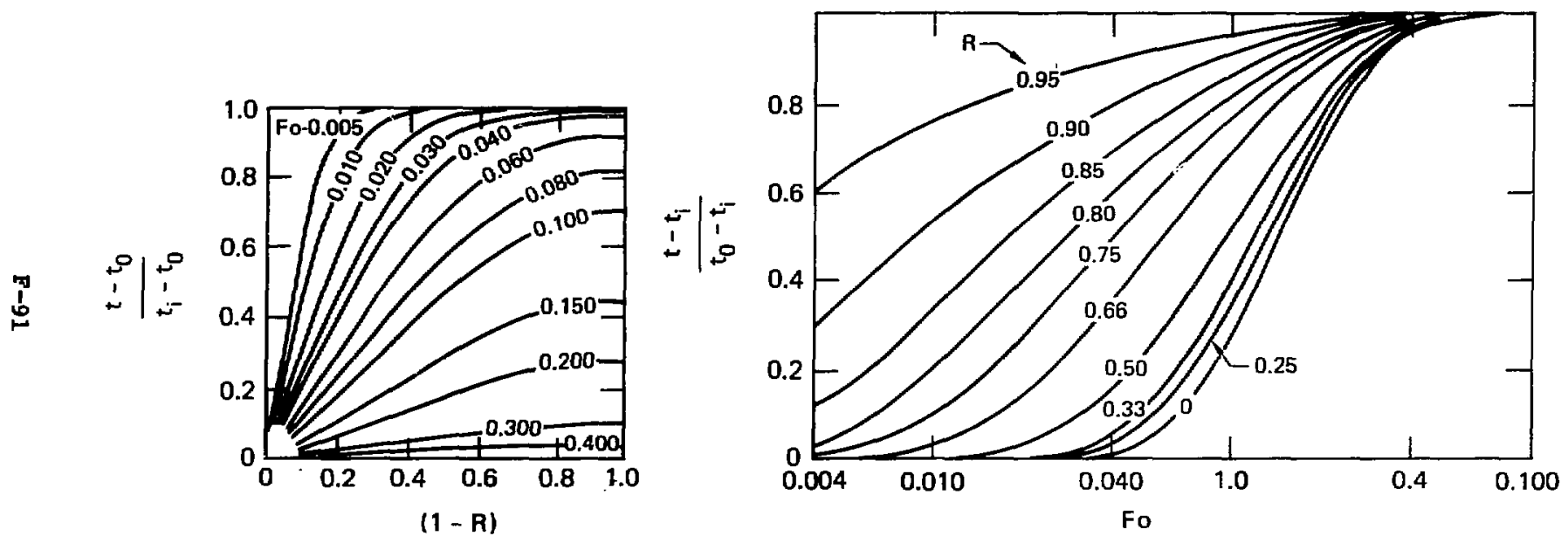

FIG. 10.1. Temperature distribution in a sphere of radius $r_{0}$ with initial temperature $t_{i}$ and surface temperature $t_{0}$ (case 10.1.1, source: Ref. 74, p. 127, Figs. 4.15 and 4.16). 


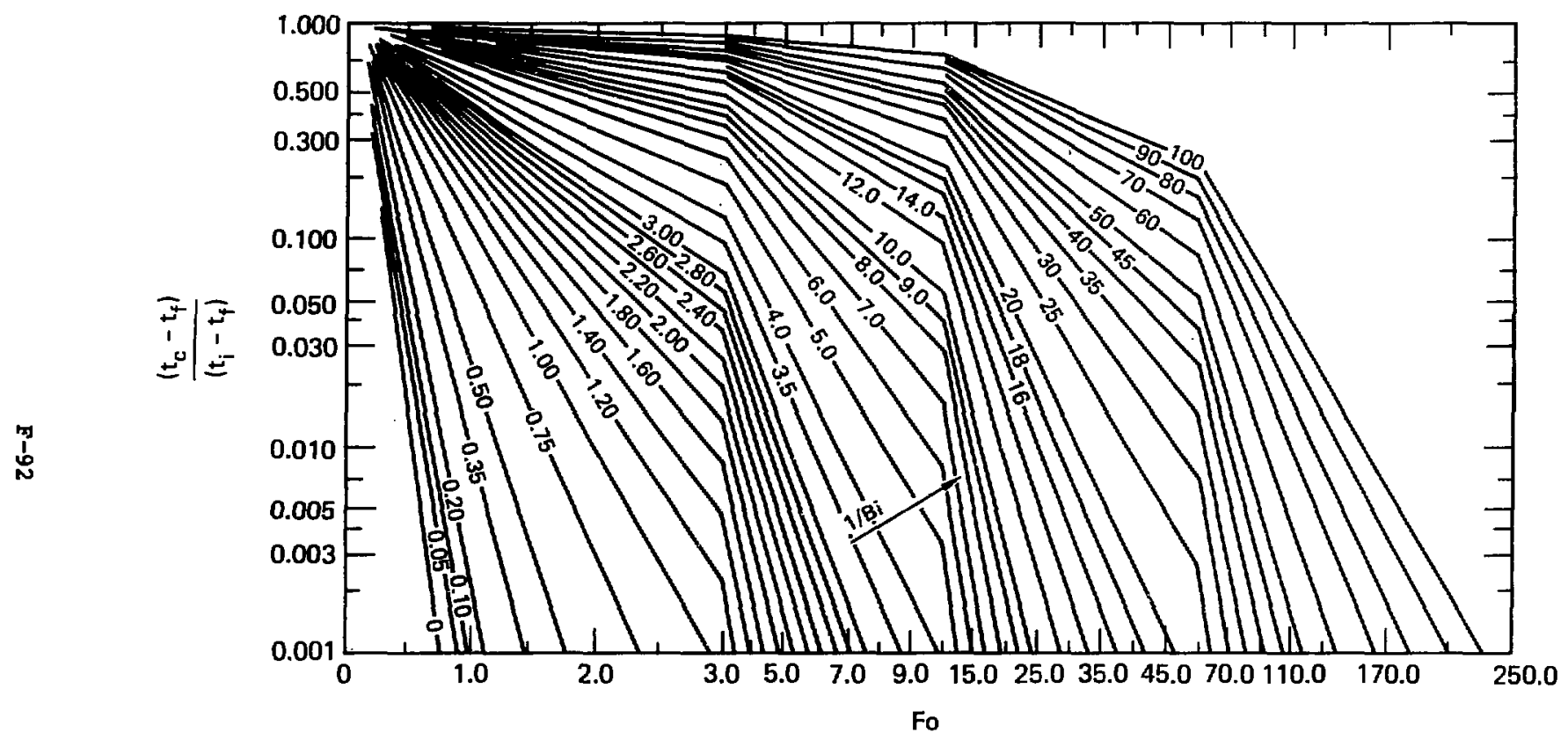

FIG. 10.2a. Center temperature of a convectively cooled sphere (case 10.1.2, source: Ref. 5, p. 85, Fig. 4-9 and source: Ref. 12). 


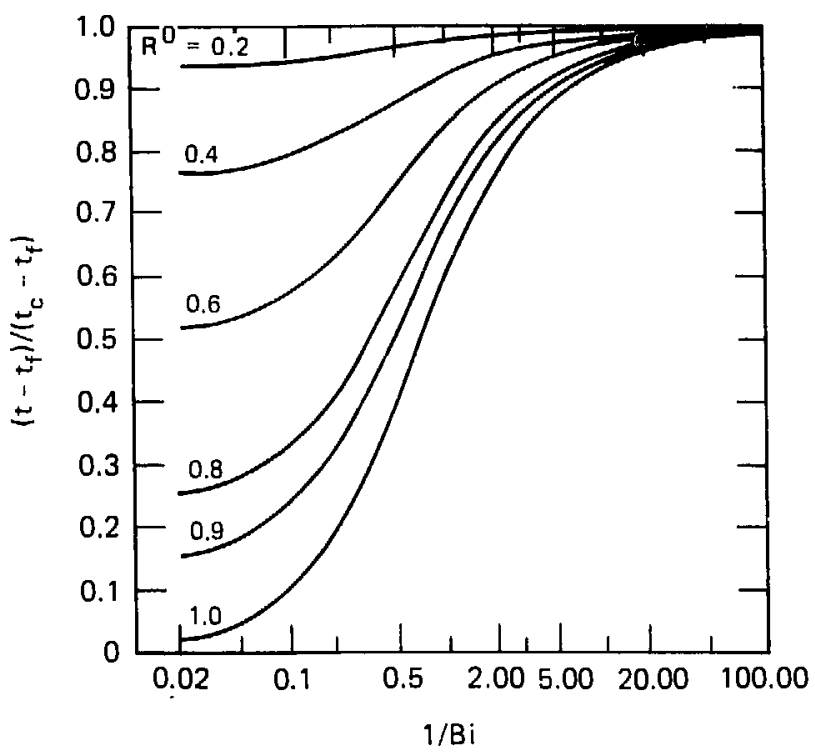

FIG. 10.2b. Temperature as a function of center temperature of a convectively cooled sphere (case 10.1.2, sour ce: Ref. 5, p. 88, Fig. 4-12, and Ref. 12).

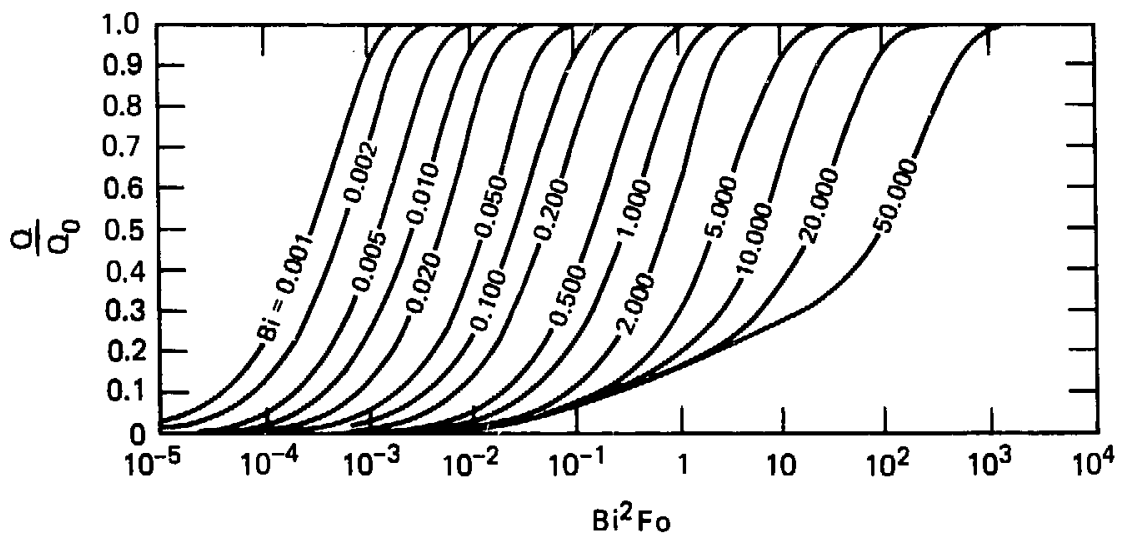

FIG. 10.2c. Dimensionless heat loss $Q / Q_{0}$ of a convectively cooled sphere (Case 10.1.2, source: Ref. 5, p. 91, Fig. 4-16, and Ref. 13). 


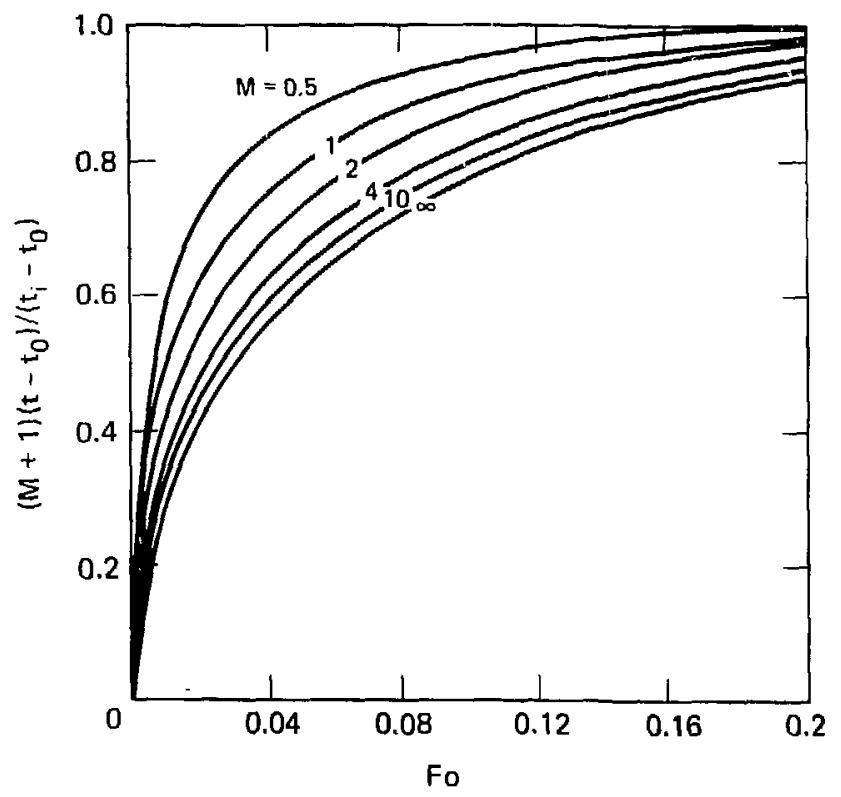

FIG. 10.3. Temperature of an infinite conductivity medium surrounding a spherical solid (case 10.1.13, source: Ref. 9, p. 241, Fig. 30).
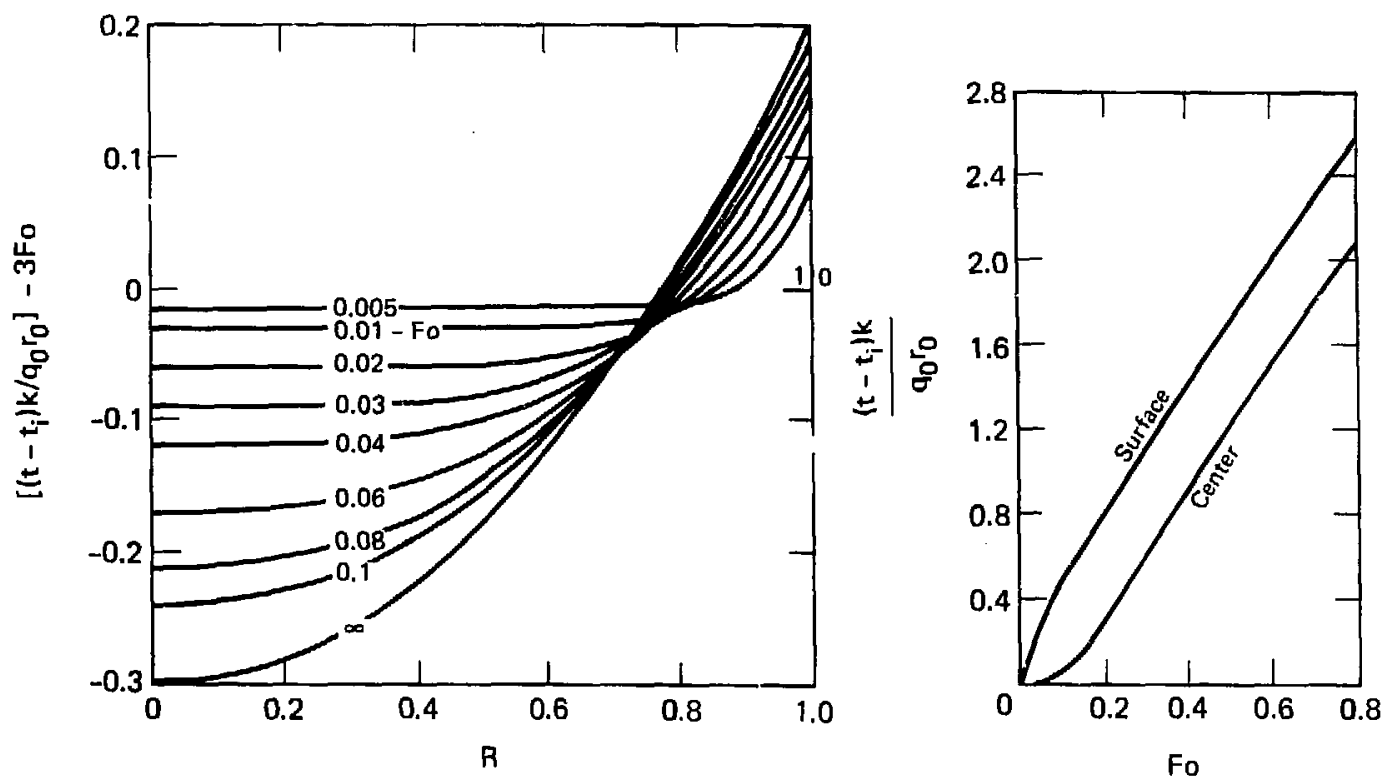

FIG. 10.4. Temperature in a sphere caused by a steady surface heat flux (case 10.1.15, source: Ref. 9, p. 242, Fig. 31). 


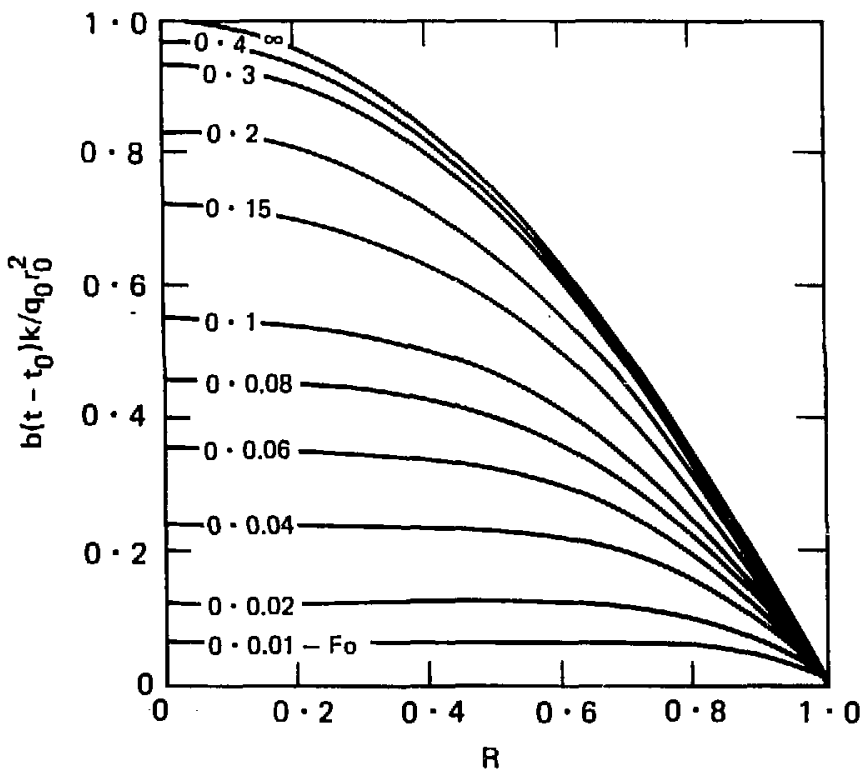

FIG. 10.5. Temperature distribution in a sphere with steady internal heating (case 10.2.1, source: Ref. 9, P. 244, Fig. 32). 

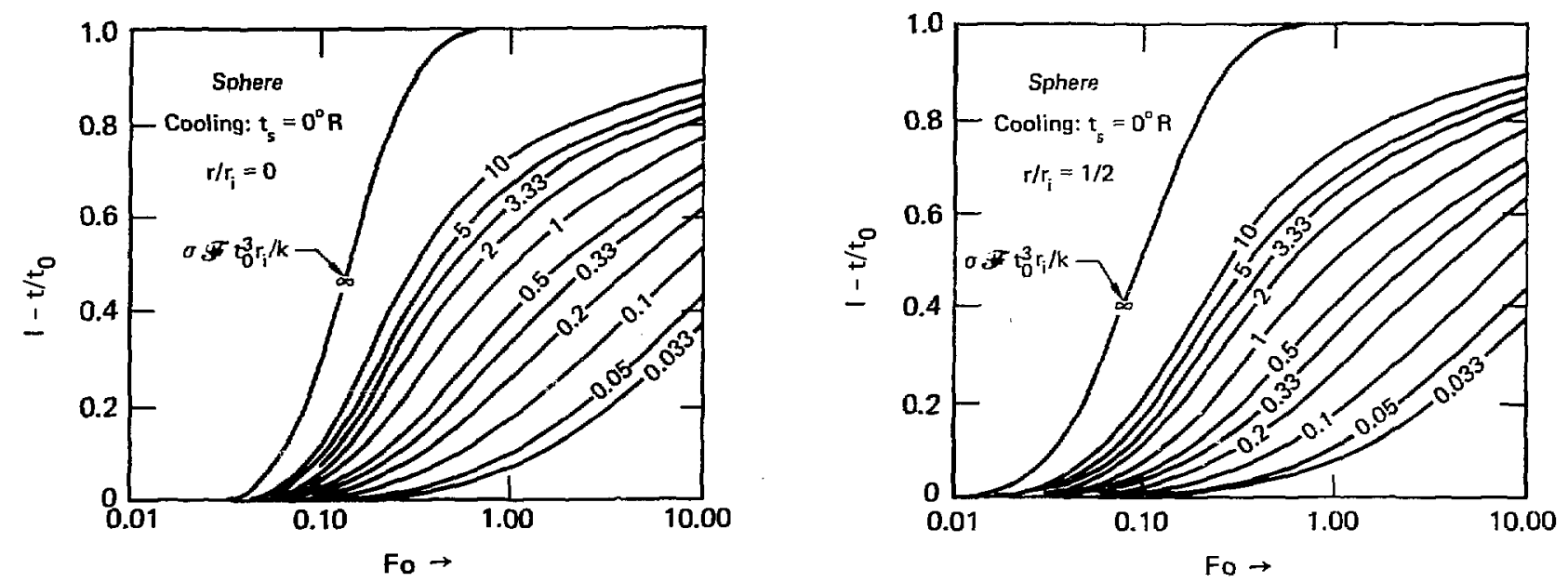

尔

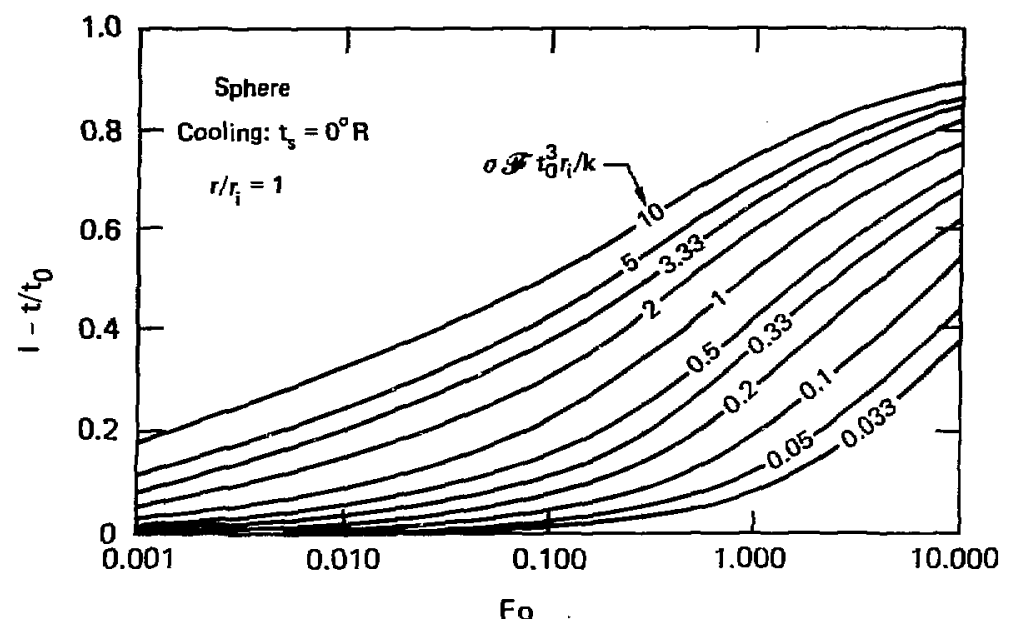

FIG. 10.6. Temperature response of solid sphere $\left(0 \leq r \leq r_{1}\right)$ cooling by radiation to a sink temperature of $0^{\circ}$ : (a) $r / r_{1}=0$, (b) $r / r_{1}=1 / 2$, (c) $r / r_{1}=1$ (case 10.1.20, source: Ref. 19, pp. 3-57, Fig. 30), 


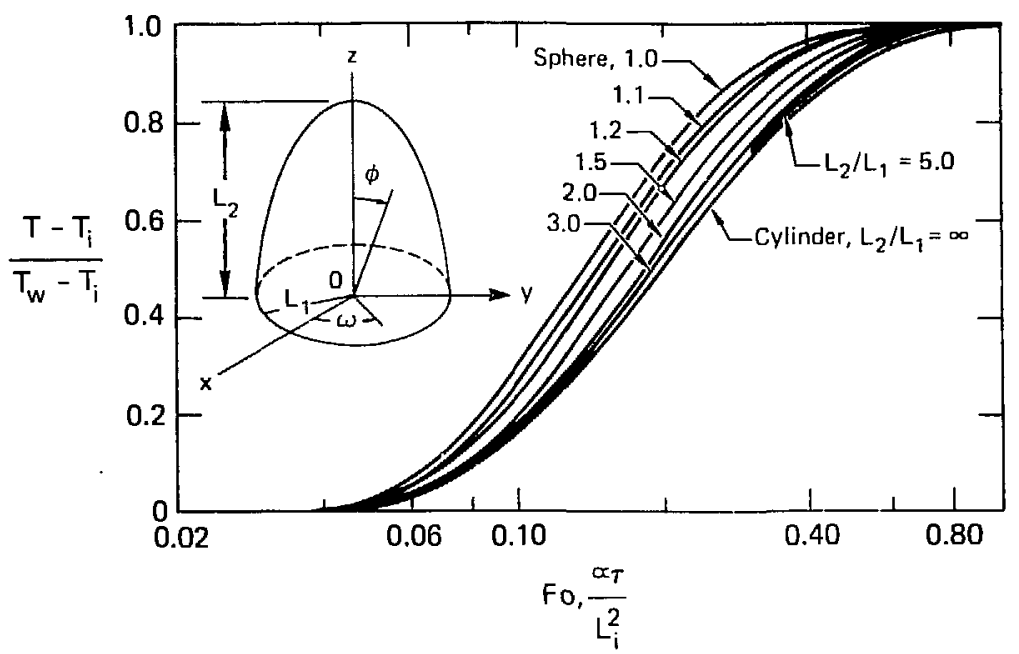

FIG. 10.7a. Transient temperature distribution at the center point $(x=y$ $=\mathbf{z}=0$ ) for various prolate spheroids (case 10.1.21, source: Raf. 72, Fig. 1).

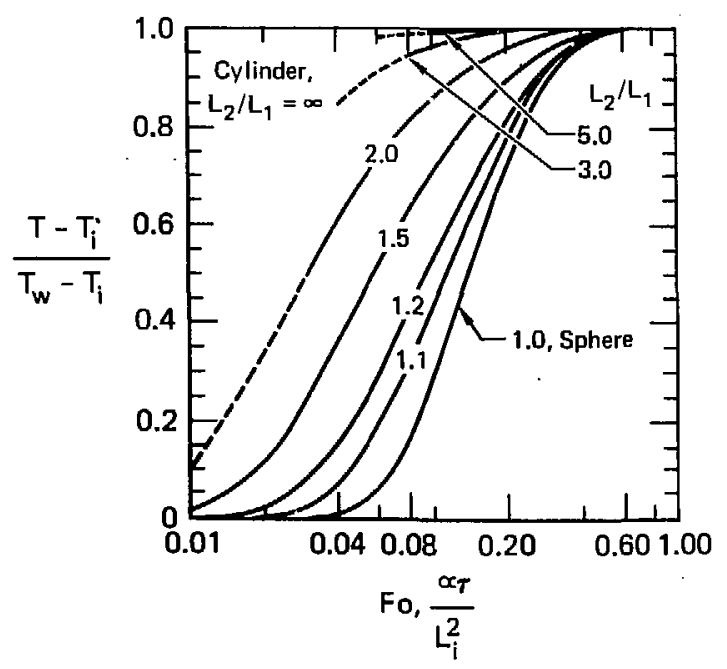

FIG. 10.7b. Transient temperature distribution at the focal point $(x=y=0$, $\mathrm{z}=\mathrm{I}_{2}$ ) for various prolate solidB (case 1.0.1.21, source: Ref, 72, Fig. 2). 


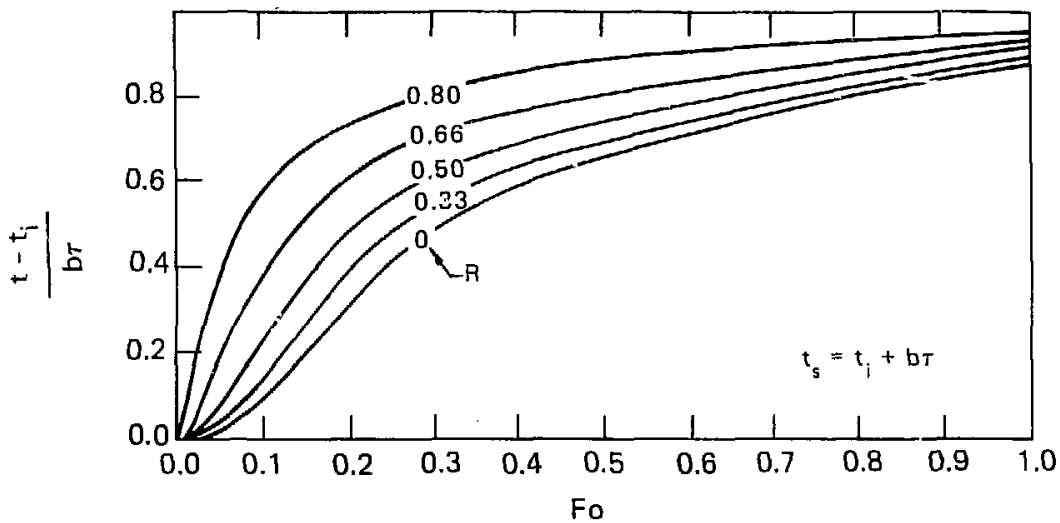

FIG. 10.8. Temperature of a sphere having a ramp surface tenperature equal to $t_{s}=t_{i}+b \tau$ (case 10.1.4, source: Ref. 74, p. 310, Fig. 7.. i.

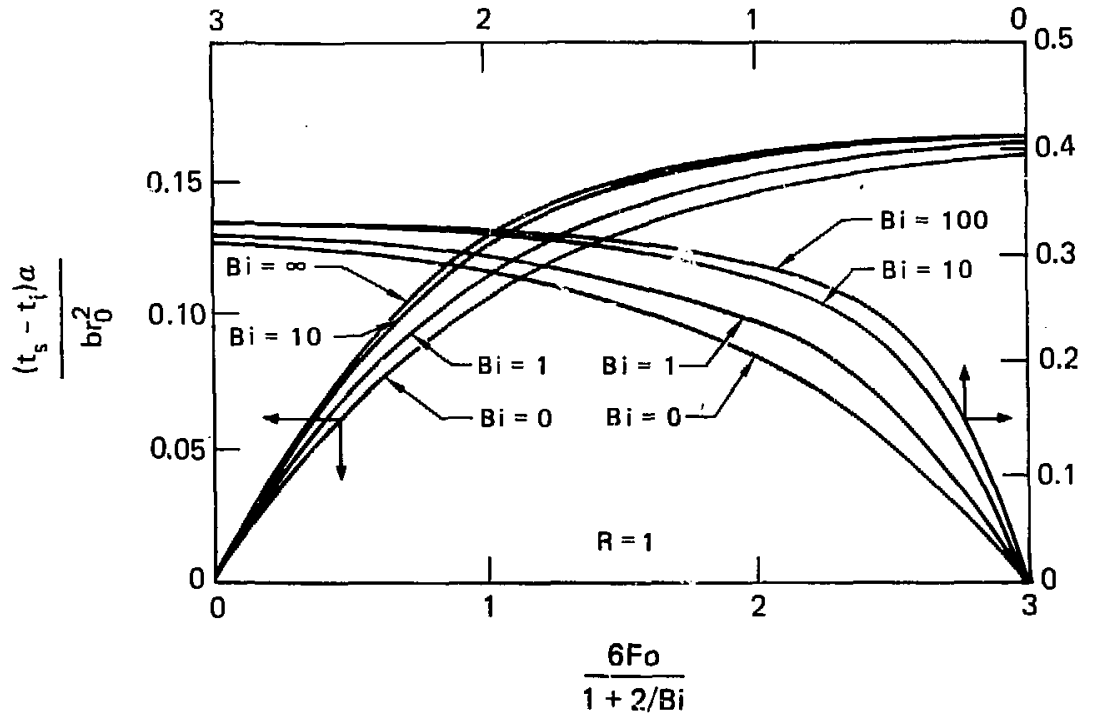

FIG. 10.9. Transient surface temperature of a sphere convectively coupled to a linearly changing environment temperature equal to $t_{f}=t_{1}+b \tau$ (case 10.1 .11 , source: Ref. 89 ). 


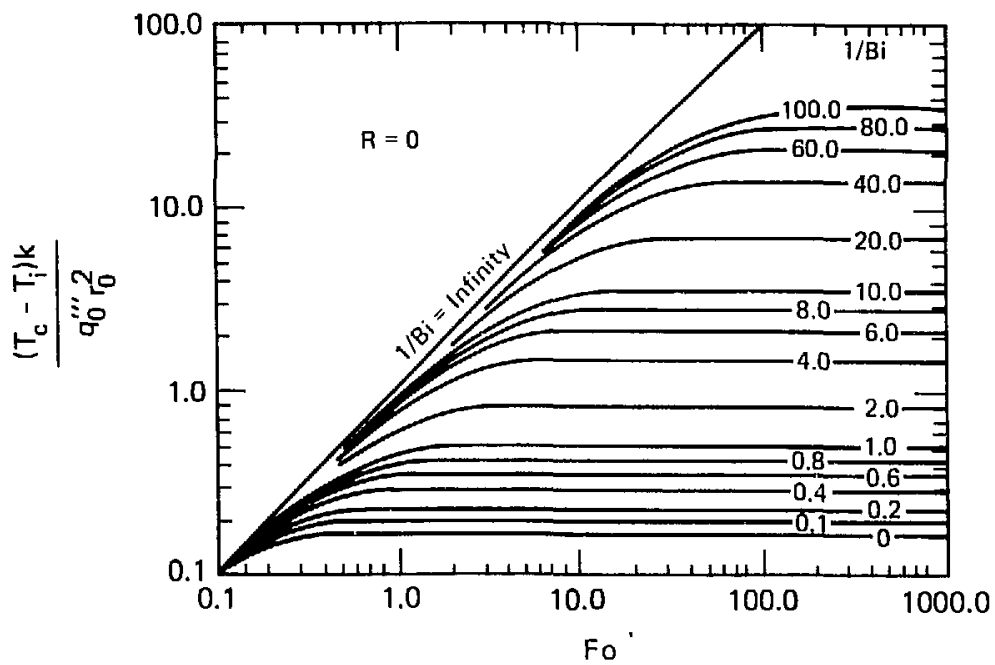

FIG. 10.10. Central temperature of a sphere with uniform internal heating and convection boundary (case 10.2.9, source: Ref. 90).

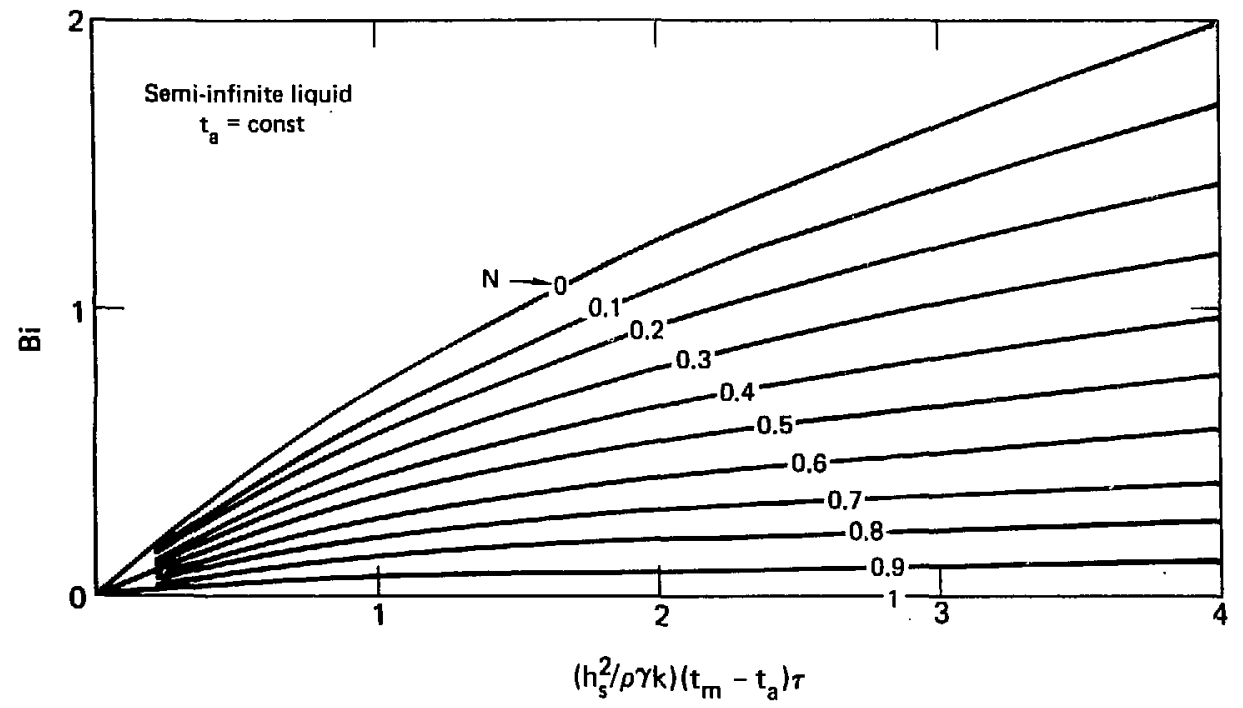

FIG. 11.1. Solidification depth in semi-infinite liquid for which solidified phase has negligible thermal capacity and is exposed to convective environments $t_{a}$ at free surface $x=0$ and $t_{0}$ at interface $x=w$ (case 11.1.10, source:

Ref. 19, pg. 3-88, Fig. 54). 

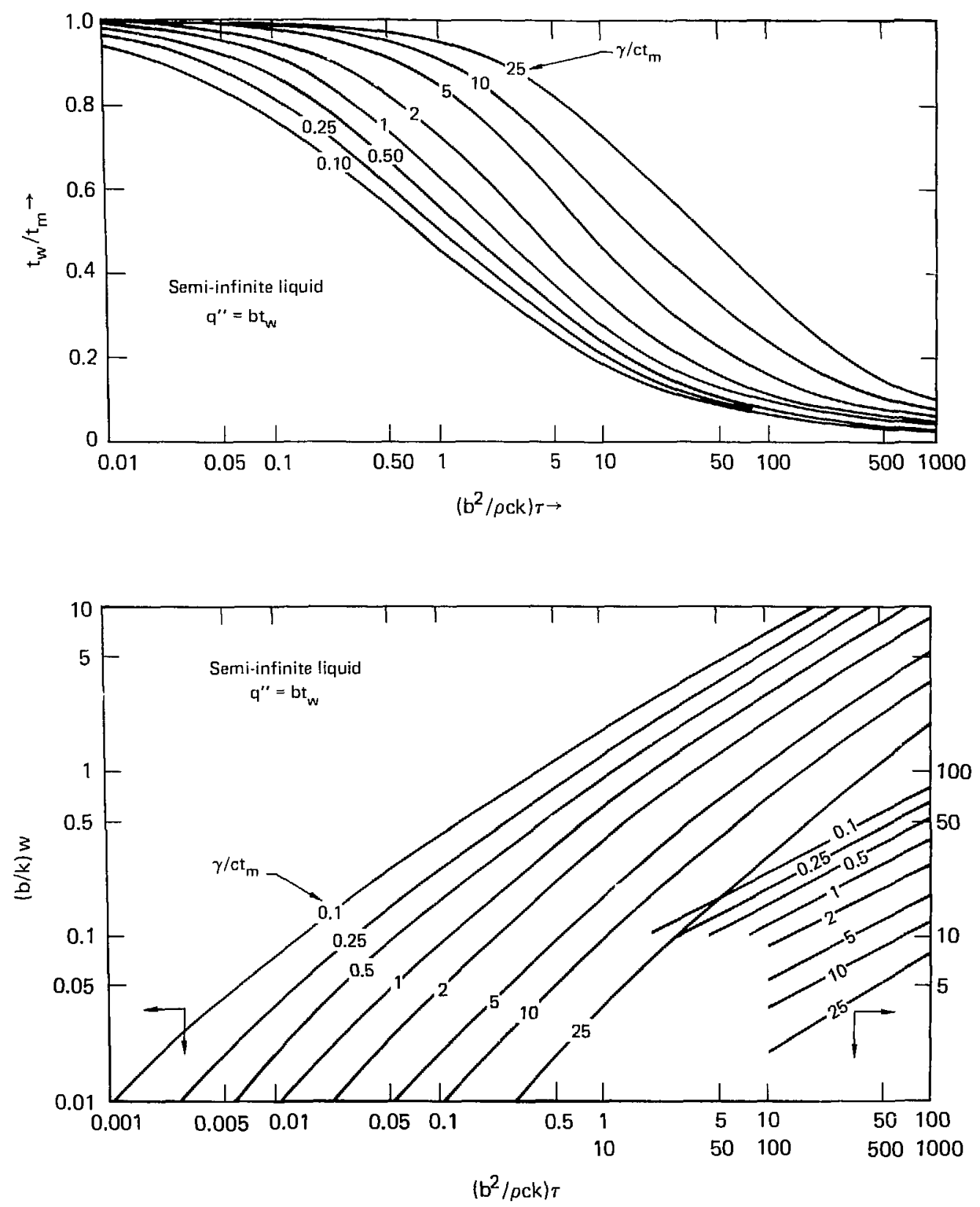

FIG. 11.2. Temperature response and solidification depth in semi-infinite liquid initially at $t_{m}$ (a) surface temperature for convective cooling $q=b t_{w}$ at $x=0$, (b) solidification depth (case 11.1.11, source: Ref. 19, pp. 3-89, Fig. 55). 

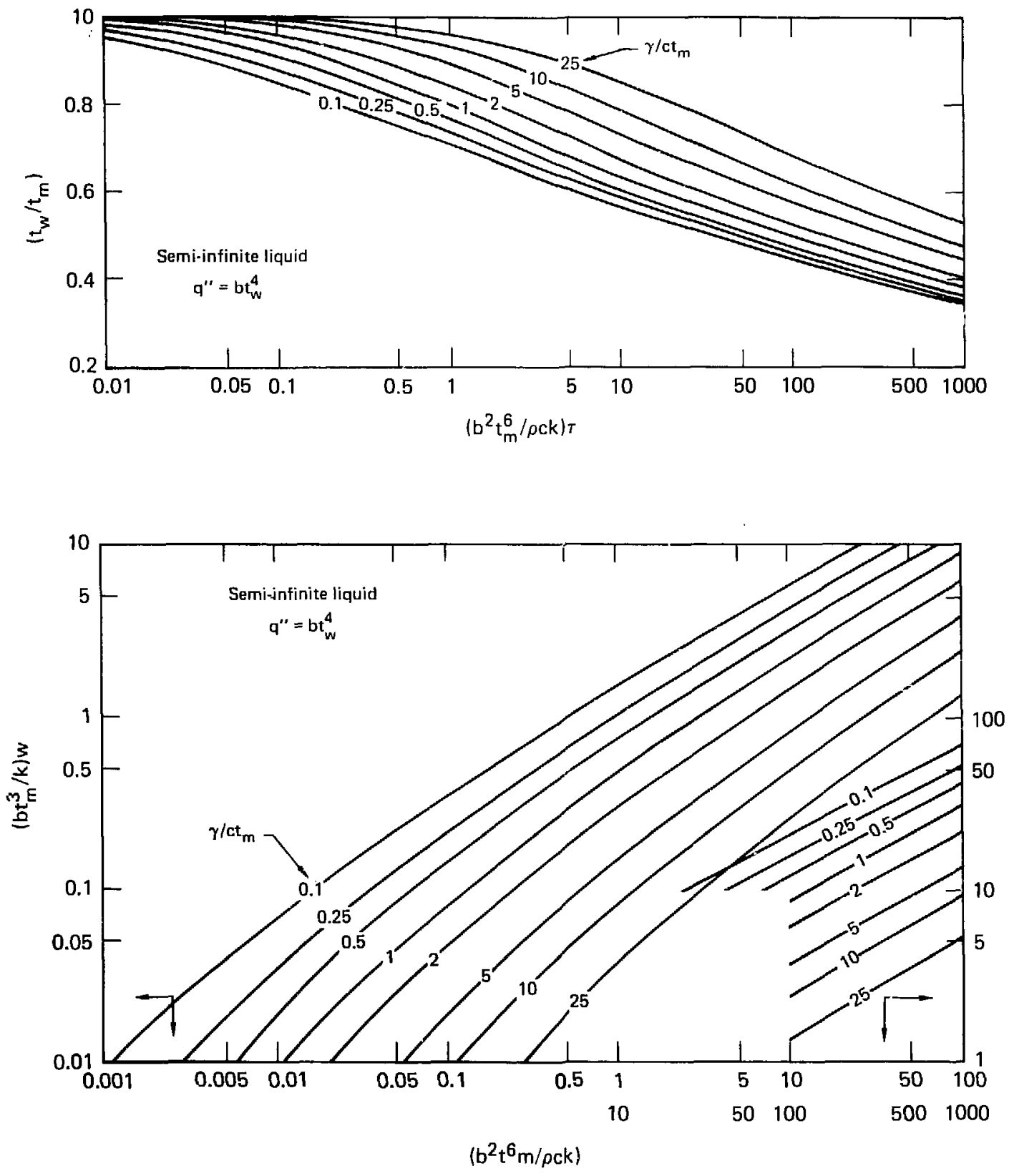

FIG. 11.3. Temperature response and solidification depth in semi-infinite liquid initially at $t_{\mathrm{T}}$ with radiative cooling $q=b t_{w}^{4}$ at $x=0$ (a) surface temperature at $x=0$, (b) solidification depth (case 11.1.12, source: Ref. 19, pp. 3-90, Fig. 55) . 

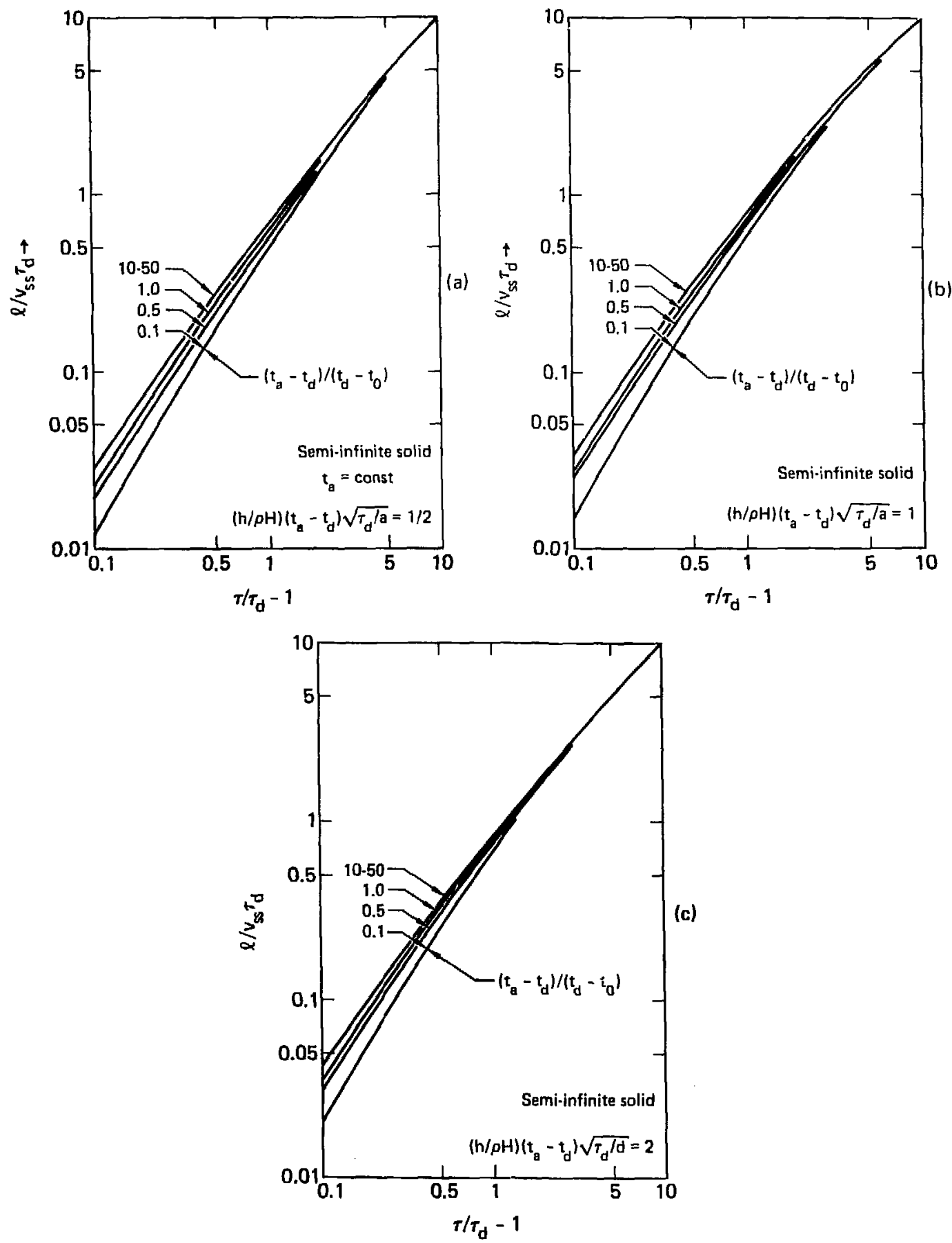

FIG. 11.5. Ablation depth of semi-infinite solid $(x \geq 0)$ after sudden exposure to convective environment $t_{a}\left(>t_{d}\right):$ (a) $(h / \overline{p H})\left(t_{a}-t_{d}\right)$ $\sqrt{\tau_{d} / \alpha}=1 / 2$, (b) $(h / \rho H)\left(t_{a}-t_{d}\right) \sqrt{\tau_{d} / \alpha}=1$, (c) $(h / \rho \theta)\left(t_{a}-t_{d}\right)$ $\sqrt{\tau_{d} / \alpha}=2$ (case 11.1.14, source: Ref. 19, pp. 3-94, Fig. 58). 


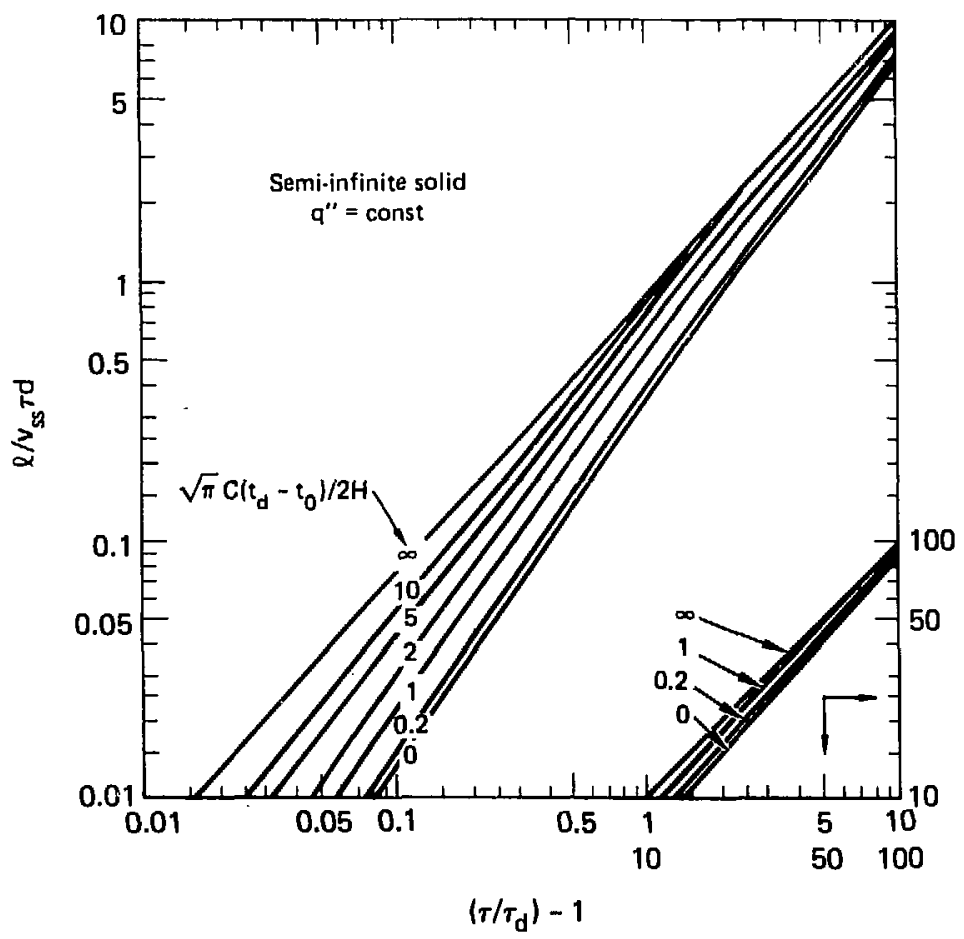

FIG. 11.6. Ablation depth of semi-infinite solid ( $x \geq 0)$ after sudden exposure to constant surface heat input $q^{\prime \prime}$ (case 11.1.15, source: Ref. 19, pp. 3-96, Fig. 59). 
TABLE 1.1. Mean and maximum temperature excesses and their ratio for electrical coils of rectangular and circular cross sections, $\theta_{m}=t_{m e a n}-t_{0}$, $\theta_{0}=t_{\max }-t_{0}$ (case 1.2.8, source: Ref. 3, p. 220, Table 10-3).

\begin{tabular}{|c|c|c|c|c|c|c|c|}
\hline \multirow{2}{*}{$\begin{array}{c}\text { Cross Section, } \\
\text { b/a }\end{array}$} & \multicolumn{6}{|c|}{ Rectangular } & Circular, \\
\hline & $\infty$ & 10 & 5 & 2.5 & 1.5 & 1 & $r=2 a / \sqrt{\pi}$ \\
\hline$\left(2 \mathrm{k} / \mathrm{q} \cdot{ }^{\prime} \cdot \mathrm{a}^{2}\right) \theta_{\mathrm{m}}$ & 0.66 & 0.625 & 0.58 & & & 0.28 & 0.32 \\
\hline$\left(2 k / q^{\prime}+a^{2}\right) \theta_{0}$ & 1.00 & 1.00 & 1.00 & & & 0.59 & 0.64 \\
\hline$\phi=\theta_{m} / \theta_{0}$ & 0.66 & 0.625 & 0.58 & 0.52 & 0.485 & 0.475 & 0.50 \\
\hline
\end{tabular}

TABLE 1.2a. Values of $\left(t-t_{1}\right) /\left(t_{f}-t_{1}\right)$ on the surface $y=0$ of a semi-infinite strip (case 1.1.29, source: Ref. 15, Table 2).

\begin{tabular}{lccccc}
\hline $\mathrm{x} / \mathrm{w}$ & $\begin{array}{c}h w / k \\
=100\end{array}$ & $\begin{array}{c}h w / k \\
=10\end{array}$ & $\begin{array}{c}\mathrm{hw} / \mathrm{k} \\
=1\end{array}$ & $\begin{array}{c}\mathrm{hw/k} \\
-0.10\end{array}$ & $\begin{array}{c}\mathrm{hw} / \mathrm{k} \\
=0.01\end{array}$ \\
\hline 0.05 & 0.889 & 0.448 & 0.094 & 0.011 & 0.001 \\
0.10 & 0.931 & 0.594 & 0.146 & 0.018 & 0.002 \\
0.20 & 0.964 & 0.725 & 0.212 & 0.027 & 0.003 \\
0.30 & 0.973 & 0.781 & 0.250 & 0.032 & 0.003 \\
0.40 & 0.977 & 0.807 & 0.270 & 0.035 & 0.004 \\
0.50 & 0.978 & 0.814 & 0.277 & 0.036 & 0.004 \\
\hline
\end{tabular}

TABLE 1.2b. Heat transfer rates through the surface $y=0$ of a semi-infinite stcip (case 1.1.29, source: Ref, 15, Table 1$) \theta=\left(t_{I}-t_{E}\right)$.

\begin{tabular}{llllllllll}
\hline & 1000 & 200 & 100 & 20 & 10 & 1.0 & 0.1 & 0.01 & 0.001 \\
\hline $\begin{array}{l}\ell / k \theta_{g} \\
8 \text { Error }\end{array} 1.8$ & 8.603 & 6.827 & 5.984 & 3.971 & 3.107 & 0.792 & 0.097 & 0.01 & 0.001 \\
\hline
\end{tabular}


TABLE 1.3. Conductance data for heat flow normal to wall cuts in an infinite plate (case 1.1.37, source: Ref. 19, p. 3-124). These groups of data are for the four kinds of wall plates shown in case 1.1.37.

\begin{tabular}{lccc}
\hline (a) & \multicolumn{3}{c}{$\mathrm{K} / \mathrm{K}$ uncut } \\
\cline { 3 - 5 } $\mathrm{c} / \mathrm{a}$ & $\mathrm{b} / \mathrm{a}=1 / 2$ & $1 / 4$ & $1 / 8$ \\
\cline { 2 - 4 } 4 & 0.902 & 0.760 & 0.646 \\
2 & 0.818 & 0.618 & 0.480 \\
1 & 0.704 & 0.465 & 0.339 \\
$1 / 2$ & 0.610 & 0.363 & 0.235 \\
$1 / 4$ & 0.564 & 0.303 & 0.182 \\
\hline
\end{tabular}

(b)

$\frac{c i a}{3 / 2}$

$3 / 4$

$1 / 2$

$1 / 3$

0.182

\begin{tabular}{|c|c|c|c|c|c|c|c|c|c|}
\hline \multicolumn{2}{|c|}{ (c) } & \multicolumn{3}{|c|}{$\mathrm{K} / \mathrm{K}_{\text {uncut }}$} & \multicolumn{2}{|c|}{ (d) } & \multicolumn{3}{|c|}{$\mathbf{K} / \mathbf{K}_{\text {uncut }}$} \\
\hline$c / a$ & $\mathrm{~d} / \mathrm{a}$ & $b / a=1 / 2$ & $1 / 4$ & $1 / 8$ & $c / a$ & $d / a$ & $b / a=1 / 4$ & $1 / 8$ & $1 / 16$ \\
\hline \multirow{4}{*}{3} & $1 / 8$ & 0.822 & 0.634 & 0.478 & & 0.402 & 0.507 & & \\
\hline & $3 / 8$ & 0.767 & 0.546 & 0.374 & $3 / 2$ & 0.469 & & 0.362 & \\
\hline & $5 / 8$ & 0.721 & 0.480 & 0.311 & & 0.502 & & & 0.310 \\
\hline & $12 / 8$ & 0.599 & 0.339 & 0.190 & & 0.866 & 0.479 & & \\
\hline \multirow{4}{*}{$3 / 2$} & $1 / 16$ & 0.740 & 0.486 & 0.342 & & 1.010 & & 0.338 & \\
\hline & $3 / 16$ & 0.698 & 0.430 & 0.284 & & 1.083 & & & 0.254 \\
\hline & $5 / 16$ & 0.661 & 0.390 & 0.242 & $1 / 2$ & $1 / 2$ & 0.329 & 0.213 & 0.141 \\
\hline & $12 / 16$ & 0.570 & 0.299 & 0.164 & & & & & \\
\hline \multirow{3}{*}{$1 / 2$} & 0 & 0.627 & 0.403 & 0.270 & & & & & \\
\hline & $1 / 16$ & 0.561 & 0.312 & 0.186 & & & & & \\
\hline & $3 / 16$ & 0.527 & 0.276 & 0.152 & & & & & \\
\hline
\end{tabular}


TABrE 2.1. Values of $\Delta_{j}$ for a triangular cooling array of cylinders (case 2.2.18, source: RE.. 64, Table 2).

\begin{tabular}{|c|c|c|c|c|c|c|c|}
\hline$s / r_{0}$ & $\Delta_{1}$ & $\Delta_{2}$ & $\Delta_{3}$ & $\Delta_{4}$ & $\Delta_{5}$ & $\Delta_{6}$ & $4_{7}$ \\
\hline 4.0 & $-5.05072\left(10^{-2}\right)$ & $-8.0891\left(10^{-4}\right)$ & $-1.262\left(10^{-5}\right)$ & $-1.97\left(10^{-7}\right)$ & $-3.08\left(10^{-9}\right)$ & $-4.8\left(10^{-11}\right)$ & $-7\left(10^{-13}\right)$ \\
\hline 2.0 & $-5.04988\left(10^{-2}\right)$ & $-8.0542\left(10^{-4}\right)$ & $-1.217\left(10^{-5}\right)$ & $-1.64\left(10^{-7}\right)$ & $-1.33\left(10^{-9}\right)$ & $2.8\left(10^{-11}\right)$ & $2\left(10^{-12}\right)$ \\
\hline 1.5 & $-5.02447\left(10^{-2}\right)$ & $-6.9920\left(10^{-4}\right)$ & $-1.374\left(10^{-6}\right)$ & $8.34\left(10^{-7}\right)$ & $5.23\left(10^{-7}\right)$ & $2.4\left(10^{-9}\right)$ & $8\left(10^{-11}\right)$ \\
\hline 1.3 & $-4.90792\left(10^{-2}\right)$ & $-2.1203\left(10^{-4}\right)$ & $6.354\left(10^{-5}\right)$ & $5.42\left(10^{-6}\right)$ & $2.99\left(10^{-7}\right)$ & $1.3\left(10^{-8}\right)$ & $4\left(10^{-10}\right)$ \\
\hline 1.2 & $-4.69398\left(10^{-2}\right)$ & $6.7812\left(10^{-4}\right)$ & $1.749\left(10^{-4}\right)$ & $1.31\left(10^{-5}\right)$ & $6.27\left(10^{-7}\right)$ & $1.7\left(10^{-8}\right\}$ & $-1\left(10^{-10}\right)$ \\
\hline 1.175 & $-4.60073\left(10^{-2}\right)$ & $1.0619\left(10^{-3}\right)$ & $2.205\left(10^{-4}\right)$ & $+1.56\left(10^{-5}\right)$ & $6.32\left(10^{-7}\right)$ & $9.8\left(10^{-10}\right)$ & $-2\left(10^{-9}\right)$ \\
\hline 1.15 & $-4.48345\left(10^{-2}\right)$ & $1.5384\left(10^{-3}\right)$ & $2.736\left(10^{-4}\right)$ & $1.76\left(10^{-5}\right)$ & $4.21\left(10^{-7}\right)$ & $-5 \quad\left(10^{-8}\right)$ & $-7\left(10^{-9}\right)$ \\
\hline 1.10 & $-4.15694\left(10^{-2}\right)$ & $2.8029\left(10^{-3}\right)$ & $3.782\left(10^{-4}\right)$ & $1.08\left(10^{-5}\right)$ & $-2.40\left(10^{-6}\right)$ & $-5 \quad\left(10^{-7}\right)$ & $-5\left(10^{-9}\right)$ \\
\hline 1.05 & $-3.68059\left(10^{-2}\right)$ & $4.3358\left(10^{-3}\right)$ & $3.169\left(10^{-4}\right)$ & $5.67\left(10^{-5}\right)$ & $-1.51\left(10^{-5}\right)$ & $-1.7\left(10^{-6}\right)$ & $-9\left(10^{-8}\right)$ \\
\hline
\end{tabular}

TABLE 2.2. Values of $\delta_{j}$ for a square cooling array of cylinders (case 2.2.19, source: Ref. 64, Table 1).

\begin{tabular}{|c|c|c|c|c|c|c|c|}
\hline$s / r_{0}$ & $\delta_{1}$ & $\delta_{2}$ & $\delta_{3}$ & $\delta_{4}$ & $\delta_{5}$ & $\delta_{6}$ & $\delta_{7}$ \\
\hline 4.0 & $-1.25382\left(10^{-1}\right)$ & $-1.0583\left(10^{-2}\right)$ & $-6.120\left(10^{-4}\right)$ & $-3.898\left(10^{-5}\right)$ & $-2.42\left(10^{-6}\right)$ & $-1.5\left(10^{-7}\right)$ & $\left(10^{-9}\right)$ \\
\hline 2.0 & $-1.25098\left(10^{-1}\right)$ & $-1.0428\left(10^{-2}\right)$ & $-5.713\left(10^{-4}\right)$ & $-3.177\left(10^{-5}\right)$ & $-1.39\left(10^{-6}\right)$ & $-2 \quad\left(10^{-8}\right)$ & $\left(10^{-9}\right)$ \\
\hline 1.5 & $-1.22597\left(10^{-1}\right)$ & $-9.060\left(10^{-3}\right)$ & $-2.08\left(10^{-4}\right)$ & $3.33\left(10^{-5}\right)$ & $8.1\left(10^{-6}\right)$ & $\left(10^{-6}\right)$ & $\left(10^{-7}\right)$ \\
\hline 1.3 & $-1.17022\left(10^{-1}\right)$ & $-5.995\left(10^{-3}\right)$ & $6.13\left(10^{-4}\right)$ & $1.83\left(10^{-4}\right)$ & $3.1\left(10^{-5}\right)$ & $\left(10^{-6}\right)$ & $\left(10^{-7}\right)$ \\
\hline 1.2 & $-1.10421\left(10^{-1}\right)$ & $-2.387\left(10^{-3}\right)$ & $1.566\left(10^{-3}\right)$ & $3.50\left(10^{-4}\right)$ & $5.3\left(10^{-5}\right)$ & $6\left(10^{-6}\right)$ & $\left(10^{-7}\right)$ \\
\hline 1.15 & $-1.05352\left(10^{-1}\right)$ & $3.172\left(10^{-4}\right)$ & $2.233\left(10^{-3}\right)$ & $4.44\left(10^{-4}\right)$ & $5.8\left(10^{-5}\right)$ & $4.3\left(10^{-6}\right)$ & $\left(10^{-8}\right)$ \\
\hline 1.1 & $-9.8721\left(10^{-2}\right)$ & $3.6596\left(10^{-3}\right)$ & $2.911\left(10^{-3}\right)$ & $4.655\left(10^{-4}\right)$ & $2.44\left(10^{-5}\right)$ & $-1.1\left(10^{-5}\right)$ & $-5.3\left(10^{-6}\right)$ \\
\hline 1.05 & $-9.0358\left(10^{-2}\right)$ & $7.310\left(10^{-3}\right)$ & $3.210\left(10^{-3}\right)$ & $2.06\left(10^{-4}\right)$ & $-1.20\left(10^{-4}\right)$ & $-5.8\left(10^{-5}\right)$ & $-1.6\left(10^{-5}\right)$ \\
\hline
\end{tabular}


TABLE 8.1. Values of $\left(t-t_{i}\right) /\left(t_{s}-t_{i}\right)$ for an infinitely wide plate whose surface temperature increases proportionally to time, $t_{s}=t_{i}+b \tau$ (case 8.1.8, source: Ref. 1, p. 268, Table 13.5).

\begin{tabular}{llllllllll}
\hline $\mathrm{x}$ & \multicolumn{7}{c}{ FO } \\
\cline { 2 - 9 } 0 & 0.08 & 0.16 & 0.20 & 0.32 & 0.40 & 0.80 & 1.60 & 2.00 & 4.00 \\
\hline 0.33 & 0.004 & 0.045 & 0.074 & 0.170 & 0.231 & 0.464 & 0.694 & 0.752 & 0.875 \\
0.5 & 0.082 & 0.194 & 0.238 & 0.346 & 0.402 & 0.594 & 0.770 & 0.814 & 0.906 \\
0.66 & 0.214 & 0.353 & 0.398 & 0.498 & 0.545 & 0.698 & 0.830 & 0.862 & 0.930 \\
0.8 & 0.418 & 0.548 & 0.586 & 0.664 & 0.699 & 0.802 & 0.889 & 0.911 & 0.955 \\
1 & 1 & 1 & 1 & 1 & 1 & 1 & 1 & 1 & 1 \\
\hline
\end{tabular}

TABLE 8.2. Values of the function $\zeta$ (case 8.1.12, source: Ref. 1 , p. 299).

\begin{tabular}{llllllllll}
\hline & \multicolumn{1}{c}{$x$} & \multicolumn{1}{c}{$x$} & & & & & \\
\cline { 2 - 8 } & 0 & $1 / 8$ & $2 / 8$ & $3 / 8$ & $4 / 8$ & $5 / 8$ & $6 / 8$ & $7 / 8$ & 1 \\
\hline 0 & 1 & 1 & 1 & 1 & 1 & 1 & 1 & 1 & 1 \\
0.5 & 0.98 & 0.98 & 0.98 & 0.98 & 0.98 & 0.98 & 0.98 & 0.99 & 1 \\
1.0 & 0.77 & 0.77 & 0.77 & 0.78 & 0.79 & 0.81 & 0.85 & 0.91 & 1 \\
1.5 & 0.47 & 0.47 & 0.47 & 0.48 & 0.52 & 0.58 & 0.68 & 0.83 & 1 \\
2.0 & 0.27 & 0.27 & 0.28 & 0.30 & 0.36 & 0.45 & 0.58 & 0.77 & 1 \\
4.0 & 0.04 & 0.04 & 0.07 & 0.08 & 0.13 & 0.22 & 0.37 & 0.64 & 1 \\
8.0 & 0.00 & 0.00 & 0.01 & 0.01 & 0.02 & 0.05 & 0.14 & 0.36 & 1 \\
$\infty$ & 0 & 0 & 0 & 0 & 0 & 0 & 0 & 0 & \\
\hline
\end{tabular}


TABLE 9.1. Values of $\left(t-t_{i}\right) /\left(t_{s}-t_{i}\right)$

for an infinitely long cylinder whose

surface increases proportionally to time

$\left(t_{s}-t_{i}=c t\right)$ (case 9.1.2, source:

Ref. 1, p. 269).

\begin{tabular}{lllll}
\hline \multirow{2}{*}{$R$} & \multicolumn{5}{c}{ FO } \\
\cline { 2 - 5 } & 0.08 & 0.16 & 0.32 & 0.80 \\
\hline 0 & 0.016 & 0.123 & 0.354 & 0.691 \\
0.33 & 0.054 & 0.191 & 0.420 & 0.725 \\
0.5 & 0.122 & 0.287 & 0.505 & 0.768 \\
0.66 & 0.368 & 0.443 & 0.628 & 0.828 \\
0.8 & 0.470 & 0.621 & 0.755 & 0.888 \\
1 & 1 & 1 & 1 & 1 \\
\hline
\end{tabular}

TABLE 10.1. Temperatures for a sphere whose surface temperature increases proportionally to time $\left(t_{0}=t_{i}+b \tau\right)$ (case 10.1 .4 , source: Ref. 1, p. 269, Table 13-7).

\begin{tabular}{llllll}
\hline \multirow{2}{*}{$R$} & \multicolumn{6}{c}{ Fo } \\
\cline { 2 - 6 } & 0.016 & 0.08 & 0.16 & 0.32 & 0.80 \\
\hline 0 & 0.00 & 0.054 & 0.219 & 0.506 & 0.792 \\
0.33 & 0.00 & 0.090 & 0.290 & 0.560 & 0.725 \\
0.5 & 0.00 & 0.162 & 0.385 & 0.626 & 0.844 \\
0.66 & 0.02 & 0.312 & 0.529 & 0.722 & 0.884 \\
0.8 & 0.14 & 0.516 & 0.686 & 0.819 & 0.925 \\
1 & 1 & 1 & 1 & 1 & 1 \\
\hline
\end{tabular}


13.1. The Error Function and Related Functions.

$$
\begin{aligned}
& \operatorname{erf}(x)=\frac{2}{\sqrt{\pi}} \int_{0}^{x} \exp \left(-z^{2}\right) d z \\
& =\frac{2}{\sqrt{i}} \sum_{n=0}^{\infty} \frac{(-1)^{n}(x)^{2 n+1}}{(2 n+1) n l} \text {, for small values of } x \\
& =1-\frac{\exp \left(-x^{2}\right)}{\sqrt{\pi}}\left(\frac{1}{x}-\frac{1}{2 x^{3}}+\frac{1}{2^{2}} \cdot \frac{3}{x^{5}}-\frac{1}{2^{3}}-\frac{3}{x^{7}} \cdot 5+\ldots\right) \cdot \begin{array}{l}
\text { for } \\
\text { large } \\
\text { values } \\
\text { of } x
\end{array} \\
& \operatorname{erf}(0)=0, \operatorname{erf}(\infty)=1 \\
& \operatorname{erf}(-x)=-\operatorname{erf}(x) \\
& \operatorname{erfc}(x)=1-\operatorname{erf}(x) \\
& i^{n} \operatorname{erfc}(x)=\int_{x}^{\infty} i^{n-1} \operatorname{erfc}(z) d z, n=1,2, \ldots, \\
& 1^{0} \operatorname{erfc}(x)=\operatorname{erfc}(x) \\
& \operatorname{ier} f c(x)=\frac{1}{\sqrt{\pi}} \exp \left(-x^{2}\right)-x \operatorname{erfc}(x) \\
& 1^{2} \operatorname{erfc}(x)=\frac{1}{4}[\operatorname{erfc}(x)-2 x \operatorname{ler} f(x)] \\
& 2 n 1^{n} \operatorname{erfc}(x)=1^{n-2} \operatorname{erfc}(x)-2 x 1^{n-1} \operatorname{erfc}(x) \\
& \frac{d}{d x} \operatorname{erf}(x)=\frac{2}{\sqrt{\pi}} \exp \left(-x^{2}\right) \\
& \frac{d^{2}}{d x^{2}} \operatorname{erf}(x)=\frac{4 x}{\sqrt{\pi}} \exp \left(-x^{2}\right)
\end{aligned}
$$


|

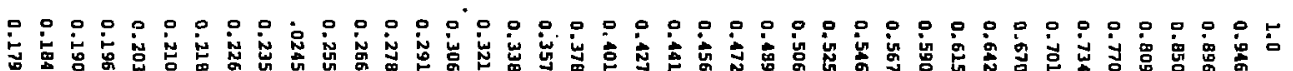

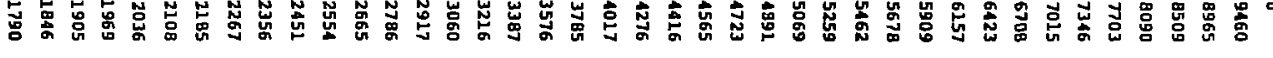

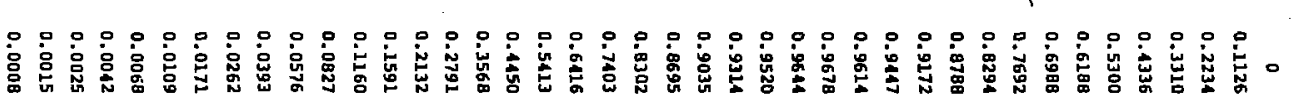

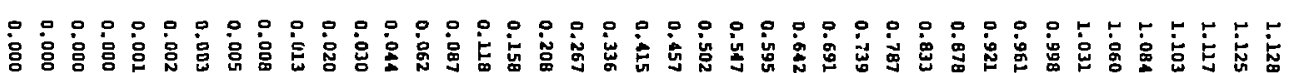
(1)

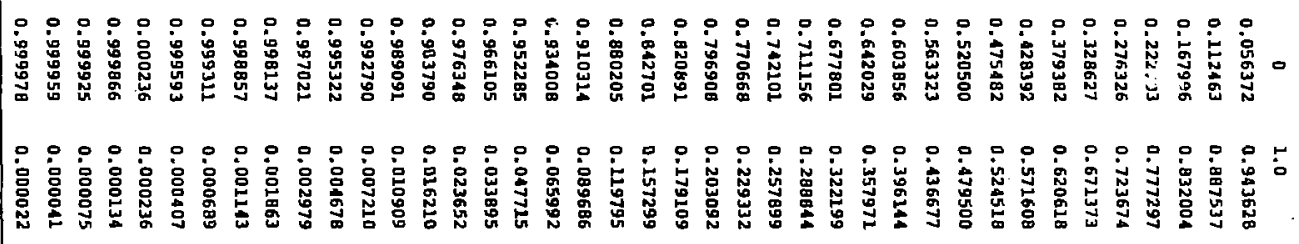

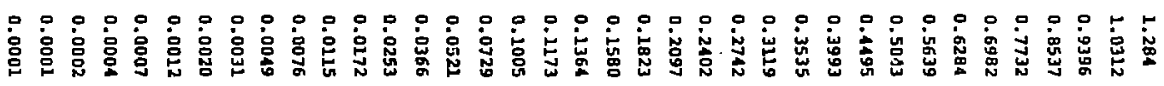

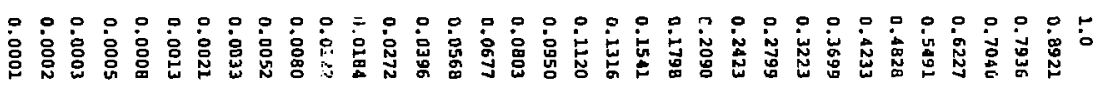

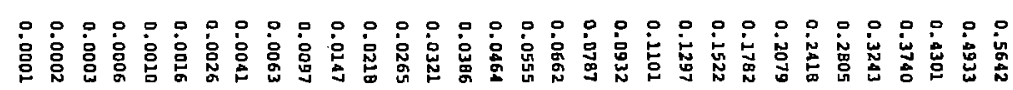

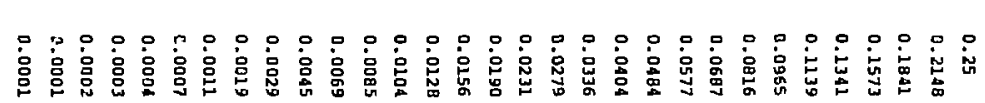

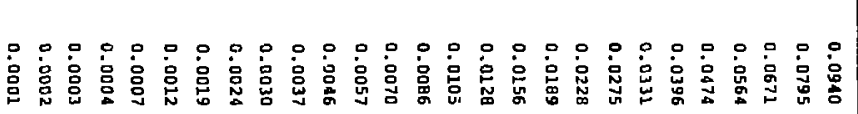

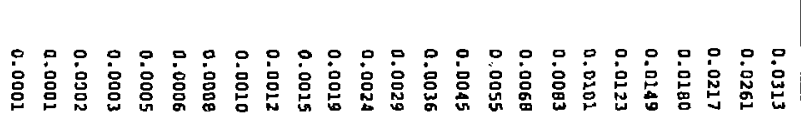


13.2. Exponential and Hyperbolic Functions.

$e=2.71828 .$.
$\exp (u)=e^{u}$
$\sinh (u)=\frac{e^{u}-e^{-u}}{2}, \sinh (0)=0, \sinh (\infty)=\infty$

$\cosh (u)=\frac{e^{u}+e^{-u}}{2}, \cosh (0)=1, \cosh (\infty)=\infty$

$\tanh (u)=\frac{e^{2 u}-1}{e^{2 u}+1}, \tanh (0)=0, \tanh (\infty)=1$ 
TABLE 13.2. Exponential functions (source: Ref. 2, pp. 367-9).

\begin{tabular}{|c|c|c|c|c|c|c|c|c|}
\hline $\mathbf{u}$ & $e^{u}$ & $e^{-u}$ & $\mathbf{u}$ & $e^{u}$ & $e^{-u}$ & $\mathbf{u}$ & $e^{u}$ & $e^{-u}$ \\
\hline 0.00 & 1.000 & 1.000 & 0.60 & 1.822 & 0.549 & 1.20 & 3.320 & 0.301 \\
\hline 0.02 & 1.020 & 0.980 & 0.62 & 1.859 & 0.538 & 1.22 & 3.387 & 0.295 \\
\hline 0.04 & 1.041 & 0.961 & 0.64 & 1.896 & 0.527 & 1.24 & 3.456 & 0.289 \\
\hline 0.06 & 1.062 & 0.942 & 0.66 & 1.935 & 0.517 & 1.26 & 3.525 & 0.284 \\
\hline 0.08 & 1.083 & 0.923 & 0.68 & 1.974 & 0.507 & 1.28 & 3.597 & 0.278 \\
\hline 0.10 & 1.105 & 0.905 & 0.70 & 2.014 & 0.497 & 1.30 & 3.669 & 0.272 \\
\hline 0.12 & 1.128 & 0.887 & 0.72 & 2.054 & 0.487 & 1.32 & 3.743 & 0.267 \\
\hline 0.14 & 1.150 & 0.869 & 0.74 & 2.096 & 0.477 & 1.34 & 3.819 & 0.262 \\
\hline 0.16 & 1.174 & 0.852 & 0.76 & 2.138 & 0.468 & 1.36 & 3.896 & 0.257 \\
\hline 0.18 & 1.197 & 0.835 & 0.78 & 2.182 & 0.458 & 1.38 & 3.975 & 0.252 \\
\hline 0.20 & 1.221 & 0.819 & 0.80 & 2.226 & 0.449 & 1.40 & 4.055 & 0.247 \\
\hline 0.22 & 1.246 & 0.802 & 0.82 & 2.270 & 0.440 & 1.42 & 4.137 & 0.242 \\
\hline 0.24 & 1.271 & 0.787 & 0.84 & 2.316 & 0.432 & 1.44 & 4.221 & 0.237 \\
\hline 0.26 & 1.297 & 0.771 & 0.86 & 2.363 & 0.423 & 1.46 & 4.306 & 2.232 \\
\hline 0.28 & 1.323 & 0.756 & 0.88 & 2.411 & 0.415 & 1.48 & 4.393 & 0.228 \\
\hline 0.30 & 1.350 & 0.741 & 0.90 & 2.460 & 0.407 & 1.50 & 4.482 & 0.223 \\
\hline 0.32 & 1.377 & 0.726 & 0.92 & 2.509 & 0.398 & 1.52 & 4.572 & 0.219 \\
\hline 0.34 & 1.405 & 0.712 & 0.94 & 2.560 & 0.391 & 1.54 & 4.665 & 0.214 \\
\hline 0.36 & 1.433 & 0.698 & 0.96 & 2.612 & 0.383 & 1.56 & 4.759 & 0.210 \\
\hline 0.38 & 1.462 & 0.684 & 0.98 & 2.664 & 0.375 & 1.58 & 4.855 & 0.206 \\
\hline 0.40 & 1.492 & 0.670 & 1.00 & 2.718 & 0.368 & 1.60 & 4.953 & 0.202 \\
\hline 0.42 & 1.522 & 0.657 & 1.02 & 2.773 & 0.361 & 1.62 & 5.053 & 0.198 \\
\hline 0.44 & 1.553 & 0.644 & 1.04 & 2.829 & 0.353 & 1.64 & 5.155 & 0.194 \\
\hline 0.46 & 1.584 & 0.631 & 1.06 & 2.886 & 0.346 & 1.66 & 5.259 & 0.190 \\
\hline 0.48 & 1.616 & 0.619 & 1.08 & 2.945 & 0.340 & 1.68 & 5.366 & 0.186 \\
\hline 0.50 & 1.649 & 0.606 & 1.10 & 3.004 & 0.333 & 1.70 & 5.474 & 0.183 \\
\hline 0.52 & 1.682 & 0.594 & 1.12 & 3.065 & 0.326 & 1.72 & 5.584 & 0.179 \\
\hline 0.54 & 1.716 & 0.583 & 1.14 & 3.127 & 0.320 & 1.74 & 5.697 & 0.176 \\
\hline 0.56 & 1.751 & 0.571 & 1.16 & 3.190 & 0.313 & 1.76 & 5.812 & 0.172 \\
\hline 0.58 & 1.786 & 0.560 & 1.18 & 3.254 & 0.307 & 1.78 & 5.930 & 0.169 \\
\hline
\end{tabular}


TABLE 13.2. (Continued).

\begin{tabular}{|c|c|c|c|c|c|c|c|c|}
\hline $\mathbf{u}$ & $e^{u}$ & $e^{-u}$ & $u$ & $e^{u}$ & $e^{-u}$ & $\mathbf{u}$ & $e^{u}$ & $e^{-u}$ \\
\hline 1.80 & 6.050 & 0.165 & 2.40 & 11.023 & 0.091 & 3.00 & 20.08 & 0.050 \\
\hline 1.82 & 6.172 & 0.162 & 2.42 & 11.246 & 0.089 & 3.10 & 22.20 & 0.045 \\
\hline 1.84 & 6.296 & 0.159 & 2.44 & 11.473 & 0.087 & 3.20 & 24.53 & 0.041 \\
\hline 1.86 & 6.424 & 0.156 & 2.46 & 11.705 & 0.085 & 3.30 & 27.11 & 0.037 \\
\hline 1.88 & 6.554 & 0.153 & 2.48 & 11.941 & 0.084 & 3.40 & 29.96 & 0.033 \\
\hline 1.90 & 6.686 & 0.150 & 2.50 & 12.18 & 0.082 & 3.50 & 33.11 & 0.030 \\
\hline 1.92 & 6.821 & 0.147 & 2.52 & 12.43 & 0.080 & 3.60 & 36.60 & 0.027 \\
\hline 1.94 & 6.959 & 0.144 & 2.54 & 12.68 & 0.079 & 3.70 & 40.45 & 0.025 \\
\hline 1.96 & 7.039 & 0.141 & 2.56 & 12.94 & 0.077 & 3.80 & 44.70 & 0.022 \\
\hline 1.98 & 7.243 & 0.138 & 2.58 & 13.20 & 0.076 & 3.90 & 49.40 & 0.020 \\
\hline 2.00 & 7.389 & 0.135 & 2.60 & 13.46 & 0.074 & 4.00 & 54.60 & 0.018 \\
\hline 2.02 & 7.538 & 0.133 & 2.62 & 13.74 & 0.073 & 4.20 & 66.69 & 0.015 \\
\hline 2.04 & 7.691 & 0.130 & 2.64 & 14.01 & 0.071 & 4.40 & 81.45 & 0.012 \\
\hline 2.06 & 7.846 & 0.127 & 2.66 & 14.30 & 0.070 & 4.60 & 99.48 & 0.010 \\
\hline 2.08 & 8.004 & 0.125 & 2.68 & 14.58 & 0.069 & 4.80 & 121.51 & 0.008 \\
\hline 2.10 & 8.166 & 0.122 & 2.70 & 14.88 & 0.067 & 5.00 & 148.4 & 0.007 \\
\hline 2.12 & 8.331 & 0.120 & 2.72 & 15.18 & 0.066 & 5.20 & 181.3 & 0.006 \\
\hline 2.14 & 8.499 & 0.118 & 2.74 & 15.49 & 0.065 & 5.40 & 221.4 & 0.004 \\
\hline 2.16 & 8.671 & 0.115 & 2.76 & 15.80 & 0.063 & 5.60 & 270.4 & 0.004 \\
\hline 2.18 & 8.846 & 0.113 & 2.78 & 16.12 & 0.062 & 5.80 & 330.3 & 0.003 \\
\hline 2.20 & 9.025 & 0.111 & 2.80 & 16.44 & 0.061 & 6.00 & 403.4 & 0.002 \\
\hline 2.22 & 9.207 & 0.109 & 2.82 & 16.78 & 0.060 & 6.50 & 665.1 & 0.002 \\
\hline 2.24 & 9.393 & 0.106 & 2.84 & 17.12 & 0.058 & 7.00 & 1096.6 & 0.001 \\
\hline 2.26 & 9.583 & 0.104 & 2.86 & 17.46 & 0.057 & 7.50 & 1808.0 & 0.001 \\
\hline 2.28 & 9.777 & 0.102 & 2.88 & 17.81 & 0.056 & 8.00 & 2981.0 & 0.000 \\
\hline 2.30 & 9.974 & 0.100 & 2.90 & 18.17 & 0.055 & 8.50 & 4914.8 & 0.000 \\
\hline 2.32 & 10.176 & 0.098 & 2.92 & 18.54 & 0.054 & 9.00 & 8103.1 & 0.000 \\
\hline 2.34 & 10.381 & 0.096 & 2.94 & 18.92 & 0.053 & 9.50 & 13360 & 0.000 \\
\hline 2.36 & 10.591 & 0.094 & 2.96 & 19.30 & 0.052 & 10.00 & 22026 & 0.000 \\
\hline 2.38 & 10.805 & 0.093 & 2.98 & 19.69 & 0.051 & & $\cdot$ & \\
\hline
\end{tabular}


13.3. The Garma Function.

$$
\begin{aligned}
& \Gamma(x)=\int_{0}^{\infty} u^{x-1} e^{-u} d u, x>0 \\
& \Gamma(x) \simeq x^{x} e^{-x} \sqrt{\frac{2 \pi}{x}}\left[1+\frac{1}{12 x}+\frac{1}{288 x^{2}}-\frac{139}{5184 x^{3}}-\frac{571}{2488320 x^{4}}+\ldots\right],
\end{aligned}
$$

for large positive values of $x$

$$
\Gamma(x+1)=x \Gamma(x)
$$

\begin{tabular}{|c|c|c|c|c|c|c|c|c|c|c|c|c|c|c|}
\hline \multirow{2}{*}{$\frac{x}{1.01}$} & \multicolumn{2}{|c|}{$\Gamma(x)$} & \multirow{2}{*}{$\frac{x}{1.21}$} & \multicolumn{2}{|c|}{$\Gamma(x)$} & \multirow{2}{*}{$\frac{x}{1.41}$} & \multicolumn{2}{|c|}{$\Gamma(x)$} & \multirow{2}{*}{$\frac{x}{1.61}$} & \multicolumn{2}{|c|}{$\Gamma(\mathrm{x})$} & \multirow{2}{*}{$\frac{x}{1.81}$} & \multicolumn{2}{|c|}{$\Gamma(x)$} \\
\hline & 0.99 & 433 & & 0.91 & 558 & & 0.88 & 676 & & 0.89 & 468 & & 0.93 & 408 \\
\hline 1.02 & 0.98 & 884 & 1.22 & 0.91 & 311 & 1.42 & 0.88 & 636 & 1.62 & 0.89 & 592 & 1.82 & 0.93 & 685 \\
\hline 1.03 & 0.98 & 355 & 1.23 & 0.91 & 075 & 1.43 & 0.88 & 604 & 1.63 & 0.89 & 724 & 1.83 & 0.93 & 969 \\
\hline 1.04 & 0.97 & 844 & 1.24 & 0.90 & 852 & 1.44 & 0.88 & 581 & 1.64 & 0.89 & 864 & 1.84 & 0.94 & 261 \\
\hline 1.05 & 0.97 & 350 & 1.25 & 0.90 & 640 & 1.45 & 0.88 & 566 & 1.65 & 0.90 & 012 & 1.85 & 0.94 & 561 \\
\hline 1.06 & 0.97 & 874 & 1.26 & 0.90 & 440 & 1.46 & 0.88 & 560 & 1.66 & 0.90 & 167 & 1.86 & 0.94 & 869 \\
\hline 1.07 & 0.96 & 415 & 1.27 & 0.90 & 250 & 1.47 & 0.88 & 563 & 1.67 & 0.90 & 330 & 1.87 & 0.95 & 184 \\
\hline 1.08 & 0.95 & 973 & 1.28 & 0.90 & 072 & 1.48 & 0.88 & 575 & 1.68 & 0.90 & 500 & $1 . B B$ & 0.95 & 507 \\
\hline 1.09 & 0.95 & 546 & 1.29 & 0.89 & 904 & 1.49 & 0.88 & 595 & 1.69 & 0.90 & 678 & 1.89 & 0.95 & 838 \\
\hline 1.10 & 0.95 & 135 & 1.30 & 0.89 & 747 & 1.50 & 0.88 & 623 & 1.70 & 0.90 & 864 & 1.90 & 0.96 & 177 \\
\hline 1.11 & 0.94 & 740 & 1.31 & 0.89 & 600 & 1.51 & 0.88 & 659 & 1.71 & 0.91 & 057 & 1.91 & 0.96 & 523 \\
\hline 1.12 & 0.94 & 359 & 1.32 & 0.89 & 464 & 1.52 & 0.88 & 704 & 1.72 & 0.91 & 258 & 1.92 & 0.96 & 877 \\
\hline 1.13 & 0.93 & 993 & 1.33 & 0.89 & 338 & 1.53 & 0.88 & 757 & 1.73 & 0.91 & 467 & 1.93 & 0.97 & 240 \\
\hline 1.14 & 0.93 & 642 & 1.34 & 0.89 & 222 & 1.54 & 0.88 & 818 & 1.74 & 0.91 & 683 & 1.94 & 0.97 & 610 \\
\hline 1.15 & 0.93 & 304 & 1.35 & 0.89 & 115 & 1.55 & 0.88 & 887 & 1.75 & 0.91 & 906 & 1.95 & 0.97 & 988 \\
\hline 1.16 & 0.92 & 980 & 1.36 & 0.89 & 018 & 1.56 & 0.88 & 964 & 1.76 & 0.92 & 137 & 1.96 & 0.98 & 374 \\
\hline 1.17 & 0.92 & 670 & 1.37 & 0.88 & 931 & 1.57 & 0.89 & 049 & 1.77 & 0.92 & 376 & 1.97 & 0.98 & 768 \\
\hline 1.18 & 0.92 & 373 & 1.38 & 0.88 & 854 & 1.58 & 0.89 & 142 & 1.78 & 0.92 & 623 & 1.98 & 0.99 & 171 \\
\hline 1.19 & 0.92 & 089 & 1.39 & 0.88 & 785 & 1.59 & 0.89 & 243 & 1.79 & 0.92 & 877 & 1.99 & 0.99 & 581 \\
\hline 1.20 & 0.91 & 817 & 1.40 & 0.88 & 726 & 1.60 & 0.89 & 352 & 1.80 & 0.93 & 138 & 2.00 & 1.00 & 000 \\
\hline
\end{tabular}

TABLE 13.3. The gamma function (source: Ref. 17, p. 136). 
the positive sign used if $-\pi / 2<\arg u<3 \pi / 2$ and the negative sign used if $-3 \pi / 2<\arg u<\pi / 2$.

$$
K_{n}(u)=\frac{\pi}{2} \frac{I_{-n}(u)-I_{n}(u)}{\sin (n \pi)} .
$$

For $n$ any positive integer:

$$
\begin{aligned}
K_{n}(u)=(-1)^{n+1}|\ln (u / 2)+\gamma| I_{n}(u)+\frac{(-1)^{n}}{2} \sum_{j=0}^{\infty} \frac{(u / 2)^{n+2 j}}{j !(n+j) !} \\
\times\left[\sum_{m=1}^{n+j} m^{-1}+\sum_{m=1}^{j} m^{-1}\right]+\frac{1}{2} \sum_{j=0}^{\infty}(-1)^{j}(u / 2)^{-n+2 j} \frac{(n-j-1) !}{j !},
\end{aligned}
$$

where for $j=0, \sum_{m=1}^{j} m^{-1}=0$.

For large values of u:

$$
\begin{aligned}
& x_{n}(u)=\sqrt{\frac{\pi}{2 u}} e^{-u}\left\{1+\frac{4 n^{2}-1^{2}}{1 ! 3 u}+\frac{\left(4 n^{2}-1^{2}\right)\left(4 n^{2}-3^{2}\right)}{2 !(8 u)}+o\left(u^{-3}\right)\right\} \\
& \frac{d}{d u} J_{n}(u)=J_{n-1}(u)-\frac{n}{u} J_{n}(u)=\frac{n}{u} J_{n}(u)-J_{n+1}(u) \\
& \frac{d}{d u} Y_{n}(u)=Y_{n-1}(u)-\frac{n}{u} Y_{n}(u)=\frac{n}{u} Y_{n}(u)-Y_{n+1}(u) \\
& \frac{d}{d u} I_{n}(u)=I_{n-1}(u)-\frac{n}{u} I_{n}(u)=I_{n+1}(u)+\frac{n}{u} I_{n}(u) \\
& \frac{d}{d u} K_{n}(u)=-K_{n-1}(u)-\frac{n}{u} K_{n}(u)=\frac{n}{u} K_{n}(u)-K_{n+1}(u)
\end{aligned}
$$


13.4. Bessel Functions.

$$
\begin{aligned}
& J_{n}(u)=\sum_{j=0}^{\infty} \frac{(-1)^{j}(u / 2)^{n+2 j}}{j ! \Gamma(n+j+1)}, n \text { is real and } u \text { may be complex } \\
& J_{n}(u)=(-1)^{n} J_{-n}(u) \text {, if } n \text { is an integer } \\
& Y_{n}(u)=\frac{J_{n}(u) \cos (n \pi)-J_{-n}(u)}{\sin (n \pi)} \\
& \pi Y_{n}(u)=2|\ln (u / 2)+\gamma| J_{n}(u)-\sum_{j=0}^{\infty} \frac{(-1)^{j}(u / 2)^{n+2 j}}{j !(n+j) !} \\
& x\left[\sum_{m=1}^{j+n} m^{-1}+\sum_{m=1}^{j} m^{-1}\right]-\sum_{j=0}^{n-1}(u / 2)^{-n+2 j} \frac{(n-j-1) !}{j !}
\end{aligned}
$$

whera $Y=0.5772 \ldots$ is Euler's constant, and $n$ is any positive integer

$$
\begin{aligned}
& \frac{\pi}{2} Y_{0}(u)=\{\ln (u / 2)+\gamma\} J_{0}(u)+(u / 2)^{2}-\left(1+\frac{1}{2}\right) \frac{(u / 2)^{4}}{(2 !)^{2}} \\
& +\left(1+\frac{1}{2}+\frac{1}{3}\right) \frac{(u / 2)^{6}}{(3 !)^{2}}-\ldots \\
& I_{n}(u)=\sum_{j=0}^{\infty} \frac{(u / 2)^{n+2 j}}{j ! \Gamma(n+j+1)}
\end{aligned}
$$

For large values of u:

$$
\begin{array}{r}
I_{n}(u)=\frac{1}{\sqrt{2 \pi u}} \exp (u)\left\{1-\frac{4 n^{2}-1^{2}}{118 u}+\frac{\left(4 n^{2}-1^{2}\right)\left(4 n^{2}-3^{2}\right)}{2 !(8 u)^{2}}+0\left(u^{-3}\right)\right\} \\
\quad+\frac{1}{\sqrt{2 \pi u}} \exp \left[-u \pm\left(n+\frac{1}{2}\right) \pi i\right]\left\{1+0\left(u^{-1}\right)\right\},
\end{array}
$$


TABLE 13.4.1. Zero and first-order Bessel functions of the first kind (source: Ref 2, PP. 369-71).

\begin{tabular}{|c|c|c|c|c|c|}
\hline $\mathbf{u}$ & $J_{0}(u)$ & $J_{1}(u)$ & $\mathbf{u}$ & $J_{0}(u)$ & $\mathrm{J}_{1}(\mathrm{u})$ \\
\hline 0.0 & 1.0000 & 0.0000 & 3.0 & -0.2600 & 0.3391 \\
\hline 0.1 & 0.9975 & 0.0499 & 3.1 & -0.2921 & 0.3009 \\
\hline 0.2 & 0.9900 & 0.0995 & 3.2 & -0.3202 & 0.2613 \\
\hline 0.3 & 0.9776 & 0.1483 & 3.3 & -0.3443 & 0.2207 \\
\hline 0.4 & 0.9604 & 0.1960 & 3.4 & -0.3643 & 0.1792 \\
\hline 0.5 & 0.9385 & 0.2423 & 3.5 & -0.3801 & 0.1374 \\
\hline 0.6 & 0.9120 & 0.2867 & 3.6 & -0.3918 & 0.0955 \\
\hline 0.7 & 0.8812 & 0.3290 & 3.7 & -0.3992 & 0.0538 \\
\hline 0.8 & 0.8463 & 0.3688 & 3.8 & -0.4026 & 0.0128 \\
\hline 0.9 & 0.8075 & 0.4059 & 3.9 & -0.4018 & -0.0272 \\
\hline 1.0 & 0.7652 & 0.4400 & 4.0 & -0.3971 & -0.0660 \\
\hline 1.1 & $0.7190^{\circ}$ & 0.4709 & 4.1 & -0.3887 & -0.1033 \\
\hline 1.2 & 0.6711 & 0.4983 & 4.2 & -0.3766 & -0.1385 \\
\hline 1.3 & 0.6201 & 0.5220 & 4.3 & -0.3610 & -0.1719 \\
\hline 1.4 & 0.5669 & 0.5419 & 4.4 & -0.3423 & -0.2028 \\
\hline 1.5 & 0.5118 & 0.5579 & 4.5 & -0.3205 & -0.2311 \\
\hline 1.6 & 0.4554 & 0.5699 & 4.6 & $-0.296 \mathrm{~J}$ & -0.2566 \\
\hline 1.7 & 0.3980 & 0.5778 & 4.7 & -0.2693 & -0.2791 \\
\hline 1.8 & 0.3400 & 0.5815 & 4.8 & -0.2404 & -0.2985 \\
\hline 1.9 & 0.2818 & 0.5812 & 4.9 & -0.2097 & -0.3147 \\
\hline 2.0 & 0.2239 & 0.5767 & 5.0 & -0.1776 & -0.3276 \\
\hline 2.1 & 0.1666 & 0.5683 & 5.1 & -0.1443 & -0.3371 \\
\hline 2.2 & 0.1104 & 0.5560 & 5.2 & -0.1103 & -0.3432 \\
\hline 2.3 & 0.0555 & 0.5399 & 5.3 & -0.0758 & -0.3460 \\
\hline 2.4 & 0.0025 & 0.5202 & 5.4 & -0.0412 & -0.3453 \\
\hline 2.5 & -0.0484 & 0.4971 & 5.5 & -0.0068 & -0.3414 \\
\hline 2.6 & -0.0968 & 0.4708 & 5.6 & 0.0270 & -0.3343 \\
\hline 2.7 & -0.1424 & 0.4416 & 5.7 & 0.0599 & -0.3241 \\
\hline 2.8 & -0.1850 & 0.4097 & 5.8 & 0.0917 & -0.3110 \\
\hline 2.9 & -0.2243 & 0.3754 & 5.9 & 0.1220 & -0.2951 \\
\hline
\end{tabular}


TABLE 13.4.1. (Continued.)

\begin{tabular}{|c|c|c|c|c|c|}
\hline $\mathbf{u}$ & $\mathrm{J}_{0}(\mathrm{u})$ & $J_{1}(u)$ & $\mathbf{u}$ & $J_{0}(u)$ & $J_{1}(u)$ \\
\hline 6.0 & 0.1506 & -0.2767 & 9.0 & -0.0903 & 0.2453 \\
\hline 6.1 & 0.1773 & -0.2559 & 9.1 & -0.1142 & 0.2324 \\
\hline 6.2 & 0.2017 & -0.2329 & 9.2 & -0.1368 & 0.2174 \\
\hline 6.3 & 0.2238 & -0.2081 & 9.3 & -0.1577 & 0.2004 \\
\hline 6.4 & 0.2433 & -0.1816 & 9.4 & -0.1768 & 0.1816 \\
\hline 6.5 & 0.2601 & -0.1538 & 9.5 & -0.1939 & 0.1613 \\
\hline 6.6 & 0.2740 & -0.1250 & 9.6 & -0.2090 & 0.1395 \\
\hline 6.7 & 0.2851 & -0.0953 & 9.7 & -0.2218 & 0.1166 \\
\hline 6.8 & 0.2931 & -0.0652 & 9.8 & -0.2323 & 0.0928 \\
\hline 6.9 & 0.2981 & -0.0349 & 9.9 & -0.2403 & 0.0634 \\
\hline 7.0 & 0.3001 & -0.0047 & 10.0 & -0.2459 & 0.0435 \\
\hline 7.1 & 0.2991 & 0.0252 & 10.1 & -0.2490 & 0.0184 \\
\hline 7.2 & 0.2951 & 0.0543 & 10.2 & -0.2496 & -0.0066 \\
\hline 7.3 & 0.2882 & 0.0826 & 10.3 & -0.2477 & -0.0313 \\
\hline 7.4 & 0.2786 & 0.1096 & 10.4 & -0.2434 & -0.0555 \\
\hline 7.5 & 0.2663 & 0.1352 & 10.5 & -0.2366 & -0.0788 \\
\hline 7.6 & 0.2516 & 0.1592 & 10.6 & -0.2276 & -0.1012 \\
\hline 7.7 & 0.2346 & 0.1813 & 10.7 & -0.2164 & -0.1224 \\
\hline 7.8 & 0.2154 & 0.2014 & 10.8 & -0.2032 & -0.1422 \\
\hline 7.9 & 0.1944 & 0.2192 & 10.9 & -0.1881 & -0.1604 \\
\hline 8.0 & 0.1716 & 0.2346 & 11.0 & -0.1712 & -0.1768 \\
\hline 8.1 & 0.1475 & 0.2476 & 11.1 & -0.1528 & -0.1913 \\
\hline 8.2 & 0.1222 & 0.2580 & 11.2 & -0.1330 & -0.2028 \\
\hline 8.3 & 0.0960 & 0.2657 & 11.3 & -0.1121 & -0.2143 \\
\hline 8.4 & 0.0692 & 0.2708 & 11.4 & -0.0902 & -0.2224 \\
\hline 8.5 & 0.0419 & 0.2731 & 11.5 & -0.0677 & -0.2284 \\
\hline 8.6 & 0.0146 & 0.2728 & 11.6 & -0.0446 & -0.2320 \\
\hline 8.7 & -0.0125 & 0.2697 & 11.7 & -0.0213 & -0.2333 \\
\hline 8.8 & -0.0392 & 0.2641 & 11.8 & 0.0020 & -0.2323 \\
\hline 8. 9 & -0.0652 & 0.2559 & 11.9 & 0.0250 & -0.2290 \\
\hline
\end{tabular}


TABLE 13.4.1. (Continued.)

\begin{tabular}{llllll}
\hline$u$ & $J_{0}(u)$ & $J_{1}(u)$ & $u$ & $J_{0}(u)$ & $J_{1}(u)$ \\
\hline 12.0 & 0.0477 & -0.2234 & 13.6 & 0.2101 & 0.0590 \\
12.1 & 0.0697 & -0.2158 & 13.7 & 0.2032 & 0.0791 \\
12.2 & 0.0908 & -0.2060 & 13.8 & 0.1943 & 0.0984 \\
12.3 & 0.1108 & -0.1943 & 13.9 & 0.1836 & 0.1165 \\
12.4 & 0.1296 & -0.1807 & 14.0 & 0.1711 & 0.1334 \\
12.5 & 0.1469 & -0.1655 & 14.1 & 0.1570 & 0.1488 \\
12.6 & 0.1626 & -0.1487 & 14.2 & 0.1414 & 0.1626 \\
12.7 & 0.1766 & -0.1307 & 14.3 & 0.1245 & 0.1747 \\
12.8 & 0.1887 & -0.1114 & 14.4 & 0.1065 & 0.1850 \\
12.9 & 0.1988 & -0.0912 & 14.5 & 0.0875 & 0.1934 \\
13.0 & 0.2069 & -0.0703 & 14.6 & 0.0679 & 0.1998 \\
13.1 & 0.2129 & -0.0488 & 14.7 & 0.0476 & 0.2043 \\
13.2 & 0.2167 & -0.0271 & 14.8 & 0.0271 & 0.2066 \\
13.3 & 0.2183 & -0.0052 & 14.9 & 0.0064 & 0.2069 \\
13.4 & 0.2177 & 0.0166 & 15.0 & 0.0142 & 0.2051 \\
13.5 & 0.2150 & 0.0380 & & & \\
\hline
\end{tabular}


TABLE 13.4.2. Zero and first-order Bessel functions of the seciond kind (source: Ref. 2 p. 371-3).

\begin{tabular}{|c|c|c|c|c|c|}
\hline $\mathbf{u}$ & $Y_{0}(u)$ & $y_{1}(u)$ & u & $Y_{0}(u)$ & $Y_{1}(u)$ \\
\hline 0.0 & $-\infty$ & $-\infty$ & 3.0 & 0.3768 & 0.3247 \\
\hline 0.1 & -1.5342 & -6.4590 & 3.1 & 0.3431 & 0.3496 \\
\hline 0.2 & -1.0811 & -3.3238 & 3.2 & 0.3070 & 0.3707 \\
\hline 0.3 & 0.8073 & -2.2931 & 3.3 & 0.2691 & 0.3878 \\
\hline 0.4 & -0.6060 & -1.7809 & 3.4 & 0.2296 & 0.4010 \\
\hline 0.5 & -0.4445 & -1.4715 & 3.5 & 0.1890 & 0.4102 \\
\hline 0.6 & -0.3085 & -1.2604 & 3.6 & 0.1477 & 0.4154 \\
\hline 0.7 & -0.1907 & -0.1032 & 3.7 & 0.1061 & 0.4167 \\
\hline 0.8 & -0.0868 & -0.9781 & 3.8 & 0.0645 & 0.4141 \\
\hline 0.9 & 0.0056 & -0.8731 & 3.9 & 0.0234 & 0.4078 \\
\hline 1.0 & 0.0883 & -0.7812 & 4.0 & -0.0169 & 0.3979 \\
\hline 1,1 & 0.1622 & -0.6981 & 4.1 & -0.0561 & 0.3846 \\
\hline 1.2 & 0.2281 & -0.6211 & 4.2 & -0.0938 & 0.3680 \\
\hline 1.3 & 0.2865 & -0.5485 & 4.3 & -0.1296 & 0.3484 \\
\hline 1.4 & 0.3379 & -0.4791 & 4.4 & -0.1633 & 0.3260 \\
\hline 1.5 & 0.3824 & -0.4123 & 4.5 & -0.1947 & 0.3010 \\
\hline 1.6 & 0.4204 & -0.3476 & 4.6 & -0.2235 & 0.2737 \\
\hline 1.7 & 0.4520 & -0.2847 & 4.7 & -0.2494 & 0.2445 \\
\hline 1.8 & 0.4774 & -0.2237 & 4.8 & -0.2723 & 0.2136 \\
\hline 1.9 & 0.4968 & -0.1644 & 4.9 & -0.2921 & 0.1812 \\
\hline 2.0 & 0.5104 & -0.1070 & 5.0 & -0.3085 & 0.1479 \\
\hline 2.1 & 0.5183 & -0.0517 & 5.1 & -0.3216 & 0.1137 \\
\hline 2.2 & 0.5208 & 0.0015 & 5.2 & -0.3312 & 0.0792 \\
\hline 2.3 & 0.5181 & 0.0523 & 5.3 & -0.3374 & 0.0445 \\
\hline 2.4 & 0.5104 & 0.1005 & 5.4 & -0.3102 & 0.0101 \\
\hline 2.5 & 0.4981 & 0.1459 & 5.5 & -0.3395 & -0.0238 \\
\hline 2.6 & 0.4813 & 0.1884 & 5.6 & -0.3354 & -0.0568 \\
\hline 2.7 & 0.4605 & 0.2276 & 5.7 & -0.3282 & -0.0887 \\
\hline 2.8 & 0.4359 & 0.2635 & 5.8 & -0.3177 & -0.1192 \\
\hline 2.9 & 0.4079 & 0.2959 & 5.9 & -0.3044 & -0.1481 \\
\hline
\end{tabular}


TABLE 13.4.2. (Continued.)

\begin{tabular}{|c|c|c|c|c|c|}
\hline $\mathbf{u}$ & $x_{0}(u)$ & $x_{1}(u)$ & $\mathbf{u}$ & $Y_{0}(u)$ & $Y_{1}(u)$ \\
\hline 6.0 & -0.2882 & -0.1750 & 9.0 & 0.2499 & 0.1043 \\
\hline 6.1 & -0.2694 & -0.1998 & 9.1 & 0.2383 & 0.1275 \\
\hline 6.2 & -0.2483 & -0.2223 & 9.2 & 0.2245 & 0.1491 \\
\hline 6.3 & -0.2251 & -0.2422 & 9.3 & 0.2086 & 0.1691 \\
\hline 6.4 & -0.2000 & -0.2596 & 9.4 & 0.1907 & 0.1871 \\
\hline 6.5 & -0.1732 & -0.2741 & 9.5 & 0.1712 & 0.2032 \\
\hline 6.6 & -0.1452 & -0.2857 & 9.6 & 0.1502 & 0.2171 \\
\hline 6.7 & -0.1162 & -0.2945 & 9.7 & 0.1279 & 0.2287 \\
\hline 6.8 & -0.0864 & -0.3002 & 9.8 & 0.1045 & 0.2379 \\
\hline 6.9 & -0.0562 & -0.3029 & 9.9 & 0.0804 & 0.2447 \\
\hline 7.0 & -0.0260 & -0.3027 & 10.0 & 0.0557 & 0.2490 \\
\hline 7.1 & 0.0042 & -0.2995 & 10.1 & 0.0307 & 0.2508 \\
\hline 7.2 & 0.0338 & -0.2934 & 10.2 & 0.0056 & 0.2502 \\
\hline 7.3 & 0.0628 & -0.2846 & 10.3 & -0.0193 & 0.2471 \\
\hline 7.4 & 0.0907 & -0.2731 & 10.4 & -0.0437 & 0.2416 \\
\hline 7.5 & 0.1173 & -0.2591 & 10.5 & -0.0675 & 0.2337 \\
\hline 7.6 & 0.1424 & -0.2428 & 10.6 & -0.0904 & 0.2236 \\
\hline 7.7 & 0.1658 & -0.2243 & 10.7 & -0.1122 & 0.2114 \\
\hline 7.8 & 0.1872 & -0.2039 & 10.8 & -0.1326 & 0.1973 \\
\hline 7.9 & 0.2065 & -0.1817 & 10.9 & -0.1516 & 0.1813 \\
\hline 8.0 & 0.2235 & -0.1581 & 11.0 & -0.1688 & 0.1637 \\
\hline 8.1 & 0.2381 & -0.1332 & 11.1 & -0.1843 & 0.1446 \\
\hline 8.2 & 0.2501 & -0.1072 & 11.2 & -0.1977 & 0.1243 \\
\hline 8.3 & 0.2595 & -0.0806 & 11.3 & -0.2091 & 0.1029 \\
\hline 8.4 & 0.2662 & -0.0535 & 11.4 & -0.2183 & 0.0807 \\
\hline 8.5 & 0.2702 & -0.0262 & 11.5 & -0.2252 & 0.0579 \\
\hline 8.6 & 0.2715 & 0.0011 & 11.6 & -0.2299 & 0.0348 \\
\hline 8.7 & 0.2700 & 0.0280 & 11.7 & -0.2322 & 0.0114 \\
\hline 8.8 & 0.2659 & 0.0544 & 11.8 & -0.2322 & -0.0118 \\
\hline 8.9 & 0.2592 & 0.0799 & 11.9 & -0.2298 & -0.0347 \\
\hline
\end{tabular}


TABLE 13.4.2. (Continued.)

\begin{tabular}{|c|c|c|c|c|c|}
\hline $\mathbf{u}$ & $y_{0}(u)$ & $Y_{1}(u)$ & $\mathbf{u}$ & $Y_{0}(u)$ & $y_{1}(u)$ \\
\hline 12.0 & -0.2252 & -0.0571 & 13.5 & 0.0301 & -0.2140 \\
\hline 12.1 & -0.2184 & -0.0787 & 13.6 & 0.0512 & -0.2084 \\
\hline 12.2 & -0.2095 & -0.0994 & 13.7 & 0.0717 & -0.2007 \\
\hline 12.3 & -0.1986 & -0.1190 & 13.8 & 0.0913 & -0.1912 \\
\hline 12.4 & -0.1858 & -0.1371 & 13.9 & 0.1099 & -0.1798 \\
\hline 12.5 & -0.1712 & -0.1538 & 14.0 & 0.1272 & -0.1666 \\
\hline 12.6 & -0.1551 & -0.1689 & 14.1 & 0.1431 & -0.1520 \\
\hline 12.7 & -0.1375 & -0.1821 & 14.2 & 0.1575 & -0.1359 \\
\hline 12.8 & -0.1187 & -0.1935 & 14.3 & 0.1703 & -0.1186 \\
\hline 12.9 & -0.0989 & -0.2028 & 14.4 & 0.1812 & -0.1003 \\
\hline 13.0 & -0.0782 & -0.2101 & 14.5 & 0.1903 & -0.0810 \\
\hline 13.1 & -0.0569 & -0.2152 & 14.6 & 0.1974 & -0.0612 \\
\hline 13.2 & -0.0352 & -0.2182 & 14.7 & 0.2025 & -0.0408 \\
\hline 13.3 & $-0,0134$ & -0.2190 & 14.8 & 0.2056 & -0.0202 \\
\hline 13.4 & 0.0085 & -0.2176 & 14.9 & 0.2066 & 0.0005 \\
\hline
\end{tabular}


TABLE 13.4.3. Zero and first order modified Bessel functions of the first kind (source: Ref. 2, p. 373-4).

\begin{tabular}{|c|c|c|c|c|c|}
\hline u & $I_{0}(u)$ & $I_{1}(u)$ & u & $I_{0}(u)$ & $I_{1}(u)$ \\
\hline 0.0 & 1. 2000 & 0.0000 & 3.0 & 4.881 & 3.953 \\
\hline 0.1 & 1.0025 & 0.0501 & 3.1 & 5.294 & 4.326 \\
\hline 0.2 & 1.0100 & 0.1005 & 3.2 & 5.747 & 4.734 \\
\hline 0.3 & 1.0226 & 0.1517 & 3.3 & 6.243 & 5.181 \\
\hline 0.4 & 1.0404 & 0.2040 & 3.4 & 6.785 & 5.670 \\
\hline 0.5 & 1.0635 & 0.2579 & 3.5 & 7.378 & 6.206 \\
\hline 0.6 & 1.0920 & 0.3137 & 3.6 & 8.028 & 6.793 \\
\hline 0.7 & 1.1263 & 0.3719 & 3.7 & 8.739 & 7.436 \\
\hline 0.8 & 1.1665 & 0.4329 & 3.8 & 9.517 & 8.140 \\
\hline 0.9 & 1.2130 & 0.4971 & 3.9 & 10.369 & 8.913 \\
\hline 1.0 & 1.2661 & 0.5652 & 4.0 & 11.30 & 9.76 \\
\hline 1.1 & 1.3262 & 0.6375 & 4.1 & 12.32 & 10.69 \\
\hline 1.2 & 1.3937 & 0.7147 & 4.2 & 13.44 & 11.71 \\
\hline 1.3 & 1.4693 & 0.7973 & 4.3 & 14.67 & 12.82 \\
\hline 1.4 & 1.5534 & 0.8861 & 4.4 & 16.01 & 14.05 \\
\hline 1.5 & 1.6467 & 0.9817 & 4.5 & 17.48 & 15.39 \\
\hline 1.6 & 1.7500 & 1.0848 & 4.6 & 19.09 & 16.86 \\
\hline 1.7 & 0.8640 & 1.1963 & 4.7 & 20.86 & 18.48 \\
\hline 1.8 & 1.9806 & 1.3172 & 4.8 & 22.79 & 20.25 \\
\hline 1.9 & 2.1277 & 1.4482 & 4.9 & 24.91 & 22.20 \\
\hline 2.0 & 2.280 & 1.591 & 5.0 & 27.24 & 24.34 \\
\hline 2.1 & 2.446 & 1.746 & 5.1 & 29.79 & 26.68 \\
\hline 2.2 & 2.629 & 1.914 & 5.2 & 32.58 & 29.25 \\
\hline 2.3 & 2.830 & 2.098 & 5.3 & 35.65 & 32.08 \\
\hline 2.4 & 3.049 & 2.298 & 5.4 & 39.01 & 35.18 \\
\hline 2.5 & 3.290 & 2.517 & 5.5 & 42.70 & 38.59 \\
\hline 2.6 & 3.553 & 2.755 & 5.6 & 46.74 & 42.33 \\
\hline 2.7 & 3.842 & 3.016 & 5.7 & 51.17 & 46.44 \\
\hline 2.8 & 4.157 & 3.301 & 5.8 & 56.04 & 50.95 \\
\hline 2.9 & 4.503 & 3.613 & 5.9 & 61.38 & 55.90 \\
\hline
\end{tabular}


TABLE 13.4.4. Zero and first order modified Bessel functions of the second kind (source: Ref. 2, p. 374-5).

$\mathrm{u} \quad \frac{2}{\pi} \mathrm{K}_{0}(u) \quad \frac{2}{\pi_{1}}(u) \quad u \quad \frac{2}{\pi_{0}(u)} \quad \frac{2}{\pi_{1}}(u)$

\begin{tabular}{|c|c|c|c|c|c|}
\hline 0.0 & $\infty$ & $\infty$ & 2.0 & 0.072 & 0.089 \\
\hline 0.1 & 1.545 & 6.270 & 2.1 & 0.064 & 0.078 \\
\hline 0.2 & 1.116 & 3.040 & 2.2 & 0.057 & 0.069 \\
\hline 0.3 & 0.874 & 1.946 & 2.3 & 0.050 & 0.060 \\
\hline 0.4 & 0.710 & 1.391 & 2.4 & 0.045 & 0.053 \\
\hline 0.5 & 0.588 & 1.054 & 2.5 & 0.040 & 0.047 \\
\hline 0.6 & 0.495 & 0.829 & 2.6 & 0.035 & 0.042 \\
\hline 0.7 & 0.420 & 0.669 & 2.7 & 0.031 & 0.037 \\
\hline 0.8 & 0.360 & 0.549 & 2.8 & 0.028 & 0.032 \\
\hline 0.9 & 0.310 & 0.456 & 2.9 & 0.025 & 0.029 \\
\hline 1.0 & 0.268 & 0.383 & 3.0 & 0.022 & 0.026 \\
\hline 1.1 & 0.233 & 0.324 & 3.1 & 0.020 & 0.023 \\
\hline 1.2 & 0.203 & 0.277 & 3.2 & 0.018 & 0.020 \\
\hline 1.3 & 0.177 & 0.237 & 3.3 & 0.016 & 0.018 \\
\hline 1.4 & 0.155 & 0.204 & 3.4 & 0.014 & 0.016 \\
\hline 1.5 & 0.136 & 0.177 & 3.5 & 0.012 & 0.014 \\
\hline 1.6 & 0.120 & 0.153 & 3.6 & 0.011 & 0.013 \\
\hline 1.7 & 0.105 & 0.133 & 3.7 & 0.010 & 0.011 \\
\hline 1.8 & 0.093 & 0.116 & 3.8 & 0.009 & 0.010 \\
\hline 1.9 & 0.082 & 0.102 & 3.9 & 0.008 & 0.009 \\
\hline
\end{tabular}


13.5. Legendre Polynomials

The Legendre polynomial of degree $n$, of the first kind:

$$
\begin{aligned}
& P_{n}(u)=(-1)^{n / 2} \frac{1 \cdot 3 \cdot 5 \cdots(n-1)}{2 \cdot 4 \cdot 6 \cdots n}\left[1-\frac{n(n+1)}{2 !} u^{2}\right. \\
& \left.+\frac{n(n-2)(n+1)(n+3)}{4 !} u^{4}+\cdots\right], n=2,4,6, \ldots \\
& P_{n}(u)=(-1)^{(n-1) / 2} \frac{1 \cdot 3 \cdot 5 \cdot \cdots n}{2 \cdot 4 \cdot 6 \cdot \cdots(n-1)}\left[u-\frac{(n-1)(n+2)}{3 !} u^{3}\right. \\
& \left.\div \frac{(n-1)(n-3)(n+2)(n+4)}{5 !} u^{5}+\cdots\right], n=3,5,7, \ldots,|u|<1 \\
& P_{0}(u)=1, P_{1}(u)=u, P_{2}(u)=\left\langle 3 u^{2}-1\right\rangle / 2, \\
& P_{3}(u)=\left(5 u^{3}-3 u\right) / 2, P_{4}(u)=\left(35 u^{4}-30 u^{2}+3\right) / 8 \text {, } \\
& \mathrm{g}_{5}(u)=\left(63 u^{5}-70 u^{3}+15 u\right) / B,|u|<1
\end{aligned}
$$

The Legendre polynomial of degree $n$, of the second kind:

$$
\begin{aligned}
Q_{n}(u)= & (-1)^{(n+1) / 2} \frac{2 \cdot 4 \cdot 6 \cdots(n-1)}{1 \cdot \frac{6}{3} \cdot n}\left[1-\frac{n(n+1)}{2 !} u^{2}\right. \\
& \left.+\frac{n(n-2)(n+1)(n+3)}{4 !} u^{4}+\cdots \cdots\right], n=3,5,7, \ldots, \\
Q_{n}(u)= & (-1)^{n / 2} \frac{2 \cdot 4 \cdot 6 \cdots n}{1 \cdot 3 \cdot 5 \cdots(n-1)}\left[u-\frac{(n-1)(n+2)}{3 !} u^{3}\right. \\
& \left.+\frac{(n-1)(n-3)(n+2)(n+4)}{5 !} u^{5}+\cdots\right], n=2,4,6, \ldots,
\end{aligned}
$$




$$
\begin{aligned}
& Q_{0}(u)=\left\{\begin{array}{l}
\frac{1}{2} \ln \left(\frac{1+u}{1-u}\right),|u|<1 \\
\frac{1}{2} \ln \left(\frac{u+1}{u-1}\right),|u|<1
\end{array}\right. \\
& Q_{1}(u)=Q_{0}(u) P_{1}(u)-1, \\
& Q_{2}(u)=Q_{0}(u) P_{2}(u)-3 u / 2 \\
& Q_{3}(u)=Q_{0}(u) P_{3}(u)-5 u^{2} / 2+\frac{2}{3}, \\
& Q_{4}(u)=Q_{0}(u) P_{4}(u)-35 u^{3} / 8+55 u^{2} / 24, \\
& Q_{n}(u)=Q_{0}(u) P_{n}(u)-\frac{(2 n-1)}{1-n} P_{n-1}(u)-\frac{(2 n-5)}{3(n-1)} P_{n-3}(u)-\ldots,
\end{aligned}
$$


TABLE 1:.5. The first five Legendre polynomials of the first kind (source: Ref. 2 , p. $375-7$ ).

\begin{tabular}{|c|c|c|c|c|c|}
\hline $\mathbf{u}$ & $P_{1}(u)$ & $\mathrm{P}_{2}(\mathrm{u})$ & $\mathrm{P}_{3}(\mathrm{u})$ & $\mathrm{P}_{4}(\mathrm{u})$ & $\mathrm{P}_{5}(\mathrm{u})$ \\
\hline 0.00 & 0.0000 & -0.5000 & 0.0000 & 0.3750 & 0.0000 \\
\hline 0.01 & 0.0100 & -0.4998 & -0.0150 & 0.3746 & 0.0187 \\
\hline 0.02 & 0.0200 & -0.4994 & -0.0300 & 0.3735 & 0.0374 \\
\hline 0.03 & 0.0300 & -0.4986 & -0.0449 & 0.3716 & 0.0560 \\
\hline 0.04 & 0.0400 & -0.4976 & -0.0598 & 0.3690 & 0.0744 \\
\hline 0.05 & 0.0500 & -0.4962 & -0.0747 & 0.3657 & 0.0927 \\
\hline 0.06 & 0.0600 & -0.4946 & -0.0895 & 0.3616 & 0.1106 \\
\hline 0.07 & 0.0700 & -0.4926 & -0.1041 & 0.3567 & 0.1283 \\
\hline 0.08 & 0.0800 & -0.4904 & -0.1187 & 0.3512 & 0.1455 \\
\hline 0.09 & 0.0900 & -0.4878 & -0.1332 & 0.3449 & 0.1624 \\
\hline 0.10 & 0.1000 & -0.4850 & -0.1475 & 0.3379 & $0.178 B$ \\
\hline 0.11 & 0.1100 & -0.4818 & -0.1617 & 0.3303 & 0.1947 \\
\hline 0.12 & 0.1200 & -0.4784 & -0.1757 & 0.3219 & 0.2101 \\
\hline 0.13 & 0.1300 & -0.4746 & -0.1895 & 0.3129 & 0.2248 \\
\hline 0.14 & 0.1400 & -0.4906 & -0.2031 & 0.3032 & 0.2389 \\
\hline 0.15 & 0.1500 & -0.4662 & -0.2166 & 0.2928 & 0.2523 \\
\hline 0.16 & 0.1600 & -0.4616 & $-0.2 \div 98$ & 0.2819 & 0.2650 \\
\hline 0.17 & 0.1700 & -0.4566 & -0.2427 & 0.2703 & 0.2769 \\
\hline 0.18 & 0.1800 & -0.4514 & -0.2554 & 0.2581 & 0.2880 \\
\hline 0.19 & 0.1900 & -0.4458 & -0.2679 & 0.2453 & 0.2982 \\
\hline 0.20 & 0.2000 & -0.4400 & -0.2800 & 0.2320 & 0.3075 \\
\hline 0.21 & 0.2100 & -0.4338 & -0.2918 & 0.2181 & 0.3159 \\
\hline 0.22 & 0.2300 & -0.4274 & -0.3034 & 0.2037 & 0.3234 \\
\hline 0.23 & 0.2300 & -0.4206 & -0.3146 & 0.1889 & 0.3299 \\
\hline 0.24 & 0.2400 & -0.4136 & -0.3254 & 0.1735 & 0.3353 \\
\hline 0.25 & 0.2500 & -0.4062 & -0.3359 & 0.1577 & 0.3397 \\
\hline 0.26 & 0.2600 & -0.3986 & -0.3461 & 0.1415 & 0.3431 \\
\hline 0.27 & 0.2700 & -0.3906 & -0.3558 & 0.1249 & 0.3453 \\
\hline 0.28 & 0.2800 & -0.3824 & -0.3651 & 0.1079 & 0.3465 \\
\hline 0.29 & 0.2900 & -0.3738 & -0.3740 & 0.0905 & 0.3465 \\
\hline
\end{tabular}


TABLE 13.5. (Continued.)

\begin{tabular}{|c|c|c|c|c|c|}
\hline $\mathbf{u}$ & $P_{1}(u)$ & $P_{2}(u)$ & $P_{3}(u)$ & $P_{4}(u)$ & $P_{5}(u)$ \\
\hline 0.30 & 0.3000 & -0.3650 & -0.3825 & 0.0729 & 0.3454 \\
\hline 0.31 & 0.3100 & -0.3558 & -0.3905 & 0.0550 & 0.3431 \\
\hline 0.32 & 0.3200 & -0.3464 & -0.3981 & 0.0369 & 0.3397 \\
\hline 0.33 & 0.3300 & -0.3366 & -0.4052 & 0.0185 & 0.3351 \\
\hline 0.34 & 0.3400 & -0.3266 & -0.4117 & 0.0000 & 0.3294 \\
\hline 0.35 & 0.3500 & -0.3162 & -0.4178 & -0.0187 & 0.3225 \\
\hline 0.36 & 0.3600 & -0.3056 & -0.4234 & -0.0375 & 0.3144 \\
\hline 0.37 & 0.3700 & -0.2946 & -0.4284 & -0.0564 & 0.3051 \\
\hline 0.38 & 0.3800 & -0.2834 & -0.4328 & $-0.075 ?$ & 0.2948 \\
\hline 0.39 & 0.3900 & -0.2718 & -0.4367 & -0.0942 & 0.2833 \\
\hline 0.40 & 0.4000 & -0.2600 & -0.4400 & -0.1130 & 0.2706 \\
\hline 0.41 & 0.4100 & -0.2478 & -0.4427 & -0.1317 & 0.2569 \\
\hline 0.42 & 0.4200 & -0.2354 & -0.4448 & -0.1504 & 0.2421 \\
\hline 0.43 & 0.4300 & -0.2226 & -0.4462 & -0.1688 & 0.2263 \\
\hline 0.44 & 0.4400 & -0.2096 & -0.4470 & -0.1870 & 0.2095 \\
\hline 0.45 & 0.4500 & -0.1962 & -0.4472 & -0.2050 & 0.1917 \\
\hline 0.46 & 0.4600 & -0.1826 & -0.4467 & -0.2226 & 0.1730 \\
\hline 0.47 & 0.4700 & -0.1686 & -0.4454 & -0.2399 & 0.1534 \\
\hline 0.48 & 0.4800 & -0.1544 & -0.4435 & -0.2568 & 0.1330 \\
\hline 0.49 & 0.4900 & -0.1398 & -0.4409 & -0.2732 & 0.1118 \\
\hline 0.50 & 0.5000 & -0.1250 & -0.4375 & -0.2891 & 0.0898 \\
\hline 0.51 & 0.5100 & -0.1098 & -0.4334 & -0.3044 & 0.0673 \\
\hline 0.52 & 0.5200 & -0.0944 & -0.4285 & -0.3191 & 0.0441 \\
\hline 0.53 & 0.5300 & -0.0786 & -0.4228 & -0.3332 & 0.0204 \\
\hline 0.54 & 0.5400 & -0.0626 & -0.4163 & -0.3465 & -0.0037 \\
\hline 0.55 & 0.5500 & -0.0462 & -0.4091 & -0.3590 & -0.0282 \\
\hline 0.56 & 0.5600 & -0.0296 & -0.4010 & -0.3707 & -0.0529 \\
\hline 0.57 & 0.5700 & -0.0126 & -0.3920 & -0.3815 & -0.0779 \\
\hline 0.58 & 0.5800 & 0.0046 & -0.3822 & -0.3914 & -0.1028 \\
\hline 0.59 & 0.5900 & 0.0222 & -0.3716 & -0.4002 & -0.1278 \\
\hline
\end{tabular}


TABLE 13.5. (Continued.)

\begin{tabular}{|c|c|c|c|c|c|}
\hline $\mathbf{u}$ & $P_{1}(u)$ & $P_{2}(u)$ & $P_{3}(u)$ & $P_{4}(u)$ & $P_{5}(u)$ \\
\hline 0.60 & 0.6000 & 0.0400 & -0.3600 & -0.4080 & -0.1526 \\
\hline 0.61 & 0.6100 & 0.0582 & -0.3475 & -0.4146 & -0.1772 \\
\hline 0.62 & 0.6200 & 0.0766 & -0.3342 & -0.4200 & -0.2014 \\
\hline 0.63 & 0.6300 & 0.0954 & -0.3199 & -0.4242 & -0.2251 \\
\hline 0.64 & 0.6400 & 0.1144 & -0.3046 & -0.4270 & -0.2482 \\
\hline 0.65 & 0.6500 & 0.1338 & -0.2884 & -0.4284 & -0.2705 \\
\hline 0.66 & 0.6600 & 0.1534 & -0.2713 & -0.4284 & -0.2919 \\
\hline 0.67 & 0.6700 & 0.1734 & -0.2531 & -0.4268 & -0.3122 \\
\hline 0.68 & 0.6800 & 0.1936 & -0.2339 & -0.4236 & -0.3313 \\
\hline 0.69 & 0.6900 & 0.2142 & -0.2137 & -0.4187 & -0.3490 \\
\hline 0.70 & 0.7000 & 0.2350 & -0.1925 & -0.4121 & -0.3652 \\
\hline 0.71 & 0.7100 & 0.2562 & -0.1702 & -0.4036 & -0.3796 \\
\hline 0.72 & 0.7200 & 0.2776 & -0.1469 & -0.3933 & -0.3922 \\
\hline 0.73 & 0.7300 & 0.2994 & -0.1225 & -0.3810 & -0.4026 \\
\hline 0.74 & 0.7400 & 0.3214 & -0.0969 & -0.3666 & -0.4107 \\
\hline 0.75 & 0.7500 & 0.3438 & -0.0703 & -0.3501 & -0.4164 \\
\hline 0.76 & 0.7600 & 0.3664 & -0.0426 & -0.3314 & -0.4193 \\
\hline 0.77 & 0.7700 & 0.3894 & -0.0137 & -0.3104 & -0.4193 \\
\hline 0.78 & 0.7800 & 0.4126 & 0.0164 & -0.2871 & -0.4162 \\
\hline 0.79 & 0.7900 & 0.4362 & 0.0476 & -0.2613 & -0.4097 \\
\hline 0.80 & 0.8000 & 0.4600 & 0.0900 & -0.2330 & -0.3995 \\
\hline 0.81 & 0.8100 & 0.4842 & 0.1136 & -0.2021 & -0.3855 \\
\hline 0.82 & 0.8200 & 0.5086 & 0.1484 & -0.1685 & -0.3674 \\
\hline 0.83 & 0.8300 & 0.5334 & 0.1845 & $-0.132 i$ & -0.3449 \\
\hline 0.84 & 0.8400 & 0.5584 & 0.2218 & -0.0928 & -0.3177 \\
\hline 0.85 & 0.8500 & 0.5838 & 0.2603 & -0.0506 & -0.2857 \\
\hline 0.86 & 0.8600 & 0.6094 & 0.3001 & -0.0053 & -0.2484 \\
\hline 0.87 & 0.8700 & 0.6354 & 0.3413 & 0.0431 & -0.2056 \\
\hline 0.88 & 0.8800 & 0.6616 & 0.3837 & 0.0947 & -0.1570 \\
\hline 0.89 & 0.8900 & 0.6882 & 0.4274 & 0.1496 & -0.1023 \\
\hline
\end{tabular}


TABLE 13.5. (Continued.)

\begin{tabular}{llllll}
\hline$u$ & $P_{1}(u)$ & $P_{2}(u)$ & $P_{3}(u)$ & $P_{4}(u)$ & $P_{5}(u)$ \\
\hline 0.90 & 0.9000 & 0.7150 & 0.4725 & 0.2079 & -0.0411 \\
0.91 & 0.9100 & 0.7422 & 0.5189 & 0.2698 & 0.0268 \\
0.92 & 0.9200 & 0.7696 & 0.5667 & 0.3352 & 0.1017 \\
0.93 & 0.9300 & 0.7974 & 0.6159 & 0.4044 & 0.1842 \\
0.94 & 0.9400 & 0.8254 & 0.6665 & 0.4773 & 0.2744 \\
0.95 & 0.9500 & 0.8538 & 0.7184 & 0.5541 & 0.3727 \\
0.96 & 0.9600 & 0.8824 & 0.7718 & 0.6349 & 0.4796 \\
0.97 & 0.9700 & 0.9114 & 0.8267 & 0.7198 & 0.5954 \\
0.98 & 0.9800 & 0.9406 & 0.8830 & 0.8089 & 0.7204 \\
0.99 & 0.9900 & 0.9702 & 0.9407 & 0.9022 & 0.8552 \\
1.00 & 1.0000 & 1.0000 & 1.0000 & 1.0000 & 1.0000 \\
\hline
\end{tabular}


13.6. Sine, Cosine, and Exponential Integrals.

Sine integral: $\operatorname{si}(x)=\int_{0}^{x} \frac{\text { sir. }(u)}{u} d u$,

$\operatorname{Si}(\infty)=\pi / 2$

Cosine integral: $\quad \operatorname{Ci}(x)=-\int_{x}^{\infty} \frac{\cos (u)}{u} d u=\ell n(\gamma x)-\int_{0}^{x} \frac{1-\cos (u)}{u} d u$

$$
\text { ln } Y=0.577215 \ldots \text { (Euler's constant) }
$$

Exponential integral: $E i(x)=-\int_{x}^{\infty} \frac{e^{-u}}{u} d u,\langle 0, \infty>x>0$

Logarithmic integral: $\quad l i(x)=\int_{0}^{x} \frac{d u}{\ln (u)}=\overline{E i}(f a x)$

$$
\ell i\left(e^{x}\right)=\overline{E i}(x)
$$

For small values of $x$ :

$$
\begin{aligned}
& s i(x) \simeq x \\
& \operatorname{li}(x) \simeq-x / \ln (1 / x)
\end{aligned}
$$

$$
\operatorname{Ci}(x) \simeq \operatorname{Ei}(-x) \simeq \overline{E i}(x) \simeq \ln (1 / \gamma x)=\gamma+\ln (x)-x+\left(x^{2} / 4\right)+O\left(x^{3}\right)
$$

For large values of $x$ :

$$
\begin{aligned}
& \operatorname{Si}(x) \simeq \pi / 2-\cos (x) / x \\
& \operatorname{Ci}(x)=\sin (x) / x \\
& \overline{E i}(x)=\left(e^{x} / x\right)(1+11 / x+21 / x+31 / x+\cdots),|x|>>1
\end{aligned}
$$


TABLE 12.6. Values of the sine, cosine, logarithmic, and exponential integrals. (source: Ref. 18, p. 6-9.)

\begin{tabular}{|c|c|c|c|c|}
\hline $\mathbf{x}$ & $S i(x)$ & $C i(x)$ & $\overline{\operatorname{Ei}}(x)$ & Ei $(-x)$ \\
\hline 0.00 & +0.000000 & $-\infty$ & $-\infty$ & $\infty$ \\
\hline 0.01 & +0.010000 & -4.0280 & -4.0179 & -4.0379 \\
\hline 0.02 & +0.019999 & -3.3349 & -3.3147 & -3.3547 \\
\hline 0.03 & +0.029998 & -2.9296 & -2.8991 & -2.9591 \\
\hline 0.04 & +0.039996 & -2.6421 & -2.6013 & -2.5813 \\
\hline 0.05 & +0.04999 & -2.4191 & -2.3679 & -2.4679 \\
\hline 0.06 & +0.05999 & -2.2371 & -2.1753 & -2.2953 \\
\hline 0.07 & +0.06998 & -2.0833 & -2.0108 & -2.1508 \\
\hline 0.08 & +0.07997 & -1.9501 & -1.8669 & -2.0269 \\
\hline 0.09 & +0.08996 & -1.8328 & -1.7387 & -1.9187 \\
\hline 0.10 & +0.09994 & -1.7279 & -1.6228 & -1.8229 \\
\hline 0.11 & +0.10993 & -1.6331 & -1.5170 & -1.7371 \\
\hline 0.12 & +0.11990 & -1.5466 & -1.4193 & -1.6595 \\
\hline 0.13 & +0.12988 & -1.4672 & -1.3287 & -1.5889 \\
\hline 0.14 & +0.13985 & -1.3938 & -1.2438 & -1.5241 \\
\hline 0.15 & +0.14981 & -1.3255 & -1.1641 & -1.4645 \\
\hline 0.16 & +0.15977 & -1.2618 & -1.0887 & -1.4092 \\
\hline 0.17 & +0.16973 & -1.2020 & -1.0172 & -1.3578 \\
\hline 0.18 & +0.1797 & -1.1457 & -0.9491 & -1.3098 \\
\hline 0.19 & +0.1896 & -1.0925 & -0.8841 & -1.2649 \\
\hline 0.20 & +0.1996 & -1.0422 & -0.8218 & -1.2227 \\
\hline 0.21 & +0.2095 & -0.9944 & -0.7619 & -1.1829 \\
\hline 0.22 & +0.2194 & -0.9490 & -0.7042 & -1.1454 \\
\hline 0.23 & +0.2293 & -0.9057 & -0.6485 & -1.1099 \\
\hline 0.24 & +0.2392 & -0.8643 & -0.5947 & -1.0762 \\
\hline 0.25 & +0.2491 & -0.8247 & -0.5425 & -1.0443 \\
\hline 0.26 & +0.2590 & -0.7867 & -0.4919 & -1.0139 \\
\hline 0.27 & +0.2689 & -0.7503 & -0.4427 & -0.9849 \\
\hline 0.28 & +0.2788 & 0.7153 & -0.3949 & -0.9573 \\
\hline 0.29 & +0.2886 & -0.6816 & -0.3482 & -0.9309 \\
\hline
\end{tabular}


TABLE 13.6. (Continued.)

\begin{tabular}{|c|c|c|c|c|}
\hline $\mathbf{x}$ & $\operatorname{si}(x)$ & $\mathrm{Ci}(\mathrm{x})$ & $\overline{E i}(x)$ & $\operatorname{Ei}(-x)$ \\
\hline 0.30 & +0.2985 & -0.6492 & -0.3027 & -0.9057 \\
\hline 0.31 & +0.3083 & -0.6179 & -0.2582 & -0.8815 \\
\hline 0.32 & +0.3182 & -0.5877 & -0.2147 & -0.8583 \\
\hline 0.33 & +0.3280 & -0.5585 & -0.17210 & -0.8361 \\
\hline 0.34 & +0.3378 & -0.5304 & -0.13036 & -0.8147 \\
\hline 0.35 & +0.3476 & -0.5031 & -0.08943 & -0.7942 \\
\hline 0.35 & +0.3574 & -0.4767 & -0.04926 & -0.7745 \\
\hline 0.37 & +0.3672 & -0.4511 & -0.00979 & -0.7554 \\
\hline 0.38 & +0.3770 & -0.4263 & +0.02901 & -0.7371 \\
\hline 0.39 & +0.3867 & -0.4022 & +0.06718 & -0.7194 \\
\hline 0.40 & +0.3965 & -0.3788 & +0.10477 & -0.7024 \\
\hline 0,41 & +0.4062 & -0.3561 & +0.14179 & -0.6859 \\
\hline 0.42 & +0.4159 & -0.3341 & +0.17828 & -0.6700 \\
\hline 0.43 & +0.4256 & -0.3126 & +0.2143 & -0.6546 \\
\hline 0.44 & +0.4353 & -0.2918 & +0.2498 & -0.6397 \\
\hline 0.45 & +0.4450 & -0.2715 & +0.2849 & -0.6253 \\
\hline 0.46 & +0.4546 & -0.2517 & +0.3195 & -0.6114 \\
\hline 0.47 & +0.4643 & -0.2325 & +0.3537 & -0.5979 \\
\hline 0.48 & +0.4739 & -0.2138 & +0.3876 & -0.5848 \\
\hline 0.49 & +0.4835 & -0.1956 & +0.4211 & -0.5721 \\
\hline 0.50 & +0.4931 & -0.17778 & $+0,4542$ & -0.5598 \\
\hline 0.51 & +0.5027 & -0.16045 & +0.4870 & -0.5478 \\
\hline 0.52 & +0.5123 & -0.14355 & +0.5195 & -0.5362 \\
\hline 0.53 & +0.5218 & -0.12707 & +0.5517 & -0.5250 \\
\hline 0.54 & +0.5313 & $-0,11099$ & +0.5836 & -0.5140 \\
\hline 0.55 & +0.5408 & -0.09530 & +0.6153 & -0.5034 \\
\hline 0.56 & +0.5503 & -0.07999 & +0.6467 & -0.4930 \\
\hline 0.57 & +0.5598 & -0.06504 & +0.6778 & -0.4830 \\
\hline 0.58 & +0.5693 & -0.05044 & +0.7087 & -0.4732 \\
\hline 0.59 & +0.5787 & -0.03619 & +0.7394 & -0.4636 \\
\hline
\end{tabular}


TABLE 13.6. (Continued.)

\begin{tabular}{|c|c|c|c|c|}
\hline$x$ & $S i(x)$ & $C i(x)$ & $\overline{\mathrm{E}} \overline{\mathrm{i}}(\mathrm{x})$ & $\operatorname{Ei}(-\mathbf{x})$ \\
\hline 0.60 & +0.5881 & -0.02227 & +0.7699 & -0.4544 \\
\hline 0.61 & +0.5975 & -0.008675 & $+0.800 \bar{z}$ & -0.4454 \\
\hline 0.62 & +0.6069 & +0.004606 & +0.8302 & -0.4366 \\
\hline 0.63 & +0.6163 & +0.01758 & +0.8601 & -0.4280 \\
\hline 0.64 & +0.6256 & .0 .03026 & +0.8898 & -0.4197 \\
\hline 0.65 & +0.6349 & +0.04265 & +0.9194 & -0.4115 \\
\hline 0.66 & +0.6442 & +0.05476 & +0.9488 & -0.4036 \\
\hline 0.67 & +0.6535 & +0.06659 & +0.9780 & -0.3959 \\
\hline 0.68 & +0.6628 & +0.07816 & +1.0071 & -0.3883 \\
\hline 0.69 & +0.6720 & +0.08946 & +1.0361 & -0.3810 \\
\hline 0.70 & +0.6812 & +0.10051 & +1.0649 & -0.3738 \\
\hline 0.71 & +0.6904 & +0.11132 & +1.0936 & -0.3668 \\
\hline 0.72 & +0.6996 & +0.12188 & +1.1222 & -0.3599 \\
\hline 0.73 & +0.7087 & +0.13220 & +1.1507 & -0.3532 \\
\hline 0.74 & +0.7179 & +0.14230 & +1.1791 & -0.3467 \\
\hline 0.75 & +0.7270 & +0.15216 & +1.2073 & -0.3403 \\
\hline 0.76 & +0.7360 & +0.16181 & +1.2355 & -0.3341 \\
\hline 0.77 & +0.7451 & +0.17124 & +1.2636 & -0.3280 \\
\hline 0.78 & +0.7541 & +0.1805 & +1.2916 & -0.3221 \\
\hline 0.79 & +0.7631 & +0.1895 & +1.3195 & -0.3163 \\
\hline 0.80 & +0.7721 & +0.1983 & +1.3474 & -0.3106 \\
\hline 0.81 & +0.7811 & +0.2069 & +1.3752 & -0.3050 \\
\hline 0.82 & +0.7900 & +0.2153 & +1.4029 & -0.2996 \\
\hline 0.83 & +0.7989 & +0.2235 & +1.4306 & -0.2943 \\
\hline 0.84 & +0.8078 & +0.2316 & +1.4582 & -0.2891 \\
\hline 0.85 & +0.8166 & +0.2394 & +1.4857 & -0.2840 \\
\hline 0.86 & +0.8254 & +0.2471 & +1.5132 & -0.2790 \\
\hline 0.87 & +0.8342 & +0.2546 & +1.5407 & -0.2742 \\
\hline 0.88 & +0.8430 & +0.2619 & +1.5681 & -0.2694 \\
\hline 0.89 & +0.8518 & +0.2691 & +1.5955 & -0.2647 \\
\hline
\end{tabular}


TABLE 13.6. (Continued.)

\begin{tabular}{|c|c|c|c|c|}
\hline $\mathbf{x}$ & Si (x) & $\operatorname{Ci}(x)$ & $\overline{E i}(x)$ & $\operatorname{Ei}(-x)$ \\
\hline 0.90 & +0.8605 & +0.2761 & +1.6228 & -0.2602 \\
\hline 0.91 & +0.8692 & +0.2829 & +1.6501 & -0.2557 \\
\hline 0.92 & +0.8778 & +0.2896 & +1.6774 & -0.2513 \\
\hline 0.93 & +0.8865 & +0.2961 & +1.7047 & -0.2470 \\
\hline 0.94 & +0.8951 & +0.3024 & +1.7319 & -0.2429 \\
\hline 0.95 & +0.9036 & +0.3086 & 11.7591 & -0.2387 \\
\hline 0.96 & +0.9122 & +0.3147 & +1.7864 & -0.2347 \\
\hline 0.97 & +0.9207 & +0.3205 & +1.8136 & -0.2308 \\
\hline 0.98 & +0.9292 & +0.3263 & +1.8407 & -0.2269 \\
\hline 0.99 & +0.9377 & +0.3319 & +1.8679 & -0.2231 \\
\hline 1.00 & +0.9461 & +0.3374 & +1.8951 & -0.2194 \\
\hline 1.0 & +0.9461 & +0.3374 & +1.8951 & -0.2194 \\
\hline 1.1 & +1.0287 & +0.3849 & +2.1674 & -0.1860 \\
\hline 1.2 & +1.1080 & +0.4205 & +2.4421 & -0.1584 \\
\hline 1.3 & +1.1840 & +0.4457 & +2.7214 & -0.1355 \\
\hline 1.4 & +1.2562 & +0.4620 & +3.0072 & -0.1162 \\
\hline 1.5 & +1.3247 & +0.4704 & +3.3013 & -0.1000 \\
\hline 1.6 & +1.3892 & +0.4717 & +3.6053 & -0.08631 \\
\hline 1.7 & +1.4496 & +0.4670 & +3.9210 & -0.07465 \\
\hline 1.8 & +1.5058 & +0.4568 & +4.2499 & -0.06471 \\
\hline 1.9 & +1.5578 & +0.4419 & +4.5937 & -0.05620 \\
\hline 2.0 & +1.6054 & +0.4230 & +4.9542 & -0.04890 \\
\hline 2.1 & +1.6487 & +0.4005 & +5.3332 & -0.04261 \\
\hline 2.2 & +1.6876 & +0.3751 & +5.7326 & -0.03719 \\
\hline 2.3 & +1.7222 & +0.3472 & +6.1544 & -0.03250 \\
\hline 2.4 & +1.7525 & +0.3173 & +6.6007 & -0.02844 \\
\hline 2.5 & +1.7785 & +0.2859 & +7.0738 & -0.02491 \\
\hline 2.6 & +1.8004 & +0.2533 & +7.5761 & -0.02185 \\
\hline 2.7 & +1.8182 & +0.2201 & +8.1103 & -0.01918 \\
\hline 2.8 & +1.8321 & +0.1865 & +8.6793 & -0.01686 \\
\hline 2.9 & +1.8422 & +0.1529 & +9.2860 & -0.01482 \\
\hline 3.0 & +1.8487 & +0.1196 & +9.9338 & -0.01304 \\
\hline
\end{tabular}


TABLE 13.6. (Continued.)

\begin{tabular}{|c|c|c|c|c|}
\hline $\mathbf{x}$ & Si $(x)$ & $\operatorname{Ci}(x)$ & $\overline{\mathrm{E}} \mathbf{I}(x)$ & $\operatorname{Ei}(-x)$ \\
\hline 3.1 & +1.8517 & +0.08699 & +10.6263 & -0.01149 \\
\hline 3.2 & +1.8514 & +0.05526 & +11.3673 & -0.01013 \\
\hline 3.3 & +1.8481 & +0.02468 & +12.1610 & $-0.0^{2} 8939$ \\
\hline 3.4 & +1.8419 & +0.004518 & +13.0121 & $-0.0^{2} 7890$ \\
\hline 3.5 & +1.8331 & -0.03213 & +13.9254 & $-0.0^{2} 6970$ \\
\hline 3.6 & +1.8219 & -0.05797 & +14.9063 & $-0.0^{2} 6160$ \\
\hline 3.7 & +1.8086 & -0.08190 & +15.9606 & $-0.0^{2} 5448$ \\
\hline 3.8 & +1.7934 & -0.1038 & +17.0948 & $-0.0^{2} 4820$ \\
\hline 3.9 & +1.7765 & -0.1235 & +18.3157 & $-0.0^{2} 4267$ \\
\hline 4.0 & +1.7582 & -0.1410 & +19.6309 & $-0.0^{2} 3779$ \\
\hline 4.1 & +1.7387 & -0.1562 & +21.0485 & $-0.0^{2} 3349$ \\
\hline 4.2 & +1.7184 & -0.1690 & +22.5774 & $-0.0^{2} 2969$ \\
\hline 4.3 & +1.6973 & -0.1795 & +24.2274 & $-0.0^{2} 2633$ \\
\hline 4.4 & +1.6758 & -0.1877 & +26.0090 & $-0.0^{2} 2336$ \\
\hline 4.5 & +1.6541 & -0.1935 & +27.9337 & $-0.0^{2} 2073$ \\
\hline 4.6 & +1.6325 & -0.1970 & +30.0141 & $-0.0^{2} 1841$ \\
\hline 4.7 & +1.6110 & -0.1984 & +32.2639 & $-0.0^{2} 1635$ \\
\hline 4.8 & +1.5900 & -0.1976 & +34.6979 & $-0.0^{2} 1453$ \\
\hline 4.9 & +1.5696 & -0.1948 & +37.3325 & $-0.0^{2} 1291$ \\
\hline 5.0 & +1.5499 & -0.1900 & +40.1853 & $-0.0^{2} 1148$ \\
\hline 6 & +1.4247 & -0.06806 & +85.9898 & $-0.0^{3} 3601$ \\
\hline 7 & +1.4546 & +0.07670 & +191.505 & $-0.0^{3} 1155$ \\
\hline 8 & +1.5742 & +0.1224 & +440.380 & $-0.0^{4} 3767$ \\
\hline 9 & +1.6650 & +0.05535 & +1037.88 & $-0.0^{4} 1245$ \\
\hline 10 & +1.6583 & -0.04546 & +2492.23 & $-0.0^{5} 4157$ \\
\hline 11 & +1.5783 & -0.08956 & +6071.41 & $-0.0^{5} 1400$ \\
\hline 12 & +1.5050 & -0.04978 & +14959.5 & $-0.0^{6} 4751$ \\
\hline 13 & +1.4994 & +0.02676 & +37197.7 & $-0.0^{6} 1622$ \\
\hline 14 & +1.5562 & +0.06940 & +93192.5 & $-0.0^{7} 5566$ \\
\hline 15 & +1.6182 & +0.04628 & +234956 & $-0.0^{7} 1918$ \\
\hline
\end{tabular}


TABLE 13.6. (Continued.)

\begin{tabular}{|c|c|c|c|c|c|}
\hline$x$ & $s i(x)$ & $C i(x)$ & $x$ & $S i(x)$ & $C i(x)$ \\
\hline 20 & +1.5482 & +0.04442 & 140 & +1.5722 & +0.007011 \\
\hline 25 & +1.5315 & -0.00685 & 150 & +1.5662 & -0.004800 \\
\hline 30 & +1.5668 & -0.03303 & 160 & +1.5769 & +0.001409 \\
\hline 35 & +1.5969 & -0.01148 & 170 & +1.5653 & +0.002010 \\
\hline 40 & +1.5870 & +0.01902 & 180 & +1.5741 & -0.004432 \\
\hline 45 & +1.5587 & +0.01863 & 190 & +1.5704 & +0.005250 \\
\hline 50 & +1.5516 & -0.00563 & 200 & +1.5684 & -0.004378 \\
\hline 55 & +1.5707 & -0.01817 & 300 & +1.5709 & -0.003332 \\
\hline 60 & +1.5867 & -0.00481 & 400 & +1.5721 & -0.002124 \\
\hline 65 & +1.5792 & +0.01285 & 500 & $\dot{+1.5726}$ & -0.0009320 \\
\hline 70 & +1.5616 & +0.01092 & 600 & +1.5725 & +0.0000764 \\
\hline 75 & +1.5586 & -0.00533 & 700 & +1.5720 & +0.0007788 \\
\hline 80 & +1.5723 & -0.01240 & 800 & +1.5714 & +0.001118 \\
\hline 85 & +1.5824 & -0.001935 & 900 & +1.5707 & +0.001109 \\
\hline 90 & +1.5757 & +0.009986 & $10^{2}$ & +1.5702 & +0.000826 \\
\hline 95 & +1.5630 & +0.007110 & $10^{4}$ & +1.5709 & -0.0000306 \\
\hline 100 & +1.5622 & -0.005149 & $10^{5}$ & +1.5708 & +0.0000004 \\
\hline 110 & +1.5799 & -0.000320 & $10^{6}$ & +1.5708 & -0.0000004 \\
\hline 120 & +1.5640 & +0.004781 & $10^{7}$ & +1.5708 & +0.0 \\
\hline 130 & +1.5737 & -0.007132 & $\infty$ & $1 / 2 \pi$ & 0.0 \\
\hline
\end{tabular}


SECTION 14. ROOTS OF SOME CHARACTERISTIC EQUATIONS

TABIE 14.1. First six roots of $\lambda_{n} \tan \lambda_{n}=C$. (Source: Ref. 74, p. 217.)

\begin{tabular}{|c|c|c|c|c|c|c|}
\hline$c$ & $\lambda_{1}$ & $\lambda_{2}$ & $\lambda_{3}$ & $\lambda_{4}$ & $\lambda_{5}$ & $\lambda_{6}$ \\
\hline 0 & 0.0000 & 3.1416 & 6.2832 & 9.4248 & 12.5664 & 15.7080 \\
\hline 0.001 & 0.0316 & 3.1419 & 6.2833 & 9.4249 & 12.5665 & 15.7080 \\
\hline 0.002 & 0.0447 & 3.1422 & 6.2835 & 9.4250 & 12.5665 & 15.7081 \\
\hline 0.004 & 0.0632 & 3.1429 & 6.2838 & 9.4252 & 12.5667 & 15.7082 \\
\hline 0.006 & 0.0774 & 3.1435 & 6.2841 & 9.4254 & 12.5668 & 15.7083 \\
\hline 0.008 & 0.0893 & 3.1441 & 6.2845 & 9.4256 & 12.5670 & 15.7085 \\
\hline 0.01 & 0.0998 & 3.1448 & $6.2 B 48$ & 9.4258 & 12.5672 & 15.7096 \\
\hline 0.02 & 0.1410 & 3.1479 & 6.2864 & 9.4269 & 12.5680 & 15.7092 \\
\hline 0.04 & 0.1987 & 3.1543 & 6.2895 & 9.4290 & 12.5696 & 15.7105 \\
\hline 0.06 & 0.2425 & 3.1606 & 6.2927 & 9.4311 & 12.5711 & 15.7118 \\
\hline 0.08 & 0.2791 & 3.1668 & 6.2959 & 9.4333 & 12.5727 & 15.7131 \\
\hline 0.1 & 0.0311 & 3.1731 & 6.2991 & 9.4354 & 12.5743 & 15.7143 \\
\hline 0.2 & 0.4328 & 3.2039 & 6.3148 & 9.4459 & 12.5823 & 15.7207 \\
\hline 0.3 & 0.5218 & 3.2341 & 6.3305 & 9.4565 & 12.5902 & 15.7270 \\
\hline 0.4 & 0.5932 & 3.2636 & 6.3461 & 9.4670 & 12.5981 & 15.7334 \\
\hline 0.5 & 0.6533 & 3.2923 & 6.3616 & 9.4775 & 12.6060 & 15.7397 \\
\hline 0.6 & 0.7051 & 3.3204 & 6.3770 & 9.4879 & 12.6139 & 15.7460 \\
\hline 0.7 & 0.7506 & 3.3477 & 6.3923 & 9.4983 & 12.6218 & 15.7524 \\
\hline 0.8 & 0.7910 & 3.3744 & 6.4074 & 9.5087 & 12.6296 & 15.7587 \\
\hline 0.9 & 0.8274 & 3.4003 & 6.4224 & 9.5190 & 12.6375 & 15.7650 \\
\hline 1.0 & 0.8603 & 3.4256 & 6.4373 & 9.5293 & 12.6453 & 15.7713 \\
\hline 1.5 & 0.9882 & 3.5422 & 6.5097 & 9.5801 & 12.6841 & 3.5 .8026 \\
\hline 2.0 & 1.0769 & 3.6436 & 6.5783 & 9.6296 & 12.7223 & 15.8336 \\
\hline 3.0 & 1.1925 & 3.8088 & 6.7040 & 9.7240 & 12.7966 & 15.8945 \\
\hline 4.0 & 1.2646 & 3.9352 & 6.8140 & 9.8119 & 12.8678 & 15.9536 \\
\hline 5.0 & 1.3138 & 4.0336 & 6.9096 & 9.8928 & 12.9352 & 16.0107 \\
\hline 6.0 & 1.3496 & 4.1116 & 6.9924 & 9.9667 & 12.9988 & 16.0654 \\
\hline 7.0 & 1.3766 & 4.1746 & 7.0640 & 10.0339 & 13.0584 & 16.1177 \\
\hline 8.0 & 1.3978 & 4.2264 & 7.1263 & 10.0949 & 13.1141 & 16.1675 \\
\hline 9.0 & 1.4149 & 4.2694 & 7.1806 & 10.1502 & 13.1660 & 16.2147 \\
\hline 10.0 & 1.4289 & 4.3058 & 7.2281 & 10.2003 & 13.2142 & 16.2594 \\
\hline
\end{tabular}


TABLE 14.1. (Continued.)

\begin{tabular}{ccccccc}
\hline $\mathrm{C}$ & $\lambda_{1}$ & $\lambda_{2}$ & $\lambda_{3}$ & $\lambda_{4}$ & $\lambda_{5}$ & $\lambda_{6}$ \\
\hline 15.0 & 1.4729 & 4.4255 & 7.3959 & 10.3898 & 13.4078 & 16.4474 \\
20.0 & 1.4961 & 4.4915 & 7.4954 & 10.5117 & 13.5420 & 16.5864 \\
30.0 & 1.5202 & 4.5615 & 7.6057 & 10.6543 & 13.7085 & 16.7691 \\
40.0 & 1.5325 & 4.5979 & 7.6647 & 10.7334 & 13.8048 & 16.8794 \\
50.0 & 1.5400 & 4.6202 & 7.7012 & 10.7832 & 13.8666 & 16.9519 \\
60.0 & 1.5451 & 4.6353 & 7.7259 & 10.8172 & 13.9094 & 17.0026 \\
80.0 & 1.5514 & 4.6543 & 7.7573 & 10.8606 & 13.9644 & 17.0686 \\
100.0 & 1.5552 & 4.6658 & 7.7764 & 10.8871 & 13.9981 & 17.1093 \\
$\infty$ & 1.5708 & 4.7124 & 7.8540 & 10.9956 & 14.1372 & 17.2788 \\
\hline
\end{tabular}


TABLE 14.2. First five roots of $1 \cdots \lambda_{n} \cot \lambda_{n}=c$. (source: Ref. 20 p. 442.)

\begin{tabular}{|c|c|c|c|c|c|}
\hline$c$ & $\lambda_{1}$ & $\lambda_{2}$ & $\lambda_{3}$ & $\lambda_{4}$ & $\lambda_{5}$ \\
\hline 0.000 & 0.0000 & 4.4934 & 7.7253 & 10.9041 & 14.0662 \\
\hline 0.005 & 0.1224 & 4.4945 & 7.7259 & 10.9046 & 14.0666 \\
\hline 0.010 & 0.1730 & 4.4956 & 7.7265 & 10.9050 & 14.0669 \\
\hline 0.020 & 0.2445 & 4.4979 & 7.7278 & 10.9060 & 14.0676 \\
\hline 0.030 & 0.2991 & 4.5001 & 7.7291 & 10.9069 & $14 . \overline{6} 683$ \\
\hline 0.040 & 0.3450 & 4.5023 & 7.7304 & 10.9078 & 14.0690 \\
\hline 0.050 & 0.3854 & 4.5045 & 7.7317 & 10.9087 & 14.0697 \\
\hline 0.060 & 0.4217 & 4.5068 & 7.7330 & 10.9096 & 14.0705 \\
\hline 0.070 & 0.4551 & 4.5090 & 7.7343 & 10.9105 & 14.0712 \\
\hline 0.080 & 0.4860 & 4.5112 & 7.7356 & 10.9115 & 14.0719 \\
\hline 0.090 & 0.5150 & 4.5134 & 7.7369 & 10.9124 & 14.0726 \\
\hline 0.100 & 0.5423 & 4.5157 & 7.7382 & 10.9133 & 14.0733 \\
\hline 0.200 & 0.7593 & 4.5379 & 7.7511 & 10.9225 & 14.0804 \\
\hline 0.300 & 0.9208 & 4.5601 & 7.7641 & 10.9316 & 14.0875 \\
\hline 0.400 & 1.0528 & 4.5822 & 7.7770 & 10.9408 & 14.0946 \\
\hline 0.500 & 1.1656 & 4.6042 & 7.7899 & 10.9499 & 14.1017 \\
\hline 0.600 & 1.2644 & 4.6261 & 7.8028 & 10.9591 & 14.1088 \\
\hline 0.700 & 1.3525 & 4.6479 & 7.8156 & 10.9682 & 14.1159 \\
\hline 0.800 & 1.4320 & 4.6696 & 7.8284 & 10.9774 & 14.1230 \\
\hline 0.900 & 1.5044 & 4.6911 & 7.8412 & 10.9865 & 14.1301 \\
\hline 1.000 & 1.5708 & 4.7124 & 7.8540 & 10.9956 & 14.1372 \\
\hline 1.500 & 1.8366 & 4.8158 & 7.9171 & 11.0409 & 14.1724 \\
\hline 2.000 & 2.0288 & 4.9132 & 7.9787 & 11.0856 & 14.2075 \\
\hline 3.000 & 2.2889 & 5.0870 & 8.0962 & 11.1727 & 14.2764 \\
\hline 4.000 & 2.4557 & 5.2329 & 8.2045 & 11.2560 & 14.3434 \\
\hline 5.000 & 2.5704 & 5.3540 & 8.3029 & 11.3349 & 14.4080 \\
\hline 6.000 & 2.6537 & 5.4544 & 8.3914 & 11.4086 & 14.4699 \\
\hline 7.000 & 2.7165 & 5.5378 & 8.4703 & 11.4773 & 14.5288 \\
\hline 8.000 & 2.7654 & 5.6078 & 8.5406 & 11.5408 & 14.5847 \\
\hline 9.000 & 2.8044 & 5.6669 & 8.6031 & 11.5994 & 14.6374 \\
\hline 10.000 & 2.8363 & 5.7172 & 8.6587 & 11.6532 & 14.6870 \\
\hline 11.000 & 2.8628 & 5.7606 & 8.7083 & 11.7027 & 14.7335 \\
\hline 16.000 & 2.9476 & 5.9080 & 8. 8898 & 11.8959 & 14.9251 \\
\hline 21.000 & 2.9930 & 5.9921 & 9.0019 & 12.0250 & 15.0625 \\
\hline
\end{tabular}


TABLE 14.2. (Continued.)

\begin{tabular}{cccccc}
\hline$c$ & $\lambda_{1}$ & $\lambda_{2}$ & $\lambda_{3}$ & $\lambda_{4}$ & $\lambda_{5}$ \\
\hline 31.000 & 3.0406 & 6.0831 & 9.1294 & 12.1807 & 15.2380 \\
41.000 & 3.0651 & 6.1311 & 9.1987 & 12.2688 & 15.3417 \\
51.000 & 3.0801 & 6.1606 & 9.2420 & 12.3247 & 15.4090 \\
101.000 & 3.1105 & 6.2211 & 9.3317 & 12.4426 & 15.5537 \\
\hline
\end{tabular}

TABLE 14.3. First five roots of $J_{m}\left(\lambda_{n}\right)=0$. (Source: Ref. 20, p. 443.)

\begin{tabular}{llllll}
\hline $\mathrm{m}$ & $\lambda_{1}$ & $\lambda_{2}$ & $\lambda_{3}$ & $\lambda_{4}$ & $\lambda_{5}$ \\
\hline 0 & 2.4048 & 5.5201 & 8.6537 & 11.7915 & 14.9309 \\
1 & 3.8317 & 7.0156 & 1.0 .1735 & 13.3237 & 16.4706 \\
2 & 5.1356 & 8.4172 & 11.6198 & 14.7960 & 17.9598 \\
3 & 6.3802 & 9.7610 & 13.0152 & 16.2235 & 19.4094 \\
4 & 7.5883 & 11.0647 & 14.3725 & 17.6160 & 20.8269 \\
\hline
\end{tabular}


TABLE 14.4. First six roots of $\lambda_{n} J_{1}\left(\lambda_{n}\right)-\mathrm{CJ}_{0}\left(\lambda_{n}\right)=0$. (Source: Ref. 74. p. 217)

\begin{tabular}{|c|c|c|c|c|c|c|}
\hline C & $\lambda_{1}$ & $\lambda_{2}$ & $\lambda_{3}$ & $\lambda_{4}$ & $\lambda_{5}$ & $\lambda_{6}$ \\
\hline 0 & 0 & 3.8317 & 7.0156 & 10.1735 & 13.3237 & 16.4706 \\
\hline 0.01 & 0.1412 & 3.8343 & 7.0170 & 10.1745 & 13.3244 & 16.4712 \\
\hline 0.02 & 0.1995 & 3.8369 & 7.0184 & 10.1754 & 13.3252 & 16.4718 \\
\hline 0.04 & 0.2814 & 3.8421 & 7.0213 & 10.1774 & 13.3267 & 16.4731 \\
\hline$\Gamma 06$ & 0.3438 & 3.8473 & 7.0241 & 10.1794 & 13.3282 & 16.4743 \\
\hline 0.08 & 0.3960 & 3.8525 & 7.0270 & 10.1813 & 13.3297 & 16.4755 \\
\hline 0.1 & 0.4417 & 3.8577 & 7.0298 & 10.1833 & 13.3312 & 16.4767 \\
\hline 0.15 & 0.5376 & 3.8706 & 7.0369 & 10.1882 & 13.3349 & 16.4797 \\
\hline 0.2 & 0.6170 & 3.8835 & 7.0440 & 10.1931 & 13.3387 & 16.4828 \\
\hline 0.3 & 0.7465 & 3.9091 & 7.0582 & 10.2029 & 13.3462 & $16.488 B$ \\
\hline 0.4 & 0.8516 & 3.9344 & 7.0723 & 10.2127 & 13.3537 & 16.4949 \\
\hline 0.5 & 0.9408 & 3.9594 & 7.0864 & 10.2225 & 13.3611 & 16.5010 \\
\hline 0.6 & 1.0184 & 3.9841 & 7.1004 & 10.2322 & 13.3686 & 16.5070 \\
\hline 0.7 & 1.0873 & 4.0085 & 7.1143 & 10.2419 & 13.3761 & 16.5131 \\
\hline 0.8 & 1.1490 & 4.0325 & 7.1282 & 10.2516 & 13.3835 & 16.5191 \\
\hline 0.9 & 1.2048 & 4.0562 & 7.1421 & 10.2613 & 13.3910 & 16.5251 \\
\hline 1.0 & 1.2558 & 4.0795 & 7.1558 & 10.2710 & 13.3984 & 16.5312 \\
\hline 1.5 & 1.4569 & 4.1902 & 7.2233 & 10.3188 & 13.4353 & 16.5612 \\
\hline 2.0 & 1.5994 & 4.2910 & 7.2884 & 10.3658 & 13.4719 & 16.5910 \\
\hline 3.0 & 1.7887 & 4.4634 & 7.4103 & 10.4566 & 13.5434 & 16.6499 \\
\hline 4.0 & 1.9081 & 4.6018 & 7.5201 & 10.5423 & 13.6125 & 16.7073 \\
\hline 5.0 & 1.9898 & 4.7131 & 7.6177 & 10.6223 & 13.6786 & 16.7630 \\
\hline 6.0 & 2.0490 & 4.8033 & 7.7039 & 10.6964 & 13.7414 & 16.8168 \\
\hline 7.0 & 2.0937 & 4.8772 & 7.7797 & 10.7646 & 13.8008 & 16.8684 \\
\hline 8.0 & 2.1286 & 4.9384 & 7.8464 & 10.8271 & 13.8566 & 16.9179 \\
\hline 9.0 & 2.1566 & 4. 9897 & 7.9051 & 10.8842 & 13.9090 & 16.9650 \\
\hline 10.0 & 2.1795 & 5.0332 & 7.9569 & 10.9363 & 13.9580 & 17.0099 \\
\hline 15.0 & 2.2509 & 5.1773 & B. 1422 & 11.1367 & 14.1576 & 17.2008 \\
\hline 20.0 & 2.2880 & 5.2568 & 8.2534 & 11.2677 & 14.2983 & 17.3442 \\
\hline 30.0 & 2.3261 & 5.3410 & 8.3771 & 11.4221 & 14.4748 & 17.5348 \\
\hline 40.0 & 2.3455 & 5.3846 & 8.4432 & 11.5081 & 14.5774 & 17.6508 \\
\hline 50.0 & 2.3572 & 5.4112 & 8.4840 & 11.5621 & 14.6433 & 17.7272 \\
\hline 60.0 & 2.3651 & 5.4291 & 8.5116 & 11.5990 & 14.6889 & 17.7807 \\
\hline 80.0 & 2.3750 & 5.4516 & 8.5466 & 11.6461 & 14.7475 & 17.8502 \\
\hline 100.0 & 2.3809 & 5.4652 & 8.5678 & 11.6747 & 14.7834 & 17.8931 \\
\hline$\infty$ & 2.4048 & 5.5201 & 8.6537 & 11.7915 & 14.9309 & 18.0711 \\
\hline
\end{tabular}


TABLE 14.5. First five roots of $J_{0}\left(\lambda_{n}\right) Y_{0}\left(C \lambda_{n}\right)=Y_{0}\left(\lambda_{n}\right) J_{0}\left(C \lambda_{n}\right)$. (Source:

Ref. 9, p. 493.)

\begin{tabular}{lrrrrr}
\hline$c$ & \multicolumn{1}{c}{$\lambda_{1}$} & $\lambda_{2}$ & $\lambda_{3}$ & $\lambda_{4}$ & $\lambda_{5}$ \\
\hline 1.2 & 15.7014 & 31.4126 & 47.1217 & 62.8302 & 78.5385 \\
1.5 & 6.2702 & 12.5598 & 18.8451 & 25.1294 & 31.4133 \\
2.0 & 3.1230 & 6.2734 & 9.4182 & 12.5614 & 15.7040 \\
2.5 & 2.0732 & 4.1773 & 6.2754 & 8.3717 & 10.4672 \\
3.0 & 1.5485 & 3.1291 & 4.7038 & 6.2767 & 7.8487 \\
3.5 & 1.2339 & 2.5002 & 3.7608 & 5.0196 & 6.2776 \\
4.0 & 1.0244 & 2.0809 & 3.1322 & 4.1816 & 5.2301 \\
\hline
\end{tabular}


TABLE 14.6. First six roots of $\tan \left(\lambda_{n}\right)=-\lambda_{n} /$ C. (Source: Ref. 74, p. 322.)

\begin{tabular}{|c|c|c|c|c|c|c|}
\hline c & $\lambda_{1}$ & $\lambda_{2}$ & $\lambda_{3}$ & $\lambda_{4}$ & $\lambda_{5}$ & $\lambda_{6}$ \\
\hline 0 & 1.5708 & 4.7124 & 7.8540 & 10.9956 & 14.1372 & 17.2738 \\
\hline 0.1 & 1.6320 & 4.7335 & 7.8667 & 11.0047 & 14.1443 & 17.2845 \\
\hline 0.2 & 1.6887 & 4.7544 & 7.8794 & 11.0137 & 14.1513 & 17.2903 \\
\hline 0.3 & 1.7414 & 4.7751 & 7.8920 & 11.0228 & 14.1584 & 17.2961 \\
\hline 0.4 & 1.7906 & 4.7956 & 7.9046 & 11.0318 & 14.1654 & 17.7019 \\
\hline 0.5 & 1.8366 & 4.8158 & 7.9171 & 11.0409 & 14.1724 & 17.3076 \\
\hline 0.6 & 1.8798 & 4.8358 & 7.9295 & 11.0498 & 14.1795 & 17.3134 \\
\hline 0.7 & 1.9203 & 4.8556 & 7.9419 & 11.0588 & 14.1865 & 17.3192 \\
\hline 0.8 & 1.9586 & 4.8751 & 7.9542 & 11.0677 & 14.1935 & 17.3249 \\
\hline 0.9 & 1.9947 & 4.8943 & 7.9665 & 11.0767 & 14.2005 & 17.3306 \\
\hline 1.0 & 2.0288 & 4.9132 & 7.9787 & 11.0856 & 14.2075 & 17.3364 \\
\hline 1.5 & 2.1746 & 5.0037 & 8.0385 & 11.1296 & 14.2421 & 17.3649 \\
\hline 2.0 & 2.2889 & 5.0870 & 8.0962 & 11.1727 & 14.2764 & 17.3932 \\
\hline 3.0 & 2.4557 & 5.2329 & 8.2045 & 11.2560 & 14.3434 & 17.4490 \\
\hline 4.0 & 2.5704 & 5.3540 & 8.3029 & 11.3349 & 14.4080 & 17.5034 \\
\hline 5.0 & 2.6537 & 5.4544 & 8.3914 & 11.4086 & 14.4699 & 17.5562 \\
\hline 6.0 & 2.7165 & 5.5373 & 8.4703 & 11.4773 & 14.5288 & 17.6072 \\
\hline 7.0 & 2.7654 & 5.6078 & 8.5406 & 11.5408 & 14.5847 & 17.6562 \\
\hline 8.0 & 2.8044 & 5.6669 & 8.6031 & 11.5994 & 14.6374 & 17.7032 \\
\hline 9.0 & 2.8363 & 5.7172 & 8.6587 & 11.6532 & 14.6870 & 17.7481 \\
\hline 10.0 & 2.8628 & 5.7606 & 8.7083 & 11.7027 & 14.7335 & 17.7908 \\
\hline 15.0 & 2.9476 & 5.9080 & 8.8898 & 11.8959 & 14.9231 & 17.9742 \\
\hline 20.0 & 2.9930 & 5.9921 & 9.0019 & 12.0250 & 15.0625 & 18.1136 \\
\hline 30.0 & 3.0406 & 6.0831 & 9.1294 & 12.1807 & 15.2380 & 18.3018 \\
\hline 40.0 & 3.0651 & 6.1311 & 9.1986 & 12.2688 & 15.3417 & 18.4180 \\
\hline 50.0 & 3.0801 & 6.1606 & 9.2420 & 12.3247 & 15.4090 & 18.4953 \\
\hline 60.0 & 3.0901 & 6.1805 & 9.2715 & 12.3632 & 15.4559 & 18.5497 \\
\hline 80.0 & 3.1028 & 6.2058 & 9.3089 & 12.4124 & 15.5164 & 18.6209 \\
\hline 100.0 & 3.1105 & 6.2211 & 9.3317 & 12.4426 & 15.5537 & 18.6650 \\
\hline$\infty$ & 3.1416 & 6.2832 & 9.4248 & 12.5664 & 15.7080 & 18.8496 \\
\hline
\end{tabular}


SECTION 15. CONSTANTS AND CONVERSION FACTORS.

15.1. Mathematical Constants.

$$
\begin{aligned}
e & =2.7182818 \ldots \\
\ln 10 & =2.3025851 \ldots \\
\pi & =3.1415926 \ldots \\
\gamma & =0.5772156 \ldots
\end{aligned}
$$

15.2. Physical Constants.

Standard acceleration

of gravity

Joule's constant

Stefan-Bol tzmann

constant

$$
\begin{aligned}
\sigma & =1.355 \times 10^{-12} \mathrm{cal} / \mathrm{s} \cdot \mathrm{cm}^{2}-\mathrm{R}^{4} \\
& =1712 \times 10^{-12} \mathrm{Btu} / \mathrm{hr} \cdot \mathrm{Ft}^{2}-\mathrm{R}^{4} \\
& =5.673 \times 10^{-8} \mathrm{~W} / \mathrm{m}^{2} \cdot \mathrm{R}^{4}
\end{aligned}
$$

Universal gas
constant

Universal gas
constant

$$
\begin{aligned}
g_{0} & =9.80665 \mathrm{~m} / \mathrm{s}^{2} \\
& =32.1742 \mathrm{ft} / \mathrm{s}^{2}
\end{aligned}
$$

$$
\begin{aligned}
J_{c} & =1.0 \mathrm{~N} \cdot \mathrm{m} / \mathrm{J} \\
& =778.16 \mathrm{ft} \cdot 1 \mathrm{bE} / \mathrm{Btu}
\end{aligned}
$$


15.3. Conversion Factors.

TABLE 15.1. Conversion factors for length.

\begin{tabular}{|c|c|c|c|c|c|c|c|}
\hline & $\mathbf{m}$ & $\operatorname{cin}$ & $\mu \mathrm{m}$ & $\AA$ & in. & ft & yd \\
\hline $1 \mathrm{~m}$ & $=1$ & 100 & $10^{6}$ & $10^{10}$ & 39.37 & 3.280 & 1.0936 \\
\hline $1 \mathrm{~cm}$ & $=0.01$ & 1 & $10^{4}$ & $10^{8}$ & 0.3937 & 0.0328 & 0.0109 \\
\hline $1 \mu \mathrm{m}$ & $=10^{-6}$ & $10^{-4}$ & 1 & $10^{4}$ & $0.3937 \times 10^{-4}$ & $0.0328 \times 10^{-4}$ & $0.0109 \times 10^{-4}$ \\
\hline $1 \AA$ & $=10^{-10}$ & $10^{-8}$ & $10^{-4}$ & 1 & $0.3937 \times 10^{-8}$ & $0.0328 \times 10^{-4}$ & $0.0109 \times 10^{-8}$ \\
\hline 1 in. & $=0.0254$ & 2.540 & $25.4 \times 10^{4}$ & $2.540 \times 10^{8}$ & 1 & 0.0833 & 0.0277 \\
\hline $1 \mathrm{ft}$ & $=0.3048$ & 30.48 & $30.48 \times 10^{4}$ & $30.43 \times 10^{8}$ & 12 & 1 & 0.3333 \\
\hline $1 \mathrm{yd}$ & $=0.9144$ & 91.440 & $91.440 \times 10^{4}$ & $91.440 \times 10^{8}$ & 36 & 3 & 1 \\
\hline
\end{tabular}

岀

TABLE 15.2. Conversion factors for area.

\begin{tabular}{|c|c|c|c|c|c|c|}
\hline & & $\mathrm{cm}^{2}$ & $\mathrm{~m}^{2}$ & in. ${ }^{2}$ & $\mathrm{ft}^{2}$ & $y d^{2}$ \\
\hline $1 \mathrm{~cm}^{2}$ & $=$ & 1 & $10^{-4}$ & 0.1550 & 1.07639 & $1.1960 \times 10^{-4}$ \\
\hline $1 \mathrm{~m}^{2}$ & $=$ & $10^{4}$ & 1 & 1550 & 10.7639 & 1.1960 \\
\hline 1 in. & $=$ & 6.4516 & $6.4516 \times 10^{-4}$ & 1 & 6.9444 & $7.7160 \times 10^{-4}$ \\
\hline $1 \mathrm{ft}^{2}$ & $=$ & 929.034 & $929.034 \times 10^{-4}$ & 144 & 1 & 0.11111 \\
\hline $1 \mathrm{yd}^{2}$ & $=$ & 8361.307 & $8361.307 \times 10^{-4}$ & 1296 & 9 & 1 \\
\hline
\end{tabular}


TABLE 15.3. Conversion factors for volume.

\begin{tabular}{lllllll}
\hline & \multicolumn{1}{c}{$\mathrm{cm}^{3}$} & \multicolumn{1}{c}{ in. $^{3}$} & $\mathrm{ft}^{3}$ & ml & liter \\
\hline $1 \mathrm{~cm}^{3}$ & $=1$ & $610.23 \times 10^{-4}$ & $35.3145 \times 10^{-4}$ & $999.972 \times 10^{-3}$ & $999.972 \times 10^{-6}$ & $264.170 \times 10^{-6}$ \\
$1 \mathrm{ina}^{3}$ & $=16.3872$ & 1 & $5.7870 \times 10^{-4}$ & 16.3867 & $16.3867 \times 10^{-3}$ & $432.900 \times 10^{-5}$ \\
$1 \mathrm{ft}^{3}$ & $=283.170 \times 10^{2}$ & 1728 & 1 & 7.4805 & 28.3162 & 0.001 \\
$1 \mathrm{ml}$ & $=1.000028$ & $610.251 \times 10^{-4}$ & $353.154 \times 10^{-7}$ & 1 & 1 & $264.178 \times 10^{-6}$ \\
$1 \mathrm{liter}$ & $=1000.028$ & 61.0251 & $353.154 \times 10^{-4}$ & 1000 & $3162 \times 10^{3}$ & $264.178 \times 10^{-3}$ \\
$1 \mathrm{gal}$ & $=3785.434$ & 231 & $133.680 \times 10^{-3}$ & 3785.329 & 3.785329 & 1 \\
\hline
\end{tabular}

崔

TABLE 15.4. Conversion factors for mass.

\begin{tabular}{llllll}
\hline & \multicolumn{1}{c}{ lb } & \multicolumn{1}{c}{ slugs } & \multicolumn{1}{c}{$\mathrm{g}$} & \multicolumn{1}{c}{$\mathrm{kg}$} & \multicolumn{1}{c}{ ton } \\
\hline $1 \mathrm{lb}$ & $=1$ & 0.03108 & 453.59 & 0.45359 & 0.0005 \\
$1 \mathrm{slug}$ & $=32.174$ & 1 & $1.4594 \times 10^{4}$ & 14.594 & 0.016087 \\
$1 \mathrm{~g}$ & $=2.2046 \times 10^{-3}$ & $6.8521 \times 10^{-5}$ & 1 & $10^{-3}$ & $1.1023 \times 10^{-6}$ \\
$1 \mathrm{~kg}$ & $=2.2046$ & $6.8521 \times 10^{-3}$ & $10^{3}$ & 1 & $1.1023 \times 10^{-3}$ \\
$1 \mathrm{ton}$ & $=2000$ & 62.162 & $9.0718 \times 10^{5}$ & 907.18 & 1 \\
\hline
\end{tabular}


TABLE 15.5. Conversion factors for density.

\begin{tabular}{|c|c|c|c|c|c|c|}
\hline & & $1 \mathrm{bm} / \mathrm{ft} \mathrm{t}^{3}$ & slug/in. ${ }^{3}$ & $1 \mathrm{bm} / \mathrm{in.}^{3}$ & $1 \mathrm{bm} / \mathrm{gal}$ & $\mathrm{g} / \mathrm{cm}^{3}$ \\
\hline $1 \mathrm{lbm} / \mathrm{ft}^{3}$ & $=$ & 1 & 0.03108 & $5.787 \times 10^{-4}$ & 0.13386 & 0.01602 \\
\hline 1 slug/ft ${ }^{3}$ & $=$ & 32.174 & 1 & 0.01862 & 4.3010 & 0.51543 \\
\hline $11 \mathrm{bm} /$ in. $^{3}$ & $=$ & 1728 & 53.706 & 1 & 231 & 27.680 \\
\hline $1 \mathrm{lbm} / \mathrm{gal}$ & $=$ & 7.4805 & 0.2325 & $4.329 \times 10^{-3}$ & 1 & 0.11983 \\
\hline $1 \mathrm{~g} / \mathrm{cos}^{3}$ & $\Rightarrow$ & 62.428 & 1.9403 & 0.03613 & 8.345 & 1 \\
\hline
\end{tabular}


TABLE 15.6. Conversion factors for pressure.

\begin{tabular}{|c|c|c|c|c|c|c|c|c|}
\hline & lbf/in. ${ }^{2}$ & dyne/ $\operatorname{con}^{2}$ & $\mathrm{kgf} / \mathrm{cm}^{2}$ & in. Hg & $\min \mathbf{~ H g}$ & in. $\mathrm{H}_{2} \mathrm{O}$ & atm & bar \\
\hline 1 lbf/in. ${ }^{2}$ & $=1$ & $\begin{array}{l}689.473 \\
\times 10^{2}\end{array}$ & 0.07031 & 2.0360 & 51.715 & 27.71 & 0.06805 & 0.06895 \\
\hline 1 dyne/cm ${ }^{2}$ & $\begin{array}{c}=145.0383 \\
\times 10^{-7}\end{array}$ & 1 & $\begin{array}{l}101.972 \\
\times 10^{-8}\end{array}$ & $\begin{array}{l}295.299 \\
\times 10^{-7}\end{array}$ & $\begin{array}{l}750.062 \\
\times 10^{-6}\end{array}$ & $\begin{array}{r}4.0188 \\
\times 10^{-4}\end{array}$ & $\begin{array}{l}986.923 \\
\times 10^{-9}\end{array}$ & $10^{-6}$ \\
\hline $1 \mathrm{kgf} / \mathrm{cm}^{2}$ & $=14.2234$ & $\begin{array}{l}980.665 \\
\times 10^{3}\end{array}$ & 1 & 28.959 & 735.559 & 394.0918 & $\begin{array}{l}967.841 \\
\times 10^{3}\end{array}$ & $\begin{array}{l}980.665 \\
\times 10^{-3}\end{array}$ \\
\hline 1 in. $\mathrm{Hg}$ & $=0.4912$ & $\begin{array}{l}338.64 \\
\times 10^{2}\end{array}$ & 0.03453 & 1 & 25.40 & 13.608 & 0.03342 & 0.03386 \\
\hline $1 \sin \mathrm{HS}$ & $=0.01934$ & 1333.223 & $\begin{array}{r}1.3595 \\
\times 10^{-3}\end{array}$ & 0.03937 & 1 & 0.5358 & $\begin{array}{l}1.315 \\
\times 10^{-3}\end{array}$ & $\begin{array}{l}1.333 \\
\times 10^{-3}\end{array}$ \\
\hline 1 in. $\mathrm{H}_{2} \mathrm{O}$ & $=0.03609$ & $\begin{array}{l}24.883 \\
\times 10^{2}\end{array}$ & $\begin{array}{l}2.537 \\
\times 10^{-3}\end{array}$ & 0.0735 & 1.8665 & 1 & $\begin{array}{l}2.458 \\
\times 10^{-3}\end{array}$ & $\begin{array}{l}2.488 \\
\times 10^{-3}\end{array}$ \\
\hline 1 atm & $=14.6960$ & $\begin{array}{l}101.325 \\
\times 10^{4}\end{array}$ & 1.03323 & 29.9212 & 760 & 460.80 & 1 & 1.01325 \\
\hline 1 bar & $=14.5038$ & $10^{6}$ & 1.01972 & 29.5299 & 750.0617 & 401.969 & $\begin{array}{l}986.923 \\
\times 10^{-3}\end{array}$ & 1 \\
\hline
\end{tabular}


TABLE 15.7. Conversion factors for energy.

\begin{tabular}{|c|c|c|c|c|c|c|}
\hline & & $f t \cdot I b f$ & abs joule & int joule & cal & I.T. cal \\
\hline $1 \mathrm{ft} \cdot 1 \mathrm{bf}$ & $=$ & 1 & 1.35582 & 1.355597 & 0.32405 & 0.32384 \\
\hline 1 abs joule & $=$ & 0.73756 & 1 & 0.999835 & 0.23885 & 0.238849 \\
\hline 1 int joule & $=$ & 0.737682 & 1.000165 & 1 & 0.239045 & 0.238889 \\
\hline $1 \mathrm{cal}$ & $=$ & 3.08596 & 4.18401 & 4.1833 & 1 & 0.99934 \\
\hline 1 I.T. cal & $=$ & 3.08799 & 4.18676 & 4.18605 & 1.000657 & 1 \\
\hline 1 Btu & $=$ & 778.16 & 1055.045 & 1054.866 & 252.161 & 251.996 \\
\hline 1 int $k w^{* h} r$ & $=$ & $265.567 \times 10^{4}$ & $360.0612 \times 10^{4}$ & $360.000 \times 10^{4}$ & $860.565 \times 10^{3}$ & $860.000 \times 10^{3}$ \\
\hline $1 \mathrm{hp} \cdot \mathrm{hr}$ & $=$ & $198.0000 \times 10^{4}$ & $268.4525 \times 10^{4}$ & $268.082 \times 10^{4}$ & $641.615 \times 10^{3}$ & $641.194 \times 10^{3}$ \\
\hline 1 liter-atm & $=$ & 74.7354 & 101.3278 & 101.3111 & 24.2179 & 24.2020 \\
\hline & & Btu & int $k \mathrm{k} \cdot \mathrm{hr}$ & $h p \cdot h r$ & liter $\cdot a t m$ & \\
\hline $1 \mathrm{ft} \cdot \mathrm{lbf}$ & $=$ & $128.5083 \times 10^{-5}$ & $376.553 \times 10^{-9}$ & $505.051 \times 10^{-9}$ & $133.8054 \times 10^{-4}$ & \\
\hline 1 abs joule & $=$ & $947.827 \times 10^{-6}$ & $277.731 \times 10^{-9}$ & $372.505 \times 10^{-9}$ & $986.896 \times 10^{-5}$ & \\
\hline 1 int joule & $=$ & $0.947988 \times 10^{-3}$ & $2.777778 \times 10^{-7}$ & $3.7256 \times 10^{-7}$ & $9.87058 \times 10^{-3}$ & \\
\hline $1 \mathrm{cal}$ & $=$ & $396.572 \times 10^{-5}$ & $116.2028 \times 10^{-8}$ & $155.8566 \times 10^{-8}$ & $412.918 \times 10^{-4}$ & \\
\hline 1 I.T. cal & $=$ & $396.832 \times 10^{-5}$ & $i 16.2791 \times 10^{-8}$ & $155.9590 \times 10^{-8}$ & $413.189 \times 10^{-4}$ & \\
\hline 1 Btu & $=$ & 1 & $293.018 \times 10^{-6}$ & $293.010 \times 10^{-6}$ & 10.4122 & \\
\hline 1 int $k W \cdot h r$ & $=$ & 3412.76 & 1 & 1.3412 & $255.343 \times 10^{2}$ & \\
\hline $1 \mathrm{hp} \cdot \mathrm{hr}$ & $=$ & 2544.46 & 0.74558 & 1 & $264.935 \times 10^{2}$ & \\
\hline 1 liter $\cdot a t m$ & $=$ & 0.09604 & $281.718 \times 10^{-7}$ & $377.452 \times 10^{-7}$ & 1 & \\
\hline
\end{tabular}


Table 15.8. Conversion factors for specific energy.

\begin{tabular}{|c|c|c|c|c|c|c|c|c|c|}
\hline & & abs joule/h & $\mathrm{cal} / \mathrm{g}$ & I.T. $\mathrm{cal} / \mathrm{gm}$ & Btu/lb & $\mathrm{ft} \cdot \mathrm{lbf} / 1 \mathrm{bm}$ & int. $\mathrm{kH} \mathrm{hr} / \mathrm{g}$ & $h p h r / l b$ & $\mathrm{ft}^{2} / \mathrm{s}^{2}$ \\
\hline abs joule/g & - & 1 & 0.2390 & 0.2388 & 0.4299 & 334.53 & $2.777 \times 10^{-7}$ & $1.690 \times 10^{-4}$ & 10763 \\
\hline $\mathrm{cal} / \mathrm{g}$ & $=$ & 4.184 & 1 & 0.9993 & 1.7988 & 1399.75 & $1.162 \times 10^{-6}$ & $7.069 \times 10^{-4}$ & $4.504 \times 10^{4}$ \\
\hline I.T. $\mathrm{cal} / \mathrm{g}$ & - & 4.186 & 1.0007 & 1 & 1.8 & 1400.69 & $1.163 \times 10^{-6}$ & $7.074 \times 10^{-4}$ & $4.506 \times 10^{4}$ \\
\hline Btu/lo & - & 2.326 & 0.5559 & $0.5550^{\circ}$ & 1 & 778.16 & $6.460 \times 10^{-7}$ & $3.930 \times 10^{-4}$ & 25,037 \\
\hline$f t=1 b f / 1 b m$ & $=$ & $2.989 \times 10^{-3}$ & $7.144 \times 10^{-4}$ & $7.139 \times 10^{-4}$ & $1.285 \times 10^{-2}$ & 1 & $8.302 \times 10^{-10}$ & $5.051 \times 10^{-7}$ & 32.174 \\
\hline int. $k w \cdot h r / g$ & - & $3.610 \times 10^{6}$ & 860,565 & $86:, 000$ & $\therefore .548 \times 10^{6}$ & $1.2046 \times 10^{9}$ & 1 & 608.4 & $3.876 \times 10^{10}$ \\
\hline$h p=h r / 1 b$ & - & 5919 & 1414.5 & 1413.6 & 2545 & $1.980 \times 10^{16}$ & 0.001644 & 1 & $6.370 \times 10^{7}$ \\
\hline$f t^{2} / \mathrm{s}^{2}$ & - & $9.291 \times 10^{-5}$ & $2.220 \times 10^{-5}$ & $2.219 \times 10^{-5}$ & $3.994 \times 10^{-5}$ & 0.03108 & $2.580 \times 10^{-11}$ & $1.567 \times 10^{-8}$ & 1 \\
\hline
\end{tabular}

Table 15.9. Conversion factors for specific energy per degree.

\begin{tabular}{|c|c|c|c|c|c|c|}
\hline & & abs joule/g•k & $\mathrm{Cal} / \mathrm{g} \cdot \mathrm{K}$ & I.T. $\mathrm{cal} / \mathrm{g} \cdot \mathrm{K}$ & $B t u / l b \cdot R$ & $\mathrm{~W} \cdot \mathrm{s} / \mathrm{kg} \cdot \mathrm{K}$ \\
\hline abs joule $/ g \cdot r$ & $=$ & 1 & 0.2390 & 0.2388 & 0.2388 & $10^{3}$ \\
\hline $\mathrm{cal} / \mathrm{g} \cdot \mathbf{K}$ & $=$ & 4.184 & 1 & 0.9993 & 0.9993 & 4184 \\
\hline I.T. $\operatorname{cal} / g \cdot \mathrm{K}$ & $=$ & 4.186 & 1.0007 & 1 & 1 & 4186 \\
\hline $\mathbf{b t u} / \mathbf{l b} \cdot \mathbf{R}$ & $=$ & 4.186 & 1.0007 & 1 & 1 & 4186 \\
\hline$w \cdot s / \mathrm{kg} \cdot \mathrm{K}$ & $=$ & $10^{-3}$ & $2.390 \times 10^{-4}$ & $2.388 \times 10^{-4}$ & $2.388 \times 10^{-4}$ & 1 \\
\hline
\end{tabular}


Table 15.10. Conversion factors for thermal conductivity.

\begin{tabular}{llcccc}
\hline & & $\mathrm{cal} / \mathrm{s}^{\cdot \mathrm{Cm}-{ }^{\circ} \mathrm{C}}$ & $\mathrm{Btu} / \mathrm{hr} \cdot \mathrm{ft} \cdot{ }^{\circ} \mathrm{F}$ & $\mathrm{Btu} / \mathrm{hr} \cdot \mathrm{ft}{ }^{2} \cdot{ }^{\circ} \mathrm{F} / \mathrm{in}$. & $\mathrm{W} / \mathrm{m} \cdot{ }^{\circ} \mathrm{C}$ \\
\hline $1 \mathrm{cal} / \mathrm{s} \cdot \mathrm{cm} \cdot \mathrm{C}$ & $=$ & 1 & 241.9 & 2903 & 418.6 \\
$1 \mathrm{Btu} / \mathrm{hr} \cdot \mathrm{ft}-\mathrm{F}$ & $=$ & $4.13 \times 10^{-3}$ & 1 & 12 & 1.73 \\
$1 \mathrm{Btu} / \mathrm{hr} \cdot \mathrm{ft}^{2}-\mathrm{F} / \mathrm{in}$. & $=$ & $3.45 \times 10^{-4}$ & 0.0833 & 1 & $1.44 \times 10^{-1}$ \\
$1 \mathrm{~W} / \mathrm{m} \mathrm{C}$ & $=$ & 23.89 & 5780 & 69350 & 1 \\
\hline
\end{tabular}

住

Table 15.11. Conversion fa. ${ }^{-}$for thermal and momentum diffusivities

\begin{tabular}{lcccc}
\hline & $\mathrm{ft}^{2} / \mathrm{hr}$ & stokes & $\mathrm{m}^{2} / \mathrm{hr}$ & $\mathrm{m}^{2} / \mathrm{s}$ \\
\hline $\mathrm{ft}^{2} / \mathrm{hr}=$ & 1 & 0.25806 & 0.092903 & $2.58 \times 10^{-5}$ \\
stokes $=$ & 3.885 & 1 & 0.36 & $10^{-4}$ \\
$\mathrm{~m}^{2} / \mathrm{hr}=$ & 10.764 & 2.778 & 1 & $1.778 \times 10^{-4}$ \\
$\mathrm{~m}^{2} / \mathrm{s}=$ & 38,750 & $10^{4}$ & 3600 & 1 \\
\hline
\end{tabular}


TABLE 15.12. Conversion factors for heat flux.

\begin{tabular}{|c|c|c|c|c|c|}
\hline & \multirow{2}{*}{$\frac{\text { Btu }}{f t^{2} \cdot h r}$} & \multirow{2}{*}{$\frac{W}{m^{2}}$} & \multirow{2}{*}{$\frac{\mathrm{kcal}}{\mathrm{hr} \cdot \mathrm{m}^{2}}$} & cal \\
\hline & & & & & $s \cdot c m^{2}$ \\
\hline$B t u / f t^{2} \cdot h r$ & $=$ & 1 & 3.154 & 2.713 & $7.536 \times 10^{-5}$ \\
\hline$w / m^{2}$ & $=$ & $3.170 \times 10^{7}$ & 1 & $8.600 \times 10^{7}$ & 2389 \\
\hline $\mathrm{kcal} / \mathrm{hr}-\mathrm{m}^{2}$ & $=$ & 0.3687 & 1.163 & 1 & 36000 \\
\hline $\mathrm{cal} / \mathrm{s} \cdot \mathrm{cm}^{2}=$ & & 13277 & 41868 & $2.778 \times 10^{-5}$ & 1 \\
\hline
\end{tabular}

TABLE 15.13. Conversion factors for heat transfer coefficient.

\begin{tabular}{lllll}
\hline & $\frac{\text { Btu }}{\mathrm{hr} \cdot \mathrm{ft}{ }^{2} \cdot{ }^{\circ} \mathrm{F}}$ & $\frac{\mathrm{W}}{\mathrm{m}^{2} \cdot{ }^{\circ} \mathrm{C}}$ & $\frac{\mathrm{cal}}{\mathrm{s} \cdot \mathrm{cm}^{2} \cdot{ }^{\circ} \mathrm{C}}$ & $\frac{\mathrm{kcal}}{\mathrm{hr}^{2} \cdot \mathrm{m}^{2} \cdot{ }^{\circ} \mathrm{C}}$ \\
\hline $\mathrm{Btu} / \mathrm{hr} \cdot \mathrm{ft}^{2} \cdot{ }^{\circ} \mathrm{F}$ & $=1$ & 5.678 & $1.356 \times 10^{-4}$ & 4.883 \\
$\mathrm{~W} / \mathrm{m}^{2} \cdot{ }^{\circ} \mathrm{C}$ & $=1.761 \times 10^{7}$ & 1 & 2391 & $8.600 \times 10^{7}$ \\
$\mathrm{cal} / \mathrm{sec}-\mathrm{cm}^{2}-{ }^{\circ} \mathrm{C}=$ & 7376 & $4.186 \times 10^{4}$ & 1 & 36000 \\
$\mathrm{kcal} / \mathrm{hr}-\mathrm{m}^{2}{ }^{\circ} \mathrm{C}=$ & 0.2049 & 1.163 & $2.778 \times 10^{-5}$ & 1 \\
\hline
\end{tabular}


SECTION 16. CONVECTION COEFFICIENTS

16.1. Forced Flow in Smooth Tubes.

16.1.1 Fully Developed Laminar Flow (Source: Ref. 21):

$\frac{h D}{k}=4.364$ (constant heat rate)

$\frac{h \mathrm{D}}{\mathrm{k}}=3.658$ (constant surface temp)

16.1.2 Fully Developed Turbulent Flow (Source: Ref. 21):

$\frac{h D}{k}=6.3+0.003(\operatorname{Re} \operatorname{Pr}), \operatorname{Pr}<0.1$ (constant heat rate)

$\frac{\mathrm{hD}}{\mathrm{k}}=4.8+0.003(\mathrm{Re} \mathrm{Pr}), \mathrm{Pr}<0.1$ (constant surface temp)

$\frac{\mathrm{hD}}{\mathrm{k}}=0.022 \mathrm{Pr}^{0.6} \mathrm{Re}^{0.8}, 0.5<\mathrm{Pr}<1.0$ (constant heat rate)

$\frac{h D}{k}=0.021 \operatorname{Pr}^{0.6} \mathrm{Re}^{0.8}, 0.5<\mathrm{Pr}<1.0$ (constant surface temp)

$\frac{\mathrm{hD}}{\mathrm{k}}=0.0155 \mathrm{Pr}^{0.5} \mathrm{Re}^{0.9}, 1.0<\mathrm{Pr}<20$

$\frac{h D}{k}=0.011 \mathrm{Pr}^{0.3} \mathrm{Re}^{0.9}, \operatorname{Pr}>20$

$\mathrm{Pr}=$ Prandtl number, $\mathrm{Re}=$ Reynolds number

16.2. Forced Flow Between Smooth Infinite Parallel Plates.

16.2.1. Fully developed laminar flow (Source: Ref. 21):

$\frac{\mathrm{hs}}{\mathrm{k}}=4.118$ (constant heat rate on both sides)

$\frac{h s}{k}=2.693$ (constant heat rate on one side. other side insulated) 
$\frac{h s}{k}=3.77$ (constant surface temperature on both sides)

$\frac{h s}{k}=2.43$ (constant surface temperature on one side, other side insulated)

$\mathbf{s}=$ spacing of plates

16.3. Forced Flow Parallel to Smooth Semi-Infinite Flat Plates Laminar Flow.

16.3.1. Laminar flow:

$$
\frac{h x}{k}=0.332 \mathrm{Pr}^{1 / 3} \operatorname{Re}_{x}^{1 / 2}\left[1-\left(x_{0} / x\right)^{3 / 4}\right]^{-1 / 3}, \begin{aligned}
& \text { (constant surface } \\
& \text { temperature) (Source: Ref.7) }
\end{aligned}
$$$$
x_{0}=\text { unheated starting length }
$$$$
x=\text { distance from leading edge }
$$

$$
\frac{h x}{k}=0.453 \operatorname{Pr}^{1 / 3} \operatorname{Re}_{x}^{1 / 2}\left[1-\left(x_{0} / x\right)^{3 / 4}\right]^{-1 / 3}, \text { (constant heat rate) }
$$

(Source: Ref. 21)

16.3.2. Turbulent flow (Source: Ref. 21):

$$
\begin{aligned}
& \frac{h x}{k}=0.0295 \operatorname{Pr}^{0.6} \operatorname{Re}_{x}^{0.8}\left[1-\left(x_{0} / x\right)^{9 / 10}\right]^{-1 / 9}, \begin{array}{c}
\text { (constant aurface } \\
\text { temperature) }
\end{array} \\
& \frac{h x}{k}=0.0307 \mathrm{Pr}^{0.6} \operatorname{Re}_{x} 0.8\left[1-\left(x_{0} / x\right)^{9 / 10}\right]^{-1 / 9}, \text { (constant heat rate) }
\end{aligned}
$$

16.4. Fully Developed Flow in Smooth Tube Annuli.

16.4.1. Laminar flow (Source: Ref. 21):

$$
\begin{aligned}
& \frac{h_{i}\left(d_{0}-d_{i}\right)}{k}=\frac{N u_{11}}{1-\left(q_{0} / q_{1}\right) \theta_{i}^{+}} \\
& \frac{h_{0}\left(d_{0}-d_{1}\right)}{k}=\frac{N u_{\infty}}{1-\left(q_{1} / q_{0}\right) \theta_{0}^{*}}
\end{aligned}
$$

(Subscripts 1 and $o$ refer to inner and outer surfaces, $q$ is surface heat flux, $\mathrm{Nu}_{\text {if }}$ and $\mathrm{Nu}_{\infty}$ are imner and outer surface Nusselt numbers when only one surface is heated and $\theta$ is an influence coefficient given in Table 16.1.) 
TABLE 16.1. Tube-annulus solutions for constant heat rate in fully developed laminar flow and temperature profiles.

\begin{tabular}{lcccc}
\hline$r_{i} r_{0}$ & $N_{i i}$ & $N_{00}$ & $\theta_{i}^{*}$ & $\theta_{0}^{*}$ \\
\hline 0 & $\infty$ & 4.364 & $\infty$ & 0 \\
0.2 & 8.499 & 4.883 & 0.905 & 0.1041 \\
0.4 & 6.583 & 4.979 & 0.603 & 0.1823 \\
0.6 & 5.912 & 5.099 & 0.473 & 0.2455 \\
0.8 & 5.580 & 5.240 & 0.401 & 0.299 \\
1.0 & 5.385 & 5.385 & 0.346 & 0.346 \\
\hline
\end{tabular}

16.4.2. Turbulent flow (Source: Ref. 22):

$$
\frac{h}{k}\left(d_{0}-d_{i}\right)-0.023 \operatorname{Re}_{\Delta d}^{0.8} \operatorname{Pr}^{0.4}\left(d_{0} / d_{i}\right)^{0.45}, \operatorname{Re} d_{\Delta d} 10^{4}, \Delta d=d_{0}-d_{i}
$$

16.5. Forced Flow Normal to Circular Cylinders.

16.5.1. Local Coefficients (Source: Ref. 22):

$$
\begin{gathered}
\frac{\text { hd }}{\mathrm{k}}=1.14 \mathrm{Pr}^{0.4} \operatorname{Re}_{\mathrm{d}}^{0.5}\left[1-(\theta / 90)^{3}\right], 0<\theta<80^{\circ} \\
\theta=\text { cylinder angle Erom stagnation point }
\end{gathered}
$$

16.5.2. Average Coefficients (Source: Ref. 7):

$$
\frac{\text { hd }}{k}=0.43+C \operatorname{Re}_{d}^{\mathrm{m}} \operatorname{Pr}^{0.31}
$$

TABLE 16.2.

\begin{tabular}{ccc}
\hline $\operatorname{Re}_{\mathrm{d}}$ & $\mathrm{C}$ & $\mathrm{m}$ \\
\hline $1-4,000$ & 0.533 & 0.500 \\
$4,000-40,000$ & 0.193 & 0.618 \\
$40,000-400,000$ & 0.0265 & 0.805 \\
\hline
\end{tabular}


16.6. Forced Flow Normal to Spheres.

16.6.1. Average coefficients (Source: Ref. 7):

$$
\begin{aligned}
& \frac{h d}{k}=0.37 \operatorname{Re}_{d}^{0.6} \operatorname{Pr}^{0.33}, 20<\operatorname{Re}_{d}<150,000 \\
& \frac{h d}{k}=2+0.37 \operatorname{Re}_{d}^{0.6} \operatorname{Pr}^{0.33}, \operatorname{Re}_{d}<20
\end{aligned}
$$

16.7. Free Convection on Vertical Plates and Cylinders.

16.7.1. Local Coefficients (Source: Ref. 7):

$$
\begin{aligned}
& \frac{\mathrm{hx}}{\mathrm{k}}=0.508(\mathrm{Pr})^{1 / 2}(0.952+\mathrm{Pr})^{-1 / 4}\left(\mathrm{Gr}_{\mathrm{x}}\right)^{1 / 4}, \mathrm{GrPr}<10^{4} \\
& \frac{\mathrm{hx}}{\mathrm{k}}=0.0295(\mathrm{Pr})^{7 / 5}\left[1+0.494(\mathrm{Pr})^{2 / 3}\right]^{-2 / 5}(\mathrm{Gr})^{2 / 5}, \mathrm{GrPr}>10^{4}
\end{aligned}
$$

16.8. Free Convection on Horizontal Cylinders.

16.8.1. Average Coefflcients (Source: Ref. 23):

$$
\frac{h d}{k}=c\left(G r_{d} P r\right)^{m}
$$

TABLE 16.3 .

\begin{tabular}{lll}
\hline \multicolumn{1}{c}{$\mathrm{Gr} \mathrm{d}^{\mathrm{Pr}}$} & $\mathrm{c}$ & $\mathrm{m}$ \\
\hline $0-10^{-5}$ & 0.40 & 0 \\
$10^{-5}-10^{-1}$ & 0.97 & $1 / 16$ \\
$10^{-1}-10^{-4}$ & 1.14 & $1 / 7$ \\
$10^{4}-10^{9}$ & 0.53 & $1 / 4$ \\
$10^{9}-10^{12}$ & 0.13 & $1 / 3$ \\
\hline
\end{tabular}


16.9. Free Convection From Horizontal Square Plates.

16.9.1. Average Coefficients (Source: Ref. 23):

$$
\frac{h L}{k}=c\left(G r_{L} P r\right)^{m}, L=\text { plate dimension }
$$

TABLE 16.4.

\begin{tabular}{lccc}
\hline \multicolumn{1}{c}{ Condition } & Gr Lr $_{\text {L }}$ & C & m \\
\hline $\begin{array}{l}\text { Upper surface heated or } \\
\text { lower surface cooled } \\
\text { Lower surface heated or } \\
\text { upper surface cooled }\end{array}$ & $10^{5}-2 \times 10^{7}$ & 0.54 & $1 / 4$ \\
$\begin{array}{l}\text { Upper surface heated or } \\
\text { lower surface cooled }\end{array}$ & $3 \times 10^{5}-3 \times 10^{10}$ & 0.27 & $1 / 4$ \\
\hline
\end{tabular}

16.10. Free Convection from Spheres.

16.10.1. Average Coefficienta (Source: (Ref. 5):

$$
\frac{h d}{k}=2+0.43\left(\mathrm{Gr}_{\mathrm{d}} \mathrm{Pr}\right)^{1 / 4}, 10^{\circ}<\mathrm{Gr}_{\mathrm{d}} \mathrm{Pr}<10^{5}
$$

16.11. Free Convection in Enclosed Spaces.

16.11.1. Enclosed Vertical Air Spaces (Source: Ref. 1):

$$
\frac{k_{e}}{k}= \begin{cases}1 & G r_{s}<2,000 \\ 0.18\left(G_{g}\right)^{1 / 4}(s / L) & 1 / 9,2,000<G r_{s}<20,000 \\ 0.065\left(G r_{g}\right)^{1 / 3}(s / L)^{1 / 9}, 20,000<G r_{s}<10^{7}\end{cases}
$$


$k_{e}=$ effective thermal conductivity

$s=$ width of air space

$\mathrm{L}=$ length of air space

16.11.2. Enclosed Horizontal Air Spaces (Source: Ref. 1):

$\frac{k_{e}}{k}=\left\{\begin{array}{l}0.195\left(\mathrm{Gr}_{\mathrm{s}}\right)^{1 / 4}, 10^{4}<\mathrm{Gr}<4 \times 10^{5} \\ 0.068\left(\mathrm{Gr}_{\mathrm{s}}\right)^{1 / 3}, 4 \times 10^{5}<\mathrm{Gr} s\end{array}\right.$

16.12. Film Condensation.

16.12.1. Vertical Plates-Average Coefficients (Source: Ref. 5):

$\frac{h}{k_{\ell}}\left[\frac{\mu_{\ell}}{\rho_{\ell}\left(\rho_{\ell}-\rho_{v}\right) g}\right]^{1 / 3}=1.47 \mathrm{Re}_{\ell}{ }^{-1 / 3}, \mathrm{Re}_{\ell}<1800$

$\frac{h}{k_{\ell}}\left[\frac{\mu{ }_{\ell}}{\rho_{\ell}\left(\rho_{\ell}-\rho_{v}\right) g}\right]^{1 / 3}=0.007 \operatorname{Re}_{\ell}{ }^{0.4}, R_{\ell}>1800$

$\operatorname{Re}_{\ell}=\left(4 h \Delta t_{v w}\right) / i_{\ell v} \mu_{\ell}$

$\Delta t_{v w}=$ temp difference between saturated vapor and wall

$i_{\ell v}=$ heat of evaporation

$\mu_{\ell}, k_{\ell}, \rho_{\ell}=v i s c o s i t y$, thermal conductivity and density of Ilquid at saturation vapor temp

16.12.2. Horizontal Tubes--Average Coefficiente (Source: Ref. 5):

$\frac{h d}{k_{\ell}}=0.725\left[\frac{\rho_{\ell}\left(\rho_{\ell}-\rho_{l}^{v}\right) g i_{\ell v} d^{3}}{k_{\ell} \mu_{\ell} \Delta t}\right]^{1 / 4}$ 
16.13. Pool Boiling,

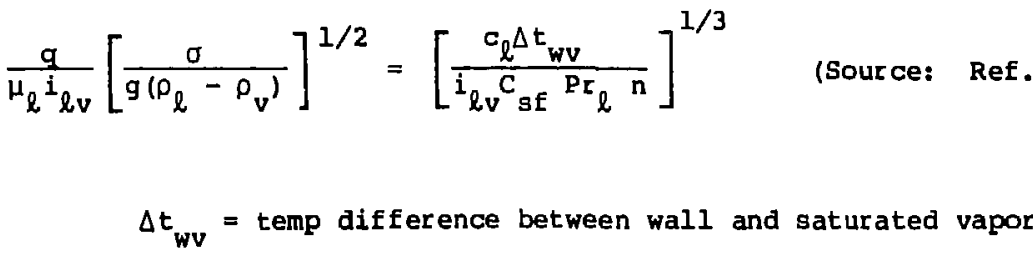

$$
\begin{aligned}
& c_{\ell}, \mu_{\ell}, \rho_{\ell} \cdot \mathrm{Pr}_{\ell}=\text { speciflc heat, viscosity, density and Prandtl } \\
& \text { number at saturated liquid temperature } \\
& \sigma=\text { surface tension at liquid vapor interface } \\
& 1_{\ell v}=\text { heat of evaporation } \\
& c_{\text {sf }}=\text { surface coefficient (see Table 16.5) }
\end{aligned}
$$

\begin{tabular}{|c|c|c|}
\hline Surface-fluid combination & $\mathrm{c}_{\mathrm{sf}}$ & $\mathbf{n}$ \\
\hline Water-nickel & 0.006 & 1.0 \\
\hline Water-platinum & 0.013 & 1.0 \\
\hline Hater-copper & 0.013 & 1.0 \\
\hline Water-brass & 0.006 & 1.0 \\
\hline $\mathrm{CCl}_{4}$-copper & 0.013 & 1.7 \\
\hline Benzene-chromium & 0.101 & 1.7 \\
\hline n--Pentane-chromium & 0.015 & 1.7 \\
\hline Ethyl alcohol-chromium & 0.0027 & 1.7 \\
\hline Isopropyl alcohol-copper & 0.0025 & 1.7 \\
\hline $35 \% \mathrm{~K}_{2} \mathrm{CO}_{3}-$ copper & 0.0054 & 1.7 \\
\hline $508 \mathrm{~K}_{2} \mathrm{CO}_{3}-$ copper & 0.0027 & 1.7 \\
\hline n-Butyl a'cohol-copper & 0.0030 & 1.7 \\
\hline
\end{tabular}

TABLE 16.5. Values of $\mathrm{C}_{\mathrm{sf}}$ and $\mathrm{n}$ (source: Ref. 19). 


\section{SECTION 17. CONTACT COEFFICIENTS}

An empirical correlation developed by Shevts and Dyban (Ref. 24) gives estimated thermal contact coefficients for many conmon ferrous and non-ferrous metals in contact, including dissimilar metals.

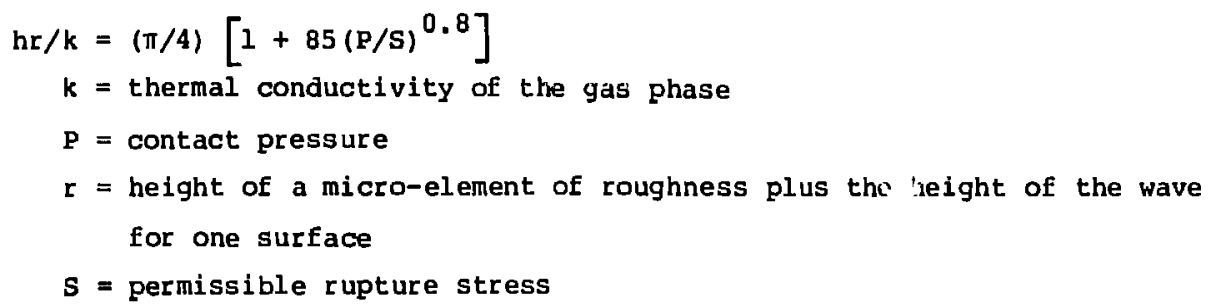

A theoretical approximation of contact coefficients developed by French and Rohsenow (Ref. 25) can be found by using Fig. 17.1 and the following properties:

$$
\begin{aligned}
& C=\text { constriction number, } P / M \\
& P=\text { contact pressure } \\
& M=\text { Meyer hardness of the softer contact material } \\
& B=\text { gap number }=0.335 \mathrm{c}^{\mathrm{m}} \\
& \mathbf{m}=0.315(\sqrt{\mathrm{A} / \mathrm{J}}) 0.137 \\
& A=\text { interface area (one side) } \\
& l=\text { effective gap thickness, } 3.56\left(l_{1}+l_{2}\right) \text { if }\left(l_{1}+l_{2}\right)<280 \\
& \text { Hin. (smooth contacts), or } 0.46\left(l_{1}+l_{2}\right)>280 \mu \text { in. (rough } \\
& \text { contacts) } \\
& \ell_{1}, l_{2}=\text { mean (or rms) depths of surface roughness } \\
& \mathbf{k}_{f}=\text { equivalent conductivity of interstitial fluid, for liquids use } \\
& k_{f}=k_{0} \text { evaluated at } t=\left(t_{1}+t_{2}\right) / 2 \text {, for gases: } \\
& k_{f}=\frac{k_{0}}{1+8 \gamma(v / \bar{v})\left(a_{1}+a_{2}-a_{1}-a_{2}\right) / P r(\gamma+1) \ell a_{1}+a_{2}} \\
& +\frac{4 \sigma l \varepsilon_{1} \varepsilon_{2} t^{3}}{\varepsilon_{1}+\varepsilon_{2}-\varepsilon_{1} \varepsilon_{2}}
\end{aligned}
$$




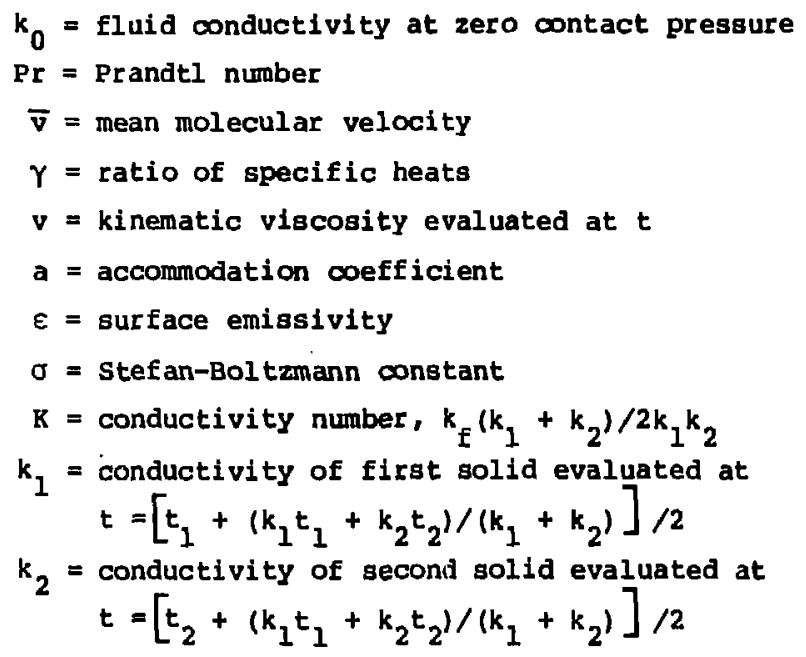

Same thermal contact data assembled by P. J. Schneider from various sources (Ref. 19) is given in FIg. 17.2. Also, some additional data from Ref. 26 showing the effects of machining processes and surface matching is given in Fig. 17.3. 
TABLE 17.1. Interface conditions for contact data given in Fig. 17.2.

\begin{tabular}{|c|c|c|c|c|}
\hline Curve & Material pair & $\begin{array}{l}\text { RMS surface } \\
\text { finish ( } \mu \text { in.) }\end{array}$ & Gap material & $\begin{array}{l}\text { Mean contact } \\
\text { temp }\left({ }^{\circ} \mathrm{F}\right)\end{array}$ \\
\hline 1 & aleminum (2024-T3) & $48-65$ & vacuum $\left(10^{-4} \mathrm{~mm} \mathrm{Hg}\right)$ & 110 \\
\hline 2 & aluminum (2024-T3) & $8-18$ & vacuum $\left(10^{-4} \mathrm{~mm} \mathrm{Hg}\right)$ & 110 \\
\hline 3 & aluminum (2024-T3 & $6-0$ (not flat) & vacuum $\left(10^{-4} \operatorname{mon} \mathrm{Hg}\right)$ & 110 \\
\hline 4 & aluminum (75S-T6) & 120 & air & 200 \\
\hline 5 & aluminum (75s-r6) & 65 & air & 200 \\
\hline 6 & aluminum (75s-T6) & 10 & air & 200 \\
\hline 7 & alumi num $(2024-\mathrm{T} 3)$ & $6-8$ (not flat) & lead foil $(0.048$ in.) & 110 \\
\hline 8 & aluminum (75s-T6) & 120 & brass foil $(0.001$ in.) & 200 \\
\hline 9 & stainless (304) & $42-60$ & vacuum $\left(10^{-4}\right.$ mun $\left.\mathrm{Hg}\right)$ & 85 \\
\hline 10 & stainless (304) & $10-15$ & vacuum $\left(10^{-4} \mathrm{~mm} \mathrm{Hg}\right)$ & 85 \\
\hline 11 & stainless (416) & 100 & air & 200 \\
\hline 12 & stainless (4.16) & 100 & brass foil $(0.001$ in.) & 200 \\
\hline 13 & magnesium (Az-31B) & $50-60$ (oxidized) & vacuum $\left(10^{-4} \mathrm{mrn} \mathrm{Hg}\right)$ & 85 \\
\hline 14 & magnesium (Az-31B) & 8-16 (oxidized) & vacuum $\left(10^{-4} \operatorname{mon} \mathrm{Hg}\right)$ & 85 \\
\hline 15 & copper (OFAC) & $7-9$ & vacuum $\left(10^{-4} \mathrm{~mm} \mathrm{Hg}\right)$ & 115 \\
\hline 16 & stainless/aluminum & $30-65$ & air & 200 \\
\hline 17 & i ron/aluminum & - & air & 80 \\
\hline 18 & tungsten/graphite & - & air & 270 \\
\hline
\end{tabular}


TABLE 17.2. Interface conditions for contact data given in Fig. 17.3.

\begin{tabular}{|c|c|c|c|c|c|c|}
\hline \multirow[b]{2}{*}{ Curve } & \multirow[b]{2}{*}{ Material } & \multirow[b]{2}{*}{ Finish } & $\begin{array}{l}\text { Roughness } \\
\frac{\text { RMS ( } \mu \text { in.) }}{\text { Block }}\end{array}$ & \multirow{2}{*}{$\begin{array}{l}\text { Fluid } \\
\text { in } \\
\text { Gap }\end{array}$} & \multirow{2}{*}{$\begin{array}{l}\text { Temp } \\
\left({ }^{\circ} \mathrm{F}\right)\end{array}$} & \multirow[b]{2}{*}{ Condition } \\
\hline & & & 1 & & & \\
\hline $\mathbf{a}$ & Cold rolled steel & Shaped & $1000-1000$ & Air & 200 & Parallel cuts, rusted \\
\hline b & Cold rolled steel & Shaped & $1000-1000$ & Air & 200 & Parallel cuts, clean \\
\hline$c$ & Cold rolled steel & shaped & $1000-1000$ & Air & 200 & Perpendicular cuts, clean \\
\hline d & Cold rolled steel & Milled & $125-125$ & Air & 200 & Parallel cuts, rusted \\
\hline e & Cold rolled steel & Milled & $125-125$ & Aìr & 200 & Parallel cuts, clean \\
\hline $\mathbf{E}$ & Cold rolled steel & Shaped & $63-63$ & Air & 200 & Perpendicular cuts, clean \\
\hline $\mathbf{g}$ & Cold rolled steel & shaped & $63-63$ & Air & 200 & Parallel cuts, clean \\
\hline $\mathbf{h}$ & Cold rolled steel & Lapped & $4-4$ & Air & 200 & clean \\
\hline$i$ & 416 Stainless & Ground & $100-100$ & Air & 200 & \\
\hline$j$ & 416 Stainless & Ground & $100-100$ & Air & 400 & \\
\hline $\mathbf{k}$ & 416 stæinless & Ground & $30-30$ & Air & 200 & \\
\hline$I$ & 416 Stainless & Ground & $30-30$ & Air & 400 & \\
\hline $\mathbf{m}$ & Stainless $^{6}$ & Milled & $195-195$ & Air & & Clean \\
\hline
\end{tabular}




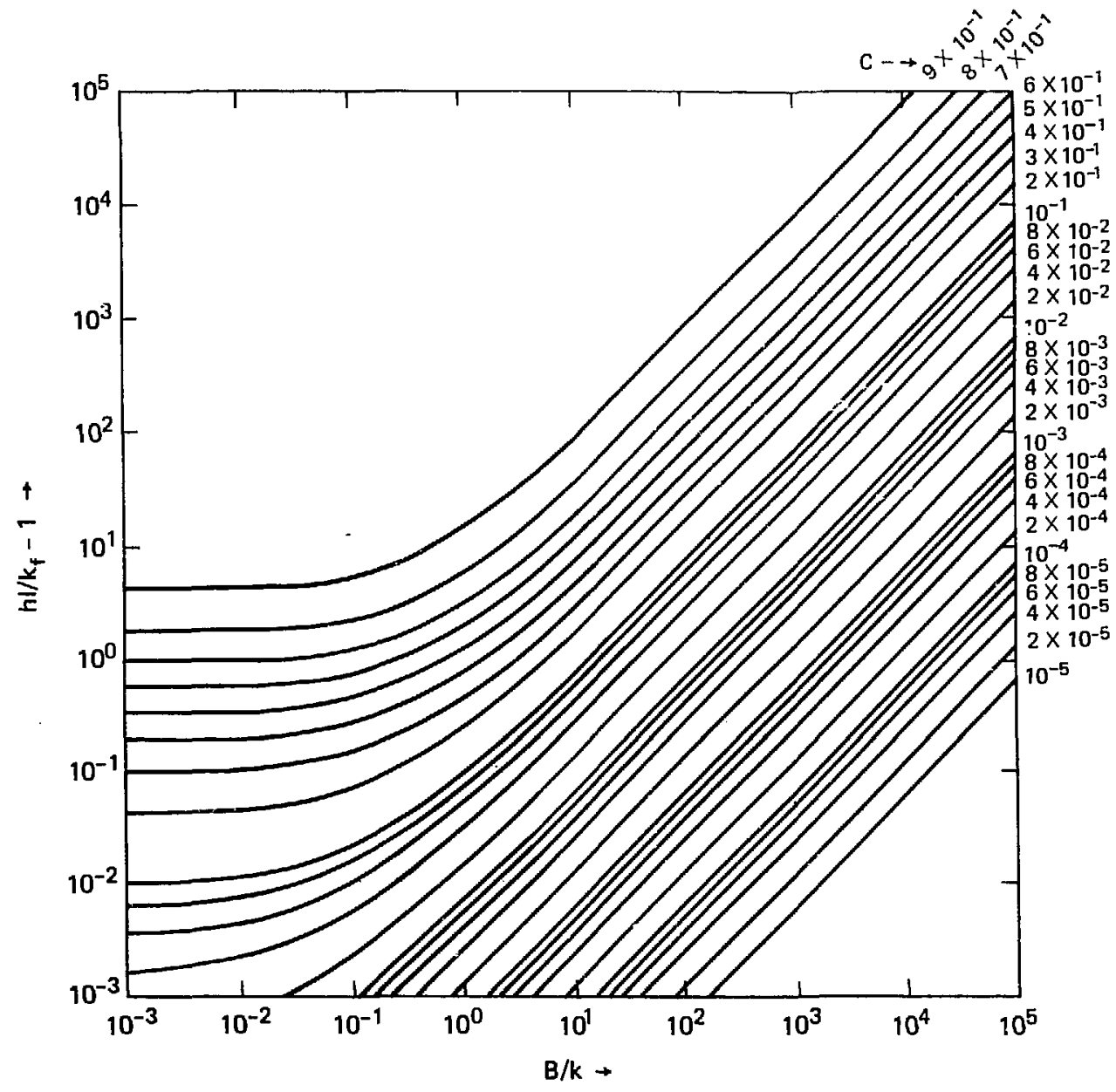

FIG. 17.1. Thermal contact coefficient from theory (source: Ref. 25). 


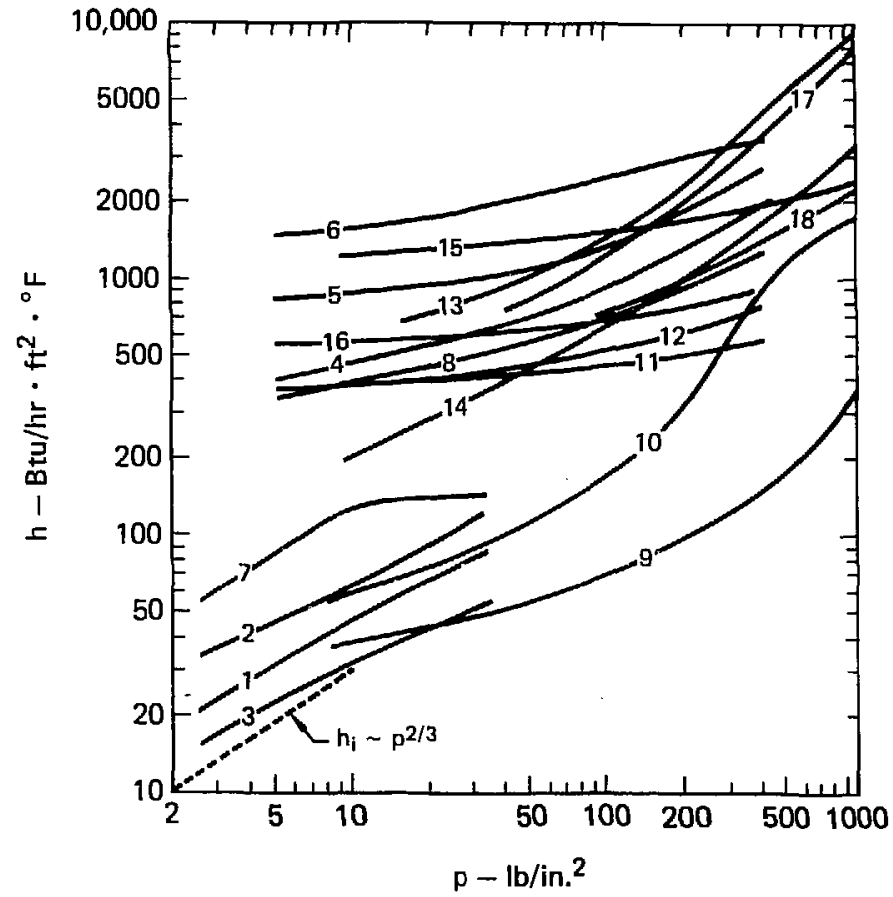

FIG. 17.2. Thermal contact coefficient data (source: Ref. 19). 


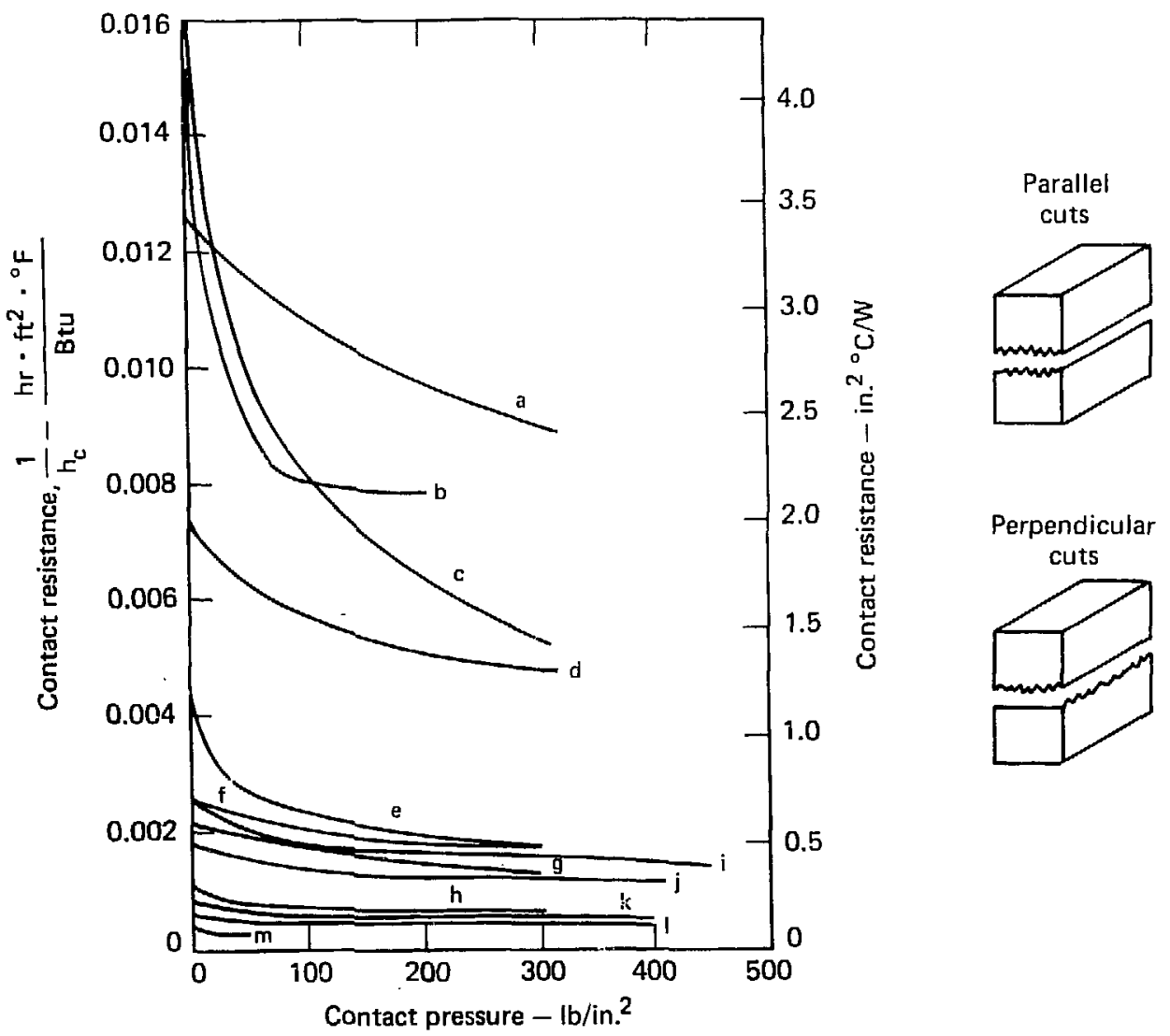

FIG. 17.3. Thermal contact coefficient for bare steel surfaces, (source: Ref. 26, section G502.5, p. 5). 
Table 18.1 Thermal properties of selected metals (source: Refs. 35 to 39 ).

\begin{tabular}{|c|c|c|c|c|c|c|c|c|c|c|c|c|c|c|}
\hline \multirow[b]{2}{*}{ Metal } & \multicolumn{4}{|c|}{ Properties at $300 \mathrm{~K}$} & \multicolumn{10}{|c|}{ Thermal conductivity $(k),(w / m \cdot x) \times 10^{-2}$} \\
\hline & $\left(\mathrm{kg} / \mathrm{m}^{3}\right) \times 10^{-3}$ & $\frac{c_{1}}{(\mathrm{~J} / \mathrm{kg} \cdot \mathrm{K}) \times 10^{-3}}$ & $(k / m-k)^{k} \times 10^{-2}$ & $\left\{\mathrm{~m}^{2} / \mathrm{s}\right)^{\alpha,} \times 10^{4}$ & $10 x$ & 20 & 50 & 100 & 200 & 400 & 600 & 800 & 1000 & 1200 \\
\hline Aluminum: Pure & 2,70 & 0.88 & 2.4 & 1.01 & 235. & 117. & 12.3 & 3.0 & 2.4 & 2.4 & 2.3 & 2.2 & & \\
\hline $2024(4.5 x \mathrm{Cu}, 1.5 x \mathrm{Mg})$ & 2.77 & 0.84 & 1.3 & 0.56 & 0.08 & 0.17 & $0.40^{\circ}$ & 0.65 & 1.0 & 1.4 & & & & \\
\hline 5086 (4.1x Mg, 0.5\% Mn\}) & 2.65 & 0.90 & 1.3 & 0.55 & 0.08 & 0.17 & 0.40 & 0.63 & 0.85 & & & & & \\
\hline $6063(0.7 x \mathrm{Mg}, 0.4 x \mathrm{Si})$ & 2.70 & 0.92 & 2.1 & 0.85 & 0.86 & 1.7 & 2.8 & 2.1 & 2.0 & & & & & \\
\hline Beryllium: Pure & 1.85 & 1.84 & 2.0 & 0.59 & $18.0^{\prime}$ & 34.8 & 40.0 & 9.9 & 3.01 & 7.61 & 1.26 & 1.07 & 0.89 & 0.73 \\
\hline Chromium: Pure & $\therefore 19$ & 4.56 & 0.90 & 0.03 & 3.9 & 6.0 & 3.2 & 1.6 & 1.1 & 0.87 & 0.81 & 0.71 & 0.65 & 0.62 \\
\hline Copper: Pure & 8.96 & 0.38 & 4.0 & 1.17 & 196. & 105. & 12.2 & 4.8 & 4.1 & 3.9 & 3.8 & 3.7 & 3.6 & 3.4 \\
\hline Comnercial bronze $(10 \times 2 \pi)$ & B.80 & 0.41 & 1.8 & 0.50 & 0.20 & 0.42 & 0.81 & 1.2 & & & & & & \\
\hline Yellow brass $(35 x 2 n)$ & 8.47 & 0.41 & 1.2 & 0.35 & & & & 0.59 & 0.94 & 1.3 & 1.5 & & & \\
\hline Naval brass $(39 x \mathrm{Zn}, 0.8 x \mathrm{Sn})$ & 8.41 & 0.38 & 0.75 & 0.23 & & & & & & 0.85 & 1.01 & & & \\
\hline German silver (22x Іл, $15 \%$ Mi) & B.55 & 0.40 & 0.25 & 0.07 & 0.028 & 0.07 & 0.15 & 0.17 & 0.20 & & & & & \\
\hline Cupronicke1 (20X Ni, 2X $2 \pi$ ) & 8.94 & 0.38 & 1.5 & 0.44 & & 0.11 & 0.20 & 0.38 & 0.92 & & & & & \\
\hline Constantan (40K $\mathrm{Ni})$ & 8.90 & 0.42 & 0.23 & 0.05 & & 0.08 & 0.15 & 0,17 & 0.19 & & & & & \\
\hline Manganin (13\% $\mathrm{Mn}, 4 \times \mathrm{Mi})$ & 8.19 & 0.40 & 0.22 & 0.07 & 0.015 & 0.032 & 0.082 & 0.13 & 0.17 & 0.28 & & & & \\
\hline Gold: Pure & 19.32 & 0.13 & 3.2 & 1.27 & 28.2 & 15.0 & 4.2 & 3.5 & 3.3 & 3.1 & 3.0 & 2.9 & 2.8 & 2.6 \\
\hline Iron: Pure & 7.87 & 0.45 & 0.80 & 0.23 & 7.1 & 10.0 & 3.7 & 1.3 & 0.94 & 0.69 & 0.55 & 0.43 & 0.33 & 0.28 \\
\hline Wraught iran $\langle 0.5 \% \mathrm{C}\}$ & 7.87 & 0.46 & 0.60 & 0.17 & & & & & & 0.58 & 0.49 & 0.39 & & \\
\hline Gray cast iron $(3.0 \times C, 0.6751)$ & 7.15 & 0.42 & 0.29 & 0.10 & & & & & & 0.31 & 0.34 & 0.29 & 0.22 & 0.20 \\
\hline SAE l0S5 stee? $(0.9 x \mathrm{C}, 0.3 \times$ 而 $)$ & 7.83 & 0.47 & 0.44 & 0.12 & & 0.08 & 0.22 & 0.34 & 0.43 & & & & & \\
\hline AISI 4340 steel $(1.9 \% \mathrm{Ni}, 0.7 \% \mathrm{Cr})$ & 7.84 & 0.46 & 0.36 & 0.10 & & & & 0.26 & 0.31 & 0.38 & 0.38 & 0.34 & & \\
\hline Nickel steel (9x $N 1,0.8 \times$ m) & 7,96 & 0.46 & 0.29 & 0.08 & & & & 0.18 & 0.24 & 0.33 & 0.36 & 0.33 & & \\
\hline Invar $(31 \% \mathrm{Ht}, 5 \mathrm{CO})$ & 8.00 & 0.46 & 0.14 & & & & & 0.08 & 0.12 & 0.16 & 0.19 & 0.22 & & \\
\hline SAE 4130 steel $(1 \times \mathrm{Cr}, 0.56 \mathrm{Mn}$ ) & 3.86 & 0.46 & 0.41 & 0.11 & & 0.06 & 0.16 & 0.17 & 0.37 & & & & & \\
\hline AISI 304 stainless $(19 x \mathrm{Cr}, 10 x \mathrm{Ni})$ & 7.90 & 0.38 & 0.15 & 0.05 & 0.008 & 0.021 & 0.06 & 0.09 & 0.13 & 0.17 & 0.20 & 0.22 & 0.25 & 0.28 \\
\hline AISI 316 stainless $(17 x \mathrm{Cr}, 12 x \mathrm{Ni})$ & 8.00 & 0.46 & 0.14 & 0.40 & 0.008 & 0.021 & 0.06 & 0.09 & 0.12 & 0.15 & 0.18 & 0.21 & 0.23 & 0.25 \\
\hline Lead: Pure & 11.34 & 0.13 & 0.35 & 0.24 & 1.78 & 0.59 & 0.44 & 0.40 & 0.37 & 0.34 & 0.31 & & & \\
\hline Solder $(80 \% \mathrm{~Pb}, 20 \% \mathrm{Sn})$ & 10.20 & & 0.37 & & & & & & & & & & & \\
\hline Solder (50x $\mathrm{Pb}, 50 x \mathrm{Sn})$ & 8.89 & 0.18 & 0.46 & 0.29 & & & & & & & & & & \\
\hline Lithiun: Pure & 0.53 & 3.97 & 0.77 & 0.37 & 6.1 & 7.2 & 2.3 & 1.1 & 0.88 & 0.72 & & & & \\
\hline Magnesium: Pure & 1.74 & 1.00 & 1.56 & 0.90 & 11.7 & 13.9 & 3.8 & 1.7 & 1.6 & 1.5 & 1.5 & 1.5 & & \\
\hline$(6 \times A 1,2 \times 5 i)$ & 1.84 & 1.00 & 0.70 & 0.38 & & & & 0.50 & 0.62 & 9.80 & & & & \\
\hline (12* A1, 2* S1) & 1.81 & 0.99 & 0.56 & 0.31 & & & & 0.30 & 0.44 & 0.68 & & & & \\
\hline Molybednum: Pure & 10.22 & 0.25 & 1.4 & 0.55 & 1.45 & 2.77 & 3.0 & 1.8 & 1.4 & 1 & 1.3 & 1.2 & 1.1 & 1.0 \\
\hline
\end{tabular}


Table 18.1 (Continued).

\begin{tabular}{|c|c|c|c|c|c|c|c|c|c|c|c|c|c|c|}
\hline \multirow[b]{2}{*}{ Meta 1} & \multicolumn{4}{|c|}{ Properties at $300 \mathrm{~K}$} & \multicolumn{10}{|c|}{ Thermal conductivity $(k),(w / m \cdot k) \times 10^{-2}$} \\
\hline & $\left(\mathrm{kg} / \mathrm{m}^{3}\right) \times 10^{\rho}$ & $\frac{\mathrm{C}}{(\mathrm{J} / \mathrm{kg}-\mathrm{K}) \times 10^{-3}}$ & $\frac{k \cdot}{(w / m-k) \times 10^{-2}}$ & $\left(\mathrm{~m}^{2} / 5\right) \times 10^{4}$ & $10 \mathrm{k}$ & 20 & 50 & 100 & 200 & 400 & 600 & 800 & 1000 & 1200 \\
\hline Nícke1: Pure & 8.90 & 0.45 & 0.91 & 0.23 & 6.0 & B.6 & 3.4 & 1.6 & 1.1 & 0.80 & 0.66 & 0.67 & $0.7 ?$ & 0.76 \\
\hline Duranickel (4.5\% Al) & 8.26 & 0.54 & 0.19 & 0.04 & & & & & & & & & & \\
\hline Monel (30\% $\mathrm{Cu}, 1.4 \% \mathrm{Fe})$ & 8.84 & 0.43 & 0.23 & 0.05 & 0.08 & 0.14 & 0.17 & 0.20 & & & & & & \\
\hline Inconel $x-750(16 \% \mathrm{Cr}, 8 \% \mathrm{Fe})$ & 8.51 & 0.46 & 0.12 & 0.03 & 0.010 & 0.025 & 0.065 & 0.085 & 0.10 & 0.13 & 0.17 & 0.21 & 0.25 & 0.29 \\
\hline Nichrame $(20 \% \mathrm{Cr})$ & 8.40 & 0.45 & 0.13 & 0.03 & & & & & & 0.14 & 0.17 & 0.21 & 0.25 & 0.28 \\
\hline Nichrome $V(24 x \mathrm{Fe}, 16 x \mathrm{Cr})$ & 8.25 & 0.44 & 0.10 & 0.03 & & & & & & 0.12 & 0.15 & 0.19 & 0.22 & 0.26 \\
\hline Niobium: Pure & 8.57 & 0.27 & 0.54 & 0.23 & 2.2 & 2.3 & 0.76 & 0.55 & 0.53 & 0.55 & 0.58 & 0.61 & 0.64 & 0.68 \\
\hline Platinum: Pure & 21.45 & 0.13 & 0.71 & 0.25 & 12.3 & 4.9 & 1.1 & 0.78 & 0.72 & 0.72 & 0.73 & 0.76 & 0.79 & 0.83 \\
\hline Plutanium: Pure & 19.84 & 0.15 & 0.67 & 0.23 & & & & 0.03 & 0.48 & 0.90 & & & & \\
\hline Rhenium: Pure & 21.04 & 0.14 & 0.48 & 0.16 & 14.0 & 8.4 & 0.96 & 0.60 & 0.51 & 0.46 & 0.44 & 0.44 & 0.45 & 0.46 \\
\hline Silvar: Pure & 10.49 & 0.24 & 4.3 & 1.71 & 168. & 51. & 7.0 & 4.5 & 4.3 & 4.2 & 4.1 & 3.9 & 3.7 & 3.6 \\
\hline Sterling $(7.5 \% \mathrm{Cu})$ & 10.38 & & 3.5 & & & & & & & & & & & \\
\hline Eutectic $\{28 x \mathrm{Cu}\rangle$ & 10.66 & & $\cdot 3.3$ & & & & & & & & & & & \\
\hline Tin: Pure & 7.30 & 0.22 & 0.67 & 0.42 & 19.3 & 3.2 & 1.2 & 0.85 & 0.73 & 0.62 & & & & \\
\hline Solder $\{40 \times \mathrm{Pb}\}$ & 8.44 & 0.19 & 0.51 & $0.3 ?$ & 0.43 & 0.56 & 0.52 & 0.54 & 0.52 & & & & & \\
\hline Tantalum: Pure & 16.6 & 0.14 & 0.58 & 0.25 & 1.08 & 1.5 & 0.72 & 0.59 & 0.58 & 0.58 & 0.59 & 0.59 & 0.50 & 0.61 \\
\hline Tungsten: Pure & 19.3 & 0.13 & 1.8 & 0.72 & 82. & 32. & 4.7 & 2.4 & 2.0 & 1.6 & $T .4$ & 1.3 & 1.2 & 1.1 \\
\hline Titanium: Pure & 4.51 & 0.50 & 0.22 & 0.10 & 0.14 & 0.28 & 0.40 & 0.31 & 0.25 & 0.20 & 0.19 & 0.20 & 0.21 & $0.2 ?$ \\
\hline A- 110 AT $(5 \times$ A1, $2.5 \times \mathrm{Sn})$ & 4.46 & 0.53 & 0.075 & 0.03 & & & & & & 0.088 & 0.11 & 0.14 & & \\
\hline Uranium: Pure & 19.07 & 0.12 & 0.28 & 0.12 & 0.098 & 0.16 & 0.19 & 0.22 & 0.25 & 0.30 & 0.34 & 0.39 & 0.44 & 0.49 \\
\hline Zine: Pure & 7.13 & 0.39 & 1.2 & 0.43 & 43. & 10.7 & 2.1 & 1.2 & 1.2 & 1.2 & 1.1 & & & \\
\hline
\end{tabular}




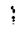

Tagte 18.2. Theraul properties of miscellaneous aollds (sources Refs. 35 to 38).

\begin{tabular}{|c|c|c|c|c|c|c|c|c|c|c|c|c|c|c|}
\hline \multirow[b]{2}{*}{ Mater 1al } & \multicolumn{4}{|c|}{ Propertiea at $300 \Omega$} & \multicolumn{10}{|c|}{ Thermal conduget $1 v 1$ ty $(k),(w / m-\pi) \times 10$} \\
\hline & $\left(\mathrm{kg} / \mathrm{m}^{\mathbf{3}^{3}}\right)^{p_{1}} \times 10^{-3}$ & {$[3 / \mathrm{kg}-\pi] \times 10^{-3}$} & $\begin{array}{c}k, \\
w / m \cdot \mathbf{x}\end{array}$ & $\frac{a,}{\left(m^{2} / 8\right) \times 10^{4}}$ & $10 \mathrm{~K}$ & 20 & so & 100 & 200 & 400 & 600 & 800 & 1000 & 1200 \\
\hline \multicolumn{15}{|l|}{$\begin{array}{l}\text { Car anics: (Polycryatalline, } 99.51 \\
\text { pur } 1 \text { ty, } 98 \text { oolld) }\end{array}$} \\
\hline$\underset{\mathrm{B}=\mathrm{O}}{\mathrm{N}_{2} \mathrm{O}_{3}}$ & $\begin{array}{l}3.84 \\
2.97\end{array}$ & $\begin{array}{l}0.79 \\
1.00\end{array}$ & $\begin{array}{r}36.0 \\
272.0\end{array}$ & $\begin{array}{l}0.12 \\
0.92\end{array}$ & & . & & 1330. & $\begin{array}{l}550 . \\
1240 .\end{array}$ & $\begin{array}{l}260 . \\
1960 .\end{array}$ & $\begin{array}{l}160 . \\
1110 .\end{array}$ & $\begin{array}{l}100 . \\
700 .\end{array}$ & $\begin{array}{l}80 . \\
470 .\end{array}$ & \\
\hline HgO & 3.21 & 0.92 & 49.0 & 0.16 & & & & & 750. & 350. & 220. & 140. & 90. & \\
\hline S10 2 (high purity fused] & 2.21 & 0,75 & 1.4 & 0.008 & 1.3 & 1.5 & 3.4 & 6.9 & 11. & 15. & 18. & 22. & 29. & \\
\hline $\mathrm{THO}_{2}$ & 9.58 & 0,23 & 13.0 & 0.06 & & & & & 180. & 100. & 70. & 50. & 40. & \\
\hline $\mathrm{THO}_{2}$ & 3.91 & 0.71 & 9.0 & 0.03 & & & & & 100. & 70. & 50. & so. & 30. & \\
\hline $\mathrm{zro}_{2}$ & 5.28 & 0.46 & 1.6 & 0.007 & & & & & & 17. & 18. & 19. & 19. & \\
\hline \multicolumn{15}{|l|}{ Claseea: } \\
\hline Borondlicate (Pyrex) & 2.21 & 0.72 & 2.1 & 0.007 & & & . & 6. & 9. & 22. & 25. & 29. & & \\
\hline Soda $1100\left(75151 \mathrm{~S}_{2}\right)$ & 2.52 & 0.65 & 0.9 & 0.005 & & & & & a. & 11. & & & & \\
\hline Vitreous aillea $\left\{100 \mathrm{~s} \mathrm{SO}_{2}\right\}$ & 2.21 & 1.00 & 1.4 & 0.006 & & & & в. & 11. & 15. & & & & \\
\hline 21 e crom $\left.(65) 510_{2}\right)$ & 2.60 & 0.67 & 1.1 & 0.006 & & & & & 9. & 13. & & & & \\
\hline \multicolumn{15}{|l|}{ Ingulationg; (For high tempa.) } \\
\hline Aluinh, fused $\left(901 \mathrm{Al}_{2} \mathrm{O}_{3}\right)$ & 2.70 & 0.83 & 5.2 & 0.02 & . & & & & & 56. & 60. & 66. & 23. & \\
\hline Aabastas paper (lamil nated) & 0.35 & 0.84 & 0.048 & 8.802 & & & & & & 0.73 & & & & \\
\hline $\begin{array}{l}\text { Diatoseceous earth } \\
\text { gilica (powder) }\end{array}$ & 0.30 & & 0.054 & & & & & & & 0.79 & 1.0 & 1.3 & & \\
\hline Pirabrick (581 $\mathrm{SIO}_{2}$ & 0.82 & 0.92 & 0.34 & 0.005 & & & & & & & 5.2 & 6.6 & 8.4 & 10. \\
\hline Magnesite $(850 \mathrm{MgO})$ & 0.19 & 1.13 & 0.050 & 0.002 & & & & & & 0.61 & & & & \\
\hline Mlaro quarta fiber (blankat) & 0.05 & 0.84 & 0.036 & 0.009 & & & & & & 0.48 & 0.89 & 1.3 & 1.7 & \\
\hline Rockwool (loose) & 0.16 & & 0.046 & & & & & & & 0.66 & 1.0 & 1.3 & & \\
\hline ziroonia (grain) & 1.81 & 0.50 & 0.18 & 0.002 & & & & & & 1.9 & 2.1 & 2.2 & 2.8 & 3.5 \\
\hline \multicolumn{15}{|l|}{ Ingulations: (Por $10 \mathrm{~m}$ tempse) } \\
\hline Expanded glass (Pannglas) & 0.17 & & 0.062 & & & & & 0.31 & 0.51 & & & & & \\
\hline Qiberglass (board) & 0.18 & & 0.040 & & & & & 0.12 & 0.24 & & & & & \\
\hline Flberglass [blanket] & 0.05 & & 0.038 & & & & & & & & & & & \\
\hline sillos atrogel (powden) & $0.0 \mathrm{~B}$ & & 0.022 & & & & & & 0.17 & & & & & \\
\hline pol ystyrene foan (1atmaba) & 0.08 & . & 0.038 & & & 0.05 & 0.07 & 0.12 & 0.24 & & & & & \\
\hline Polystyrene foam $\left\{10^{-3}\right.$ atrabo & 0.08 & & 0.017 & & & & & & 0.12 & & & & & \\
\hline
\end{tabular}


TABLE IB.2. \{Continued.।

\begin{tabular}{|c|c|c|c|c|c|c|c|c|c|c|c|c|c|c|}
\hline \multirow[b]{2}{*}{ Mater ial } & \multicolumn{4}{|c|}{ Properties at $300 \mathrm{~K}$} & \multicolumn{10}{|c|}{ Therael conduceivity $(k),(v / m x) \times 10^{-2}$} \\
\hline & $\left(\mathrm{kg} / \mathrm{m}^{3}\right)^{\mathrm{p}} \times 10^{-3}$ & $\begin{array}{c}c, \\
(\mathrm{~J} / \mathrm{kg} \cdot \pi) \times 10^{-3}\end{array}$ & $\begin{array}{c}k, \\
\omega / m=k\end{array}$ & $\stackrel{\alpha_{r}}{\left(\pi^{2} / 8\right) \times 10^{4}}$ & $20 x$ & 20 & 50 & 100 & 200 & 400 & 600 & 800 & 1000 & 1200 \\
\hline \multicolumn{15}{|l|}{ Plastica: } \\
\hline Aerylic, Prat (Plexiglas) & $1.1 \mathrm{a}$ & 1.46 & 0.26 & 0.001 & 0.6 & 0.7 & & & 1.2 & & & & & \\
\hline Mylan 6 & 1.26 & 1.59 & 0.25 & 0.001 & 8.3 & 0.9 & & & & & & & & \\
\hline Polyvinyl chlaride (EIgld) & 1.40 & 1.00 & 0.15 & 0.001 & & & & 1.5 & 1.6 & 1.5 & & & & \\
\hline Teflon, PTFE & 2.18 & 1.05 & 0.40 & 0.002 & 1.0 & 1.5 & 2.0 & 2.5 & & 4.5 & 5.5 & & & \\
\hline Polyetrylene, high density & 0.95 & 2.30 & 0.50 & 0.002 & & & & & & & & & & \\
\hline \multicolumn{15}{|l|}{ Rocks: } \\
\hline Granite & 2.60 & 0.79 & 3.4 & 0.017 & & & & & & 30. & $2 n$. & & & \\
\hline Marble & 2.50 & 0.88 & 1.8 & 0.008 & & & & & & 17. & 11. & & & \\
\hline Sendstone & 2.20 & 0.92 & 5.3 & 0.026 & & & & & & 44. & 30. & & & \\
\hline strale & 2.60 & 0.71 & 1.8 & 0.010 & & & & & & 15. & 14. & & & \\
\hline \multicolumn{15}{|l|}{ woods: (across graln, oven dry) } \\
\hline Bassa & 0.16 & & 0.059 & & & & & & & & & & & \\
\hline Douglas fir & 0.46 & 2.72 & 0.11 & 0.001 & & & & & & & & & & \\
\hline Oak, red & 0.67 & 2.38 & 0.17 & 0.001 & & & & & & & & & & \\
\hline Pine, uhite & 0.10 & 2.60 & 0.10 & 0.001 & $\cdot$ & & & & & & & & & \\
\hline Redwood & 0.42 & 2.90 & 0.11 & 0.001 & & & & & & & & & & \\
\hline \multicolumn{15}{|l|}{ Miscellareous: } \\
\hline Carbon black (powdor) & 0.19 & 0.84 & 0.021 & 0.001 & & & & & & & & & & \\
\hline \multicolumn{15}{|l|}{ Graphl te (nTJ) } \\
\hline (1) to graing) & 1.73 & 0.84 & 129.0 & 0.89 & 5. & 25. & 170. & s8o. & 1200. & 1180. & 950. & 770. & 640. & \\
\hline (1 to grains) & 1.73 & 0.84 & 90.0 & 0.67 & 4. & 19. & 130. & 420. & 860. & 900. & 730. & 590. & 490. & \\
\hline \multicolumn{15}{|l|}{ Graphite (pyrolytia) } \\
\hline (I to graira) & 3.20 & 0.84 & 2000.0 & 10.8 & B10. & 4200 & 23000 & 50000 & 32000. & 14600. & 9300. & 6800 & 5300 & \\
\hline (l to graina) & 2.20 & 0.84 & 9.0 & 0.05 & 270. & 1100. & 1000 & 390. & 150. & 70. & 40. & 30. & 20. & \\
\hline conerete & 2.28 & 0.67 & 3.8 & 0.012 & & & & & & & & & & \\
\hline Hica & 1.95 & 0.88 & 0.43 & 0.002 & & & & & & & & & & \\
\hline Rubber (hard) & 1.19 & 1.88 & 0.16 & 0.001 & & & & & & & & & & \\
\hline Gypace board & 0.82 & & 0.11 & & & & $\cdot$ & & & & & & & \\
\hline Sand $(\mathrm{dr} y)$ & 1.52 & 0.80 & 0.33 & 0.003 & & & & & & & & & & \\
\hline
\end{tabular}


TABLE 18.3. Thermal properties of some metals at $10 \mathrm{~K}$ above their melting point (source: Refs. 35 and 38 ).

\begin{tabular}{|c|c|c|c|c|c|}
\hline Metal & $\begin{array}{c}\mathrm{mp}, \\
\mathrm{k}\end{array}$ & $\begin{array}{c}k, \\
W / m \cdot k\end{array}$ & $\begin{array}{c}\rho_{1} \\
\mathrm{~kg} / \mathrm{m}^{3}\end{array}$ & $\begin{array}{c}c, \\
\mathrm{~kJ} / \mathrm{kg} \cdot \mathrm{k}\end{array}$ & $\begin{array}{c}\gamma \\
\mathrm{kJ} / \mathrm{kg}\end{array}$ \\
\hline Aluminum & 933.2 & 91 & 2390 & 1.09 & 395.4 \\
\hline Copper & 1356 & 167 & 7940 & 0.49 & 205. \\
\hline Iron & 1810. & 41 & 7020 & 0.82 & 281.6 \\
\hline Lead & 600.6 & 16 & 10700 & 0.21 & 24.7 \\
\hline Iithium & 453.7 & 43 & 520 & 4.25 & 663.2 \\
\hline Mer cury & 234.3 & 7 & 13650 & 1.34 & 11.3 \\
\hline Potassium & 336.8 & 54 & 820 & 0.84 & 61.5 \\
\hline Sodium & 371.0 & 87 & 930 & 1.34 & 114.6 \\
\hline NaK (eutectic) & 262. & 13 & 850 & 1.00 & -- \\
\hline Tin & 505.1 & 31 & 6980 & 0.23 & 60.2 \\
\hline zine & 692.7 & 50 & 6640 & 0.50 & 102.1 \\
\hline
\end{tabular}


TABLE 18.4. Room temperature total emissivities (source: kef. 37).

\begin{tabular}{llll}
\hline Silver (highly polished) & 0.02 & Brass (polished) & 0.60 \\
Platinum (highly polished) & 0.05 & Oxidized copper & 0.60 \\
ainc (highly polished) & 0.05 & Oxidized steel & 0.70 \\
Aluminum (highly polished) & 0.08 & Bronze paint & 0.80 \\
Monel metal (polished) & 0.09 & Black gloss paint & 0.90 \\
Nickel (polisied) & 0.12 & White lacguer & 0.95 \\
Copper (poldued) & 0.15 & White vitreous enamel & 0.95 \\
Stellite (polished) & 0.18 & Asbestos paper & 0.95 \\
Cast iron (polisheri) & 0.25 & Green paint & 0.95 \\
Monel motal (oxidized) & 0.43 & Gray paint & 0.95 \\
Aluminum paint & 0.55 & Lamp black & 0.95 \\
\hline
\end{tabular}


TABLE 18.5. Total emissivities of miscellaneous materials (source: Ref. 37).

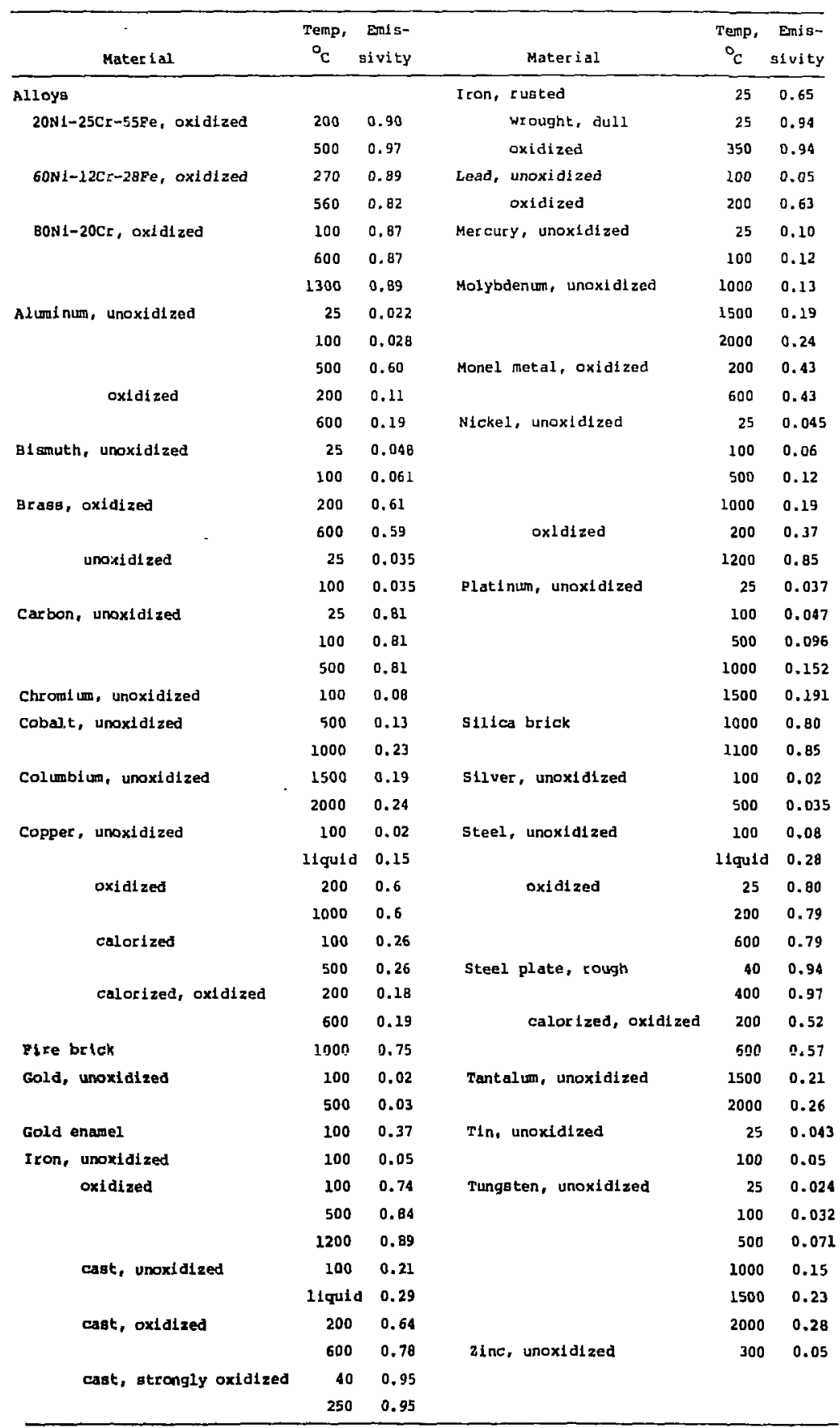


TABLE 18.6. Electrical resigtivity of scme common metals (source: Ref. 37 ).

\begin{tabular}{|c|c|c|c|c|}
\hline Metal & $\begin{array}{c}\text { Resistivity, } \\
\mu \Omega^{\circ} \mathrm{cm} \\
20^{\circ} \mathrm{C}\end{array}$ & $\begin{array}{l}\text { Temp. } \\
\text { coefficient } \\
20^{\circ} \mathrm{C}\end{array}$ & $\begin{array}{l}\text { specific } \\
\text { gravity, } \\
g / \mathrm{cm}^{3}\end{array}$ & $\begin{array}{l}\text { Melting } \\
\text { point, } \\
{ }^{\circ}\end{array}$ \\
\hline \multicolumn{5}{|l|}{ Advance. See constantan } \\
\hline Aluminum & 2.824 & 0.0039 & 2.70 & 659 \\
\hline Antimony & 4.1.7 & .0036 & 6.6 & 630 \\
\hline Arsenic & 33.3 & .0042 & 5.73 & -- \\
\hline B ismuth & 120 & .004 & 9.8 & 271 \\
\hline Brass & 7 & .002 & 8.6 & 900 \\
\hline Cadmlun & 7.6 & .0038 & 8.6 & 321 \\
\hline \multicolumn{5}{|l|}{ Calido. See nichrome } \\
\hline Climax & 87 & .0007 & 0.1 & 1250 \\
\hline Cobalt & 9.8 & .0033 & 8.71 & 1480 \\
\hline Constantan & 49 & .00001 & 8.9 & 1190 \\
\hline Copper: annealed & 1.7241 & .00393 & 8.89 & 1003 \\
\hline hard-drawn & 1.771 & .00382 & 8.89 & -- \\
\hline \multicolumn{5}{|l|}{ Eureka. See constantan } \\
\hline Excello & 92 & .00016 & 8.9 & 1500 \\
\hline Gas carbon & 5000 & -.0005 & $-\infty$ & 3500 \\
\hline German silver, $18 \% \mathrm{Ni}$ & 33 & .0004 & 8.4 & 1100 \\
\hline Gold & 2.44 & .0034 & 19.3 & 1063 \\
\hline \multicolumn{5}{|l|}{ Ideal. See constantan } \\
\hline Iron, 99.988 pure & 10 & .005 & 7.8 & 1530 \\
\hline Lead & 22 & .0039 & 11.4 & 327 \\
\hline Magnesi um & 4.6 & .004 & 1.74 & 651 \\
\hline Manganin & 44 & .00001 & 8.4 & 910 \\
\hline Mercury & 95.783 & .00089 & 13.546 & -38.9 \\
\hline Molybdenum, drawn & 5.7 & .004 & 9.0 & 2500 \\
\hline Monel metal & 42 & .0020 & 8.9 & 1300 \\
\hline Nichrome $e^{a}$ & 100 & .0004 & B.2 & 1500 \\
\hline Nickel & 7.8 & .006 & 8.9 & 1452 \\
\hline Palladium & 11 & .0033 & 12.2 & 1550 \\
\hline Phosphor bronze & 7.8 & .0018 & 8.9 & 750 \\
\hline Plati num & 10 & .003 & 21.4 & 1755 \\
\hline S11ver & 1.59 & .0038 & 10.5 & 960 \\
\hline Steel, E. B. B. & 10.4 & .005 & 7.7 & 1510 \\
\hline Steel, B. B. & 11.9 & .004 & 7.7 & 1510 \\
\hline steel, siemens-Martin & 18 & .003 & 7.7 & 1510 \\
\hline Steel, manganese & 70 & .001 & 7.5 & 1260 \\
\hline Tantalum & 15.5 & .0031 & 16.6 & 2850 \\
\hline Ther $10^{a}$ & 47 & .00001 & 8.2 & -- \\
\hline Tin & 11.5 & .0042 & 7.3 & 232 \\
\hline Tungsten, drasm & 5.6 & .0045 & 19 & 3400 \\
\hline ZInc & 5.8 & .0037 & 7.1 & 419 \\
\hline
\end{tabular}

Trade mark. 
IABLE 18.7. Thermal conductivity integrols of alscellaneous materials (source: Ref. 76).

$$
Q=\frac{\pi}{L}\left(k_{I}\right), k_{I}=\int_{4 x}^{t} x d t, w / m \times 10^{-2}
$$

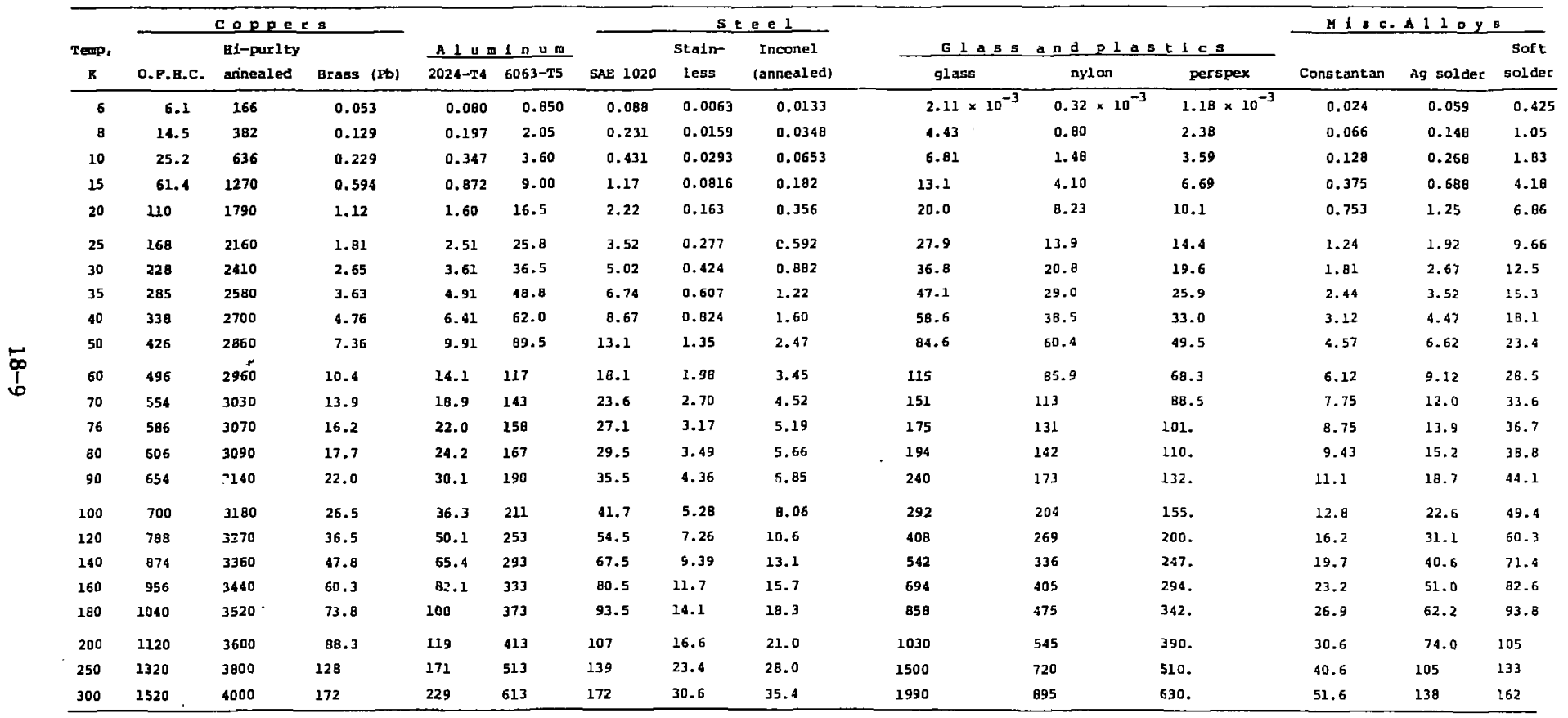


lable 18.8 Specific heat of misccllaneous solids (source: Rets. 35 to 38 ).

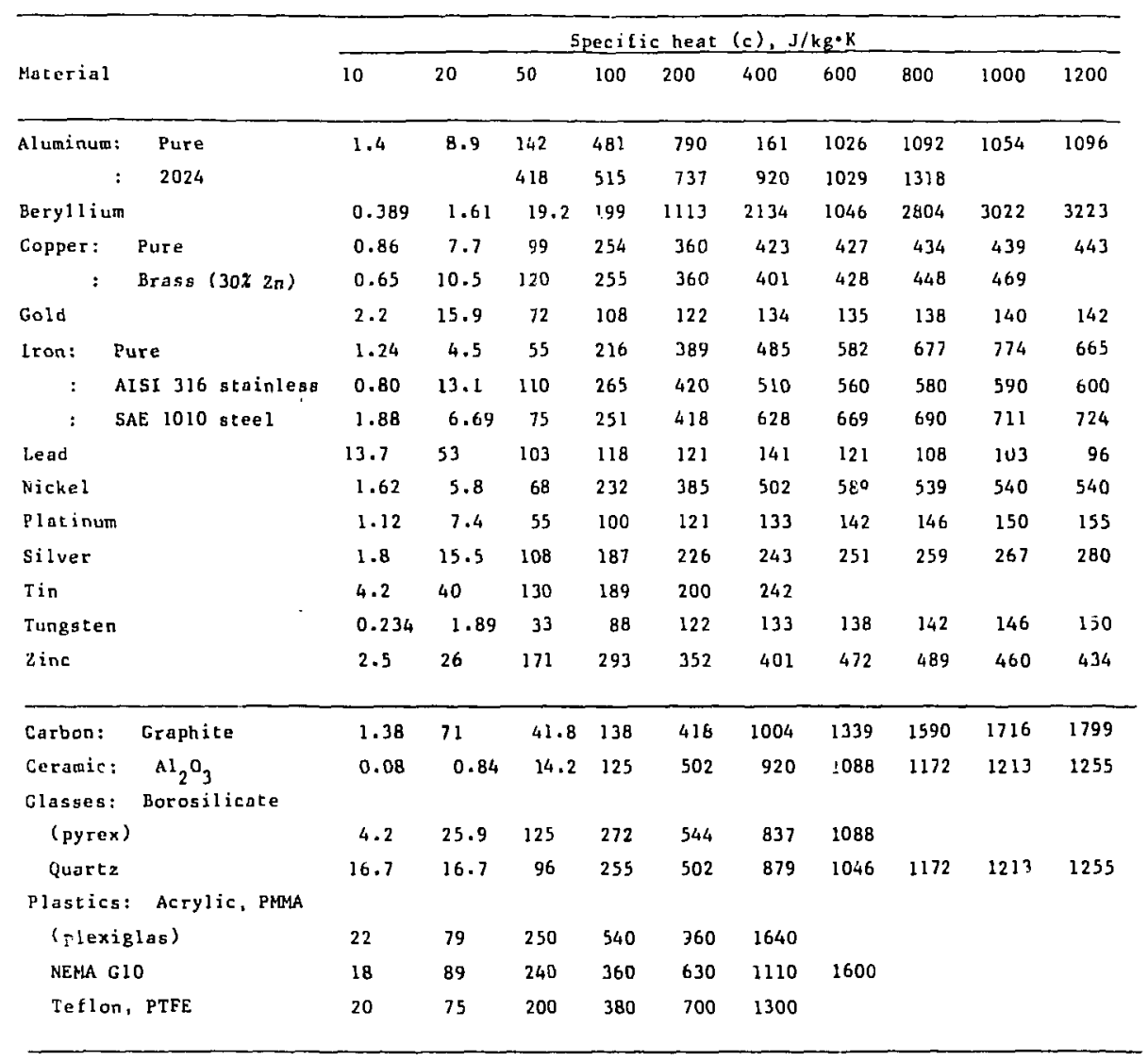


1. M. Jakob, Heat Transfer, Vol. I (Wiley, New York, 1949).

2. P. J. Schneider, Conduction Heat Transfer (Addison-Wesley, Reading, 1955 ).

3. V. S. Arpaci, Conduction Heat Transfer (Addison-Wesley, Reading, 1966).

4. S. S. Kutateladze and V. M. Borishanskii, A Concise Encyclopedia of Heat Transfer (Perganon, New York, 1966).

5. J. R. Holman, Heat Transfer (MCGraw-Hill, New York, 1963).

6. R. B. Bird, W. E. Stewart, and E. N. Lightfoot, Transport Phenomena (Wiley, New York, 1960).

7. E. R. G. Eckert and R. M. Drake Jr., Heat and Mass Transfer (McGraw-Hill, New York, 1959).

8. K. A. Gardner, "Efficiency of Extended Surfaces," Trans. ASIME 67, $621-631$ (1945).

9. H. S. Carslaw and J. C. Jaeger, Conduction of Heat In Solids, 2nd Ed. (Oxford Eress, London, 1959).

10. D. Q. Kern and A. D. Kraus, Extended Surface Heat Transfer (McGraw-Hill, New York, 1972).

11. E. M. Sparrow and S. H. Lin, Int. J. Heat Mass Transfer $7,951-952$ (1964).

12. M. P. Heisler, "Temperature Charts for Induction and Constant Temperature Heating," Trans. ASME 69, 227-236 (3.947).

13. H. Gröber, S. Erk, and U. Grigul1, Eundamentals of Heat Transfer (MoGraw-Hill, New York, 1961).

14. R. E. D. Stewart and F. C. Wessling Jr., On Heat Conduction in Solids Having Temperature Dependent Properties," Sandia Labs, Albuquerque, SC-RR-70-334 (1970).

15. J. H. Vansant and M. B. Larson, "Heat Transfer From a Sen-Infinite Rectangular Strip," J. Heat Transfer $\underline{85}, 191-192$ (1963).

16. J. H. Vansant and J. H. Pitts, "Variable Property Effects on the Inside Surface Temperature of a Heated Tube," J. Heat Transfer $\underline{88}$, 340-341 (1966).

17. B. O. Peirce and R. M. Foster, A short lable of Integrals (Ginn, Naw Yurk, 1956).

18. E. Jahnke and F. Emde, Tables of Functions, 4th Ed. (Dover, New York, 1945). 
19. W. M. Rohsenow and J. P. Hartnett, Eds., Handbook of Heat Transfer (Mograw-Hill, New York, 1973).

2. G. E. Myers, Analytical Methods in Conduction Heat Transfer (McGraw-Hill, Hew York, 1971.).

21. W. M. Kays, Convective Heat and Mass Transfer (McGraw-Hill, New York, 1966).

22. J. G. Knudsen and D. L. Katz, Fluid Dynamics and Heat Transfer (MCGraw-Hill, New York, 1958).

23. W. H. McAdams, Heat Transmission, 3rd Ed. (MCGraw-Hill, New York, 1954).

24. I. I. Shevts and E. P. Dyban, "Contact Heat Transfer Between Plane Metal Surfaces," Int. Chem. Eng. 4, 621-241 (1964).

25. H. Fenech and W. H. Rohsenow "Prediction of Thermal Conductane of Metallic Surfaces in Contact," J. Heat Transfer 85, 15-24 (1963).

26. N. D. Fitzroy, Ed., Heat Transfer Data Book (General Electric Co., Schenectady, 1970).

27. J. H. VanSant, "Temperature Variations on the Surface of a Strip-Heated Flat Plate," J. Heat Transfer 89, 372-373 (1967).

28. J. H. VanSant, Temperature of a Flat Plate Having Alternating Adiabatic and Constant Heat Flux Strips on One Side, Lawrence Livermore Laboratory, Livermore, CA, UCRL-7990 (1.964).

29. J. H. VanSant, "The Spot Insulated Piate," Nuc. Eng. Design 8, 247-250 (1968).

30. N. R. Des Ruisseau and R. D. Zerkle, "Temperature in Semi-Infinite and Cylindrical Bodies Subjected to Moving Heat Sources and Surface Cooling," J. Heat Transfer $92,456-464$ (1970).

31. K. Kotan and 0. A. Arnar, "On the Optimization of the Design Paraneters of Parabolic Radiating Fins," ASME Paper 65-HT-42 (1965).

32. M. J. Pascual, S. D. Beck, and J. E. Zweig, Temperature Field In a Slab Containing An Insulating Filament, Watervliet Arsenal, Watervliet, N.Y., HVT-6511 (1965).

33. K. O. Beatty, Jr. and A. A. Armstrong, The Measurement of Thermal Properties of Nonmecallia Materials at Elevated Temperatures, U.S. Atomic Energy Comm,, Oak Ridge, TN, ORO-170 (1956).

34. R. G. Watts, "Temperature Distributions in Solid and Hollow Cylinders Due to a Moving Circumferential Ring Heat Source," J. Heat Tra: isfer $\underline{\text { S1, }}$ 465-470 (1969). 
35. Y. S. Touloukian, et al., Thermophysical Properties of Matter, Vols. 1, 2.4\&5 (IFI/Plenum, New York, 1970).

36. ^. Parrish, Ed., Mechanical Engineer's Reference Book, llth Ed. (CRC Press, Cleveland, 1973).

37. R. C. Weast, Ed., Handbook of Chemistry and Physics, 55th Ed. (CRC Press, Cleveland, 1974).

38. T. Baurister, Ed., Standard Handbook for Mechanical Engineers, 7th Ed. (MoGraw-Hill, New York, 1967).

39. T. Lyman, Ed., Metals Handbook 8th Ed., Vol. 1, "Properties and Selection of Metals" (American Society for Metals, Metals Park, OH, 1961).

40. D. M. Tellep, Temperature Response of Thin Skins Under Low Heat Flux Conditions, Lockheed Missiles and Space Co., Sunnyvale, CA, IAD 349, (1958) .

41. M, P. Heisler, "Temperature Charts for Internal Heat Generation," Trans. ASME 78, 1187-1192 (1956).

42. W. H. Holter and J. H. G:over, "Insulation Temperature for the Transient Heating of an Insulated Infinite Metal Slab," J. ARS 30, 907-908 (1960).

43. J. H. Grover and $\mathbf{W}$. H. Holter, "Solution of the Transient Heat Conduction Equation for an Insulated Infinite Metal Slab," Jet Propulsion 27, 1249-1252 (1957).

44. R. D. Zerkle and J. E. Sunderland, "The Transient Temperature Distribution in a Slab Subject to rhermal Radiation," J. Heat Transfer 87, 117-133 (1965).

45. D. L. Ayers, "Transient Cooling of a Sphere in Space," J. Heat Transfer 92, 180-182 (1970).

46. N. Konopliv and E. M. Sparrow, "Transient Heat Conduction in Nonh mogeneous Sphezical Systems," Wärme-und Sloffübertragung 3 , 197-210 0).

47. B. B. Newman, "The Temperature-Time Relations Resulting From the Electrical Heating of the Face of a Slab," Trans. AICHE 3 , 598-613 (1934).

48. M. D. Mikheylow, "Transient Temperature Fields in Shells," NASA, Waahington D.C., TT F-552,(1969).

49. C. D. Coulbert et al., Temperature Response of Infinite Plate and Slabs to Heat Inputs of Short Duration at One Surface, Dept. Eng., Univ. Calif., Los Angeles (1951). 
50. J. A. Kuhn, Temperature Distribution in a Finite Slab Caused by a Heat Rate of the Form $q(\theta)=\left(q_{\max } / 2\right)(1-\operatorname{Cos} \omega \theta)$, Lockheed Missiles and Space Co., Sunnyvale, CA, TXN/548 (1961).

51. A. R. Mendelsohn, "Transient Temperature of a Porous-Cooled Wall," J. AIAA 1, 1449-1451 (1963).

52. H. Halle and D.E. Taylor, Transient Heating of Two-Element Slabs Exposed to a Plane Heat Source, Chicago Midway Labs, Chicago, CML-59-M-1 (1959).

53. P. J. Schneider and C.F. Wilson Jr., "Pulse Heating and Equilibration of an Insulated Substructure," Spacecraft and Rockets 7, 1457-1460 (1970).

54. H. C. Hottel and C. C. Williams III, "Transient Heat Flow in Organic Materials Exposed to High-Intensity Thermal Radiation," ASME Heat Transfer and Fluid Mechanics Institute (1954).

55. A. L. London and R. A. Seban, "Rate of Ice Formation," Trans. ASME 65, $771-778$ (1943).

56. R. R. Cullom and W. H. Robins, One-Dimensional Heat Transfer Analysis of Thermal-Energy Storage for Solar Direct-Energy-Conversion Systems, NASA, Washington D.C., TN D-2110 (1964).

57. J. E. Sunderland and R. J. Grosh, "Transient Temperatures in a Melting Solid," J. Heat Transfer 83 , 409-414 (1961).

58. H. G. Landau, "Heat Conduction in a Melting Solid," guart. Appl. Math. $\underline{8}$, $8194(1950)$.

59. J. W. Tatom, "Shell Radiation," ASME Paper 60-WA-234 (1960).

60. S. Katsoff, similitude in Thermal Models of Spacecraft, NASA, Washington D.C., TN D-1631 (1963).

61. E. L. Knuth, "An Engineering Approximation for Resistance of Certain TwoDimensional Conductors," Int. J. Heat Mass Transfer I, 270-272 (1964).

62. J. G. Bartas, "Gas side-Wall Temperature in Rib-Backed Liquid-Cooled Combustion Chambers," Jet Propulsion 27, 784-786 (1957).

63. J. E. Schmidt and G. Sonnemann, "Transient Temperatures and Thermal Stresses in Hollow Cylinders Due to Heat Generation," J. Heat Transfer 82 , 273-279 (1960).

64. E. M. Sparrow, "Temperature Distribution and Heat-Transfer Results for an Internally Cooled, Heat-Generating Solid," J. Heat Transfer 82, 389-392 (1.960). 
65. D. R. Burnett, "Transient Temperature Measurement Errors in Heated slabs for Thermocouples Located at the Insulated Surfaces," J. Heat Transfer 83, 505-506 (1961).

66. J. J. Brogan and P. J. Scheider, "Heat Conduction in a Series Composite Wall," J. Heat Transfer $83,506-507$ (1961).

67. M. R. El-Saden, "Heat Conduction in an Eccentrically Hollow Infinitely Long Cylinder with Internal Heat Generation," J. Heat Transfer 83, 510-512 (1961).

68. R. S. Fairall, R. A. Belcher, "Unsteady-State Heat Transfer in solids with Radiation at One Boundary," J. Heat Transfer 84, 266-267 (1962).

69. Y. T. Tsui and F. K. Tsou, "Ratio of Radial to Total heat Flow in a Circular Rod," J. Heat Transfer 85, 285-286 (1963).

70. G. Horvay and M. Dacosta, "Temperature Distribution in a Cylindrical Rod Moving From a Chamber at One Temperature to a Chamber at Another Temperature," J. Heat Transfer 86, 265-270 (1964).

71. F. Jackson, "Moving Heat Sources with Change of Phase," J. Heat Transfer 87, 329-339 (I965).

72. A. Hiji-Sheikh and E. M. Sparrow, "Transient Heat Conduction in a Prolate Spheroidal Solid," J. Heat Transfer $88,331-333$ (1966).

73. J. C. Rowley and J. B. Payne, "Steady State Temperature Solution for a Heat Generating Circular Cylinder Cooled by a Ring of Holes," J. Heat Transfer $86,531-536$ (1964).

74. A. V. Luikov, Analytical Heat Diffusion Theory (Academic Press, New York, $1968)$.

75. R. B. Stewart and V. J. Johnson, A Compendium of the Properties of Materials at Low Temperatures, National Bureau of Standards, Boulder, WADD Tech Report 60-56, Part IV (1961).

76. Handbook on Materials for Superconducting Machinery (Metals and Ceramics Information Center, Battelle, Columbus, Jan. 1977).

77. K. J. Mody and M. N. Ozisik, "Melting of a Semi-Infinite Solid with Non-Linear Heat Transfer at the Boundary," Letters in Heat and Mass Transfer, $\underline{2}, 487-494$ (1975).

78. B. T. F. Chung and L. T. Yeh, "Freezing and Melting of Materials with Variable Properties and Arbitrary Heat Fluxes," AIAA Journ., 14, No. 3, 388-390 (1976). 
79. E. Hahne and U. Grigull, "Formfaktor Und Form-widerstand Der Stationaren Mehrdimensionalen Warmeleitung," Int. J. Heat Mass Transfer, 18 , $751-767$ (1975).

80. A. A. Irlev, "Steady State Heat Conduction in an Eccentric Annulus," Heat Transfer-Soviet Research, 8 , no. 3, 164-171 (1976).

81. A. Muzzio, "Approximate Solution for Corrective Fins with Variable Thermal Conductivity," J. Heat Transfer, 680-682 (1976).

82. J. J. Brogan and P. J. Schneider, "Heat Conduction in a Series Composite Wall," J. Heat Transfer, 506-508, Nov. 1961.

83. C. Kippenham and D. F. Schnell, "A Note on Heat Transfer Through sheet Fins," J. Heat Transfer, 293-295 (1964).

84. C. C. Sih, "Heat Conduction in the Infinite Medium with Lines of Discontinuities," J. Heat Transfer, 293-298 (1965).

85. W. Fagan and 5 . Leipziger, "Nonuniform Cooling of a Heat-Generating Cylinder or Sphere," J. Heat Transfer, 257-264, Aug. 1966.

86. G. E. Schneider, "Thermal Resistance of a Cylinder with Two Diametrically Opposite, Symmetrical Isothermal Caps," J. Heat Transfer, 465-467, 1975.

87. E. I. Griggs, D. R. Pitts, and A. B. Goyal, "Conductive Shape Factors for a Circular Cylinder Centered in a Rectangular Slab Having one and Two Adiabatic Surfaces," J. Heat Transfer, 129-130 (1973).

88. R. A. Schmitz, "Heat Flux Through a Strip Heated Flat Plate," J. Heat Transfer, 201-202 (1970).

89. A. M. Poindexter, "Temperature Charts for Simple Shapes in a Linearly Changing Envi ronment," J. Heat Transfer, 562-564 (1970).

90. F. M. Young and C. R. Savino, "Time-Temperature Charts for One-Dimensional Conduction with Uniform Internal Heat Generation," J. Heat Transfer, 207-210 (1970).

91. P. J. Smith and J. Sucec, "Efficiency of Circular Fins of Triangular Profile," J. Heat Transfer, 181-182 (1969).

92. J. H. Van Sant, Lawrence Livermore National Laboratory, unpublished work. 